\title{
Abgehängt, chancenlos, unwillig?
}

\section{Eine empirische Reorientierung von Integrationstheorien zu MigrantInnen der zweiten Generation in Deutschland}

Dissertation zur Erlangung des Grades des Doktors / der Doktorin der Philosophie (Dr. phil.) am Fachbereich Politik- und Sozialwissenschaften

- Otto-Suhr-Institut für Politikwissenschaft der Freien Universität Berlin 


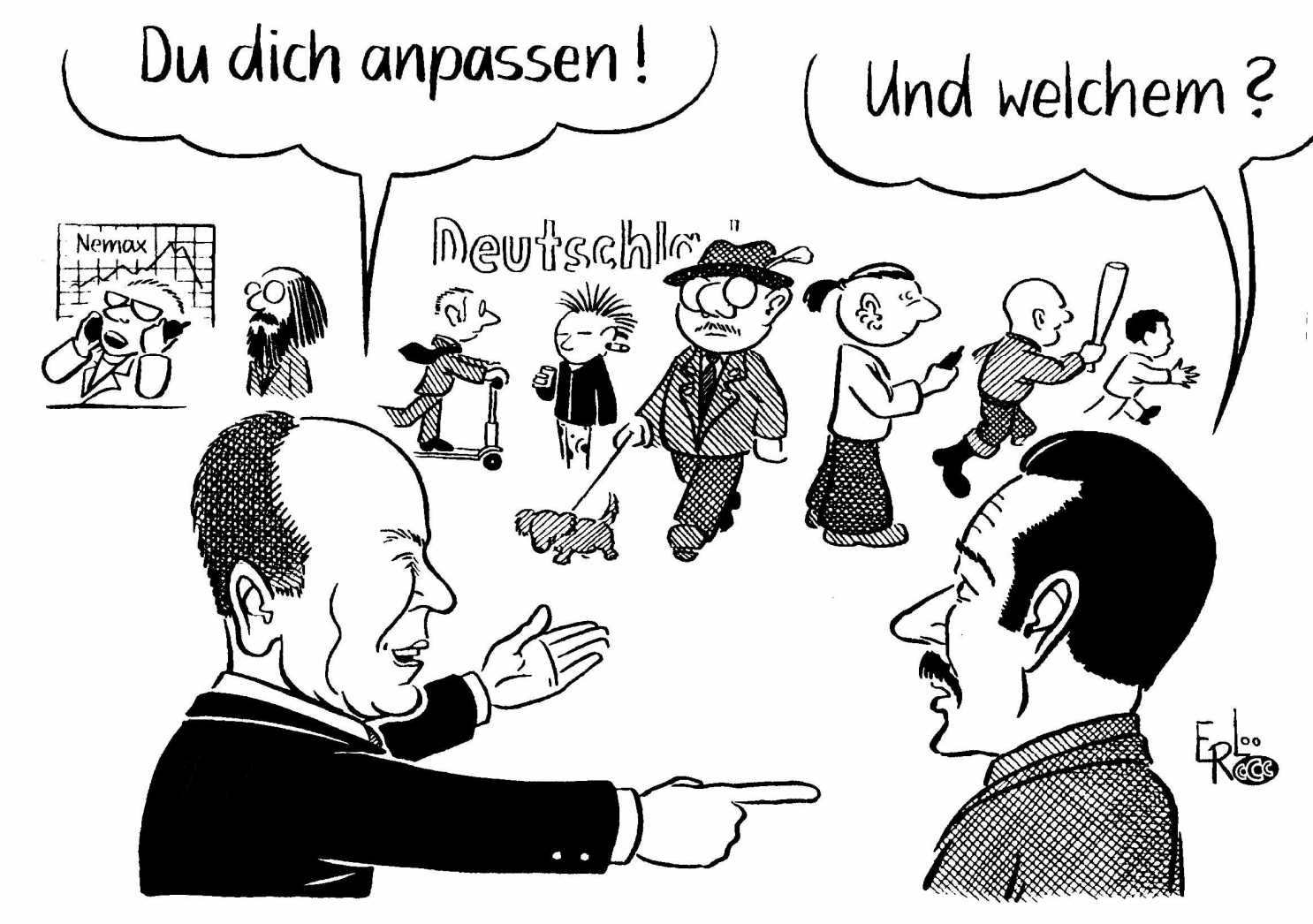

Und welchem?

Quelle: Martin Erl / CCC, www.c5.net

Erstgutachter: Prof. Hajo Funke

Zweitgutachter: Prof. Thomas Faist

Tag der Disputation: 15. Juli 2008 


\section{Inhaltsverzeichnis}

1 EINLEITUNG …........................................................................................................................ 5

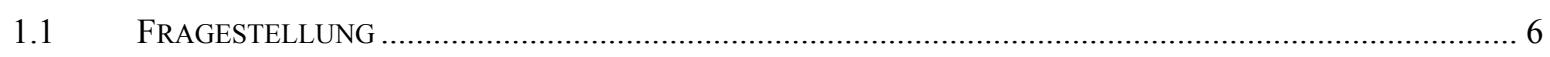

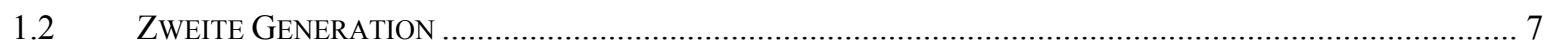

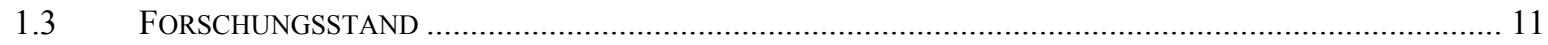

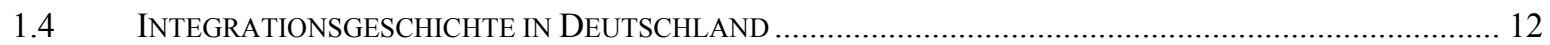

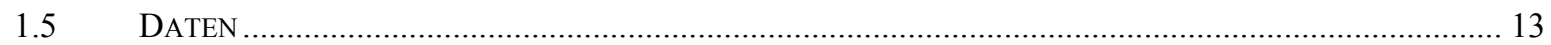

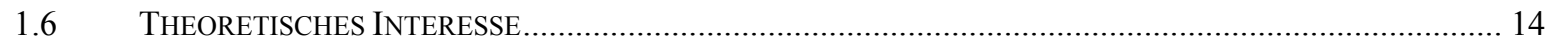

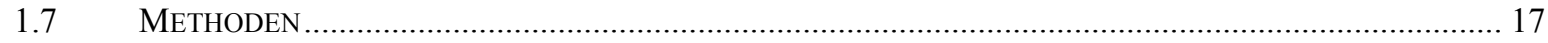

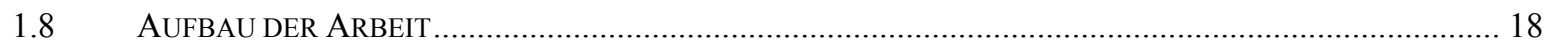

2 INTEGRATIONSTHEORIEN: EINE REORIENTIERUNG............................................................ 19

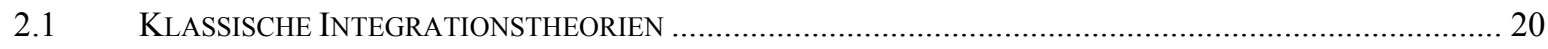

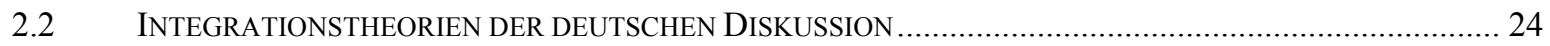

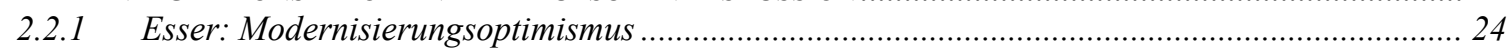

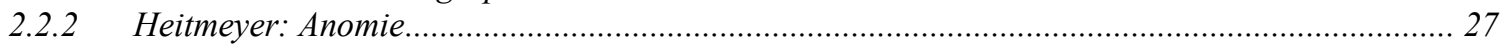

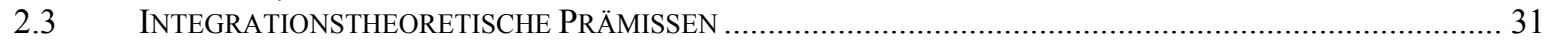

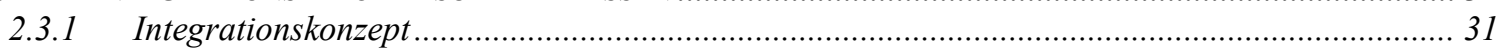

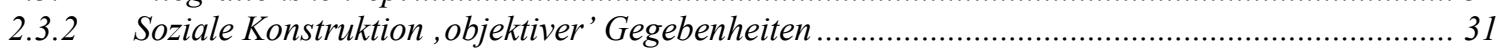

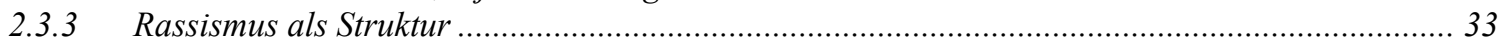

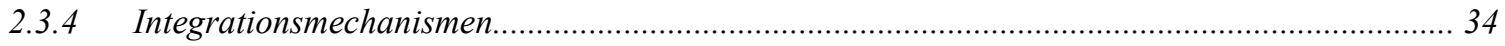

$2.4 \quad$ STRÖMUNGEN DER US-AMERIKANISCHEN INTEGRATIONSDEBATTE ……....................................... 37

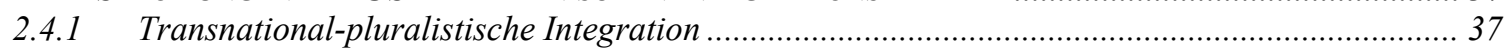

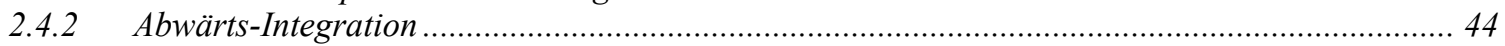

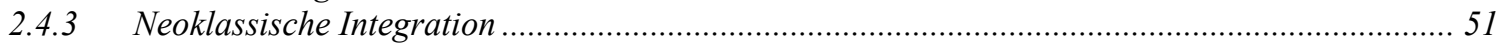

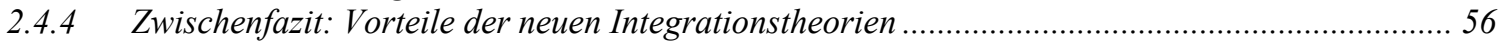

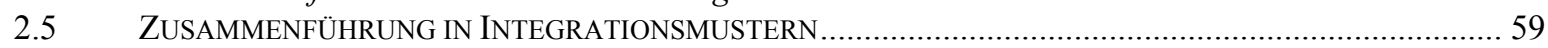

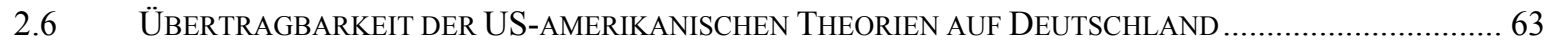

3 EMPIRIE I: INDIVIDUALEBENE DER MIGRANTINNEN - SPRACHE, BILDUNG, BERUF,

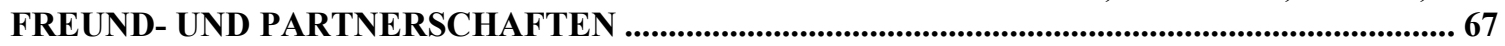

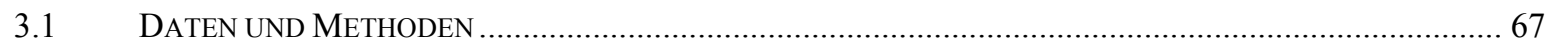

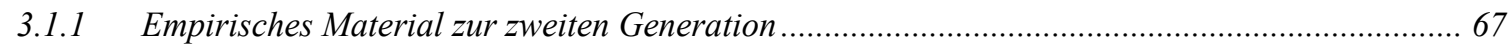

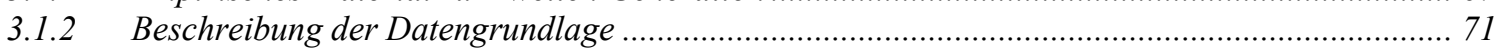

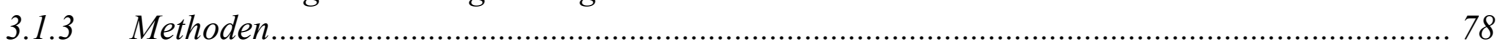

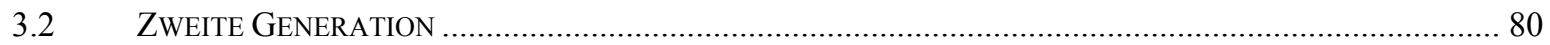

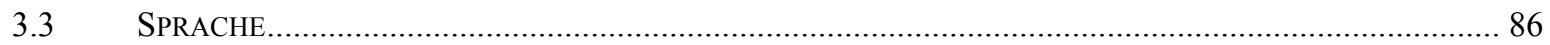

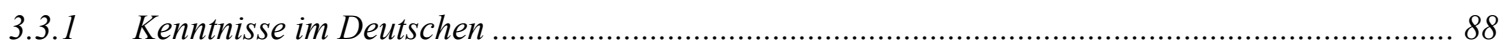

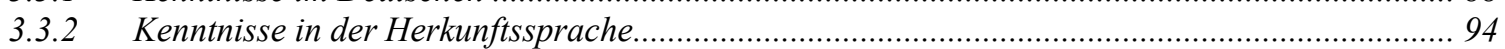

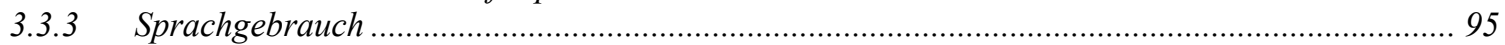

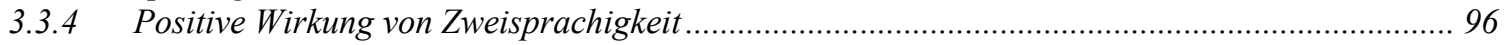

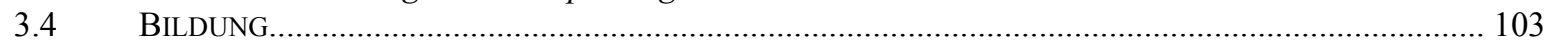

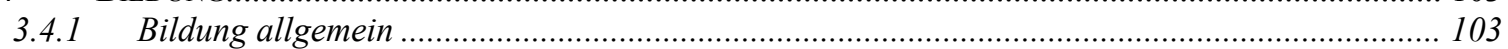

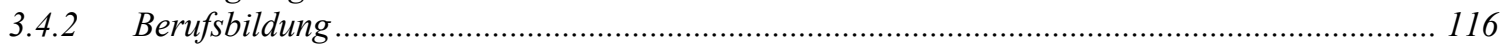

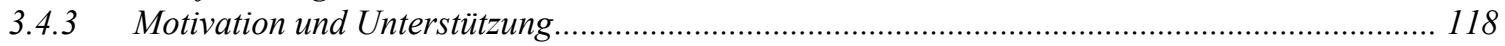

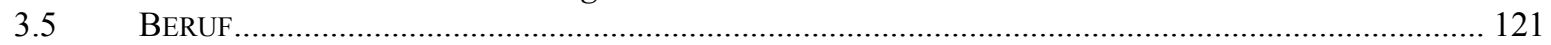

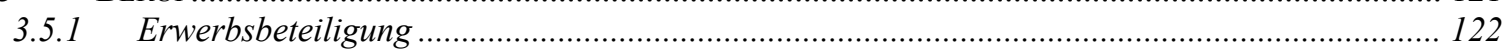

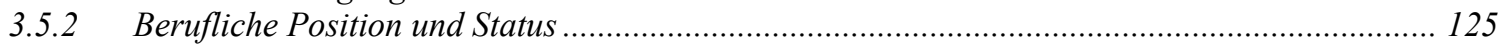

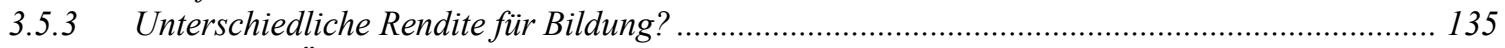

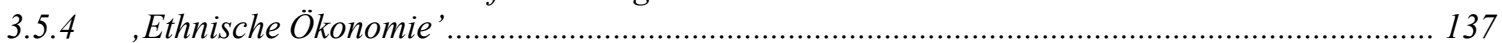

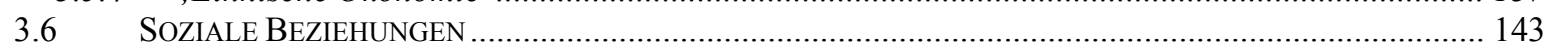

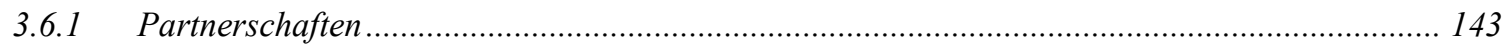

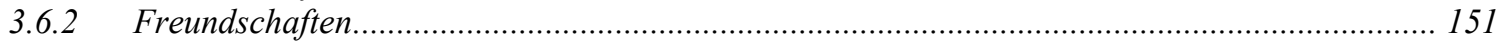

3.6.3 Positive Konsequenzen, ko-ethnischer' Freundschaften ...................................................... 156

4 EMPIRIE II: INDIVIDUALEBENE DER MIGRANTINNEN - INTEGRATIONSMUSTER ....... 160

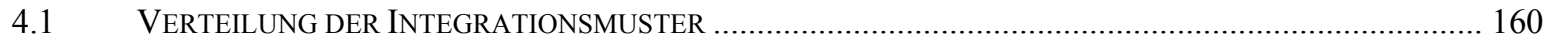




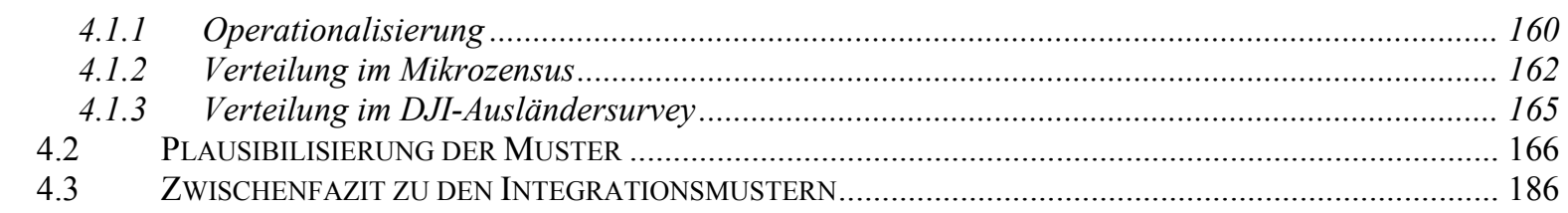

5 EMPIRIE III: DOMINANZBEVÖLKERUNG - ÄHNLICHKEITSWAHRNEHMUNG UND

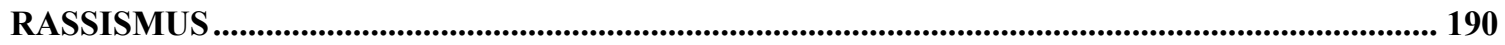

5.1 WAHRNEHMUNG VON ÄHNLICHKEIT DURCH DIE DOMINANZBEVÖLKERUNG ................................... 191

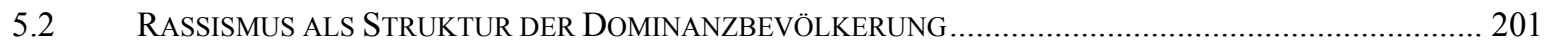

6 DISKUSSION: EMPIRISCHE BEFUNDE UND PLAUSIBILISIERUNG DER THEORIEN ....... 219

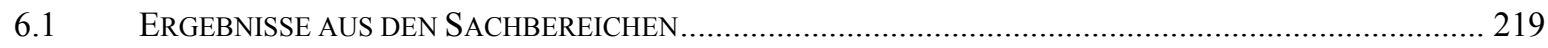

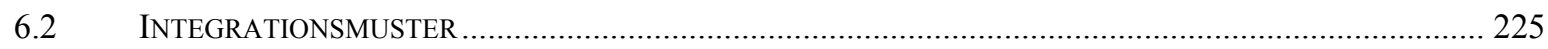

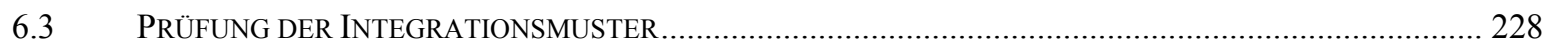

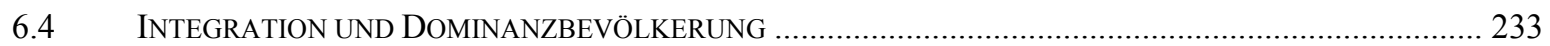

7 FAZIT: MIGRANTINNEN DER ZWEITEN GENERATION - ABGEHÄNGT, CHANCENLOS,

UNWILLIG?......................................................................................................................... 240

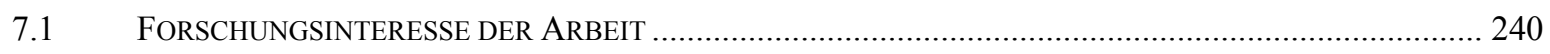

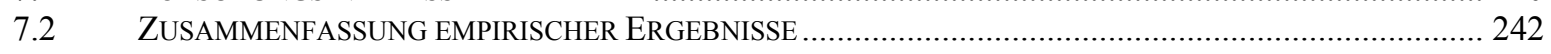

7.3 THEORETISCHE VERORTUNG IN DEN MIGRATIONSWISSENSCHAFTEN ……........................................ 248

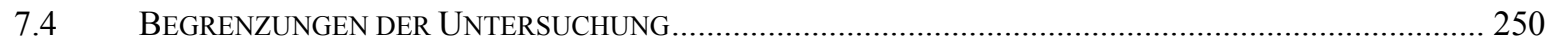

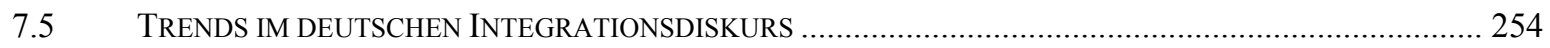

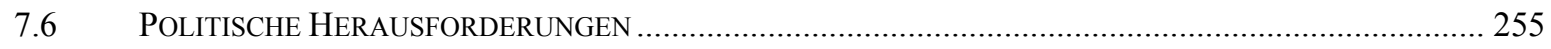

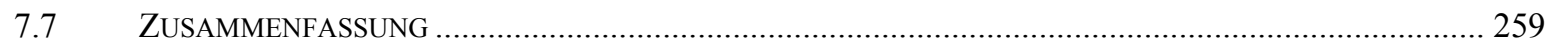

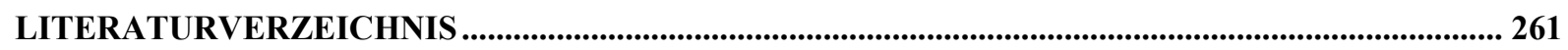

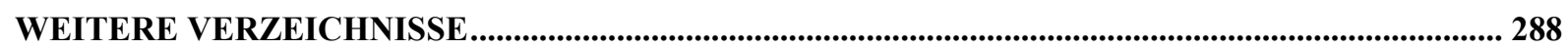

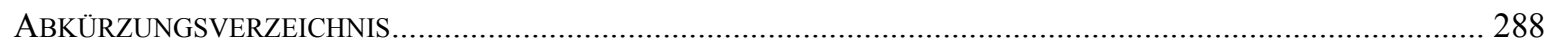

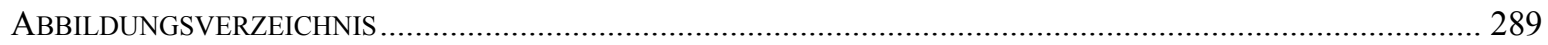

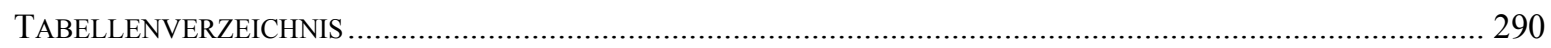




\section{Einleitung}

„Die zweite Generation wird abgehängt. [...] Die zweite Generation kann deutlich schlechter lesen und rechnen als die erste Generation. " (Spiegel Online, 15.05.2006)

„Organisierte Verweigerung. Viele Muslime lehnen Bemühungen um Integration ab - Gefahr einer islamischen Parallelgesellschaft “ (Süddeutsche Zeitung, 13.11.2004, S. 2)

Scheitern MigrantInnen der zweiten Generation? Sind sie abgehängt, chancenlos und (integrations-) unwillig - wie obige Zitate nahelegen? Wissenschaftliche und journalistische Berichte nennen erschreckende Zahlen: So liegt die Quote der ausländischen Jugendlichen, die die Schule nicht beenden, mit 13,6\% mehr als doppelt so hoch wie die deutscher Jugendlicher ohne Migrationshintergrund (5,4\%) (Bildungsberichterstattung 2008: 274). In Bezug auf Arbeitslosigkeit sind die Verhältnisse ähnlich. In Berlin beträgt beispielsweise die Arbeitslosigkeit bei Deutschen ohne Migrationshintergrund 16,5\%, bei ausländischen StaatsbürgerInnen jedoch 35\%, in einigen Stadtvierteln sogar weit über 40\%. (Hillmann 2006). 81\% der ausländischen Arbeitslosen haben keine Berufsausbildung, 54\% verfügen über keinerlei Schulabschluss (Bundesagentur 2005). Treffen diese alarmierenden Zahlen auch für die zweite Generation von MigrantInnen zu? Seit der Veröffentlichung der Ergebnisse der PISAUntersuchung (Programme for International Student Assessment) wird die These vom ,Niedergang' der zweiten Generation vertreten, d.h. die Annahme, dass die zweite Migrantengeneration schlechtere Bildungsabschlüsse und einen prekäreren Status auf dem Arbeitsmarkt aufweist als die erste. Dem medialen Diskurs über ,AusländerInnen', die sich in ,Parallelgesellschaften' abschotten, über familiäre Gewalt und SchulabbrecherInnen entspricht ein Strang der (Des-) Integrationsforschung um Wilhelm Heitmeyer, der für die gegenwärtige deutsche Gesellschaft ein hohes Maß an Anomie und Diskriminierung ausmacht sowie den Wunsch nach eindeutigen und einfachen Erklärungen, wie sie fundamentalistische Ideologien bieten (Heitmeyer, et al. 1997). Gemäß dieser Sicht scheitert Integration zur Zeit. Dagegen geht der bekannteste deutsche Integrationstheoretiker Hartmut Esser von Integration als Regelfall aus, die als Prozess des Verschmelzens der ,Fremden' zu einer ,ethnisch' homogenen Gesellschaft verstanden wird (Esser 1980). Hinter beiden Theorien steht ein Verständnis von Integration $^{1}$ als einem Prozess, bei dem MigrantInnen die ihnen zugeschriebenen Defizite ü-

\footnotetext{
${ }^{1}$ Neben ,Integration' wird in diesem Zusammenhang auch der Begriff der ,Assimilation' verwendet. Die Frage nach den Begrifflichkeiten für das zu beschreibende Phänomen ist nicht trivial. Die nordamerikanische Forschung verwendet den schon zu Beginn des 20. Jahrhunderts geprägten Assimilationsbegriff, unabhängig davon, ob sie die Annahmen der klassischen Assimilationstheorien wie etwa die Forderung nach einer einseitigen Anpassung der MigrantInnen an die kulturellen Maßstäbe der Aufnahmegesellschaft teilt oder im Gegenteil vom
} 
berwinden, um als vollwertige Mitglieder der Gesellschaft anerkannt zu werden. Die Bevölkerung ohne Migrationshintergrund gilt grundsätzlich als integriert. Aber muss Integration so aufgefasst werden? Und bestätigt eine breitere statistische Erhebung, die auch deutsche StaatsbürgerInnen mit Migrationshintergrund einschließt, die oben angeführten negativen Zahlen zu (jungen) AusländerInnen, auch für die zweite Generation?

\section{$1.1 \quad$ Fragestellung}

Die vorliegende Arbeit geht diesem Thema anhand von zwei Forschungsfragen nach:

- Inwiefern sind MigrantInnen der zweiten Generation in Deutschland integriert?

- Was sind Schwachstellen bisheriger Integrationstheorien (und wie können sie überwunden werden)? Welche Integrationstheorien eignen sich für die Analyse der zweiten Generation in Deutschland?

Diese beiden Fragenstellungen differenzieren sich in die folgenden Unteraspekte: Wie ist die zweite Generation in den Bereichen Sprachkenntnisse, Bildung, Beruf und Freund- sowie Partnerschaften $^{2}$ im Vergleich zur ersten und zur anderthalbten Generation sowie zu NichtMigrantInnen der gleichen Altersgruppe positioniert? Wie verändert sich das Bild durch den Einschluss von MigrantInnen der zweiten Generation mit deutscher Staatsbürgerschaft (Eingebürgerte oder seit Geburt Deutsche, Kinder von (Spät-)AussiedlerInnen ${ }^{3}$ )? Unterscheiden sich die Integrationsergebnisse je nach Herkunftsgruppen, bzw. weichen auch innerhalb der Gruppen die individuellen Ergebnisse stark von einander ab? Integrieren sich alle in der gleichen Art und Weise oder lassen sich verschiedene Muster finden? Geht ein gesicherter sozioökonomischer Status immer mit kultureller Anpassung und umgekehrt ein prekärer mit einem

\footnotetext{
Entstehen eines gemeinsamen Neuen ausgeht. In Deutschland wird dagegen ausschließlich von Integration gesprochen. ,Assimilation' hat - nicht zuletzt aufgrund der nationalsozialistischen Geschichte - einen xenophoben Beiklang. Doch auch bei dem Begriff ,Integration' erweist sich bei näherer Betrachtung, dass dahinter in Deutschland nicht selten klassische Assimilationskonzepte stehen. Dennoch verwende ich aufgrund der historischen Sensibilitäten in dieser Arbeit den Begriff Integration. In Kapitel 2.3 werden diese Überlegungen weitergeführt und sozialkonstruktivistische Integrationsprämissen entwickelt, welche diese Arbeit anleiten.

${ }^{2}$ In der Integrationsforschung wird gemeinhin zwischen strukturellen, kulturellen und psychologischen Aspekten der Integration unterschieden (Rumbaut 2001; DeSipio/de la Garza 1998; Gerstle/Mollenkopf 2001). In diesem Buch sollen folgende zentrale sozioökonomische und kulturelle Bereiche betrachtet werden: Sprachkompetenz, Bildung, Beruf, Freund- und Partnerschaften. Da Freund- und Partnerschaften immanent auf Zweiseitigkeit basieren, können sie zudem in Teilen als Indikator von Integration gewertet werden. Psychologische Aspekte wie Identitätsbeschreibungen wurden außer Acht gelassen, da sie fluide sind und stark von externen Ereignissen abhängen. Zudem können sie verschiedenste Referenzpunkte annehmen (z.B. Identität über Staatsbürgerschaft, Wohnort, Beruf, Hobby), was es erschwert, ihren Zusammenhang zu Integration einzuschätzen.

${ }^{3}$ Nach dem Bundesvertriebenengesetz sind AussiedlerInnen deutschstämmige Personen aus historischen Siedlungsgebieten in Osteuropa, Südeuropa, Asien oder den Regionen, in welche sie vertrieben wurden. Sie leben zumeist seit Generationen in diesen Ländern und wandern nun nach Deutschland zu. Erfolgte die Zuwanderung nach dem 1.1.1993, werden sie als SpätaussiedlerInnen bezeichnet.
} 
Festhalten an der ,Herkunftskultur' einher? Welche Rolle spielt Rassismus bei der Integration von MigrantInnen der zweiten Generation?

Entgegen der oben dargestellten Dichotomie in der Diskussion von Integration versus Desintegration vertrete ich die These, dass die zweite Generation von MigrantInnen in Deutschland in sozioökonomischer und kultureller Hinsicht eine große Vielfalt an Positionierungen aufweist und zudem im Allgemeinen ressourcenreichere Positionen einnimmt als die erste und die anderthalbte Migrantengeneration. Diese Verbesserung rührt - so wird gezeigt werden in Teilen von dem Einschluss deutscher StaatsbürgerInnen mit Migrationshintergrund in die Datensätze.

Dabei ist in einer sich diversifizierenden deutschen Gesellschaft zunehmend unklar, woran MigrantInnen sich anpassen sollen (siehe Cartoon). Empirisch lässt sich mehr als ein Integrationsmuster finden. Es existieren auch Formen der Integration, bei denen kulturelle Anpassung mit einem sozioökonomischen Abstieg einhergeht, Integration also mit reduzierten Lebenschancen verbunden ist, oder ein nicht-prekärer sozioökonomischer Status ohne kulturelle Anpassung erlangt wird. Diese Arbeit wird zeigen, dass Integration als (mindestens) zweiseitiger Prozess verstanden werden sollte. Der aktuell praktizierte ausschließliche Blick auf die MigrantInnen und ihre vermeintlichen Defizite verkürzt die Komplexität des Integrationsprozesses. Dieser hängt auch von strukturellem Rassismus in der deutschen Gesellschaft und rassistischen Praktiken ab, welche die Einnahme bestimmter Positionen durch MigrantInnen und das Maß der Wahrnehmung von Ähnlichkeit zwischen MigrantInnen und Nicht-MigrantInnen auf Seiten der Dominanzbevölkerung beeinflussen.

\subsection{Zweite Generation}

Während in der deutschen Migrationsforschung bisher von ,AusländerInnen' und ,Ausländerkindern' (vormals ,GastarbeiterInnen', ,Gastarbeiterkindern') gesprochen wurde (z.B. Glatzer 2004; Lajios 1991), wird inzwischen zunehmend die als politisch korrekt angesehene Formulierung Menschen ,mit Migrationshintergrund' verwendet. ${ }^{4}$ Dies trägt dem Faktum Rechnung, dass bei weitem nicht alle MigrantInnen ausländische StaatsbürgerInnen sind. Allerdings werden in den entsprechenden empirischen Untersuchungen häufig doch wieder nur sozial-

\footnotetext{
${ }^{4}$ Der Begriff ,mit Migrationshintergrund' hat einen regelrechten Siegeszug angetreten. So zeigt eine entsprechende Stichwortsuche in den Schlagzeilen deutschsprachiger Zeitungen, die in der Datenbank Lexis-Nexis enthalten sind, dass der Begriff erstmals im Jahr 2002 in einer Schlagzeile auftaucht und auch 2003 nur zweimal, im Jahr 2004 dann aber schon acht Mal. 2005 taucht er in 13 Schlagzeilen auf, 2006 in 34 Schlagzeilen, 2007 in 40 und allein in den ersten drei Monaten des Jahres 2008 in 37 Schlagzeilen. Der Begriff hat sich also durchgesetzt. Daneben wird im Bildungskontext auch der Ausdruck ,Personen nicht-deutscher Herkunftssprache’ verwendet.
} 
strukturelle Merkmale von ausländischen StaatsbürgerInnen analysiert (für Beispiele siehe Diefenbach 2005). Überdies wird in den deutschsprachigen Migrationswissenschaften weiterhin wenig nach Migrantengeneration unterschieden. Wer sind also MigrantInnen der zweiten Generation?

Die zweite Generation ${ }^{5}$ wird in diesem Buch definiert als jene Gruppe von Personen, deren Eltern beide MigrantInnen waren (d.h. deren Geburtsort im Ausland liegt) und die selbst in Deutschland geboren oder bis zum Alter von sechs Jahren (Einschulung) nach Deutschland gekommen sind (Crul/Vermeulen 2003: 971). Als erste Generation werden hingegen diejenigen bezeichnet, die erst im Erwachsenenalter nach Deutschland zugezogen sind, als anderthalbte Generation diejenigen, die zwischen dem 7. und dem 15. Lebensjahr zugewandert sind. Die MigrantInnen der zweiten Generation haben somit ihre gesamte formale Schulbildung in Deutschland erhalten. ${ }^{6}$ Diese Definition beinhaltet nicht Kinder und Jugendliche mit nur einem zugewanderten Elternteil wie z.B. bei Farley und Alba (Farley/Alba 2002: 679), da angenommen wird, dass Kinder mit einem Elternteil ohne Migrationshintergrund andere kulturelle und sprachliche Ressourcen erhalten als Kinder mit zwei migrantischen Eltern und daher die Herausforderungen, vor denen die beiden Gruppen stehen, nicht identisch sind. ${ }^{7}$

Inwiefern ist die Untersuchung der zweiten Generation aufschlussreich? Obwohl die zweite Generation keine homogene Gruppe darstellt, sondern sich hinsichtlich des sozioökonomischen Status der Eltern, der kulturellen Praktiken in den Familien und der Narrationen ihrer Gruppe über die Geschichte der Migration und der Aufnahme in Deutschland unterscheidet, gibt es doch einende Merkmale im Unterschied zur ersten Generation: ${ }^{8}$ Während die erste Generation der ,GastarbeiterInnen', Flüchtlinge und anderer MigrantInnen häufig auf eine Rückkehr in ihre Ursprungsländer hofft, beabsichtigen MigrantInnen der zweiten Generation zum überwiegenden Teil, im Aufnahmeland zu verbleiben. Im Gegensatz zu ihren Eltern richten sie ihre Wünsche und Ziele auf die dortige Lebenssituation. Der Rückkehr ins Heimatland

\footnotetext{
${ }^{5}$ Der Generationenbegriff in der Soziologie ist geprägt von Karl Mannheims Aufsatz Das Problem der Generation (Mannheim 1964). In den Migrationswissenschaften ist der Generationenbegriff hingegen unabhängig von der Alterskohorte, da die eigene oder indirekte Migrationserfahrung als einschneidendes Erlebnis vergleichbar einem historischen Ereignis verstanden wird. In meiner empirischen Untersuchung schränke ich jedoch die Generation auf eine ungefähre Alterskohorte ein, da ich Mannheim hinsichtlich der Bedeutung des historischen Kontextes mit seinen rechtlichen, diskursiven und sozioökonomischen Besonderheiten zustimme.

${ }^{6}$ Eine Ausnahme hiervon sind MigrantInnen der zweiten Generation, die während ihrer Schulzeit für längere Zeitperioden in die Herkunftsländer ihrer Eltern zurückgeschickt wurden und dort zur Schule gingen.

${ }^{7}$ Diese Definition der zweiten Generation grenzt sich zudem ab von Untersuchungen, die den Begriff allgemein für jüngere Immigrationswellen verwenden (Klinkhammer 2000: 16) oder aber für alle Kinder und Jugendlichen mit Migrationshintergrund unabhängig davon, wann diese nach Deutschland gekommen sind (z.B. Lajios 1991).

${ }^{8}$ Lüttinger hat in seiner Untersuchung der Integration von deutschen Flüchtlingen und Vertriebenen gezeigt, wie selbst in dieser Gruppe, die allgemein als Musterbeispiel einer gelungen Integration angeführt wird, sozioökonomische Benachteiligungen durch die Migration bis in die zweite Generation hinein identifizierbar sind, und dies trotz des wirtschaftlichen Aufschwungs in Deutschland in den 1950er und 1960er Jahren (Lüttinger 1989).
} 
der Eltern kommt eher symbolische Bedeutung zu: ihre praktische Umsetzung ist nicht zuletzt wegen fehlender Sprachkenntnisse schwierig. (Sen, et al. 2001: 8) Da MigrantInnen der zweiten Generation zudem ihre Schulbildung vollständig in Deutschland erhalten (haben), müssen sie im Unterschied zur ersten Generation nicht um die Anerkennung ihrer Abschlüsse bangen (Chiswick 1978; Friedberg 2000). Weiterhin sind die Kenntnisse in der Sprache des Aufnahmelandes bei der zweiten Generation im Allgemeinen besser als bei der ersten Generation. Dies liegt zum einen daran, dass es aus kognitiven Gründen für Kinder leichter ist, sich eine neue Sprache akzentfrei anzueignen (Lenneberg 1967: 53; Bialystok 1994), zum anderen an den aufgrund des Schulbesuchs zahlreicheren Gelegenheiten, Deutsch zu lernen. Zudem machen MigrantInnen der zweiten Generation zwar ebenfalls Erfahrungen der NichtAnerkennung und Diskriminierung, sind jedoch im Vergleich zur ersten Generation durchschnittlich weniger gewillt, solche Fremdheitszuschreibungen und fehlenden Respekt zu akzeptieren. Vielmehr erscheint ihnen eine vollständige Teilhabegerechtigkeit angemessen.

Auf der anderen Seite verbindet sie gegenüber Nicht-MigrantInnen derselben Altersgruppe ihre eigene frühe oder die über die Eltern vermittelte Migrationserfahrung. Für selbstverständlich gehaltene Lebenseinstellungen werden durch die im Zuge der Migration gemachten Erfahrungen in Frage gestellt, was zu Verunsicherungen und Konflikten führen kann. MigrantInnen der zweiten Generation müssen sich zumeist bewusster mit widersprüchlichen kulturellen Anforderungen auseinander setzen als Menschen ohne Migrationshintergrund. Dadurch können aber auch alternative Ressourcen zum Umgang mit Problemen in Deutschland freigesetzt werden, die den meisten Deutschen ohne Migrationshintergrund nicht zur Verfügung stehen. (Lauria-Perricelli 1992: 257) Migrantenkinder der zweiten Generation wachsen zudem in mehr oder weniger zweisprachigen Familien auf. Weiterhin gehen sie zwar gemeinsam mit Kindern und Jugendlichen ohne Migrationshintergrund in deutsche Schulen, aber sie erhalten von ihren Eltern wegen deren fehlender eigener Erfahrung im deutschen Bildungssystem und des durchschnittlich niedrigeren sozioökonomischen Status im Allgemeinen weniger Unterstützung (Wissen, Tipps, Hilfe, Orientierung, Netzwerke).

Schließlich verbinden MigrantInnen der zweiten Generation unabhängig von ihrem Staatsbürgerschaftsstatus auch Diskriminierungserfahrungen. Obwohl die Diskriminierung entlang einer Reihe von Faktoren variiert und MigrantInnen je nach Gender sowie nach Hautfarbe und anderen Markierungen der Fremdheit in unterschiedlichem Maße betrifft, erfolgen Fremdheitszuschreibungen durch die Dominanzbevölkerung ${ }^{9}$ zu einzelnen Zeitpunkten doch für al-

\footnotetext{
${ }^{9}$ In Anlehnung an Rommelspacher wird der Begriff der Dominanzbevölkerung zusammen mit dem der nichtmigrantischen Bevölkerung, der Bevölkerung ohne Migrationshintergrund oder auch der Nicht-MigrantInnen
} 
le. Da rassistische Diskriminierung trotz rechtlich formaler Gleichheit auch deutsche StaatsbürgerInnen mit Migrationshintergrund trifft, ist es angemessen, auch bei dieser Gruppe weiterhin von MigrantInnen zu sprechen. Befürchtungen, dass der Einschluss der zweiten Generation (v.a. mit deutscher Staatsbürgerschaft) in die Gruppe der MigrantInnen ihren Status als ,Fremde' fortschreibt (Martiniello 2005), sind zwar ernst zu nehmen, gehen in meinen Augen aber an der Realität vorbei: Solange MigrantInnen der zweiten Generation diskriminiert werden (siehe Kapitel 5) und sich ihre Teilhabechancen durchschnittlich von denen von Deutschen ohne Migrationshintergrund unterscheiden, muss dies auch untersucht und thematisiert werden. Freilich sollten solche Untersuchungen vermeiden, selbst Fremdheitszuschreibungen zu verfestigen.

Bei der Analyse von Integration nach Generationen zu unterscheiden ist deswegen sinnvoll, weil Integration vor allem ein intergenerationeller Prozess ist: Der Extremfall einer Assimilation kann auch dann eintreten, wenn sich einzelne Individuen nicht verändern, sondern nur die Nachgeborenen andere Lebensformen annehmen (Brubaker 2003: 52). Daher erlaubt der Blick auf die zweite Generation und das Verhältnis der Dominanzbevölkerung zu ihr aussagekräftigere Prognosen über die langfristige Integrationsentwicklung in einem Land als die Untersuchung aller Zuwanderergruppen zusammen. ${ }^{10}$

Zur zweiten Generation in Deutschland gehören:

- 1,5 Millionen in Deutschland geborene und wohnhafte AusländerInnen, die zwei im Ausland geborene Elternteile haben (Bundesregierung 2004b: 82); ${ }^{11}$

- ein Großteil der 1 Million deutscher StaatsbürgerInnen mit zwei im Ausland geborenen Elternteilen (Unabhängige Kommission 2001: 31), und zwar alle jene, die in Deutschland geboren wurden oder bis zum 6. Lebensjahr nach Deutschland gekommen sind;

- mindestens 400.000 (Spät-)AussiedlerInnen der zweiten Generation ${ }^{12}$ (Bildungsberichterstattung 2006: 140);

verwendet. Damit wird zum Ausdruck gebracht, dass es sich nicht nur um verschiedene Bevölkerungsteile handelt, sondern dass diese in einem hierarchischen Verhältnis zueinander stehen. Die fälschliche Homogenisierung innerhalb der Dominanzbevölkerung wird dabei zugunsten der analytischen Schärfe aus der Aufdeckung von Hierarchieverhältnissen in Kauf genommen.

${ }^{10}$ Selbstverständlich ist Integration keinesfalls in der zweiten Generation abgeschlossen, sondern beschäftigt auch noch die dritte und vierte Generation (Gans 1999: 161). Allerdings ist die dritte Generation in Deutschland noch zu jung, als dass über ihre Situation im Bildungssystem und v.a. auf dem Arbeitsmarkt Aussagen gemacht werden könnten. Daher untersucht die vorliegende Arbeit Faktoren, die die weitere Entwicklung beeinflussen können. Diese sollte aber nicht als bereits feststehender Prozess mit unabänderlichen Ergebnissen missverstanden werden.

${ }^{11}$ Hinzu kommen noch ausländische Staatsangehörige mit zwei im Ausland geborenen Elternteilen, die bis zum Alter von sechs Jahren zugewandert sind. Für diese liegen keine gesonderten Zahlen vor. 
- 280.000 seit 2000 (bzw. durch Übergangsregelung seit 1991) in Deutschland geborene Kinder von zwei im Ausland geborenen Elternteilen mit ausländischer Staatsangehörigkeit, die bei Geburt die deutsche Staatsbürgerschaft erhalten und bis maximal zum 23. Lebensjahr über eine doppelte Staatsbürgerschaft verfügen dürfen (Bildungsberichterstattung 2006: 140).

Zudem steigt der relative Anteil der zweiten Generation an allen MigrantInnen. Solange die vor allem aus demografischen und marktwirtschaftlichen Gründen geforderte Liberalisierung der Zuwanderungspolitik ausbleibt und die Zahl der Neuzuwanderung auf so niedrigem Stand liegt (Wanderungssaldo 2005: 79.000 (Bundesamt 2008: 57)), gewinnt die Gruppe der MigrantInnen der zweiten Generation innerhalb der gesamten Migrantengruppe an Bedeutung.

Von dieser zweiten Generation wird häufig behauptet, dass sie gegenüber dem sozioökonomischen Status ihrer Eltern keine Verbesserungen erziele oder gar dahinter zurückfalle. Die Kenntnisse in der Hochsprache des Aufnahmelandes seien mangelhaft und die MigrantInnen tendierten dazu, sich vom Rest der Gesellschaft abzuschotten. Bei muslimischen MigrantInnen der zweiten Generation wird befürchtet, dass sie aus dieser Gemengelage heraus anfällig für islamistischen Extremismus sind (siehe Zitate oben). Unter diesem Blickwinkel werden die MigrantInnen der zweiten Generation, die praktisch ihr ganzes Leben in europäischen Aufnahmeländern verbracht haben und zum Teil die Staatsbürgerschaft des Wohnlandes besitzen, zu Fremden, die als nicht integrierbar gelten oder sich vorgeblich zu großen Anteilen dieser Integration verweigern. Treffen diese Einschätzungen zu?

\subsection{Forschungsstand}

Wenn man zur Beantwortung dieser Frage die bisherige Forschung heranzieht, fällt auf, dass bislang wenige Arbeiten vorliegen und die meisten Untersuchungen zur zweiten Generation sich zudem einzelnen Bereichen (z.B. Grundschulbildung) oder Gruppen widmen.

Die Sozialwissenschaften haben die in Europa geborenen Kinder der MigrantInnen erst spät entdeckt. Während in den USA die Integration der zweiten Generation bereits seit Anfang der 1990er Jahre rege diskutiert wird (Zolberg 1989, Gans 1992, Portes/Zhou 1993, Waters 1996,

\footnotetext{
${ }^{12}$ Von 1950 bis 1987 kamen 1,4 Millionen AussiedlerInnen und Angehörige nach Deutschland, nach dem Ende des Kalten Krieges von 1988 bis 2000 nochmals 2,7 Millionen (Spät-)AussiedlerInnen (Unabhängige Kommission 2001: 178). Dabei von 400.000 MigrantInnen der zweiten Generation auszugehen, die entweder selbst bis zum Alter von sechs Jahren zuwanderten oder als Kinder eines Aussiedlerelternpaares geboren worden, basiert auf konservativen Operationalisierungen und wird den tatsächlichen Umfang der Gruppe eher unterschätzen.
} 
Farley/Alba 2002), begann die Debatte in Europa erst vor wenigen Jahren: 2003 brachte die International Migration Review ein Heft zur zweiten Generation in Europa heraus (Crul/Vermeulen 2003), die EU-Kommission finanzierte von 1998 bis 2000 das mehrere Länder umfassende Projekt Effectiveness of National Integration Strategies Towards Second Generation Migrant Youth in a Comparative European Perspective (EFFNATIS) (efms 2001), welches in Deutschland Daten für Bamberg erhob und seit 2007 unterstützt sie das Forschungsnetzwerk The Integration of the European Second Generation (TIES), welches die zweite Generation in Österreich, Belgien, Frankreich, Deutschland, den Niederlanden, Spanien, Schweden und der Schweiz vergleichend in den Blick nimmt.

Darüber hinaus existieren für Deutschland kleinere quantitative Studien zu einzelnen Themenfeldern, die mehrheitlich die zweite Generation, jedoch nur der ausländischen Staatsangehörigen, betrachten: z.B. zu Bildung (Kristen 2002), Beruf (Granato/Kalter 2001; Granato 2003; Kalter 2004), Religion (Worbs/Heckmann 2003), Identitätsbildung (Nauck/Steinbach 2001) und Partizipation (Diehl 2002). Auch bestehende deskriptive Untersuchungen unterscheiden zumeist nicht zwischen MigrantInnen mit ausländischer und jenden mit deutscher Staatsbürgerschaft und argumentieren entweder aggregiert abstrakt oder beschränken die empirische Untersuchung auf eine Gruppe in einer Stadt/Region (z.B. Akbulut 1993; Bade/Oltmer 1999; Herwartz-Emden/Westphal 2002; Karakasoglu-Aydin 2000; Kristen 2004).

\subsection{Integrationsgeschichte in Deutschland}

Der späte Beginn der Forschung zur zweiten Generation in Deutschland lässt sich teilweise aus der deutschen Migrationsgeschichte erklären. So war zwar bei den Flüchtlingen nach dem Zweiten Weltkrieg und den AussiedlerInnen in den folgenden Jahren Integration das vorrangiges Ziel. ${ }^{13}$ Bei den durch ,Gastarbeiter'verträge ${ }^{14}$ angeworbenen MigrantInnen war sie jedoch nicht erwünscht: Es wurde davon ausgegangen, dass die ,Gastarbeiter'familien sich nur befristet in Deutschland aufhielten und daher auf die Rückkehr in ihre Heimatländer vorbereitet werden müssten, z.B. durch die Einrichtung von griechischen, türkischen, italienischen u.a. Schulen in Deutschland sowie durch gesonderte ,Ausländerklassen' (Engelmann 2001) ${ }^{15}$.

\footnotetext{
${ }^{13}$ Allerdings wurde hier die Hauptschwierigkeit im materiellen Bereich gesehen; der anders geartete kulturelle Hintergrund der AussiedlerInnen und SpätaussiedlerInnen wurde lange Zeit ausgeblendet.

${ }^{14}$ Diese bilateralen, staatlichen Verträge wurden mit folgenden Staaten abgeschlossen: Italien (1955), Spanien und Griechenland (1960), Türkei (1961), Marokko (1963), Portugal (1964), Tunesien (1965) und Jugoslawien (1968) (Bade 1983; Ohliger, et al. 1999).

${ }^{15}$ Ein Konzept zur interkulturellen Bildung wurde erst 1996 von der Kultusministerkonferenz verabschiedet (KMK 1996).
} 
1973 wurde die Anwerbung von Arbeitskräften von außerhalb der Europäischen Gemeinschaft gestoppt. Die Bundesregierung förderte die Rückkehr von ,GastarbeiterInnen' und ihren Familien. Allerdings hatte der Anwerbestopp unbeabsichtigte Folgen: Er beendete die vorherige Pendelmigration und schaffte für die zeitlich befristeten MigrantInnen Anreize, sich fest in Deutschland niederzulassen. Während in der öffentlichen Diskussion betont wurde, dass Deutschland kein Einwanderungsland sei und die ,GastarbeiterInnen ${ }^{16}$ irgendwann nach „Hause' zurückkehren würden, geschah das genaue Gegenteil: Viele zunächst temporäre MigrantInnen gewöhnten sich an die Idee, langfristig in Deutschland zu bleiben und holten im Rahmen von Familienzusammenführung ihre Angehörigen nach, so dass die Zahl der MigrantInnen in den 1980er Jahren stark stieg (Unabhängige Kommission 2001: 14; Mahnig 2001: 176). Dennoch wurde erst im Zuge der Diskussion um das Zuwanderungsgesetz seit Ende der 1990er Jahre anerkannt, dass Deutschland ein Einwanderungsland ist, und Integration zu einer der wichtigsten Aufgaben der Zuwanderungspolitik erklärt (Bundesregierung 2004a). ${ }^{17}$

\subsection{Daten}

Neben diesen aus der jüngsten Migrationsgeschichte und ihrer politischen Verarbeitung resultierenden Gründen für die bislang wenig umfangreiche Forschung zur zweiten Generation liegt eine weitere Ursache in bisherigen Datenproblemen. So konnten MigrantInnen der zweiten Generation bis vor kurzem nur über ihre ausländische Staatsbürgerschaft identifiziert werden und/oder darüber, dass sie bei ihren ausländischen Eltern wohnten (z.B. im Soziooekonomischen Panel). Alternativ kamen nur zwei gesondert erhobene kleinere Datensets in Frage: Zum einen untersuchte der Integrationssurvey, der vom Bundesinstitut für Bevölkerungswissenschaften im Jahr 2000 in Auftrag gegeben wurde (von Below 2003; Glatzer 2004), die Situation der MigrantInnen italienischer, spanischer und türkischer Herkunft sowie einer deutschen Kontrollgruppe in den Bereichen Sprachkenntnisse, Bildung und Beruf sowie Identität und soziale Beziehungen. In diesem Datenset kann die zweite Generation identifiziert werden. Das Sampling für den Survey wies allerdings Probleme auf, so dass die Repräsentativität eingeschränkt ist (Mammey/Sattig 2002). Zum anderen erlaubt der Ausländersur-

\footnotetext{
${ }^{16}$ Eryilmaz beschreibt die Geschichte der ,GastarbeiterInnen' und ihre schwierigen Arbeits- und Lebensverhältnisse eindrücklich Eryilmaz/Jamin 1998. Für eine Untersuchung der in der Forschung häufig vernachlässigten Situation der weiblichen ,Gastarbeiterinnen' siehe Mattes 2005; zur Rolle von Diskriminierung und Rechtsextremismus, speziell in den 1990er Jahren Funke 2002.

${ }^{17}$ Bade spricht daher für Deutschland von einer ,,nachholenden Integrationspolitik“, bei der Integration im internationalen Vergleich verspätet auf die politische Agenda gesetzt wird und zu einem Zeitpunkt an Bedeutung gewinnt, an dem es nur wenig Neuzuwanderung nach Deutschland gibt (Bade 2007).
} 
vey des Deutschen Jugendinstituts (DJI) Analysen zu MigrantInnen der zweiten Generation türkischer, italienischer und griechischer Herkunft (Weidacher 2000).

Im Jahr 2005 wurde jedoch der Mikrozensus, eine jährliche Umfrage bei 1\% der Haushalte in Deutschland, reformiert. Er enthielt 2005 erstmals Fragen zu vorherigen Staatsbürgerschaften und dem Migrationshintergrund der Eltern (Zuwanderungsjahr, Staatsbürgerschaften). Zudem kommen für die meisten Herkunftsgruppen ausreichend große Fallzahlen zusammen - auch in den sonst unterrepräsentierten höheren Altersgruppen. Damit ist es jetzt problemlos möglich, auch die berufliche Situation von MigrantInnen der zweiten Generation zu untersuchen, die nicht mehr im Haushalt ihrer Eltern leben. Das Wichtigste jedoch ist, dass durch diese neuen Fragen MigrantInnen der zweiten Generation unabhängig vom Vorliegen einer ausländischen Staatsbürgerschaft identifiziert werden können. Salentin und Wilkening haben gezeigt, dass die Gruppe der AusländerInnen nicht länger repräsentativ für alle MigrantInnen ist, weil MigrantInnen mit höherer Bildung und höherem sozioökonomischen Status häufiger deutsche StaatsbürgerInnen werden als MigrantInnen mit niedrigem sozioökonomischen Status (Salentin/Wilkening 2003). Die übliche Verzerrung der ,AusländerInnen'-Untersuchungen kann nun also vermieden werden. Und schließlich sorgt der Mikrozensus durch die Art seiner Erhebung für eine relative Repräsentativität auch bei der migrantischen Bevölkerung (mehr zum Mikrozensus und dem DJI-Ausländersurvey in Kapitel 3.1.2).

In dieser Arbeit wird zur Beantwortung der Frage nach der Integration der zweiten Generation in Deutschland auf die Daten des Mikrozensus 2005 und des DJI-Ausländersurveys zurückgegriffen. Diese werden in einzelnen Fragen ergänzt durch Zweitauswertungen vorhandener Analysen (z.B. der PISA-Untersuchung). Für die Untersuchung eventueller Veränderungen in der Wahrnehmung bei der deutschen Dominanzbevölkerung wird die Allgemeine Bevölkerungsumfrage (ALLBUS) herangezogen.

\subsection{Theoretisches Interesse}

Die empirische Analyse ist geleitet von einem theoretischen Gerüst, das aus der Kritik an den klassischen Integrationstheorien in den Migrationswissenschaften (Park 1930, Warner/Srole 1945, Gordon 1964) und den darauf basierenden Theorien von Esser und Heitmeyer entwickelt wird. In Abgrenzung zu deren dichotomer Sichtweise, ihrer Ablehnung positiver Effekte von ,ethnischem' Sozialkapitals und Bikulturalismus für Integration sowie ihren ausgeprägten Homogenitätsannahmen über die Dominanzbevölkerung wird in diesem Buch ein sozialkonstruktivistisches Integrationskonzept entwickelt, gemäß dem Integration als die Zunahme der 
wahrgenommenen Ähnlichkeit auf Seiten der Dominanzbevölkerung gegenüber MigrantInnen definiert ist. Dadurch wird Integration als zweiseitiger Prozess mit kontingenten, im Vorhinein nicht absehbaren Ergebnissen aufgefasst. Integration ist keinesfalls unvermeidlich oder ein für alle Mal erreicht oder gescheitert, sondern in einer heterogenen Gesellschaft immer wieder neu auszuhandeln. ${ }^{18}$

Aufbauend auf diesem Integrationsverständnis werden aus der aktuellen Debatte zur Integration der zweiten Generation in den USA drei Integrationstheorien herauskristallisiert (pluralistische Integration, Abwärts-Integration und neoklassische Integration) und daraus drei Integrationsmuster in den Bereichen Sprache, Bildung, Beruf und Freund-/Partnerschaft abgeleitet: Neoklassische Integrationstheorien (Alba/Nee 2003) gehen davon aus, dass MigrantInnen sich ihnen bietende Chancen individuell ergreifen und dadurch einen nicht-prekären sozioökonomischen Status erlangen. Herkunftsbezogene Unterschiede werden dadurch in Zukunft an Sichtbarkeit verlieren und der Umfang der gesellschaftlichen Bereiche, in denen Fremdheitsmarkierungen über das Kriterium der Herkunftsgruppe noch relevant sind, wird zurückgehen. Dagegen beschreibt Abwärts-Integration (Portes/Rumbaut 2001; Portes 1996; Gans 1992), wie die Kinder von MigrantInnen mit geringem sozioökonomischem Status und einem Mangel an familiärem und herkunftsgruppenbezogenem Sozialkapital in urbanen Brennpunkten Teil nicht-migrantischer, sozial marginalisierter Gruppen werden und dadurch Aufstiegsmotivation und -chancen verlieren. Pluralistische Theorien in der Integrationsforschung schließlich sehen bikulturelle Kompetenzen und herkunftsgruppenbezogene Netzwerke nicht primär als Bedrohung der Integration, sondern als Chance. Sie gehen davon aus, dass die Verbindungen zum Herkunftsland der Eltern und zu herkunftsbezogenen Gruppen im Aufnahmeland alternative wirtschaftliche, soziale und normative Ressourcen für einen sozioökonomischen Aufstieg im Aufnahmeland bereitstellen. (Levitt 2001; Levitt/Waters 2002a; Itzigsohn, et al. 1999; Guarnizo 2001) Die drei Theorien geben also unterschiedliche Antworten auf die Frage nach der Referenzgruppe und den Mechanismen der Ähnlichkeitswahrnehmung: Soll sich die Ähnlichkeitswahrnehmung bei einer imaginierten ,Mitte' der Gesellschaft vergrößern oder bei einem Teil der Bevölkerung ohne Migrationshintergrund, der selbst sozial marginalisiert ist?

\footnotetext{
${ }^{18}$ Aus dieser Konzeptionalisierung von Integration folgt, dass die Untersuchung nicht darauf abzielt, einen Integrationsindikator zu schaffen, bei dem das Erreichen bestimmter Positionen in einzelnen Sachbereichen mit Integration gleich gesetzt wird. Erzielt eine Herkunftsgruppe also beispielsweise höhere Bildungsabschlüsse, dann ist sie gemäß des Integrationsindikators besser integriert als eine Herkunftsgruppe, die durchschnittlich niedrigere Bildungsabschlüsse erwirbt. Eine solche sozioökonomische Positionierung muss sich in meinem Konzept aufgrund von Diskriminierung und Rassismus jedoch nicht in vermehrte Ähnlichkeitswahrnehmung umsetzen. Daher erlauben Integrationsindikatoren allein - solange sie nicht die Messung der Einstellungen der nichtmigrantischen Bevölkerung einschließen - kaum gültige Aussagen zum Integrationsstand und bergen vielmehr die Gefahr, bei geringer Integration ausschließlich Migranten in die Verantwortung zu nehmen. Das vorliegende Buch betont dagegen den wechselseitigen und kontingenten Charakter von Integration.
} 
Und wodurch soll die Ähnlichkeitswahrnehmung erreicht werden - durch Mechanismen im kulturellen oder im sozioökonomischen Feld? (Kapitel 2.4)

Inwieweit sind nun diese Integrationstheorien aus dem klassischen Einwanderungsland USA auf Deutschland übertragbar? Die beiden Länder stehen in einer globalisierten Welt zunehmend vor den gleichen Herausforderungen; dazu zählt v.a. der Umgang mit wachsender sozioökonomischer und kultureller sowie ,ethnischer' und ,rassischer, ${ }^{19}$ Diversifizierung (Archdeacon 1992: 544). Während in ihrer Zuwanderungspolitik ehemals große Unterschiede bestanden, haben sie sich in den letzten Jahren politisch angenähert. Ein wichtiger Unterschied besteht jedoch weiter: Zwar gibt es auch in Deutschland marginalisierte soziale Schichten, die in städtischen Problembezirken v.a. aus bildungsfernen Langzeitarbeitslosen bestehen, welche auf Leistungen des Sozialstaats angewiesen sind (Alisch/Dangschat 1998; Keller 1999; Kronauer 2002), doch unterliegen diese Schichten im Gegensatz zu den USA nicht einer phänotypisch basierten Fremdheitsmarkierung. Es erscheint daher plausibel, dass die Dynamik um die Fremdheitsmarkierungen ${ }^{20}$ bei der Integration von MigrantInnen in Deutschland eine andere ist als in den USA (Hammar 1989). Eine weitere Besonderheit des europäischen Kontextes liegt in der Möglichkeit für MigrantInnen, innerhalb der Europäischen Union Unterstützung und Anerkennung für plurale Lebensweisen zu finden. ${ }^{21}$

Während alle drei oben beschriebenen Theorien Integrationstheorien sind, da sie sozioökonomische oder kulturelle Prozesse beschreiben, die zu einer größeren wahrgenommenen Ähnlichkeit auf Seiten der Dominanzbevölkerung gegenüber MigrantInnen führen können, sind die Integrationsmuster, die sie beschreiben unter einem normativen Blickwinkel sozialer Gerechtigkeit unterschiedlich zu bewerten: Überspitzt gesagt beschreibt die neoklassische Theorie der Integration, wie MigrantInnen der zweiten Generation der Mehrheitsbevölkerung ähnlich werden und damit gleichen Status erreichen, pluralistische Theorien interessieren sich dafür, wie MigrantInnen anders bleiben und darüber gleichen Status erlangen, und die ,Abwärts-Integration' beschreibt, wie MigrantInnen einem bestimmten Segment der Dominanz-

\footnotetext{
${ }^{19}$,Rasse', ,Ethnizität', ,Schwarz', ,westlich', Parallelgesellschaft, , GastarbeiterIn', AusländerIn usw. werden in dieser Arbeit grundsätzlich als soziale Konstruktionen verstanden, auch wenn die Begriffe nicht in Anführungszeichen gesetzt sind. Die Frage nach der Existenz von ,Ethnien' und ihren ,natürlichen' Eigenschaften wird also als falsch zurückgewiesen; vielmehr geht es um die Bedeutung, die einer solchen sozialen Konstruktion zukommt und die wandelbar, aber keinesfalls beliebig und in ihrer Wirkungsmächtigkeit real ist. So führten Drake und Cayton schon 1945 in ihrem Buch Black Metropolis aus, dass ,Rasse' kein biologisches Merkmal sei, sondern ein soziales, weil sozial konstruierte Sichtbarkeit zu Diskriminierung führe (Drake/Cayton 1945).

${ }^{20} \mathrm{Ob}$ für die erfolgreiche Integration von nicht-weißen MigrantInnen die Existenz einer einheimischen Gruppe der ,Anderen' nötig ist, in Abgrenzung zu welcher die MigrantInnen als Teil der Dominanzbevölkerung wahrgenommen werden, bleibt eine offene Frage (Alba/Nee 2003: 120). Zu den Unterscheidungen zwischen ,Rasse' und Ethnizität im US-amerikanischen Diskurs siehe Bös 2005.

${ }^{21}$ Diese Binnenintegration wird komplementiert durch die Exklusion von als ,fremd Markierten' außerhalb der Union.
} 
bevölkerung ähnlich werden und dadurch einen geringeren Status als die Dominanzbevölkerung insgesamt erlangen. Das theoretische Gerüst der Arbeit, welches diese drei Theorien vereinigt, enthält somit die Möglichkeit, dass Integration nicht immer positiv bewertet werden

kann. Dabei argumentieren die drei Theorien ausschließlich aus der Sicht der MigrantInnen. Sie richten ihr Augenmerk auf die Teilhabechancen von MigrantInnen der zweiten Generation in sich diversifizierenden Gesellschaften; die Interessen der Mehrheitsgesellschaft, z.B. nach sozialer Kohäsion, finden keine Beachtung.

Mit welchen Methoden wird die Arbeit den oben beschriebenen theoretisch angeleiteten Fragestellungen nachgehen?

\subsection{Methoden}

Die vorliegende Arbeit wendet quantitative Methoden der empirischen Sozialforschung an. Wo es sinnvoll erscheint, werden qualitative Untersuchungen durch eine Sekundärauswertung ebenfalls rezipiert. Im Sinne einer „Data Triangulation“ (Denzin 1970) baut der empirische Teil der Arbeit auf drei verschiedenen Datenquellen auf:

(1) Das Schwergewicht liegt auf einer statistischen Untersuchung mit Hilfe der Daten des Mikrozensus 2005 und des DJI-Ausländersurveys. Mit deskriptiven und analytischen Verfahren werden die Verteilungen verschiedener Herkunftsgruppen und Generationen hinsichtlich relevanter Merkmale in Kreuztabellen erhoben und die Streuungen innerhalb der Generationen und Herkunftsgruppen über Dispersionsmaße, z.B. den Interquartilbereich, gemessen. Um Herkunftsgruppenunterschiede zu quantifizieren und zum Generationenstatus in Beziehung zu setzen sowie einzelne von den Theorien hypothetisierte Zusammenhänge zu überprüfen, werden zudem in den Sachbereichen Sprache, Bildung, Beruf und Freund-/Partnerschaften Regressionen durchgeführt. Anschließend werden die Integrationsmuster aus den vier Sachbereichen heraus operationalisiert und ihre Verteilung über Generationen und Herkunftsgruppen deskriptiv untersucht. Darüber hinaus stellt sich die Frage, warum und auch wie diese Muster entstehen. Die drei aus der US-amerikanischen Diskussion herausgearbeiteten Theorien benennen hierfür Kausalitätsmechanismen. Diese werden durch den Vergleich theorierelevanter Variablen über die drei Integrationsmuster und das Muster der Segregation plausibilisiert oder abgelehnt. Die biografische Entstehung der Muster auf der individuellen Ebene kann damit in ihrer Komplexität jedoch nicht überprüft werden. 
(2) Ergänzt wird diese statistische Untersuchung durch Sekundärauswertungen vorliegender quantitativer Untersuchungen (z.B. PISA-E 2000 und 2003, DESI, Sprachstandserhebungen) und einzelner qualitativer Studien.

(3) Schließlich werden mit Blick auf die Dominanzbevökerung die aggregierten Daten der Allgemeinen Bevölkerungsumfrage (ALLBUS) analysiert und bestehende Experimentalstudien zu Diskriminierung zweitausgewertet.

\subsection{Aufbau des Buches}

Im nächsten Kapitel wird das theoretische Gerüst dieser Arbeit mit seinen sozialkonstruktivistischen Integrationsprämissen und den drei aus der aktuellen US-amerikanischen Debatte herauskristallisierten Integrationstheorien (und ihren Integrationsmustern) entwickelt (Kapitel 2). Anschließend wird davon angeleitet die empirische Untersuchung in den vier Sachbereichen Sprache, Bildung, Beruf und Freund-/Partnerschaften (Kapitel 3) sowie die Zusammenführung in den Integrationsmustern in Kapitel 4 vorgenommen. Danach geht es um die Frage, ob dem durchschnittlich verbesserten sozioökonomischen Status und der durchschnittlich stärkeren kulturellen Anpassung in der zweiten Generation auch eine größere Ähnlichkeitswahrnehmung von dieser Gruppe durch die Dominanzbevölkerung entspricht (Kapitel 5). Dabei werden auch struktureller Rassismus und rassistische Praktiken untersucht. In Kapitel 6 werden die empirischen Ergebnisse diskutiert. Kapitel 7 fasst die Ergebnisse zusammen, verweist auf Beschränkungen der Untersuchung und zeigt die sich aus dieser Arbeit ergebenden Herausforderungen für Wissenschaft, Integrationsdiskurs und Politik auf. 


\section{Integrationstheorien: Eine Reorientierung}

In diesem Kapitel wird der theoretische Rahmen der Arbeit entwickelt. Dazu werden zunächst die klassischen Integrationstheorien aus den Vereinigten Staaten (Park, Warner/Srole, Gordon, Glazer/Moynihan) diskutiert und die darauf basierenden Integrationsmodelle von Esser und Heitmeyer vorgestellt, die die deutsche Debatte beherrschen. In Abgrenzung zu diesen ,Klassikern' werden sozialkonstruktivistische Integrationsprämissen entwickelt, auf denen die weitere Arbeit aufbaut. Anschließend werden aus der gegenwärtigen US-amerikanischen Diskussion zur Integration der Kinder von MigrantInnen drei Theorien herauskristallisiert, die an diese sozialkonstruktivistischen Integrationsprämissen anschlussfähig sind und mit denen Schwachstellen der klassischen Integrationstheorien sowie der Modelle von Esser und Heitmeyer überwunden werden können. Schließlich werden aus diesen drei Theorien Integrationsmuster für die Bereiche Sprache, Bildung, Beruf sowie Freund- und Partnerschaften abgeleitet, deren empirische Existenz in den Kapiteln 3.3 bis 3.6 überprüft wird.

Folgende Fragen leiten dabei die Kurzdarstellung der klassischen Theorien sowie der auf ihnen aufbauenden Ansätze von Esser und Heitmeyer an:

1. Zielvorstellung: Wird Integration als progressives Durchleben verschiedener Integrationsstufen verstanden, welches unvermeidlich zu Integration in allen Bereichen führt? Findet Integration zwangsläufig in einen nationalen ,Mainstream' statt oder kann sie auch in eine lokale Kultur erfolgen ${ }^{22}$

2. Bild des ,Mainstreams': Wie wird der ,Mainstream', in den MigrantInnen und ihre Nachfahren sich integrieren, dargestellt: Als anglo-saxonische Mittelschicht? ${ }^{23}$ Schmelztiegel? Als pluralistische Mischung? Sind Menschen mit nicht-weißer Hautfarbe vom ,Mainstream' ausgeschlossen?

3. Richtung des Wandels: Untersucht die Theorie ausschließlich Veränderungen der MigrantInnen und ihrer Nachfahren oder werden auch Transformationen des, Mainstreams' betrachtet?

\footnotetext{
${ }^{22}$ In ihrer historischen Arbeit zu MigrantInnen deutscher Herkunft in den USA beschreibt Conzen, wie die MigrantInnen lokale Gepflogenheiten veränderten und anglo-saxonische EinwohnerInnen sich an den so entstehenden neuen lokalen ,Mainstream' anpassten. Die Deutschen waren daher lokal vollständig assimiliert, auf einer nationalen Ebene jedoch nicht (Conzen 1991).

${ }^{23}$ Als WASP (white anglo-saxon protestant) wird eine gesellschaftlich und politisch einflussreiche Gruppe der Nachfahren der britischen KolonialistInnen in Abgrenzung zu späteren katholischen MigrantInnen (z.B. IrInnen, ItalienerInnen) sowie den neuen Zuwanderungswellen seit den 1965 Jahren bezeichnet (Baltzell 1964).
} 
4. Status sozialer Kategorien: Hält die Theorie die ,Ethnizität' der MigrantInnen für gegeben oder glaubt sie, dass ,Ethnizität' sich im Prozess der Migration verändert oder in ihrer konkreten Ausprägung sogar erst während der Migration entsteht? Schließen sich verschiedene Kulturen wechselseitig aus oder kann es Bikulturalismus geben? Wie beeinflussen Geschlecht, ,Ethnizität', Klasse, ,Rasse' u.a. den Integrationsprozesse?

Nachfolgend wird untersucht, wie klassische Integrationstheorien sowie die in Deutschland weit verbreiteten Ansätze von Esser und Heitmeyer diese Fragen beantworten und welche Probleme sich hieraus ergeben. Im Weiteren wird dann die Frage behandelt, ob diese Probleme sich durch ein neues Integrationsverständnis beheben lassen.

\subsection{Klassische Integrationstheorien}

Die Integration von MigrantInnen wurde in größerem Umfang zum ersten Mal zu Beginn des 20. Jahrhunderts wissenschaftlich untersucht. Masseneinwanderung in die USA und die große Zahl von MigrantInnen der ersten und zweiten Generation v.a. in den Städten weckten ein dringliches Interesse an der Frage, ob diese ZuwanderInnen ,gute AmerikanerInnen' werden würden. (Herrmann 1996)

Ab den 1920er Jahren forschten Robert Ezra Park und seine MitarbeiterInnen und Studierenden in Chicago zur Integration von MigrantInnen, speziell der neuen Herkunftsgruppen aus Süd- und Osteuropa, und den Beziehungen zwischen Menschen unterschiedlicher ,Rassen’. Park entwickelte einen ,Rassen’beziehungszyklus (,race relation cycle“624) bestehend aus den Phasen Wettkampf, Konflikt, Akkomodation und Integration. ${ }^{25}$ Gemäß diesem Zyklus findet in der ersten Phase ein ständiger und allgemeiner Wettkampf um Ressourcen statt, wohingegen im Stadium des Konfliktes bewusst Auseinandersetzungen zur Statusgewinnung geführt werden (Park/Burgess 1921 (2nd ed. 1924): 504ff, 574ff ). Während der Akkomodation werden diese Auseinandersetzungen temporär ausgesetzt, da die sozialen Beziehungen sich an die neuen Gegebenheiten anpassen (Park/Burgess 1921 (2nd ed. 1924): 664f, 735). Diese Prozesse der ersten drei Phasen finden in einer Vielzahl von Kontexten statt und betreffen die Frage der sozialen Ordnung allgemein. Das Stadium der Integration bezieht sich hingegen speziell auf die Beziehungen zwischen ,ethnischen’ Gruppen und ,Rassen’: „Assimilation is a

\footnotetext{
${ }^{24}$ Park selbst verwendete den Begriff „race relation cycle” allerdings nur selten (Rumbaut 2001: 845).

${ }^{25}$ In den klassischen Integrationstheorien wird grundsätzlich das Wort ,Assimilation' verwendet. Um in der Terminologie dieser Arbeit zu bleiben, wird es hier mit ,Integration' übersetzt. Der Begriff ,Assimilation' taucht also nur in Zitaten auf oder um zu verdeutlichen, dass über allgemeine Annahmen der klassischen Integrationstheorien weitreichendere Anpassungsleistungen gefordert werden.
} 
process of interpenetration and fusion in which persons and groups acquire the memories, sentiments, and attitudes of other persons and groups, and, by sharing their experience and history, are incorporated with them in a common cultural life" (Park/Burgess 1921 (2nd ed. 1924): 735).

Während Akkomodation rasch und absichtlich statt findet, ist Integration ein inkrementeller und unbewusster Prozess, der sich überwiegend in persönlichen und intimen Beziehungen vollzieht. Nach dem ,Rassen'beziehungszyklus werden die verschiedenen Phasen sukzessive durchlaufen, Integration ist das irreversible Ergebnis dieses Prozesses (Park 1950: 150). ${ }^{26}$ In Bezug auf die Frage, ob der ,Rassen'beziehungszyklus notwendigerweise zu Integration führt, erscheint Parks Haltung inkonsistent: Auf der einen Seite bezeichnet er Integration als „final perfect outcome" des Prozesses (Park/Burgess 1921 (2nd ed. 1924): 736). Andererseits erscheint Integration nur als eines von mehreren möglichen Ergebnissen: „They will take the form of a caste system, as in India; they will terminate in complete assimilation, as in China; or the unassimilated race will constitute a permanent racial minority within the limits of a national state, as in the case of the Jews in Europe [...] All three types of change are involved [...] in what we may describe as the "race relations cycle" (Park 1937: xii). ${ }^{27}$

Bezüglich der Gesellschaft, in die MigrantInnen und ihre Kinder sich integrieren, ist der ,Rassen'beziehungszyklus sowohl mit dem Paradigma der anglo-saxonischen Konformität wie auch mit dem Schmelztiegelmodel vereinbar (Park 1937: 734f). Allerdings vertritt Park ausdrücklich die Haltung, dass die Menschen in einer Gesellschaft durch eine einzige Kultur verbunden sein sollten. Darüber hinaus hält er den marginalen Mensch (,marginal man'), der in zwei Kulturen lebt und sie verkörpert, für ein interessantes Studienobjekt, das jedoch ein AuBenseiter bleibt (Park 1950, Coser 1977: 365f).

Warner und Srole entwickeln in ihrem Buch The Social Systems of American Ethnic Groups Parks Ansichten weiter, indem sie der Integrationstheorie Variablen für spezifische ,Ethnien’ und ,Rassen' hinzufügen. Sie beschreiben, wie Integration durch Unterschiede in Sprache, Religion und ,Rasse' von der dominanten Gruppe bestimmt wird. Während ,ethnische' Charakteristika verschwänden und nur für eine Transitionsphase hilfreich seien, ,until these members ,unlearn' what they have been taught and successfully learn the new way of life necessary for full acceptance in the host society" (Warner/Srole 1945: 285), seien ,Rassenunterschiede' wandlungsresistenter: „,T] he physical traits which have become symbols of

\footnotetext{
${ }^{26}$ Akkomodation, die zur Beruhigung eines Konfliktes gefunden wurde, kann jedoch scheitern, und Konflikt dadurch wieder entfacht werden, der dann auf einem höheren Niveau das nächste Mal überkommen werden wird.

${ }^{27}$ Für eine ausführlichere Diskussion dieser Frage siehe Schmitter Heisler 1992: 626.
} 
inferior status are permanent. Unless the host society changes its methods of evaluation these racial groups are doomed to a permanent inferior ranking" (Warner/Srole 1945: 285). ${ }^{28}$ Dennoch stellen Warner und Srole fest, dass das amerikanische politische und wirtschaftliche System sowie das Bildungssystem und die Klassenstruktur der amerikanischen Gesellschaft notwendigerweise $\mathrm{zu}$ Integration durch sozioökonomischen Aufstieg und Akkulturation sowie gemischte Partnerschaften führen. Gleichzeitig wird der Zeitrahmen für die Integration von Menschen mit afrikanischer, lateinamerikanischer oder asiatischer Herkunft wesentlich größer angesetzt als derjenige für die Integration von EuropäerInnen. An manchen Stellen bezweifeln Warner und Srole sogar, dass Integration für diesen Personenkreis überhaupt möglich ist. Die Autoren stellen somit ein lineares Modell der Integration in einen anglo-saxonischen ,Mainstream' vor, in dem Bikulturalismus keinen Platz hat. Sie schreiben ,Rassen'unterschieden eine größere Bedeutung im Integrationsprozess zu als Park.

Im Unterschied zu Park sowie zu Warner und Srole unterteilt Milton Gordon Integration in mehrere Bestandteile. Integration ist nicht länger ein monolithisches Konzept, sondern sie umfasst unterschiedliche Aspekte, z.B. Kultur, soziale Partizipation, intime Beziehungen, Einstellungen. Gordon differenziert zwischen sieben Integrationsaspekten: kulturelle Integration (,[c]hange of cultural patterns to those of host society“ (Gordon 1964: 71)), strukturelle Integration (,entrance of the minority group into the cliques, clubs, and institutions of the core society at the primary level“ (Gordon 1964: 80)), Heiratsintegration, identifikatorische Integration, Übernahme der Einstellungen (,,attitude receptional assimilation”), Übernahme des Verhaltens des ,Mainstreams' sowie staatsbürgerliche Integration. Die letzten drei Phasen setzen die Abwesenheit von Vorurteilen, Diskriminierung und Wertkonflikten voraus. Diese sieben Aspekte der Integration müssen nicht zwangsläufig aufeinander folgen (Gordon 1964: 71): Zwar findet kulturelle Integration zuerst statt, sie garantiert jedoch keinesfalls eine nachfolgende strukturelle Integration (Gordon 1964: 77f). Hat strukturelle Integration als ,the keystone of the arch of assimilation" stattgefunden, folgen andere Integrationsschritte allerdings schnell (Gordon 1964: 80f).

In konzeptioneller Hinsicht stimmt Gordon mit den beiden zuvor dargestellten Theorien überein, dass Integration in einen Schmelztiegel oder in kulturellen Pluralismus stattfinden kann (Gordon 1964: 74f). Empirisch jedoch findet ihm zufolge Integration in eine anglo-saxonische Kerngruppe hinein statt (Gordon 1964: 72). Die Einflüsse später in die USA gekommener Kulturen auf diese anglo-saxonische Kultur seien gering gewesen (Gordon 1964: 72f). Im

\footnotetext{
${ }^{28}$ In diesem Zitat äußert sich erstmals auch eine sozialkonstruktivistische Sichtweise von ,Rasse'. Diese wird in Kapitel 2.3 aufgegriffen.
} 
Gegensatz zu Park und Warner/Srole, stellt Gordon den Integrationsprozess nicht als linear dar. Das Endergebnis ist nicht notwendigerweise eine vollständige Integration. Bikulturelle Lebensweisen werden jedoch ebenfalls als unwahrscheinlich und nicht wünschenswert zurückgewiesen.

Glazer und Moynihan erklären in ihrem Buch Beyond the Melting Pot (1963) Integration hauptsächlich über den Faktor ,Rasse'. Sie starten somit am gleichen Punkt wie Warner und Srole kommen aber zu kritischeren Schlussfolgerungen. Die Autoren schreiben: „[r]eligion and race seem to define the major groups into which American society is evolving as the specifically national aspect of ethnicity declines" (Glazer/Moynihan 1963: 314). Indem sie die vorrangige Bedeutung von Charakteristika der Religion und ,Rasse' gegenüber ,ethnischen' Faktoren für den Integrationsprozess betonen, stellen Glazer und Moynihan rückblickend die Integrationsfähigkeit des anglo-saxonischen Kerns der amerikanischen Gesellschaft in Frage: „But these test cases merely reveal to us how partial was the power of the old American type of assimilation - it assimilated its ethnic cousins" (Glazer/Moynihan 1963: 20). Damit lehnen sie eine zentrale Annahme der klassischen Integrationstheorien, nämlich die Integrationsleistung des ,Mainstreams' ab. Beyond the Melting Pot läutet das Ende der klassischen Integrationstheorien ein.

Hinsichtlich der eingangs gestellten Fragen eint also alle klassischen Integrationstheorien die Zielvorstellung, dass Integration in den ,Mainstream' der amerikanischen Gesellschaft möglich und wünschenswert ist. Bei Park sowie bei Warner und Srole führt ein Prozess durch verschiedene Stadien unvermeidlich zu Integration, während nach Gordon volle Integration nicht notwendigerweise erreicht werden muss. Weiterhin erfolgt Integration im empirischen Regelfall in einen anglo-saxonischen, Mainstream'. Sie geschieht aufgrund rationaler KostenNutzen-Kalkulationen, wobei die nicht-migrantische Bevölkerung auf die ZuwandererInnen und ihre Kinder Druck ausübt, sich zu assimilieren. Alle vier dargestellten Theorien gehen davon aus, dass es einen ,Mainstream' der amerikanischen Bevölkerung mit bestimmten kulturellen Werten und sozioökonomischen Chancen gibt und dass sich dieser im Zuge der Eingliederung der MigrantInnen kaum verändert.

Die vier Theorien unterscheiden sich hinsichtlich der Bedeutung von ,Rasse' für den Integrationsprozess: Während Warner und Srole an der Integrationsfähigkeit von ,Schwarzen’ zweifeln, betont Gordon die Notwendigkeit der Bereitschaft des ,Mainstreams', auch jene MigrantInnen aufzunehmen, die einer anderen ,Rasse' angehören. Glazer und Moynihan schließlich bestreiten eben diese Integrationsbereitschaft des ,Mainstreams' gegenüber nicht-weißen 
,Rassen'. ,Ethnizität' wird in allen klassischen Integrationstheorien als eine feste Kategorie betrachtet, die Individuen im Zuge der Integration ablegen, die sich aber als solche nicht verändert. Ein Leben in zwei Kulturen halten die klassischen Integrationstheorien für eine Ausnahmeerscheinung und für wenig wünschenswert. Andere soziale Kategorien als ,Ethnizität' und ,Rasse', wie z.B. die sozioökonomische Klassenzugehörigkeit und das Geschlecht der MigrantInnen, finden in den klassischen Integrationstheorien keine Beachtung. Diese Theorien beschreiben Integration somit vor allem als einseitige Leistung, die MigrantInnen erbringen müssen.

Alle vier Theorien gehen davon aus, dass es einen ,Mainstream' der amerikanischen Gesellschaft gibt. In den modernen pluralistischen Gesellschaften erscheint es aber schwierig festzuhalten, wodurch der ,Mainstream' sowohl in kultureller als auch in sozioökonomischer Hinsicht gekennzeichnet ist. Und selbst wenn der ,Mainstream' bestimmt werden kann, unterliegt er über die Jahre einem Wandel. Weiterhin stellen alle hier vorgestellten Theorien kulturelle Faktoren in den Mittelpunkt des Integrationsgeschehens. Die Annahme, dass kulturelle Gleichheit mit sozioökonomischer Ähnlichkeit identisch sei oder zu dieser führe, lässt sich jedoch in modernen, differenzierten Gesellschaften schwerlich aufrecht erhalten.

Inwieweit basieren nun die in der gegenwärtigen deutschen Theoriediskussion vorherrschenden Theorien ebenfalls auf diesen Annahmen? Dieser Frage wird im folgenden Abschnitt nachgegangen.

\subsection{Integrationstheorien der deutschen Diskussion}

Die Diskussion über den Integrationsstand von MigrantInnen der zweiten Generation in Deutschland bewegt sich zwischen dem optimistischen Ansatz von Hartmut Esser und dem Menetekel von Wilhelm Heitmeyer. Während Esser Integration langfristig für unausweichlich hält, beschreibt Heitmeyer einen Prozess der Fundamentalisierung und zunehmenden Gewaltbereitschaft bei Jugendlichen der zweiten Generation. Beide Theorien sollen im Folgenden dargestellt werden. Dabei wird gezeigt, dass sie sich trotz ihrer Unterschiede in ihren Verkürzungen gleichen.

\subsubsection{Esser: Modernisierungsoptimismus}

Hartmut Essers Habilitationsschrift Aspekte der Wanderungssoziologie (Esser 1980; Esser 1979 ) ist der Klassiker der deutschen Integrationsforschung. Esser geht davon aus, dass Integration in funktional differenzierten Gesellschaften sich am besten handlungstheoretisch- 
individualistisch erklären lässt (Esser 1980: 240f, 257): Die Akteure analysieren ihre jeweilige Situation, wählen ein Verhalten, und die verschiedenen Entscheidungen aggregieren sich zu einem gesellschaftlichen Ergebnis. Dieser Dreischritt von Situationslogik, Wahllogik und Aggregationslogik wird getragen von rationaler Wahl (,rational choice'), die auf dem geschätzten Nutzwert eines Verhaltens basiert (Esser 2003: 9). Entscheidungen werden dabei als Folge von Lernerfahrungen aufgefasst, „wobei die jeweilige (vergangene oder gegenwärtige) Handlungsumgebung die Hauptdeterminante der Lernerfahrung ist"“ (Esser 1979: 85). Gesellschaftliche, politische und wirtschaftliche Strukturen beeinflussen die Akteure, determinieren aber nicht ihr Verhalten.

Unter Integration versteht Esser den „Zusammenhalt von Teilen in einem ,systemischen’ Ganzen [...], gleichgültig zunächst worauf dieser Zusammenhalt beruht. Die Teile müssen ein nicht wegzudenkender, ein, wie man auch sagen könnte, ,integraler' Bestandteil des Ganzen sein“ (Esser 2001: 2). Kennzeichen der Integration ist also eine Interdependenz, die auf materieller Abhängigkeit, Institutionen und/oder Kultur basieren kann (Esser 2001: 3). Das Gegenteil hierzu ist Segmentation, die durch ein beziehungsloses Nebeneinander gekennzeichnet ist (Esser 2001: 3). Laut Esser ist das Nebeneinander aber meist nicht gleichwertig, sondern mit hierarchischen Unterschieden verbunden, so dass ,ethnisch' differenzierte Gesellschaften zugleich ,ethnische' Schichtungen sind (Esser 2001: 32). Diese sind gekennzeichnet durch ,das Vorliegen systematischer vertikaler sozialer Ungleichheiten zwischen den verschiedenen ethnischen Gruppen [...] insbesondere in der Verfügung über besonders interessante Ressourcen und Markt- bzw. Organisationsmacht“ (Esser 2001: 3). Eine Beschränkung allein auf horizontale Ungleichheiten, also auf Unterschiede, die nicht eine Höher- oder Niedrigbewertung zur Folge haben, ist unwahrscheinlich.

In modernen Gesellschaften spielt nach Esser bei der Systemintegration der Markt die größte Rolle (Esser 2001: 4). Hinzukommen muss gemäß der Theorie jedoch die Sozialintegration, nämlich der „Einbezug der Akteure in das gesellschaftliche Geschehen, etwa in Form der Gewährung von Rechten, des Erwerbs von Sprachkenntnissen, der Beteiligung am Bildungssystem und am Arbeitsmarkt, der Entstehung sozialer Akzeptanz, der Aufnahme von interethnischen Freundschaften, der Beteiligung am öffentlichen und am politischen Leben und auch der emotionalen Identifikation mit dem Aufnahmeland“" (Esser 2001: 8). Da Esser die Möglichkeit einer Mehrfachintegration in verschiedene Gesellschaften oder Kulturen ablehnt, kann Sozialintegration für ihn nur in Form von ,Assimilation' stattfinden (Esser 2001: 19f); darunter versteht er „die Auflösung systematischer Unterschiede zwischen den verschiedenen Gruppen und Kategorien“ (Esser 2001: 20). Als politisches Ziel sieht er ,ethnische’ Homoge- 
nität (Esser 2001: 17). Zwar erkennt er an, dass die Annäherung sowohl von Seiten der MigrantInnen als auch seitens der Mehrheitsbevölkerung erfolgen kann. Jedoch hält er letztere nur in Randbereichen wie Essensgewohnheiten für praktisch möglich (Esser 2001: 20f). „[W]enigstens grosso modo und über gewisse Zeiträume hinweg [ist] die Assimilation in die jeweils aufnehmende Gesellschaft der empirische Regelfall.“ (Esser 2001: 21) Diese Schlussfolgerung wird gestützt durch die Einschätzung, dass ,ethnische Schichtung' nicht dem kapitalistischen System entspricht: „Ethnic stratifications, and certainly ethnic (neo-)feudalism, are structurally incompatible with global capitalism" (Esser 2003: 32). Allerdings befürchtet Esser, dass die Anreize einer Situation MigrantInnen dazu führen könnten, in herkunftsgruppenbezogenes Kapital zu investieren statt in universell verwertbares Kapital (z.B. Esser 2003). Eine solche Entscheidung führe zu Fehlentscheidungen, die zu einem späteren Zeitpunkt nur noch mit unverhältnismäßig hohen Kosten korrigiert werden könnten. In Anlehnung an Norbert F. Wileys soziale Mobilitätsfalle (Wiley 1967) konstatiert Esser: „Ethnische Gemeinden bilden eine - oft nahezug unwiderstehliche - strukturelle Versuchung für das Tappen in solche Mobilitätsfallen.“ (Esser 2001: 36).

Essers Stärke besteht somit darin, eine umfassende Theorie entwickelt zu haben, die Mikround Makromechanismen zusammenbringt und Integration unter unterschiedlichen Bedingungen zu erklären sucht. Er muss dabei keine Integration in einen ,Mainstream' postulieren, da es ihm um die Auflösung objektiver sozioökonomischer und kultureller Unterschiede geht (2001: 17, 20). Sein binäres Konzept, bei dem am Ende des Integrationsprozesses entweder ,Assimilation' oder ,ethnische Schichtung' steht, führt auch zu einer strikten Ablehnung von Mehrfachintegration, welche Esser außer für Diplomatenkinder für nicht realisierbar hält (Esser 2001: 19). Kulturelle Identitäten und Kompetenzen sind für ihn ein Nullsummenspiel, bei dem das Erlernen einer neuen Kultur mit dem Verlernen der alten Hand in Hand geht (Esser 2001: 9). Nicht beachtet wird dabei, dass kulturelle Kompetenzen synergetisch wirken können und dass die Herkunftskulturen der MigrantInnen kaum völlig gegensätzlich zur Kultur der Mehrheitsgesellschaft im Aufnahmeland sein wird. Esser scheint zu unterstellen, dass die eine modern und die anderen rückwärts gewandt sind und es daher keine Wertüberschneidungen gibt. Die Ablehnung bikultureller Kompetenz ist mit dem Verständnis einer homogenen Kultur der Mehrheitsgesellschaft verbunden: Esser postuliert eine kohärente Mehrheitskultur, die von allen Mitgliedern der Mehrheitsgesellschaft gelebt und beherrscht wird. Bei modernen, differenzierten Gesellschaften ist es jedoch plausibler, von verschiedenen kulturellen Milieus und sehr unterschiedlichen kulturellen Kompetenzen auszugehen (Beck/BeckGernsheim 1994). Problematisch erscheint zudem, dass Esser durch den Blick auf vermeint- 
lich objektive Faktoren wie ,Ethnizität' und Kultur Diskriminierung aus den Augen verliert. Zum Beispiel beschreibt er die Situation von AfroamerikanerInnen in den USA als ein Problem der ,ethnischen' Segmentation (2001: 32). Dabei kann hier schwerlich von der Inkompatibilität einer ,schwarzen' Kultur mit einer, weißen' Mehrheitskultur ausgegangen werden. Eine größere Erklärungskraft kommt dem Faktor ,Rasse’ und rassistischer Diskriminierung zu. Zudem verstellt dieser Fokus auf Kultur den Blick auf soziale Ausschließungsprozesse. So ist bei Essers Beschreibung der ,ethnischen' Schichtung in Deutschland unklar, inwieweit diese zwar aus Fehlinvestitionen, aber dennoch freiwillig entsteht und inwieweit sie eine Folge von Diskriminierung und Ausgrenzung durch die Mehrheitsgesellschaft ist (Esser 2003). Esser beschreibt den Prozess insgesamt eher als einen freiwilligen, was sich nicht zuletzt in der Wortwahl äußert, z.B. „Hineintappen“ in Mobilitätsfallen versus Hineingedrängt-Werden (Esser 2001: 36). Eine solche Sichtweise schreibt die Verantwortung für Integrationsprozesse vorwiegend den MigrantInnen zu und vernachlässigt die Bedeutung einer diskriminierungsfreien Umwelt. Erstaunlich ist in diesem Zusammenhang, wie Esser die Rolle des globalen Kapitalismus einschätzt: Lokale oder transnationale Segmentation wird durch Kapitalismus zwangsläufig überwunden (Esser 2003: 32). ,Ethnische' Stratifizierung widerspreche dem kapitalistischen System. Damit lehnt Esser sich an neoliberale Theorien der Wertschöpfung an (Friedmann 1962) und ignoriert die sozioökonomische Sinnhaftigkeit verfestigter globaler und nationaler Macht- und Hierarchieverhältnisse zur Unterstützung kapitalistischer Strukturen.

Insgesamt kann es für Esser somit nur zwei Integrationsergebnisse geben: ,ethnische' Schichtung oder klassische, Assimilation'. Empirisch hält er letztere für wesentlich wahrscheinlicher. Eine Unterscheidung verschiedener Migrantengenerationen wird zwar erwähnt, aber die Ausführungen dazu sind nicht konsistent: So soll auf der einen Seite eine Segmentation der ersten Generation sich in der zweiten Generation verstärken und zu Ghettos mit massiven Konflikten führen (2001: 36); auf der anderen wird es für möglich gehalten, dass die erste Generation eine ,ethnische' Unterschicht bildet und die Folgegenerationen sich integrieren (Esser 2001: 23).

\subsubsection{Heitmeyer: Anomie}

Ein Teil dieser Probleme trifft auch für Heitmeyers Integrationsansatz zu, den er u.a. in seinem Buch Verlockender Fundamentalismus darstellt und der hier exemplarisch für einen in der deutschen Diskussion verbreiteten kulturell-defizitären Ansatz dargestellt wird (siehe auch Kelek 2002; Heitmeyer/Anhut 2000). Heitmeyer geht davon aus, dass jugendliche MigrantIn- 
nen in Deutschland unter enormem Erwartungsdruck von Seiten der Eltern, der ,ethnischen Gemeinschaft $^{29}$ und der Mehrheitsgesellschaft stehen. Gleichzeitig erhalten sie nur wenig Hilfe und Ressourcen zur Erfüllung der an sie gerichteten Ansprüche und zum Umgang mit widersprüchlichen Erwartungen. Durch die Verschlechterung der sozioökonomischen Situation in Deutschland seit den 1980er Jahren haben sich die Handlungsspielräume der Jugendlichen mit Migrationshintergrund verringert. Zudem reagiert die Mehrheitsgesellschaft selbst auf Integrationsbemühungen immer wieder mit Diskriminierung und fremdenfeindlicher Gewalt (Heitmeyer, et al. 1997: 26). Die Realisierung der ambitionierten Ziele der MigrantInnen der zweiten Generation wird somit unwahrscheinlich: „Die Chancen auf eigenständige Lebensgestaltung nehmen generell zu, während für immer größere Teilgruppen die Realisierungsmöglichkeiten abnehmen.“ (Heitmeyer, et al. 1997: 24) Aus der Orientierung an der Familie und traditionellen Werten, ,ethnischer' Identifikation und Rückzug in die ,ethnische Gemeinschaft', geringer schulischer Qualifikation, ausbleibendem Statusgewinn und Diskriminierungserfahrungen sowie Verweigerung einer kollektiven Identität durch die Mehrheitsgesellschaft entsteht der Wunsch nach fundamentalistischen Ideologien (Heitmeyer, et al. 1997: 146, 179ff, 183), die durch einfache Orientierungsmuster Halt und Selbstwertgefühl bieten. Solche Formen entdifferenzierender religiös-kultureller Orientierungsmuster befürworten im Kontext einer modernen Gesellschaft meist Gewalt zur Durchsetzung ihrer ideologischen Ziele (Heitmeyer, et al. 1997: 41). Daraus entsteht nach Heitmeyer eine ernstzunehmende Gefahr der Desintegration - auch für die Mehrheitsgesellschaft.

Die Stärke von Heitmeyers Analyse liegt darin, die Entwicklungen der letzten Jahre bei Jugendlichen türkischer Herkunft in Deutschland in einen größeren soziologischen Kontext der Entstehung von Gewalt und der Desintegration von Gesellschaften einzubetten. So sind z.B. seine empirischen Beobachtungen, dass Personen in einer sich verschlechternden sozialen Lage überproportional häufig Minderheiten abwerten und einer Ideologie der Ungleichheit anhängen (Heitmeyer 2004: 4), auf seine Untersuchung der Jugendlichen türkischer Herkunft übertragbar. Im Unterschied zu Esser, der die MigrantInnen in Mobilitätsfallen hineintappen sieht, weist Heitmeyer der Mehrheitsgesellschaft Verantwortung zu und ist modernisierungskritisch: Wo Esser in der Moderne systemimmanente Lösungen sieht, beschreibt Heitmeyer, wie Desintegrationspotenziale erst durch aktuelle wirtschaftliche und gesellschaftliche Entwicklungen entstehen. Der Markt löst diese Probleme nicht, er schafft sie.

\footnotetext{
${ }^{29}$ Durch Begriffe wie ,ethnische Gemeinschaft' und ,ethnisches Sozialkapital' wird die Normalität von ,Weiß'Sein und Deutsch-Sein reifiziert. Selbstverständlich stellen auch Deutsche eine ,ethnische Gemeinschaft' dar, diese wird jedoch für normal gehalten, so dass z.B. nur die türkische ,ethnische Gemeinschaft' erwähnenswert ist. Für eine kritische Diskussion solcher Mechanismen, wie sie in den ,Whiteness Studies' erfolgt, siehe Wachendorfer 2001.
} 
In der empirischen Umsetzung der Studie treten allerdings eine Reihe von Problemen auf: So setzt Heitmeyer, wie schon Esser, MigrantInnen mit AusländerInnen gleich und trennt nicht nach Generationen: Zwar erwähnt er die dritte Generation, definiert sie jedoch nicht, und soweit sich dies einschätzen lässt, - haben an der Studie keine MigrantInnen der dritten Generation im üblichen Sinn teilgenommen (Heitmeyer, et al. 1995: 406). Obwohl in Verlockender Fundamentalismus ausschließlich türkische Jugendliche in Deutschland betrachtet werden, scheint zudem der Anspruch durch, (Des-) Integrationsmuster für Migrantenjugendliche aller Herkunftsgruppen in Deutschland zu beschreiben. Hinsichtlich des empirischen Vorgehens ist der fehlende Vergleich mit deutschen Jugendlichen ohne Migrationshintergrund problematisch. Deren Erfahrungen mit Leistungsdruck, Versagensängsten und Gewalt werden nicht beleuchtet, auch weiß man nicht, inwieweit sie sich ebenfalls nach vereinfachenden Orientierungsmustern sehnen (Heitmeyer, et al. 1997: 41, 50-62). ${ }^{30}$ Da ein solcher Vergleich fehlt, kann Heitmeyer seine Ergebnisse an idealen Vorstellungen messen. In der daraus folgenden defizittheoretischen Argumentation liegt der Fokus auf dem, was Jugendlichen türkischer Herkunft fehlt, und nicht auf ihrer Andersartigkeit, die auch Ressourcen beinhalten könnte. ${ }^{31}$

Auf der theoretischen Ebene verhindert diese defizitorientierte Argumentation eine nichtbinäre Sicht: Die jeweilige ,ethnische' Kultur und Identität steht angeblich im Widerspruch zu einer ,westlichen' Identität mit kohärenten ,westlichen' Werten (Heitmeyer, et al. 1997: 43, 92). ${ }^{32}$ Zudem lehnt Heitmeyer die Möglichkeit bikultureller Kompetenz bei Jugendlichen mit Migrationshintergrund ab: Die Jugendlichen säßen zwischen den Stühlen, sie erhielten weder in Deutschland noch in der Türkei noch in einem transnationalen Raum Identitätsangebote (Heitmeyer, et al. 1997: 152). Einen Gewinn aus dieser Auseinandersetzung mit zwei Kulturen sieht Heitmeyer nicht. So weist er auch den Wert herkunftsgruppenbezogenen Sozialkapitals zurück. Integration kann aus seiner Sicht nicht über einen kollektiven Weg der Stärkung der Herkunftsgruppen erfolgen: „Die angeblich positiven Effekte von Segregation stehen auf tönernen Füßen; statt dessen werden gruppenspezifische Eigeninteressen erkennbar, die die

\footnotetext{
${ }^{30}$ Einzig in einer anderen Studie zu Gewalt lässt sich ein solcher Vergleich für einige Items durchführen. Allerdings war hier die Migrantengruppe mit 313 Personen sehr klein (Heitmeyer, et al. 1995: 401ff).

${ }^{31}$ Zudem legt Heitmeyer eine pessimistische Deutung von Situationen an den Tag: So zieht er zum Beispiel daraus, dass die meisten türkischen Jugendlichen einen hinsichtlich des Migrationsstatus gemischten Freundeskreis haben und sich intensiveren Kontakt zu Deutschen wünschen (Heitmeyer, et al. 1997: 89f), den Schluss, dass Deutsche sich dem Kontakt verweigern. „Von daher überrascht es nicht, daß deutsche und ausländische Jugendliche fast nur noch dort etwas miteinander zu tun haben, wo sie etwas miteinander zu tun haben müssen, also in der Schule und in der beruflichen Ausbildung bzw. im Beruf." (Heitmeyer, et al. 1997: 89f)

${ }^{32}$ Dies ist jedoch selten der Fall und für das Wertespektrum in der Türkei nicht zutreffend. Weiterhin ist die hohe Zustimmung zu den Werten Leistungsbereitschaft, Ordentlichkeit/Fleiß, Achtung vor den Eltern und Gehorsam, wie sie Heitmeyer für Jugendliche türkischer Herkunft konstatiert (Heitmeyer, et al. 1997: 97), zwar nicht postmodern, aber auch unter Angehörigen der Dominanzbevölkerung weit verbreitet. So fanden $75 \%$ der für die Shell Studie 2002 befragten Jugendlichen Fleiß und Ehrgeiz wichtig (Albert, et al. 2004: 3).
} 
Desintegration forcieren und Schließungsprozesse nach innen mitsamt Konflikt an den ,Grenzen’ von Stadtvierteln und ethnischen Communities fördern.“(Heitmeyer 1998: 452)

Damit ergeben sich trotz der unterschiedlichen Prognosen von Essers und Heitmeyers Theorie hinsichtlich der eingangs formulierten Fragen mehrere Gemeinsamkeiten:

1. Beide halten im Sinne eines progressiven Integrationsprozesses kulturelle Anpassung (Sprache, Werte) für unabdingbar, um sozioökonomisch aufzusteigen. Während jedoch Esser Integration als Endpunkt dieses Prozesses modernisierungsoptimistisch für unausweichlich hält, ist Heitmeyer skeptischer: Für ihn ist auch Desintegration ein mögliches Ergebnis.

2. Die Mehrheitsbevölkerung ist für beide kulturell homogen, bei Heitmeyer aber sozioökonomisch gespalten. Mit der unterstellten Homogenität des ,Mainstreams' orientieren sich beide Theorien eher an der , anglo-saxonischen' Konzeptionalisierung denn am Konzept eines Schmelztiegels.

3. Veränderungen werden nur bei den MigrantInnen erwartet. Bei der Mehrheitsbevölkerung würden sich höchstens Essgewohnheiten wandeln, bei Heitmeyer außerdem über längere Zeiträume auch die sozioökonomischen Verhältnisse im ,Mainstream'.

4. Beide sehen Kultur und damit auch ,Ethnizität' als etwas Festes und Gegebenes an. Ihr gehört man entweder ganz oder gar nicht an. Bikulturelle Kompetenzen werden höchstens für eine kleine Elite für möglich gehalten. Der Aufbau von ,ethnischem' Sozialkapital erschwert Integration.

In empirischer Hinsicht schließlich sprechen Esser und Heitmeyer zwar von MigrantInnen allgemein, betrachten aber nur spezifische Herkunftsgruppen (Heitmeyer: MigrantInnen türkischer Herkunft, Esser: MigrantInnen türkischer, italienischer und jugoslawischer Herkunft). Weiterhin unterscheiden sie nicht systematisch nach Generationen.

Im folgenden Kapitel werden aufbauend auf dieser kritischen Analyse sozialkonstruktivistische Prämissen von Integration entwickelt mit dem Ziel, die hier festgestellten Beschränkungen der dargestellten Theorien zu überwinden. 


\subsection{Integrationstheoretische Prämissen}

In Abgrenzung zu den Annahmen der klassischen Integrationstheorien, die sich auch in den auf Deutschland bezogenen Studien von Esser und Heitmeyer wiederfinden, wird in diesem Kapitel ein sozialkonstruktivistisches Verständnis von Integration entwickelt, das nicht auf objektiven Kriterien, sondern auf ihrer Wahrnehmung basiert und nicht ,Defizite' auf Seiten der MigrantInnen analysiert, sondern Integration als einen wechselseitigen Prozess zwischen MigrantInnen und Nicht-MigrantInnen versteht.

\subsubsection{Integrationskonzept}

Integration soll in dieser Arbeit nicht als zwangsläufiger und unumkehrbarer Prozess verstanden werden. In Anlehnung an Abramson, der Integration als Prozesse definiert, die zu größerer Homogenität in der Gesellschaft führen (Abramson 1980: 150), wird Integration hier sozialkonstruktivistisch gewendet und als Zunahme der wahrgenommenen Ähnlichkeit der migrantischen Bevölkerung auf Seiten der Dominanzbevölkerung verstanden. ${ }^{33}$ Es geht also nicht mehr um vermeintlich objektive Homogenität oder Ähnlichkeit, sondern darum, wie diese wahrgenommen wird. Der Begriff ,Dominanzbevölkerung' wird in Anlehnung an Rommelspacher 1995 für die nicht-migrantische Bevölkerung in Deutschland verwendet, um zum Ausdruck zu bringen, dass die beiden Bevölkerungsgruppe aufgrund von rassistischen Strukturen in der Gesellschaft in einem hierarchischen Verhältnis zueinander stehen. Zugunsten der klareren Abgrenzung wird in Kauf genommen, dass dieser Begriff die nichtmigrantische Bevölkerung über Gebühr homogenisiert und weitere darin wirkende Machtachsen (z.B. Gender, Klasse) dadurch verdeckt werden.

\subsubsection{Soziale Konstruktion ,objektiver' Gegebenheiten}

In sozialkonstruktivistischer Tradition gehe ich davon aus, dass es Homogenität oder (Un-) Ähnlichkeit nicht unabhängig von sozialen Wahrnehmungen, Bewertungen und Beurteilungen gibt: ,[M]aterial resources only acquire meaning for human action through the structure of shared knowledge in which they are embedded“" (Wendt 1995: 73). Dies bedeutet nicht, dass

\footnotetext{
${ }^{33}$ Dabei wird nur die Wahrnehmung auf Seiten der Dominanzbevölkerung untersucht, weil in einem hierarchisch strukturierten System die Wahrnehmung der statushöheren Gruppe über die realen Lebenschancen von Angehörigen der statusniedrigeren Gruppe entscheidet, während dies umgekehrt kaum der Fall ist.
} 
es keine materielle Welt ${ }^{34}$ gibt, aber ihre soziale und damit auch individuelle Bedeutung entsteht erst durch soziale Bewertung und Sinnzuschreibung an diese Gegebenheiten: „Welt, offene Welt, ist eine anthropologische Dimension und fungiert als unausschöpflicher Hintergrund für jede Art von artikulierter Wirklichkeit“ (Plesner in: Berger/Luckmann 1977: x). Individuen haben bei der Artikulierung von Realität jedoch weder freie Hand, noch können sie die soziale Realität einfach verändern, da die Kriterien der Sinngebung sozialer Natur und intersubjektiv sind. Dem Individuum erscheint soziale Realität damit zumeist als objektiv gegeben (Berger/Luckmann 1977: 22ff). Für diese Arbeit bedeutsam ist, dass Kategorien wie ,Ethnizität', ,Rasse', Migrationsstatus, Geschlecht nicht aus sich heraus eine objektive, unveränderliche, natürliche Bedeutung haben, sondern dass ihnen im Rahmen intersubjektiver Verständigung und deren verfestigter Fortschreibung in Institutionen und Sprache eine Bedeutung zugewiesen wird. ${ }^{35}$ Die ihnen zugeschriebene Bedeutung kann sich also im Laufe der Zeit wandeln. ${ }^{36}$ Zudem bedingen Struktur und AkteurInnen einander gegenseitig. So hat schon Schütz in seiner phänomenologischen Soziologie darauf hingewiesen, dass Individuen in eine vorstrukturierte soziale Welt hineingeboren werden, diese Welt aber durch ihre Interpretationen auch rekonstruieren und verändern (Schütz 1932; Schütz 1984).

Für meine Arbeit bedeutet dies, dass ,objektive' Grundlagen wichtig sind, weil sie mit verfestigten, intersubjektiven Bewertungen und mit Normen verbunden sind (z.B. Norm einer Nationalsprache, Bedeutung eines nicht-prekären beruflichen Status, Höherbewertung formaler Bildungsabschlüsse zu Lasten lebensweltlicher Erfahrung), sie aber dennoch nicht automatisch, also quasi natürlich, zu bestimmten Bewertungen führen müssen. Wenn MigrantInnen akzentfrei Deutsch sprechen und gesicherte Erwerbsarbeit haben, sind sie deswegen nicht zwangsläufig integriert. Ob ihr Status in eine Wahrnehmung von Ähnlichkeit überführt wird, hängt von einer Reihe von Faktoren ab, u.a. vom Rassismus in der Gesellschaft.

\footnotetext{
${ }^{34}$ Die Bewertung dieses ,Rumpfmaterialismus' ist in Bereichen der Politikwissenschaft umstritten. Für eine Diskussion siehe Adler 2001: 96 und Wendt 1995: 73.

${ }^{35}$ Dies wird in der empirischen Untersuchung britischer IndustriearbeiterInnen von Elias und Scotson auf eindrückliche Weise veranschaulicht. In die von ihnen untersuchte Siedlung zog eine große Gruppe von ArbeiterInnen neu zu. Alteingesessene BewohnerInnen und Neuankömmlinge waren derselben nationalen und ethnischen Herkunft, verfügten über den gleichen Beruf, die gleiche Bildung und das gleiche Einkommen. Sie unterschieden sich einzig hinsichtlich der Wohndauer in der Siedlung. Das darauf basierende Machtungleichgewicht führte zu Spannungen und erlaubte der etablierten Gruppe, die Außenseitergruppe als „,faul“, „,stinkend“, ,anders“ zu stigmatisieren. Die verfestigten Abwertungen gewannen über die Zeit den Status einer objektiven Differenz und äußerten sich in realen verringerten Lebenschancen der Diskriminierten. (Elias/Scotson 1993: 7ff)

${ }^{36}$ Das gleiche gilt für Kultur, die ebenfalls nur in einem intersubjektiven Prozess Bedeutung erlangt und im Sinn von Swidler als ,Werkzeugkasten' verstanden wird, mit Hilfe dessen Menschen verschiedene Handlungsstrategien entwickeln: „Culture influences action not by providing the ultimate values toward which actions are oriented, but by shaping a repertoire or ,tool kit' of habits, skills, and styles from which people construct ,strategies of action'“(Swidler 1986: 273).
} 


\subsubsection{Rassismus als Struktur}

Fremdheitsmarkierungen werden in dieser sozialkonstruktivistischen Konzeptionalisierung als soziale Grenzen verstanden, die sowohl der Distanzierung als auch der Selbstvergewisserung der Dominanzbevölkerung dienen (Claessens 1991). In einem hierarchischen System ist damit zumeist eine Über- und Unterordnung verbunden. Die sozialen Grenzen beinhalten sowohl eine symbolische Komponente - Distinktionsstrategien und normative Diskurse (Espiritu 2001) - als auch eine soziale Beschränkung des Zugangs zu gesellschaftlichen Ressourcen. Die symbolische Dimension ist eine notwendige, aber nicht hinreichende Bedingung für die Verfestigung sozialer Grenzen (Lamont/Molnar 2002).

In Gesellschaften ${ }^{37}$ gibt es eine ganze Reihe unterschiedlicher Machtverhältnisse: zwischen MigrantInnen und Nicht-MigrantInnen, zwischen Männern und Frauen, zwischen Alten und Jungen, zwischen Gesunden und Kranken, zwischen Armen und Reichen, zwischen Menschen verschiedener sexueller Orientierungen usw. Diese Machtverhältnisse wirken auf jedes einzelne Individuum in unterschiedlicher Weise ein. Das Zusammenwirken verschiedener Faktoren zu untersuchen, ist eine komplexe Aufgabe. ${ }^{38}$ In dieser Arbeit liegt der Fokus auf Diskriminierung aufgrund einer Markierung als MigrantIn und/oder ,fremd aussehende' Person. Für diese Diskriminierung wird der Begriff des Rassismus verwendet, um zum Ausdruck zu bringen, dass es sich hierbei nicht allein um individuelle Einstellungen und Verhaltensweisen handelt - dies wird z.B. suggeriert, wenn Rechtsextremismus in Ostdeutschland als Problem gewaltbereiter Jugendlicher marginalisierter sozialer Schichten erklärt wird. Es soll zum Ausdruck gebracht werden, dass es sich vielmehr um Einstellungen und Verhaltensweisen handelt, die sich aus gesellschaftlichen Machtverhältnissen ergeben. Diese Konzeptionalisierung verdeutlicht die Formulierung von Fanon: „une société est raciste ou ne l'est pas“ (Fanon 1969: 69). Es geht also um die Gesellschaft als Ganzes, die bestimmte Einstellungen und Handlungen von Individuen erst ermöglicht. Im Anschluss an Fanon versteht Anja Weiß in ihrem Buch Rassismus wider Willen Rassismus als Praktiken symbolischer Delegitimierung, die sich mit der Zeit zu negativem symbolischem Kapital verfestigt und damit zu einer Dimension von Ungleichheit wird (Weiß 2001: 351). Dadurch verschiebt sich der Fokus vom individuellen Verhalten $\mathrm{zu}$ den es ermöglichenden Strukturen, und Formen des nicht-

\footnotetext{
${ }^{37}$ Gesellschaften sind dabei nicht als in sich geschlossene Entitäten zu betrachten. Globale Machtverhältnisse und verfestigte Hierarchien beeinflussen auch die Machtbalance innerhalb von Staaten.

${ }^{38}$ In der feministischen Forschung wird dies zum Teil unter dem Stichwort der Intersektionalität versucht; siehe z.B. hooks 1989; Collins 1990 oder für den deutschsprachigen Raum z.B. Castro Varela 2007; Klinger 2003; Knapp/Wetterer 2003.
} 
intendierten und des institutionellen Rassismus geraten ins Blickfeld. ${ }^{39}$ Weiß zeigt zudem, dass Rassismus zwei Komponenten enthält: symbolische Abwertung und Festschreibung in sozialen Strukturen. Diese beiden Komponenten verstärken einander wechselseitig. Weiß greift hier auf die Kapitalientheorie von Bourdieu zurück, der zwischen ökonomischem, kulturellem, sozialem und symbolischem Kapital als akkumulierbaren und ineinander überführbaren Ressourcen unterscheidet, die für das soziale Leben einer Person ausschlaggebend sind (Bourdieu 1987).

\subsubsection{Integrationsmechanismen}

MigrantInnen erwerben einen gewissen Status und nehmen kulturelle Anpassungsleistungen in einem strukturellen Kontext von Rassismus vor, der sie in unterschiedlicher Weise trifft. Die sozioökonomischen und kulturellen Ressourcen setzen sie dann in Interaktionen mit Mitgliedern der Dominanzbevölkerung ein. Diese Interaktionen werden von der Dominanzbevölkerung als status(un)gleich und/oder kultur(un)gleich markiert. ${ }^{40}$ Daraus ergibt sich eine vergrößerte Wahrnehmung von Ähnlichkeit (respektive Unähnlichkeit) bei der Dominanzbevölkerung. Auf der einen Seite sind die Interaktion und die Art und Weise, wie sie wahrgenommen werden, vom umgebenden strukturellen Kontext des Rassismus beeinflusst, auf der anderen Seite trägt die Wahrnehmung von Ähnlichkeit oder Unähnlichkeit zum Abbau bzw. zur Reproduktion von Rassismus bei. Rassistische Praktiken sind also nicht primär individualpsychologisch zu erklären, aber auch nicht ausschließlich strukturell, da Spielräume für individuelle Entscheidungen bestehen.

Status- und kulturgleich markiert meint dabei keinesfalls, dass es sich um einen objektiv gleichen Status oder Kultur handelt. Dies wird durch das Wort ,markiert' zum Ausdruck gebracht. Darüber hinaus basieren solche Markierungen aber auch auf Normen, Normen davon, was einen guten und erstrebenswerten sozioökonomischen Status ausmacht. Es geht also nicht darum, dass beispielsweise ein Mitglied der Dominanzbevölkerung mit Realschulabschluss nur MigrantInnen mit eben diesem Schulabschluss als ähnlich wahrnehmen kann, sondern dass in seiner Wahrnehmung eine bestimmte Norm erfüllt wird, z.B. „Bildung ist wertvoll““ Dies eröffnet auch die Möglichkeit, dass in einer gesellschaftlichen Subgruppe eine andere

\footnotetext{
${ }^{39}$ Die vorliegende Arbeit untersucht dabei jedoch nicht die Gründe, die zur Entstehung von Rassismus in der Dominanzgesellschaft führen (z.B. symbolische Sicherung von Privilegien, sozialpsychologische Identitätsbedürfnisse)

${ }^{40}$ Diese Mechanismen der Ähnlichkeitswahrnehmung werden von Shibutani und Kwan beschrieben: Sozioökonomische Ressourcen führen zum Einschluss in Kommunikationskanäle, wodurch sich bestimmte kulturelle Ressourcen entwickeln. Kulturelle Ressourcen können jedoch auch unabhängig von sozioökonomischen eingesetzt werden (Shibutani/Kwan 1965: 573f).
} 
Norm als in der Gesamtgesellschaft gilt. Zudem wird hier wiederum deutlich, dass es sich keinesfalls um subjektive, individuell willkürliche Prozesse handelt, sondern um sozial, d.h. intersubjektiv konstruierte.

Wie oben erläutert, wird mit Fremdheitsmarkierungen eine soziale Grenze geschaffen. Solche Grenzen können nach Bauböck 1995 auf drei verschiedene Weisen überwunden werden:

,Boundary Crossing' bezeichnet den Prozess, bei dem eine einzelne Person soziale und symbolische Grenzen überwindet, zumeist indem sie als eine andere durchgeht (,passing'). Die Grenze selbst bleibt jedoch bestehen. Ein Beispiel hierfür ist eine Deutsche in den USA, die akzentfrei Englisch spricht, im Auftreten für eine Amerikanerin gehalten wird und ihren Geburtsort nicht erwähnt. Beim ,Boundary Shifting' bleibt die Grenze ebenfalls bestehen, jedoch verändert sich ihre Kontur im Verlauf. Sie wird so verlegt, dass eine ganze Gruppe von der Seite des Fremden (,die Anderen') auf die Seite des Ähnlichen (,Wir') gelangt. In der amerikanischen Migrationsgeschichte hat dies beispielsweise für die irische Gruppe stattgefunden, die zunächst außerhalb des anglo-saxonischen ,Mainstreams' verortet und erst nach einem langwierigen Prozess als Teil des ,Mainstreams' betrachtet wurde. ${ }^{41}$,Boundary Blurring' schließlich bezeichnet den weitreichendsten der drei Prozesse, bei dem die Grenze selbst sich verändert, indem sie ,schwammig' wird und so mehrdeutige Lokalisierungen eines Individuums in Bezug auf die Grenze zulässt (Bauböck 1995; Zolberg/Woon 1999). Bei diesem Prozess verändern sich die Kriterien, anhand derer Markierungen als fremd oder ähnlich vorgenommen werden: Einige verlieren an Bedeutung wohingegen andere an Gewicht zunehmen. Zum Beispiel hat in den USA Religion als Kriterium der Fremdheitszuschreibung in den letzten fünfzig Jahren an Schärfe abgenommen. Differenzen bestehen weiter, werden jedoch nicht länger als Kristallisationspunkt für Fremdheitsmarkierungen verwendet. ${ }^{42}$ Im ,schwammigen' Bereich wird eine Fremdheits- oder Ähnlichkeitszuschreibung nicht mehr anhand eines Kriteriums vorgenommen. Stattdessen löst sich die Dichotomie in eine Multiplexität von sich teilweise überschneidenden, aber auch widersprüchlichen Gruppenzugehörigkeiten auf.

Welche Vorteile bergen diese sozialkonstruktivistischen Integrationsprämissen, gemäß denen Integration als Zunahme der wahrgenommenen Ähnlichkeit der migrantischen Bevölkerung auf Seiten der Dominanzbevölkerung verstanden wird, gegenüber den zuvor behandelten klassischen Integrationstheorien?

1. Wo vorherige klassische Theorien (Park; Warner und Srole) Anpassung und Integration als unvermeidlichen, wenn auch langwierigen Prozess verstanden, verweist die so-

\footnotetext{
${ }^{41}$ Diesen Prozess zeichnet Ignatiev in seinem Buch How the Irish Became White nach (Ignatiev 1995).

${ }^{42}$ Allerdings ist dieser Trend nach den Anschlägen des 11. September 2001 zum Stoppen gekommen.
} 
zialkonstruktivistische Auffassung auf die Labilität der wahrgenommenen Ähnlichkeit. Diese kann sowohl zu- als auch abnehmen: So werden beispielsweise Menschen arabischer Herkunft in ,westlichen' Staaten seit den Anschlägen des 11. September 2001 stärker als fremd wahrgenommen als vor den Terrorakten (Deane/Fears 2006; CBS 2006). Integration ist keine Einbahnstraße, bei der Veränderungen nur zu einer vergrößerten und nicht zu einer verminderten Ähnlichkeitswahrnehmung führen können. Barry spricht deswegen auch von Integration als „two-way street” (Barry 2001: $85)$.

2. Integration wird nicht länger wie bei Warner und Srole, Gordon sowie Esser und Heitmeyer als ausschließliche Anpassungsleistung der MigrantInnen verstanden, sondern als ein Prozess, der in allen gesellschaftlichen Gruppen, auch der Nichtmigrantenbevölkerung, stattfindet. Zum einen wandeln sich die Einstellungen und Praktiken der Dominanzgesellschaft ohnehin über die Zeit hinweg, zum anderen müssen sie sich verändern, um die Aufnahme von MigrantInnen zu ermöglichen, d.h. die Dominanzgesellschaft muss MigrantInnen ein Regelwerk und ein institutionelles Gefüge anbieten, das deren prinzipiellen Einschluss, ihre Anerkennung und relative Diskriminierungsfreiheit ermöglicht. ${ }^{43}$

3. Diese neue Konzeptionalisierung eröffnet zudem die Möglichkeit, der Vielfalt auf Seiten der Dominanzbevölkerung gerecht zu werden: Statt um objektive Kriterien der Ähnlichkeit geht es um auf Normen basierende Wahrnehmung. Es wird also nicht länger davon ausgegangen, dass die nicht-migrantische Bevölkerung auf jeden Fall durch einen herrschenden Konsens integriert sei.

4. Dies bedeutet auch, dass im Gegensatz zu den klassischen Integrationstheorien und denen von Esser und Heitmeyer kulturelle Unterschiede nicht als objektiv gegeben betrachtet werden, sondern als Ergebnis eines intersubjektiven Aushandlungsprozesses. Damit muss aber auch eine Verankerung in mehr als einer Kultur nicht zwangsläufig zu Zerrissenheit und Problemen führen.

In den folgenden Kapiteln werden drei aus der US-amerikanischen Diskussion herausgefilterten Theorien vorgestellt, die, wie sich zeigen wird, mit dem hier vorgestellten Integrationskonzept kompatibel sind. Diese Theorien stehen grundsätzlich in Konkurrenz zueinander. Da sie jedoch von verschiedenen Kontexten ausgehen, ergänzen sie sich, insofern diese Rahmenbedingungen auf verschiedene Migrantengruppen unterschiedlich stark zutreffen.

\footnotetext{
${ }^{43}$ Wenn zum Beispiel die Integration einer muslimischen Migrantengruppe erwünscht ist, dann darf die Dominanzbevölkerung nicht eine Leitkultur aufstellen, die die Werte des Christentums einfordert, ohne diese explizit auszuformulieren oder universell zu begründen.
} 


\subsection{Strömungen der US-amerikanischen Integrationsdebatte}

In der US-amerikanischen Forschung begann Anfang der 1990er Jahre eine lebhafte Diskussion über die Kinder der nach Hautfarbe und Herkunftsregion vielfältigen ImmigrantInnen seit 1965. Im Folgenden wird diese Debatte strukturiert, indem die drei Hauptströmungen der gegenwärtigen Auseinandersetzung über die Integrationschancen und Herausforderungen dieser ,neuen' MigrantInnen herausgearbeitet und als drei konkurrierende Integrationstheorien gebündelt werden. Diese drei Theorien werden systematisiert dargestellt und auf die Anforderungen der sozialkonstruktivistischen Integrationsprämissen aus Kapitel 2.3 abgestimmt.

In der Vorstellung der herausgearbeiteten Theorien wird jeweils zuerst eine Verortung in der akademischen Debatte vorgenommen. An welche Stränge knüpft die Theorie an? Welche Kritiken greift sie auf? Und was sind ihre wissenschaftstheoretischen Ziele? Es folgt eine Diskussion des der Theorie zugrunde liegenden Integrationsbegriffs. Darauf wird beleuchtet, auf welche gesellschaftlichen Veränderungen der letzten Jahre die Theorie reagiert: Welche institutionellen und gesellschaftspolitischen Entwicklungen werden von ihr hervorgehoben? Abschließend wird erläutert, wie gemäß der herausgearbeiteten Theorie der Integrationsprozess abläuft und welches Integrationsmuster danach erwartbar ist.

\subsubsection{Transnational-pluralistische Integration}

\section{Verortung}

Theorien eines transnationalen Pluralismus greifen auf die durch die Globalisierung entstehenden Möglichkeiten für verstärkte transnationale Kontakte zurück und beschreiben, wie dadurch auch für MigrantInnen in der zweiten Generation ein pluralistisches Leben mit transnationalen Bezügen in den Aufnahmeländern möglich wird. In wissenschaftlicher Hinsicht entstanden transnationale Theorien als eine Strömung im Forschungsbereich der Internationalen Beziehungen, wo sie die sich vermehrenden Beziehungen und Netzwerke nicht nur zwischen staatlichen Akteure sondern auch zwischen gesellschaftlichen Gruppen erfassen sollen (Haas 1992; Risse 2002). Transnationalen Theorien der Internationalen Beziehungen zufolge entsteht ein zivilgesellschaftlicher Raum über staatliche Grenzen hinweg in den Bereichen Wirtschaft, Kultur und Wissenschaft. Während die ersten Ansätze (Deutsch 1957, Keohane/Nye 1972) sich auf Verbindungen zwischen Ländern der ersten Welt konzentrierten, gerieten mit der Globalisierungsdebatte zunehmend Schwellen- und Entwicklungsländer in den Blick. Parallel dazu wuchs Ende der 1980er und Anfang der 1990er Jahre in der Anthropologie das Interesse an transnationalen Fragestellungen (Glick Schiller, et al. 1992a; Basch, et al. 1994; 
Appadurai 1996; Wakeman 1988). Beide Entwicklungen griffen Ende der 1990er Jahre auf die Migrationsforschung über (Guarnizo 2001, Faist 2000c, Faist 2004a, Itzigsohn, et al. 1999, Levitt 2001, Portes, et al. 1999). Dabei stand zunächst die Frage im Vordergrund, ob transnationale Migrationsprozesse ein neuartiges oder ein altbekanntes Phänomen seien (Lauria-Perricelli 1992: 251; Foner 1997; Smith 2003). Nachdem sowohl Kontinuitäten als auch Neuartigkeiten, z.B. durch den größeren Umfang der aktuellen transnationalen Migrationsströme, herausgearbeitet worden sind, geht es nun um die Auswirkungen transnationaler Migration in den Herkunftsregionen: Wie beeinflusst der Transfer von Geld und Normen ${ }^{44}$ die Entwicklung im Herkunftsland (Levitt 2001)? Und wie versuchen Emigrationsländer ihre transnationalen MigrantInnen zu unterstützen und zu beeinflussen (Faist 2004b: 343f)? Untersucht werden auch die spiegelbildlichen Konsequenzen im Aufnahmeland wie verstärkter bilateraler Handel und Wachstum in bestimmten Wirtschaftsnischen (Faist 2004a). Vor allem aber interessiert die Frage, wie transnationale Netzwerke und Räume aussehen und funktionieren (Faist 2004a).

Die Frage, wie Transnationalismus Integrationsprozesse beeinflusst, wurde lange Zeit nicht untersucht. Es wurde angenommen, dass transnationale Migrationsströme das Ende von Integration und kultureller Anpassung bedeuten. In dieser Sichtweise wird Transnationalismus zum Gegenparadigma der vorherrschenden Integrationstheorien. So argumentiert Pries, man müsse den Integrationsfokus verlassen und sich auf das neue Verhältnis von sozialem und geografischem Raum konzentrieren (Pries 1997). Ein anderer Strang der Transnationalismusforschung hält transnationale Räume für ein dauerhaftes Phänomen; sie würden auch von der im Aufnahmeland verwurzelten zweiten Generation genutzt (Morawska 2003). Aus dieser Perspektive kann Transnationalismus zur Erklärung der Integration von MigrantInnen in die Aufnahmegesellschaften herangezogen werden, auch wenn sich eine transnational pluralistische Integration anders gestaltet, als die klassischen Integrationstheorien dies vermuten (Levitt 2001; Levitt/Waters 2002a; Portes, et al. 1999; Benton 2003).

Gemäß diesem transnational pluralistischen Forschungsstrang nutzen die MigrantInnen die Ressourcen des transnationalen Raums, um auf diesem alternativen Weg einen gesicherten sozioökonomischen Status zu erlangen. Publikationen spezifisch zur Integration der zweiten Generation aus transnationalistischer Sicht gibt es jedoch erst seit kurzem (z.B. Levitt/Waters 2002a, Smith 1998, Vasquez/Gomez 2001).

\footnotetext{
${ }^{44}$ Für letztere prägte Peggy Levitt analog zu den ,economic remittances' den Begriff der ,social remittances' (Levitt 2001).
} 
Wie unterscheiden sich diese transnational pluralistischen Integrationstheorien von der Theorie des Multikulturalismus? Dem Multikulturalismus zufolge kann Integration von MigrantInnen nur über eine Stärkung der, ethnischen' Gemeinschaften und deren Integration stattfinden. Er macht präskriptiv die Beibehaltung der verschiedenen Kulturen zur Pflicht. Transnational pluralistische Theorien dagegen halten den transnationalen Raum für einen alternativen Weg zum Ressourcenerwerb, der besonders dann wichtig wird, wenn sonstige Wege verschlossen sind. Es gibt jedoch weder eine implizite Annahme der Höherwertigkeit der Herkunftskultur noch die Annahme, dass man erst in einer Kultur gefestigt sein muss, bevor man sich anderen Kulturen öffnen kann. Für transnational-pluralistische Theorien sind unterschiedliche Kulturen kein Wert an sich, sondern lebendige Systeme, die sich über die Zeit wandeln.

\section{Definition von Integration}

Transnationaler Pluralismus bezieht sich auf ,the processes by which immigrants forge and sustain multi-stranded social relations that link together their societies of origin and settlement" (Glick Schiller, et al. 1992b: 7). Diese sozialen Beziehungen werden selbst dann unterhalten, wenn die MigrantInnen langfristig im Aufnahmeland leben. Sie finden im wirtschaftlichen, politischen und soziokulturellen Bereich statt, ihre Institutionalisierung variiert (Portes, et al. 1999: 222). Die dadurch entstehenden transnationalen ${ }^{45}$ Räume bezeichnen ,,verdichtete ökonomische, politische und kulturelle Beziehungen zwischen Personen und Kollektiven, die Grenzen von souveränen Staaten überschreiten. Sie verbinden Menschen, Netzwerke und Organisationen in mehreren Orten über die jeweiligen Staatsgrenzen hinweg. Eine hohe Dichte, Häufigkeit, eine gewisse Stabilität und Langlebigkeit kennzeichnen diese Beziehungen unterhalb bzw. neben der Regierungsebene“ (Faist 2000a: 10).

Mit dieser Definition grenzt sich die vorliegende Arbeit von engen TransnationalismusVerständnissen ab, die auf kontinuierliche Pendelbewegungen abzielen. ${ }^{46}$ Stattdessen geht es um die Existenz eines transnationalen Raumes mit herkunftsgruppenbezogenen Netzwerken,

\footnotetext{
${ }^{45}$ Faist zieht den Begriff des transstaatlichen Raumes vor, um darauf zu verweisen, dass es sich um einen Raum in und zwischen Staaten handelt, bei dem Nationen keine besondere Bedeutung zukommt (Faist 2000a: 14).

${ }^{46}$ So definiert z.B. Portes Transnationalismus als Engagement in ,occupations and activities that require regular and sustained social contacts over time across national borders for their implementation"; danach sind weder Geldtransfers noch Hausbesitz relevante Kriterien für Transnationalismus (Portes, et al. 1999: 219). Die selbe Unterscheidung in engen und breiten Transnationalismus wird auch von Itzigsohn vorgenommen: ,Transnationalism in a ,narrow' sense refers to those people involved in economic, political, social or cultural practices that involve a regular movement within the geographic transnational field, a high level of institutionalization, or constant personal involvement. Transnationality in a ,broad' sense refers to a series of material and symbolic practices in which people engage that involve only sporadic physical movement between the two countries, a low level of institutionalization, or just occasional personal involvement, but nevertheless includes both countries as reference points." (Itzigsohn, et al. 1999: 323)
} 
welche als Referenzpunkt fungieren und Ressourcen für ein pluralistisches Leben im Aufnahmeland bereithalten. Die sich daraus möglicherweise ergebenden sozioökonomischen Statusgewinne für die zweite Generation werden in Interaktionen mit der Dominanzbevölkerung als Ressourcen eingesetzt und vergrößern bei Abwesenheit einer starken kulturellen Fremdheitsmarkierung die wahrgenommene Ähnlichkeit. Das genaue Ausmaß und die Nachhaltigkeit transnational pluralistischer Aktivitäten der zweiten Generation bleiben offen; sie fallen jedoch wahrscheinlich hinter das Engagement der Eltern zurück.

Laut den transnational pluralistischen Theorien können transnationale Verbindungen den Integrationsprozess positiv beeinflussen, da sie Ressourcen bieten, die MigrantInnen helfen, ihre sozioökonomischen, kulturellen, politischen und Identitätsbedürfnisse zu erfüllen, und so u.a. zu einem gesicherten sozioökonomischen Status führen können (Portes 1999: 469; Portes, et al. 1999: 229). Zu diesen Ressourcen des transnationalen Raumes gehören Möglichkeiten für Handel, Dienstleistungsideen, spezifisches Wissen, Identitätsangebote sowie Netzwerke und Vereinigungen im Aufnahmeland (Portes, et al. 1999: 218; Zhou 2004). Die Beibehaltung und Entwicklung, ethnischer' Verbindungen wird als Pfad zur Ressourcenproduktion und verteilung betrachtet und nicht, wie vormals in den klassischen Theorien, als Hindernis für eine erfolgreiche Integration (Sanders 2002). Damit wenden sich transnational pluralistische Theorien auch gegen die alarmistische Argumentation von kulturalistischen Autoren wie Samuel Huntington. Huntington warnt vor einer, Überschwemmung' der Vereinigten Staaten durch lateinamerikanische EinwanderInnen und einer damit einhergehenden Bedrohung der Vorherrschaft des Englischen sowie dem Verlust der ,anglo-saxonischen' Werte (Huntington 2004). Den neuen MigrantInnen wirft Huntington vor, dass sie weder bereit seien, Englisch zu lernen und ihre spanische Muttersprache aufzugeben, noch dazu, Werte wie Pflichtbewusstsein, Ehre und individuelles Erfolgsstreben zu übernehmen. Stattdessen pendelten sie häufig zwischen den USA und ihrem Herkunftsland bzw. dem Herkunftsland der Eltern und verbrächten einen Großteil ihres Lebens in den USA in herkunftsgruppenbezogenen Netzwerken. (Huntington 2004) Dagegen argumentieren pluralistische TheoretikerInnen, dass das Leben in transnational pluralistischen Strukturen mittelfristig zu einer gleichberechtigteren Teilhabe am amerikanischen Leben führen könne (Portes 1999: 472).

\section{Gesellschaftlicher Kontext}

Kennzeichen des aktuellen Migrationsumfeldes ist, dass Immigration in die USA heute kontinuierlich stattfindet. Jedes Jahr kommen neue MigrantInnen, die einen anhaltenden Zufluss in die ,ethnischen Gemeinschaften' in den Aufnahmeländern garantieren. Zuwanderungsstopps, 
wie z.B. 1924 in den USA, gibt es aktuell nicht (Faist 2000b: 341). ${ }^{47}$ Zudem pluralisieren sich kulturelle Normen und Lebensstile in liberalen Gesellschaften der, westlichen' Welt, was die Etablierung transnationaler Verbindungen erleichtert (Faist 2000a: 43).

Damit ein transnationaler Pluralismus funktionieren kann, müssen eine Reihe von Bedingungen erfüllt sein: Moderne Informationstechnologien, Satelliten- oder Kabelfernsehen, Telefonverbindungen und Fernreisen müssen einfach verfügbar und relativ kostengünstig sein, so dass MigrantInnen langfristig im Kontakt mit den Herkunftsorten ihrer Eltern bleiben und über die dortigen Entwicklungen informiert sein können (Portes, et al. 1999: 223; Faist 2004a; Glick Schiller, et al. 1997: 126). Zudem müssen zwischen Aufnahme- und Sendeland zumindest freundliche Beziehungen bestehen, damit Waren, Dienstleistungen und Personen frei ausgetauscht werden können. Bilaterale Verträge, die zum Beispiel die Anerkennung von Schulabschlüssen regeln, fördern transnationale Verhaltensmuster (Faist 2000b; Faist 2004a). Jegliche Institutionalisierung transnationalen Kapitals der ersten Generation fördert auch in der zweiten Generation einen transnational pluralistischen Lebensstil.

\section{Integrationsprozess}

Unter diesen Kontextbedingungen entwickeln MigrantInnen der zweiten Generation transnational pluralistische Lebensweisen, wenn die Möglichkeiten des transnationalen Raumes und der herkunftsgruppenbezogenen Gemeinschaft die des ,Mainstreams' überragen. Dies ist besonders dann der Fall, wenn (a) das Herkunftsland gro $\beta$ ist und viele Chancen bietet, (b) die Möglichkeiten des ,Mainstreams' stark limitiert sind oder (c) der transnationale Raum gro $\beta$ ist, da es eine umfangreiche Migrantenbevölkerung aus dem Herkunftsland gibt und enge wirtschaftliche Verflechtungen zwischen beiden Ländern/Regionen bestehen (Faist 2004a: 28ff; Portes, et al. 1999: 231; Waldinger/Fitzgerald 2004: 1177, 1185). MigrantInnen der zweiten Generation werden in diesen Fällen versuchen, ihre Bedürfnisse nach sozioökonomischem Aufstieg, Anerkennung und Identitätsbestimmung im transnationalen Raum und in ,ethnischen Netzwerken' zu befriedigen (Portes, et al. 1999: 229, Portes 1999: 469). Das dort vorhandene soziale, ökonomische und kulturelle Kapital erlaubt ihnen den Zugang zu sozioökonomischen Ressourcen, Informationen, sozialer Unterstützung, Vertrauen und Anerkennung. Die in der herkunftsgruppenbezogenen Gemeinschaft vorhandenen Normen ${ }^{48}$ und die damit einhergehende soziale Kontrolle können bei der Überwindung von Problemen in der

\footnotetext{
${ }^{47}$ Der Johnson-Reed Act von 1924 führte nationale Herkunftsquoten ein und beschränkte die jährliche Immigration in die USA auf 154000 Personen. Die Immigration aus der, westlichen' Hemisphäre (d.h. Kanada, Mexiko und anderen lateinamerikanischen Staaten) blieb unbeschränkt. (Alba, et al. 1994: 168f)

48 Diese Normen müssen nicht zwangsläufig mit denen des Herkunftslandes identisch sein. Levitt weist darauf hin, dass sie sich im Migrationsprozess verändern können (Levitt 2001: 54).
} 
Kindererziehung oder in einer Partnerschaft helfen. In diesem Sinne stellt der transnationale Raum ,Coping'-Strategien für die Lebensgestaltung zur Verfügung. (Portes 1999: 469) Durch all diese Mechanismen kann es transnational orientierten MigrantInnen gelingen, Status zu gewinnen und sie zunächst benachteiligende Stratifikationsregime zu überwinden (Goldring 1997).

Wenig diskutiert wird in transnational pluralistischen Untersuchungen allerdings die Frage, ob eine solche ,ethnische Ökonomie ${ }^{49}$ sozialen Aufstieg nachhaltig ermöglichen kann. ${ }^{50}$ Übersetzen sich diese durch den transnationalen Pluralismus erworbenen Ressourcen in den Interaktionen mit der Dominanzbevölkerung auch in eine vergrößerte Ähnlichkeitswahrnehmung? Erst dann wäre gemäß den hier angewandten Prämissen Integration erzielt. Oder kann die Markierung als statusgleich in den Interaktionen keine Wirkung erzielen, weil anhand anderer Kriterien eine Markierung als kulturungleich vorgenommen wird, die alle anderen Faktoren dominiert? Das Funktionieren transnational pluralistischer Integrationsmuster wird also vom Grad des Rassismus in der Gesellschaft abhängen und davon, welche Kriterien der Fremdheitsmarkierung schon ,schwammig' geworden sind und welche weiterhin für klare Grenzziehungen sorgen.

\section{Integrationsmuster}

Gelingt eine transnational pluralistische Integration, dann zeigt sich das folgende Muster: Die MigrantInnen der zweiten Generation besitzen sehr gute Kenntnisse der Kultur und Sprache des elterlichen Herkunftslandes. Pluralistisch integrierte Familien leiden daher weniger unter Konflikten zwischen Kindern und Eltern als andere Migrantenfamilien und sind stärker in herkunftsgruppenbezogene Netzwerke eingebunden. Gleichzeitig verfügen MigrantInnen der zweiten Generation aber zumindest über hinreichende Kenntnisse der Aufnahmegesellschaft, um sich in dieser erfolgreich zu bewegen, wenn ihre pluralistischen Aktivitäten dies verlangen. Bikulturelle Kompetenzen sind weit verbreitet. Wichtig ist, dass die MigrantInnen laut dieser Theorie keinesfalls eine Rückkehrperspektive beibehalten müssen. ,Institutional completeness' (Breton 1964), d.h. das Vorhandensein einer herkunftsgruppenbezogenen Gemeinschaft, die alle notwendigen Dienstleistungen anbietet, erlaubt den MigrantInnen, sich trotz eindeutiger räumlicher Verankerung im Aufnahmeland in einer Reihe von transnationalen Aktivitäten zu engagieren. (Levitt/Waters 2002b: 20)

\footnotetext{
${ }^{49}$ Zum Konzept der ,ethnischen Ökonomie' siehe Light 1972; Light, et al. 1994; Bonacich 1987 und für einen neueren Überblick Fong/Ooka 2002.

${ }^{50}$ Für die Diskussion der vielseitigen Konsequenzen einer ,ethnischen Ökonomie', die auch diese Frage kurz anspricht, vergleiche Zhou 2004.
} 
Im Einzelnen sind also sehr gute Kenntnisse der Hochsprache des Herkunftslandes der Eltern zu erwarten. Der Umfang der Sprachkenntnisse des Aufnahmelandes variiert mit dem ergriffenen Beruf und der institutionellen Vollständigkeit der Gemeinschaft. (Portes/Rumbaut 2001: 54) Freundschaften werden überwiegend mit Personen der gleichen Herkunftsgruppe unterhalten (Portes/Rumbaut 2001: 54), und auch Partnerschaften gehen pluralistische MigrantInnen der zweiten Generation häufig mit Personen aus dem Herkunftsland der Eltern ein. Hinsichtlich der Bildungsabschlüsse erzielen MigrantInnen der zweiten Generation mit starken transnationalen Netzwerken bessere Ergebnisse segregiert lebenden MigrantInnen. Ihr Erfolg hängt dabei von den transnational pluralistischen Normen hinsichtlich der Bedeutung von Bildung ab, z.B. davon, ob für Jungen und Mädchen unterschiedliche Bildungsnormen gelten. Denn das ,ethnische Sozialkapital' und die starke Kontrolle der Kinder durch die Eltern stärken alle elterlichen Normen, auch bildungsabwertende und geschlechtsdiskriminierende.

Weiterhin geht der positive Effekt des transnationalen Netzwerkes auf die im transnationalen Raum verfügbaren Ressourcen zurück, welche durch die Ausstattung der jeweiligen Mitglieder und ihrer sozialen Lage bestimmt werden. Transnational pluralistische Theorien nehmen jedoch an, dass sich auch in einem ressourcenmäßig schlecht ausgestatteten Raum noch positive Effekte erzielen lassen, besonders wenn die Institutionen und Netzwerke des Aufnahmelandes den MigrantInnen vergleichsweise wenig Chancen bieten. (Portes/Rumbaut 2001: 48) Beruflich sind pluralistisch integrierte MigrantInnen der zweiten Generation v.a. in einer ,ethnischen Ökonomie' beschäftigt, z.B. im Import/Exportbereich, Einzelhandel, Dienstleistungssektor und der Gastronomie. ${ }^{51}$

\section{Getrennte Möglichkeiten, aber gleiche Ergebnisse?}

Transnational pluralistische Integrationstheorien behaupten, dass der transnationale Raum alternative Ressourcen bereithält, die MigrantInnen der zweiten Generation einen gesicherten sozioökonomischen Status ermöglichen. Die individuell akquirierten Ressourcen werden in solche Interaktionen mit der Dominanzbevölkerung überführt, bei denen eine hohe Wahrscheinlichkeit besteht, dass sie von dieser als statusgleich markiert werden. Der transnationale Raum inklusive herkunftsgruppenbezogener Netzwerke im Aufnahmeland kompensiert also

\footnotetext{
${ }^{51}$ Eine transnationale Lebensweise stellt für MigrantInnen der zweiten Generation sowohl eine Chance als auch eine schwierige Herausforderung dar: Sie profitieren von erweiterten wirtschaftlichen, politischen und kulturellen Möglichkeiten, können sich aber auf der anderen Seite davon überfordert fühlen, ihren Weg in dem komplexen Beziehungsnetz finden zu müssen. Transnational pluralistische Theorien sollten einen transnationalen Lebensstil daher nicht verklären (Vertovec 1999).
} 
für dort möglicherweise bestehende Nachteile. Die alternativen Ressourcen stehen allerdings nur für diejenigen MigrantInnen der zweiten Generation zur Verfügung, die sich kulturell nicht vollständig an die Normen des Aufnahmelandes anpassen oder zumindest eine bikulturelle Kompetenz bewahren. Der ausschlaggebende Mechanismus für Integration, also für eine vergrößerte Ähnlichkeitswahrnehmung auf Seiten der Dominanzbevölkerung, ist ein sozioökonomisch gesicherter Status. Der transnationale Pluralismus behauptet somit, dass über getrennte Möglichkeiten gleiche Ergebnisse entstehen können.

\subsubsection{Abwärts-Integration}

\section{Verortung}

Während sowohl die klassischen Integrationstheorien als auch die Theorie des transnationalen Pluralismus Integration für positiv halten, wird in einer anderen Strömung, die hier als Abwärts-Integration zusammengeführt wird, ein Pfad beschrieben, bei dem die Integration der MigrantInnen der zweiten Generation in ein bestimmtes Segment der Gesellschaft zu verringerten Lebenschancen und somit zu einem normativ gesehen negativen Ergebnis führt (Gans 1992; Portes/Zhou 1993; Portes/Rumbaut 1996; Portes 1996; Portes/Rumbaut 2001). Die Theorie der Abwärts-Integration erwächst aus der Diskussion um die soziale Polarisierung der Gesellschaft, die Tertiarisierung der Wirtschaft und die Benachteiligungen, denen ,schwarze' MigrantInnen, auch aus Lateinamerika, in den USA ausgesetzt sind. Sie grenzt sich damit von einem positiven Bild der idealtypischen Integration in die Mittelschicht ab und zeichnet ein ambivalentes Bild des Aufnahmelandes. Diese gesellschaftlichen Veränderungen unterscheiden gemäß der Theorie der Abwärts-Integration grundsätzlich die Integrationspfade früherer Migrantengruppen von den Wegen, die die Kinder von MigrantInnen nach 1965 in den Vereinigten Staaten heute einschlagen.

\section{Definition}

Laut Portes durchläuft die zweite Generation einen Prozess der „segmented assimilation where outcomes vary across immigrant minorities and where rapid integration and acceptance into the American mainstream represent just one possible alternative" (Portes/Rumbaut 2001: 45). Abwärts-Integration beschreibt den $\mathrm{Pfad}^{52}$, auf dem MigrantInnen der zweiten Generation hinter die Errungenschaften ihrer Eltern zurückfallen und Teil einer einheimischen ,rain-

\footnotetext{
${ }^{52}$ Zur Theorie der ,Segmented Assimilation' gehören noch zwei weitere Pfade: , upward assimilation combined with biculturalism' und ,mostly upward assimilation; blocked at times by discrimination' (Portes/Rumbaut 2001: 63). Diese überschneiden sich jeweils in Teilen mit transnational-pluralistischen und neoklassischen Theorien.
} 
bow underclass ${ }^{53}$ werden. Sie teilen nicht länger das Erfolgsstreben ihrer Eltern und deren Bereitschaft, zurückzustecken, sondern nehmen die ,Oppositionskultur' innerstädtischer Ghettos an. (Portes/Rumbaut 2001: 45) Die Frage ist somit nicht länger, ob diese MigrantInnen der zweiten Generation sich integrieren, sondern in welches Segment der amerikanischen Gesellschaft sie sich integrieren (Portes/Rumbaut 2001: 55).

\section{Gesellschaftlicher Kontext}

Die Theorie der Abwärts-Integration soll die Integrationsergebnisse bestimmter Migrantengruppen unter den Kontextbedingungen von Rassismus, zunehmender Spaltung des amerikanischen Arbeitsmarkts und der Existenz einer marginalisierten Bevölkerung in den Innenstädten erklären (Portes/Rumbaut 2001: 55). ${ }^{54}$

Trotz Bürgerrechtsbewegung, ,equal opportunities' und ,affirmative action' hält laut Portes Rassismus in den USA an (Portes/Rumbaut 2001: 55). Rassistische Diskriminierung wird besonders im Fall der doppelten Benachteiligung (d.h. arm und ,schwarz') virulent. Es lässt sich zudem feststellen, dass die Trennlinie zwischen den ,Rassen' sich von ,Weiß versus Nichtweiß' zu ,Nichtschwarz versus Schwarz' verschiebt (Waters 1999: 344). Wo z.B. asiatische MigrantInnen vormals nicht als, weiß' wahrgenommen wurden, werden sie nun als Teil der dominanten ,nichtschwarzen' Bevölkerung aufgefasst, während sich für dunkelhäutige MigrantInnen die Wahrnehmung als Teil der US-amerikanischen ,Schwarzen' verfestigt und sie entsprechende Diskriminierung erleben.

Im Zuge der Globalisierung entwickelt sich die Wirtschaft weg von industrieller Produktion hin zu postindustrieller Dienstleistung. Dadurch sinken die Arbeitsmöglichkeiten für Ungelernte oder Niedrigqualifizierte und die Schere zwischen Niedrig- und Hochlohnsektoren geht weiter auf (Wilson 1987: 142). Die meisten Jobs im Dienstleistungssektor setzen eine Reihe von ,soft skills' voraus (Teamfähigkeit, Kundenkontakt, Höflichkeit, sprachliche Fertigkeiten) und speziell bei den schlechter bezahlten Dienstleistungen zudem die Bereitschaft, sich unterzuordnen und die Kunden mit Zuvorkommenheit zu behandeln, unabhängig davon, wie diese sich verhalten. ${ }^{55}$

Während es viele Jahre lang möglich war, sich in einem Unternehmen hochzuarbeiten und so trotz geringer Bildung sozial aufzusteigen, werden in der heutigen ,Hourglass economy’ hö-

\footnotetext{
${ }^{53}$ Mit dem Begriff des Regenbogens soll erfasst werden, dass sich in diesem Gesellschaftssegment Menschen unterschiedlicher Herkunftsgruppen und Hautfarben wiederfinden.

${ }^{54}$ Für eine Diskussion dieser sozioökonomischen Veränderungen in Europa siehe (Castel 2000).

${ }^{55}$ Schneider stellt in seinem Buch zu New Yorker Jugendbands dar, wie die Anforderung ,femininen', wenn nicht gar untertänigen Verhaltens in vielen Dienstleistungsjobs von männlichen Angehörigen sozial marginalisierter Schichten als eine Bedrohung ihrer Ehre und Männlichkeit betrachtet wird (Schneider 1999).
} 
here Positionen in Unternehmen von außen besetzt. Niedrigqualifizierte und schlecht bezahlte Jobs bieten heute kaum mehr Möglichkeiten für Aufstieg und Verbesserung (Kazis/Miller 2001). Die Umstrukturierung der Wirtschaft mit dem Verlust von klassischen Arbeiterjobs (,blue collar') und der zunehmenden Bedeutung von formaler Bildung hat zudem eine Gruppe von ArbeitnehmerInnen entstehen lassen, deren Familien trotz regelmäßiger Erwerbstätigkeit in Armut leben. Die Jobs dieser , working poor ${ }^{56}$ werden schlecht entlohnt, sind prekär und instabil, enthalten keine zusätzlichen Leistungen (z.B. Krankenversicherung) und verlangen flexible Arbeitszeiten. Darüber hinaus verschaffen diese Jobs den ArbeitnehmerInnen keinerlei sozialen Status; im Gegenteil, vielen dieser niedrigqualifizierten Jobs haftet ein soziales Stigma an. Die ,working poor' werden dabei sogar von zwei Seiten diskriminiert: Sie werden weder von arbeitslosen NachbarInnen noch von ArbeitnehmerInnen der Mittelklasse akzeptiert. (Newman 1999: 93ff)

Neben anhaltendem Rassismus und den Veränderungen auf dem Arbeitsmarkt kommt einer weiteren Veränderung eine bedeutsame Rolle zu: Die soziale Durchmischung der amerikanischen Innenstädte hat seit der Bürgerrechtsbewegung und dem Wegzug der schwarzen Mittelschicht abgenommen. Seitdem konzentriert sich in den benachteiligten Stadtvierteln eine arme ,schwarze’ Bevölkerung, die Wilson ,urban underclass' nennt: „Included in this group are individuals who lack training and skills and either experience long-term unemployment or are not members of the labor force, individuals who are engaged in street crime and other forms of aberrant behavior, and families that experience long-term spells of poverty and/or welfare dependency." (Wilson 1987: 8) Dabei ist für Wilson die räumliche Segregation nicht nur Ausdruck der verschiedenen Lebenslagen, sondern zugleich Ursache des marginalisierten Status (Wilson 1980; Wilson 1987; Wilson 1996): Menschen, die in einer Nachbarschaft leben, deren Bevölkerung zum größten Teil sozial benachteiligt ist, werden qua sozialräumliche Effekte selbst vom sozialen Leben ausgeschlossen, erhalten kaum Informationen über Arbeitsmöglichkeiten und werden erst recht nicht für konkrete Jobs empfohlen (Wilson 1987: 57). Zudem fehlen ihnen Rollenvorbilder (Wilson 1987: 144). Die BewohnerInnen dieser Stadtviertel haben im Durchschnitt weniger Sozialkapital und die sozialen Beziehungen, über die sie verfügen, bieten wenig Ressourcen, weil FreundInnen und Bekannte selbst niedrigqualifiziert und arbeitslos sind (Wacquant/Wilson 1989). Dabei sind es gerade lose Bekanntschaften mit Menschen, die in anderen Netzwerken verankert sind, welche wertvolle Informationen bieten (Granovetter 1973). Die Armut der innerstädtischen Bevölkerung ist also nicht nur eine mone-

\footnotetext{
${ }^{56}$ Unter , working poor' werden in den USA Familien verstanden, in denen die Erwachsenen mindestens die Hälfte des Jahres arbeiten und die dennoch nicht mehr als das Doppelte des auf Bundesebene festgelegten Armutsniveaus verdienen (Acs, et al. 2000: 6).
} 
täre, sondern auch eine soziale. Es fehlt der Kontakt mit Individuen und Institutionen des ,Mainstreams' (Wilson 1987: 60). ${ }^{57}$

Unter diesen sozialräumlichen Bedingungen bilden sich spezifische kulturelle Praktiken heraus, zu denen neben erlernter ,sozialer Ängstlichkeit ${ }^{58}$, niedrigem Selbstwertgefühl und geringer Kontrollüberzeugung auch eine Kultur der Opposition gegen die Werte des ,weißen Mainstreams' gehört. Obgleich diese kulturellen Faktoren zu einer Perpetuierung der schwierigen Lebensumstände beitragen, sind sie für Wilson nicht Auslöser, sondern Folge der Situation (Wilson 1987: 14): ,culture is a response to social structural constraints and opportunities" (Wilson 1987: 61). Kulturelle Werte entstehen aus bestimmten Umständen und reflektieren die Position ihrer Träger in der Klassenstruktur (Wilson 1987: 158). Für diese innerstädtischen Nachbarschaften gilt, dass sich die sozialen Problemlagen verfestigen und von einer Generation auf die nächste vererben. Sozialer Aufstieg ist diesen Gruppen zunehmend nachhaltig verwehrt.

\section{Integrationsprozess}

Wie läuft der Integrationsprozess unter diesen Bedingungen ab? Der Mechanismus von Abwärts-Integration ist ,dissonante Akkulturation': MigrantInnen der zweiten Generation lernen Englisch schneller als ihre Eltern und kommen in der Aufnahmegesellschaft besser zurecht als diese. Gleichzeitig verlernen sie die Sprache des Herkunftslandes der Eltern. Durch diese Situation entstehen in den Familien Fehlkommunikation und Missverständnisse. Verfügen die Eltern nicht über externe Ressourcen (FreundInnen, Bekannte, staatliche und private Hilfen), sind sie beim Treffen wichtiger Entscheidungen auf ihre Kinder angewiesen. Dies hat einen Rollentausch zur Folge, durch den die Kinder teilweise die Fürsorge für die Eltern übernehmen und der elterlichen Kontrolle entgleiten. (Portes/Rumbaut 2001: 49ff) Das Phänomen des Rollentausches hat besonders in armen, innerstädtischen Bezirken Konsequenzen, wo Jugendliche ohne Migrationshintergrund aus sozial marginalisierten Schichten die MigrantInnen der zweiten Generation negativ beeinflussen: Durch Sozialisierung übernehmen die MigrantInnen die feindliche Haltung, die viele ,schwarze' Jugendlichen ohne Migrationshintergrund in diesen Stadtvierteln gegenüber dem ,Establishment' haben (Portes/Rumbaut 1996: 248f). Diese

\footnotetext{
${ }^{57} \mathrm{Zu}$ den Problemen, die sich aus der wirtschaftlichen Umstrukturierung und ressourcenarmen Netzwerken ergeben, kommt die schlechte Qualität der Schulen in diesen Stadtvierteln, da die Schulen in den USA großenteils über das lokale Steueraufkommen finanziert werden (McGuire/Papke 2008). Dies bedeutet, dass die Bildungsinstitutionen in armen Nachbarschaften im Allgemeinen unterfinanziert sind und ihre Schüler dementsprechend nicht ausreichend fördern können.

${ }^{58}$ So Heinz Bude in seinem Vortrag beim 12. Haniel Forum Auf der Suche nach neuen Wegen, soziale Herausforderungen zu lösen am 24./25. November 2005 in der Haniel Akademie in Duisburg-Ruhrort.
} 
,Oppositionskultur ${ }^{59}$ toleriert kein Verhalten, das als typisch für eine ,weiße’ Mittelklasse wahrgenommen wird, sei es hinsichtlich der Sprache, der Kleidung, einer Teilnahme an ,weißen' Netzwerken oder der Ausübung sozialer Praktiken, die als Unterwerfungsgesten verstanden werden (Lundy 2003: 463). Ein Brennpunkt der Auseinandersetzung ist die Schule: Das Bildungssystem in den USA wird von vielen, schwarzen' Jugendlichen als Werkzeug zur Durchsetzung einer dominanten Kultur wahrgenommen, welche ihre eigene kulturelle Identität abwertet. Diese Gruppe von Jugendlichen entwickelt Verhaltensmuster, welche im Kontrast zu den vorherrschenden kulturellen Praktiken stehen und diese herausfordern. ,Acting white' ist dann ein schwerwiegender Vorwurf, den Angehörige dieser Gruppe ihren Peers machen, wenn sie vermuten, dass diese die dominanten Praktiken der, weißen' Mittelklasse übernehmen: „To behave in the manner defined as falling within a White cultural frame of reference is to ,act White' and is negatively sanctioned" (Fordham/Ogbu 1986: 181). ${ }^{60}$ Erfolg im amerikanischen Bildungssystem hängt aber von der Übernahme dieser Werte ab, und so wird im Umkehrschluss akademische Leistung selbst als, acting white' diffamiert (Fordham/Ogbu 1986). Die Kinder lernen, diese Leistung nicht zu erbringen (,learning not to learn“" (Suarez-Orozco 1987: 164)). ${ }^{61}$

Zum Misserfolg im Bildungssystem trägt auch eine Kultur der Armut (,culture of poverty’) bei (Lewis 1966; Lewis 1998; Lewis 1965): Arme Kinder lernen in ihren Familien nicht die kulturellen Muster kennen, welche für eine erfolgreiche Schulkarriere benötigt werden (z.B. sprachliche Fähigkeiten, Verhandlung mit Autoritäten, Selbstmotivierung). Zudem stehen einige der Eltern dem Bildungssystem kritisch gegenüber: Zwar wird der Wert von Bildung hochgehalten, doch befürchten einige Eltern gleichzeitig, dass ihre Kinder, wenn sie immer mehr Bildung erwerben, ihnen zunehmend fremd werden.

Durch diese Mechanismen erreichen MigrantInnen der zweiten Generation mit geringer Ressourcenausstattung in diesen Stadtvierteln nur niedrige formale Bildungsabschlüsse, was ih-

\footnotetext{
${ }^{59}$ Zur ,Oppositionskultur' siehe v.a. Fordham/Ogbu 1986. Eine Kritik und Weiterentwicklungen des Konzepts findet sich bei Gould 1999 und Lundy 2003. Letzterer weist vor allem darauf hin, dass dem Schulsystem ,weiße' Werte zugrunde liegen, es diese aber als rassen- und kulturmäßig neutral verkauft.

${ }^{60}$ Kritiker der Theorie der ,Oppositionskultur' führen an, dass das Protestverhalten ein vorübergehendes Phänomen sei: Es beschränke sich auf die Kinder- und Jugendjahre und werde mit zunehmendem Alter abgelegt (Sullivan 1989, Osterman 1980). Dies mag stimmen; es wäre aber ein Fehlschluss zu glauben, dass der Effekt der ,Oppositionskultur' damit auch zu Ende sei: Bei der zunehmenden Bedeutung formaler Bildung für den Zugang zu qualifizierten Arbeitsplätzen ist für die meisten jungen Menschen der Zug abgefahren, wenn sie mit 20 Jahren einen Nachholbedarf an schulischer Bildung konstatieren; die früheren Versäumnisse lassen sich nur schwer aufholen.

${ }^{61}$ Ogbu beschreibt darüber hinaus das geringe Bildungsstreben vieler ,Schwarzer' als funktionale und rationale Anpassung an ihre wahrscheinliche spätere Arbeitsposition. Da die meisten armen ,Schwarzen' nicht davon ausgehen können, einmal einen höherwertigen Beruf ergreifen zu können, sei ihre geringe Investition in Bildung nur rational und eine Anpassung an die realistischen Erwartungen hinsichtlich ihres zukünftigen Status. (Ogbu 1991)
} 
nen in den meisten Fällen nur sehr beschränkte Verdienstmöglichkeiten auf dem Arbeitsmarkt eröffnet. Es gelingt ihnen nicht, ihre in den USA entwickelten materiellen Ansprüche einzulösen (Portes/Zhou 1993: 85). Auf der anderen Seite ist die zweite Generation nicht länger willens, schlecht bezahlte Migrantenjobs auszuüben, wie dies ihre Eltern getan haben: „They come to the world of work with American standards, and may not even be familiar with the old country conditions. [...] Nor do they have the long-range goals that persuaded their parents to work long hours at low wages. [...] From their perspective, immigrant jobs are demeaning." (Gans 1992: 182)

Auf der Basis des niedrigen sozioökonomischen Status und der subkulturellen Anpassung wird eine vergrößerte Ähnlichkeitswahrnehmung auf Seiten der, einheimischen schwarzen’ Bevölkerung in diesen Stadtbezirken von Personen im Muster der Abwärts-Integration wahrscheinlich. Unterstützt wird dieser Prozess durch die in den Vereinigten Staaten wirkenden ,Rassendynamiken' mit anhaltendem Rassismus gegenüber ,Schwarzen'. ${ }^{62}$ Die Ähnlichkeitswahrnehmung stellt sich hier also bei einem Teil der Nicht-Migranten-Bevölkerung ein, der selbst marginalisiert ist. Mitglieder der Dominanzbevölkerung nehmen nicht eine vergrößerte Ähnlichkeit zu sich selber wahr, sondern zur ,einheimischen' marginalisierten sozialen Schicht.

Der Integrationspfad ist somit abhängig von der Immigrationsgeschichte der ersten Generation, der Geschwindigkeit der Akkulturation bei Eltern und Kindern, kulturellen und wirtschaftlichen Barrieren, mit denen die Kinder konfrontiert werden, sowie von den familiären sowie herkunftsgruppenbezogenen Ressourcen (Portes/Rumbaut 2001: 45). Abwärts-Integration betrifft vor allem Kinder von schwarzen Alleinerziehenden, die nur geringes Humankapital haben und nicht in ein ,ethnisches Netzwerk' eingebunden sind (Portes/Rumbaut 2001: 55).

\section{Integrationsmuster}

$\mathrm{Zu}$ welchen Integrationsmustern führt nun dieser Prozess? Bereits erwähnt wurden die geringen Bildungsabschlüsse dieser Gruppe und die hohe Zahl der Schulabbrecher. Die Eltern sind nicht in der Lage, ihre Kinder in deren schulischer Entwicklung zu unterstützen. Ihr niedriges formales Bildungsniveau trägt dazu bei, dass die zweite Generation kaum Zugang zu höher qualifizierten und damit besser bezahlten Jobs erhält; so arbeiten viele MigrantInnen der zweiten Generation aus dieser Gruppe in niedrigqualifizierten und schlechtbezahlten Jobs o-

\footnotetext{
${ }^{62}$ Waters analysiert, wie manche ,schwarze' MigrantInnen gezielt versuchen, eine Markierung als MigrantIn beizubehalten (über einen Akzent oder die Kleidung), um sich von ,schwarzen' AmerikanerInnen abzuheben und so weniger Diskriminierung zu erleben (Waters 1999).
} 
der sind arbeitslos. Der informelle Sektor mit seinem Versprechen von schnellem Geld übt eine große Attraktion aus. Zudem sind viele MigrantInnen der zweiten Generation von staatlichen Transferleistungen abhängig. Die Kinder, Jugendlichen und jungen Erwachsenen sprechen die Sprache des Aufnahmelandes fließend, allerdings häufig nicht die Hochsprache, sondern einen gruppenspezifischen Slang der Straße. Ihr schriftliches Ausdrucksvermögen ist begrenzt. In der Muttersprache der Eltern haben die meisten dieser MigrantInnen nur sehr beschränkte Kenntnisse. Konflikte zwischen den Generationen sind ausgeprägt.

FreundInnen und Bekannte kommen aus der nachbarschaftlichen Peergroup, der zumeist auch der/die PartnerIn entstammt. Dabei ist die Peergroup hinsichtlich der Ethnien und Nationalitäten gemischt, bezogen auf den sozioökonomischen Status allerdings homogen.

\section{Gleiche Möglichkeiten und doch ungleiche Ergebnisse?}

Während im Modell der Abwärts-Integration die MigrantInnen der zweiten Generation einem Segment der Bevölkerung ohne Migrationshintergrund ähnlicher werden, das selbst unter Diskriminierung leidet, verringern sich ihre relativen Entwicklungsmöglichkeiten. Sie erhalten also die gleichen Möglichkeiten wie die Nicht-Migrantenbevölkerung dieses Segments und erzielen doch im Vergleich mit der Gesamtbevölkerung ungleiche Ergebnisse. Es geht also nicht wie bei Hoffmann-Nowotny um eine ,Unterschichtung', bei der alle MigrantInnen sich in einer nur aus MigrantInnen bestehenden sozialen Schicht wiederfinden, die unterhalb aller anderen sozialen Schichten der Nicht-MigrantInnen liegt (Hoffmann-Nowotny 1973: 53). Portes zeichnet mit Abwärts-Integration vielmehr einen Pfad, über den ein Teil der MigrantInnen in eine sozial marginalisierte Gruppe eingeht, die aber auch Angehörige der Bevölkerung ohne Migrationshintergrund umfasst.

Abwärts-Integration beschreibt den Integrationsprozess unter den Bedingungen von Umstrukturierungen auf dem Arbeitsmarkt, städtischer Segregation und anhaltendem Rassismus, v.a. für Menschen, die zudem arm sind. Die soziale Ungleichheit verfestigt sich. Während es für andere soziale Schichten Auf- und Abstiege gibt, lässt sich für diese unteren sozialen Gruppen eine soziale Schließung konstatieren.

Ein solcher Abstiegsweg ist für diejenigen MigrantInnen der zweiten Generation wahrscheinlich, deren Eltern einen geringen sozioökonomischen Status haben und sozial isoliert leben. Wenn die Kinder in Problemstadtvierteln aufwachsen, verstärken sich die negativen Effekte von Rollentausch und fehlender Unterstützung sowie unzureichender Kontrolle. Portes' Argument zielt dabei auf die Ressourcenbasis der MigrantInnen sowie auf strukturelle Faktoren 
der Umwelt ab und beschreibt, wie sich daraus aufstiegsbehindernde kulturelle Muster ergeben. $^{63}$

\subsubsection{Neoklassische Integration}

\section{Verortung}

Die in Kapitel 2.1 vorgestellten klassischen Integrationstheorien mit ihrer Forderung nach einer einseitigen Anpassungsleistung der MigrantInnen waren in den Vereinigten Staaten bis in die 1960er Jahre die dominanten Theorien zur Erklärung des Verhaltens von MigrantInnen im Aufnahmeland. Die Bürgerrechtsbewegung in den 1960er und 1970er Jahren stärkte jedoch den Wert von Pluralismus in der amerikanischen Gesellschaft. Integrationstheorien, die eine einseitige Anpassung forderten, wurden nicht länger als politisch korrekt betrachtet und gerieten in Verruf, weil sie zu sehr an die Zwangsamerikanisierung vom Beginn des 20. Jahrhunderts erinnerten (Herrmann 1996). Auch empirisch wurde bezweifelt, dass solche klassischen Integrationstheorien die Integrationsperspektiven der nach Hautfarben und Herkunftsgruppen heterogenen ImmigrantInnen seit $1965^{64}$ beschreiben könnten (Bonacich 1972; Portes/Rumbaut 1996). Als Reaktion hierauf setzte eine Strömung, die in dieser Arbeit als neoklassisch bezeichnet wird, sich zum Ziel, den Integrationsbegriff zu rehabilitieren und seine Nützlichkeit für die aktuelle zweite Generation zu belegen.

\section{Definition}

Unter Integration verstehen Alba und Nee ,the decline of an ethnic distinction and its corollary cultural and social differences": Unterschiede verlieren an Salienz, Ethnizität als Kristallisationspunkt der Markierung von Fremdheit ist für eine immer geringere Anzahl von Ereignissen relevant und beschränkt sich auf zunehmend weniger Bereiche der Gesellschaft (Alba/Nee 2003: 11). Der ,Mainstream', definiert als „that part of the society within which ethnic and racial origins have at most minor impacts on life chances or opportunities”, ist also

\footnotetext{
${ }^{63}$ Allerdings berücksichtigt die Theorie der Abwärts-Integration nicht mögliche Genderunterschiede: So ist oppositionelles Verhalten unter männlichen Jugendlichen weiter verbreitet als unter weiblichen. Die Aufstiegschancen von jungen Frauen und Mädchen der zweiten Generation müssen daher für besser gehalten werden (Waldinger/Feliciano 2004: 380ff). Und schließlich stellt sich die Frage, ob Abwärts-Integration möglicherweise einen Selektionsfehler beinhaltet: So stellen Kasinitz et al. (Kasinitz, et al. 2004) fest, dass das beschriebene aufbegehrende Verhalten von vielen Jugendlichen an den Tag gelehnt werde. Es sei daher nicht das Verhalten selbst, welches die innerstädtischen Teenager von anderen unterscheide, sondern die strengere Sanktionierung solchen Verhaltens bei Jugendlichen aus sozial marginalisierten Schichten. Dies spricht dafür, in die empirische Untersuchung eine Vergleichsgruppe der Dominanzbevölkerung aus nicht-marginalisierten sozialen Schichten einzufügen.

${ }^{64}$ In diesem Jahr wurde das Hart-Cellar-Gesetz verabschiedet, welches die nationalen Quoten von 1924 aufhob (Alba, et al. 1994: 174f).
} 
auch in Bewegung $^{65}$ (Alba/Nee 2003: 12, 11). Ein ,Schwammigwerden' der Grenzen wie in dem oben vorgestellten Konzept des ,Boundary Blurring' (s. Kapitel 2.3) wird gemäß Alba und Nee begünstigt durch ,the sustained equal-status contact between the members of different groups that is produced on a substantial scale by the socioeconomic and residential opportunities available to many members of the new groups - supported by institutional mechanisms enforcing equal rights" (Alba/Nee 2003: 62). Ein gesicherter sozioökonomischer Status wird also als besonders wichtig für Integration eingeschätzt; er soll erlangt werden durch ,minority participation in mainstream socioeconomic institutions (e.g. labor market, education) on the basis of parity with ethnic-majority individuals of similar socio-economic origins" (Alba/Nee 2003: 28). Verbesserungen in der wirtschaftlichen Lage machen Interaktionen, die als statusgleich markiert werden, wahrscheinlich, zudem werden durch die Teilnahme in ,Mainstream'-Organisationen häufig auch kulturelle Anpassungen vorgenommen, die wiederum die Markierung von Interaktionen als kulturgleich erleichtern.

\section{Gesellschaftlicher Kontext}

Alba und Nee betonen in ihrer Theorie die gegenseitige Beeinflussung von Akteuren und Strukturen (Alba/Nee 2003: 36). Obgleich sie die sozioökonomischen Merkmale des einzelnen MigrantInnen in den Mittelpunkt ihrer empirischen Analyse stellen, ist es ihnen wichtig, die strukturelle Eingebundenheit der Akteure zu berücksichtigen. Als wichtige institutionelle Faktoren betrachten sie dabei:

- die gesellschaftlichen Veränderungen durch die Bürgerrechtsbewegung, die neben der ursprünglichen Zielgruppe der, einheimischen' Minderheiten nun MigrantInnen zugute kommen, wie z.B. Maßnahmen der , affirmative action' und eine weitreichende Anti-Diskriminierungsgesetzgebung (Alba/Nee 2003: 54, 120, 278f);

- durch die Bildungsexpansion früherer Jahre und das Wirtschaftswachstum bedingte Aufstiegschancen v.a. für MigrantInnen der zweiten Generation. Gestützt werden diese durch ein im Allgemeinen meritokratisches Gesellschaftssystem, in dem es mehr Auf- und Abwärtsbewegungen für alle Gruppen gibt: ,,[A]n individual's socioeconomic origins are now less determinative of his or her adult position than they used to be“" (Alba/Nee 2003: 138).

\footnotetext{
${ }^{65}$ Alba und Nee geben einige Beispiele für Veränderungen des Mainstreams durch die MigrantInnen (im Bereich der Essgewohnheiten, der Religion, der Vorstellungen über Schönheit, der Demographie, der ethnischen Diversität) (Alba/Nee 2003: 12f); grundsätzlich gilt aber, dass der Mainstream sich langsamer verändert, als die/der einzelne MigrantIn dies tut.
} 
Offen lassen Alba und Nee, ob die erfolgreiche Integration von MigrantInnen einer einheimischen Minderheit bedarf, die als die ,Anderen' fungiert, und verglichen mit denen die MigrantInnen sich als Teil der Mehrheitsgesellschaft fühlen können (Alba/Nee 2003: 120).

Allgemein gilt, dass der Staat günstige Bedingungen für die Integration von MigrantInnen schaffen kann (z.B. über die Gewährung von Einbürgerungsrechten und Sozialleistungen zur Eingliederung), aber auch Bildungsorganisationen, Unternehmen und die öffentliche Meinung Integration erleichtern können (Alba/Nee 2003: 108).

\section{Integrationsprozess}

Wie läuft nun dieses ,Schwammigwerden' der Grenzen ab? Für Alba und Nee ist kulturelle Anpassung und Statuserwerb nicht eine einmalige, willentliche Entscheidung, sondern das „,cumulative by-product of choices made by individuals seeking to take advantage of opportunities to improve their life chances and well-being through purposive action" (Alba/Nee 2003: 71). Nach diesem Modell führen also viele kleine rationale Schritte zu einem großen Ergebnis, für das man sich nicht im Voraus wissentlich entschieden hat: „Assimilation [...] is something that frequently enough happens to people while they are making other plans“ (Alba/Nee 2003: 282). Die Identitäten der MigrantInnen werden im Integrationsprozess verändert, Integration wird unbewusst vollzogen.

Deutlich wird in diesem Zitat, dass Alba und Nee in ihrer Theorie zunächst die einzelnen, autonomen MigrantInnen betrachten. Der Familie oder der ,ethnischen Gemeinschaft' messen sie keine größere Bedeutung bei. (Alba/Nee 2003: 35f) Es wird angenommen, dass alle MigrantInnen nach Erfolg streben, unabhängig von ihrem kulturellen Hintergrund. ${ }^{66}$

Das absichtsvolle Handeln der MigrantInnen zur Verbesserung ihres sozioökonomischen Status wird in den praktischen Ausführungen von Alba und Nee daher vor allem von den relativen Kosten der Teilnahme an der ,Mainstream'- versus der ,ethnischen Ökonomie' bestimmt (Alba/Nee 2003: 36). Um vom ,Mainstream' angebotene Möglichkeiten nutzen zu können, werden die Kinder von MigrantInnen die dafür notwendigen Sprachkenntnisse und kulturellen Fähigkeiten erwerben (Alba/Nee 2003: 122). Den MigrantInnen werden hohe sozioökonomische Aspirationen sowie langfristige Ambitionen im Aufnahmeland unterstellt.

\footnotetext{
${ }^{66}$ Zwar betonen Alba und Nee, dass kulturelle Annahmen, Beziehungen und Pfadabhängigkeit das zielgerichtete Handeln der MigrantInnen beeinflussen. Somit kommt neben den relativen Kosten der verschiedenen Optionen auch diesen Faktoren Gewicht zu (Alba/Nee 2003: 36). Praktische Konsequenzen für ihr Modell ziehen die Autoren jedoch aus der Bedeutung dieser sozialstrukturellen Faktoren nicht: Ihr Homo oeconomicus ist zwar sozial eingebettet, doch dies hindert ihn nicht daran, sich bietende Chancen jederzeit zu ergreifen.
} 
Ressourcenerwerb und v.a. die Veränderung der Wahrnehmung bei der Dominanzbevölkerung sind für Alba und Nee eine langfristige Angelegenheit, so dass die Prozesse in der zweiten Generation keinesfalls abgeschlossen sein werden (Alba/Nee 2003: 30). Gemäß dem sozialkonstruktivistischen Integrationsverständnis kann zudem über die objektiven Anforderungen für eine Integration keine Aussage getroffen werden, da die Kriterien für eine Ähnlichkeitswahrnehmung selbst sich verändern können. Allerdings ist dies ein eher langwieriger Prozess, so dass kurzfristig gesehen die meisten MigrantInnen sich an bestehende Normen zumindest in Teilen anpassen (Alba/Nee 2003: 275). Diese Anpassung ist aber nicht gleichbedeutend mit Integration, da für diese eine vergrößerte Ähnlichkeitswahrnehmung in als status- oder kulturgleich markierten Interaktionen nötig ist. Die Anpassung erleichtert jedoch eine solche Markierung als status- oder kulturgleich und macht eine Ähnlichkeitswahrnehmung daher wahrscheinlicher. Die Ähnlichkeitswahrnehmung/Integration hängt darüber hinaus vom strukturellen Rassismus und den rassistischen Praktiken in der Gesellschaft ab, die die Möglichkeiten des ,Blurrings' oder umgekehrt der Verfestigung bestimmter Kriterien der Fremdheitsmarkierung bestimmen. Systematische Diskriminierung und ausgeprägten Rassismus halten Alba und Nee jedoch für die US-amerikanische Gesellschaft für eine Ausnahme. Trete diese auf, bedürfe es kollektiver Strategien der MigrantInnen, damit es zu einer politischen Veränderung der Kriterien für Fremdheitsmarkierungen kommt. (Alba/Nee 2003: 45)

\section{Integrationsmuster}

Aufbauend auf der sozialkonstruktivistischen Offenheit des Integrationsbegriffs, der allerdings in der empirischen Realität kurzfristig eher durch eine größere Anpassung auf Seiten der MigrantInnen als durch ein ,Schwammigwerden' der Kriterien auf Seiten der Dominanzbevölkerung gefüllt wird, stellen Alba und Nee fest:

Assimilation refers to the results of long-term processes that have gradually whittled away the social foundations for ethnic distinctions: diminishing cultural differences that serve to signal ethnic membership to others and to sustain ethnic solidarity; bringing about a rough parity of life chances to attain socioeconomic goods such as educational credentials and remunerative jobs while loosening the attachment of ethnicity to specific economic niches; shifting residence away from central-city ethnic neighborhoods to ethnically mixed suburbs and urban neighborhoods; and, finally, fostering relatively easy social intercourse across ethnic lines, resulting ultimately in high rates of ethnic intermarriage and mixed ancestry (Alba/Nee 2003: 70f). 
Aus diesem Zitat ergibt sich ein Integrationsmuster, nach dem MigrantInnen der zweiten Generation die Sprache des Aufnahmelandes fließend sprechen. Die Kenntnisse der Herkunftssprache werden mit der Zeit zurückgehen, außer wenn diese auch für die Aufnahmegesellschaft relevant ist. Formale Bildungsabschlüsse werden geschätzt und die zweite Generation wird im Vergleich zur ersten höhere Bildungsabschlüsse erzielen, v.a. wenn die erste Generation nur gering qualifiziert war. Hinsichtlich Bildung und Beruf spielen das ,ethnische Sozialkapital' der Familie und ,ethnische Netzwerke' keine wichtige Rolle. Die Bildungsergebnisse von Jungen und Mädchen weisen nur die Unterschiede auf, die auch in der NichtMigrantenbevölkerung zu finden sind. MigrantInnen der zweiten Generation haben hohe soziale Erwartungen und werden auf dem Arbeitsmarkt entsprechend den Nicht-MigrantInnen des gleichen sozioökonomischen Status aufgestellt sein. Sie üben verschiedene Berufe aus und konzentrieren sich nicht auf eine bestimmte Nische. Von Arbeitslosigkeit sind sie im gleichen Maß betroffen wie Nicht-MigrantInnen. Die Bevorzugung von Menschen der gleichen Herkunftsgruppe für Freund- und Partnerschaften wird über die Zeit zurückgehen. Die Ähnlichkeitswahrnehmung von MigrantInnen in diesem Muster auf Seiten der Dominanzbevölkerung nimmt sowohl durch status- als auch durch kulturgleich markierte Interaktionen mit der Zeit zu. $^{67}$ (Alba/Nee 2003; Alba/Nee 1997: 849ff)

\section{Gleiche Möglichkeiten und daher gleiche Ergebnisse?}

Alba und Nee glauben, dass gleiche Möglichkeiten für MigrantInnen zu gleichen Ergebnissen führen - innerhalb der Bandbreite von Ergebnissen, die typisch für die Mehrheitsbevölkerung sind. Daher gehen sie davon aus, dass Integration mit einer gewissen kultureller Anpassung das dominante Muster auch für die Immigration nach 1965 sein wird. Diese Annahme wird jedoch in dem Fall problematisch, wo MigrantInnen systematisch über geringere sozioökonomische Ressourcen verfügen als die Mehrheitsbevölkerung. Gleichbehandlung (,equality of treatment ${ }^{68}$ ) führt dann nicht zu gleichen Ergebnissen.

\footnotetext{
${ }^{67}$ Die neoklassische Integrationstheorie verlangt also von MigrantInnen, dass sie z.B. die Sprache des Aufnahmelandes lernen und die im Aufnahmeland dominanten Normen (z.B. individuelle Verantwortung, Ehrgeiz, ein bestimmtes Zeit- und Pünktlichkeitsverständnis) übernehmen. Gleichzeitig können sie andere Aspekte ihrer Identität beibehalten (z.B. Essen, Religion, Musik, die Sprache der Eltern zusätzlich zu der des Aufnahmelandes). Integration zwingt MigrantInnen somit keinesfalls, ihr kulturelles Erbe aufzugeben. Kulturelle Unterschiede werden zwar an Sichtbarkeit verlieren, dies kann aber auf dreierlei Arten geschehen: dadurch, dass MigrantInnen Teile ihre kulturellen Gewohnheiten aufgeben, dadurch, dass die Nicht-Migrantenbevölkerung neue kulturelle Ausdrucksmöglichkeiten übernimmt oder dadurch, dass Kultur zur Markierung von Differenz allgemein an Bedeutung verliert. (Alba/Nee 2003: 62)

${ }^{68}$ Siehe die übliche Unterscheidung von, equality of treatment' als gleiche Behandlung, ,equality of opportunity' als Gleichbehandlung, die ungleiche Startpositionen berücksichtigt und ausgleicht, und ,equality of outcome', wodurch die gleichen Ergebnisse (,outcomes') für alle erreicht werden sollen (Sen 2000).
} 
Insgesamt ist diese neue Integrationstheorie somit hinsichtlich der Bildungs- und Berufsmöglichkeiten für MigrantInnen der zweiten Generation optimistisch. Weder individuelle Faktoren wie geringes Humankapital, ,Rasse', Gender oder fehlende familiäre Unterstützung noch der institutionelle Kontext von Rassismus, steigender Ungleichheit und prekären Bedingungen in Teilen des Arbeitsmarktes werden in den USA für so bedeutsam gehalten, dass sie Integration nachhaltig verhindern könnten.

\subsubsection{Zwischenfazit: Vorteile der neuen Integrationstheorien}

Als Zwischenfazit lässt sich festhalten, dass die drei aus der gegenwärtigen Debatte in den Vereinigten Staaten herausgearbeiteten Strömungen die Probleme der klassischen Integrationstheorien und den auf diesen basierenden Ansätzen von Esser und Heitmeyer, die in Kapitel 2.2 analysiert wurden, vermeiden. Sie geben damit folgende Antworten auf die vier in der Einleitung dieses Kapitels gestellten Fragen:

1. Die neuen Theorien begreifen Integration nicht länger als zwangsläufigen, fortwährenden Prozess, der ohne Umkehrungen und Rückschläge stattfindet. Wahrgenommene Ähnlichkeiten können sich auch wieder verringern, und Integration kann unter bestimmten Umständen völlig ausbleiben. Zudem halten die neuen Theorien das Ergebnis des Integrationsprozesses nicht für von vornherein festgelegt: Die Annahme einer ,straight line'-Integration (Warner/Srole 1945) in eine Mittelschicht wird in Frage gestellt, in der Theorie der Abwärts-Integration ist Integration sogar mit sozialem Abstieg und dem Verlust von Entwicklungschancen verbunden.

2. Eine Homogenität des ,Mainstreams' wird von allen drei Theorien abgelehnt. Am ehesten ist ein solches Bild in der neoklassischen Theorie zu finden, die von einer wenig gespaltenen und relativ diskriminierungsfreien Gesellschaft ausgeht. In der Theorie der Abwärts-Integration hingegen ist der ,Mainstream' sozioökonomisch tief gespalten und kulturell pluralistisch, in der transnational-pluralistischen Theorie wird wie schon der Name der Theorie sagt - ebenso von einer kulturell pluralistischen Gesellschaft ausgegangen.

3. Zudem konzentrieren sich die neuen Theorien nicht einseitig auf die MigrantInnen: Wo die klassischen Integrationstheorien es als die alleinige Pflicht der ImmigrantInnen betrachteten, sich so schnell wie möglich an alle Aspekte der Lebensweise im Aufnahmeland anzupassen, verweisen die hier herausgearbeiteten Strömungen darauf, dass Integration in einer Gesellschaft mit Rassismus auch Veränderungen der Bewertungskriterien bei der Dominanzbevölkerung selbst verlangt. Zwar sind auf Seiten der 
MigrantInnen Anpassungen an bestimmte sozioökonomische und kulturelle Normen für Integration förderlich, aber sie sind weder eine notwendige noch eine hinreichende Bedingung für Integration. Mit den hier angewendeten sozialkonstruktivistischen Prämissen ließe sich auch der Fall denken, dass die wahrgenommene Ähnlichkeit sich vergrößert, ohne dass es eine sozioökonomische oder kulturelle Anpassung gegeben hätte, weil sich die Kristallisationspunkte für Fremdheitsmarkierungen verändert haben. Im Einzelnen werden die Gewichtungen jedoch ein wenig unterschiedlich gesetzt: So sehen Alba und Nee kurzfristig v.a. Veränderungen auf migrantischer Seite als wahrscheinlich an, halten mittelfristig aber ein ,Blurring' bestimmter Kriterien auf Seiten der Dominanzbevölkerung für notwendig. Bei der Abwärts-Integration wird ebenfalls v.a. die sozioökonomische und kulturelle Anpassung der MigrantInnen, allerdings an eine sozial marginalisierte Schicht, betont sowie die Bereitschaft von Angehörigen dieser marginalisierten Schicht, MigrantInnen als ähnlich wahrzunehmen. Auch sozial bessergestellte Mitglieder der Dominanzbevölkerung sehen Ähnlichkeiten zwischen diesen MigrantInnen und den nicht-migrantischen Mitgliedern der sozial marginalisierten Schicht. Die pluralistische Theorie hingegen basiert nur auf einer sozioökonomischen Anpassung der MigrantInnen, für erfolgreiche Integration wird jedoch ein ,Blurring' bestimmter kultureller Kriterien für Fremdheitsmarkierungen auf Seiten der Dominanzbevölkerung nötig.

4. Im Unterschied zu Heitmeyer und Esser gehen die drei hier herausgearbeiteten Strömungen nicht länger davon aus, dass sich unterschiedliche Kulturen gegenseitig ausschließen und MigrantInnen sich zwischen den Kulturen entscheiden müssen. In Heitmeyers und Essers Sicht ist ein ,Verhaftetsein' in der Kultur der Eltern ein Hindernis für ein erfolgreiches Leben im Aufnahmeland. Dagegen betrachten die drei neuen Theorien Kompetenz in der Kultur des Herkunftslandes entweder neutral oder sogar als Ressource. Überhaupt halten sie sozioökonomische und kulturelle Normen für Konstruktionen, die sich innerhalb eines von Rassismus geprägten Systems immer wieder verändern. Die Grenzziehungen sind in der pluralistischen Theorie in sozioökonomischer Hinsicht stark, in kultureller jedoch fließend. In der neoklassischen Theorie wird von einem zunehmenden ,Blurring' verschiedener sozioökonomischer und kultureller Kriterien ausgegangen, und nur in der Abwärts-Integration werden sowohl kulturelle als auch sozioökonomische Grenzziehungen in einer Gesellschaft mit starkem Rassismus für verfestigt gehalten. 
Damit ließen sich diese drei Theorien mit den in Kapitel 2.3 vorgestellten integrationstheoretischen Prämissen kompatibel machen und untersuchen vor allem die Mechanismen, durch die es entweder zu als status(un)gleich oder als kultur(un)gleich markierten Interaktionen kommt. Für eine vergrößerte Ähnlichkeitswahrnehmung wird in allen drei Theorien dem ökonomischen Ressourcenerwerb ein leichtes Übergewicht gegeben. Die drei Theorien messen im Vergleich mit den klassischen Integrationstheorien sowie den Theorien von Heitmeyer und Esser kulturellen Faktoren weniger Gewicht bei. Vor allem aber nehmen sie nicht länger an, dass kulturelle Anpassung mit einem gesicherten sozioökonomischen Status Hand in Hand geht. Abbildung 1 verortet die drei neuen Theorien und den Fall der Segregation, d.h. der fehlenden Integration, in einem durch die Achsen ,kulturelle Anpassung' und ,sozioökonomischer' Status aufgespannten Feld: Rechts oben ist die neoklassische Integration mit gesichertem sozioökonomischem Status und einer gewissen kulturellen Anpassung zu finden, darunter die Abwärts-Integration, bei der kulturelle Anpassung an eine marginalisierte soziale Schicht mit einem prekären wirtschaftlichen Status zusammengeht. Links unten folgt der Fall fehlender Integration, hier als Segregation bezeichnet, bei der ganz im Sinne klassischer Integrationstheorien und der Modelle von Heitmeyer und Esser fehlende kulturelle Anpassung mit einem prekären sozioökonomischen Status verbunden ist. Links oben sind schließlich die pluralistischen Theorien verortet, bei denen ein gesicherter sozioökonomischer Status mit nur geringer kultureller Anpassung Hand in Hand geht.

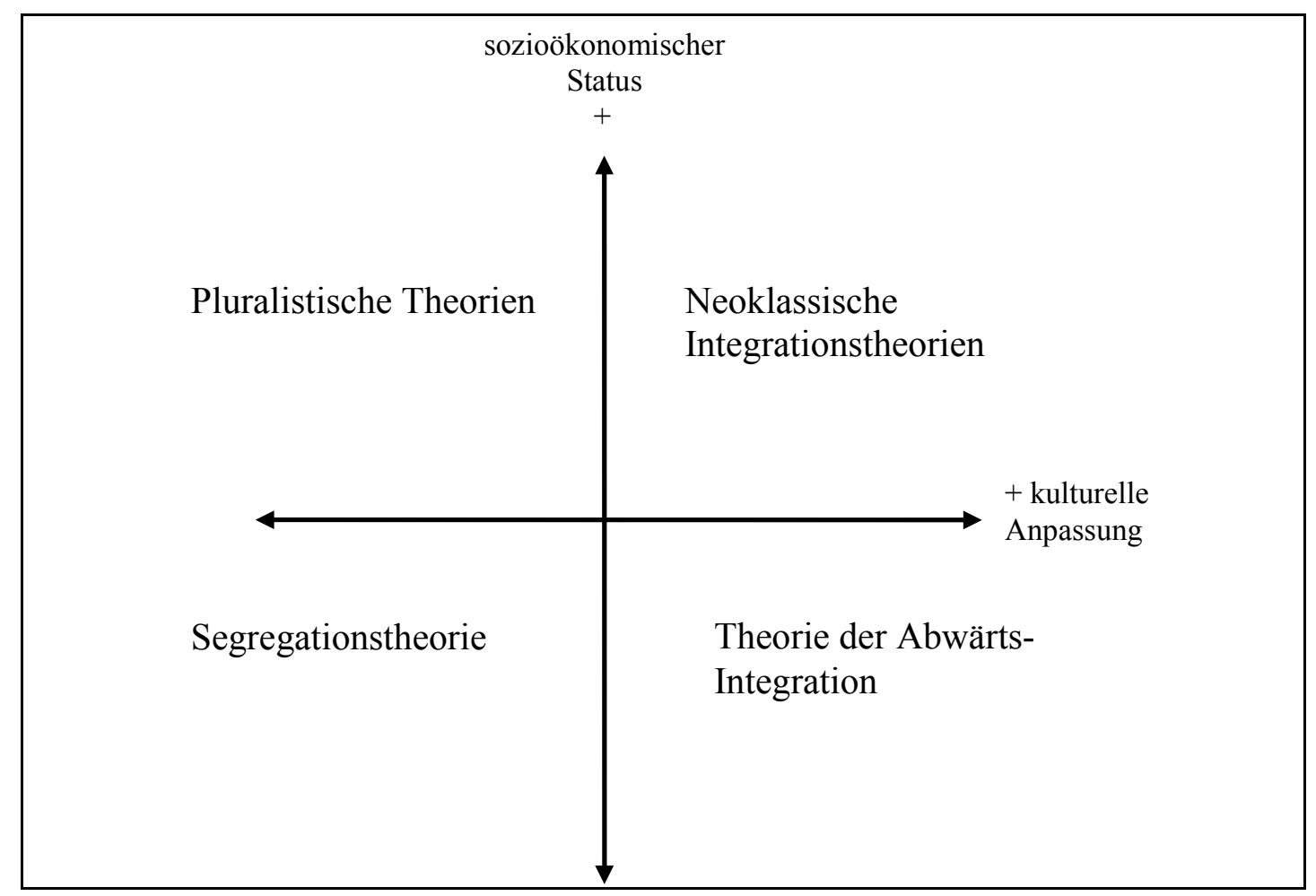

Abbildung 1 - Vier-Felder-Matrix 
Im nächsten Kapitel werden aus den drei Theorien Integrationsmuster für die Bereiche Sprache, Bildung, Beruf und Freund-/Partnerschaften entwickelt, bevor die Übertragbarkeit der hier vorgestellten US-amerikanischen Theorieströmungen auf Deutschland kritisch diskutiert wird.

\subsection{Zusammenführung in Integrationsmustern}

In diesem Abschnitt sollen aus den im vorherigen Kapitel beschriebenen Theorien Integrationsmuster abgeleitet werden. Die nachfolgende Tabelle 1 fasst für Deutschland die Integrationsmuster über die Lebensbereiche Sprachkenntnisse, Bildung, Beruf, Freund- und Partnerschaften zusammen und kontrastiert sie mit dem Muster fehlender Integration. Wieso werden gerade diese vier Bereiche untersucht? Sprachkenntnisse sind eine Vorbedingung der Verständigung mit anderen Gruppen; sie bestimmen, mit wem prinzipiell interagiert werden kann. Formale Bildungsabschlüsse sind ausschlaggebend für den Zugang zum Arbeitsmarkt und schaffen darüber hinaus Ressourcen, die es erlauben, das eigene Leben (mehr oder weniger) aktiv zu gestalten. Die berufliche Situation ist ein zentrales Kriterium, da Integration als statusgleich markierter Interaktionen bedarf. Der Beruf kann sowohl über den finanziellen Verdienst als auch über den Status der ausgeübten Tätigkeit Ressourcen generieren, die eine solche Markierung zwar nicht garantieren, aber wahrscheinlicher machen. Freundschaften und Partnerschaften haben eine doppelte Verbindung zu Fremd- und Ähnlichkeitswahrnehmungen: Die Wahl einer/s bestimmten PartnerIn kann nicht nur als begünstigendes Kriterium für eine Ähnlichkeitswahrnehmung verstanden werden, sondern auch als Indikator einer stattgefundenen Ähnlichkeitswahrnehmung. Aus diesen Gründen eignen sich die vier Bereiche Sprachkenntnisse, Bildung, Beruf und Freund- sowie Partnerschaften besonders gut, um anhand ihrer die Bandbreite der Integrationsmuster zu untersuchen. Überträgt man die Theorien auf Deutschland, lassen sich folgende Muster erwarten:

Hinsichtlich der Sprachkenntnisse sagt die transnational-pluralistische Theorie für MigrantInnen der zweiten Generation sehr gute Kenntnisse in der Sprache der Eltern voraus, wohingegen die Deutschkenntnisse sich je nach den Anforderungen des Arbeitsmarktes auf einem mittleren bis hohen Niveau bewegen. MigrantInnen im Muster der Abwärts-Integration verfügen über fließende Deutschkenntnisse, allerdings nur in einer mündlichen Alltagssprache, die mit Slang durchsetzt ist. Im Hoch- und Schriftdeutsch sind ihre Kenntnisse mangelhaft. Die Sprache der Eltern wird schlecht bis gar nicht gesprochen. Dagegen sprechen und schreiben MigrantInnen der zweiten Generation im neoklassischen Muster Deutsch sehr gut. Die 
Kenntnisse der elterlichen Sprache schlafen mit der Zeit ein. Segregierte MigrantInnen der zweiten Generation verfügen nur über geringe Deutschkenntnisse. Die Sprache der Eltern sprechen sie in einer zumindest für die Alltagskommunikation hinreichenden Form.

Bei ihrer Bildungskarriere erhalten MigrantInnen der zweiten Generation im pluralistischen Muster Unterstützung durch ,ethnische Netzwerke'. Die Eltern kontrollieren das Handeln ihrer Kinder und versuchen, es an den Normen des Herkunftslandes auszurichten. Wenn möglich, besuchen die Kinder Schulen, die vom Herkunftsland der Eltern unterstützt werden, oder verbringen sogar einen Teil ihrer Schulzeit im Geburtsland der Eltern. Dagegen entwickeln MigrantInnen der zweiten Generation auf dem Weg der Abwärts-Integration eine Opposition gegen das Wertesystem der deutschen Mittelklasse, wie es z.B. das Schulsystem verkörpert. Ihnen fehlt außerdem im Bildungsbereich jegliche Unterstützung durch die Eltern, und sie erzielen somit nur geringe Bildungsabschlüsse. Neoklassisch integrierte MigrantInnen erzielen durchschnittlich höhere Bildungsabschlüsse als ihre Eltern. Segregierte MigrantInnen sind hingegen im deutschen Bildungssystem nicht erfolgreich, sie erreichen nur geringe oder gar keine Schulabschlüsse.

Laut der pluralistischen Theorie erlaubt eine Beschäftigung in der ,ethnischen Ökonomie', beispielsweise im Großhandel, Verkauf, Dienstleistungs- und Nahrungsmittelbereich, einen gesicherten sozioökonomischen Status. Das pluralistische Muster unterscheidet sich damit vom Muster der Abwärts-Integration: In diesem nehmen die MigrantInnen der zweiten Generation nur eingeschränkt am formalen Arbeitsmarkt in Deutschland teil. Wenn sie es tun, dann zumeist in niedrigqualifizierten und schlechtbezahlten Jobs. Während die Beteiligung an der Schattenwirtschaft signifikant ist, lebt ein erheblicher Teil dieser MigrantInnen der zweiten Generation von staatlichen Transferleistungen. Im neoklassischen Muster passt sich die Beschäftigungssituation von MigrantInnen der zweiten Generation mittelfristig der der Deutschen ohne Migrationshintergrund an. Die MigrantInnen streben nach sozialem Aufstieg und sind erfolgsorientiert und motiviert. MigrantInnen im Muster der Segregation arbeiten entweder nicht oder aber in der, ethnischen Ökonomie', ohne dort allerdings soziale Aufstiegsmöglichkeiten nutzen zu können.

FreundInnen und PartnerInnen sind im pluralistischen Muster stark herkunftsgruppenbezogen und transnational geprägt: Sie kommen häufig aus dem gleichen Herkunftsland der Eltern oder sogar der gleichen Region, dem gleichen Dorf. Laut der Theorie der AbwärtsIntegration steht für MigrantInnen die Kultur der Peergruppe im Mittelpunkt: Sowohl FreundInnen als auch PartnerInnen stammen aus der gleichen Subkultur. Die Zugehörigkeit zur 
Peergruppe ist dabei wichtiger als die Zugehörigkeit zu einer Herkunftsgruppe. Im neoklassischen Muster kommt es mittelfristig zu einer statistischen Normalverteilung von Freund- und Partnerschaftsmustern. Kurzfristig kommt der Ethnizität bei der Auswahl von FreundInnen und potenziellen PartnerInnen weiterhin Bedeutung zu. Eine Partnerschaft mit einer/m nachziehenden PartnerIn aus dem Herkunftsland der Eltern bleibt die Ausnahme. Dagegen schließen segregierte MigrantInnen der zweiten Generation Freund- und Partnerschaften mit Angehörigen der gleichen Herkunftsgruppe; sie sind dabei aber nicht wie MigrantInnen im pluralistischen Muster in transnationale oder herkunftsgruppenbezogene Netzwerke mit entsprechenden Kapitalien eingebunden.

Hinsichtlich der Integrationsmechanismen stellt die pluralistische Theorie von der Dominanzbevölkerung als statusgleich markierte Interaktionen in den Vordergrund, wohingegen im Muster der Abwärts-Integration die Referenzgruppe eine sozial marginalisierte Schicht innerhalb der Dominanzbevölkerung ist, die Interaktionen mit MigrantInnen der zweiten Generation, die in dieses Muster fallen, als status- und kulturgleich markiert. Bei der neoklassischen Integration sind ebenfalls sowohl status- als auch kulturgleich markierte Interaktionen relevant, allerdings müssen diese Markierungen nicht von einer Untergruppe, sondern von der allgemeinen Dominanzbevölkerung vorgenommen werden. Im Muster der Segregation schließlich werden Interaktionen mit MigrantInnen weder als kultur- noch als statusgleich markiert.

Zusammen beschreiben die drei Integrationstheorien und der Fall der Segregation vier verschiedene Pfade mit sehr unterschiedlichen Entwicklungsmöglichkeiten für MigrantInnen in Deutschland. Sie decken somit das gesamte Spektrum möglicher Integrationsergebnisse ab. Tabelle 1 fasst diese Muster überblicksartig zusammen.

Abschließend wird auf die Frage eingegangen, inwieweit sich die drei Theorien aus den USA auf den deutschen Kontext übertragen lassen und was dabei zu beachten ist. 
Tabelle 1 - Prognostizierte Integrationsmuster

\begin{tabular}{|c|c|c|c|c|}
\hline & PLURALISTISCHE INTEGRATION & ABWÄRTS-INTEGRATION & NEOKLASSISCHE INTEGRATION & SEGREGATION \\
\hline $\begin{array}{l}\text { SPRACH- } \\
\text { KENNTNISSE }\end{array}$ & $\begin{array}{l}\text { Mittlere bis gute Deutschkenntnisse. } \\
\text { Sehr gute Kenntnisse der Herkunftsspra- } \\
\text { che. }\end{array}$ & $\begin{array}{l}\text { Fließend in Deutsch, aber nur Umgangs- } \\
\text { sprache/Slang. } \\
\text { Geringe Kenntnisse der Herkunftsspra- } \\
\text { che. }\end{array}$ & $\begin{array}{l}\text { Sehr gute Deutschkenntnisse. } \\
\text { Wahrscheinlich geringe Kenntnisse in der } \\
\text { Sprache der Eltern (Theorie macht keine } \\
\text { definitive Aussage). }\end{array}$ & $\begin{array}{l}\text { Keine bis geringe Deutsch- } \\
\text { kenntnisse. } \\
\text { Kenntnisse der Herkunftssprache } \\
\text { sind vorhanden. }\end{array}$ \\
\hline BILDUNG & $\begin{array}{c}\text { Wenn möglich, Besuch von Schulen, die } \\
\text { vom Herkunftsland der Eltern unterstützt } \\
\text { werden. Schulbesuch findet teilweise im } \\
\text { Herkunftsland der Eltern statt. Normen } \\
\text { des elterlichen Herkunftslandes prägen } \\
\text { die Einstellung zu Bildung. }\end{array}$ & $\begin{array}{l}\text { Oppositionsverhalten behindert schuli- } \\
\text { schen Erfolg. Sehr niedrige deutsche Bil- } \\
\text { dungsabschlüsse. Eltern sind nicht in der } \\
\text { Lage, schulische Aktivitäten ihrer Kinder } \\
\text { zu begleiten. }\end{array}$ & $\begin{array}{l}\text { Große Wertschätzung von Bildungserfol- } \\
\text { gen. Fortschritte im Bildungserfolg von } \\
\text { einer Generation zur nächsten sind min- } \\
\text { destens genauso groß wie bei der deut- } \\
\text { schen Nicht-Migrantenbevölkerung (bei } \\
\text { Kontrolle der sozioökonomischen Fakto- } \\
\text { ren). }\end{array}$ & $\begin{array}{l}\text { Sehr niedrige Bildungsabschlüs- } \\
\text { se an deutschen Schulen. Hoher } \\
\text { Anteil an Schulabbrechern. }\end{array}$ \\
\hline BERUF & $\begin{array}{c}\text { Arbeit in der ,ethnischen Ökonomie' } \\
\text { (Handel, Verkauf...), darüber z.T. Inter- } \\
\text { aktion mit ,Mainstream'. Arbeit ermög- } \\
\text { licht gesicherten sozioökonomischen Sta- } \\
\text { tus. }\end{array}$ & $\begin{array}{l}\text { Prekäre Teilnahme am Arbeitsmarkt, grö- } \\
\text { ßere Beteiligung an der Schattenwirt- } \\
\text { schaft. Abhängigkeit von staatlichen So- } \\
\text { zialleistungen. }\end{array}$ & $\begin{array}{l}\text { Streben nach sozialem Aufstieg, hohe Ar- } \\
\text { beitsmotivation. } \\
\text { Gesicherte Teilhabe am deutschen Ar- } \\
\text { beitsmarkt. }\end{array}$ & $\begin{array}{l}\text { Keine Arbeit oder Arbeit in der } \\
\text {,ethnischen Ökonomie' ohne } \\
\text { soziale Aufstiegsmöglichkeiten. }\end{array}$ \\
\hline $\begin{array}{l}\text { FREUND-/ PART- } \\
\text { NER-SCHAFT }\end{array}$ & $\begin{array}{l}\text { PartnerIn kommt häufig aus dem Her- } \\
\text { kunftsland der Eltern (oder sogar der glei- } \\
\text { chen Region). Freundschafts- und Famili- } \\
\text { enmuster sind stark von Herkunftsgrup- } \\
\text { pen und transnationalen Verbindungen } \\
\text { geprägt, können aber auch Mitglieder des } \\
\text {,Mainstreams' einschließen. }\end{array}$ & $\begin{array}{l}\text { Im Mittelpunkt steht die Kultur der Cli- } \\
\text { que. Beziehungen mit PartnerInnen aus } \\
\text { der gleichen Subkultur, unabhängig von } \\
\text {,ethnischer' Herkunft. Partnerschaft mit } \\
\text { einer/m PartnerIn aus dem Herkunftsland } \\
\text { der Eltern bleibt die Ausnahme. }\end{array}$ & $\begin{array}{l}\text { Langfristig kommt es zu einer statisti- } \\
\text { schen Normalverteilung von Freund- } \\
\text { schafts- und Partnerschaftsmustern. Kurz- } \\
\text { fristig kommt der Zugehörigkeit zu einer } \\
\text { Herkunftsgruppe weiterhin Bedeutung zu, } \\
\text { jedoch ebenso der sozialen Schicht oder } \\
\text { der Wohnnachbarschaft. Partnerschaft mit } \\
\text { PartnerIn aus dem Herkunftsland der El- } \\
\text { tern bleibt die Ausnahme. }\end{array}$ & $\begin{array}{l}\text { Freundschaften und Partner- } \\
\text { schaften mit Angehörigen der } \\
\text { gleichen Herkunftsgruppe. }\end{array}$ \\
\hline $\begin{array}{l}\text { DARAUF AUF- } \\
\text { BAUEND: } \\
\text { INTEGRATIONS- } \\
\text { MECHANISMEN }\end{array}$ & $\begin{array}{l}\text { Von Dominanzbevölkerung als status- } \\
\text { gleich markierte Interaktionen stehen im } \\
\text { Zentrum. }\end{array}$ & $\begin{array}{l}\text { Von sozial marginalisierter Schicht inner- } \\
\text { halb der Dominanzbevölkerung als status- } \\
\text { und kulturgleich markierte Interaktionen. }\end{array}$ & $\begin{array}{l}\text { Von Dominanzbevölkerung als status- } \\
\text { und kulturgleich markierte Interaktionen. }\end{array}$ & $\begin{array}{l}\text { Weder status- noch kulturgleich } \\
\text { markierte Interaktionen. }\end{array}$ \\
\hline
\end{tabular}




\section{6 Übertragbarkeit der US-amerikanischen Theorien auf Deutsch- land}

Auf den ersten Blick gibt es zwischen Deutschland und den USA so viele Unterschiede, dass es fraglich erscheinen mag, ob die in der Forschung über die USA entwickelten Theorien auf Deutschland übertragen werden können.

So sind die USA von ihrem Selbstverständnis und ihrer historischen Entwicklung ein Einwanderungsland, während Deutschland als „verspäteter Nationalstaat“ (Plessner 1959) lange Zeit ein ,ethnisches' Volksverständnis hatte. Sklaverei, indigene Bevölkerung und frühe Einwanderung haben dazu geführt, dass die USA von Anfang an ein hinsichtlich der ,Ethnien’ und ,Rassen' vielfältiges Land waren. Diese Vielfalt wurde im Zuge der Bürgerrechtsbewegung anerkannt und zunehmend positiv bewertet. Eine solche Entwicklung hat in Deutschland nicht stattgefunden.

Diese Betonung der Unterschiede zwischen Deutschland und den USA vernachlässigt Deutschlands Geschichte alter und zunehmender Migration und macht sich die politisch lange Zeit vorherrschende Aussage, Deutschland sei kein Einwanderungsland, unhinterfragt zu eigen: So übersieht sie die Migration v.a. von Polen und osteuropäischen Juden und Jüdinnen nach Deutschland zu Beginn des 20. Jahrhunderts (Oenning 1990) sowie die bestehende ,ethnische' Vielfalt v.a. in den deutschen Großstädten (Kapphan 2001).

Vor allem aber verkennt eine Hervorhebung der Unterschiede, dass sich die Integrationsentwicklung in Deutschland und den USA in den letzten Jahren angeglichen hat und dies weiter tut: Mit der Reform des Staatsbürgerrechts wurden auch in Deutschland Ansätze eines ius soli eingeführt (Hailbronner/Renner 2005). ${ }^{69}$ Die wirtschaftliche Struktur beider Länder verändert sich hin zu einer Dienstleistungs- und Wissensgesellschaft (Bell 1976; Bell 1994; Beck 1999). Im Zuge der Globalisierung kommt es zu kulturellen Annäherungen und die technologischen Möglichkeiten vergrößern sich (Altvater/Mahnkopf 2002). Schließlich machen die Vorgaben der EU Antidiskriminierungsmaßnahmen erforderlich, die die Pluralisierung der Gesellschaften in Europa vorantreiben (EU 2004). Und MigrantInnen in Deutschland verstehen sich keinesfalls mehr als kurzfristige ,GastarbeiterInnen', sondern haben die gleichen Bleibeabsichten wie in den USA. Die zweite Generation beansprucht zudem vom deutschen Staat gleichbe-

\footnotetext{
${ }^{69}$ In dem Aufruhr um die Leitkulturdebatte (Merz 2000) ist untergegangen, dass diese Debatte im Kern zunächst einmal die ,ethnische' Vielfalt in Deutschland anerkennt, welche es nötig macht, eine Diskussion über das verbindende Gemeinsame der in Deutschland lebenden Menschen zu führen.
} 
rechtigte Teilhabe. (Lau 2008; vgl. Tabelle 7) Daher sind die von den drei amerikanischen Theorien geforderten Rahmenbedingungen in zunehmendem Maße auch in Deutschland gegeben.

Im Einzelnen setzen die Theorien folgende Prämissen:

Die transnational-pluralistische Theorie setzt Möglichkeiten im Bereich der Informationstechnologien und Verkehrsmittel wie billige Telefonverbindungen, Kabel- und Satellitenfernsehen, Internet und preiswerte Flugreisen voraus. Solche Verbindungen und günstigen Reisemöglichkeiten bestehen von Deutschland aus zu den meisten Herkunftsstaaten der MigrantInnen - ebenso wie in den USA. Regionale und internationale Wirtschaftsabkommen vergrößern die Handelsmöglichkeiten und schaffen neue Arbeits- und Dienstleistungsoptionen. Bestehende Kooperationsbeziehungen, wie z.B. bei der gegenseitigen Anerkennung von Schulabschlüssen oder Pensionsansprüchen, erleichtern die Herausbildung transnationaler Lebensweisen (Fijalkowski 1997; Linsenmann 2004: 335-337; Dicke 2004: 230-232). Hybridität, mehrfache Loyalitäten und kulturelle Pluralität werden dadurch grundsätzlich ermöglicht.

Die Theorie der Abwärts-Integration setzt als Rahmenbedingung weitreichende Veränderungen in der Wirtschaft und der Stadtstruktur voraus. Für die deutsche Wirtschaft gilt ebenso wie für die US-amerikanische, dass sie einem Prozess der Tertiarisierung unterliegt und die entstehenden Dienstleistungsökonomien immer höhere Anforderungen an das Ausbildungsniveau stellen (Häußermann 1998: 165). So werden selbst für schlechtbezahlte Dienstleistungstätigkeiten z.B. in Callcenters ein hohes Maß an Sozialkompetenz und verbaler Ausdrucksfähigkeit verlangt. Niedrigqualifizierte haben daher große Schwierigkeiten, den Lebensunterhalt sichernde Arbeit zu bekommen. Zudem wächst die Zahl der geringfügig oder ,schwarz' beschäftigten Personen, die auf Basis ihrer Tätigkeiten keinen sozialen Aufstieg schaffen (Pilz 2004: 133, 136). Dementsprechend nimmt die Zahl der Menschen, die arm sind, obwohl sie Vollzeit arbeiten (,working poor'), auch in Deutschland zu (Schäfer 2003). Der Aufstieg vom ungelernten Arbeiter über den Facharbeiter ins Management wird immer unwahrscheinlicher, da statushöhere Tätigkeiten Bildungszertifikate voraussetzen und mit externen KandidatInnen besetzt werden. Durch die Bildungsexpansion in Deutschland in den 1970er und 1980er Jahren kommt fehlender formaler Bildung und beruflicher Qualifizierung in Deutschland zunehmend ein Signaleffekt zu: Strukturelle Benachteiligungen werden in einem seinem Selbstverständnis nach meritokratischen System zu individuellen Problemen erklärt und auf fehlenden Fleiß und schlechte Charaktereigenschaften zurückgeführt. (Solga 2005) Insgesamt führt dies dazu, dass es auch in Deutschland eine Schicht von sozial benachteiligten Einheimischen gibt, 
die von staatlichen Sozialleistungen abhängig und deren Arbeitsbiografien mehrfach gebrochen sind. ${ }^{70}$ Diese Gruppe der sozial Marginalisierten konzentriert sich in bestimmten Stadtvierteln, was unter anderem daran liegt, dass seit der Diskreditierung des sozialen Wohnungsbaus in den 1980er Jahren (Skandal um Neue Heimat) die öffentlichen Investitionen in diesem Bereich signifikant gekürzt wurden. Dadurch besteht sozial gebundener Wohnraum heute fast nur noch in den Schlafstädten der Vororte. Die für Deutschland vormals typische soziale Durchmischung der Quartiere geht zurück; Häußermann prophezeit eine zunehmende Amerikanisierung, d.h. Segregierung, in den deutschen Städten (Häußermann 1998: 163, 168, 173). Schließlich hängt die Qualität der schulischen Bildung von der Nachbarschaft ab. Schließlich ist rassistisch motivierte Diskriminierung in Deutschland weiter verbreitet, als meist angenommen wird (Goldberg, et al. 1995; Menschenrechtskommission 1996). Die Prämissen, auf denen Abwärts-Integration basiert, sind also auch in Deutschland weitestgehend erfüllt. Der größte Unterschied zwischen den beiden Ländern besteht im Fehlen einer phänotypisch differenten Minderheit in Deutschland, die keine Migrationsgeschichte hat, wie dies für AfroamerikanerInnen in den USA der Fall ist. MigrantInnen in den USA können in Abgrenzung zu diesen ,Anderen' Teil des US-amerikanischen ,Mainstreams' werden. Bei der empirischen Analyse wird daher zu betrachten sein, ob und wie sich das Fehlen dieser einheimischen Minderheit auf Integrationsprozesse in Deutschland auswirkt.

Die dritte der vorgestellten US-amerikanischen Theorien, die neoklassische Integrationstheorie, setzt ein generell meritokratisches System voraus, bei dem prinzipiell alle Bildungswege und Berufe für die zweite Generation offen sind. Rechtlich gesehen gilt dies in Deutschland bis auf den Beamtenstatus, und auch praktisch nimmt die ,ethnische' Vielfalt in der Arbeitsbevölkerung und im öffentlichen Leben zu (z.B. Bundestagsabgeordnete, FernsehmoderatorInnen). Dies bedeutet, dass Fremdheit in Deutschland am Beginn des 21. Jahrhunderts zumindest in den großen Städten nicht mehr so eindeutig konnotiert ist wie zuvor; die SchlieBung der deutschen Gesellschaft zeigt Ansätze von Lockerung (Ha 1999; Koopmans/Statham 1999; Joppke 1996).

Insgesamt sind daher die Rahmenbedingungen für die drei Integrationstheorien trotz der unterschiedlichen historischen Ausgangsbedingungen mittlerweile auch in Deutschland erfüllt. Eine Übertragung auf die Situation in Deutschland ist somit möglich. Die Tragfähigkeit der Theorien muss sich in der empirischen Überprüfung beweisen. Nachdem in den vorherigen Kapiteln die verschiedenen Strömungen der gegenwärtigen Integrationsdebatte in den Verei-

\footnotetext{
${ }^{70}$ Diese Gruppen sind somit das Äquivalent der von Wilson beschriebenen ,underclass' - ein Begriff, den die meisten SoziologInnen für Deutschland ablehnen (Andreß 1997).
} 
nigten Staaten zu drei Theorien verdichtet und aus ihnen Integrationsmuster für Deutschland abgeleitet wurden, untersucht der nun folgende zweite Teil der Arbeit, ob sich diese Muster, anhand einfacher Indikatoren operationalisiert, in der Empirie finden lassen. 


\section{Empirie I: Individualebene der MigrantInnen - Sprache, Bil- dung, Beruf, Freund- und Partnerschaften}

Nachdem im vorigen Kapitel das theoretische Gerüst dieser Arbeit entwickelt wurde, werden in diesem Kapitel die Sprachkenntnisse, Bildungsergebnisse, die Beteiligung am Arbeitsmarkt sowie Freund- und Partnerschaften von MigrantInnen der zweiten Generation in Deutschland analysiert. Dabei werden Indikatoren vorgestellt, die anschließend in Kapitel 4 zur Operationalisierung der oben abgeleiteten Integrationsmuster verwendet werden. Anhand dieser Muster wird in Kapitel 5 untersucht, ob einer durchschnittlichen sozioökonomischen Verbesserung der zweiten Generation und verstärkter kultureller Anpassung auch eine Wahrnehmung größerer Ähnlichkeit auf Seiten der Dominanzbevölkerung entspricht.

Zunächst wird der empirische Zugang der Arbeit erläutert und die Datenauswahl sowie das methodische Vorgehen begründet. In den Kapiteln 3.3, 3.4, 3.5 und 3.6 erfolgt die eigene empirische Untersuchung, getrennt nach den Sachbereichen Sprache, Bildung, Beruf und Freund- sowie Partnerschaften.

\subsection{Daten und Methoden}

In diesem Kapitel wird beschrieben, welche Daten sich bei einer quantitativen Untersuchung der zweiten Generation heranziehen lassen. Zudem wird diskutiert, welche Methoden für die theoretisch angeleitete empirische Untersuchung besonders geeignet sind.

\subsubsection{Empirisches Material zur zweiten Generation}

Die Debatte um die Integration der zweiten Generation ist emotional aufgeladen und wird nicht selten als ,Gefühlsdebatte ${ }^{71}$ geführt, in der PraktikerInnen, PolitikerInnen, MigrantInnen, ExpertInnen und interessierte Öffentlichkeit darüber berichten, für wie gut oder schlecht integriert sie die zweite Generation in Deutschland halten. Nicht selten verzichtet diese Diskussion ganz auf empirische Belege oder es werden geografisch oder auf bestimmte Gruppen beschränkte Untersuchungen als repräsentativ für die zweite Generation herangezogen. Begründet und gefördert wird diese Tendenz durch die bisherige Kargheit empirischen Materials

\footnotetext{
${ }^{71}$ So der Berliner Beauftragte für Integration und Migration Günther Piening in seiner Rede auf der Tagung Governing Immigrant Integration in Europe: Monitoring and Benchmarking via Indicators? in Berlin am 29. Mai 2006.
} 
zur zweiten Generation. Eine solche ,Gefühlsdebatte' führt jedoch häufig zu voreingenommenen Diskussionen über vermutete oder erwünschte Sachverhalte, die nicht unbedingt der Realität entsprechen. Aus diesem Grund habe ich mich für eine breit angelegte quantitative Untersuchung entschieden, die Repräsentativität anstrebt, statt den Fokus auf positive oder negative Extremgruppen zu legen. Ein quantitativer Zugang erlaubt zudem, Deutsche ohne Migrationshintergrund in die Analyse einzuschließen und ihre Durchschnittswerte sowie Streuungen hinsichtlich verschiedener Variablen mit denen der MigrantInnen zu vergleichen. Denn nicht selten wird nur die Gruppe der MigrantInnen analysiert und mit einer unhinterfragt als integriert geltenden deutschen Gruppe kontrastiert, ohne dass für Letztere Vergleichszahlen vorlägen. Diese Tendenz wird beispielsweise deutlich, wenn es um Identitätsfragen geht. So impliziert die an MigrantInnen gestellte Frage, ob sie sich eher als Deutsche oder als TürkInnen/GriechInnen/ItalienerInnen fühlen (Heitmeyer, et al. 1997), dass Deutsche ohne Migrationshintergrund sich selbstverständlich als Deutsche identifizieren. Forschungen im Bereich der politischen Kultur haben jedoch gezeigt, dass dies häufig nicht der Fall ist, sondern stattdessen Identitäten auf regionaler Ebene (NürnbergerIn, BayerIn, EuropäerIn) oder oberhalb solch partikularer Identifikationssysteme (als Frau, als Mensch) angenommen werden. Die gleiche Tendenz der Homogenisierung und Normalisierung der Nicht-Migrantenbevölkerung wird bei der Analyse der Sprachkenntnisse von MigrantInnen deutlich: Defizite im schriftlichen Ausdrucksvermögen der MigrantInnen werden nicht empirisch mit denen der Deutschen ohne Migrationshintergrund verglichen, sondern an einer Norm gemessen, gemäß derer Deutsche ohne Migrationshintergrund qua nationale Zugehörigkeit über ausreichende Sprachkenntnisse verfügen. Wo immer möglich, versucht die vorliegende Arbeit diese implizit normierenden Tendenzen aufzubrechen.

Insgesamt gibt es bisher relativ wenig Untersuchungen, die speziell die zweite Generation in Deutschland betrachten (für Ausnahmen siehe z.B. Alba, et al. 1994; Diehl 2002; efms 2001; Granato 2003; Kalter 2004; Kristen 2002; Nauck 2001; Straßburger 2004; Worbs 2003), wobei das Fehlen speziell von quantitativen Studien auch der Datenlage geschuldet ist. Das Untersuchungsobjekt der quantitativen Integrationsforschung waren bis vor kurzem ,AusländerInnen' - eine Tendenz, die in der staatlichen Berichterstattung noch anhält: z.B. im Bericht der Bundesregierung zur Situation der ausländischen Arbeitnehmer und ihrer Familienangehörigen in der Bundesrepublik Deutschland von 2002 (BMAS 1996) und im 7. Bericht der Beauftragten der Bundesregierung für Migration, Flüchtlinge und Integration über die Lage der Ausländerinnen und Ausländer in Deutschland vom Dezember 2007 (Beauftragte der Bundesregierung für Migration 2007). Und obgleich Ende der 1990er Jahre begonnen wurde, 
von MigrantInnen zu sprechen, kamen in den quantitativen Stichproben manchmal nur AusländerInnen vor (für Beispiele vgl. Diefenbach 2005). Trotz dieser Limitierung der Untersuchung auf AusländerInnen wäre es jedoch möglich gewesen, die Ergebnisse nach Generationen getrennt zu präsentieren. Dies geschah aber zumeist nicht: So wurden MigrantInnen, die (nahezu) ihr gesamtes Leben in Deutschland verbracht haben, nicht unterschieden von denen, die erst kürzlich im Rahmen des Familiennachzugs, der Arbeitsaufnahme oder für die Ausbildung nach Deutschland gekommen sind.

Ausnahmen zu dieser fehlenden Differenzierung zwischen den Generationen liegen beim Integrationssurvey des Bundesinstituts für Bevölkerungswissenschaften (BiB), dem Ausländersurvey des Deutschen Jugendinstituts (DJI) und dem europäischen EFFNATIS-Projekt (Effectiveness of National Integration Strategies Towards Second Generation Migrant Youth in a Comparative European Perspective) vor. ${ }^{72}$ Das letztgenannte Projekt wurde in Deutschland in Bamberg durchgeführt. Auch einige spezifischere Studien, die Primärdaten erhoben haben, nehmen eine Unterscheidung nach Generationen vor, so z.B. Diehl in ihrer Untersuchung der politischen und sozialen Partizipation von MigrantInnen in Deutschland (Diehl 1998). Alba/Handle (Alba, et al. 1994) und Kalter (Kalter 2004) tun dies ebenfalls in ihren bundesweiten quantitativen Untersuchungen, die das Sozio-oekonomische Panel respektive den Mikrozensus benutzen. Aufgrund der Struktur dieser Datensets beschränken sich ihre Studien allerdings auf solche MigrantInnen der zweiten Generation, die noch bei ihren Eltern zu Hause wohnen.

Diese Limitierung ist nun durch Änderungen im Mikrozensus aufgehoben worden. Die vorliegende Untersuchung wird daher für die quantitative Analyse auf den neuen Mikrozensus des Jahres 2005 zurückgreifen, da dieser Daten für ganz Deutschland enthält und relativ große Fallzahlen in verschiedenen Herkunftsgruppen und allen Generationen bietet. Dabei sind sowohl ausländische als auch deutsche StaatsbürgerInnen mit Migrationshintergrund identifizierbar und zwar in allen Altersgruppen. Durch die altersbezogene Ausweitung der zweiten Generation über die zuvor ausschließlich erfassten Jugendlichen, die noch bei ihren Eltern wohnen, hinaus bis hin zu den 45-Jährigen rückt Partizipation auf dem Arbeitsmarkt als einem wichtigen Feld des Ressourcenerwerbs in den Blick. Dabei können über die Berufsausbildung hinaus berufliche Stellung, Prestige der Arbeit und Einkommen sowie Erwerbsbeteiligung und Arbeitslosigkeiten untersucht werden. Ein weiterer Vorteil des Mikrozensus ist die aufgrund seiner aufwendigen Anlage gegebene Repräsentanz hinsichtlich der Daten zum so-

\footnotetext{
${ }^{72}$ Speziell die zweite Generation betrachtet auch das aktuell laufende TIES-Projekt (The Integration of the Second Generation in Europe), welches von der EU finanziert wird.
} 
zioökonomischen Status. Ergänzend zum Mikrozensus analysiere ich die Daten des DJIAusländersurveys, um im Mikrozensus nicht enthaltene Variablen wie Sprache, Freundschaftsbeziehungen und Werteinstellungen zu untersuchen. Darüber hinaus werden an einigen Stellen vorliegende Studien wie die erweiterten PISA-Untersuchungen ${ }^{73}$ von 2000 und 2003, Spracherhebungen und die Studie Deutsch Englisch Schülerleistung International (DESI) herangezogen. ${ }^{74}$

Mit der Entscheidung für eine quantitative Untersuchung sind jedoch auch spezifische Nachteile verbunden. Erstens sind quantitative Umfragen nicht theoriefrei: Den Kategorien der Erhebung liegen immer auch integrationstheoretische Annahmen zugrunde. Folgende Beispiele aus den von mir ausgewählten Datensets belegen dies:

- So wird im Mikrozensus das Zuzugsjahr über den Zuzug nach Deutschland nach einem mindestens sechsmonatigen Auslandsaufenthalt erhoben. Menschen, die über längere Zeiträume in zwei Staaten leben, würden somit als frisch Zugezogene erfasst werden. Eine transnationale Pendelmigration kann also nicht erkannt werden. Im Mikrozensus wird offensichtlich davon ausgegangen, dass ein solches ,Leben in zwei Welten' nicht in signifikantem Umfang existiert oder aber nicht von Interesse ist (MZ Frage 98, 99).

- Sowohl im Mikrozensus als auch im DJI-Ausländersurvey wird nur nach der Staatsbürgerschaft gefragt, nicht nach ,ethnischer' oder anderer Zugehörigkeit. Damit folgt die Umfrage Integrationstheorien, die nationale Zugehörigkeit als wichtigste Identifikations- und Fremdheitsmarkierung verstehen.

- Im DJI-Ausländersurvey wird ein mögliches Verwandtschaftsverhältnis zwischen den PartnerInnen abgefragt und die möglichen Antworten detailliert in elf Optionen aufgeschlüsselt (Sohn von Mutters Bruder, Sohn von Mutters Schwester, ...) (Frage 98 DJIAusländersurvey). Damit werden bestimmte integrationstheoretische Annahmen über

\footnotetext{
${ }^{73}$ Die PISA-Daten 2003 aus dem erweiterten nationalen Sample würden sich auch für eine Analyse eignen, speziell um Einstellungs- und Motivationszusammenhänge im Bildungsbereich zu überprüfen. Sie stehen allerdings für wissenschaftliche Analysen noch nicht zur Verfügung (Stand: Sommer 2007).

${ }^{74}$ Ideal wäre selbstverständlich eine eigene Erhebung gewesen, bei der die Instrumente der sozialen Umfragenforschung an das Erkenntnisinteresse der vorliegenden Arbeit angepasst und zusätzliche theorierelevante Fragen eingefügt werden. Eine solche eigene Untersuchung war jedoch aus Kosten- und Zeitgründen nicht möglich. Ein repräsentatives Sampling der Migrantenbevölkerung unterliegt besonderen Problemen, da die Standardverfahren der Gewinnung einer Stichprobe unter MigrantInnen aus dem Melderegister oder über eine Auswahl ,migrantischer' Nachnamen aus dem Telefonbuch mit Problemen der Repräsentativität einhergehen: Über das Melderegister lassen sich nur AusländerInnen sampeln, zudem sind die Angaben häufig veraltet, und bei Umfragen auf der Basis von Telefonbucheintragungen ist die Response sehr gering. Untersuchungen basierend auf dem Schneeballprinzip sind jedoch noch weniger repräsentativ. Neben diesen Problemen des Samplings sprachen Kostengründe gegen eine eigene Erhebung. Auch die Beschränkung auf einen Stadtteil oder eine Stadt schien bei dem gegebenen Forschungsinteresse nicht ratsam.
} 
soziale Realitäten nahegelegt, in diesem Fall die hohe Wahrscheinlichkeit einer Partnerwahl innerhalb der eigenen Familie.

Zweitens erlauben diese bundesweiten quantitativen Umfragen keine Analyse kleinräumiger Sozialstrukturen, da hierfür nicht genügend Personen in einem Stadtviertel befragt werden und die Datensätze in wissenschaftlichen Zweitanalysen nur auf einem bestimmten Aggregationsniveau untersucht werden dürfen (z.B. Bezirksebene). Auch dies hat integrationstheoretische Rückwirkungen, da nur solche Untersuchungen möglich sind, die sozialräumlichen Faktoren und Interaktionen bei der Integration keinen entscheidenden Einfluss zuschreiben.

Drittens sind umfangreiche quantitative Umfragen auch in den Aspekten limitiert, zu denen sie Fragen stellen. Eine Dichte und Tiefe des Materials, wie sie qualitative Untersuchungen üblicherweise bieten, können quantitative Studien somit nicht erreichen.

Damit hängt der vierte Punkt zusammen, dass quantitative Studien Zusammenhänge beschreiben können, aber nicht die Bedeutung und den Sinn, den MigrantInnen ihnen geben. Ein sinnhaftes Verstehen im Sinne von Max Weber ist daher nicht direkt möglich. ${ }^{75}$ Trotz dieser Nachteile quantitativer Untersuchungen wird aus den oben erläuterten Gründen für diese Arbeit mit ihrer spezifischen Fragestellung ein quantitativer Zugang mit großen sozialstatistischen Datensätzen gewählt. Durch den Einschluss vereinzelter qualitativer Untersuchungen wird versucht, einige problematische Aspekte abzuschwächen. Vor- und Nachteile eines solchen Zugangs werden im Schlusskapitel diskutiert.

\subsubsection{Beschreibung der Datengrundlage}

Die eigene empirische Auswertung in den nächsten Kapitel kombiniert also die Repräsentativität des Mikrozensus 2005 mit der Tiefe des Ausländersurveys des Deutschen Jugendinstituts. ${ }^{76}$ Für Daten zur Wahrnehmungsveränderung bei der Dominanzbevölkerung (Kapitel 5) wird die ALLBUS herangezogen. ${ }^{77}$ Ergebnisse kleinerer, spezialisierterer Untersuchungen

\footnotetext{
${ }^{75}$ Weber hat Soziologie sogar definiert als „Wissenschaft, welche soziales Handeln deutend verstehen und dadurch in seinem Ablauf und seinen Wirkungen ursächlich erklären will“" (Weber 1985: § 1).

${ }^{76}$ Andere große Sozialumfragen wie der European Social Survey (ESS) kamen wegen zu geringer Fallzahlen nicht in Frage. Das Sozio-Oekonomische Panel (SOEP) wäre noch am ehesten als Datensatz in Betracht gekommen. Das Migrantensample darin (=Sample B) umfasste 2003 knapp 400 Deutsche und 1130 AusländerInnen, von denen ein Teil der zweiten Generation angehört. Allerdings schien der Vorteil weiterer Informationen zu den Sprachkenntnissen die Nachteile, die sich aus relativ geringen Fallzahlen (nicht mehr als 600 Personen in der zweiten Generation insgesamt), einer Dominanz der türkischen Herkunftsgruppe und der Schwierigkeit, die zweite Generation unabhängig von der Staatsbürgerschaft und dem Zusammenwohnen mit den Eltern zu identifizieren, ergaben, nicht aufzuwiegen.

${ }^{77}$ Für eine Analyse auf der Individualebene in den Sachbereichen konnte die ALLBUS jedoch wegen zu geringer Fallzahlen nicht herangezogen werden: Er umfasst jedes Jahr nur 2400 Interviewte in West- und 1100 Interviewte in Ostdeutschland, darunter insgesamt kaum mehr als 150 MigrantInnen der zweiten Generation.
} 
werden in der empirischen Analyse eingespeist, sofern sie über das Material des Mikrozensus und des DJI-Ausländersurveys hinausgehende Aussagen erlauben und speziell für die Musteranalyse relevant sind. Das Design solcher zusätzlicher Studien wird an den betreffenden Stellen vorgestellt; der Mikrozensus und der Ausländersurvey des DJI werden im folgenden Abschnitt ausführlicher dargestellt.

\section{Mikrozensus}

Jährlich wird 1\% der Haushalte in Deutschland im Rahmen des Mikrozensus befragt. Die Haushalte werden von den Statistischen Landesämtern mit Hilfe einer Flächenstichprobe zufällig ausgewählt. Die Ergebnisse des Mikrozensus dienen der jährlichen Anpassung der letzten Volkszählung (1987). Das Datenset enthält einen Hochrechnungsfaktor, mit dem, basierend auf Geschlecht, Alter und Nationalität, in den räumlichen Erhebungskreisen eine Gewichtung des Samples zur Hochrechnung auf die gesamte Bevölkerung in Deutschland vorgenommen werden kann. Die Beantwortung des Großteils der Fragen ist verpflichtend, und auch bei den freiwilligen Angaben ist die Auskunftsbereitschaft sehr hoch.

Die Fragenkomplexe betreffen v.a. sozialstrukturelle Merkmale wie Erwerbsbeteiligung und tätigkeit, Arbeitssuche und Arbeitswege, Schul-, Aus- und Fortbildungen, Altersvorsorge, Einkommen, Fragen zur Gesundheit, und seit 2005 ist ein stark erweiterter Komplex zu Staatsbürgerschaft und Zuzugsjahren enthalten. Die meisten der Fragen werden jedes Jahr gestellt und sind hinsichtlich ihrer Validität ausgiebig geprüft. Fragen zu den Sozialbeziehungen (FreundInnen, Freizeitverhalten) sowie eine Erhebung von Sprachkenntnissen und Einstellungen und Werten fehlen jedoch.

Da die Fragebögen des Mikrozensus nur in Deutsch vorliegen und die Statistischen Landesämter nicht speziell InterviewerInnen mit Fremdsprachenkenntnissen einstellen, könnte angenommen werden, dass der Mikrozensus für die Subpopulation der MigrantInnen nicht repräsentativ ist. Eine ausführliche Untersuchung dieser Frage liegt bisher nicht vor. Allerdings scheint die Auskunftspflicht den sonst zu erwartenden Ausfall durch fehlende Sprachkenntnisse zu verhindern: MigrantInnen, die des Deutschen nicht hinreichend mächtig sind, finden Verwandte oder FreundInnen, die ihnen beim Beantworten der Fragen behilflich sind. ${ }^{78} \mathrm{Ab}-$ gesehen von der generellen Tendenz von Umfragen, Menschen aus stark marginalisierten sozialen Schichten (z.B. Obdachlose) nur ungenügend einzuschließen, kann daher davon ausge-

\footnotetext{
${ }^{78}$ So die Einschätzung des Zentrums für Umfragen, Methoden und Analysen (ZUMA) (telefonische Auskunft von Herrn Schimpl-Neimanns, ZUMA, 28.08.06.).
} 
gangen werden, dass der Mikrozensus ein repräsentatives Segment der MigrantInnen in Deutschland erfasst.

Der Mikrozensus 2005 wurde bis März 2006 erhoben. Ab Ende August 2006 konnte ich nach Bewilligung meines Antrags eine anonymisierte 70\%-Stichprobe im Berliner Forschungsdatenzentrum analysieren. Der Scientific Use File, der ebenfalls 70\% der Fälle enthält, war erst ab April 2007 erhältlich. Über die Ergebnisse dieser Untersuchungen durften jedoch keine Aufzeichnungen angefertigt werden. Daher wurden diese Analysen vor Ort durch eine kontrollierte Datenfernverarbeitung ergänzt, bei der die entwickelte Syntax ${ }^{79}$ über den vollständigen Datensatz lief; zur Verfügung gestellt wurde anschließend die zweifach geprüfte Ausgabedatei, in der sämtliche Ergebnisse, die zu geringe Fallzahlen beinhalteten oder Rückschlüsse auf Einzelfälle erlaubten, gelöscht waren. Der unübertreffliche Vorteil der kontrollierten Datenfernverarbeitung besteht also darin, dass die Ergebnisse auf dem 100\%-Sample des Mikrozensus beruhen.

Tabelle 2 gibt einen Überblick über die Verteilung der MigrantInnen der verschiedenen Herkunftsgruppen auf die Generationen. Die Zahlen sind anhand des Mikrozensus für Deutschland hochgerechnet und umfassen die Altersgruppe von 20 bis 45 Jahren. Die zweite Generation wurde dabei entlang der Definition von Crul und Vermeulen (Crul/Vermeulen 2003: 679, s. Kapitel 1.2) operationalisiert: Sie umfasst die in Deutschland geborenen Kinder von zwei zugewanderten Eltern. Gibt es Informationen nur zu einem Elternteil, so ist dieser ausschlaggebend. Kinder, die innerhalb der ersten sechs Lebensjahre zugewandert sind, fallen ebenfalls in diese Gruppe. Die Herkunft wird dabei über die aktuelle oder vormalige ausländische Staatsbürgerschaft des Vaters erhoben, fehlen Informationen hierzu, über die der Mutter. ${ }^{80}$ MigrantInnen der ersten Generation sind dementsprechend Personen, die nach dem 16. Lebensjahr nach Deutschland kamen. Ihre Herkunftsgruppe wird über ihre eigene aktuelle oder ehemalige Staatsbürgerschaft bestimmt. Als Kontrollgruppe werden MigrantInnen der anderthalbten Generation eingefügt, die im Alter von 7 bis 15 Jahren nach Deutschland zugewandert sind.

\footnotetext{
${ }^{79}$ Unter Syntax wird die schriftliche Mitteilung der Rechenschritte an das Programm verstanden. Sie ist eine Programmiersprache, die die Befehle für die anzufertigenden Berechnungen und den Programmablauf enthält. Da sie separat von den Daten gespeichert wird, kann über sie die Herleitung der statistischen Ergebnisse nachvollzogen und kontrolliert werden.

${ }^{80}$ Eine Kontrolluntersuchung ergab, dass die beiden Staatsbürgerschaften sich in den meisten Fällen entsprechen.
} 
Die Analyse beschränkt sich auf MigrantInnen türkischer, italienischer, griechischer, exjugoslawischer ${ }^{81}$ und spanisch-portugiesischer ${ }^{82}$ Herkunft, da diese wie in Tabelle 2 zu sehen ist, die größten Herkunftsgruppen stellen. ${ }^{83}$ Zudem werden zwei Vergleichsgruppen eingeführt: MigrantInnen aus, westlichen' Nicht-Anwerbestaaten und (Kinder von) AussiedlerInnen. AussiedlerInnen sind nach dem Bundesvertriebenengesetz deutschstämmige Personen aus historischen Siedlungsgebieten in Osteuropa, Südeuropa, Asien oder den Regionen, in welche sie vertrieben wurden, die zum Teil seit Generationen in diesen Ländern leben und nun nach Deutschland migrieren. Personen, die nach dem 31.12.1992 zugewandert sind, werden als SpätaussiedlerInnen bezeichnet ( $\$ 4$ Abs. 1,2 Bundesvertriebenengesetz). ${ }^{84}$ Da bis Sommer 2007 keine Typologisierung des Statistischen Bundesamtes für (Spät)AussiedlerInnen im Mikrozensus 2005 vorlag, wurden MigrantInnen aus den möglichen Herkunftsländern (Russland, Kasachstan, Polen, Rumänien etc.), die nach ihrer Zuwanderung nach Deutschland ohne Einbürgerung die deutsche Staatsbürgerschaft erworben haben, als (Spät-)AussiedlerInnen eingeordnet. Weiterhin wurden solche Personen zu den (Spät)AussiedlerInnen gezählt, die zwar angaben, eingebürgert worden zu sein, deren Einbürgerung aber innerhalb eines Jahres nach Ankunft in Deutschland erfolgt ist. Ein solch schneller Erwerb der Staatsbürgerschaft ist für ausländische Staatsangehörige aus den oben genannten Ländern nicht möglich, auch nicht bei Heirat mit einem/r Deutschen. Die Einbürgerung innerhalb eines Jahres nach Ankunft verweist daher ebenfalls auf einen (Spät-)Aussiedlerstatus und legt nahe, dass die (Spät-)AussiedlerInnen den Erhalt des deutschen Passes subjektiv als Einbürgerung verstanden haben.

Die Analyse wird, um Informationen zum Arbeitsmarktgeschehen zu erlangen, auf die Altersgruppe der 20- bis 45-Jährigen limitiert. Damit unterscheidet sich das ausgewählte Alterssegment erheblich von dem in anderen Integrationsdatensätzen, die sich zumeist auf Jugendliche

\footnotetext{
${ }^{81}$ Da in dieser Untersuchung nur die von 1960 bis 1985 geborene zweite Generation betrachtet wird, besteht für diese Gruppe nicht das sonst übliche Problem, Personen mit sehr heterogener Migrationsgeschichte zu vermischen (z.B. ArbeitsmigrantInnen aus den 1960er und 1970er Jahren und Bürgerkriegsflüchtlinge der 1990er Jahre).

${ }^{82}$ Die spanische und die portugiesische Herkunftsgruppe mussten wegen zu geringer Fallzahlen in der Analyse zusammen betrachtet werden. Die beiden Gruppen stimmen aber hinsichtlich ihres durchschnittlichen sozioökonomischen Status überein, wie eine hier nicht extra belegte Kontrolluntersuchung zeigte.

${ }^{83}$ Dabei decken diese Gruppen mit Ausnahme der PolInnen, RussInnen und UkrainerInnen auch die 15 größten Gruppen ausländischer StaatsbürgerInnen in Deutschland im Jahr 2003 ab (http://www.zuwanderung.de/tabellen/1_02.html (24.04.2006)). MigrantInnen polnischer, russischer und ukrainischer Herkunft gehören zudem überwiegend der ersten Migrantengeneration an.

${ }^{84} \mathrm{Da}$ in meine Untersuchung der zweiten Generation nur MigrantInnen eingeschlossen wurden, die im Jahr 2005 zwischen 20 und 45 Jahre alt waren, gibt es unter ihnen keine Kinder von SpätaussiedlerInnen, sondern nur Aussiedlerkinder.
} 
und manchmal auf junge Erwachsene bis maximal 25 Jahre konzentrieren. ${ }^{85}$ Letztere Gruppe ist auch Gegenstand des DJI-Ausländersurveys, der nun vorgestellt wird.

\footnotetext{
${ }^{85}$ So hat Haug basierend auf dem Integrationssurvey gezeigt, dass viele MigrantInnen wie Mitglieder der Dominanzbevölkerung mit 18, spätestens mit 20 Jahren ihre Elternhäuser verlassen (Haug 2004: 171) und dann darüber nicht mehr erhoben werden können.
} 
Tabelle 2 - MigrantInnen: Generationen und Herkunftsgruppen

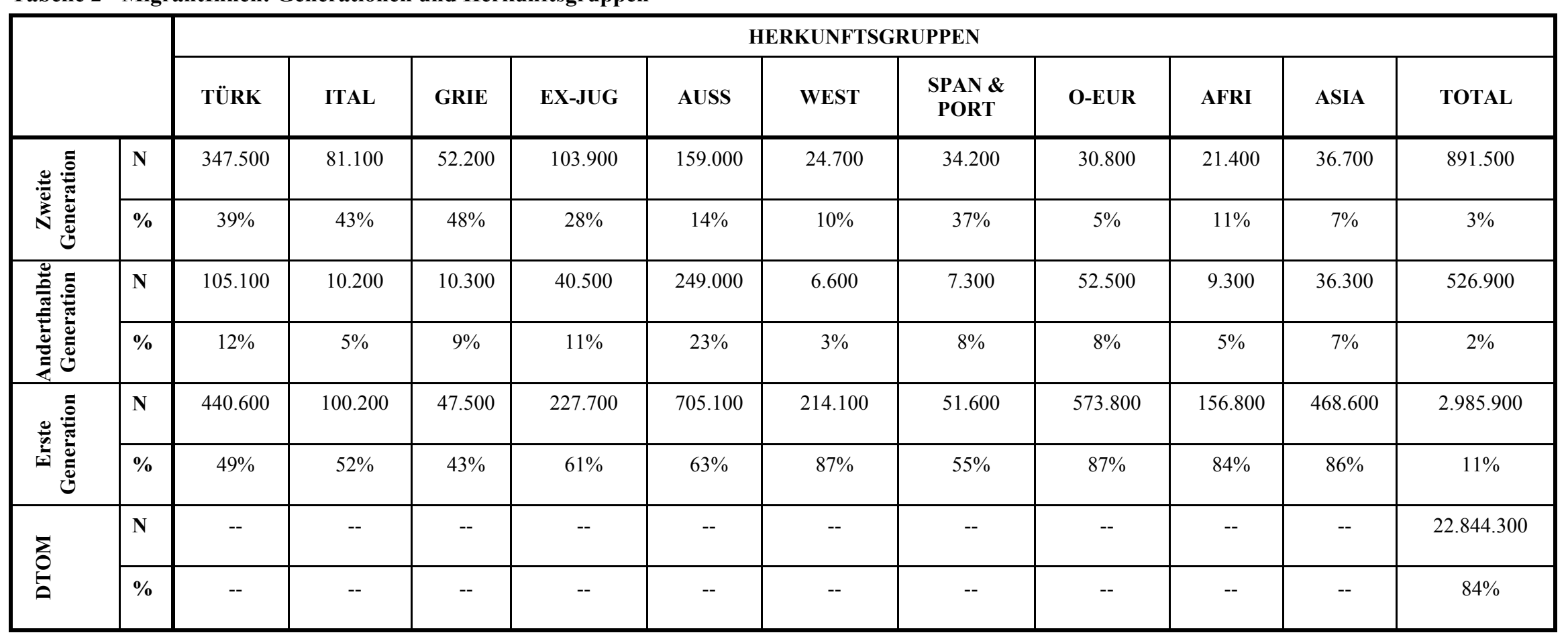

Mikrozensus 2005, FDZ der Statistischen Ämter des Bundes und der Länder, Eigene Berechnungen 


\section{DJI-Ausländersurvey}

Der Ausländersurvey 1997 wurde parallel zum Jugendsurvey 1997 vom Deutschen Jugendinstitut in München in Zusammenarbeit mit der Technischen Universität Chemnitz entwickelt. Die Daten wurden von November 1996 bis Februar 1997 von der Marplan Forschungsgesellschaft nach einem Quotenplan, der auf Nationalität, Geschlecht, Alter, Familienstruktur, Befragungsgebiet und Verstädterungsgrad basiert, erhoben. Hierzu wurden persönliche mündliche Interviews anhand vollstrukturierter Fragebögen durchgeführt, die jeweils sowohl in Deutsch als auch in der Muttersprache der Eltern vorlagen. (Mittag/Weidacher 2000: 273ff) Befragt wurden MigrantInnen türkischer, italienischer und griechischer Herkunft der Altersgruppe von 18 bis 25 Jahren. Die Untersuchung war limitiert auf die ,alten' Bundesländer, da kaum MigrantInnen dieser Herkunftsländer in den ,neuen’ Bundesländern wohnen. Der Ausländersurvey enthält neben den Standardfragen zu Sozialstruktur (Bildung, Berufsbildung, Arbeitstätigkeit, Einkommen, Partnerschaftsstatus, Kinder) viele Items zu sozialen Interaktionen, Freundesnetzwerken, Einstellungen, Werten und Sprachkenntnissen. Dadurch ergänzt er den Mikrozensus gut. Die Analyse wurde mit dem anonymisierten Scientific Use File durchgeführt, der die volle Stichprobe enthält.

Die Einteilung der MigrantInnen in erste, anderthalbte und zweite Generation erfolgte entlang dem zuvor vorgestellten Schema. Die wenigen Fälle, bei denen nur einer der beiden Elternteile im Ausland geboren war, wurden von der Analyse ausgeschlossen. Tabelle 3 zeigt, dass der Schwerpunkt des Datensets bei der zweiten Generation liegt, in allen Generationen aber ausreichende Fallzahlen vorliegen. Die Zuweisung der Herkunftsgruppen liegt im Datensatz schon als Variable vor. Allerdings sind alle Befragten ausländische StaatsbürgerInnen. Dies war von den ForscherInnen des DJI nicht intendiert, sie nahmen sogar eine Frage zur Einbürgerung in den Bogen auf. Es verdeutlicht jedoch die Schwierigkeit, MigrantInnen mit deutscher Staatsbürgerschaft in kleineren Untersuchungen zu erfassen. Tabelle 3 stellt die Fallzahlen über Herkunftsgruppen und Generation dar.

Tabelle 3 - Fallzahlen im DJI-Ausländersurvey nach Generationen und Herkunftsgruppen

\begin{tabular}{|l|c|c|c|c|}
\hline & TÜRK & ITAL & GRIE & GESAMT \\
\hline Zweite Generation & 603 & 592 & 576 & 1.771 \\
\hline Anderthalbte Generation & 127 & 134 & 134 & 395 \\
\hline Erste Generation & 87 & 77 & 68 & 232 \\
\hline Gesamt & 817 & 803 & 778 & 2.398 \\
\hline
\end{tabular}

Ausländersurvey 1997 des Deutschen Jugendinstituts, Eigene Berechnungen 
Im Folgenden wird das methodische Vorgehen der Arbeit erläutert.

\subsubsection{Methoden}

In der empirischen Untersuchung werden zunächst mit Methoden der deskriptiven Statistik Verteilungen von MigrantInnen verschiedener Herkunftsgruppen und Generationen hinsichtlich relevanter Merkmale in den Bereichen Sprache, Bildung, Beruf und soziale Beziehungen analysiert. ${ }^{86}$ Um eventuelle Unterschiede zwischen den Generationen und Herkunftsgruppen (inklusive der Vergleichsgruppe der Deutschen ohne Migrationshintergrund) zu erheben, werden diese Verteilungen in zwei- und dreischichtigen Kreuztabellen dargestellt. Zwecks Überprüfung der Streuung in den Bildungs- und Berufsergebnissen wurden die Interquartilbereiche verschiedener Variablen nach Herkunftsgruppen und Generationen getrennt untersucht. Der Interquartilbereich untersucht, wie ähnlich oder unterschiedlich sich die Integrationsergebnisse von MigrantInnen innerhalb einer Herkunftsgruppe (oder einer Generation) verhalten. Er gibt als Dispersionsmaß die Breite der Verteilung zwischen 75\%-Perzentil und 25\%Perzentil an, also für die 50\% der Personen, die sich hinsichtlich der Verteilung in der Mitte befinden. Dadurch ist der Interquartilbereich unempfindlich gegenüber Ausreißern und erfordert keine Normalverteilung der Variablen. (Norusis 2003: 98ff)

Um die Herkunftsgruppenunterschiede quantifizieren und in Abhängigkeit von der Generation bringen zu können, werden in den Sachbereichen Bildung und Arbeitsmarkt Regressionen mit Herkunfts- und Generationsdummies durchgeführt, bei denen Standardvariablen der Sozialforschung (Geschlecht, Alter, Stadt-Land-Verteilung) kontrolliert werden. Die Signifikanz der durchschnittlichen Unterschiede wird ebenfalls geprüft. Dummies dienen dazu, nominale Variablen mit mehreren Ausprägungen jeweils auf eine binäre Struktur zu reduzieren, wobei eine Ausprägung als Referenzkategorie dient (z.B. italienische Herkunft/keine italienische Herkunft im Vergleich zur türkischen Herkunft), so dass sie in multivariate Verfahren eingeschlossen werden können. Die Regressionskoeffizienten B geben dann den durchschnittlichen Unterschied in der abhängigen Variable im Verhältnis zur Referenzkategorie an. Dadurch können für jede Kombination von unabhängigen Variablen Durchschnittswerte in Bezug auf die abhängige Variable berechnet werden. In den hier vorliegenden Regressionen wurden die türkische Herkunftsgruppe und die erste Migrantengeneration als Referenzkategorien gewählt; die Entscheidung für bestimmte Referenzkategorien beeinflusst nicht die Durch-

\footnotetext{
${ }^{86}$ Sprachkompetenz ist die Vorbedingung für gesellschaftliche Partizipation. In Bildung, Beruf und Familie werden Leistungsrollen eingenommen, die auf viele andere Aspekte gesellschaftlichen Lebens (z.B. politische Partizipation, gesundheitliches Wohlergehen) Einfluss haben. Identitätsfragen wurden außer Acht gelassen, da sie als volatil eingeschätzt wurden.
} 
schnittswerte. Weiterhin werden in den verschiedenen Sachbereichen Regressionen zur Überprüfung spezieller, in den Theorien hypothetisierter Zusammenhänge (z.B. positive Effekte von Zweisprachigkeit für Bildungsergebnisse) durchgeführt.

Anschließend werden die Indikatoren der verschiedenen Sachbereiche verbunden und in die vier Muster aus Kapitel 2.5 überführt. Die Operationalisierung der Statusmerkmale (Bildung und Beruf) und Kulturmerkmale (Sprache, Partnerschaften) wird in Kapitel 4.1.1 dargestellt. Die sich daraus ergebenden vier Muster werden in Kreuztabellen auf ihre Verteilung in den Generationen und Herkunftsgruppen untersucht. Um von den Theorien postulierte kausale Zusammenhänge zu plausibilisieren oder in Frage zu stellen, werden in Kapitel 4.2 Durchschnittswerte theorierelevanter Variablen über die vier Muster verglichen. Hierzu werden Boxplots der Durchschnittswerte mit einem 95\%-Konfidenzintervall ${ }^{87}$ angefertigt und grafisch dargestellt. Überschneiden sich diese Boxplots nicht, dann kann auf einem statistischen Signifikanzniveau von $\alpha \leq 0,05^{88}$ davon ausgegangen werden, dass die Durchschnittswerte voneinander abweichen. In der grafischen Darstellung lässt sich zudem sofort die Richtung der Abweichung erkennen. Ergänzt werden diese Untersuchungen durch Ergebnisse aus anderen Studien, insofern diese Aussagen zu theorierelevanten Aspekten machen, die mit den anderen Datensets nicht untersucht werden konnten. Insgesamt können darüber die Zusammenhänge in den Mustern zwar plausibilisiert oder abgelehnt werden, nicht aber das Zustandekommen eines Musters auf der Ebene des Individuums in aller Komplexität der hierfür bedeutsamen Faktoren kausal erklärt werden. Für eine solche Erklärung der Integrationspfade wäre eine Längsschnittstudie mit qualitativen Daten nötig.

In Kapitel 5 wird dann untersucht, inwieweit die in der Empirie gefundenen Muster für Deutschland auch mit einer vergrößerten bzw. verkleinerten Ähnlichkeitswahrnehmung verschiedener Gruppen der zweiten Generation bei der Dominanzbevölkerung zusammengehen. Hierfür werden Umfragedaten der ALLBUS analysiert sowie die Ergebnisse zweier quasiexperimentellen Untersuchungen zu Hilfe gezogen. Erst wenn sich neben den Mustern auch eine Erhöhung der Ähnlichkeitswahrnehmung gegenüber den entsprechenden Gruppen belegen lässt, kann von Integration gesprochen werden.

\footnotetext{
${ }^{87}$ Ein 95\%-Konfidenzintervall umfasst den Bereich um den berechneten Mittelwert der Stichprobe, innerhalb dessen der (reale) Mittelwert der Grundgesamtheit mit 95\% Wahrscheinlichkeit liegt.

${ }^{88} \alpha$ gibt die Wahrscheinlichkeit eines Fehlers erster Ordnung an, also die Wahrscheinlichkeit für einen falsch positiven. Dies sind Fälle, in denen die Nullhypothese abgelehnt wird, obwohl sie stimmt. (Schaich 1990: 188) In der hier besprochenen Untersuchung der grafischen Darstellung der Durchschnittswerte bedeutet dies, dass in maximal 5\% der Fälle ein Unterschied in den Durchschnittswerten der Muster konstatiert wird, wo eigentlich keiner vorliegt.
} 
Bevor nun die Ergebnisse der einzelnen Sachbereiche diskutiert werden, gibt Kapitel 3.2 allgemeine Informationen zur zweiten Generation in der Altersgruppe von 20 bis 45 Jahren, die sich aus meinen Datensets ergeben.

\subsection{Zweite Generation}

In diesem Kapitel wird basierend auf dem Mikrozensus die Zusammensetzung der zweiten Generation von MigrantInnen nach den von mir ausgewählten Herkunfts- und Altersgruppen sowie nach dem Anteil der deutschen Staatsbürger untersucht. Zusammen mit Informationen zur sozialräumlichen Verteilung soll dadurch ein erster Überblick über die zweite Generation gegeben werden.

Wie in Abbildung 2 zu sehen ist, ist die zweite Generation v.a. bei den unter 20-Jährigen sehr umfangreich, wo sie zwischen 12 und 20\% der Geburtsjahrgänge stellt. ${ }^{89}$ Auch wenn der jeweilige Prozentsatz des Jahrgangs sich durch eine spätere Zuwanderung von erwachsenen MigrantInnen derselben Jahrgänge etwas verringern wird, ist offensichtlich, dass die zweite Generation über die Kohorten an absoluter und relativer Größe gewinnt. Zusammen mit MigrantInnen der ersten (und zunehmend der dritten ${ }^{90}$ ) Generation sowie den Personen mit nur einem im Ausland geborenen Elternteil, die aus Tabelle 2 ausgeschlossen sind, ist der zahlenmäßige Umfang von Menschen mit Migrationshintergrund in den jüngeren Alterskohorten hoch und wird auch in Zukunft weiter steigen. Dies gilt aufgrund der ,BestandsmigrantInnen' auch dann, wenn die Zuwanderung nach Deutschland zukünftig weiter erschwert werden sollte. Insgesamt machen MigrantInnen der ersten, anderthalbten und zweiten Generation (ohne Personen mit nur einem migrantischen Elternteil) 15\% der Bevölkerung in Deutschland aus.

Es wird aber auch deutlich, dass in der Altersgruppe von 20 bis 45 Jahren, die hier untersucht wird, ein signifikanter Prozentsatz der Bevölkerung der zweiten Generation angehört, wohingegen in den älteren Segmenten kaum mehr Angehörige der zweiten Generation zu finden sind: Nur 28.000 MigrantInnen der zweiten Generation in Deutschland sind vor 1960 gebo-

\footnotetext{
${ }^{89}$ Die Differenz der Gesamtsumme über die Generationen zu den 82 Millionen Menschen Bevölkerung in Deutschland rührt aus dem Ausschluss von Personen mit einseitigem Migrationshintergrund und der dritten Generation.

${ }^{90}$ Die dritte Generation, also die Kinder von MigrantInnen der zweiten Generation, ist zumeist noch so jung, dass über sie keine Aussagen zu Bildungsabschlüssen und Arbeitsmarktpositionen getroffen werden können. Dies wird sich in den nächsten Jahren ändern. Laut einer Hochrechnung auf der Basis des Mikrozensus 2005 umfasst die dritte Generation 107.000 ausländische Staatsangehörige (Bildungsberichterstattung 2006: 140). Über die Zahl der MigrantInnen mit deutscher Staatsangehörigkeit in der dritten Generation liegen keine Zahlen vor, und aus den oben genannten Altersgründen wurden keine eigenen Zahlen berechnet.
} 
ren. Diese älteren MigrantInnen wurden ausgeschlossen, um eine Vergleichbarkeit innerhalb der Gruppen zu gewährleisten. Wie im vorangegangenen Kapitel erläutert, ist das Ziel der vorliegenden Arbeit, auch die sonst häufig vernachlässigte Arbeitsmarktbeteiligung der zweiten Generation zu untersuchen. Daher wurden MigrantInnen der zweiten Generation, die 2005 jünger als 20 Jahre waren, ebenfalls aus der Analyse ausgeschlossen.

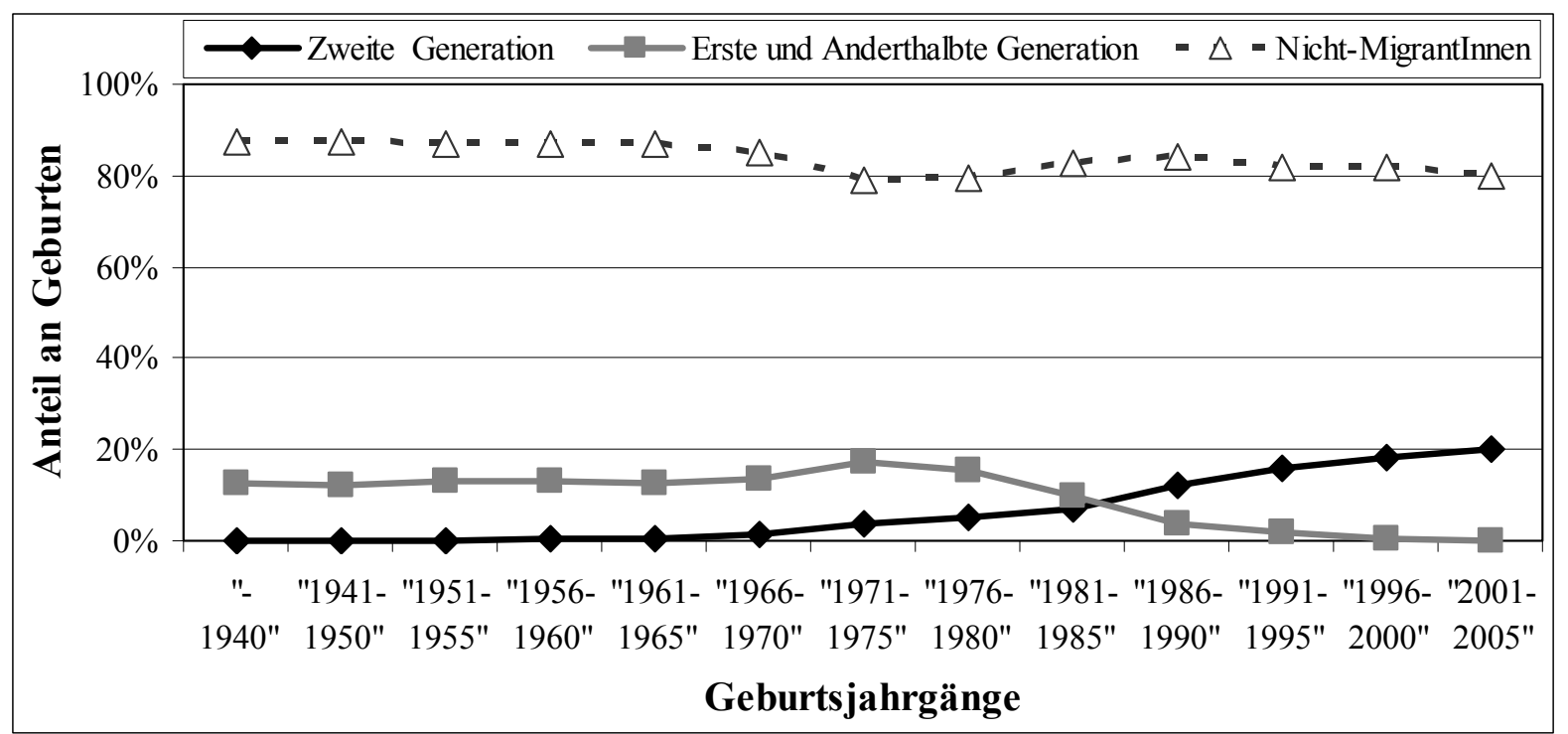

Mikrozensus 2005, FDZ der Statistischen Ämter des Bundes und der Länder, Eigene Berechnungen

Abbildung 2 - Generationen über Geburtsjahrgänge

Abbildung 3 verdeutlicht, dass MigrantInnen türkischer Herkunft die größte Gruppe der zwischen 1960 und 1985 Geborenen der zweiten Generation darstellen, gefolgt von den Kindern von AussiedlerInnen. Danach kommen MigrantInnen ex-jugoslawischer, dann italienischer, griechischer und schließlich spanisch-portugiesischer Herkunft. Diese Herkunftsgruppen sind auch in der zweiten Generation insgesamt die Bedeutsamsten und werden daher in der vorliegenden Arbeit betrachtet. Zum Vergleich zu diesen Migrantengruppen aus Ländern, mit denen Anwerbeabkommen bestanden, und der Gruppe der AussiedlerInnen wird eine Gruppe ,westlicher’ MigrantInnen aus Nicht-Anwerbeländern eingeschlossen, obwohl diese zahlenmäßig geringer ausfällt als z.B. die Gruppe von MigrantInnen der zweiten Generation, deren Eltern aus verschiedenen asiatischen Staaten stammen. 


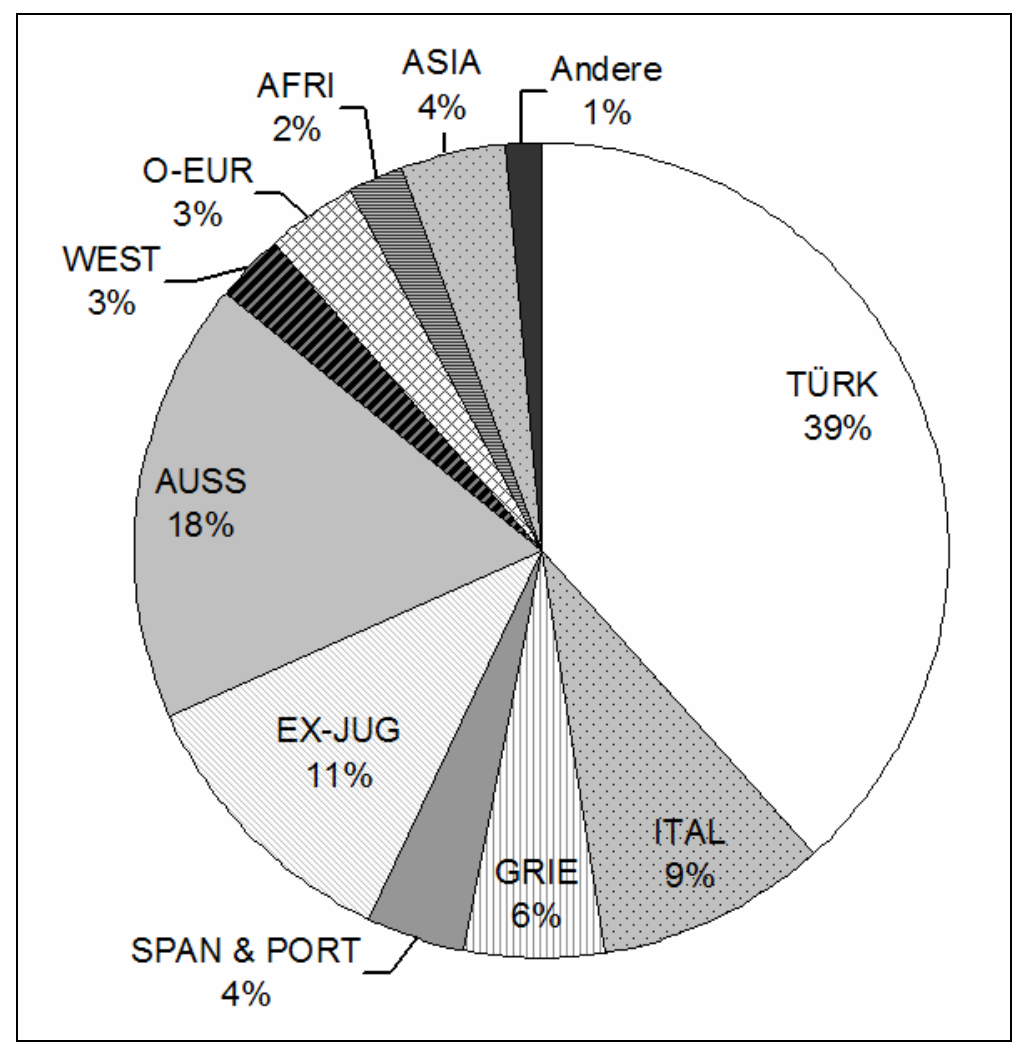

Mikrozensus 2005, FDZ der Statistischen Ämter des Bundes und der Länder, Eigene Berechnungen

Abbildung 3 - Zweite Generation nach Herkunftsgruppen

Hinsichtlich der Staatsbürgerschaften fällt bei der zweiten Migrantengeneration der 20- bis 45-Jährigen auf, dass jene mit türkischer Herkunft ${ }^{91} \mathrm{zu} 43 \%$ die deutsche Staatsbürgerschaft besitzen, wohingegen dies bei MigrantInnen italienischer, griechischer und spanischportugiesischer Herkunft nur bei unter 10\% der Fall ist (Abbildung 4). Die Zahlen schließen Personen mit doppelter Staatsbürgerschaft ein. Die Kinder von AussiedlerInnen ${ }^{92}$ haben zu 100\% (auch) die deutsche Staatsbürgerschaft, da diese konstitutiver Teil ihres Status als AussiedlerInnen ist. Bei den MigrantInnen der zweiten Generation jugoslawischer Herkunft besitzen 25\% (auch) die deutsche Staatsbürgerschaft; bei MigrantInnen, deren Eltern aus den ,westlichen' Nicht-Anwerbestaaten kamen, sind es 33\%. MigrantInnen jugoslawischer und ,westlicher' Herkunft haben zusammen mit den AussiedlerInnen mit 4\% bzw. 6\% auch den

\footnotetext{
${ }^{91}$ Dies schließt MigrantInnen kurdischer Herkunft ein. Der Mikrozensus erfasst nur die aktuelle und vormalige eigene und elterliche Staatsbürgerschaft. Ethnische Zugehörigkeiten werden nicht erfragt. Für einen Überblick speziell über die Unterschiede in der Lebenssituation von kurdischen und türkischen MigrantInnen in Deutschland siehe Senol 1992 und Falk 1998.

${ }_{92}$ Seit dem 1. 1. 1993 werden gemäß Bundesvertriebenengesetz alle ,deutschstämmigen' ZuwanderInnen als SpätaussiedlerInnen bezeichnet, zuvor als AussiedlerInnen. Da ich in meiner Analyse der zweiten Generation MigrantInnen betrachte, die im Jahr 200520 Jahre alt waren und bis spätestens zum 6. Lebensjahr zugewandert sind, können unter ihnen nur Kinder von AussiedlerInnen sein, in der ersten und anderthalbten Generation jedoch auch Kinder von SpätaussiedlerInnen.
} 
höchsten Anteil an Personen mit doppelter Staatsbürgerschaft. ${ }^{93}$ Die Quote der deutschen StaatsbürgerInnen ist also bei den MigrantInnen türkischer Herkunft am größten. Je nachdem, ob zwischen deutschen StaatsbürgerInnen und AusländerInnen signifikante Unterschiede hinsichtlich des sozioökonomischen Status und kultureller Anpassung bestehen, wären für die türkische Herkunftsgruppe die Ergebnisse bei ausschließlicher Betrachtung des ausländischen Teils der zweiten Generation am wenigsten repräsentativ. In der ersten und anderthalbten Generation fällt im Vergleich in allen Herkunftsgruppen die Zahl der deutschen StaatsbürgerInnen wesentlich niedriger aus.

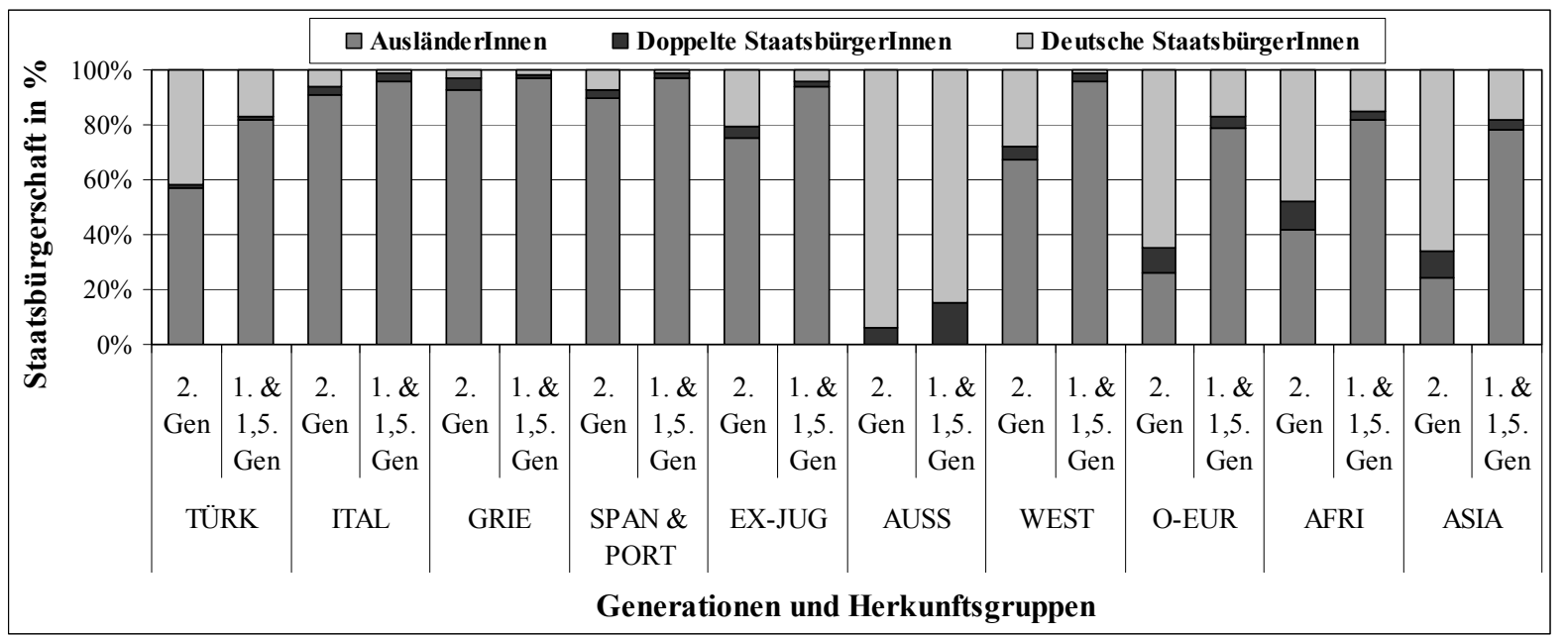

Mikrozensus 2005, FDZ der Statistischen Ämter des Bundes und der Länder, Eigene Berechnungen

Abbildung 4 - Staatsbürgerschaft: Generationen und Herkunftsgruppen

Wie sieht es mit der regionalen Verteilung der MigrantInnen in Deutschland aus? Bis auf AussiedlerInnen der zweiten Generation, deren Eltern bei Ankunft nach einem verbindlichen Schlüssel auf alle Bundesländer verteilt worden sind, wohnt in der zweiten Generation aus keiner Herkunftsgruppe ein signifikanter Prozentsatz in den ,neuen' Bundesländern, Berlin ausgenommen (Tabelle 4). Auch von den AussiedlerInnen leben in der Altersgruppe von 20 bis 45 Jahren nur knapp 5\% in Ostdeutschland. Die Gruppe der ,westlichen' MigrantInnen aus Nicht-Anwerbestaaten folgt mit 2\%. Selbst bei den afrikanischen und asiatischen Herkunftsregionen, die teilweise durch Arbeitsanwerbung und Kooperationsabkommen mit der DDR historisch eine engere Verbindung zu Ostdeutschland haben, liegen die Zahlen unter 1\%. Die Kinder der größeren Herkunftsgruppen, unter ihnen viele ArbeitsmigrantInnen, leben somit zu einem vernachlässigbaren Anteil in Ostdeutschland und können als Phänomen der westdeutschen Bundesländer (inkl. Berlin) aufgefasst werden.

\footnotetext{
${ }^{93}$ Nur bei den in dieser Arbeit nicht untersuchten MigrantInnen aus osteuropäischer, afrikanischer und asiatischer Herkunft liegt der Anteil doppelter StaatsbürgerInnen mit ca. 10\% noch höher.
} 
Tabelle 4 - Zweite Generation: Ost-West-Verteilung

\begin{tabular}{|c|c|c|c|c|c|c|c|c|c|c|c|}
\hline & TÜRK & ITAL & GRIE & $\begin{array}{l}\text { SPAN \& } \\
\text { PORT }\end{array}$ & EX-JUG & AUSS & WEST & O-EUR & AFRI & ASIA & DTOM \\
\hline $\begin{array}{l}\text { Ehemalige Bundesrepublik } \\
\text { und Berlin }\end{array}$ & $99,9 \%$ & $99,5 \%$ & $99,8 \%$ & $99,9 \%$ & $99,8 \%$ & $95,3 \%$ & $98,5 \%$ & $98,5 \%$ & $99,9 \%$ & $99,2 \%$ & $81,2 \%$ \\
\hline $\begin{array}{l}\text { Ehemalige DDR (ohne Ber- } \\
\text { lin) }\end{array}$ & $0,1 \%$ & $0,5 \%$ & $0,2 \%$ & $0,1 \%$ & $0,2 \%$ & $4,7 \%$ & $1,5 \%$ & $1,5 \%$ & $0,1 \%$ & $0,8 \%$ & $18,8 \%$ \\
\hline
\end{tabular}

Mikrozensus 2005, FDZ der Statistischen Ämter des Bundes und der Länder, Eigene Berechnungen

Tabelle 5 - Zweite Generation: Stadt-Land-Verteilung

\begin{tabular}{|c|c|c|c|c|c|c|c|c|c|c|c|}
\hline & TÜRK & ITAL & GRIE & $\begin{array}{l}\text { SPAN \& } \\
\text { PORT }\end{array}$ & EX-JUG & AUSS & WEST & O-EUR & AFRI & ASIA & DTOM \\
\hline Urban & $75 \%$ & $68 \%$ & $82 \%$ & $69 \%$ & $71 \%$ & $60 \%$ & $52 \%$ & $64 \%$ & $85 \%$ & $83 \%$ & $47 \%$ \\
\hline Semi-Urban & $21 \%$ & $30 \%$ & $16 \%$ & $28 \%$ & $26 \%$ & $30 \%$ & $32 \%$ & $30 \%$ & $14 \%$ & $14 \%$ & $33 \%$ \\
\hline Ländlich & $4 \%$ & $2 \%$ & $2 \%$ & $3 \%$ & $3 \%$ & $10 \%$ & $16 \%$ & $6 \%$ & $1 \%$ & $3 \%$ & $20 \%$ \\
\hline
\end{tabular}

Mikrozensus 2005, FDZ der Statistischen Ämter des Bundes und der Länder, Eigene Berechnungen

Tabelle 6 - Zweite Generation: Geschlechterverteilung

\begin{tabular}{|c|c|c|c|c|c|c|c|c|c|c|c|}
\hline & TÜRK & ITAL & GRIE & $\begin{array}{l}\text { SPAN \& } \\
\text { PORT }\end{array}$ & EX-JUG & AUSS & WEST & O-EUR & AFRI & ASIA & DTOM \\
\hline Frauen & $48 \%$ & $47 \%$ & $44 \%$ & $51 \%$ & $45 \%$ & $45 \%$ & $46 \%$ & $51 \%$ & $46 \%$ & $49 \%$ & $50 \%$ \\
\hline Männer & $52 \%$ & $53 \%$ & $56 \%$ & $49 \%$ & $55 \%$ & $55 \%$ & $54 \%$ & $49 \%$ & $54 \%$ & $51 \%$ & $50 \%$ \\
\hline
\end{tabular}

Mikrozensus 2005, FDZ der Statistischen Ämter des Bundes und der Länder, Eigene Berechnungen 
Tabelle 5 zeigt, dass MigrantInnen der zweiten Generation gemäß der Eurostat Definition mehrheitlich in urbanen Räumen leben. Bei den MigrantInnen der zweiten Generation ist der großstädtische Anteil bei der griechischen Herkunftsgruppe am größten (82\%), gefolgt von der türkischen Herkunftsgruppe mit 75\%. Aus der Gruppe ,westlicher' MigrantInnen aus Nicht-Anwerbestaaten leben am wenigsten MigrantInnen in Großstädten. Der Anteil der MigrantInnen der zweiten Generation, die in einem ländlichen Umfeld leben, liegt insgesamt um die 3\%. Nur bei AussiedlerInnen, bedingt auch durch die ursprüngliche Zuweisung, und bei Kindern von ,westlichen' MigrantInnen aus Nicht-Anwerbestaaten ist er höher (10 bzw. 16\%) und kommt damit dem Prozentsatz bei den Deutschen ohne Migrationshintergrund am nächsten.

Das Verhältnis von Männern zu Frauen wird für die 20- bis 45-Jährigen in der zweiten Generation in Tabelle 6 dargestellt: Abgesehen von der spanisch-portugiesischen und der osteuropäischen Kategorie ist in allen Herkunftsgruppen ein leichtes Übergewicht männlicher MigrantInnen der zweiten Generation zu verzeichnen. Die Ursachen hierfür sind unklar.

Wie sieht es mit Rückkehrabsichten bei der zweiten Generation aus? Gemäß dem Ausländersurvey des Deutschen Jugendinstituts wollen 85\% der MigrantInnen der zweiten Generation aus der türkischen, italienischen und griechischen Herkunftsgruppe in Deutschland bleiben (Tabelle 7).

Tabelle 7 - Verbleibeabsicht

\begin{tabular}{|c|c|c|c|c|}
\hline & & TÜRK & ITAL & GRIE \\
\hline \multirow{3}{*}{ 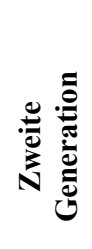 } & In Deutschland verbleiben & $85 \%$ & $86 \%$ & $85 \%$ \\
\hline & Zurückkehren & $11 \%$ & $12 \%$ & $12 \%$ \\
\hline & In drittes Land umsiedeln & $4 \%$ & $2 \%$ & $3 \%$ \\
\hline \multirow{3}{*}{ 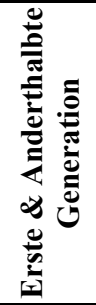 } & In Deutschland verbleiben & $66 \%$ & $49 \%$ & $49 \%$ \\
\hline & Zurückkehren & $34 \%$ & $50 \%$ & $49 \%$ \\
\hline & In drittes Land umsiedeln & $0 \%$ & $1 \%$ & $2 \%$ \\
\hline
\end{tabular}

Ausländersurvey 1997 des Deutschen Jugendinstituts, Eigene Berechnungen

Knapp über $10 \%$ dieser drei Herkunftsgruppen möchten in die Heimatländer ihrer Eltern zurückkehren, und etwa 3\% geben an, in ein drittes Land weiterwandern zu wollen. Diese Ergebnisse unterscheiden sich deutlich von denen in der ersten und anderthalbten Generation, 
von denen noch die Hälfte der MigrantInnen in die Herkunftsländer zurückkehren will, außer bei der türkischen Herkunftsgruppe. Dort wünschen sich dies nur ein Drittel der Befragten.

Dieses kurze Kapitel hat gezeigt, dass MigrantInnen der zweiten Generation eine zunehmend wichtige Gruppe der Bevölkerung in Deutschland ausmachen, deren Anteil in den jüngeren Geburtsjahrgängen steigt. Allerdings ist der Umfang der zweiten Generation bis hinauf zu den heute 45-Jährigen signifikant, so dass eine Untersuchung der zweiten Generation sich keinesfalls auf Kinder und Jugendliche beschränken sollte. Fast neun Zehntel der zweiten Generation geben an, in Deutschland verbleiben zu wollen. Auch in der Selbsteinschätzung der MigrantInnen handelt es sich also überwiegend nicht um einen temporären Aufenthalt. Daher sind Untersuchungen zur Integration und zu den Teilhabechancen der zweiten Generation wichtig.

Die zweite Generation stellt sich dabei insgesamt v.a. als ein westdeutsches und städtisches Phänomen dar. Ihre Mitglieder sind zu einem nicht unerheblichen Anteil deutsche StaatsbürgerInnen. ${ }^{94}$ Die wichtigsten Herkunftsgruppen in der zweiten Generation sind MigrantInnen, deren Eltern aus Ländern kommen, mit denen zuvor Anwerbeabkommen bestanden haben, sowie die Gruppe der AussiedlerInnen.

Im folgenden Kapitel werden nach diesen allgemeinen Informationen nun die Sprachkenntnisse der zweiten Generation sowohl im Deutschen als auch in der Herkunftssprache betrachtet.

\subsection{Sprache}

Vorausgeschickt sei, dass Sprachkenntnisse unterschiedlich bewertet werden: Einige AmerikanerInnen beispielsweise können noch nach vielen Jahren Aufenthalt in Deutschland auf Deutsch nur radebrechen. FreundInnen, Bekannte und ArbeitskollegInnen ohne Migrationshintergrund finden es manchmal sogar schick, mit ihnen Englisch zu sprechen, da Englisch

\footnotetext{
${ }^{94}$ Der Anteil der deutschen StaatsbürgerInnen hat sich durch die Reform des Staatsbürgerschaftsrechts erhöht. Seit 2001 können Kinder mit einem Elternteil, der sich seit acht Jahren rechtmäßig in Deutschland aufhält und über eine unbefristete Aufenthaltsgenehmigung verfügt, bei ihrer Geburt neben der ausländischen Staatsangehörigkeit der Eltern auch die deutsche Staatsbürgerschaft erlangen ( $\$ 4$ Abs. 3 Staatsangehörigkeitsgesetz StAG). Diese Möglichkeit wurde für einen befristeten Zeitraum auch rückwirkend bis zum Jahr 1991 gewährt. Zwischen dem 18. und dem 25. Lebensjahr müssen diese DoppelstaatlerInnen erklären, welche Staatsangehörigkeit sie behalten wollen ( 29 StAG). Entscheidend wird in dieser Hinsicht sein, wie 2014 mit den ersten Personen umgegangen wird, die bis dahin eine doppelte Staatsbürgerschaft hatten und nun eine der beiden werden aufgeben müssen. Eine Verfassungsbeschwerde gegen diesen Zwang und die mögliche Ausbürgerung ist wahrscheinlich. Und es kann darüber spekuliert werden, ob das Verfassungsgericht die zwangsweise Ausbürgerung wie im Staatsbürgerrecht vorgesehen, tatsächlich für mit dem Grundgesetz konform halten wird. Eine Ausweitung der doppelten Staatsbürgerschaft aber würde es vielen MigrantInnen erleichtern, die deutsche Staatsbürgerschaft anzunehmen (Thränhardt/Böcker 2003).
} 
weltweit wertgeschätzt wird. Integrationsunwilligkeit wird diesen AmerikanerInnen auf jeden Fall nicht unterstellt.

Für nicht-englische MuttersprachlerInnen gilt jedoch, dass bei ihnen deutsche Sprachkenntnisse als bedeutsam angesehen werden: Für jede Form der Partizipation ist Kommunikation Vorbedingung. Damit sind sprachliche Kenntnisse auch die Basis, auf der Bildungs- und Berufserfolg aufbaut, und sie stehen in enger Wechselwirkung mit sozialen Beziehungen mit Nicht-MigrantInnen oder MigrantInnen anderer Herkunftsgruppen. Da die umgekehrte Form der Anpassung der Nicht-Migrantenbevölkerung an MigrantInnen (oder von anderen MigrantInnen an eine dominante Migrantengruppe) nur in wenigen Sprachen oder erst ab einer bestimmten Größe der Migrantenbevölkerung und Konzentration (siehe türkische Schilder in Berlin-Kreuzberg) erfolgt, ist die Last der sprachlichen Anpassung ungleich verteilt.

Für Angehörige der zweiten Generation stellt sich die Frage nach der ,Anpassung' ein wenig anders dar, da sie nicht mit einer voll ausgebildeten Sprache nach Deutschland kommen und Deutsch dann additiv lernen müssen, sondern in einem unterschiedlich stark deutsch geprägten Sprachumfeld aufwachsen, je nachdem, welche Sprache mit den Eltern und Geschwistern sowie im Freundeskreis, im Kindergarten und in der Nachbarschaft gesprochen wird.

Daher untersucht dieses Kapitel v.a. mit den Zahlen des DJI-Ausländersurveys die Sprachkenntnisse im Deutschen und in der Muttersprache der Eltern bei den 18- bis 25-Jährigen türkischer, italienischer und griechischer Herkunft. Diese Zahlen gehen in Kapitel 4.1 in die Konstruktion der Muster auf individueller Ebene ein. Die zweite Generation wird mit der ersten und anderthalbten verglichen. Betrachtet werden nicht allgemein die Kenntnisse in den beiden Sprachen, sondern vielmehr das schriftliche Ausdrucksvermögen, welches für Bildung und Beruf am wichtigsten ist. Die Ergebnisse werden, soweit möglich, mit der NichtMigrantenbevölkerung verglichen, bei der fälschlicherweise zumeist davon ausgegangen wird, dass sie grundsätzlich keine sprachlichen Defizite aufweist.

Im anschließenden Abschnitt wird beleuchtet, welche Sprache mit Eltern und Geschwistern und in der Schule/auf der Arbeit gebraucht wird, um zusätzlich zu den Kenntnissen auch die Sprachpraxis zu beleuchten. Das Kapitel schließt mit einer Diskussion der positiven Effekte von Zweisprachigkeit - einem umstrittenen Thema, das in der pluralistischen Theorie eine große Rolle spielt. 


\subsubsection{Kenntnisse im Deutschen}

Die Deutschkenntnisse der zweiten Generation sind wesentlich besser als die der ersten und anderthalbten Generation: Im Vergleich mit diesen geben mehr als doppelt so viele MigrantInnen der zweiten Generation an, gute oder sehr gute schriftliche Deutschkenntnisse zu haben. Auch beim Verstehen, Sprechen oder Lesen ist in Tabelle 8 ein großer Fortschritt gegenüber der ersten und anderthalbten Generation abzulesen.

Tabelle 8 - Kenntnisse im Deutschen

\begin{tabular}{|c|c|c|c|c|}
\hline & & TÜRK & ITAL & GRIE \\
\hline \multirow{4}{*}{ 苋 } & Verstehen & $\begin{array}{c}99 \% \text {,sehr gut' oder ,gut' } \\
62 \% \text {,sehr gut' }\end{array}$ & $\begin{array}{l}99 \% \text {,sehr gut' oder , gut' } \\
79 \% \text {, sehr gut' }\end{array}$ & $\begin{array}{c}99 \% \text {,sehr gut' oder , gut' } \\
74 \% \text {,sehr gut' }\end{array}$ \\
\hline & Sprechen & $\begin{array}{c}99 \% \text {,sehr gut' oder ,gut' } \\
60 \% \text {, sehr gut' }\end{array}$ & $\begin{array}{c}99 \% \text {,sehr gut' oder , gut' } \\
78 \% \text {, sehr gut' }\end{array}$ & $\begin{array}{l}98 \% \text {,sehr gut' oder ,gut' } \\
71 \% \text {,sehr gut' }\end{array}$ \\
\hline & Lesen & $\begin{array}{c}95 \% \text {,sehr gut' oder ,gut' } \\
56 \% \text {,sehr gut' }\end{array}$ & $\begin{array}{c}97 \% \text {,sehr gut' oder , gut' } \\
73 \% \text {, sehr gut' }\end{array}$ & $\begin{array}{c}96 \% \text {, sehr gut' oder , gut' } \\
67 \% \text {,sehr gut' }\end{array}$ \\
\hline & Schreiben & $\begin{array}{l}91 \% \text {,sehr gut' oder ,gut' } \\
53 \% \text {,sehr gut' }\end{array}$ & $\begin{array}{c}95 \% \text {,sehr gut' oder , gut' } \\
70 \% \text {, sehr gut' }\end{array}$ & $\begin{array}{c}95 \% \text {,sehr gut' oder ,gut' } \\
63 \% \text {,sehr gut' }\end{array}$ \\
\hline \multirow{4}{*}{ 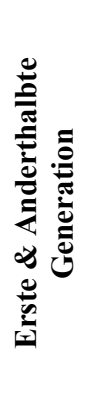 } & Verstehen & $\begin{array}{c}73 \% \text {,sehr gut' oder ,gut' } \\
16 \% \text {,sehr gut' }\end{array}$ & $\begin{array}{c}87 \% \text {,sehr gut' oder , gut' } \\
27 \% \text {, sehr gut' }\end{array}$ & $\begin{array}{c}86 \% \text {,sehr gut' oder ,gut' } \\
21 \% \text {,sehr gut' }\end{array}$ \\
\hline & Sprechen & $\begin{array}{c}66 \% \text {,sehr gut' oder ,gut' } \\
12 \% \text {,sehr gut' }\end{array}$ & $\begin{array}{c}76 \% \text {,sehr gut' oder , gut' } \\
23 \% \text {, sehr gut' }\end{array}$ & $\begin{array}{c}77 \% \text {, sehr gut' oder ,gut' } \\
17 \% \text {,sehr gut' }\end{array}$ \\
\hline & Lesen & $\begin{array}{c}54 \% \text {,sehr gut' oder , gut' } \\
10 \% \text {,sehr gut' }\end{array}$ & $\begin{array}{c}60 \% \text {,sehr gut' oder , gut' } \\
17 \% \text {, sehr gut' }\end{array}$ & $\begin{array}{c}60 \% \text {, sehr gut' oder , gut' } \\
17 \% \text {,sehr gut' }\end{array}$ \\
\hline & Schreiben & $\begin{array}{c}42 \% \text {, sehr gut' oder , gut' } \\
9 \% \text {, sehr gut' }\end{array}$ & $\begin{array}{c}46 \% \text {, sehr gut' oder , gut' } \\
16 \% \text {,sehr gut' }\end{array}$ & $\begin{array}{c}46 \% \text {,sehr gut' oder , gut' } \\
14 \% \text {,sehr gut' }\end{array}$ \\
\hline
\end{tabular}

Ausländersurvey 1997 des Deutschen Jugendinstituts, Eigene Berechnungen

Der Prozentsatz derjenigen, die sehr gute Kenntnisse angeben, steigt jeweils am stärksten. Sowohl in der ersten als auch in der zweiten Generation sind die Sprachkenntnisse der MigrantInnen italienischer Herkunft am besten, gefolgt von denen griechischer Herkunft und schließlich denen türkischer Herkunft. Die Unterschiede werden v.a. im Prozentsatz derjenigen sichtbar, die jeweils sehr gut Deutsch verstehen, sprechen, lesen und schreiben. Allerdings ist das Sprachniveau insgesamt sehr hoch. Beinahe 100\% der MigrantInnen der zweiten Generation geben an, Deutsch entweder gut oder sehr gut zu verstehen und zu sprechen. Um die 96\% sagen, dass sie Deutsch gut oder sehr gut lesen können, und zwischen 91 und 95\% beurteilen auch ihre schriftlichen Fähigkeiten so. Auf diesem hohen Niveau gibt es also eine Abstufung von der einfachsten Anforderung, dem Verstehen, bis hin zur schwierigsten Anforderung, dem Schreiben (ca. 10 Prozentpunkte weniger, die jeweils sehr gut angeben). Umgekehrt bedeutet dies aber auch, dass beinahe $50 \%$ der zweiten Generation türkischer Her- 
kunft, 40\% derjenigen griechischer Herkunft und 30\% derjenigen italienischer Herkunft ihre deutschen Schreibfähigkeiten für schlechter als ,sehr gut' halten.

Diese guten Kenntnisse der deutschen Sprache werden durch die Einschätzung der InterviewerInnen bestätigt: Diese bescheinigen 96\% der zweiten Generation italienischer und griechischer Herkunft perfekte oder gute Kenntnisse. Bei der zweiten Generation türkischer Herkunft liegt der Wert mit 92\% um vier Punkte niedriger. Obwohl die InterviewerInnen auch der jeweiligen Herkunftssprache kundig waren, wurden $88 \%$ der mit den MigrantInnen italienischer Herkunft geführten Interviews ausschließlich auf Deutsch durchgeführt, bei den MigrantInnen griechischer Herkunft waren es $89 \%$ und bei denen türkischer Herkunft $84 \%$. Wird auch der Anteil der Interviews eingerechnet, die überwiegend auf Deutsch geführt wurden, dann erhöhen sich die Werte auf beinahe $100 \%$ (97\% für die italienische, 98\% für die griechische und 97\% für die türkische Herkunftsgruppe).

Leider liegen bezüglich der Deutschkompetenz keine Vergleichszahlen von deutschen NichtMigrantInnen vor. Menschen ohne Migrationshintergrund werden weder nach ihren schriftlichen Deutschkenntnissen befragt, noch werden in Umfragen Interviewermeinungen hinsichtlich ihrer Deutschkenntnisse eingeholt. Die reifizierende und homogenisierende Annahme ist hierbei, dass Deutsche ohne Migrationshintergrund grundsätzlich sehr gute Deutschkenntnisse haben und im Deutschen sehr gut schreiben können, wohingegen MigrantInnen von dieser Norm abweichen. Dies ist jedoch nicht der Fall. Belegt wird dies zum Beispiel durch die Zahl von 4 Millionen funktionalen AnalphabetInnen in Deutschland, die keinesfalls alle innerhalb der migrantischen Bevölkerung zu finden sind (Bundesamt 2000 zitiert nach Döbert/Hubertus 2000). Eine weitere Informationsquelle zu den sprachlichen Fähigkeiten bei der Dominanzbevölkerung sind die seit ein paar Jahren in einigen Bundesländern durchgeführten Sprachstandserhebungen vor der Einschulung. Dadurch sollen Kinder mit sprachlichen Defiziten noch vor der Einschulung gezielt gefördert werden. In manchen Bundesländern wird diese Untersuchung nur bei Kindern mit Migrationshintergrund durchgeführt, in anderen ist sie für alle Kinder zur Einschulung verpflichtend (z.B. Berlin, Hamburg, Niedersachsen, NordrheinWestfalen). Als erstes Bundesland hat Berlin 2002 mit der verpflichtenden Sprachstandserhebung bei allen Vorschulkindern begonnen. Die Berliner Zahlen ermöglichen somit einen Vergleich der Sprachkenntnisse von Kindern mit und ohne Migrationshintergrund. Der Indikator für den Migrationshintergrund ist dabei die nicht-deutsche Herkunftssprache, d.h. die Eigenangabe, dass zu Hause überwiegend eine andere Sprache als Deutsch gesprochen wird. (Senatsverwaltung für Bildung 2003; Senatsverwaltung für Bildung 2006b) 
Tabelle 9 zeigt, dass sprachliche Defizite keinesfalls nur bei Vorschulkindern nicht-deutscher Herkunftssprache bestehen, sondern auch bei Kindern deutscher Herkunftssprache: Durchschnittlich knapp über $10 \%$ der Kinder mit deutscher Herkunftssprache weisen im Test Deutsch Plus Sprachprobleme auf und können ohne weitere Förderung nicht eingeschult werden. Bei den Kindern nicht-deutscher Herkunftssprache liegt die Quote bei etwas über 50\%. Sie weisen somit fünfmal so häufig sprachliche Defizite auf wie Kinder deutscher Herkunftssprache. Bei der Gruppe der Kinder mit Migrationshintergrund insgesamt werden die entsprechenden Zahlen ein wenig niedriger liegen, da diejenigen unter ihnen mit sehr guten Deutschkenntnissen möglicherweise in Teilen auch zu Hause Deutsch sprechen und daher in der Untersuchung in die Gruppe mit deutscher Herkunftssprache rutschen. Es existiert also ein gewisser Selektionsfehler, der das Ausmaß des Förderungsbedarfs für Migrantenkinder überschätzen lässt.

Die Daten von Deutsch Plus belegen aber auch eine große Heterogenität der Ergebnisse. Schwierigkeiten im Deutschen scheinen sowohl bei Nicht-MigrantInnen als auch bei MigrantInnen mit dem sozialen Status zusammenzuhängen. Zwar wurde der soziale Status der Familie im Sprachtest nicht direkt erhoben, allerdings oszillieren die Berliner Ergebnisse stark nach Stadtteilen: von 7\% bis zu 21\% Förderbedarf bei Kindern deutscher Herkunftssprache und $37 \%$ bis $68 \%$ bei Kindern nicht-deutscher Herkunftssprache im Jahr 2005. Die geringen Prozentzahlen finden sich dabei in überdurchschnittlich wohlhabenden Stadtteilen wie Charlottenburg/Wilmersdorf, Steglitz/Zehlendorf und Pankow, wohingegen der höchste Förderbedarf für Kinder aller Herkunftssprachen in Neukölln zu finden ist, gefolgt vom Bezirk Mitte (Senatsverwaltung für Bildung 2006b; Senatsverwaltung für Bildung 2006a). ${ }^{95}$ Hinsichtlich der Zusammensetzung der Eingangsschulklassen ergibt sich somit die Tendenz, dass dort, wo besonders viele sprachlich Förderungsbedürftige mit Migrationshintergrund eingeschult werden, auch besonders viele Kinder ohne Migrationshintergrund mit Förderungsbedarf zu finden sind. Aufgrund der sozioökonomischen Segregation nach Stadtvierteln entsteht somit eine sozialstrukturelle Benachteiligung schon bei Schuleintritt.

\footnotetext{
${ }^{95}$ Für Angaben zu den Arbeitslosen- und Sozialhilfequoten sowie zum Haushalts- und Pro-Kopf-Einkommen in den Berliner Bezirken siehe Häußermann/Kapphan 2002, OECD 2003.
} 
Tabelle 9 - Förderbedarf im Deutschen: Stadtviertel und Kitabesuch

\begin{tabular}{|c|c|c|c|c|c|c|c|c|c|}
\hline & \multicolumn{4}{|c|}{ DEUTSCHE HERKUNFTSSPRACHE } & \multicolumn{4}{|c|}{ NICHT-DEUTSCHE HERKUNFTSSPRACHE } & \multirow{2}{*}{$\begin{array}{c}\text { DEUTSCHE UND } \\
\text { NICHT-DEUTSCHE } \\
\text { HERKUNFTS- } \\
\text { SPRACHE ZUSAM- } \\
\text { MEN } \\
\text { ANTEIL FÖRDER- } \\
\text { BEDARF }\end{array}$} \\
\hline & $\begin{array}{l}\text { ANTEIL } \\
\text { FÖRDER- } \\
\text { BEDARF }\end{array}$ & $\begin{array}{c}\text { ANTEIL IN } \\
\text { STADTTEIL } \\
\text { MIT NIE- } \\
\text { DRIGSTEM } \\
\text { FÖRDER- } \\
\text { BEDARF }\end{array}$ & $\begin{array}{c}\text { ANTEIL IN } \\
\text { STADTTEIL } \\
\text { MIT } \\
\text { HÖCHSTEM } \\
\text { FÖRDER- } \\
\text { BEDARF }\end{array}$ & $\begin{array}{c}\text { ANTEIL } \\
\text { FÖRDER- } \\
\text { BEDARF } \\
\text { OHNE KITA- } \\
\text { BESUCH }\end{array}$ & $\begin{array}{l}\text { ANTEIL } \\
\text { FÖRDER- } \\
\text { BEDARF }\end{array}$ & $\begin{array}{c}\text { ANTEIL IN } \\
\text { STADTTEIL } \\
\text { MIT NIE- } \\
\text { DRIGSTEM } \\
\text { FÖRDER- } \\
\text { BEDARF }\end{array}$ & $\begin{array}{c}\text { ANTEIL IN } \\
\text { STADTTEIL } \\
\text { MIT } \\
\text { HÖCHSTEM } \\
\text { FÖRDER- } \\
\text { BEDARF }\end{array}$ & $\begin{array}{c}\text { ANTEIL } \\
\text { FÖRDER- } \\
\text { BEDARF } \\
\text { OHNE KITA- } \\
\text { BESUCH }\end{array}$ & \\
\hline $\begin{array}{l}\text { Bärenstark } \\
2003\end{array}$ & $29 \%$ & & & & $80 \%$ & & & & $45 \%$ \\
\hline $\begin{array}{l}\text { Deutsch plus } \\
2005\end{array}$ & $12 \%$ & $7 \%$ & $21 \%$ & & $57 \%$ & $37 \%$ & $68 \%$ & & $25 \%$ \\
\hline $\begin{array}{l}\text { Deutsch plus } \\
2006\end{array}$ & $11 \%$ & $6 \%$ & $20 \%$ & $29 \%$ & $54 \%$ & $37 \%$ & $65 \%$ & $72 \%$ & $24 \%$ \\
\hline
\end{tabular}

Ergebnisse Deutsch Plus und Bärenstark Senatsverwaltung für Bildung, Jugend und Sport Berlin (Senatsverwaltung für Bildung (2003); Senatsverwaltung für Bildung (2006)), Eigene Berechnungen 
Weiterhin zeigt sich, dass ein Kindergartenbesuch mit durchschnittlich besseren Deutschkenntnissen einhergeht. Kinder, die keinen Kindergarten besuchen - egal ob deutscher oder nicht-deutscher Herkunftssprache - weisen im Durchschnitt einen stark erhöhten Förderungsbedarf auf: 29\% für Kinder deutscher Herkunftssprache und 72\% für Kinder nicht-deutscher Herkunftssprache. Auch dies ist ein Hinweis auf den Zusammenhang zwischen Deutschkompetenz und sozialem Status, und zwar sowohl für MigrantInnen als auch für NichtMigrantInnen, da Kinder aus marginalisierten sozialen Schichten überproportional häufig nicht in den Kindergarten gehen (Ehmann 2001).

Bärenstark, der Vorläufertest von Deutsch Plus, arbeitete mit einem anspruchsvolleren Sprachniveau und fand bei den Vorschulkindern einen dementsprechend höheren Prozentsatz von Förderungsbedürftigen. Insgesamt konnten bei dem Sprachtest maximal 100 Punkte erreicht werden. Bei Ergebnissen unter 80 Punkten wurde ein Förderbedarf diagnostiziert, bei weniger als 60 Punkten ein intensiver Förderbedarf. Demnach weisen 29\% der Kinder deutscher Herkunftssprache einen Förderbedarf auf (8\% davon einen intensiven) und $80 \%$ der Kinder nicht-deutscher Herkunftssprache, davon 44\% einen intensiven Förderbedarf. Die nachfolgenden Abbildungen zeigen allerdings auch die große Streuung der nach Kindern deutscher und nicht-deutscher Herkunftssprache getrennten Ergebnisse. Dabei fällt schon in der Grafik auf, dass die Standardabweichung für Kinder nicht-deutscher Herkunftssprache höher ist als für Kinder deutscher Herkunftssprache, ihre Ergebnisse also heterogener sind (Abbildung 5, Abbildung 6) - ein Effekt, der durch den Vergleich der Bezirke belegt werden kann: Überall sind nicht nur die Mittelwerte der Kinder nicht-deutscher Herkunftssprache geringer als die der Kinder deutscher Herkunftssprache, sondern auch die Standardabweichungen der Kinder nicht-deutscher Herkunftssprache wesentlich größer (Mengering 2005: 251f). ${ }^{96}$ Dies verweist auf die große Diversität innerhalb der Migrantengruppe: Die Ergebnisse der Kinder nicht-deutscher Herkunftssprache strecken sich über das volle Spektrum von 0 bis 100 Punkten. Bei den Kindern deutscher Herkunftssprache ist die Normalverteilung nach oben verschoben und stärker gedeckelt, allerdings gibt es auch hier vereinzelt Kinder, die 0 Punkte erzielten.

\footnotetext{
${ }^{96}$ Von den 23 Ortsteilen ist nur in Hellersdorf die Standardabweichung der Kinder deutscher Herkunftssprache größer als die der Kinder nicht-deutscher Herkunftssprache, bei einer insgesamt geringen Migrantenfallzahl $(\mathrm{N}=58)$.
} 


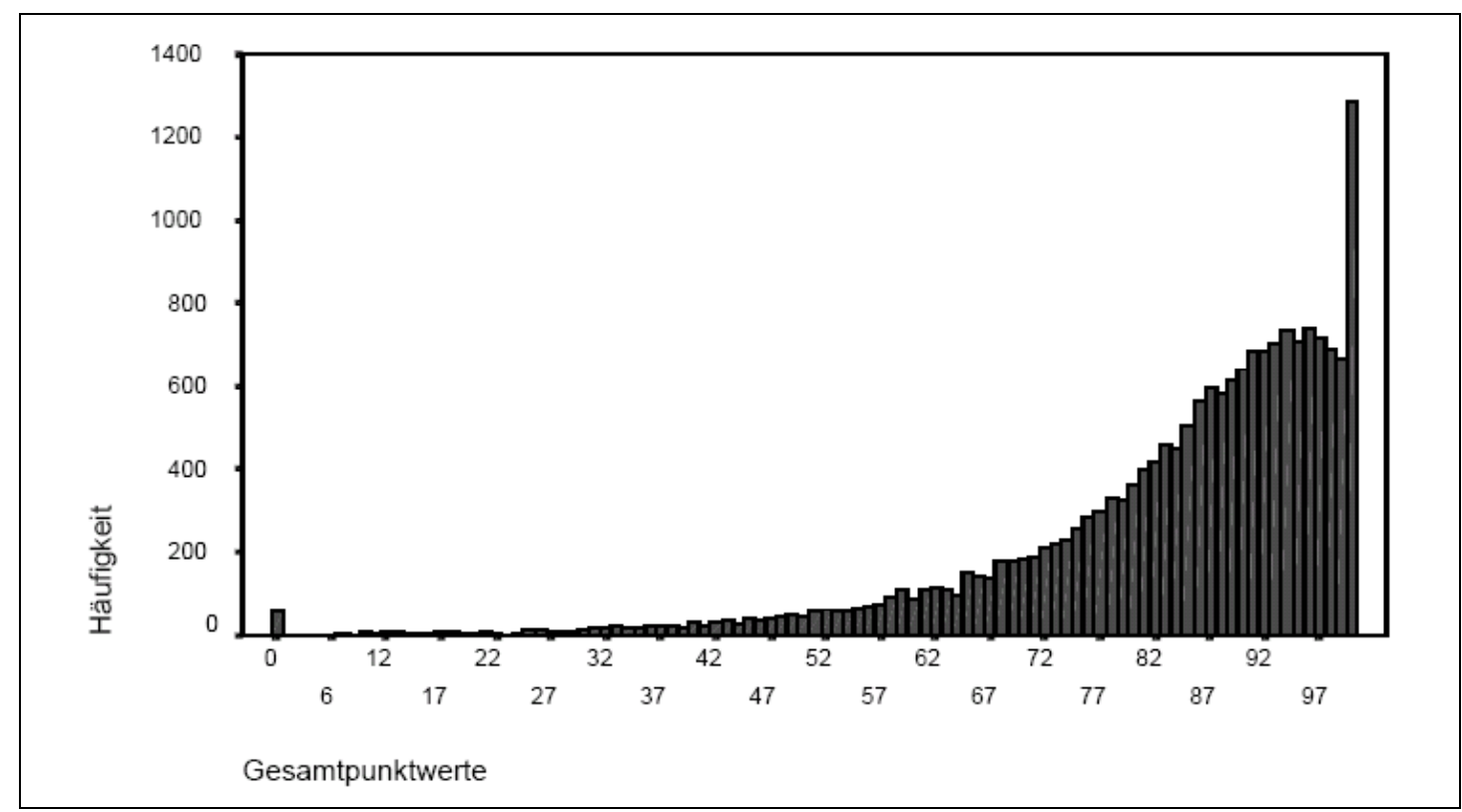

Bärenstark 2003 (N=18457) (Mengering (2005): 251)

Abbildung 5 - Förderbedarf im Deutschen: Punktwerte der Kinder deutscher Herkunftssprache

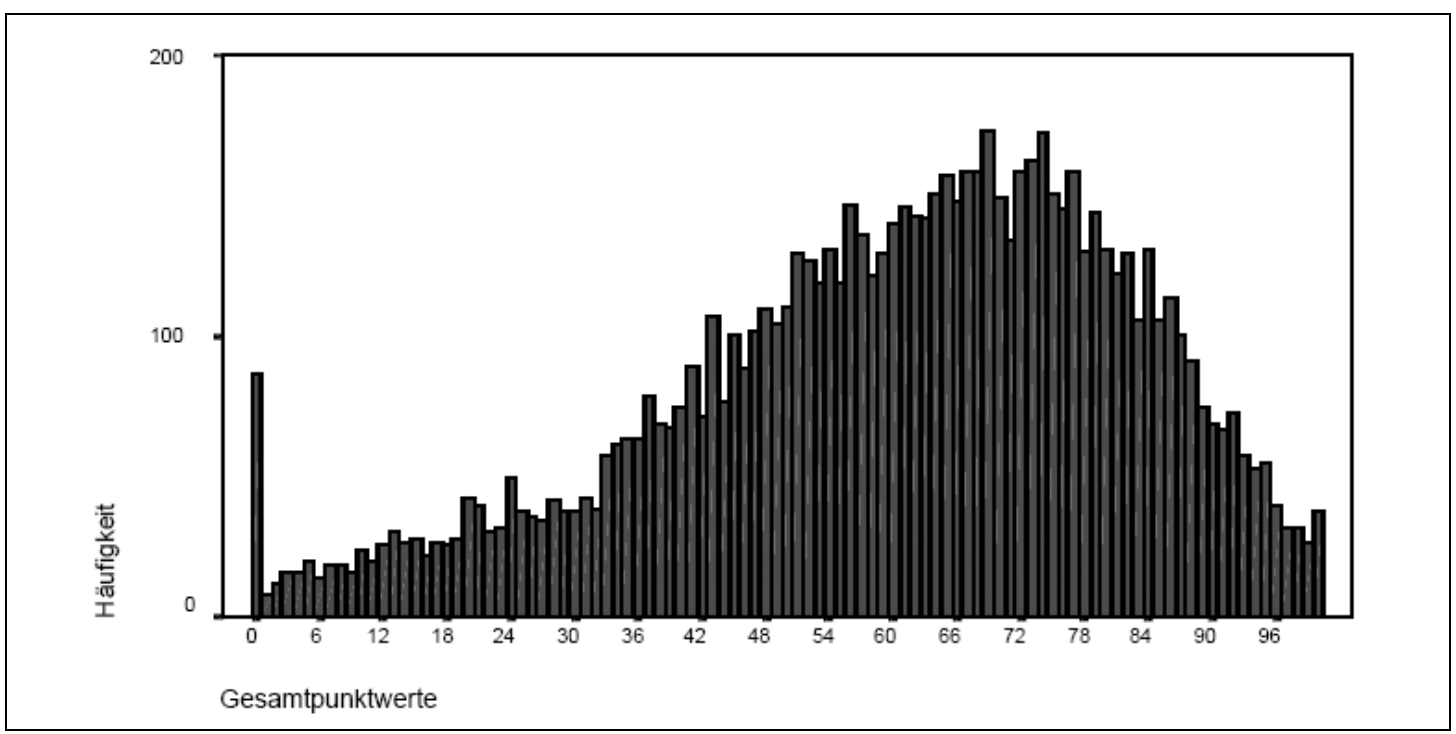

Bärenstark 2003 (N=8263) (Mengering (2005): 251)

Abbildung 6 - Förderbedarf im Deutschen: Punktwerte der Kinder nicht-deutscher Herkunftssprache

Die Sprachstandserhebungen zeigen somit, dass auch bei Kindern deutscher Herkunftssprache sprachlicher Förderbedarf besteht, der Förderbedarf bei allen Kindern mit dem sozioökonomischen Status zusammenhängt, die Diversität bei MigrantInnen groß ist, Kindergartenbesuch das Sprachniveau verbessert und eine Erhebung nach familiären Herkunftssprachen das Maß der sprachlich hochqualifizierten Migrantenkinder unterschätzt, da diese teilweise der NichtMigrantengruppe zugeschlagen werden.

Wenn also, wie mit den Daten des DJI-Ausländersurveys gezeigt, bei den 18- bis 25-Jährigen die sehr guten Sprachkenntnisse überwiegen, bedeutet dies nicht, dass bei ihnen allen auch 
schon bei der Einschulung die Deutschkenntnisse gut bis sehr gut waren. Dies wird unter anderem von der Umgangssprache zu Hause und im Freundeskreis sowie einem eventuellen Kindergartenbesuch abhängen. Sprachliche Probleme bei der Einschulung aber führen im aktuellen deutschen Schulsystem häufig zu einer Zurückstellung oder gar zur Überweisung an die Sonderschule. SchülerInnen mit Defiziten haben es zudem schwerer, dem monolingualen Unterricht zu folgen und sich den Schulstoff anzueignen. Aus diesen Gründen können mangelnde Deutschkenntnisse bei Fünf-, Sechs- und Siebenjährigen zu kumulativen negativen Effekten in der Schulkarriere führen - und dies auch bei jenen Personen, die als Teenager nicht durch Sprachdefizite auffallen.

\subsubsection{Kenntnisse in der Herkunftssprache}

Die zweite Generation verfügt in der Herkunftssprache der Eltern wie erwartet über geringere Fähigkeiten als die erste und anderthalbte Generation (Tabelle 10). Die Kenntnisse bleiben allerdings recht hoch, wobei die Unterschiede zwischen der geringeren Anforderungen des Verstehens und der hohen Anforderung des Schreibens in der Sprache der Eltern größer sind als im Deutschen. Entgegen üblichen medialen Erwartungen sind es nicht die MigrantInnen der zweiten Generation türkischer Herkunft, die die besten Kenntnisse in der Sprache der Eltern haben. Bei der ausschließlichen Betrachtung der sehr guten Kenntnisse liegen sie durchweg auf dem letzten Platz.

Tabelle 10 - Kenntnisse in Herkunftssprache

\begin{tabular}{|c|c|c|c|c|}
\hline & & ITAL & GRIE & TÜRK \\
\hline \multirow{4}{*}{ 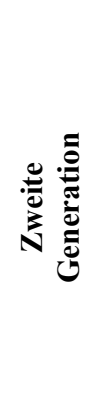 } & Verstehen & $\begin{array}{l}98 \% \text {,sehr gut' oder , gut' } \\
60 \% \text {,sehr gut' }\end{array}$ & $\begin{array}{c}96 \% \text {,sehr gut' oder , gut' } \\
58 \% \text {, sehr gut' }\end{array}$ & $\begin{array}{l}99 \% \text {,sehr gut' oder ,gut' } \\
53 \% \text {,sehr gut' }\end{array}$ \\
\hline & Sprechen & $\begin{array}{l}96 \% \text {,sehr gut' oder , gut' } \\
57 \% \text {,sehr gut' }\end{array}$ & $\begin{array}{l}94 \% \text {,sehr gut' oder , gut' } \\
55 \% \text {, sehr gut' }\end{array}$ & $\begin{array}{l}97 \% \text {,sehr gut' oder , gut' } \\
51 \% \text {,sehr gut' }\end{array}$ \\
\hline & Lesen & $\begin{array}{l}88 \% \text {, sehr gut' oder , gut' } \\
46 \% \text {,sehr gut' }\end{array}$ & $\begin{array}{l}80 \% \text {,sehr gut' oder ,gut' } \\
41 \% \text {,sehr gut' }\end{array}$ & $\begin{array}{c}86 \% \text {,sehr gut' oder , gut' } \\
39 \% \text {,sehr gut' }\end{array}$ \\
\hline & Schreiben & $\begin{array}{l}82 \% \text {, sehr gut' oder , gut' } \\
42 \% \text {, sehr gut' }\end{array}$ & $\begin{array}{l}74 \% \text {,sehr gut' oder ,gut' } \\
36 \% \text {, sehr gut' }\end{array}$ & $\begin{array}{c}72 \% \text {,sehr gut' oder , gut' } \\
33 \% \text {,sehr gut' }\end{array}$ \\
\hline \multirow{4}{*}{ 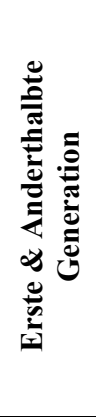 } & Verstehen & $\begin{array}{c}100 \% \text {,sehr gut' oder , gut' } \\
70 \% \text {, sehr gut' }\end{array}$ & $\begin{array}{l}99 \% \text {,sehr gut' oder ,gut' } \\
68 \% \text {,sehr gut' }\end{array}$ & $\begin{array}{c}99 \% \text {,sehr gut' oder , gut' } \\
62 \% \text {,sehr gut' }\end{array}$ \\
\hline & Sprechen & $\begin{array}{c}100 \% \text {,sehr gut' oder , gut' } \\
69 \% \text {, sehr gut' }\end{array}$ & $\begin{array}{l}99 \% \text {,sehr gut' oder ,gut' } \\
69 \% \text {,sehr gut' }\end{array}$ & $\begin{array}{l}99 \% \text {,sehr gut' oder ,gut' } \\
61 \% \text {,sehr gut' }\end{array}$ \\
\hline & Lesen & $\begin{array}{l}96 \% \text {,sehr gut' oder , gut' } \\
60 \% \text {,sehr gut' }\end{array}$ & $\begin{array}{l}97 \% \text {,sehr gut' oder , gut' } \\
60 \% \text {,sehr gut' }\end{array}$ & $\begin{array}{c}91 \% \text {,sehr gut' oder , gut' } \\
50 \% \text {,sehr gut' }\end{array}$ \\
\hline & Schreiben & $\begin{array}{l}93 \% \text {,sehr gut' oder ,gut' } \\
57 \% \text {,sehr gut' }\end{array}$ & $\begin{array}{l}94 \% \text {,sehr gut' oder , gut' } \\
57 \% \text {, sehr gut' }\end{array}$ & $\begin{array}{l}87 \% \text {,sehr gut' oder ,gut' } \\
47 \% \text {,sehr gut' }\end{array}$ \\
\hline
\end{tabular}

Ausländersurvey 1997 des Deutschen Jugendinstituts, Eigene Berechnungen 
Im Vergleich besitzen MigrantInnen der zweiten Generation damit wesentlich bessere Kenntnisse im Deutschen als in der Sprache der Eltern. Dieser Effekt verstärkt sich mit zunehmendem Schwierigkeitsgrad von Verstehen zu Schreiben, beim Schreiben beträgt der Unterschied zwischen $12 \%$ und 21\%. Dabei ist der Effekt noch stärker ausgeprägt, wenn nur diejenigen betrachtet werden, die jeweils sehr gut verstehen, sprechen, lesen und schreiben: Beispielsweise liegt der Anteil der MigrantInnen, die die Sprache der Eltern sehr gut schreiben, durchschnittlich 25 Prozentpunkte niedriger als der Anteil derjenigen, die deutsch sehr gut schreiben. Trotz der Dominanz des Deutschen bestehen in der zweiten Generation hohe Kenntnisse der Sprache der Eltern fort: So liegt zum Beispiel der Prozentsatz derer, die in dieser Sprache sehr gut schreiben (was für berufliche Kontakte nötig wäre), zwischen 33\% und 42\% je nach Herkunftsgruppe. Dies ermöglicht das Aufrechthalten transnationaler Verbindungen.

Auch die Kenntnisse in der Herkunftssprache werden von den InterviewerInnen bestätigt. Die Eigeneinschätzungen der Interviewten sind sogar schlechter als die durch die InterviewerInnen: Perfekt in der Herkunftssprache der Eltern lesen können laut Einschätzung der InterviewerInnen 56\% der italienischen, 53\% der griechischen und 45\% der türkischen Herkunftsgruppe der zweiten Generation. Nur 46\%, 41\% bzw. 39\% denken dies selbst von. Werden auch gute Fähigkeiten eingeschlossen, steigt der Anteil nach Interviewereinschätzung auf 95\% für die italienische, 93\% für die griechische und 94\% für die türkische Herkunftsgruppe (Eigeneinschätzung: $88 \%, 80 \%$ und $86 \%$ ).

\subsubsection{Sprachgebrauch}

Wie spiegeln sich diese Kenntnisse im tatsächlichen Sprachgebrauch wider? 76\% der MigrantInnen der zweiten Generation italienischer Herkunft sprechen mit ihren Eltern überwiegend die Muttersprache, bei der griechischen Herkunftsgruppe sind es 79\% und bei der türkischen 87\% (Tabelle 11). Umgekehrt bedeutet dies aber auch, dass ein Viertel der MigrantInnen italienischer Herkunft und ein Fünftel derjenigen griechischer Herkunft in der zweiten Generation überwiegend Deutsch mit ihren Eltern sprechen. Mit den Geschwistern teilt sich die überwiegende Konversationssprache ungefähr gleich auf, bei MigrantInnen griechischer Herkunft und noch etwas stärker bei denen türkischer Herkunft überwiegt die Herkunftssprache der Eltern. In der Schule und am Arbeitsplatz wird hingegen zu rund 90\% überwiegend Deutsch gesprochen: bei den MigrantInnen italienischer Herkunft zu 93\%, denen griechischer Herkunft zu 91\% und denen türkischer Herkunft zu 86\%. 
Tabelle 11 - Konversationssprache

\begin{tabular}{|c|c|c|c|c|c|}
\hline & & & TÜRK & ITAL & GRIE \\
\hline \multirow{6}{*}{ 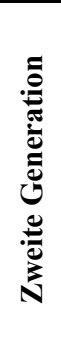 } & \multirow{2}{*}{$\begin{array}{l}\text { Konversationssprache } \\
\text { Eltern Kind }\end{array}$} & Überwiegend Herkunftssprache & $87 \%$ & $76 \%$ & $79 \%$ \\
\hline & & Überwiegend Deutsch & $13 \%$ & $24 \%$ & $21 \%$ \\
\hline & \multirow{2}{*}{$\begin{array}{l}\text { Konversationssprache } \\
\text { Geschwister }\end{array}$} & Überwiegend Herkunftssprache & $61 \%$ & $48 \%$ & $54 \%$ \\
\hline & & Überwiegend Deutsch & $39 \%$ & $52 \%$ & $46 \%$ \\
\hline & \multirow{2}{*}{$\begin{array}{l}\text { Konversationssprache } \\
\text { Schule/Arbeit in Pausen }\end{array}$} & Überwiegend Herkunftssprache & $14 \%$ & $7 \%$ & $9 \%$ \\
\hline & & Überwiegend Deutsch & $86 \%$ & $93 \%$ & $91 \%$ \\
\hline \multirow{6}{*}{ 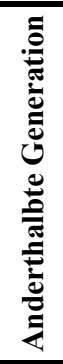 } & \multirow{2}{*}{$\begin{array}{l}\text { Konversationssprache } \\
\text { Eltern Kind }\end{array}$} & Überwiegend Herkunftssprache & $95 \%$ & $86 \%$ & $95 \%$ \\
\hline & & Überwiegend Deutsch & $5 \%$ & $14 \%$ & $5 \%$ \\
\hline & \multirow{2}{*}{$\begin{array}{l}\text { Konversationssprache } \\
\text { Geschwister }\end{array}$} & Überwiegend Herkunftssprache & $85 \%$ & $78 \%$ & $88 \%$ \\
\hline & & Überwiegend Deutsch & $15 \%$ & $22 \%$ & $12 \%$ \\
\hline & \multirow{2}{*}{$\begin{array}{l}\text { Konversationssprache } \\
\text { Schule/Arbeit in Pausen }\end{array}$} & Überwiegend Herkunftssprache & $24 \%$ & $16 \%$ & $29 \%$ \\
\hline & & Überwiegend Deutsch & $76 \%$ & $84 \%$ & $71 \%$ \\
\hline \multirow{6}{*}{ 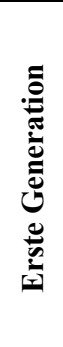 } & \multirow{2}{*}{$\begin{array}{l}\text { Konversationssprache } \\
\text { Eltern Kind }\end{array}$} & Überwiegend Herkunftssprache & $99 \%$ & $100 \%$ & $100 \%$ \\
\hline & & Überwiegend Deutsch & $1 \%$ & $0 \%$ & $0 \%$ \\
\hline & \multirow{2}{*}{$\begin{array}{l}\text { Konversationssprache } \\
\text { Geschwister }\end{array}$} & Überwiegend Herkunftssprache & $99 \%$ & $97 \%$ & $100 \%$ \\
\hline & & Überwiegend Deutsch & $1 \%$ & $3 \%$ & $0 \%$ \\
\hline & \multirow{2}{*}{$\begin{array}{l}\text { Konversationssprache } \\
\text { Schule/Arbeit in Pausen }\end{array}$} & Überwiegend Herkunftssprache & $68 \%$ & $58 \%$ & $46 \%$ \\
\hline & & Überwiegend Deutsch & $32 \%$ & $42 \%$ & $54 \%$ \\
\hline
\end{tabular}

Ausländersurvey 1997 des Deutschen Jugendinstituts, Eigene Berechnungen

Darüber hinaus zeigt sich eine weitgehende Pluralität, wenn die Elternteile einzeln betrachtet werden, wie in der Spracherhebung an Essener Grundschulen 2002. Dieser zufolge sprechen von den Kindern 15\% mit beiden Elternteilen Deutsch, 38\% mit einem Elternteil Deutsch, mit dem anderen jedoch nicht, und 43\% sprechen mit beiden Eltern die gleiche nicht-deutsche Sprache, während 1,4\% unterschiedliche nicht-deutsche Sprachen sprechen; beim Rest $(2,6 \%)$ fehlte bei mindestens einem Elternteil die Angabe. (Chlosta/Ostermann 2005: 62) Dies deutet auf ein nicht unerhebliches Maß an Multilingualität schon in der Familie hin.

\subsubsection{Positive Wirkung von Zweisprachigkeit}

Die Frage, ob und in welchem Umfang Bilingualität positive Wirkungen hat, wird kontrovers diskutiert (Esser 2006). Schon was genau unter Bilingualismus zu verstehen ist, bleibt umstritten. Für die hier untersuchte zweite Generation von MigrantInnen soll Zweisprachigkeit Kompetenz in mehr als einer Sprache auf sehr gutem Niveau, auch in der Schriftsprache bedeuten. 
Eine positive Dividende einer solchen Kompetenz könnte über vier Mechanismen entstehen:

- Erleichterter Erwerb der Sprache des Aufnahmelandes: Wenn Kinder, die zunächst eine hohe Sprachkompetenz in der Herkunftssprache erworben haben, dann leichter die Sprache des Aufnahmelandes erlernen und damit im Ergebnis zweisprachig sind, dann ist dies ein Vorteil gegenüber Kindern, die möglicherweise aufgrund geringer Kompetenz in der Herkunftssprache auch die Aufnahmesprache nicht vollständig erwerben.

- Kognitive Vorteile: Eine sprachliche Doppelkompetenz könnte Zugewinne in den kognitiven Fähigkeiten bewirken.

- Positive psychologische Effekte: Wenn die Beherrschung der Muttersprache der Eltern mit einer gefestigteren Identität, einem vermehrten Selbstbewusstsein, einem gestärkten Selbstwertgefühl und einer größeren Resilienz gegenüber Diskriminierung einhergeht, dann kann Zweisprachigkeit eine nicht-sprachliche Rendite haben.

- Konfliktfreiere und emotional positiver besetzte Familienbeziehungen: Sehr gute Kenntnisse der Muttersprache der Eltern können die Kommunikation mit den Eltern verbessern.

Die Annahme ist also, dass diese vier Mechanismen einzeln oder zusammen dazu führen, dass zweisprachige MigrantInnen der zweiten Generation bessere Ergebnisse im Bildungs- und Arbeitsbereich erzielen als monolinguale oder solche, die beide Sprachen nur unvollständig sprechen. Möglicherweise könnten auch die gleichen Ressourcen (z.B. Bildungsabschlüsse) aufgrund des größeren Selbstbewusstseins oder der positiven Familienbeziehungen gewinnbringender eingesetzt werden.

Wie sehen die Befunde hinsichtlich dieser vier Mechanismen im DJI-Datenset aus? Um diese Frage zu klären, werden innerhalb der zweiten Migrantengeneration drei Gruppen gebildet:

- MigrantInnen, die sowohl im Deutschen als auch in der Muttersprache sehr gute schriftliche Kenntnisse haben (zweisprachige Gruppe),

- MigrantInnen, die sehr gute schriftliche Deutschkenntnisse haben, aber schlechtere schriftliche Fähigkeiten in der Muttersprache (deutschsprachige Gruppe) und

- eine Restgruppe, die entweder hinsichtlich beider Sprachen in der Schriftform maximal gute Kenntnisse hat oder aber in der Herkunftssprache der Eltern sehr gute Kenntnisse hat und im Deutschen nicht.

$\mathrm{Zu}$ den ersten beiden der oben genannten Mechanismen lassen sich mit dem DJI-Datenset keine Aussagen treffen, da Längsschnittdaten und direkte kognitive Indikatoren fehlen. Daher sei an dieser Stelle auf die Ergebnisse einiger größerer Untersuchungen verwiesen: Skutnabb- 
Kangas und Toukomaa zeigten 1976, dass bei den finnischen EinwanderInnen nach Schweden diejenigen die Zweitsprache besser erlernten, die über hervorragende Kenntnisse in der Muttersprache verfügten. Auch ein positiver Einfluss auf die Fähigkeiten in Mathematik und auf den Erwerb der zweiten Zweitsprache (hier: Englisch) wurden festgestellt (SkutnabbKangas/Toukomaa 1976). Auf solche positiven kognitiven Konsequenzen hat auch Cummins in einer Studie von 1979 hingewiesen (Cummins 1979b; Cummins 1979a).

Hinsichtlich des dritten Mechanismus der psychologischen Effekte zeigt sich in Abbildung 7, dass Zweisprachige häufiger als beide andere Gruppen von sich sagen, dass sie in der Lage sind, sich vor Pech zu schützen. Auch gehen sie stärker als die dritte Gruppe der Semilingualen und derjenigen, die nur die Herkunftssprache der Eltern sehr gut schreiben können, davon aus, dass Erfolg von den eigenen Leistungen abhängt (Abbildung 8). Jedoch denkt dies die deutschsprachige Gruppe noch etwas stärker, auch wenn der Unterschied nicht signifikant ist, wie an der Überschneidung der Boxplots abzulesen ist. Hinsichtlich der Indikatoren des Selbstwertgefühls erzielen bilinguale MigrantInnen der zweiten Generation also höhere Werte als die dritte Gruppe. Im Vergleich zur deutschsprachigen, zweiten Gruppe erzielen sie einmal etwas bessere, einmal etwas schlechtere Werte. Bilinguale geben zudem seltener als die beiden anderen Gruppen an, wegen ihrer Nationalität benachteiligt zu werden, was eher auf eine höhere Resilienz als auf eine tatsächlich geringere Diskriminierungsrate zurückzuführen ist (Abbildung 9). Insgesamt besteht also ein positiver Zusammenhang zwischen Bilingualität und den oben beschriebenen psychologischen Effekten in der zweiten Generation.

Was die Qualität und Konfliktfreiheit der Familienbeziehungen angeht, kann diese nur über den Indikator der Wichtigkeit von Familie und Geschwistern gemessen werden. Für diesen gibt es relativ große Streuungen in allen drei Gruppen und keine signifikanten Unterschiede (Abbildung 10). Ein anderer Indikator für die Qualität der Beziehungen und den Umfang intergenerationaler Konflikte liegt leider nicht vor. Ein höheres Selbstwertgefühl, weniger wahrgenommene Diskriminierung sowie ein besseres Familienklima sollten sich laut den oben genannten Mechanismen in einem höheren sozioökonomischen Status äußern. In dieser Hinsicht ergibt der Vergleich im DJI-Ausländersurvey eine marginale, aber bei weitem nicht signifikante Besserstellung der Zweisprachigen (Abbildung 11). Das gleiche Ergebnis zeigt sich auch in der Regression, bei Kontrolle von anderen Standardmerkmalen (Tabelle 12). 


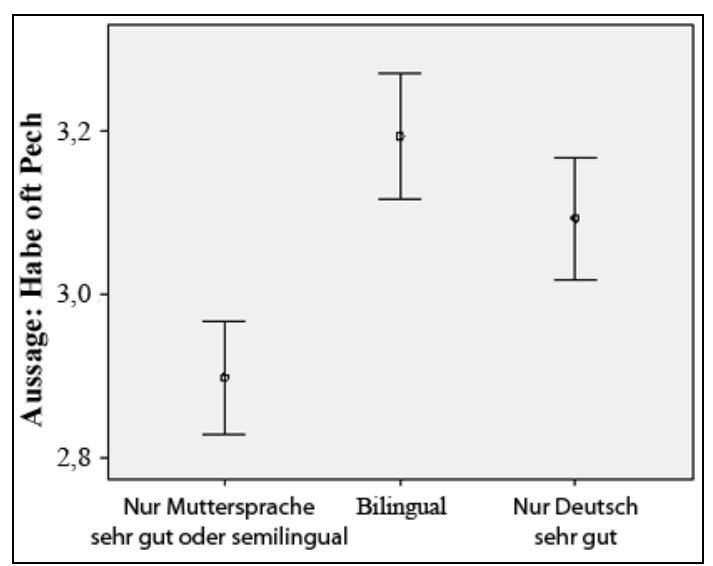

Abbildung 7 - Zweisprachigkeit: Kontrollüberzeugung

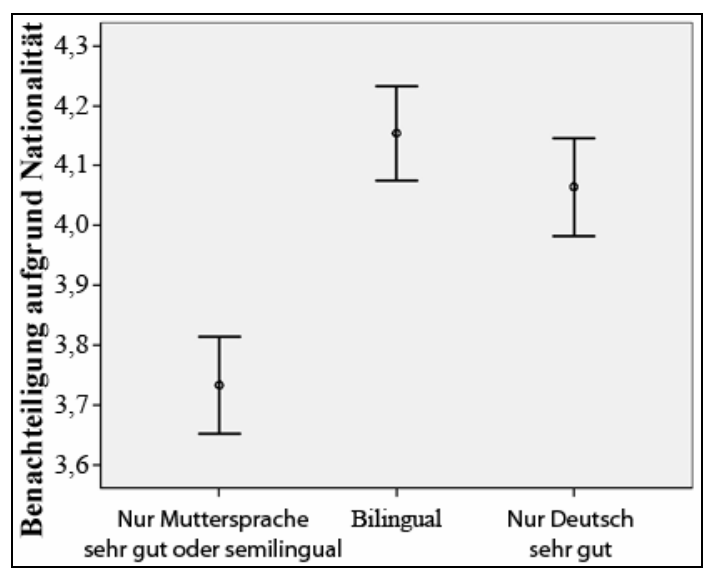

Abbildung 9 - Zweisprachigkeit: Benachteiligung aufgrund Nationalität

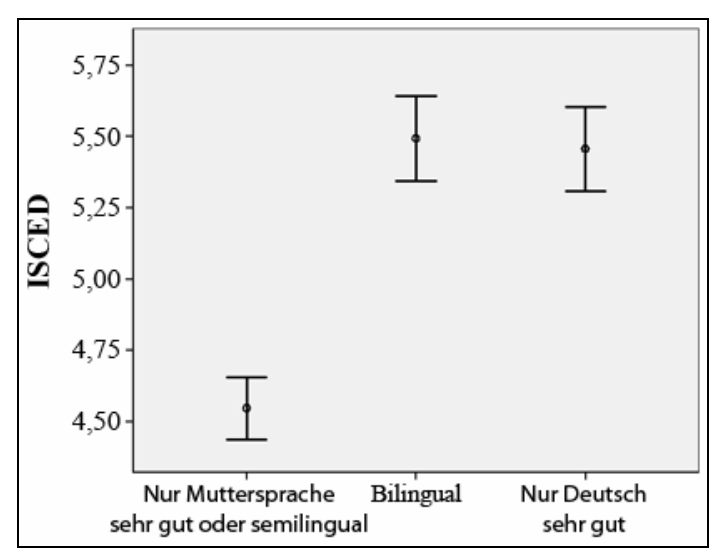

Abbildung 11 - Zweisprachigkeit: Bildungsabschlüsse

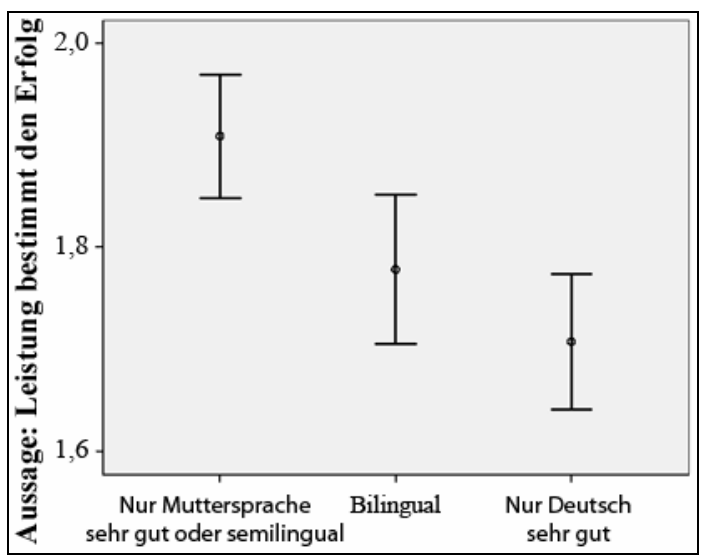

Abbildung 8 - Zweisprachigkeit: Selbstwirksamkeit

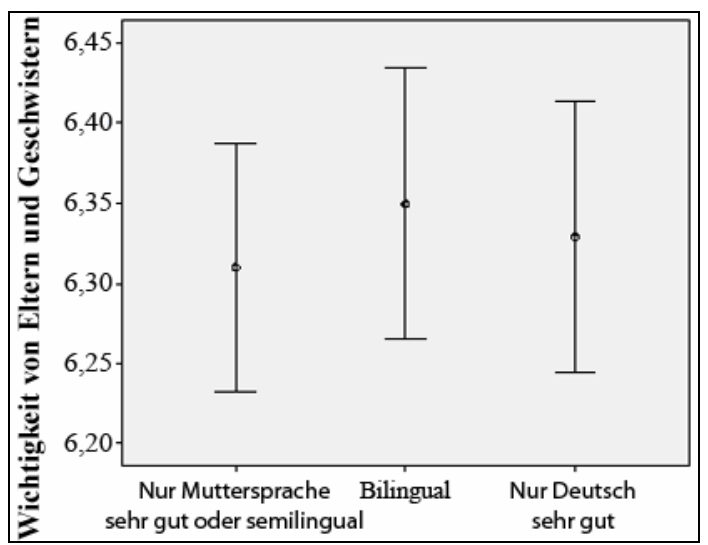

Abbildung 10 - Zweisprachigkeit: Wichtigkeit von Eltern und Geschwistern

Abbildung 7: „Ich habe oft keine Möglichkeit, mich vor Pech zu schützen“ (Skala von 1 „stimme voll

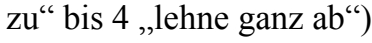

Abbildung 8: „Erfolg in Schule und Beruf hängt in erster Linie von eigenen Leistungen ab" (Skala von 1 ,stimme voll zu“ bis 4 ,lehne ganz ab“)

Abbildung 9: „Es kommt vor, dass man im Leben benachteiligt wird. Können Sie mir sagen, wie häufig Sie aus folgenden Gründen Benachteiligungen erfahren haben. War das jeweils sehr oft, oft, manchmal, selten oder nie? - wegen Ihrer Nationalität" (Skala von 1 „,sehr oft" bis 5 ,nie“)

Abbildung 10: „Wie wichtig sind für Sie persönlich die einzelnen Lebensbereiche [hier: Eltern und Geschwister]?“ (Skala von 1 ,"̈̈berhaupt nicht wichtig“" bis 7 ,sehr wichtig“)

Abbildung 11: ISCED (Skala von 1 „,niedrigster Wert" bis 10 „höchster Wert")

Ausländersurvey 1997 des Deutschen Jugendinstituts, Eigene Berechnungen 
Tabelle 12 - Auswirkungen Zweisprachigkeit auf Bildungsabschluss (ISCED)

\begin{tabular}{|l|c|}
\hline & B (S.F.) \\
\hline ITAL - &, 07 n.s. $(, 09)$ \\
GRIE - &, 13 n.s. $(, 09)$ \\
Beruf EItern (in 6 Stufen) &, 07 n.s. $(, 03)$ \\
Bildung EItern (in 4 Stufen) &, $82^{* *}(, 06)$ \\
Frauen $^{\circ}$ &,- 10 n.s. $(, 07)$ \\
Bilingual $^{a}$ &, 10 n.s. $(, 09)$ \\
Nur Muttersprache sehr gut (o- $_{\text {der semilingual) }}{ }^{\alpha}$ &,$- 76^{* *}(, 09)$ \\
\hline Konstante $^{\text {Korrigiertes } \mathbf{R}^{2}}$ & 3,10 \\
\hline
\end{tabular}

Koeffizienten (B) linearer Regression, Abhängige Variable Bildungsabschluss (ISCED in 5 Stufen)

- Referenzkategorie: TÜRK; ${ }^{\circ}$ Referenzkategorie: Männer; o Referenzkategorie: Nur Deutsch sehr gut

** statistisch signifikant bei $\mathrm{p}<0.01,{ }^{*}$ statistisch signifikant bei $\mathrm{p}<0.05$, n.s. nicht signifikant

Ausländersurvey 1997 des Deutschen Jugendinstituts, Eigene Berechnungen

Insgesamt lassen sich somit die meisten Mechanismen bestätigen, wenn auch die Gruppe der Bilingualen nur wenig von der zweiten, deutschsprachigen Gruppe abweicht. Bei den schulischen Ergebnisse ist zwar ein Vorsprung der Bilingualen vor der deutschsprachigen Gruppe vorhanden, jedoch ist dieser statistisch nicht signifikant.

Als eine von wenigen Studien ist die Untersuchung Deutsch Englisch Schülerleistung International (DESI) der Wirkung von Bilingualität auf schulische Leistungen methodisch kontrolliert nachgegangen: Es wurden 11.000 SchülerInnen der 9. Klasse bundesweit befragt, von denen $6 \%$ in Haushalten aufwachsen, in denen Deutsch und eine andere Sprache gesprochen werden, und 13\% in Haushalten, in denen nur eine andere Sprache gesprochen wird. Hinsichtlich des durchschnittlichen sozioökonomischen Status liegen die Familien der SchülerInnen nicht-deutscher Erstsprache bei 40 Punkten auf der ISEI-Skala, ${ }^{97}$ die der deutschsprachigen bei 50 Punkten und die der mehrsprachigen bei 47 Punkten. Die Vermutung, dass nur Kinder von sozial hochgestellte Familien Zweisprachigkeit erzielen könnten, bestätigt sich also nicht.

Werden die Schulleistungen im Deutschen betrachtet, dann liegen über alle Schultypen deutschsprachige Kinder vor mehrsprachigen und diese vor denjenigen, die zu Hause nicht Deutsch sprechen. Bei den Englischleistungen verkehrt sich dieser Befund, Mehrsprachige schneiden grundsätzlich besser ab als nur Deutsch Sprechende. Weiterhin haben Migrantenkinder, die zu Hause nur die Sprache der Eltern sprechen, an Haupt- und Realschulen höhere

\footnotetext{
${ }^{97}$ Der International Socio-Economic Index of Occupational Status ist ein Standardmaß des ökonomischen Status und umfasst als Index Einkommen, Bildung und Beruf. ISEI baut auf der Annahme auf, dass bestimmte Bildungsergebnisse sich vermittelt über die Berufe in bestimmte Einkommen umwandeln lassen. Für nähere Angaben siehe Ganzeboom, et al. 1992.
} 
Leistungen im Englischen als nur Deutsch Sprechende, an Gesamtschulen und Gymnasien jedoch nicht. Wird allerdings der sozioökonomische Status kontrolliert, dann sind auch diese Kinder an allen Schultypen im Englischen besser als nur Deutsch Sprechende. Für Mehrsprachige wiederum verstärken sich bei der Kontrolle des sozioökonomischen Status die positiven Effekte. Die Kontrolle kognitiver Fähigkeiten ändert nichts an diesen Ergebnissen. (Klieme, et al. 2006)

Die DESI-Studie verweist somit auf positive Effekte von Bilingualität ${ }^{98}$ in dem schulisch wichtigen Bereich des Fremdsprachenerwerbs: Bilinguale schneiden besser ab als nur Deutsch Sprechende, erst recht, wenn ihr sozioökonomischer Status kontrolliert wird. Diejenigen, die sich kulturell nicht vollständig anpassen, sind also im Vorteil. Dies belegt auch die Bedeutung methodischer Faktoren wie der Kontrolle des sozioökonomischen Status oder genereller kognitiver Fähigkeiten ${ }^{99}$ für die Güte der Untersuchungen. Eine umfassende Wirkungsanalyse müsste neben diesen Kontrollen jedoch als Längsschnitt angelegt sein, um die jeweiligen Einflüsse auf die Entwicklung zu untersuchen: Möglicherweise hätten z.B. Kinder ohne die sehr guten Kenntnisse in der Herkunftssprache der Eltern niemals so gut Deutsch gelernt. Oder sie hätten sich stärker diskriminiert gefühlt und sich daraufhin zurückgezogen. Da es kaum möglich ist, so viele Faktoren im Nachhinein zu kontrollieren, wäre es wünschenswert, Kinder beim Erwerb ihrer Sprachkompetenz(en) zu begleiten und dabei vermutete Mechanismen zu überprüfen. Sicherlich wäre die alternative Entwicklungsoption nicht für alle bilingualen Kinder der Erwerb perfekter Deutschkenntnisse gewesen, sondern bei einigen das Entstehen semilingualer Fähigkeiten. Semilingualismus bedeutet ungenügende Kenntnisse sowohl der Herkunftssprache der Eltern als auch der Zweitsprache. Kenntnisse auf alltagssprachlichem Niveau sind vorhanden, aber die in der Schule und an den meisten Arbeitsplätzen geforderten Sprachfähigkeiten werden nicht erbracht. Semilinguale Jugendliche haben Probleme im Ausdruck, mit der Grammatik und mit abstrakten Begriffen. Damit stellt Semilingualismus ein ernsthaftes Problem bei der Erlangung eines abgesicherten sozialen Status dar. Was führt aber entweder zu Bi- oder zu Semilingualismus? Aus der Forschung ist bekannt, dass Bilingualität durch Wertschätzung auch der weniger gesprochenen Sprache geför-

\footnotetext{
${ }^{98}$ Welchen Grad der Bilingualität die Kinder erlangt haben, lässt sich aus der Einteilung der Elternhäuser nicht zweifelsfrei ablesen: Bei den Kindern, die zu Hause zweisprachig aufwachsen, kann vermutet werden, dass sie in beiden Sprachen über ein sehr hohes Sprachniveau verfügen. Unter den Kindern, die mit einer anderen Herkunftssprache zu Hause aufwachsen, dürften sich allerdings auch einige bilinguale befinden. Eine direkte Erhebung der Bilingualität der Jugendlichen wurde in der DESI-Studien nicht vorgenommen.

${ }^{99}$ So hat Fürstenau in ihrer qualitativen Untersuchung gezeigt, dass Lehrer je nach schulischen Leistungen der MigrantInnen deren Herkunftssprachenkenntnisse unterschiedlich stark wertschätzen. Leistungsstarke SchülerInnen erhalten wesentlich häufiger eine Anerkennung ihrer Mehrsprachigkeit, wohingegen diese bei leistungsschwachen SchülerInnen im Gegenteil als eine der Ursachen für geringen Schulerfolg ausgemacht wird. (Fürstenau 2004: 111)
} 
dert wird (Reich/Roth 2002: 16). In ihrem Buch Der monolinguale Habitus der multilingualen Schule zeigt Ingrid Gogolin, wie an deutschen Schulen bilinguale Fähigkeiten systematisch ignoriert, wenn nicht gar abgewertet werden (Gogolin 1994). Diese strukturellen Rahmenfaktoren müssen mit in Betracht gezogen werden, wenn auf der individuellen Ebene nach positiven Effekten einer Kompetenz in der Herkunftssprache gefragt wird. Dazu kommt, dass wie eingangs erwähnt, sprachliche Kompetenzen in westeuropäischen Sprachen zumeist honoriert, andere abgewertet werden: Kenntnisse in EU-Sprachen werden dabei nicht zuletzt aufgrund der Programme zur Mobilitätsförderung der EU-Kommission zunehmend anerkannt (Fürstenau 2004: 273, 283).

Dieser Abschnitt hat gezeigt, dass MigrantInnen der zweiten Generation bis auf wenige Ausnahmen über gute bis sehr gute Kenntnisse im Deutschen verfügen, auch im anspruchsvollsten Bereich der Schriftsprache. Wichtig bleibt allerdings die Frage nach dem genauen Zeitpunkt, zu dem diese erreicht wurden: Ist dies erst nach der Einschulung der Fall, könnten schulische Leistungen unter dem Aufholprozess leiden. Es wurde aber auch deutlich, dass sprachliche Defizite bei der Einschulung in Deutschland auch bei Kindern ohne Migrationshintergrund bestehen. Demzufolge sind Sprachprobleme auch ein Schichtproblem. Die Kenntnisse in der Herkunftssprache der Eltern fallen bei der zweiten Generation durchschnittlich schlechter aus als die Deutschkenntnisse. Während im Umgang mit den Eltern überwiegend deren Muttersprache gesprochen wird, teilt sich der Sprachgebrauch mit den Geschwistern ungefähr gleich auf: Mit diesen spricht die eine Hälfte überwiegend Deutsch, die andere überwiegend die Muttersprache der Eltern. Dagegen dominiert bei der Arbeit und in der Schule fast ausschließlich das Deutsche. Die in diesen Zahlen zum Ausdruck kommende Mehrsprachigkeit nicht weniger MigrantInnen der zweiten Generation übersetzt sich in leicht erhöhte Durchschnitte bei verschiedenen psychologischen Ressourcen und in etwas höhere Bildungsabschlüsse, wobei der Unterschied zu denen, die nur Deutsch sehr gut sprechen statistisch nicht signifikant ist. Im Hinblick auf die fremdsprachlichen Leistungen in der Schule werden die Vorteile für diejenigen mit Herkunftssprachenkenntnissen in der DESI-Studie jedoch deutlich.

Das nächste Kapitel beleuchtet nun allgemein die Bildungsabschlüsse der MigrantInnen der zweiten Generation. 


\subsection{Bildung}

Der Erwerb formaler Bildungszertifikate wird als wichtigster Schlüssel zu sozioökonomischem Aufstieg in modernen Gesellschaften betrachtet, da in ihnen das Leistungsprinzip gelte. ${ }^{100}$ Wie meritokratisch beispielsweise ,westliche' Gesellschaften tatsächlich sind, kann an dieser Stelle nicht diskutiert werden. In jedem Fall kommt Bildung zumindest als einem Faktor unter anderen bei dem Versuch, wirtschaftliche Verbesserung zu erlangen, große Bedeutung zu. Der nachfolgende Abschnitt untersucht den individuellen Bildungserfolg (schulische Bildung, Berufsbildung) und die Bildungsaspiration von MigrantInnen. Strukturelle Faktoren, die den Rahmen für die Entwicklung individueller Ergebnisse bilden, werden nicht analysiert; wo es nötig ist, wird kurz auf sie verwiesen. Fragen der institutionellen Diskriminierung werden in Kapitel 5.2 ausführlicher behandelt. Das nachfolgende Kapitel dient zudem der Vorstellung des Bildungsindikators, der später zur Operationalisierung der Muster herangezogen wird.

\subsubsection{Bildung allgemein}

In diesem Abschnitt wird der Schulerfolg von MigrantInnen der Jahrgänge 1961-1985 (Mikrozensus) und 1970-1979 (DJI-Ausländersurvey) mit Hilfe der Internationalen Standardklassifikation von Bildung (ISCED 1997) untersucht.

\section{ISCED Definition}

Die International Standard Classification of Education (ISCED) umfasst jegliche Form organisierter und dauerhafter Bildung für Kinder, Jugendliche und Erwachsene (UNESCO 2000, OECD 1999, Schroedter, et al. 2006). Sie enthält sowohl schulische als auch berufliche Bildung und ist daher aussagekräftiger als andere Indikatoren wie beispielsweise die Anzahl der absolvierten Schuljahre oder Berufsausbildungstitel. Der ISCED-Indikator wurde mittels eines Umwandlungsprogramms (Routine) aus den im Mikrozensus enthaltenen Variablen geschaffen. Die Routine für diese Arbeit und die Gegebenheiten des Mikrozensus 2005 entwickelte ich auf der Grundlage einer Version für frühere Mikrozensen von der Universität Mannheim (ZUMA). Für den DJI-Ausländersurvey schrieb ich eine eigene Routine. Um einen direkten Vergleich der Bildungsniveaus der jüngeren Alterskohorten (Anfang 20) und der älteren in den dreißiger und Anfang der vierziger Jahre zu ermöglichen, behandelt diese veränderte Routine Personen, die sich gegenwärtig in einem Bildungsprogramm befinden so, als ob

\footnotetext{
${ }^{100}$ Für einen Überblick über die Bildungstheorien siehe Allmendinger/Aisenbrey 2002 und Kristen 1999.
} 
sie dieses schon erfolgreich abgeschlossen hätten (Schroedter, et al. 2006: 15). ${ }^{101}$ Für eine Einschätzung der ISCED-Niveaus sei auf Ergebnisse der Bildungsforschung der letzten Jahre hingewiesen, gemäß denen für eine nachhaltige Teilnahme am deutschen Arbeitsmarkt und in der Gesellschaft allgemein ein ISCED-Niveau von mindestens 3 vorliegen muss, was einem Hauptschulabschluss zusammen mit abgeschlossener Lehre entspricht (Solga 2005).

\section{Bildungsergebnisse des Mikrozensus nach ISCED}

Die nachfolgenden Kreuztabellen mit den Daten des Mikrozensus 2005 stellen die gerundeten Prozentwerte sowohl für die zweite Generation als auch für MigrantInnen der ersten und anderthalbten Generation über fünf zusammengefasste Kategorien von ISCED-Niveaus hinweg dar: dabei ist 1 die niedrigste, 6 die höchste Bildungskategorie. ${ }^{102}$ Die Prozentwerte werden sowohl für Männer und Frauen zusammen als auch getrennt ausgegeben.

Zunächst einmal zeigt Tabelle 13, dass die zweite Generation hinsichtlich ihrer Bildungsabschlüsse wesentlich besser ${ }^{103}$ gestellt ist als die erste und anderthalbte Generation und zwar in allen Herkunftsgruppen bis auf die, westlichen' MigrantInnen aus Nicht-Anwerbestaaten. Dieser Effekt ist stark und eindeutig. Hinsichtlich der Geschlechtsunterschiede sind Frauen der zweiten Generation in der italienischen und der griechischen Herkunftsgruppe sowie die Töchter von AussiedlerInnen und ,westlicher' MigrantInnen aus Nicht-Anwerbestaaten erfolgreicher als die Männer der jeweiligen Altersgruppe. In der türkischen Herkunftsgruppe liegen Männer und Frauen in der zweiten Generation nah beieinander. Dieser Effekt widerspricht der öffentlichen Wahrnehmung gemäß der weibliche Migrantinnen angeblich nur prekäre Schulleistungen erzielen. Die Tabelle zeigt auch, dass selbst bei den MigrantInnen türkischer Herkunft, die gemeinhin als die Migrantengruppe mit den problematischsten Bildungsergebnissen betrachtet wird, 14\% der Altersgruppe von 20 bis 45 Jahren tertiäre Bildung erwerben (die von einem Meisterabschluss über Berufsakademien und Verwaltungsfachhochschulen bis zu Universitäten und Promotionsprogrammen reicht). Auf der anderen Seite erwerben 5\% der zweiten Generation türkischer Herkunft gar keinen Bildungsabschluss. Die Bandbreite der Ergebnisse ist also sehr groß.

\footnotetext{
${ }^{101}$ Selbstverständlich besteht die Gefahr, dass manche dieser Personen den Ausbildungsgang noch abbrechen und damit zu Unrecht der höheren Kategorie zugerechnet werden. Allerdings überwiegen in meinen Augen die Vorteile, die sich aus der besseren Vergleichbarkeit ergeben.

${ }^{102}$ Das nachfolgende A, B oder C bezieht sich darauf, ob dieser Bildungsweg üblicherweise auf einen Verbleib im Bildungssystem oder einen Eintritt in den Arbeitsmarkt vorbereitet. Die Buchstaben dienen also innerhalb des gleichen Bildungsniveaus der weiteren Differenzierung nach Berufsperspektiven. Ihnen kommt in dieser Untersuchung keine weitere Bedeutung zu.

${ }^{103}$ Wegen der missverständlichen sprachlichen Formulierung, sei mit Nachdruck darauf hingewiesen, dass es sich nicht um individuelle oder intergenerationelle Mobilität von Eltern zu Kindern handelt. Vielmehr werden verschiedene Migrantengenerationen derselben Altersgruppen verglichen.
} 
Tabelle 13 - Bildungsabschluss (ISCED) : Generationen, Herkunftsgruppen, Geschlechter

\begin{tabular}{|c|c|c|c|c|c|c|c|c|c|c|c|c|c|c|c|c|c|c|c|c|c|c|c|c|c|}
\hline & & \multicolumn{8}{|c|}{ MÄNNER UND FRAUEN } & \multicolumn{8}{|c|}{ FRAUEN } & \multicolumn{8}{|c|}{ MÄNNER } \\
\hline & & TÜRK & ITAL & GRIE & $\begin{array}{c}\text { SPAN } \\
\& \\
\text { PORT }\end{array}$ & $\begin{array}{l}\text { EX- } \\
\text { JUG }\end{array}$ & AUSS & WEST & ртом & TÜRK & ITAL & GRIE & $\begin{array}{c}\text { SPAN } \\
\& \\
\text { PORT }\end{array}$ & $\begin{array}{l}\text { EX- } \\
\text { JUG }\end{array}$ & AUSS & WEST & Dтом & TÜRK & ITAL & GRIE & $\begin{array}{c}\text { SPAN } \\
\& \\
\text { PORT }\end{array}$ & $\begin{array}{l}\text { EX- } \\
\text { JUG }\end{array}$ & AUSS & WEST & DTOM \\
\hline \multirow{5}{*}{ ڤُ } & $1 \mathrm{~A}, \mathrm{~B}$ & $5 \%$ & $4 \%$ & $4 \%$ & $4 \%$ & $3 \%$ & $1 \%$ & $1 \%$ & $2 \%$ & $5 \%$ & $5 \%$ & $4 \%$ & $4 \%$ & $4 \%$ & $1 \%$ & $0 \%$ & $1 \%$ & $5 \%$ & $3 \%$ & $4 \%$ & $4 \%$ & $3 \%$ & $2 \%$ & $2 \%$ & $2 \%$ \\
\hline & $2 \mathrm{~A}, \mathrm{~B}$ & $34 \%$ & $30 \%$ & $23 \%$ & $20 \%$ & $18 \%$ & $20 \%$ & $10 \%$ & $11 \%$ & $37 \%$ & $27 \%$ & $19 \%$ & $19 \%$ & $21 \%$ & $19 \%$ & $6 \%$ & $12 \%$ & $31 \%$ & $32 \%$ & $26 \%$ & $21 \%$ & $16 \%$ & $20 \%$ & $13 \%$ & $10 \%$ \\
\hline & $3 \mathrm{~A}, \mathrm{~B}$ & $42 \%$ & $48 \%$ & $44 \%$ & $42 \%$ & $49 \%$ & $46 \%$ & $39 \%$ & $49 \%$ & $39 \%$ & $49 \%$ & $43 \%$ & $45 \%$ & $46 \%$ & $46 \%$ & $40 \%$ & $49 \%$ & $45 \%$ & $48 \%$ & $44 \%$ & $39 \%$ & $51 \%$ & $45 \%$ & $39 \%$ & $49 \%$ \\
\hline & $4 \mathrm{~A}$ & $5 \%$ & $5 \%$ & $10 \%$ & $14 \%$ & $9 \%$ & $6 \%$ & $7 \%$ & $8 \%$ & $5 \%$ & $7 \%$ & $11 \%$ & $17 \%$ & $11 \%$ & $7 \%$ & $10 \%$ & $10 \%$ & $5 \%$ & $4 \%$ & $10 \%$ & $12 \%$ & $7 \%$ & $5 \%$ & $4 \%$ & $7 \%$ \\
\hline & $\begin{array}{l}5 \text { A,B } \\
6\end{array}$ & $14 \%$ & $13 \%$ & $19 \%$ & $20 \%$ & $21 \%$ & $27 \%$ & $43 \%$ & $30 \%$ & $14 \%$ & $12 \%$ & $23 \%$ & $15 \%$ & $18 \%$ & $27 \%$ & $44 \%$ & $28 \%$ & $14 \%$ & $13 \%$ & $16 \%$ & $24 \%$ & $23 \%$ & $28 \%$ & $42 \%$ & $32 \%$ \\
\hline \multirow{5}{*}{ 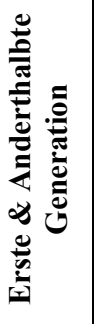 } & $1 \mathrm{~A}, \mathrm{~B}$ & $31 \%$ & $10 \%$ & $13 \%$ & $20 \%$ & $12 \%$ & $3 \%$ & $2 \%$ & - & $38 \%$ & $12 \%$ & $22 \%$ & $20 \%$ & $16 \%$ & $3 \%$ & $3 \%$ & - & $24 \%$ & $9 \%$ & $7 \%$ & $20 \%$ & $9 \%$ & $3 \%$ & $2 \%$ & - \\
\hline & $2 \mathrm{~A}, \mathrm{~B}$ & $41 \%$ & $42 \%$ & $36 \%$ & $33 \%$ & $30 \%$ & $25 \%$ & $10 \%$ & - & $44 \%$ & $42 \%$ & $31 \%$ & $34 \%$ & $32 \%$ & $25 \%$ & $12 \%$ & - & $39 \%$ & $42 \%$ & $39 \%$ & $30 \%$ & $28 \%$ & $25 \%$ & $10 \%$ & - \\
\hline & $3 \mathrm{~A}, \mathrm{~B}$ & $20 \%$ & $27 \%$ & $31 \%$ & $20 \%$ & $41 \%$ & $49 \%$ & $26 \%$ & - & $13 \%$ & $23 \%$ & $27 \%$ & $19 \%$ & $36 \%$ & $47 \%$ & $24 \%$ & - & $26 \%$ & $30 \%$ & $33 \%$ & $22 \%$ & $45 \%$ & $51 \%$ & $26 \%$ & - \\
\hline & $4 \mathrm{~A}$ & $2 \%$ & $3 \%$ & $5 \%$ & $3 \%$ & $4 \%$ & $6 \%$ & $8 \%$ & - & $2 \%$ & $3 \%$ & $5 \%$ & $5 \%$ & $4 \%$ & $8 \%$ & $6 \%$ & - & $3 \%$ & $2 \%$ & $5 \%$ & $3 \%$ & $5 \%$ & $5 \%$ & $9 \%$ & - \\
\hline & $\begin{array}{l}5 \mathrm{~A}, \mathrm{~B} \\
6\end{array}$ & $6 \%$ & $18 \%$ & $15 \%$ & $24 \%$ & $13 \%$ & $17 \%$ & $54 \%$ & - & $3 \%$ & $20 \%$ & $15 \%$ & $22 \%$ & $12 \%$ & $17 \%$ & $55 \%$ & - & $8 \%$ & $17 \%$ & $16 \%$ & $25 \%$ & $13 \%$ & $16 \%$ & $53 \%$ & - \\
\hline
\end{tabular}

Mikrozensus 2005, FDZ der Statistischen Ämter des Bundes und der Länder, Eigene Berechnungen 


\section{Bildungsergebnisse des Ausländersurveys nach ISCED}

Wie sehen die entsprechenden Ergebnisse für die jüngere Altersgruppe (18 bis 25 Jahre) im Ausländersurvey des DJI aus? Da ISCED als Bildungsindikator in die Musteranalyse einfließen wird, sollte eine grundsätzliche Vergleichbarkeit gegeben sein, auch wenn der Ausländersurvey durch die einfachere Stichprobenziehung nicht an den Grad der Repräsentativität des Mikrozensus heranreicht. Wie Tabelle 14 für Männer und Frauen zusammen zeigt, entsprechen sich die Ergebnisse der beiden Datensets weitestgehend: Es gibt eine klare Besserstellung der zweiten Generation im Verhältnis zur ersten und anderthalbten Generation und deutliche Unterschiede in den Herkunftsgruppen in der zweiten Generation (die MigrantInnen griechischer Herkunft liegen vor denen italienischer Herkunft und diese wiederum vor denen türkischer Herkunft).

Tabelle 14 - Vergleich Bildungsabschluss (ISCED) im MZ und DJI-Ausländersurvey

\begin{tabular}{|c|c|c|c|c|c|c|c|}
\hline & & \multicolumn{3}{|c|}{ DJI } & \multicolumn{3}{|c|}{ MZ } \\
\hline & & TÜRK & ITAL & GRIE & TÜRK & ITAL & GRIE \\
\hline \multirow{5}{*}{ 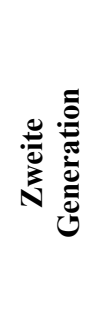 } & $1 \mathrm{~A}, \mathrm{~B}$ & $1 \%$ & - & - & $5 \%$ & $4 \%$ & $4 \%$ \\
\hline & $2 \mathrm{~A}, \mathrm{~B}$ & $35 \%$ & $25 \%$ & $25 \%$ & $34 \%$ & $30 \%$ & $23 \%$ \\
\hline & $3 \mathrm{~A}, \mathrm{~B}$ & $52 \%$ & $62 \%$ & $59 \%$ & $42 \%$ & $48 \%$ & $44 \%$ \\
\hline & $4 \mathrm{~A}$ & $2 \%$ & $2 \%$ & $2 \%$ & $5 \%$ & $5 \%$ & $10 \%$ \\
\hline & $\begin{array}{l}5 \mathrm{~A}, \mathrm{~B} \\
6\end{array}$ & $10 \%$ & $11 \%$ & $14 \%$ & $14 \%$ & $13 \%$ & $19 \%$ \\
\hline \multirow{5}{*}{ 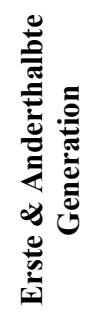 } & $1 \mathrm{~A}, \mathrm{~B}$ & $22 \%$ & $11 \%$ & $11 \%$ & $31 \%$ & $10 \%$ & $13 \%$ \\
\hline & $2 \mathrm{~A}, \mathrm{~B}$ & $41 \%$ & $29 \%$ & $31 \%$ & $41 \%$ & $42 \%$ & $36 \%$ \\
\hline & $3 \mathrm{~A}, \mathrm{~B}$ & $31 \%$ & $49 \%$ & $50 \%$ & $20 \%$ & $27 \%$ & $31 \%$ \\
\hline & $4 \mathrm{~A}$ & $1 \%$ & $1 \%$ & $1 \%$ & $2 \%$ & $3 \%$ & $5 \%$ \\
\hline & $\begin{array}{l}5 \mathrm{~A}, \mathrm{~B} \\
6\end{array}$ & $5 \%$ & $10 \%$ & $7 \%$ & $6 \%$ & $18 \%$ & $15 \%$ \\
\hline
\end{tabular}

Mikrozensus 2005, FDZ der Statistischen Ämter des Bundes und der Länder, Eigene Berechnungen und Ausländersurvey 1997 des Deutschen Jugendinstituts, Eigene Berechnungen

Die Ergebnisse der beiden Datensets unterscheiden sich darin, dass es im Ausländersurvey eine Konzentration bei den mittleren Ergebnissen gibt und sowohl hochqualifizierte als auch v.a. niedrigqualifizierte MigrantInnen weniger vertreten sind als im Mikrozensus. Dies liegt zum einen daran, dass das Sample im Ausländersurvey jünger ist und viele daher noch gar keine (Fach-)Hochschulbildung haben können. Zum anderen wird bei der Klassifikation des Bildungsniveaus aktueller SchülerInnen davon ausgegangen, dass diese den Abschluss, den sie anstreben, auch schaffen werden. Dies wird für die meisten von ihnen sicherlich der Fall 
sein, manche aber werden die Ausbildung ohne Abschluss beenden und würden dann zur niedrigsten Kategorie gehören. Diese Verzerrung entsteht im Mikrozensus wegen der von mir vorgenommenen Beschränkung auf ältere MigrantInnen weniger. Und schließlich gibt es im Ausländersurvey wie in den meisten Umfragen eine Tendenz, Niedrigqualifizierte nicht in repräsentativem Maße in die Befragung einzuschließen. Da bei der Musteranalyse der ISCED-Indikator durch Zusammenlegung verschiedener Kategorien vergröbert wird, beeinflusst diese Ausdünnung in den Extrembereichen die Vergleichbarkeit der beiden Datensets in der Musteranalyse jedoch kaum.

\section{Unterschiede bei deutscher Staatsbürgerschaft?}

Um die Generationen- und Herkunftseffekte bei Kontrolle anderer Faktoren zu überprüfen, werden im Folgenden die Ergebnisse einer Regression mit der abhängigen Variable ISCED auf einer Skala von 1 (,kein Bildungsabschluss') bis 10 (,Doktortitel') mit den Daten des Mikrozensus dargestellt. Die Regression nutzt Dummy-Variablen ${ }^{104}$ für Herkunft, Generation, Altersgruppe (20-29 Jahre versus 30-45 Jahre) Stadt/Land und Geschlecht, um Durchschnittswerte $^{105}$ für unterschiedliche Kombinationen der Dummies zu erlangen (siehe rechter Teil Tabelle 15) ${ }^{106}$. Im Unterschied zu den Kreuztabellen erlauben diese Durchschnittswerte einen einfachen Vergleich zwischen Generationen und Herkunftsgruppen bei Kontrolle der Altersgruppe.

Die Regressionen ${ }^{107}$ bestätigen die Verbesserung im ISCED-Niveau von der ersten zur anderthalbten und von der anderthalbten zur zweiten Generation. Während die vorherige Kreuztabelle zugunsten der Übersichtlichkeit die erste und anderthalbte Generation zusammenfasste, zeigt Spalte I der Regressionstabelle, dass die Erhöhung des Bildungsniveaus ein schrittweiser und dauerhafter Effekt über die drei Generationen ist: Im Vergleich zur ersten Generation (Referenzkategorie) erlangen MigrantInnen der zweiten Generation im Durchschnitt ein Bildungsniveau, das auf der ISCED-Skala eine Stufe höher liegt $(B=1,03)$. MigrantInnen der

\footnotetext{
${ }^{104}$ Dummy-Variablen sind Stellvertreter-Variablen mit den Ausprägungen 0 oder 1, die zur Aufbereitung von kategorialen Daten für Regressionen u.a. benutzt werden. Zum Beispiel wird die Variable Herkunft (italienisch, spanisch, türkisch, ...) in mehrere Herkunftsdummies umgewandelt (italienisch - nicht-italienisch, spanisch nicht-spanisch,...), welche dann unter Auslassung einer Referenzkategorie in die Regression eingeschlossen werden können.

${ }^{105}$ Durchschnittswerte bei kategorialen Daten sind selbstverständlich mit Vorsicht zu genießen. Da die ISCEDKategorien jedoch hierarchisch (ordinal) und bis zu einem gewissen Grad sogar intervallskaliert sind, können diese Durchschnittswerte reliabel interpretiert werden, auch wenn strenge StatistikerInnen sie nicht unproblematisch fänden.

${ }^{106}$ Interaktionsvariablen für Herkunftsgruppe und Generation waren nur für die Gruppe der westlichen MigrantInnen aus Nicht-Anwerbestaaten signifikant und wurden daher aus der Regression ausgeschlossen.

${ }^{107}$ Das korrigierte $\mathrm{R}^{2}$ wird nur zur Information angegeben. Der Einschluss von Nicht-MigrantInnen in die Regression senkt die erklärte Varianz, da Personen ohne Migrationshintergrund keine Varianz in der Generationsvariablen aufweisen (siehe auch Tabelle 21; Tabelle 22).
} 
anderthalbten Generation erreichen durchschnittlich Werte, die eine Viertelstufe höher liegen als die der ersten Generation ( $\mathrm{B}=0,24)$. Dieser Generationeneffekt verstärkt sich bei eingebürgerten MigrantInnen (Spalten II bis IV). Spalte V schließlich untersucht den Effekt einer ausländischen Staatsangehörigkeit. Auch hier erhöht sich das ISCED-Niveau bei der zweiten Generation um knapp einen Schritt; der Unterschied zwischen erster und anderthalbter Generation ist nicht signifikant.

Zudem haben MigrantInnen mit deutscher Staatsbürgerschaft über alle Herkunftsgruppen hinweg ein höheres durchschnittliches ISCED-Niveaus, was sich an den größeren Konstanten (3,8 versus 3,1 für AusländerInnen) ablesen lässt. Die rechte Hälfte der Tabelle 15 gibt Auskunft über die durchschnittlichen ISCED-Werte für männliche Ausländer oder männliche deutsche Staatsbürger in der ersten bzw. zweiten Generation in den verschiedenen Herkunftsgruppen. Es zeigt sich, dass Migranten der zweiten Generation mit deutscher Staatsbürgerschaft an den ISCED-Durchschnitt der Deutschen ohne Migrationshintergrund heranreichen $(6,5)$, mit Ausnahme der deutschen Staatsbürger türkischer Herkunft in der zweiten Generation, deren Durchschnittswerte niedriger bleiben (5,3). Die Werte für Frauen fallen ähnlich, jedoch etwas niedriger aus. Der durchschnittliche Unterschied zwischen ausländischen StaatsbürgerInnen und Nicht-MigrantInnen ist größer, sowohl in der ersten als auch in der zweiten Generation. Nur bei den, westlichen' MigrantInnen aus Nicht-Anwerbestaaten haben diejenigen, die weiterhin ausschließlich die ausländische Staatsbürgerschaft besitzen, im Durchschnitt einen höheren Bildungsstatus als MigrantInnen mit deutscher Staatsbürgerschaft. Schließlich zeigt Spalte V, dass die höheren Werte für deutsche StaatsbürgerInnen nicht allein durch das bessere Bildungsniveau der AussiedlerInnen erklärt werden kann, da der Effekt auch dann anhält, wenn AussiedlerInnen aus der Regression ausgeschlossen werden.

Es kann also zusammengefasst werden, dass die zweite Generation hinsichtlich des Bildungsniveaus signifikant besser gestellt ist als die anderthalbte und diese wiederum besser als die erste Generation. Diese Verbesserung im ISCED-Niveau besteht für alle Herkunftsgruppen außer für ,westliche' MigrantInnen aus Nicht-Anwerbestaaten. Eingebürgerte MigrantInnen haben höhere ISCED-Durchschnitte als ausländische StaatsbürgerInnen (wieder mit Ausnahme der, westlichen' MigrantInnen aus Nicht-Anwerbestaaten). Die Unterschiede zwischen Männern und Frauen sind gering und schwanken je nach Herkunftsgruppe. Insgesamt zeigt die bisherige Analyse die große inter- und intraethnische Diversität anhand der breiten Streuungen über die ISCED-Niveaus in allen Herkunftsgruppen. 
Tabelle 15 - Bildungsabschluss (ISCED): Generationen, Herkunftsgruppen, Staatsbürgerschaften

\begin{tabular}{|c|c|c|c|c|c|c|c|c|c|c|}
\hline & \multirow{2}{*}{$\begin{array}{c}\text { I } \\
\text { DEUT \& AUSL, } \\
\text { INKL. AUSS \& } \\
\text { DTOM }\end{array}$} & \multirow{2}{*}{$\begin{array}{c}\text { II } \\
\text { NUR DEUT } \\
\text { INKL. AUSS \& } \\
\text { DTOM }\end{array}$} & \multirow{2}{*}{$\begin{array}{c}\text { III } \\
\text { NUR DEUT } \\
\text { INKL. AUSS } \\
\text { OHNE DTOM }\end{array}$} & \multirow{2}{*}{$\begin{array}{c}\text { IV } \\
\text { NUR DEUT } \\
\text { OHNE AUSS \& } \\
\text { DTOM }\end{array}$} & \multirow{2}{*}{$\begin{array}{c}\mathrm{V} \\
\text { NUR AUSL } \\
\text { OHNE AUSS \& } \\
\text { DTOM }\end{array}$} & & \multicolumn{2}{|c|}{$\begin{array}{c}\text { MW ISCED } \\
\text { ERSTE } \\
\text { GENERATION }\end{array}$} & \multicolumn{2}{|c|}{$\begin{array}{c}\text { MW ISCED } \\
\text { ZWEITE } \\
\text { GENERATION }\end{array}$} \\
\hline & & & & & & & $\begin{array}{l}\text { AUSL IN } \\
\text { STADT }\end{array}$ & $\begin{array}{l}\text { DEUT IN } \\
\text { STADT }\end{array}$ & $\begin{array}{l}\text { AUSL IN } \\
\text { STADT }\end{array}$ & $\begin{array}{l}\text { DEUT IN } \\
\text { STADT }\end{array}$ \\
\hline & & & & & & TÜRK & 3,5 & 4,3 & 4,4 & 5,3 \\
\hline $\begin{array}{l}\text { ITAL, GRIE, } \\
\text { SPAN, PORT }\end{array}$ &, $96^{* *}$ &, $85^{* *}$ &, $82 * *$ &, $83 * *$ & $1,13 * *$ & $\begin{array}{l}\text { ITAL, GRIE, } \\
\text { SPAN, PORT }\end{array}$ & 4,6 & 5,1 & 5,5 & 6,2 \\
\hline EX-JUG- & $1,07 * *$ & $1,17 * *$ & $1,16^{* *}$ & $1,14 * *$ & $1,20 * *$ & EX-JUG & 4,7 & 5,5 & 5,6 & 6,5 \\
\hline WEST - & $3,48 * *$ & $2,06 * *$ & $2,00 * *$ & $2,10 * *$ & $3,74 * *$ & WEST & 7,3 & 6,3 & 8,1 & 7,3 \\
\hline AUSS- & $1,78^{* *}$ & $1,13^{* *}$ & $1,18^{* *}$ & -- & -- & AUSS & -- & 5,5 & -- & 6,5 \\
\hline DTOM & $2,74 * *$ & $2,07 * *$ & -- & -- & -- & DTOM & 6,5 & 6,5 & 6,5 & 6,5 \\
\hline $\begin{array}{l}\text { Zweite Genera- } \\
\text { tion } \dagger\end{array}$ & $1,03 * *$ &, $83 * *$ & $1,04 * *$ & $1,24 * *$ &, $89 * *$ & & & & & \\
\hline $\begin{array}{l}\text { Anderthalbte } \\
\text { Generation } \uparrow\end{array}$ &, $24 * *$ &, $24 * *$ &, $44 * *$ &, $63^{* *}$ & ,01 n.s. & & & & & \\
\hline 1976-1985 geb• &, $02 * *$ & ,01 n.s. &,$- 38 * *$ &,- 004 n.s. &, $29 * *$ & & & & & \\
\hline Frauen $^{\circ}$ &,$- 13 * *$ &,$- 11 * *$ & ,01 n.s. &,$- 44 * *$ &,$- 37 * *$ & & & & & \\
\hline Stadta &, $61 * *$ &, $63 * *$ &, $45^{* *}$ &, $47 * *$ &, $43 * *$ & & & & & \\
\hline Konstante & $3,13 * *$ & $3,76^{* *}$ & $3,84 * *$ & $3,75^{* *}$ & $3,08 * *$ & & & & & \\
\hline Korrigiertes $\mathbf{R}^{2}$ & 0,08 & 0,04 & 0,06 & 0,12 & 0,26 & & & & & \\
\hline
\end{tabular}

Koeffizienten (unstandardisierte Bs) linearer Regression, abhängige Variable: ISCED

$\ulcorner$ Referenzkategorie: TÜRK; † Referenzkategorie: Erste Generation; • Referenzkategorie: 1961-1975 geboren; Referenzkategorie: Männer; a Referenzkategorie: Land/Kleinstadt

Mikrozensus 2005, FDZ der Statistischen Ämter des Bundes und der Länder, Eigene Berechnungen; ** statistisch signifikant bei p<0.01, * statistisch signifikant bei $p<0.05$, n.s. nicht signifikant 


\section{Konzentrierte Unterschichtung oder breite Streuung?}

Der folgende Abschnitt beleuchtet die Frage der Streuung oder Konzentration der Bildungsergebnisse der MigrantInnen der zweiten Generation genauer. In der überwiegenden Mehrheit der medialen Berichterstattung und einem Teil der sozialwissenschaftlichen Forschung (Esser 2001) wird behauptet, MigrantInnen der zweiten Generation würden die deutsche Gesellschaft ,unterschichten'. Gemäß dieser Annahme bilden MigrantInnen eine eigene Schicht unterhalb der niedrigsten Schicht der Deutschen ohne Migrationshintergrund. Diese Annahme erscheint schon wegen der Belegung aller Felder in der Kreuztabelle nicht plausibel; sie soll hier aber noch genauer untersucht werden.

Da bei den Tests der PISA-Studie die Leistungen auf einer großen Punkteskala gemessen wurden ( 0 bis 800$)$, eignen sie sich besonders für die Untersuchung von Streuungen durch Perzentilbereiche. Zur Gewinnung von Perzentilbereichen werden die Durchschnittswerte der Höhe nach geordnet und dann der Punktwert z.B. des 5\%-Perzentils, also der Wert, unter dem 5\% der Ergebnisse im Sample liegen, von dem 95\%-Perzentil abgezogen. Dadurch erhält man die Bandbreite der Punktwerte für die 90\% des Samples, die in der Mitte liegen. Die Ausreißer werden also nicht berücksichtigt. Speziell beim Interquartilbereich wird das Spektrum der geordneten Durchschnittswerte in vier Quartile unterteilt und dann der Wert des ersten Quartils $(25 \%)$ von dem des dritten (75\%) abgezogen (vgl. Kapitel 3.1.3). In den PISA-Daten in Tabelle 16 zeigt sich, dass in Deutschland der Interquartilbereich sowie der Bereich zwischen 5\%- und 95\%-Perzentil für MigrantInnen der zweiten Generation größer ist als für Deutsche ohne Migrationshintergrund: So beträgt die Streuung im Perzentil zwischen 5\% und 95\% beim Lesen für MigrantInnen der zweiten Generation 342 Punkte, für Nicht-MigrantInnen jedoch nur 306 Punkte. In der Mathematik sind es 323 gegenüber 298 Punkten. Auch bei den $50 \%$ in der Mitte, die über den Interquartilbereich erfasst werden, ist die Streuung für MigrantInnen der zweiten Generation größer als für Nicht-MigrantInnen: beim Lesen 137 Punkte gegenüber 129 Punkten und in der Mathematik 136 gegenüber 128 Punkten. Somit bleiben die durchschnittlichen Ergebnisse zwar klar unter denen der Nicht-MigrantInnen, aber es gibt auch eine Gruppe kompetenzstarker MigrantInnen, die in die Nähe der leistungsstärksten Nicht-MigrantInnen kommt. Am unteren Ende des Spektrums liegen die Punktleistungen der MigrantInnen jedoch deutlich unter denen der Nicht-MigrantInnen. Insgesamt aber decken die Perzentilbereiche größtenteils die gleiche Fläche ab. Die Überschneidungen überwiegen, und es kann keinesfalls von einer grundsätzlichen ,Unterschichtung' ausgegangen werden. 
Tabelle 16 - Generationen auf PISA Mathematik- und Leseskala

\begin{tabular}{|l|c|c|c|c|c|c|}
\hline & \multicolumn{3}{|c|}{ MATHEMATIKSKALA } & \multicolumn{3}{c|}{ LESESKALA } \\
\cline { 2 - 7 } & $\begin{array}{c}\text { ZWEITE } \\
\text { GENERATION }\end{array}$ & $\begin{array}{c}\text { ERSTE } \\
\text { GENERATION }\end{array}$ & DTOM & $\begin{array}{c}\text { ZWEITE } \\
\text { GENERATION }\end{array}$ & $\begin{array}{c}\text { ERSTE } \\
\text { GENERATION }\end{array}$ & DTOM \\
\hline $\begin{array}{l}\text { Durchschnitt } \\
\text { (S.F.) }\end{array}$ & $432(9,1)$ & $454(7,5)$ & $525(3,5)$ & $420(9,9)$ & $431(8,9)$ & $517(3,5)$ \\
\hline $\mathbf{5 \%}$ Perzentil & 280 & 297 & 371 & 254 & 248 & 353 \\
\hline $\mathbf{2 5 \% ~ P e r z e n t i l ~}$ & 361 & 379 & 462 & 349 & 351 & 455 \\
\hline $\mathbf{7 5 \%}$ Perzentil & 497 & 528 & 590 & 486 & 514 & 584 \\
\hline $\mathbf{9 5 \%}$ Perzentil & 603 & 609 & 669 & 596 & 599 & 659 \\
\hline
\end{tabular}

Stanat (2006): 40f, $186 f$

Obgleich die These einer generellen ,Unterschichtung' durch die MigrantInnen der zweiten Generation zurückgewiesen werden kann, sollten die großen Spannbreiten nicht darüber hinwegtäuschen, dass ein Teil der zweiten Generation es nicht schafft, solche Bildungsniveaus zu erlangen, die eine uneingeschränkte gesellschaftliche Teilhabe ermöglichen. Im Bericht der PISA-Studie werden die Punktergebnisse in Kompetenzstufen überführt, wobei ein Niveau unterhalb der Kompetenzstufe 2 (mindestens 421 Punkte) als für eine nachhaltige Partizipation auf dem Arbeitsmarkt und in der Gesellschaft nicht ausreichend betrachtet wird. Der Bericht hält fest: „Besonders alarmierend ist, dass über 50 Prozent der Jugendlichen türkischer Herkunft, obwohl sie in Deutschland geboren sind, nur marginale Kompetenzen erreichen, die nicht über die Stufe 1 hinausgehen. Die Verteilung auf die Kompetenzstufen sieht für [Jugendliche der ersten Generation] aus der ehemaligen Sowjetunion zwar günstiger aus, aber über 30 Prozent von ihnen erreichen ebenfalls nur maximal die erste Kompetenzstufe." (PISA-Konsortium 2004: 264) ${ }^{108}$ Bei deutschen SchülerInnen ohne Migrationshintergrund beträgt der Anteil in der ersten Kompetenzstufe (oder darunter) hingegen nur 13\% (Stanat/Christensen 2006: 188).

Auf der anderen Seite zeigt eine Studie der Hochschulinformationssysteme, dass Studienberechtigte mit Migrationshintergrund ${ }^{109}$ häufiger an die Hochschule gehen als Studienberechtigte ohne Migrationshintergrund. Besonders stark ausgeprägt ist die Studierneigung bei denjenigen, die zu Hause kein Deutsch sprechen (83\% gegenüber 70\% bei denen ohne Migrati-

108 An dieser Stelle muss auch auf den überproportionalen Anteil ausländischer SchülerInnen an Sonderschulen in Deutschland aufmerksam gemacht werden: 4,9\% der spanischen, 6,3\% der türkischen und 8,0\% der italienischen SchülerInnen sowie 9,6\% der SchülerInnen aus dem ehemaligen Jugoslawien besuchen eine Sonderschule. Bei allen SchülerInnen in Deutschland sind dies nur 5,6\%. Zahlen, die nach Migrationsstatus unterscheiden, liegen hierzu nicht vor. (Bundesamt 2001; KMK 2005)

${ }^{109}$ Der Migrationshintergrund wird in dieser Studie über die ausländische Staatsangehörigkeit, mindestens einen im Ausland geborenen Elternteil und/oder die nicht-deutsche Sprache in der Familie operationalisiert (Bildungsberichterstattung 2006: 156-7). 
onshintergrund). In diesem Spitzensegment ist also eine starke Bildungsmotivation vorhanden. (Heine, et al. 2006 zitiert nach Bildungsberichterstattung 2006: 156-7; ähnlich auch Stanat/Christensen 2006).

Eine wichtige Beobachtung bleibt somit die der Streuung der Ergebnisse, wo Spitzenergebnisse ebenso zu verzeichnen sind wie Bildungsniveaus, die keine nachhaltige Teilhabe sichern.

\section{PISA und die Verschlechterung zwischen den Generationen?}

In der Auswertung der Daten der PISA-Studie ist auf schlechtere Testergebnisse der zweiten Generation $^{110}$ im Vergleich zur ersten hingewiesen worden (PISA-Konsortium 2004: 257). Da dies den von mir sowohl im Mikrozensus als auch im Ausländersurvey gefundenen Generationsverbesserungen von der ersten über die anderthalbte zur zweiten Generation widerspricht, soll dieses vermeintliche ,Absacken' hier genauer beleuchtet werden.

Erste und zweite Generation in der PISA-Untersuchung setzen sich aus unterschiedlichen Herkunftsgruppen zusammen: Die Eltern der zweiten Generation sind überwiegend in der Türkei geboren (63\%); bei den zugewanderten Familien der ersten Generation kommt ein großer Teil aus den Ländern der ehemaligen Sowjetunion (50\%), Polen (21\%) und dem ehemaligen Jugoslawien (6\%) (PISA-Konsortium 2004: 263). Damit konstituiert sich die erste Generation v.a. aus MigrantInnen aus ehemaligen Ostblockländern, wo der durchschnittliche Bildungsstand hoch ist, während die zweite Generation die Kinder der ,GastarbeiterInnen' umfasst, von denen viele nur über niedrige Bildungsabschlüsse verfügten. Trotzdem stellen die AuswerterInnen der PISA-Studie fest, dass die Unterschiede zwischen den Generationen nicht über die Zusammensetzung zu erklären seien: „Dieses Ergebnis kann nicht auf Unterschiede im sozialen und wirtschaftlichen Hintergrund zurückgeführt werden, weil der durchschnittliche Kennwert für die sozioökonomische Lage (HISEI ${ }^{111}[\ldots]$ ) dieser beiden Gruppen sich nur geringfügig unterscheidet (Türkei: 36, ehem. Sowjetunion: 40).“ (PISA-Konsortium 2004: 264) Allerdings erfasst der Kennwert HISEI nur den ausgeübten, nicht den erlernten Beruf. Gerade bei zugewanderten Personen weicht die ausgeübte Tätigkeit jedoch häufig von dem im Herkunftsland erworbenen Qualifizierungsniveau ab. Aus allgemeinen Bildungsuntersuchungen ist zudem bekannt, dass der Bildungsstand der Eltern für das Kind wichtiger ist als das Familieneinkommen oder der aktuelle Berufsstatus (Kristen 1999). Beim direkten

\footnotetext{
${ }^{110}$ Die Terminologie der PISA-Studie unterscheidet sich hiervon insofern, als sie von zugewanderten Jugendlichen (hier: erste Generation) und der ersten Generation (hier: zweite Generation) spricht.

${ }^{111}$ HISEI steht für ,highest ISEI“ und ist der ISEI-Wert des höhergestellten Elternteils. Zur Konzeptionalisierung von ISEI siehe Fußnote 97.
} 
Vergleich der elterlichen Bildung zeigen sich dann auch deutliche Unterschiede: $40 \%$ der Eltern von Jugendlichen der zweiten Generation haben mindestens einen Abschluss einer beruflichen Schule, Realschule, Gymnasium oder Fachhochschule. Für die Eltern der ersten Generation liegt die Zahl hingegen mit 60\% um 20 Prozentpunkte höher (PISA-Konsortium 2004: 266). Die Vermutung, dass die Schlechterstellung der zweiten Generation sich großenteils durch die unterschiedliche Zusammensetzung der beiden Generationen erklären lässt, wird schließlich auch dadurch bestätigt, dass beim direkten Vergleich innerhalb der türkischen und der ex-jugoslawischen Herkunftsgruppe jeweils die zweite Generation bessere Ergebnisse erzielt als die erste (PISA-Konsortium 2004: 268, Tabelle 9.19). Damit soll aber nicht behauptet werden, dass dieser Zusammensetzungseffekt die Abweichungen vom erwarteten Generationentrend voll und ganz erklären kann. Faktoren wie Diskriminierung, nicht-materiellen Ressourcen in den jeweiligen Netzwerken und unterschiedlichen Unterstützungsleistungen kommt ebenso Bedeutung zu, wobei SpätaussiedlerInnen und jüdische Kontingentflüchtlinge in Deutschland mehr staatliche Förderung (Eingliederungshilfen, Sprachkurse) erhalten als ArbeitsmigrantInnen und MigrantInnen im Rahmen einer Familienzusammenführung, auch wenn sich dies langsam ändert.

Damit belegen die PISA-Daten aber entgegen ihrer medialen (und z.T. wissenschaftlichen) Aufbereitung insgesamt keinesfalls ein ,Abrutschen' der zweiten Generation im Vergleich zur ersten. Es handelt sich vielmehr um unterschiedliche Populationen und damit um Zusammensetzungseffekte. Wenn innerhalb der Herkunftsgruppen ein Vergleich angestellt wird, zeigen sich im Gegenteil in Übereinstimmung mit meiner Analyse des Mikrozensus und des DJIAusländersurveys Verbesserungen von der ersten zur zweiten Generation.

\section{Innerhalb der Familie: Bildungsauf- oder abstieg?}

Wie bereits erwähnt wurde, sind Verbesserungen zwischen den Migrantengenerationen nicht gleichbedeutend mit generationellem Aufstieg innerhalb der Familie. Daher sollen an dieser Stelle die intrafamiliären Entwicklungen betrachtet werden. Die Analyse erfolgt dabei mit einem stark reduzierten Datensatz, da nur die MigrantInnen der zweiten Generation untersucht werden können, die noch in einem Haushalt mit ihren Eltern leben.

In Tabelle 17 wird das ISCED-Niveau der Kinder mit dem der Väter verglichen. Fehlen die Angaben zur väterlichen Bildung, wird mit der Mutter verglichen. ${ }^{112}$

\footnotetext{
${ }^{112} \mathrm{Da}$ die väterliche Bildung im Durchschnitt höher ist, liegt damit ein härterer Indikator vor als wenn der Durchschnitt der Bildung beider Eltern herangezogen worden wäre. Ein Vergleich der Söhne mit den Vätern und der Töchter mit den Müttern erscheint unsinnig, da darüber kulturelle Annahmen verfestigt würden, laut den Migrantinnen grundsätzlich niedrigere Bildungsabschlüsse als Migranten erwerben.
} 
Tabelle 17 - Bildungsverhältnis Eltern Kinder

\begin{tabular}{|c|c|c|c|c|c|c|c|c|c|c|c|c|c|c|c|c|}
\hline & & \multicolumn{5}{|c|}{ MÄNNER UND FRAUEN } & \multicolumn{5}{|c|}{ FRAUEN } & \multicolumn{5}{|c|}{ MÄNNER } \\
\hline & & TÜRK & $\begin{array}{l}\text { ITAL, } \\
\text { GRIE, } \\
\text { SPAN, } \\
\text { PORT }\end{array}$ & EX-JUG & AUSS & WEST & TÜRK & $\begin{array}{l}\text { ITAL, } \\
\text { GRIE, } \\
\text { SPAN, } \\
\text { PORT }\end{array}$ & EX-JUG & AUSS & WEST & TÜRK & $\begin{array}{l}\text { ITAL, } \\
\text { GRIE, } \\
\text { SPAN, } \\
\text { PORT }\end{array}$ & EX-JUG & AUSS & WEST \\
\hline \multirow{3}{*}{ 苞苛 } & $\begin{array}{l}\text { ISCED } \\
\text { MigrantIn höher }\end{array}$ & $72 \%$ & $69 \%$ & $55 \%$ & $39 \%$ & $30 \%$ & $77 \%$ & $77 \%$ & $51 \%$ & $46 \%$ & $25 \%$ & $68 \%$ & $64 \%$ & $58 \%$ & $36 \%$ & $34 \%$ \\
\hline & $\begin{array}{l}\text { ISCED } \\
\text { MigrantIn gleich }\end{array}$ & $18 \%$ & $20 \%$ & $31 \%$ & $28 \%$ & $42 \%$ & $15 \%$ & $14 \%$ & $35 \%$ & $25 \%$ & $61 \%$ & $21 \%$ & $24 \%$ & $28 \%$ & $30 \%$ & $29 \%$ \\
\hline & $\begin{array}{l}\text { ISCED } \\
\text { MigrantIn niedriger }\end{array}$ & $10 \%$ & $11 \%$ & $14 \%$ & $33 \%$ & $28 \%$ & $8 \%$ & $9 \%$ & $14 \%$ & $29 \%$ & $14 \%$ & $11 \%$ & $12 \%$ & $14 \%$ & $34 \%$ & $37 \%$ \\
\hline \multirow{3}{*}{ 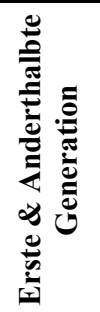 } & $\begin{array}{l}\text { ISCED } \\
\text { MigrantIn höher }\end{array}$ & $62 \%$ & $65 \%$ & $27 \%$ & $32 \%$ & $39 \%$ & $67 \%$ & $64 \%$ & $34 \%$ & $38 \%$ & - & $57 \%$ & $65 \%$ & $22 \%$ & $29 \%$ & - \\
\hline & $\begin{array}{l}\text { ISCED } \\
\text { MigrantIn gleich }\end{array}$ & $20 \%$ & $28 \%$ & $34 \%$ & $31 \%$ & $35 \%$ & $15 \%$ & $36 \%$ & $28 \%$ & $27 \%$ & - & $24 \%$ & $26 \%$ & $39 \%$ & $33 \%$ & - \\
\hline & $\begin{array}{l}\text { ISCED } \\
\text { MigrantIn niedriger }\end{array}$ & $18 \%$ & $7 \%$ & $39 \%$ & $37 \%$ & $26 \%$ & $18 \%$ & $0 \%$ & $38 \%$ & $35 \%$ & - & $19 \%$ & $9 \%$ & $39 \%$ & $38 \%$ & - \\
\hline
\end{tabular}

Mikrozensus 2005, FDZ der Statistischen Ämter des Bundes und der Länder, Eigene Berechnungen 
Der Bildungsaufstieg innerhalb der Familie fällt in der zweiten Generation stärker aus als in der ersten. Am größten ist er für die türkische Herkunftsgruppe: Dort erlangen $72 \%$ eine höhere Bildung als ihre Väter (gegebenenfalls: ihre Mütter). Auf der anderen Seite erreichen um die 30\% der MigrantInnen der zweiten Generation, deren Eltern aus ,Anwerbestaaten' kommen, keine höheren Abschlüsse als ihre Eltern. Das Bildungsniveau bleibt für diese Gruppe also von Eltern zu Kindern gleich oder verschlechtert sich sogar. Angesichts des bei den Eltern aus ,Anwerbestaaten' durchschnittlich niedrigeren Bildungsniveaus ist ein solches, Verharren' nicht unproblematisch. Im internationalen Vergleich gelingt es in anderen Ländern besser, die Kinder niedrigqualifizierter Eltern auf ein höheres Bildungsniveau zu führen (Stanat/Christensen 2006: 69). Im Vergleich von Männern und Frauen ist festzustellen, dass Frauen seltener als Männer eine niedrigere Bildung erlangen als ihre Eltern. ${ }^{113}$

Derselbe Vergleich zwischen Bildung der Eltern und der der Kinder im Ausländersurvey führt zu ähnlichen Ergebnissen und wird daher hier nicht eigens aufgeführt. Aufgrund des höheren Bildungsdurchschnitts im DJI-Sample fällt der Prozentsatz der MigrantInnen, deren Bildungsstatus sich gegenüber dem ihrer Eltern verschlechtert hat, noch geringer aus.

Diese insgesamt signifikanten Bildungsfortschritte von der Eltern- zur Kindergeneration gerade für die MigrantInnen der zweiten Generation, deren Eltern aus ,Anwerbestaaten' stammen, müssen jedoch angesichts der allgemeinen Bildungsexpansion in den 1970er Jahren im Zusammenhang mit der Entwicklung bei der Nicht-Migrantenbevölkerung betrachtet werden. Diese Bildungsexpansion hat dazu geführt, dass der Abstand zwischen MigrantInnen und Nicht-MigrantInnen sich nicht so stark verringert hat, wie man aufgrund der intergenerationalen Mobilität bei den MigrantInnen zunächst hätte annehmen können. (Kalter/Granato 2002)

\section{Bildungsergebnisse durch sozialen Status erklärbar?}

Die bisher vorgestellten Zahlen sind erstaunlich, wenn man die Vielzahl von Problemen bedenkt, vor die MigrantInnen gestellt sind: Rückstellungen bei der Einschulung, übermäßig häufiger Besuch von Sonderschulen, überdurchschnittlich viele Hauptschulempfehlungen, hohe Quoten des Sitzenbleibens und Schulabbruchs (Bildungsberichterstattung 2006: 151). So verbleiben von den Kindern mit Migrationshintergrund auf dem Gymnasium nur 77 von 100 bis zur 9. Klasse (von den Kindern ohne Migrationshintergrund 83 von 100). Auf der Realschule verbleiben $73 \%$ der Kinder mit Migrationshintergrund bis zur 9. Klasse gegenüber zu 84\% der Kinder ohne Migrationshintergrund. (Bildungsberichterstattung 2006: 152). Für

\footnotetext{
${ }^{113}$ In der ersten und anderthalbten Generation reichen die Fallzahlen in der Gruppe der westlichen MigrantInnen aus Nicht-Anwerbestaaten nicht aus, um nach Geschlechtern zu trennen.
} 
MigrantInnen ist das Risiko, eine Klasse zu wiederholen, zwei- bis dreimal so hoch wie für Kinder ohne Migrationshintergrund (Krohne, et al. 2004). Diese Negativa werden zumeist mit Migrationsspezifika erklärt. Bei Kontrolle des sozioökonomischen Status reduziert sich allerdings für die meisten Herkunftsgruppen die Wahrscheinlichkeit von Problemen auf das Maß der Nicht-MigrantInnen (Kristen 2002). Entsprechend stellt die PISA-Studie fest: „Hervorzuheben ist, dass unter Kontrolle anderer Merkmale der sozialen Herkunft der Migrationsstatus eine eher nachgeordnete Rolle bei der Vorhersage von Kompetenzunterschieden spielt.“ (PISA-Konsortium 2004: 278) In Deutschland ist jedoch der Zusammenhang zwischen sozialem Status der Eltern und Schulleistungen der Kinder besonders stark - ein Problem, das MigrantInnen und Jugendliche ohne Migrationshintergrund gleichermaßen betrifft. (PISAKonsortium 2004: 232, 273f)

Die überragende Bedeutung des sozialen Status führt u.a. dazu, dass die Variable ,alleinerziehend' bei Kontrolle des sozioökonomischen Status keinen negativen Einfluss auf die Bildungsergebnisse hat (PISA-Konsortium 2004: 228, 274, 276). In manchen Untersuchungen erlangten die Kinder von Alleinerziehenden bei Kontrolle des sozioökonomischen Status sogar bessere Bildungsergebnisse (Kristen/Granato 2005: 38).

Interessant ist schließlich, dass der soziale Status vor allem am unteren Leistungsspektrum bedeutsam wird, während im höheren Segment meritokratische Prinzipien zu gelten scheinen: So sind bei der Frage, ob eine Empfehlung für Realschule oder Gymnasium ausgesprochen wird, im Prinzip nur die Noten entscheidend. Zur Erklärung der Wahrscheinlichkeit, bei gleichen Noten eine Hauptschulempfehlung versus eine Empfehlung für eine andere Schulform zu bekommen, muss in Untersuchungen allerdings auf ,ethnische' und soziale Herkunft zurückgegriffen werden. Die Noten alleine können die größere Häufigkeit der Hauptschulempfehlung für MigrantInnen nicht erklären (Kristen 2002: 545).

Nach dieser Darstellung allgemeiner Bildungsergebnisse für MigrantInnen der zweiten Generation werden in den beiden nächsten Abschnitten zwei konkrete Teilbereiche betrachtet: zum einen die Berufsbildung, die wie kaum ein anderer Faktor über den Einstieg auf dem Arbeitsmarkt entscheidet, zum anderen Bildungsaspirationen, schulische Motivation und reale Unterstützung, also die psychologischen Hintergrundfaktoren für Erfolg in der Schule.

\subsubsection{Berufsbildung}

Im internationalen Vergleich erlangten MigrantInnen der zweiten Generation in Deutschland wegen des dualen Systems relativ gute Berufsabschlüsse (Simon 2003: 1110; Worbs 2003). 
Seitdem das duale System aber selbst kriselt, hat sich die berufliche Bildungssituation der MigrantInnen verschlechtert (BMBF 2006: 113).

In den Daten des Mikrozensus zeigen sich in der Berufsbildung Verbesserungen zwischen den Generationen und höhere Abschlüsse für deutsche StaatsbürgerInnen mit Migrationshintergrund im Vergleich zu AusländerInnen. Jedoch erlangen MigrantInnen der zweiten Generation weiterhin doppelt so häufig keinen beruflichen Abschluss wie Deutsche ohne Migrationshintergrund (Bildungsberichterstattung 2006: 148). Eine Längsschnittstudie an allen Hamburger Schulen bestätigte diese Ergebnisse: Deutsche StaatsbürgerInnen mit Migrationshintergrund erreichten höhere Berufsausbildungen als ausländische Staatsangehörige, Deutsche ohne Migrationshintergrund lagen jedoch vor beiden Gruppen. (Lehmann, et al. 2004)

Die Zahlen einer von der Bundesagentur durchgeführten Bewerberbefragung zeigen zudem, dass die Wahrscheinlichkeit, einen Ausbildungsplatz zu erhalten, für BewerberInnen mit Migrationshintergrund ${ }^{114}$ mit besseren Leistungen und einem günstigeren Umfeld steigt, jedoch konstant hinter den Chancen der BewerberInnen ohne Migrationshintergrund bleibt: So liegt die Wahrscheinlichkeit, innerhalb des ersten Jahres einen Ausbildungsplatz zu bekommen, für BewerberInnen ohne Migrationshintergrund bei $40 \%$, für MigrantInnen jedoch durchschnittlich nur bei 29\% - und zwar für MigrantInnen aus der ehemaligen Sowjetunion bei 35\% und für MigrantInnen, die in der Türkei geboren sind, bei 16\%. Die Chance auf einen Ausbildungsplatz erhöht sich bei einem mittleren Ausbildungsabschluss (bis hin zur Fachhochschulreife) auf $47 \%$ für nicht-migrantische BewerberInnen gegenüber $34 \%$ für BewerberInnen mit Migrationshintergrund. Bei guten oder sehr guten Mathematiknoten fallen die Wahrscheinlichkeiten nochmals höher aus: 64\% und 41\%. Liegt zudem die Arbeitslosenquote vor Ort unter 9\%, dann beträgt die Chance auf einen Ausbildungsplatz für Personen ohne Migrationshintergrund 71\% und für MigrantInnen 44\%. (Bildungsberichterstattung 2006: 156, Eberhard, et al. 2005, Ulrich 2006, Eberhard, et al. 2006) Dies zeigt, dass ein Zusammenhang zwischen Leistung und der Wahrscheinlichkeit für einen Ausbildungsplatz besteht, dieser für MigrantInnen aber schwächer ist als für Jugendliche ohne Migrationshintergrund. Im Umkehrschluss bedeuten diese Zahlen eine besondere Gefährdung niedrigqualifizierter MigrantInnen, langfristig vom Arbeitsmarkt ausgeschlossen zu werden: „Besonders prekär ist die Situation für Bewerber mit Migrationshintergrund, die ,nur’ über einen Hauptschulab-

\footnotetext{
${ }^{114}$ Die Gruppe der LehrstellenbewerberInnen mit Migrationshintergrund umfasst in dieser Studie alle AusländerInnen, AussiedlerInnen und Eingebürgerten und damit MigrantInnen sowohl der ersten als auch der zweiten Generation. Unter deutschen LehrstellenbewerberInnen werden diejenigen zusammengefasst, die die deutsche Staatsbürgerschaft besitzen, in Deutschland geboren sind und Deutsch als Muttersprache haben.
} 
schluss verfügen. Ihre Einmündungsquote in eine betriebliche Lehre betrug rund $25 \%$. War die letzte Mathematiknote nicht besser als ,ausreichend', reduzierte sich die Wahrscheinlichkeit auf 15\%. Und leben diese Jugendlichen in einer Region mit einer Arbeitslosenquote von neun Prozent und mehr, so waren es gerade einmal 8\%, die eine betriebliche Lehrstelle fanden“" (Ulrich/Granato 2006: 43); bei den Personen ohne Migrationshintergrund sind es doppelt so viele.

Damit klaffen für MigrantInnen, besonders am unteren Qualifikationsspektrum, Ausbildungshoffnung und Realität weit auseinander, und es besteht die Gefahr eines verfestigten Ausschlusses. So stellt Gestring et al. zufolge ein Einstieg in den Arbeitsmarkt ohne Berufsausbildung zumeist den Beginn einer ,Ausgrenzungskarriere' dar. Es folgten prekäre Beschäftigungen bei Zeitarbeitsfirmen, in randständigen Betrieben sowie lange Phasen der Arbeitslosigkeit (Gestring, et al. 2004: 9). ${ }^{115}$

\subsubsection{Motivation und Unterstützung}

Angesichts dieser gemischten Befunden im Hinblick auf die (Berufs-) Bildungssituation der MigrantInnen der zweiten Generation stellt sich die Frage, wie es mit der Motivation und der tatsächlichen Unterstützung für MigrantInnen am unteren und am oberen Qualifikationsspektrum aussieht.

Zunächst fällt auf, dass die Motivation der MigrantInnen höher oder zumindest ebenso hoch ist wie die der Nicht-MigrantInnen ist (Stanat/Christensen 2006: 8). In den PISA-Daten wies die instrumentelle Motivation für Mathematik der MigrantInnen der zweiten Generation verglichen mit der ersten Generation und mit Nicht-MigrantInnen zudem die größte Spannbreite auf, was auf eine große Diversität innerhalb der zweiten Generation hinweist (Stanat/Christensen 2006: 94). Trotz dieser hohen Motivation schätzten MigrantInnen der zweiten Generation in der PISA-Erhebung ihre Selbstwirksamkeit (,self-efficacy') geringer ein als die Nicht-MigrantInnen (Stanat/Christensen 2006: 101). Mit dem Konzept der Selbstwirksamkeit wird die Überzeugung gemessen, gewünschte Ziele unter Einsatz von Ressourcen erreichen zu können. Es wird davon ausgegangen, dass sich die angenommene Selbstwirksamkeit in tatsächliche Leistungen und Erfolge übersetzt. (Bandura 1986). In den PISADaten zeigt sich, dass die durchschnittlich angegebenen Werte der Selbstwirksamkeit nach

\footnotetext{
115 Mit dem Wandel zur Dienstleistungsgesellschaft hat die Diskriminierung gegenüber bestimmten als fremd markierten Gruppen auf dem Arbeitsmarkt zugenommen, da Angehörige der fremd markierten Gruppen seltener für Positionen mit Kundenkontakt eingestellt werden (siehe Diskussion in Kapitel 5.2).
} 
Schultypen unterschiedlich und an der Hauptschule am niedrigsten sind (Stanat/Christensen 2006: 111).

Während in den PISA-Daten die zweite Generation im Allgemeinen eine positivere Haltung gegenüber der Schule einnimmt als Nicht-MigrantInnen, gibt es auch unter ihnen eine Gruppe, die sehr negative Gefühle gegenüber der Schule hegt: 9\% der MigrantInnen der zweiten Generation stimmen (sehr) der Aussage zu, dass Schule eine Zeitverschwendung sei. Bei den deutschen SchülerInnen ohne Migrationshintergrund liegt die Zahl mit 7\% etwas niedriger. (Stanat/Christensen 2006: 106f)

Insgesamt deuten diese Zahlen entgegen üblichen Annahmen auf eine sehr hohe Motivation bei MigrantInnen der zweiten Generation hin. Gleichzeitig streuen die Werte dieser psychologischen Faktoren stärker als bei den Nicht-MigrantInnen, und es gibt ein Segment der zweiten Generation an der unteren Qualifikationsskala, welches sich abgehängt und aufgegeben fühlt, damit allerdings möglicherweise auch eine realistische Einschätzung der Situation zum Ausdruck bringt.

Wie sieht es mit der familiären Unterstützung in der Bildung aus? Bei den meisten Migranteneltern ist der Wunsch nach Bildungserfolg für ihre Kinder groß, ${ }^{116}$ wie eine Reihe von Studien zeigen; dabei bestehen Aspirationen sowohl für die Töchter als auch für die Söhne (Cavalli-Wordel 1989, Holtbrügge 1975, Neumann 1980, Rosen 1959: 78-83, Wilpert 1980). Zum Teil sind diese Erwartungen sehr hoch; die Kinder sollen das begonnene Migrationsprojekt um jeden Preis erfolgreich beenden. Nicht allen diesen MigrantInnen der zweiten Generation wird es jedoch gelingen, die von ihren Eltern anvisierten Spitzenpositionen zu erreichen. Dabei scheint der Leistungsdruck durch die Eltern umso größer zu sein, je geringer die realen Aufstiegschancen in Bildung und Beruf sind. Die gleiche Tendenz findet sich auch bei Eltern ohne Migrationshintergrund: Werden in den PISA-Daten die Eltern gemäß der Schichtzugehörigkeit in vier Quartile aufgeteilt, dann ist der Leistungsdruck gegenüber den Kindern umso höher, je niedriger das Schicht-Quartil ist. (PISA-Konsortium 2004: 243) Werden die Erwartungen der migrantischen Eltern dieses Segments enttäuscht, reagieren diese auf Misserfolge jedoch schnell mit Resignation, so das Ergebnis einer qualitativen Studie von Cavalli-Wordel. Für den schulischen Alltag bestünde kaum Interesse, und nicht wenige Eltern hätten trotz ho-

\footnotetext{
${ }^{116}$ Dem elterlichen Wunsch entspricht spiegelbildlich die hohe Wertschätzung eines guten Familienklimas auf Seiten der Kinder. So belegen mehrere qualitative Studien, wie zum Beispiel BildungsaufsteigerInnen in der zweiten Generation versuchen, trotz Reibereien und Ablösungsprozessen einen Bruch mit der Herkunftsfamilie zu vermeiden und dafür auch hohe Kosten in Kauf zu nehmen (Ofner 2003: 235, Pott 2002, Raiser 2007). Damit unterscheiden sie sich von AufsteigerInnen ohne Migrationshintergrund, denen die Aufrechterhaltung des familiären Zusammenhalts nicht so wichtig ist (Raiser 2007).
} 
her Bildungsansprüche den Eindruck, dass die Fertigkeiten, die wirklich für das Leben benötigt würden, sowieso nicht in der Schule vermittelt werden. (Cavalli-Wordel 1989: 147f) Friese stellte in seiner Untersuchung in Frankfurt fest, dass diese enttäuschten Eltern die Erziehung ihrer Kinder häufig nach außen abgeben und problematisches Verhalten der Kinder ,decken' würden. Schließlich stünden einige dieser Eltern auch in einem umgekehrten Abhängigkeitsverhältnis zu ihren Kindern. (Friese 1998)

Zusammenfassend lässt sich feststellen, dass trotz großer Bildungswünsche für die Kinder die tatsächliche Unterstützung durch die Eltern eingeschränkt ist (Karakasoglu-Aydin 2000) und für einen Teil der MigrantInnen der zweiten Generation gar übermäßiger Druck kontraproduktive Effekte zeigt. Dabei ist das System der Halbtagsschule in Deutschland stark auf Erziehungs- und Unterstützungsleistungen der Eltern angewiesen.

Wenn die Eltern als schulische UnterstützerInnen in Teilen ausfallen, bei wem holen sich die MigrantInnen der zweiten Generation dann Rat und Hilfe?

Gemäß der DESI-Studie werden 34\% der MigrantInnen der zweiten Generation bei schulischen Problemen von ihren Geschwistern unterstützt, bei den MigrantInnen türkischer Herkunft sind dies sogar 48\% (Holtappels, et al. 2007; Bildungsberichterstattung 2006: 169). Auch schulische Angebote werden, wo vorhanden, wahrgenommen: Bietet die Schule Hausaufgabenbetreuung oder zusätzliche Trainings an, so nutzen MigrantInnen aller Generationen diese häufiger als Jugendliche ohne Migrationshintergrund. Darüber hinaus sind FreundInnen für Beratung und Unterstützung wichtiger (Raiser 2007). Aspirationen der MitschülerInnen und FreundInnen werden übernommen, Informationen ausgetauscht, Lebensziele diskutiert und entwickelt. Durch diese Orientierung an FreundInnen wird die Zusammensetzung der Freundeskreise bedeutsam (Weißhaupt 1996: 60). In Deutschland kommen dabei jedoch Segregationseffekte negativ zum Tragen: 37\% der MigrantInnen der zweiten Generation gehen auf Schulen mit einem Migrantenanteil von mehr als 50\% (Stanat/Christensen 2006: 74), 7\% der SchülerInnen mit mindestens einem im Ausland geborenen Elternteil im erweiterten PISA 2003-Sample besuchen gar eine Schule, an der 75\% der SchülerInnen MigrantInnen sind (Bildungsberichterstattung 2006: H4-5web). Könnte man schon aufgrund dieser Konzentration von MigrantInnen von einem sozialen Netzwerk mit limitierten Ressourcen für soziale Mobilität in Deutschland ausgehen, so verstärkt sich der Effekt durch den durchschnittlich geringeren sozioökonomischen Status an diesen Schulen: Die PISA-Daten zeigen, dass MigrantInnen aller Generationen durchschnittlich Schulen besuchen, bei denen der sozioökonomische Status der Eltern tiefer liegt als bei den Schulen, die durchschnittlich von Nicht- 
MigrantInnen besucht werden. Der niedrigere Status an diesen Schulen betrifft dabei sowohl die Migranteneltern als auch die Eltern ohne Migrationshintergrund (Stanat/Christensen 2006: 77).

Dieses Kapitel zu Bildung und Berufsbildung hat gezeigt, dass die zweite Migrantengeneration höhere Abschlüsse erlangt als die erste. Dabei gibt es große Unterschiede zwischen den Herkunftsgruppen. Ausländische StaatsbürgerInnen erlangen zumeist niedrigere Bildungsergebnisse als deutsche StaatsbürgerInnen mit Migrationshintergrund, welche durchschnittlich nahe an die Ergebnisse von Deutschen ohne Migrationshintergrund heranreichen (oder, im Falle der, westlichen' MigrantInnen aus Nicht-Anwerbestaaten, diese überbieten). Zudem bestehen große Streuungen innerhalb der Herkunftsgruppen. Nur wenige MigrantInnen erzielen geringere Bildungsabschlüsse als ihre Eltern. Es wurde weiterhin gezeigt, dass weibliche Angehörige der zweiten Generation keinesfalls systematisch schlechtere Bildungsergebnisse erzielen als männliche Migranten. Ein nicht unerheblicher Teil der Unterschiede zwischen MigrantInnen und Nicht-MigrantInnen konnte zudem durch den durchschnittlich geringen sozioökonomischen Status der MigrantInnen (und ihrer Eltern) erklärt werden und verschwand bei Kontrolle dieser Variablen. Zudem wurde anhand einer Reihe vorliegender Studien gezeigt, dass das Bildungssystem im höheren Leistungsspektrum meritokratischer funktioniert als bei niedrigen Leistungen. Bei der Betrachtung der Berufsbildung, eines entscheidenden Kriteriums für den Zugang zum Arbeitsmarkt, wurde aber auch die Existenz eines abgehängten Segments mit sehr schlechten Chancen auf dem Lehrstellenmarkt deutlich. Und schließlich wurde gezeigt, dass Motivation und Bildungsaspirationen bei den meisten MigrantInnen hoch sind, die elterliche Unterstützung durchschnittlich aber geringer ausfällt als bei den Nicht-MigrantInnen und daher Freundschaftsnetzwerke und deren Normen und Orientierungen an Bedeutung gewinnen.

Im folgenden Kapitel wird nun der zweite Bereich des sozioökonomischen Ressourcenerwerbs, nämlich der Arbeitsmarkt, betrachtet.

\subsection{Beruf}

Wie spiegeln sich diese Bildungsergebnisse in der Situation am Arbeitsmarkt wider? In diesem Kapitel wird zunächst die Rate der Erwerbsbeteiligung am Arbeitsmarkt in der Altersgruppe von 20 bis 45 Jahren (MZ) und von 18 bis 25 Jahren (DJI) untersucht. Danach werden zwei Indikatoren vorgestellt, mit denen die Stellung auf dem Arbeitsmarkt und der Status des Berufs analysiert werden können. Anhand dieser Indikatoren werden anschließend die beruf- 
lichen Positionen der hier untersuchten Migrantengenerationen und Herkunftsgruppen anhand des Mikrozensus 2005 analysiert. Der Mikrozensus ist hierfür wegen des höheren Alterssegments und der genauen Erfassung der Erwerbsbeteiligung das bevorzugte Datenset, allerdings wird ein Vergleich zu den Ergebnissen für die jüngere Altersgruppe im DJI-Ausländersurvey gezogen. Weiterhin werden in diesem Kapitel mögliche Verbesserungen über die Generationen, inter- und intraethnische Unterschiede sowie Differenzen zwischen MigrantInnen mit deutscher und mit ausländischer Staatsbürgerschaft untersucht. Schließlich wird es um die Frage gehen, ob (Berufs-) Bildungsabschlüsse für die verschiedenen Generationen und Herkunftsgruppen unterschiedliche Renditen bringen. Abschließend werden Vor- und Nachteile einer Beschäftigung in der, ethnischen Ökonomie' untersucht.

\subsubsection{Erwerbsbeteiligung}

In der Altersgruppe von 20 bis 45 Jahren der zweiten Generation findet sich die höchste Rate der Erwerbsbeteiligung bei der spanisch-portugiesischen Herkunftsgruppe; sie liegt mit 81\% noch vor der Gruppe der Deutschen ohne Migrationshintergrund (76\%). MigrantInnen griechischer und italienischer Herkunft folgen mit 73\% und 72\%. Aber auch MigrantInnen aus ,westlichen' Nicht-Anwerbestaaten und (ex-) jugoslawischer Herkunft sowie die Kinder von AussiedlerInnen liegen nahe an diesem Satz (69 respektive 67\%). Einzig die MigrantInnen türkischer Herkunft sind nur zu 59\% am Arbeitsmarkt beteiligt. Dies erklärt sich zum Teil durch Unterschiede zwischen den Geschlechtern in den jeweiligen Herkunftsgruppen: So beteiligen nur die Hälfte aller Migrantinnen türkischer Herkunft in dieser Altersgruppe am Arbeitsmarkt, bei den Männern hingegen sind es 66\%, was jedoch immer noch der niedrigste Wert in den verschiedenen Herkunftsgruppen ist. Beinahe ebenso groß ist die Differenz zwischen Männern und Frauen italienischer Herkunft in der zweiten Generation (14 Prozentpunkte). Am geringsten fällt der Unterschied bei den Kindern von AussiedlerInnen aus: nur 3 Prozentpunkte mehr Männer als Frauen beteiligen sich am Arbeitsmarkt.

Diesen Befunden zur Erwerbsbeteiligung entsprechen die Arbeitslosenzahlen (Erwerbslose ${ }^{117}$ und arbeitssuchende Nichterwerbspersonen zusammen) inTabelle 18: Während knapp ein Fünftel der MigrantInnen der zweiten Generation türkischer Herkunft Arbeit sucht, sind dies bei den MigrantInnen spanisch-portugiesischer Herkunft und aus ,westlichen' Nicht-

\footnotetext{
${ }^{117}$ Hierunter werden nicht nur die bei der Bundesagentur für Arbeit als arbeitssuchend gemeldeten Personen verstanden sondern auch diejenigen, die angeben, dass sie weniger als eine Stunde auf dem offiziellen Arbeitsmarkt arbeiten, aber gerne mehr arbeiten wollen würden.
} 
Anwerbestaaten nur 8\%. ${ }^{118}$ Bei den Frauen liegt die Arbeitslosenquote in allen Herkunftsgruppen niedriger als bei den Männern. Dies ist teilweise durch ihre höhere Beschäftigung als Hausfrau und Mutter zu erklären. Ob eine solche Beschäftigung im privaten Bereich immer freiwillig gewählt ist oder ob sie eine rationale Wahl angesichts der schlechten Chancen auf dem offiziellen Arbeitsmarkt darstellt, kann aus diesen Zahlen nicht abgelesen werden.

In der ersten und anderthalbten Generation sind die Erwerbstätigenraten außer bei AussiedlerInnen und MigrantInnen aus, westlichen' Nicht-Anwerbestaaten geringer. Allerdings verdecken diese allgemeinen Zahlen gegensätzliche Trends für Männer und Frauen: Während bei den Frauen die Erwerbsbeteiligung von der ersten und anderthalbten Generation zur zweiten Generation steigt (mit Ausnahme der, westlichen' Herkunftsländer, wo sie beinahe gleich bleibt), sinkt sie bei den Männern (mit Ausnahme von Migranten ex-jugoslawischer Herkunft), bei MigrantInnen aus ,westlichen' Herkunftsländern sogar um 13 Prozentpunkte. Besonders auffällig ist schließlich die Differenz im Anteil der sonstigen Nichterwerbspersonen bei den Frauen zwischen der ersten und anderthalbten und der zweiten Generation. Der Prozentsatz sinkt mit Ausnahme der Aussiedlerinnen und,westlichen' Migrantinnen in allen Gruppen stark, bei den Migrantinnen türkischer Herkunft um 23 Prozentpunkte.

Damit wird deutlich, dass für die meisten Herkunftsgruppen die Erwerbsbeteiligung aufgrund der stärkeren außerhäuslichen Arbeit der Frauen in der zweiten Generation größer ist als in der ersten/anderthalbten und sich mit Ausnahme der MigrantInnen türkischer Herkunft auf einem Niveau bewegt, das relativ nah an das der Nicht-MigrantInnen heranreicht oder dieses sogar übersteigt. ${ }^{119}$

Die Daten des DJI-Ausländersurveys sind wegen des niedrigeren Durchschnittsalters nicht in gleichem Maße aussagekräftig, sie bestätigen im Trend aber die Ergebnisse des Mikrozensus und werden daher nicht gesondert vorgestellt.

\footnotetext{
${ }^{118}$ Die Statistik der Bundesagentur für Arbeit zeigt für dieses Segment zunehmend einen längeren oder sogar dauerhaften Ausschluss vom Arbeitmarkt, der zu Armut führt (Goebel, et al. 2004: 636ff).

${ }^{119}$ Historisch betrachtet war die Erwerbsbeteiligung der Migrantinnen in den 1960er, 1970er und 1980er Jahren durchgängig hoch und überstieg die der deutschen Nicht-Migrantinnen (Mattes 2005). Allerdings sind die Partizipationsraten auf dem Arbeitsmarkt bei deutschen Frauen ohne Migrationshintergrund v.a. in den jüngeren Altersgruppen in den letzten Jahren gestiegen, während sich die Chancen auf dem Arbeitsmarkt für Migrantinnen seit Anfang der 1990er Jahre verschlechtert haben.
} 
Tabelle 18 - Erwerbstätigenraten

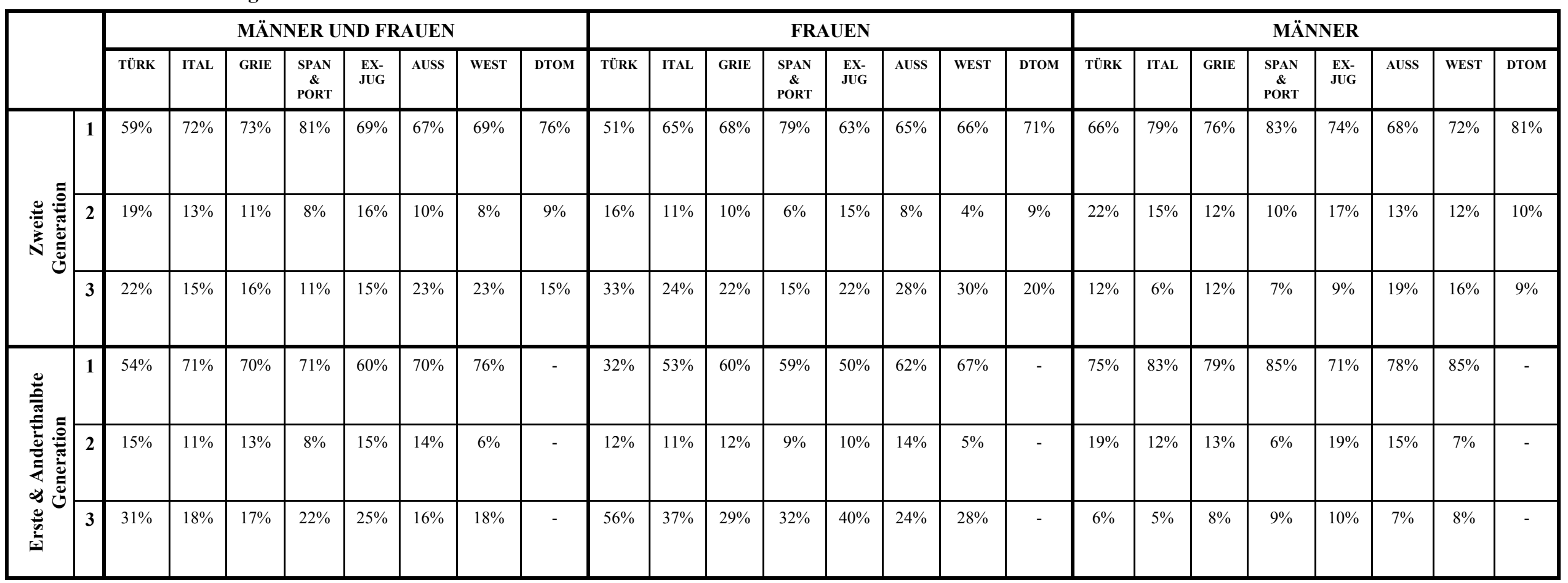

1= Erwerbstätige, 2=Erwerbslose oder Arbeitssuchende Nichterwerbspersonen, 3=Sonstige Nichterwerbspersonen (Hausfrau, in Rente)

Mikrozensus 2005, FDZ der Statistischen Ämter des Bundes und der Länder, Eigene Berechnungen 


\subsubsection{Berufliche Position und Status}

Um die Frage einer Verbesserung oder Verschlechterung der beruflichen Stellung über die Generationen zu beantworten, müssen zunächst geeignete Indikatoren für den beruflichen Status gefunden werden. Die Klassifizierung der Berufe, wie sie im Mikrozensus enthalten ist, eignet sich nämlich nicht dafür: Sie trennt die Berufe sehr detailliert mit vierstelligen Zahlen nach Wirtschaftssektoren, doch nehmen diese Zahlen keine Hierarchisierung vor und sind daher schlecht zu verwenden. Für meine Fragestellung werden deshalb zwei alternative Klassifikationsschemata benutzt: zum einen die Kategorisierung nach Blossfeld und zum anderen die Magnitude-Prestigeskala. Diese werden im Folgenden vorgestellt.

\section{Indikatoren nach Blossfeld und MPS}

Die Blossfeld-Skala konstruiert die Beschäftigungskategorien anhand der Daten der Volksund Berufszählung 1970 so, dass die in ihnen zusammengefassten Berufe hinsichtlich ihrer durchschnittlichen Bildungs- und Ausbildungsabschlüsse so homogen wie möglich sind (Blossfeld 1985: 69). ${ }^{120}$ Dadurch entstehen insgesamt zwölf Kategorien in Produktion, Dienstleistung und Verwaltung: Agrarberufe ${ }^{121}$ (AGR; typisches Beispiel: LandwirtIn), einfache manuelle Berufe (EMB; Beispiel: StraßenbauerIn), qualifizierte manuelle Berufe (QMB; Beispiele: ElektrikerIn, BuchbinderIn und SchlosserIn), TechnikerInnen (TEC; Beispiele: Bau- und VermessungstechnikerIn), IngenieurInnen (ING; Beispiele: ArchitektIn und PhysikerIn), einfache Dienste (EDI; Beispiele: GebäudereinigerIn und KellnerIn), qualifizierte Dienste (QDI, Beispiele: PolizistIn und FotografIn), Semiprofessionen (SEMI; Beispiele: KrankenpflegerIn, SozialpädagogIn und VolksschullehrerIn), Professionen (PROF; Beispiele: ÄrztIn und GymnasiallehrerIn), einfache kaufmännische Verwaltungsberufe (EVB; Beispiele: VerkäuferIn und Bürohilfskräfte), qualifizierte kaufmännische und Verwaltungsberufe (QVB; Beispiele: Großhandelskaufleute und Bankfachleute), ManagerInnen (MAN; Beispiele: UnternehmerIn und GeschäftsführerIn) (Schimpl-Neimanns 2003: 5).

Die Kategorien sind in dieser ursprünglichen Form nicht in eine Rangfolge gebracht, sondern entlang der Wirtschaftssektoren Produktion, Dienstleistung und Verwaltung aufgelistet. Da meine Analyse jedoch ein Ranking verlangt, nahm ich eine Regruppierung vor. Diese beruht

\footnotetext{
${ }^{120}$ Dies bedeutet, dass die Blossfeld-Skala im Unterschied zu anderen Indikatoren des Arbeitsmarktes nicht individuelle Bildungsvariablen einrechnet und damit eine von den konkreten Bildungsergebnissen unabhängige Einschätzung des beruflichen Status der Person erlaubt.

${ }^{121}$ Die Kategorie der Agrarberufe entfiel bei dieser Rekategorisierung, da sie Berufe mit sehr unterschiedlichem Status einschließt (familieneigene Landarbeitskräfte bis Großgrundbesitzer) und nur sehr wenige MigrantInnen in diesem Segment arbeiten. Dieser Ausschluss führte zu einem Verlust von weniger als 1\% der Fälle.
} 
auf einer Analyse der zwölf Kategorien mit dem Vollsample des Mikrozensus 1998 (SchimplNeimanns 2003: 10-14), bei der folgende Faktoren berücksichtigt wurden:

- Bildungs- und Berufsbildungsabschlüsse,

- Stellung im Betrieb und

- Einkommen.

Daraus bildete ich die folgenden Kategorien:

Kategorie 1: Erwerbslose oder arbeitssuchende Nichterwerbspersonen

Kategorie 2: EMB; EDI

Kategorie 3: QMB, EVB

Kategorie 4: QDI, TEC, QVB

Kategorie 5: SEMIPROF, MAN, ING, PROF

Diese fünf Kategorien enthalten Blossfeld-Unterkategorien, die hinsichtlich der drei genannten Kriterien so weit wie möglich homogen sind. Sie sind hierarchisch aufsteigend angeordnet.

Zusätzlich zu diesem Indikator für die berufliche Stellung wurde die Magnitude-Prestigeskala (MPS) verwendet, auf der das den Berufen zugeschriebene Prestige bewertet wird. Damit wird zusätzlich zur ,objektiven' Situation auf dem Arbeitsmarkt das gesellschaftliche Ansehen der jeweiligen Berufe gemessen, da diese beiden Faktoren gerade für die Gruppe der MigrantInnen auseinandergehen könnten. Die Magnitude-Prestigeskala wurde von Wegener entwickelt, der in einer repräsentativen Untersuchung Personen nach der sozialen Wertschätzung befragte, die sie mit verschiedenen Berufen verbinden (Wegener 1988). Aus diesen ,subjektiven' Bewertungen schuf er eine Skala, die für Regressionen genutzt werden kann. Sie reicht von einem MPS-Wert von 30 (mithelfende Landarbeitskräfte) bis zu einem Wert von 216 (ZahnärztInnen). In der empirischen Analyse verwende ich wie schon für den Bildungsindikator ISCED eine vom ZUMA entwickelte Routine, die ich an die veränderte Datenerhebung des Jahres 2005 anpasse (Frietsch/Wirth 2001).

\section{Berufsergebnisse des Mikrozensus nach Blossfeld}

Anhand der oben eingeführten Indikatoren wurde die empirische Analyse der Partizipation am Arbeitsmarkt durchgeführt, deren Ergebnisse im Folgenden dargestellt werden. Die Kreuztabelle des kategorisierten Blossfeld-Indikators getrennt nach Generationen und Herkunftsgruppen (Tabelle 19) zeigt für alle Herkunftsgruppen außer den ,westlichen' MigrantInnen aus Nicht-Anwerbestaaten eine klare Verbesserung der zweiten Generation gegenüber der ersten 
und anderthalbten Generation. Diese impliziert jedoch nicht, dass in der ersten und anderthalbten Generation alle MigrantInnen niedrig qualifiziert waren. Der durchgängig signifikante Prozentsatz von MigrantInnen der ersten und anderthalbten Generation in der höchsten Blossfeld-Kategorie belegt eine große Bandbreite in der ersten Generation. Diese Streuung vergrößert sich in der zweiten Generation: Beispielsweise ist die Hälfte der grundsätzlich erwerbstätigen MigrantInnen türkischer Herkunft entweder arbeitslos oder übt nur einfache Tätigkeiten (Kategorie EMB, EDI) aus, während sich gleichzeitig knapp ein Zehntel der zweiten Generation türkischer Herkunft in der höchsten Kategorie der (Semi-)Professionen, ManagerInnen und IngenieurInnen findet.

Hinsichtlich der Unterschiede zwischen den Herkunftsgruppen fällt auf, dass die Verteilung der MigrantInnen der zweiten Generation, deren Eltern aus Italien, Griechenland und ExJugoslawien stammen, der der MigrantInnen türkischer Herkunft ähnlich ist, der Prozentsatz der Arbeitslosen bei den MigrantInnen türkischer Herkunft den der anderen Gruppen jedoch deutlich übersteigt. Die Kinder von AussiedlerInnen und MigrantInnen spanischportugiesischer Herkunft haben eine statushöhere Verteilung. Nur, westliche' MigrantInnen aus Nicht-Anwerbestaaten und deutsche Nicht-MigrantInnen weisen eine noch höhere Blossfeld-Verteilung auf. Die Unterschiede von einem Ende des Spektrums zum anderen sind groß: Die Hälfte der MigrantInnen der zweiten Generation türkischer Herkunft partizipiert in den zwei niedrigsten Kategorien auf dem Arbeitsmarkt, gegenüber einem Viertel bei der spanischportugiesischen Herkunftsgruppe.

Geschlechterunterschiede äußern sich darin, dass Frauen in den höheren Kategorien überrepräsentiert sind. Es ist für Männer unwahrscheinlicher, in technischen Berufen, höheren Dienstleistungs- und Verwaltungsberufen sowie in den (Semi-) Professionen, in ManagerInnen- und IngenieurInnenjobs auf dem Arbeitsmarkt teilzunehmen. Dieses zunächst überraschende Ergebnis wird dadurch relativiert, dass insgesamt weniger Frauen am Arbeitsmarkt teilnehmen, da sie zu einem höheren Prozentsatz außerhalb des offiziellen Arbeitsmarktes als Hausfrau, Mutter und/oder auf dem illegalen Arbeitsmarkt (z.B. Putzen, Kranken- und Altenpflege) beschäftigt sind. 
Tabelle 19 - Berufsposition (Blossfeld): Generationen, Herkunftsgruppen, Geschlechter

\begin{tabular}{|c|c|c|c|c|c|c|c|c|c|c|c|c|c|c|c|c|c|c|c|c|c|c|c|c|c|}
\hline & & \multicolumn{8}{|c|}{ MÄNNER UND FRAUEN } & \multicolumn{8}{|c|}{ FRAUEN } & \multicolumn{8}{|c|}{ MÄNNER } \\
\hline & & $\overline{\text { TÜRK }}$ & $\overline{\text { ITAL }}$ & $\overline{\text { GRIE }}$ & $\begin{array}{c}\text { SPAN } \\
\& \\
\text { PORT }\end{array}$ & $\begin{array}{l}\text { EX- } \\
\text { JUG }\end{array}$ & $\overline{\text { AUSS }}$ & $\overline{\text { WEST }}$ & $\overline{\text { DTOM }}$ & TÜRK & $\overline{\text { ITAL }}$ & $\overline{\text { GRIE }}$ & $\begin{array}{c}\text { SPAN } \\
\& \\
\text { PORT }\end{array}$ & $\begin{array}{c}\text { EX- } \\
\text { JUG }\end{array}$ & $\overline{\text { AUSS }}$ & $\overline{\text { WEST }}$ & $\overline{\text { DTOM }}$ & $\overline{\text { TÜRK }}$ & ITAL & $\overline{\text { GRIE }}$ & $\begin{array}{c}\text { SPAN } \\
\& \\
\text { PORT }\end{array}$ & $\begin{array}{c}\text { EX- } \\
\text { JUG }\end{array}$ & $\overline{\text { AUSS }}$ & $\overline{\text { WEST }}$ & $\overline{\text { DTOM }}$ \\
\hline \multirow{5}{*}{ 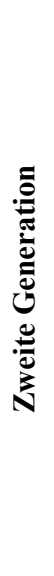 } & Arbeitslos & $24 \%$ & $14 \%$ & $15 \%$ & $9 \%$ & $18 \%$ & $13 \%$ & $9 \%$ & $10 \%$ & $20 \%$ & $12 \%$ & $13 \%$ & $6 \%$ & $16 \%$ & $10 \%$ & $5 \%$ & $10 \%$ & $26 \%$ & $17 \%$ & $17 \%$ & $11 \%$ & $18 \%$ & $16 \%$ & $12 \%$ & $11 \%$ \\
\hline & $\begin{array}{l}\text { EMB, } \\
\text { EDI }\end{array}$ & $26 \%$ & $23 \%$ & $27 \%$ & $17 \%$ & $17 \%$ & $16 \%$ & $17 \%$ & $15 \%$ & $21 \%$ & $19 \%$ & $17 \%$ & $13 \%$ & $10 \%$ & $12 \%$ & $12 \%$ & $12 \%$ & $30 \%$ & $25 \%$ & $34 \%$ & $21 \%$ & $23 \%$ & $19 \%$ & $21 \%$ & $19 \%$ \\
\hline & $\begin{array}{l}\text { QMB, } \\
\text { EVB }\end{array}$ & $21 \%$ & $25 \%$ & $20 \%$ & $19 \%$ & $24 \%$ & $20 \%$ & $18 \%$ & $20 \%$ & $17 \%$ & $14 \%$ & $19 \%$ & $17 \%$ & $21 \%$ & $11 \%$ & $11 \%$ & $15 \%$ & $24 \%$ & $33 \%$ & $22 \%$ & $20 \%$ & $27 \%$ & $26 \%$ & $23 \%$ & $24 \%$ \\
\hline & $\begin{array}{l}\text { QDI, } \\
\text { TEC, } \\
\text { QVB }\end{array}$ & $22 \%$ & $31 \%$ & $29 \%$ & $39 \%$ & $30 \%$ & $35 \%$ & $35 \%$ & $34 \%$ & $32 \%$ & $46 \%$ & $38 \%$ & $44 \%$ & $37 \%$ & $44 \%$ & $46 \%$ & $40 \%$ & $15 \%$ & $20 \%$ & $22 \%$ & $35 \%$ & $25 \%$ & $28 \%$ & $28 \%$ & $29 \%$ \\
\hline & $\begin{array}{l}\text { SEMI- } \\
\text { PROF, } \\
\text { MAN, } \\
\text { ING, } \\
\text { PROF }\end{array}$ & $7 \%$ & $7 \%$ & $9 \%$ & $16 \%$ & $11 \%$ & $16 \%$ & $21 \%$ & $21 \%$ & $10 \%$ & $9 \%$ & $13 \%$ & $20 \%$ & $16 \%$ & $23 \%$ & $26 \%$ & $23 \%$ & $5 \%$ & $5 \%$ & $5 \%$ & $13 \%$ & $7 \%$ & $11 \%$ & $16 \%$ & $17 \%$ \\
\hline \multirow{5}{*}{ 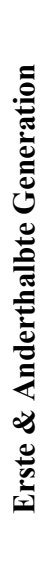 } & Arbeitslos & $21 \%$ & $14 \%$ & $15 \%$ & $8 \%$ & $19 \%$ & $16 \%$ & $7 \%$ & - & $21 \%$ & $13 \%$ & $15 \%$ & $11 \%$ & $15 \%$ & $16 \%$ & $6 \%$ & - & $21 \%$ & $15 \%$ & $14 \%$ & $7 \%$ & $22 \%$ & $16 \%$ & $8 \%$ & - \\
\hline & $\begin{array}{l}\text { EMB, } \\
\text { EDI }\end{array}$ & $47 \%$ & $38 \%$ & $49 \%$ & $46 \%$ & $40 \%$ & $32 \%$ & $14 \%$ & - & $49 \%$ & $40 \%$ & $38 \%$ & $44 \%$ & $36 \%$ & $27 \%$ & $12 \%$ & - & $47 \%$ & $36 \%$ & $55 \%$ & $47 \%$ & $43 \%$ & $37 \%$ & $16 \%$ & - \\
\hline & $\begin{array}{l}\text { QMB, } \\
\text { EVB }\end{array}$ & $22 \%$ & $24 \%$ & $20 \%$ & $20 \%$ & $22 \%$ & $22 \%$ & $13 \%$ & - & $16 \%$ & $16 \%$ & $21 \%$ & $13 \%$ & $21 \%$ & $16 \%$ & $12 \%$ & - & $24 \%$ & $28 \%$ & $20 \%$ & $25 \%$ & $23 \%$ & $29 \%$ & $14 \%$ & - \\
\hline & $\begin{array}{l}\text { QDI, } \\
\text { TEC, } \\
\text { QVB }\end{array}$ & $6 \%$ & $14 \%$ & $10 \%$ & $10 \%$ & $11 \%$ & $19 \%$ & $30 \%$ & - & $9 \%$ & $20 \%$ & $18 \%$ & $12 \%$ & $15 \%$ & $25 \%$ & $33 \%$ & - & $5 \%$ & $12 \%$ & $6 \%$ & $9 \%$ & $7 \%$ & $13 \%$ & $28 \%$ & - \\
\hline & $\begin{array}{l}\text { SEMI- } \\
\text { PROF, } \\
\text { MAN, } \\
\text { ING, } \\
\text { PROF }\end{array}$ & $4 \%$ & $10 \%$ & $6 \%$ & $16 \%$ & $8 \%$ & $11 \%$ & $36 \%$ & - & $5 \%$ & $11 \%$ & $8 \%$ & $20 \%$ & $13 \%$ & $16 \%$ & $37 \%$ & - & $3 \%$ & $9 \%$ & $5 \%$ & $12 \%$ & $5 \%$ & $5 \%$ & $34 \%$ & - \\
\hline
\end{tabular}

Mikrozensus 2005, FDZ der Statistischen Ämter des Bundes und der Länder, Eigene Berechnungen 


\section{Vergleich mit dem Ausländersurvey}

Im Ausländersurvey des DJI werden Informationen zum Beruf nicht in derselben Tiefe abgefragt wie im Mikrozensus, so dass die Anwendung des Blossfeld-Indikators oder der Magnitude-Prestigeskala hier nicht möglich ist. Aufgrund des jugendlichen Alters der Befragten sind Aussagen zum Arbeitsmarktgeschehen anhand dieser Daten allerdings ohnehin nur bedingt reliabel. Rund ein Drittel der MigrantInnen der zweiten Generation befindet sich noch in der Ausbildung, so dass für diese Gruppe keine beruflichen Angaben vorliegen. Daher werden bei den Integrationsmustern in Kapitel 4, die auf dem DJI-Ausländersurvey basieren, berufliche Angaben außer Acht gelassen. Der folgende Abschnitt belegt jedoch ähnliche Trends wie im Mikrozensus.

Die Zahlen der Tabelle 20 bestätigen, dass die zweite Generation beruflich höhere Positionen einnimmt als die erste und anderthalbte. Migrantinnen sind gemessen an ihrem höheren Anteil an den Angestellten beruflich erfolgreicher als männliche Migranten. Sie sind insbesondere seltener arbeitslos. Auch im DJI-Ausländersurvey ist dies in Teilen auf die generell geringere Erwerbstätigkeitsrate bei Frauen zurückzuführen.

Tabelle 20 - Berufsposition (Angestelltenstatus): Generationen, Herkunftsgruppen, Geschlechter

\begin{tabular}{|c|c|c|c|c|c|c|c|c|c|c|}
\hline & & \multicolumn{3}{|c|}{$\begin{array}{l}\text { MÄNNER UND } \\
\text { FRAUEN }\end{array}$} & \multicolumn{3}{|c|}{ FRAUEN } & \multicolumn{3}{|c|}{ MÄNNER } \\
\hline & & TÜRK & ITAL & GRIE & TÜRK & ITAL & GRIE & TÜRK & ITAL & GRIE \\
\hline \multirow{5}{*}{ لَّ } & Familienbetrieb & $6 \%$ & $12 \%$ & $16 \%$ & $8 \%$ & $11 \%$ & $16 \%$ & $5 \%$ & $13 \%$ & $15 \%$ \\
\hline & ArbeiterIn & $56 \%$ & $43 \%$ & $37 \%$ & $37 \%$ & $27 \%$ & $23 \%$ & $72 \%$ & $58 \%$ & $51 \%$ \\
\hline & Angestellt & $35 \%$ & $44 \%$ & $42 \%$ & $52 \%$ & $61 \%$ & $59 \%$ & $19 \%$ & $27 \%$ & $28 \%$ \\
\hline & Freiberuflich & $0 \%$ & $0 \%$ & $1 \%$ & $0 \%$ & $1 \%$ & $0 \%$ & $1 \%$ & $0 \%$ & $1 \%$ \\
\hline & Selbstständig & $3 \%$ & $1 \%$ & $4 \%$ & $3 \%$ & $0 \%$ & $2 \%$ & $3 \%$ & $2 \%$ & $5 \%$ \\
\hline \multirow{5}{*}{ 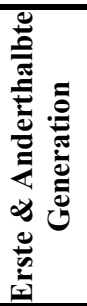 } & Familienbetrieb & $12 \%$ & $28 \%$ & $26 \%$ & $14 \%$ & $30 \%$ & $21 \%$ & $11 \%$ & $26 \%$ & $29 \%$ \\
\hline & ArbeiterIn & $65 \%$ & $40 \%$ & $47 \%$ & $55 \%$ & $28 \%$ & $39 \%$ & $71 \%$ & $48 \%$ & $52 \%$ \\
\hline & Angestellt & $18 \%$ & $30 \%$ & $23 \%$ & $31 \%$ & $42 \%$ & $38 \%$ & $11 \%$ & $22 \%$ & $14 \%$ \\
\hline & Freiberuflich & $0 \%$ & $0 \%$ & $0 \%$ & $0 \%$ & $0 \%$ & $0 \%$ & $0 \%$ & $0 \%$ & $0 \%$ \\
\hline & Selbstständig & $5 \%$ & $2 \%$ & $4 \%$ & $0 \%$ & $0 \%$ & $2 \%$ & $7 \%$ & $4 \%$ & $5 \%$ \\
\hline
\end{tabular}

Ausländersurvey 1997 des Deutschen Jugendinstituts, Eigene Berechnungen

Die Unterschiede zwischen den drei Herkunftsgruppen entsprechen den Ergebnissen des MZ: MigrantInnen italienischer und griechischer Herkunft nehmen bessere Positionen ein, wohingegen MigrantInnen türkischer Herkunft häufiger als ArbeiterInnen denn als Angestellte beschäftigt sind. In Familienbetrieben oder als Selbständige arbeiten in der zweiten Generation 9 
bis 20 Prozent - ein starker Rückgang von der ersten und anderthalbten Generation, wobei der Prozentsatz bei MigrantInnen türkischer Herkunft am niedrigsten ausfällt. Nicht signifikant ist hingegen der Prozentsatz der freiberuflich Beschäftigten. Nur bei MigrantInnen griechischer Herkunft taucht er mit 1\% überhaupt in der Statistik auf.

\section{Unterschiede bei deutscher Staatsbürgerschaft?}

Um Unterschiede zwischen MigrantInnen mit deutscher und denen mit ausländischer Staatsbürgerschaft zu erheben und die drei Generationen getrennt miteinander zu vergleichen, werden in diesem Abschnitt Regressionen mit der abhängigen Variable der modifizierten Blossfeld-Kategorien (Werte von 1 bis 5) für Herkunfts-, Generations- und Altersdummies durchgeführt. Interaktionsvariablen für Herkunft und Generation waren (mit Ausnahme der, westlichen' Herkunftsgruppe) nicht signifikant und wurden daher nicht in die Analyse eingeschlossen. Für die Regression wurde eine kombinierte Gruppe von Personen italienischer, griechischer und spanisch-portugiesischer Herkunft gebildet, da die Zahl der deutschen StaatsbürgerInnen in diesen Herkunftsgruppen zu gering ausfiel. Spalte I in Tabelle 21 führt die nichtstandardisierten Koeffizienten für alle MigrantInnen in der Altersgruppe von 20 bis 45 Jahren auf. In den nächsten Spalten ist die Analyse auf deutsche (Spalten II-IV) bzw. auf ausländische StaatsbürgerInnen beschränkt (Spalte V). Die rechte Seite der Tabelle präsentiert Durchschnittswerte abhängig vom Staatsbürgerschaftsstatus und der Generation. Die Tabelle zeigt, dass deutsche StaatsbürgerInnen mit Migrationshintergrund in der zweiten Generation auf dem Arbeitsmarkt erfolgreicher sind als ausländische StaatsbürgerInnen der zweiten Generation. Der Effekt besteht auch dann, wenn AussiedlerInnen, die qua Definition alle die deutsche Staatsbürgerschaft besitzen, aus der Analyse ausgeschlossen werden. Einzig bei ,westlichen' MigrantInnen aus Nicht-Anwerbestaaten erreichen Personen mit deutscher Staatsbürgerschaft auf der kategorisierten Blossfeld-Skala im Durchschnitt geringere Werte als Personen mit ausländischer Staatsangehörigkeit. Der Rückstand zu Deutschen ohne Migrationshintergrund verringert sich bei MigrantInnen mit deutscher Staatsbürgerschaft in der zweiten Generation erheblich, wobei MigrantInnen türkischer Herkunft die am weitesten entfernte Gruppe bleiben.

Tabelle 21weist auch den Fortschritt zwischen den Generationen nach. 
Tabelle 21 - Berufsposition (Blossfeld): Generationen, Herkunftsgruppen, Staatsbürgerschaften

\begin{tabular}{|c|c|c|c|c|c|c|c|c|c|c|}
\hline & \multirow{2}{*}{$\begin{array}{c}\text { I } \\
\text { DEUT \& AUSL, } \\
\text { INKL. AUSS \& } \\
\text { DTOM }\end{array}$} & \multirow{2}{*}{$\begin{array}{c}\text { II } \\
\text { NUR DEUT } \\
\text { INKL. AUSS } \\
\text { \& DTOM }\end{array}$} & \multirow{2}{*}{$\begin{array}{c}\text { III } \\
\text { NUR DEUT } \\
\text { INKL. AUSS } \\
\text { OHNE DTOM }\end{array}$} & \multirow{2}{*}{$\begin{array}{c}\text { IV } \\
\text { NUR DEUT } \\
\text { OHNE AUSS \& } \\
\text { DTOM }\end{array}$} & \multirow{2}{*}{$\begin{array}{c}\mathrm{V} \\
\text { NUR AUSL } \\
\text { OHNE AUSS } \\
\text { UND DTOM }\end{array}$} & & \multicolumn{2}{|c|}{$\begin{array}{c}\text { MW BLOSSFELD } \\
\text { ERSTE } \\
\text { GENERATION }\end{array}$} & \multicolumn{2}{|c|}{$\begin{array}{c}\text { MW BLOSSFELD } \\
\text { ZWEITE } \\
\text { GENERATION }\end{array}$} \\
\hline & & & & & & & $\begin{array}{c}\text { AUSL IN } \\
\text { STADT }\end{array}$ & $\begin{array}{c}\text { DEUT IN } \\
\text { STADT }\end{array}$ & $\begin{array}{c}\text { AUSL IN } \\
\text { STADT }\end{array}$ & $\begin{array}{c}\text { DEUT IN } \\
\text { STADT }\end{array}$ \\
\hline & & & & & & TÜRK & 2,1 & 2,1 & 2,5 & 2,8 \\
\hline $\begin{array}{l}\text { ITAL, GRIE, } \\
\text { SPAN \& PORT }-\end{array}$ &, $40 * *$ &, $46^{* *}$ &, $47 * *$ &, $48 * *$ &, $46^{* *}$ & $\begin{array}{l}\text { ITAL, GRIE, } \\
\text { SPAN \& PORT }\end{array}$ & 2,6 & 2,6 & 2,9 & 3,2 \\
\hline EX-JUG- &, $30 * *$ &, $28 * *$ &, $28 * *$ &, $28 * *$ &, $35^{* *}$ & EX-JUG & 2,4 & 2,4 & 2,7 & 3,0 \\
\hline WEST $\vdash$ & $1,49 * *$ &, $78 * *$ &, $80 * *$ &, $77 * *$ & $1,54 * *$ & WEST & 3,6 & 2,9 & 4,0 & 3,6 \\
\hline AUSS- &, $53^{* *}$ &, $42 * *$ &, $40^{* *}$ & -- & -- & AUSS & -- & 2,5 & -- & 3,2 \\
\hline DTOM & $1,26 * *$ & $1,20 * *$ & -- & -- & -- & DTOM & 3,4 & 3,4 & 3,4 & 3,4 \\
\hline $\begin{array}{l}\text { Zweite Generati- } \\
\text { on } \dagger\end{array}$ &, $54 * *$ &, $65^{* *}$ &, $61^{* *}$ &, $42 * *$ &, $38^{* *}$ & & & & & \\
\hline $\begin{array}{l}\text { Anderthalbte } \\
\text { Generation } \dagger\end{array}$ &, $44 * *$ &, $59 * *$ &, $56^{* *}$ &, $50 * *$ &, $11^{*}$ & & & & & \\
\hline 1976-1985 geb• & $-020 * *$ &,$- 21 * *$ &,$- 13 * *$ &,- 04 n.s. & ,01 n.s. & & & & & \\
\hline Frauen $^{\circ}$ &, $33 * *$ &, $33 * *$ &, $41 * *$ &, $29 * *$ &, $27 * *$ & & & & & \\
\hline Stadto &, $16^{* *}$ &, $17 * *$ &, $16^{* *}$ &, $11 * *$ &, $09 * *$ & & & & & \\
\hline Konstante & 1,95 & 2,02 & 1,98 & 2,17 & 2,00 & & & & & \\
\hline Korrigiertes $\mathbf{R}^{2}$ &, 06 &, 04 &, 07 & ,06 &, 18 & & & & & \\
\hline
\end{tabular}

Koeffizienten (unstandardisierte Bs) linearer Regression, abhängige Variable: Blossfeld-Indikator in 5 Stufen

$\left\ulcorner\right.$ Referenzkategorie: TÜRK; † Referenzkategorie: Erste Generation; • Referenzkategorie: 1961-1975 geboren; ${ }^{\circ}$ Referenzkategorie: Männer; a Referenzkategorie: Land/Kleinstadt

Mikrozensus 2005, FDZ der Statistischen Ämter des Bundes und der Länder, Eigene Berechnungen; ** statistisch signifikant bei p<0.01, * statistisch signifikant bei p<0.05, n.s. nicht signifikant 
Hier zeigt sich nicht nur eine Verbesserung der zweiten Generation zur ersten, sondern diese ist auch größer als zur anderthalbten Generation. Dies spricht für einen konstanten Statusgewinn über die Generationen. Nur in Spalte IV (= MigrantInnen mit deutscher Staatsbürgerschaft ohne AussiedlerInnen) fällt der Generationsdummy für die anderthalbte Generation höher aus als für die zweite. ${ }^{122}$ Schließlich sind die Durchschnitte des kategorisierten BlossfeldIndikators für die jüngere Altersgruppe leicht geringer. Dies erscheint plausibel, weil die 20bis 29-Jährigen in den meisten Fällen noch nicht so gut auf dem Arbeitsmarkt situiert sein können wie die 30- bis 45-Jährigen. StadtbewohnerInnen und Frauen erzielen durchschnittlich etwas höhere Blossfeld-Werte.

\section{Generationen- und Staatsbürgereffekte auch beim Status?}

Diese Ergebnisse für den Arbeitsmarkt bestätigen sich in der Regression mit der MagnitudePrestigeskala (MPS) als abhängiger Variable (Tabelle 22): Auch hier erreichen MigrantInnen mit deutscher Staatsbürgerschaft höhere durchschnittliche Statuswerte als MigrantInnen mit ausländischer Staatsangehörigkeit, unabhängig davon, ob AussiedlerInnen eingeschlossen werden. Eine Ausnahme bilden dabei MigrantInnen aus, westlichen' Nicht-Anwerbestaaten, bei denen ausländische Staatsangehörige höhere MPS-Durchschnitte aufweisen als deutsche StaatsbürgerInnen. ${ }^{123}$ MigrantInnen der zweiten Generation mit deutscher Staatsbürgerschaft erreichen ähnliche Werte wie Deutsche ohne Migrationshintergrund. Der größte Unterschied besteht weiterhin zu den deutschen StaatsbürgerInnen türkischer Herkunft (10 Punkte auf der MPS). Dies belegt, dass MigrantInnen, die die deutsche Staatsbürgerschaft erworben haben, hinsichtlich des Prestiges der Berufe, in denen sie beschäftigt sind, den Nicht-MigrantInnen ähnlicher sind als die MigrantInnen, die nur eine ausländische Staatsbürgerschaft besitzen. Der Anstieg der MPS-Werte von der ersten zur anderthalbten und zur zweiten Generationen ist sowohl für MigrantInnen mit deutscher als auch für jene mit ausländischer Staatsbürgerschaft signifikant. Die Angaben zum beruflichen Prestige entsprechen also den Ergebnissen zur beruflichen Positionierung der MigrantInnen. Damit gibt es keine grundsätzliche Statusverringerung für MigrantInnen, die durch die ausgeübten Berufe begründet sein könnte. Dies bedeutet jedoch nicht, dass die MigrantInnen im konkreten Fall nicht dennoch weniger berufliche Anerkennung genießen würden, nur ist dies nicht in ihrem beruflichen Status begründet.

\footnotetext{
${ }^{122}$ Allerdings gibt es in dieser Regression nur geringe Fallzahlen.

${ }^{123}$ Interaktionsvariablen für Herkunft und Generation waren wie zuvor nicht signifikant und wurden daher ausgeschlossen.
} 
Tabelle 22 - Berufsstatus (MPS): Generationen, Herkunftsgruppen, Staatsbürgerschaften

\begin{tabular}{|c|c|c|c|c|c|c|c|c|c|c|}
\hline & \multirow{2}{*}{$\begin{array}{c}\text { I } \\
\text { DEUT \& AUSL, } \\
\text { INKL. AUSS \& } \\
\text { DTOM }\end{array}$} & \multirow{2}{*}{$\begin{array}{c}\text { II } \\
\text { NUR DEUT } \\
\text { INKL. AUSS \& } \\
\text { DTOM }\end{array}$} & \multirow{2}{*}{$\begin{array}{c}\text { III } \\
\text { NUR DEUT } \\
\text { INKL. AUSS } \\
\text { OHNE DTOM }\end{array}$} & \multirow{2}{*}{$\begin{array}{c}\text { IV } \\
\text { NUR DEUT } \\
\text { OHNE AUSS \& } \\
\text { DTOM }\end{array}$} & \multirow{2}{*}{$\begin{array}{c}\text { V } \\
\text { NUR AUSL } \\
\text { OHNE AUSS } \\
\text { UND DTOM }\end{array}$} & & $\begin{array}{r}\text { M } \\
\text { ERSTE } \\
\text { NEF }\end{array}$ & $\begin{array}{l}\text { MPS GE- } \\
\text { TION }\end{array}$ & $\begin{array}{r}\text { MV } \\
\text { ZWEITE } \\
\text { NER }\end{array}$ & $\begin{array}{l}\text { MPS GE- } \\
\text { TION }\end{array}$ \\
\hline & & & & & & & $\begin{array}{c}\text { AUSL IN } \\
\text { STADT }\end{array}$ & $\begin{array}{l}\text { DEUT IN } \\
\text { STADT }\end{array}$ & $\begin{array}{l}\text { AUSL IN } \\
\text { STADT }\end{array}$ & $\begin{array}{c}\text { DEUT IN } \\
\text { STADT }\end{array}$ \\
\hline & & & & & & TÜRK & 41,9 & 42,9 & 47,1 & 54,1 \\
\hline $\begin{array}{l}\text { ITAL GRIE } \\
\text { SPAN PORT }\end{array}$ & $6,6^{* *}$ & $7,8^{* *}$ & $7,8^{* *}$ & $7,7^{* *}$ & $8,1 * *$ & $\begin{array}{l}\text { ITAL GRIE } \\
\text { SPAN PORT }\end{array}$ & 50,0 & 50,7 & 55,2 & 61,9 \\
\hline EX-JUG- & $5,0 * *$ & $6,5^{* *}$ & $6,6^{* *}$ & $6,5^{* *}$ & $5,2 * *$ & EX-JUG & 47,1 & 49,5 & 52,3 & 60,7 \\
\hline WEST - & $29,4 * *$ & $18,5^{* *}$ & $17,7^{* *}$ & $17,5^{* *}$ & $30,3 * *$ & WEST & 72,2 & 60,6 & 77,4 & 71,8 \\
\hline AUSS- & $7,9 * *$ & $5,6^{* *}$ & $4,7 * *$ & -- & -- & AUSS & -- & 47,6 & -- & 58,8 \\
\hline DTOM & $21,3 * *$ & $20,0 * *$ & -- & -- & -- & DTOM & 64,8 & 64,8 & 64,8 & 64,8 \\
\hline $\begin{array}{l}\text { Zweite Genera- } \\
\text { tion } \dagger\end{array}$ & $10,0^{* *}$ & $12,4 * *$ & $11,2 * *$ & $9,3^{* *}$ & $5,2^{* *}$ & & & & & \\
\hline $\begin{array}{l}\text { Anderthalbte } \\
\text { Generation } \uparrow\end{array}$ & $6,1^{* *}$ & $9,0^{* *}$ & $7,7^{* *}$ & $5,3^{* *}$ & $-0,52$ n.s. & & & & & \\
\hline 1976-1985 geb• & $-4,5 * *$ & $-4,8 * *$ & $-2,2 * *$ & $-1,8$ n.s. & $1,5^{* *}$ & & & & & \\
\hline Frauen $^{\circ}$ & $3,5^{* *}$ & $3,5^{* *}$ & $6,0^{* *}$ & $2,6^{* *}$ & $2,7^{* *}$ & & & & & \\
\hline Stadta & $6,8^{* *}$ & $7,0^{* *}$ & $4,3^{* *}$ & $4,6^{* *}$ & $4,2^{* *}$ & & & & & \\
\hline Konstante & $36,7 * *$ & $38,0^{* *}$ & $38,6^{* *}$ & $41,5^{* *}$ & $37,7 * *$ & & & & & \\
\hline Korrigiertes $\mathbf{R}^{2}$ &, 05 &, 04 &, 07 &, 06 & 20 & & & & & \\
\hline
\end{tabular}

Koeffizienten (unstandardisierte Bs) linearer Regression, abhängige Variable: Magnitude Prestigeskala (MPS)

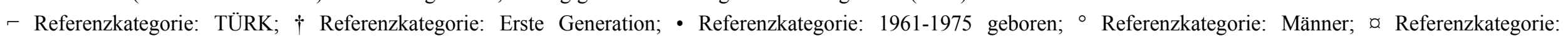
Land/Kleinstadt

Mikrozensus 2005, FDZ der Statistischen Ämter des Bundes und der Länder, Eigene Berechnungen; ** statistisch signifikant bei p<0.01, * statistisch signifikant bei $p<0.05$, n.s. nicht signifikant 


\section{Konzentrierte Unterschichtung oder breite Streuung im Berufsleben?}

In der Diskussion über die Arbeitsmarktbeteiligung der zweiten Generation wird regelmäßig behauptet, dass diese MigrantInnen sich in einigen wenigen Segmenten des Arbeitsmarktes konzentrierten, die geringes soziales Ansehen mit schlechter Bezahlung und fehlenden Aufstiegsmöglichkeiten kombinierten (von Loeffelholz/Hernold 2001). Tabelle 23 und Tabelle 24 gehen dieser Frage nach, indem sie die Streuung der durchschnittlichen MPS-Werte analysieren. Tabelle 23 zeigt, dass neben dem Mittelwert und dem Median auch der Interquartilwert über die Generationen steigt. Beim Interquartilwert wird das Spektrum der geordneten Durchschnittswerte in vier Quartile zerteilt und dann der Wert des ersten Quartils von dem des dritten Quartils abgezogen. Der Wert gibt somit die Streuung der MPS-Werte für die $50 \%$ des Samples an, die in der Mitte liegen. Während der Interquartilwert für MigrantInnen der ersten Generation hinter dem der deutschen Nicht-MigrantInnen um 4 Punkte zurückbleibt, verringert sich dieser Rückstand für die zweite Generation auf einen einzigen Punkt auf der Magnitude-Prestigeskala. Die mittleren 50\% der zweiten Generation sind also in Tätigkeiten beschäftigt, deren Prestige erheblich variiert. Sie konzentrieren sich nicht auf wenige Beschäftigungssegmente. Das Prestige ihrer Jobs streut vielmehr ähnlich wie bei den Deutschen ohne Migrationshintergrund, wenngleich auf einem niedrigeren Niveau.

Tabelle 23 - Streuung Berufsstatus (MPS): Generationen

\begin{tabular}{|l|c|c|c|c|}
\hline & $\begin{array}{c}\text { ZWEITE } \\
\text { GENERATION }\end{array}$ & $\begin{array}{c}\text { ANDERTHALBTE } \\
\text { GENERATION }\end{array}$ & $\begin{array}{c}\text { ERSTE } \\
\text { GENERATION }\end{array}$ & DTOM \\
\hline Mittelwert & 55,2 & 52,0 & 52,0 & 61,5 \\
\hline Median & 51,0 & 50,2 & 44,4 & 60,4 \\
\hline Interquartil & 24 & 22 & 26 & 25 \\
\hline
\end{tabular}

Mikrozensus 2005, FDZ der Statistischen Ämter des Bundes und der Länder, Eigene Berechnungen

Nun könnte man annehmen, dass diese Vielfalt durch die unterschiedlichen Herkunftsgruppen begründet ist, die in den Generationen vereinigt sind. Tabelle 24 zeigt jedoch, dass die Spannbreiten auch innerhalb der Herkunftsgruppen groß sind, wobei der Interquartilwert für die zweite Generation türkischer Herkunft nach der, westlichen' Herkunftsgruppe der größte ist (24 MPS-Punkte) und dem Wert der deutschen Nicht-MigrantInnen (25 MPS-Punkte) am nächsten kommt. Ein Vergleich der ersten und anderthalbten Generation (rechte Seite der Tabelle) mit der zweiten Generation (linke Seite), zeigt, dass außer bei den AussiedlerInnen und den ,westlichen' MigrantInnen aus Nicht-Anwerbestaaten die Bandbreiten über die Generationen ansteigen. 
Tabelle 24 - Streuung Berufsstatus (MPS): Herkunftsgruppen und Generationen

\begin{tabular}{|c|c|c|c|c|c|c|}
\hline & & TÜRK & $\begin{array}{l}\text { ITAL, GRIE, } \\
\text { SPAN \& PORT }\end{array}$ & EX-JUG & AUSS & WEST \\
\hline \multirow{3}{*}{ 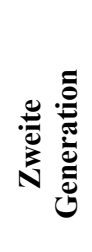 } & Mittelwert & 51,2 & 56,0 & 56,8 & 58,8 & 63,1 \\
\hline & Median & 48 & 51,5 & 51,5 & 53,9 & 60,4 \\
\hline & Interquartil & 24,5 & 20,2 & 19,3 & 21,3 & 33,2 \\
\hline \multirow{3}{*}{ 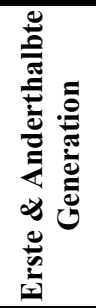 } & Mittelwert & 42,6 & 50,3 & 46,6 & 49,6 & 72,9 \\
\hline & Median & 37,1 & 43,6 & 41,1 & 44,2 & 69,1 \\
\hline & Interquartil & 16,6 & 18,8 & 16,5 & 25,3 & 37,2 \\
\hline
\end{tabular}

Mikrozensus 2005, FDZ der Statistischen Ämter des Bundes und der Länder, Eigene Berechnungen

\subsubsection{Unterschiedliche Rendite für Bildung?}

Im vorigen Kapitel wurde gezeigt, dass trotz des Fortschritts über die Generationen und einer Besserstellung deutscher StaatsbürgerInnen die Bildungsabschnitte von MigrantInnen in den meisten Herkunftsgruppen unter dem Durchschnitt der formalen Abschlüsse der NichtMigrantenbevölkerung bleiben. Wenn sich nun, wie oben gezeigt wurde, bei der beruflichen Position im Großen und Ganzen ein ähnliches Bild ergibt, dann stellt sich die Frage, ob die durchschnittlich niedrigere Position auf dem Arbeitsmarkt durch eine geringere Ausstattung mit Bildungsabschlüssen zufriedenstellend erklärt werden kann oder ob weitere Faktoren hinzugezogen werden müssen. Eine weitere Frage ist, ob sich die Rentabilität von Bildung in den verschiedenen Generationen unterscheidet.

Marc Szydliks Untersuchung mit den Daten des SOEP zeigt, dass AusländerInnen ${ }^{124}$ der zweiten Generation bessere Chancen haben, ihre Ausbildung mit der ausgeübten Tätigkeit in Übereinstimmung zu bringen, als die der ersten Generation. So nimmt der Anteil derjenigen, die für den ausgeübten Jobs überqualifiziert sind, in der zweiten Generation stark ab. Er bleibt aber dennoch über dem Niveau der Deutschen ohne Migrationshintergrund. Für diese unter ihrem Qualifikationsniveau beschäftigten MigrantInnen der zweiten Generation gilt jedoch, dass sich die Einkommenseinbußen durch die nicht angemessene Beschäftigung im Vergleich zur ersten Generation der AusländerInnen ebenfalls verringern. Bildung lohnt sich also für die

\footnotetext{
${ }^{124}$ MigrantInnen mit deutscher Staatsangehörigkeit sind nicht Teil von Szydliks Untersuchung.
} 
zweite Generation mehr als für die erste, bringt für sie allerdings immer noch geringere Erträge als für Deutsche ohne Migrationshintergrund. (Szydlik 1996: 668, 673)

Während Szydlik nicht nach Herkunftsgruppen trennt, untersuchen Granato und Kalter mit den Daten des Mikrozensus 1996 die unterschiedlichen Wahrscheinlichkeiten für eine Angestellten- versus eine Arbeiterposition bei den ausländischen StaatsbürgerInnen getrennt nach Nationalitäten. Bei Kontrolle des Bildungsniveaus finden sie nur für die Gruppe der türkischen Staatsangehörigen eine signifikant geringere Chance auf eine Angestelltenposition. Wird speziell die Wahrscheinlichkeit betrachtet, Führungsaufgaben zu übernehmen, so haben türkische und jugoslawische ArbeiterInnen niedrigere Chancen. Unterschiede im Bildungsniveau können dies wohlgemerkt nicht erklären, da sie kontrolliert werden. (Granato/Kalter 2001) Zu ähnlichen Ergebnissen kommt auch Granato (2003), die die Wahrscheinlichkeit untersucht, physisch wenig belastende Jobs im Gegensatz zu einer ,Knochenarbeit' zu bekommen. Auch hier ist bei Kontrolle der Faktoren Bildung, Geschlecht und Berufserfahrung die Zugehörigkeit zu einer Herkunftsgruppe in der zweiten Generation nicht mehr signifikant. Nur für MigrantInnen der türkischen Herkunftsgruppe und in geringerem Maße auch der osteuropäischen Herkunftsgruppe ergeben sich auch bei Kontrolle dieser Faktoren negative Effekte (Granato 2003: 129f).

Dieser Befund wird von Kalter (2004) bestätigt, der ebenfalls die Wahrscheinlichkeit untersucht, eine Angestellten- im Gegensatz zu einer Arbeiterposition einzunehmen. Er vergleicht drei Gruppen: deutsche StaatsbürgerInnen, türkische StaatsbürgerInnen (Personen türkischer Herkunft mit deutscher oder doppelter Staatsbürgerschaft werden ausgeschlossen) und eine kombinierte Gruppe von MigrantInnen mit ausländischer Staatsangehörigkeit, deren Eltern aus anderen Anwerbestaaten stammen. Wie in den oben genannten Untersuchungen verschwinden auch bei Kalter bei Kontrolle der Bildung die negativen Arbeitsmarkteffekte. Nur bei türkischen StaatsbürgerInnen bleiben diese bestehen und lassen sich auch nicht durch den sozioökonomischen Hintergrund des Vaters erklären. Erst wenn der Prozentsatz der Deutschen ohne Migrationshintergrund unter den drei besten FreundInnen in die Regression eingerechnet wird, verfügen TürkInnen über die gleiche Wahrscheinlichkeit auf eine Angestelltenposition wie Angehörige der anderen ,Gastarbeitergruppen'. Dieser gewichtige Effekt des Prozentsatzes der deutschen FreundInnen für die berufliche Position (Angestellte(r) versus ArbeiterIn) bleibt auch bei Kontrolle der Sprachkenntnisse und des Freundesnetzwerkes des Vaters bestehen. (Kalter 2004) Diese Frage nach den Auswirkungen von ,ko-ethnischen' oder gemischten Freundschaftsnetzwerken wird im nächsten Kapitel noch ausführlicher diskutiert. 
Insgesamt zahlt sich also Bildung in der zweiten Generation stärker aus als in der ersten. Allerdings bringt sie auch in der zweiten Generation nicht für alle Herkunftsgruppen die gleiche Rendite wie für Deutsche ohne Migrationshintergrund. Diese Benachteiligung betrifft besonders MigrantInnen türkischer Herkunft. Als eine mögliche Erklärung dieser Schlechterstellung wird manchmal eine verstärkte Teilhabe der MigrantInnen an einer ,ethnischen Ökonomie' herangezogen. Der nächste Abschnitt stellt daher die Frage nach den Wirkungen einer ,ethnischen Ökonomie'.

\subsection{4 ,Ethnische Ökonomie’}

Unter dem Begriff der ,ethnischen Ökonomie' werden unterschiedliche Dinge verstanden: Sie kann darüber bestimmt werden, dass ArbeitgeberInnen und Angestellte der gleichen Herkunftsgruppe angehören (Bonacich/Modell 1980). Es können aber auch nur der KollegInnenkreis (Zhou/Logan 1989) und/oder die KundInnen der gleichen Herkunftsgruppe angehören. Weiterhin können Familienbetriebe von MigrantInnen als ,ethnische Ökonomie’ verstanden werden, besonders wenn bei der Arbeit die Sprache des Herkunftslandes gesprochen wird und mögliche KundInnen und ZuliefererInnen diese ebenfalls verwenden. Schließlich kann eine ,ethnische Ökonomie' durch das Feld des Wirtschaftens selbst bestimmt sein, der Begriff kann also beispielsweise Unternehmen bezeichnen, die im Import/Export-Geschäft mit dem Herkunftsland arbeiten oder als ,ethnisch' markierte Dienstleistungen anbieten (z.B. Hamam oder griechisches Restaurant) (Portes/Bach 1985). ${ }^{125}$ Der Begriff , ethnische Ökonomie’ deckt also ein breites Spektrum unterschiedlicher wirtschaftlicher Unternehmungen ab; insofern ist es nicht verwunderlich, dass über die Wirkung dieser ,ethnischen Ökonomie' Uneinigkeit besteht.

Welche empirischen Ergebnisse gibt es nun zu dieser ,ethnischen Ökonomie' in Deutschland? Informationen hierzu sind im Unterschied zu den USA nur spärlich vorhanden, möglicherweise weil lange Zeit nicht anerkannt wurde, dass MigrantInnen sich dauerhaft in Deutschland aufhalten und sich hier eine wirtschaftliche Zukunft aufbauen. Darüber hinaus war AusländerInnen lange Zeit die Selbstständigkeit verschlossen (Zimmermann/Hinte 2004: 146ff). Die

\footnotetext{
${ }^{125}$ Selbstverständlich ist die deutsche Wirtschaft größtenteils ebenfalls eine, ethnische Ökonomie', bei der Menschen, die der deutschen Herkunftsgruppe angehören, mit anderen Deutschen handeln, Dinge produzieren oder füreinander Dienstleistungen anbieten. In diesem Fall spricht jedoch niemand von einer ,ethnischen Ökonomie', da diese Form des Wirtschaftens als ,normal' und nur die der Zuwanderer als ,anders' markiert wird. Korrekter wäre es deshalb, den Begriff z.B. durch Migrantenwirtschaft zu ersetzen. Dagegen spricht allerdings, dass dadurch zum einen der Migrantenstatus festgeschrieben würde und zum anderen die verschiedenen Herkunftsgruppen als vermeintlich homogene Gruppe von MigrantInnen behandelt würden. Daher entscheide ich mich in dieser Arbeit für die Beibehaltung des Begriffs.
} 
folgenden Zahlen trennen daher leider auch nicht nach den verschiedenen Migrantengenerationen.

Die meisten vorliegenden Untersuchungen befassen sich mit der größten Gruppe von MigrantInnen, den türkischen ZuwanderInnen und ihren Kindern. Die Zahl der türkischen Selbstständigen hat sich allein in Berlin von $22.000 \mathrm{im} \mathrm{Jahr} 1985$ auf $59.500 \mathrm{im}$ Jahr 2000 erhöht (Sen/Sauer 2005: 5). Von den Selbstständigen türkischer Herkunft hatten im Jahr 2005 knapp die Hälfte die deutsche Staatsbürgerschaft (Sen/Sauer 2005: 11). Die Selbstständigen türkischer Herkunft in Berlin verteilen sich folgendermaßen auf die Branchen: Einzelhandel 26\%, Dienstleistung 24\%, Gastronomie 19\%, handwerksähnliche Betriebe 10\%, Großhandel 8\%, Handwerk 6\%, Baugewerbe 5\% und verarbeitendes Gewerbe 2\%. Diese Aufteilung entspricht der Situation auf Bundesebene. (Sen/Sauer 2005: 23, ZfT 2003)

Hinsichtlich der KundInnen zeigt sich eine große Vielfalt: Fast drei Viertel der von Sen und den WissenschaftlerInnen des Zentrums für Türkeistudien untersuchten Unternehmen in Berlin hatten entweder hauptsächlich nicht-migrantische KundInnen oder aber gleichermaßen nicht-migrantische und migrantische KundInnen türkischer Herkunft. Der Kreis der LieferantInnen ist noch stärker gemischt. Hinsichtlich der MitarbeiterInnen - rund vier Fünftel der UnternehmerInnen türkischer Herkunft haben MitarbeiterInnen eingestellt - zeigt sich ebenfalls eine Pluralität: Bei 62\% der Unternehmen sind alle MitarbeiterInnen türkischer Herkunft, bei weiteren $22 \%$ sind sie türkischer und anderer Herkunft, und 11\% der Unternehmen beschäftigen ausschließlich Deutsche ohne Migrationshintergrund. (Sen/Sauer 2005: 26-33) Die Zahlen schwanken jedoch nach Branche: Für den Nahrungsmittelsektor in Berlin (alle Herkunftsgruppen zusammen) hat Hillmann gezeigt, dass vier Fünftel der Beschäftigten Familienangehörige sind (Hillmann 2001: 201).

Auch bei der Größe der Unternehmen wird die gesamte Bandbreite abgedeckt: So gehört die Hälfte der türkischen Unternehmen in Berlin zu den Kleinst- oder Familienbetrieben, die andere Hälfte zu mittleren oder Großbetrieben (Sen/Sauer 2005: 31). Für die UnternehmerInnen aller Herkunftsgruppen fand Pütz heraus, dass im Jahr 2002 jeder fünfte Betrieb ein Mehrbetriebsunternehmen war und eine erfolgreiche Expansion hinter sich hatte. Auf der anderen Seite war jeder siebte ,ethnische' Betrieb in Berlin ein Ein-Personen-Unternehmen. Auch mit Blick auf die notwendigen Qualifikationen ergab sich eine große Bandbreite: „Nahezu jeder achte Selbstständige führte ein Unternehmen, das entweder hohen Kapitaleinsatz erfordert [...] oder aber hohe Vorqualifikationen - hierzu zählen wissensintensive Dienstleistungen wie Unternehmensberatungen oder Kanzleien.“ (Pütz 2005: 202) 
Schließlich herrscht auch beim Einkommen eine breite Streuung (Özcan/Seifert o.J.). Die Annahme klassischer Integrationstheorien, dass sich in einer ,ethnischen' Selbstständigkeit nur jene wiederfinden, die auf dem, nicht-ethnischen' Arbeitsmarkt gescheitert sind, kann nicht bestätigt werden. Dennoch gibt es selbstverständlich einen erheblichen Prozentsatz von UnternehmerInnen, die die Niedrigeinkommensschwelle nicht überschreiten. Er liegt nach den Zahlen des SOEP bei ausländischen Staatsangehörigen oder im Ausland Geborenen bei 31\%, wohingegen nur ein Fünftel der deutschen Selbstständigen ohne Migrationshintergrund ein Einkommen unter dieser Schwelle bezieht (Tucci 2005: 188).

Nachdem diese aggregierten Fakten eine große Vielfalt hinsichtlich der Branchen-, Kunden-, Lieferanten- und Mitarbeiterstruktur sowie der Größe und der notwendigen Qualifikation gezeigt haben, wird nun der Wirkungsfrage nachgegangen: Bietet die ,ethnische Ökonomie' Aufstiegsmöglichkeiten oder ist sie eine ,Statusfalle'?

Dafür werden zunächst die Ergebnisse einer Regression jeweils mit den Daten des Mikrozensus und des DJI-Ausländersurveys vorgestellt, die untersucht, welche Konsequenzen eine Beschäftigung in der, ethnischen Ökonomie' gegenüber einer Beschäftigung als ArbeiterIn oder Angestellte(r) hat. Die ,ethnische Ökonomie' wurde dabei als Selbstständigkeit von MigrantInnen der zweiten Generation oder Arbeit als mithelfende(r) Familienangehörige(r) operationalisiert. Tabelle 25 zeigt mit den Daten des Mikrozensus einen positiven Effekt einer Beschäftigung in der ,ethnischen Ökonomie' gegenüber einer Beschäftigung als ArbeiterIn und zwar um eine gute halbe Stufe auf einer Zehner-Skala des persönlichen Nettoeinkommens, wobei das Bildungsniveau, die Zugehörigkeit zu einer Herkunftsgruppe und das Geschlecht kontrolliert werden. Dagegen fällt der positive Einkommenseffekt für eine Angestelltenposition im Vergleich zur Arbeiterposition geringer aus $(\mathrm{B}=0.3)$. MigrantInnen der zweiten Generation türkischer, italienischer, griechischer und spanischer-portugiesischer Herkunft unterscheiden sich hierbei nicht. Frauen haben durchschnittlich ein niedrigeres Nettoeinkommen, 30 bis 45 Jährige im Vergleich zu den 20 bis 29 Jährigen ein höheres. Bei gleicher Bildung und unter Kontrolle der Herkunftsgruppe, des Geschlechts und Alters ist also eine Tätigkeit in der, ethnischen Ökonomie' lukrativer als ein Arbeiter- oder Angestelltenverhältnis. 
Tabelle 25 - Auswirkung, ethnische Ökonomie' auf Einkommen, MZ

\begin{tabular}{|l|c|}
\hline & B (S.F.) \\
\hline Bildung (ISCED in 5 Stufen) &, $55^{* *}(, 04)$ \\
ITAL GRIE SPAN PORT- &, 001 n.s. $(, 10)$ \\
EX-JUG- &, $416^{* *}(, 12)$ \\
WEST- &, $42^{*}(, 23)$ \\
AUSS- &,$- 31^{* *}(, 11)$ \\
1961-1975 geb• & $2,136^{* *}(, 08)$ \\
Frauen ${ }^{\circ}$ & $-2,12^{* *}(, 08)$ \\
Selbstständig/Familienbetrieba &, $57 * *(, 16)$ \\
Angestellte(r) $\boldsymbol{Q}$ &, $30^{* *}(, 09)$ \\
\hline Konstante & $4,84(, 13)$ \\
Korrigiertes $\mathbf{R}^{\mathbf{2}}$ &, 28 \\
\hline
\end{tabular}

Koeffizienten (B) linearer Regression, abhängige Variable: Persönliches Nettoeinkommen (10-Skala), nur zweite Generation

$\left\ulcorner\right.$ Referenzkategorie: TÜRK; • Referenzkategorie: 1976-1985 geboren; ${ }^{\circ}$ Referenzkategorie: Männer; ‘ Referenzkategorie: ArbeiterIn

** statistisch signifikant bei $\mathrm{p}<0.01, *$ statistisch signifikant bei $\mathrm{p}<0.05$, n.s. nicht signifikant

Mikrozensus 2005, FDZ der Statistischen Ämter des Bundes und der Länder, Eigene Berechnungen

Diese Ergebnisse bestätigen sich in der Regression mit den Daten des DJI-Ausländersurveys (Tabelle 26): Auch hier haben Selbstständige oder mithelfende Familienangehörige ein signifikant höheres Einkommen ${ }^{126}$ als ArbeiterInnen. Die fehlende Signifikanz des Dummies für höhere Bildung mag an dem niedrigeren Alter der MigrantInnen der zweiten Generation (18 bis 25 Jahre) liegen, da sich Verdienstunterschiede durch unterschiedliche Bildung zumeist erst im höheren Alter zeigen. Sehr gute Sprachkenntnisse (in Deutsch oder in der Herkunftssprache der Eltern) waren in der Regression nicht signifikant und werden daher nicht extra ausgewiesen.

Zusammengefasst lässt sich also in beiden Datensätzen ein positiver Effekt einer Beschäftigung in der ,ethnischen Ökonomie' auf das Einkommen finden. Welche weiteren Vorteile bietet die ,ethnische Ökonomie' mit ihrer engen Verbindung zur ,ethnischen Gemeinschaft' den Selbstständigen?

\footnotetext{
${ }^{126}$ Allerdings wird im Ausländersurvey im Gegensatz zum Mikrozensus das persönliche Nettoeinkommen nicht erfragt. Daher wird das Familieneinkommen herangezogen.
} 
Tabelle 26 - Auswirkung, ethnische Ökonomie' auf Einkommen, DJI-Ausländersurvey

\begin{tabular}{|l|c|}
\hline & B (S.F.) \\
\hline Bildung (ISCED $\geq$ 3) $\dagger$ & 233 n.s. (194) \\
ITAL- & 243 n.s. (181) \\
GRIE- & $472^{* *}(191)$ \\
Alter: 23-26 Jahre• & $258^{* *}(167)$ \\
Frauen ${ }^{\circ}$ & $409^{* *}(173)$ \\
Selbstständig/Familienbetrieba & $868^{* *}(262)$ \\
Freiberuflich $\propto$ & 186 n.s. (833) \\
Angestellte(r) $\propto$ & -23 n.s. (189) \\
\hline Konstante & 2471 (228) \\
Korrigiertes $\mathbf{R}^{2}$ &, 06 \\
\hline
\end{tabular}

Koeffizienten (B) linearer Regression, abhängige Variable: Haushaltsnettoeinkommen (in DM), nur zweite Generation

$\dagger$ Referenzkategorie: Bildung (ISCED < 3); - Referenzkategorie: TÜRK; • Referenzkategorie: 18-22 Jahre;

○ Referenzkategorie: Männer; $\propto$ Referenzkategorie: ArbeiterIn

** statistisch signifikant bei $\mathrm{p}<0.01, *$ statistisch signifikant bei $\mathrm{p}<0.05$, n.s. nicht signifikant

Ausländersurvey 1997 des Deutschen Jugendinstituts, Eigene Berechnungen

Pecoud stellt in seiner Untersuchung der Berliner Selbstständigen türkischer Herkunft fest, dass die Mehrzahl der kleinen LadenbesitzerInnen ihr Geschäft nicht ohne finanzielle Hilfe des deutsch-türkischen Netzwerkes hätten eröffnen können. Selbstständigkeit garantiert, solange das Geschäft gut läuft, eine sichere Beschäftigung und bietet in vielen Fällen Ausbildungsplätze für Jugendliche aus einer herkunftsgruppenbezogenen Gemeinschaft. Zudem fließt nicht selten Know How und Arbeitskraft verschiedener Familienmitglieder in das Geschäft und hilft über schwierige Phasen hinweg. Diese Solidarität hat in den Augen der Interviewten allerdings eine Kehrseite: So finden sie es schwieriger, ,ko-ethnische' MitarbeiterInnen zu entlassen. Zudem verfügt die Kundschaft im ,ethnischen’ Kiez über beschränkte Kaufkraft, so dass es aus unternehmerischer Sicht nötig werde den Absprung in reichere Stadtviertel, in denen v.a. Personen ohne Migrationshintergrund leben, zu schaffen. (Pecoud 2004)

Wie sieht es nun mit immateriellen Vorzügen einer Beschäftigung in der ,ethnischen Ökonomie' aus? Das Projekt Ethnogeneration an der Universität Frankfurt führte Interviews mit den über 16-jährigen Kindern von migrantischen Selbstständigen sowie mit ihren LehrerInnen durch. Danach verbringen die Kinder mangels anderer Betreuungsmöglichkeiten schon von klein auf viel Zeit im Geschäft ihrer Eltern, wo sie in Maßen am Geschäftsablauf teilnehmen. Sie lernen, soziale Situationen zu durchschauen, und entwickeln Strategien für Konfliktsituationen. Dieses praktische Lernen wirkt sich auf die Beziehung zwischen Eltern und Kind aus: 
„Die Autorität der Eltern gegenüber den Kindern stärkt sich durch das Berufswissen, das weiter gegeben wird.“ (Kontos 2005: 214, 216) Aber auch das Selbstwertgefühl der Kinder wächst, indem sie sich mit dem elterlichen Betrieb identifizieren. Wurde der Betrieb erst spät, während der Adoleszenz der MigrantInnen der zweiten Generation gegründet, verändert sich die Eltern-Kind-Beziehung häufig hin zu einem partnerschaftlichen Verhältnis, die Familie wird über die neue Aufgabe des Unternehmens gestärkt. (Kontos 2005: 216) Abgesehen davon, dass manchmal auch die vermeintliche Unprofessionalität der Eltern als peinlich erlebt wird, sind dies wichtige immaterielle Vorteile.

Die LehrerInnen schätzen die untersuchten Kinder als überdurchschnittlich erfolgreich ein, was sich auch in einem überproportional häufigen Besuch von weiterführenden Schulen zeigt. Für ihre Kinder erhoffen sich die selbstständigen Migranteneltern eine bessere berufliche Position als die eigene, die Weitergabe des Betriebes wird zumeist nicht angestrebt, daher kommt es diesbezüglich kaum zum Konflikt. (Kontos 2005: 215) Die Ergebnisse im Rahmen des Projektes Ethnogeneration widersprechen somit der These einer Mobilitätsfalle: „Die Migrantenfamilie in der ethnischen Ökonomie ist eher ein Feld, in dem soziale Aufstiegsprozesse vorbereitet werden.“(Kontos 2005: 218)

Die Analyse der Partizipation auf dem Arbeitsmarkt für MigrantInnen zeigt - sowohl gemessen am kategorisierten Blossfeld als auch der Magnitude-Prestigeskala - eine deutliche Verbesserung der zweiten Generation im Vergleich zur ersten und anderthalbten. Nur bei ,westlichen' MigrantInnen aus Nicht-Anwerbestaaten verfügt die erste Generation über einen höheren Status als die zweite. MigrantInnen mit deutscher Staatsbürgerschaft erlangen durchschnittlich bessere Positionen auf dem Arbeitsmarkt als ausländische MigrantInnen. Neben der Variation zwischen den Herkunftsgruppen ist auch die Streuung der Durchschnittswerte innerhalb der Herkunftsgruppen hoch und deutet auf eine große Diversität in der zweiten Generation hin. Migrantinnen, die am Arbeitsmarkt teilnehmen, tun dies überproportional häufig in den Sektoren Technik, qualifizierte Dienste und Verwaltung sowie in (semi-) professionellen, Managerinnen- und Ingenieurinnenjobs. Daneben existiert jedoch auch ein Segment mit geringer Qualifikation, das langfristig vom Arbeitsmarkt ausgeschlossen ist. Die Einnahme einer solch risikoreichen Position hat über den beruflichen Sektor hinaus weitreichende Folgen für die gesellschaftliche Teilhabe. Obwohl sich also Bildungsinvestitionen in der zweiten Generation insgesamt wesentlich stärker rentieren als in der ersten und anderthalbten, bleiben Unterschiede zwischen Deutschen ohne Migrationshintergrund und MigrantInnen türkischer sowie teilweise denen italienischer und ex-jugoslawischer Herkunft bestehen. Die Teilnahme 
in einer ,ethnische Ökonomie' hat trotz gewisser Probleme positive Effekte auf das Einkommen und bringt eine Reihe immaterieller Vorteile.

\subsection{Soziale Beziehungen}

Partnerschaften zwischen Menschen unterschiedlicher Herkunft sind der anspruchsvollste Indikator für kulturelle Integration und stellen sich nur langsam ein. Diese besondere Sensitivität der intimen Beziehungen beschränkt sich nicht auf MigrantInnen. In Deutschland waren beispielsweise noch vor 50 Jahren Partnerschaften zwischen protestantischen und katholischen Deutschen eine Ausnahme (Teckenberg 1999). Und Paare, bei denen die Partner aus sehr unterschiedlichen sozialen Schichten stammen, sind auch heute noch selten. (Ermish, et al. 2006) Auch bei Freundschaften kommt Homophilie ${ }^{127}$ zum Tragen, gemäß derer Menschen, die einander ähnlich sind, sich enger miteinander befreunden, weil sie einander stärker vertrauen und sich näher fühlen (McPherson, et al. 2001). Diese Neigung aller Menschen zur Homophilie - verstärkt durch soziale Konventionen und materielle Interessen - sollte bei der Einschätzung der folgenden Zahlen im Hinterkopf behalten werden.

\subsubsection{Partnerschaften}

In diesem Abschnitt werden Partnerschaftsmuster für MigrantInnen in den beiden Datensets betrachtet. Im Mikrozensus 2005 werden dafür MigrantInnen im Alter von 20 bis 45 Jahren untersucht, die mit einer/m PartnerIn zusammen in einem Haushalt leben. In vielen Fällen handelt es sich um EhepartnerInnen, aber es können auch andere PartnerInnen sein. Leider enthält der Mikrozensus keine Informationen zu PartnerInnen, die nicht im selben Haushalt leben. Daher können diese nicht in die Analyse eingeschlossen werden. Beim DJIAusländersurvey können Ehen und feste Partnerschaften in die Analyse der 18- bis 25Jährigen eingeschlossen werden, unabhängig davon, ob die PartnerInnen zusammenwohnen. In beiden Datensets lebt nur ein Teil aller MigrantInnen der Altersgruppen in einer Partnerschaft. Für den Mikrozensus zeigt Tabelle 27, wie viele Personen in den verschiedenen Herkunftsgruppen und Generation jeweils mit einer/m PartnerIn im Haushalt zusammenleben: Der Prozentsatz derer mit PartnerIn ist besonders in der zweiten Generation und dort namentlich bei den AussiedlerInnen gering. Beim DJI-Ausländersurvey führt eine Beschränkung auf MigrantInnen, die eine/n PartnerIn haben, zu einem ähnlichen Rückgang der Fallzahlen.

\footnotetext{
${ }^{127}$ Der Begriff wird hier in seiner übertragenen englischen Bedeutung (,homophily') als Anziehung zwischen Menschen ähnlicher Herkunft und sozialer Schicht verstanden. Im Deutschen verengt er sich häufig auf die Anziehung zu einer/m gleichgeschlechtlichen PartnerIn.
} 
Tabelle 27 - Fallzahlen PartnerIn im Haushalt

\begin{tabular}{|c|c|c|c|c|c|c|c|c|}
\hline & & TÜRK & ITAL & GRIE & $\begin{array}{l}\text { SPAN \& } \\
\text { PORT }\end{array}$ & EX-JUG & AUSS & WEST \\
\hline \multirow{3}{*}{ 莺 } & PartnerIn im Haushalt & 1.095 & 215 & 117 & 95 & 251 & 283 & 65 \\
\hline & $\begin{array}{l}\text { Kein/e PartnerIn im } \\
\text { Haushalt }\end{array}$ & 1.306 & 297 & 184 & 109 & 396 & 972 & 94 \\
\hline & Total & 2.401 & 512 & 301 & 204 & 647 & 1.255 & 159 \\
\hline \multirow{3}{*}{ 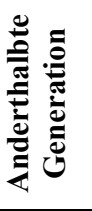 } & PartnerIn im Haushalt & 549 & 41 & 29 & 25 & 103 & 811 & 21 \\
\hline & $\begin{array}{l}\text { Kein/e PartnerIn im } \\
\text { Haushalt }\end{array}$ & 191 & 19 & 32 & 17 & 128 & 1.097 & 22 \\
\hline & Total & 740 & 60 & 61 & 42 & 231 & 1.908 & 43 \\
\hline \multirow{3}{*}{ 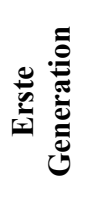 } & PartnerIn im Haushalt & 2.665 & 448 & 203 & 277 & 1.098 & 4.260 & 840 \\
\hline & $\begin{array}{l}\text { Kein/e PartnerIn im } \\
\text { Haushalt }\end{array}$ & 348 & 139 & 73 & 79 & 226 & 1.227 & 432 \\
\hline & Total & 3.013 & 587 & 276 & 306 & 1.324 & 5.487 & 1.272 \\
\hline
\end{tabular}

Mikrozensus 2005, FDZ der Statistischen Ämter des Bundes und der Länder, Eigene Berechnungen

Es stellt sich daher die Frage, ob hinsichtlich des sozioökonomischen Status zwischen der Gruppe mit PartnerInnen und der ohne PartnerInnen im Haushalt systematische Unterschiede bestehen. Tabelle 28 belegt für den Mikrozensus, dass in allen drei Generationen MigrantInnen mit einer/m PartnerIn im Haushalt etwas niedrigere durchschnittliche Werte beim ISCED und dem kategorisierten Blossfeld-Indiktor sowie auf der Magnitude-Prestigeskala haben. Bei den Nicht-MigrantInnen zeichnet sich die gegenteilige Tendenz ab. Die Unterschiede im ISCED sind dabei kleiner als die der Indikatoren des Arbeitsmarkts. Im Hinblick auf die Herkunftsgruppen in der zweiten Generation haben MigrantInnen türkischer, italienischer, griechischer, spanischer und portugiesischer Herkunft sowie die Kinder von MigrantInnen aus Ex-Jugoslawien mit PartnerInnen im Haushalt geringere MPS-Werte als MigrantInnen ohne PartnerInnen, wohingegen AussiedlerInnen und, westliche' MigrantInnen aus NichtAnwerbestaaten mit PartnerInnen höhere Werte aufweisen. Die Durchschnittswerte des kategorisierten Blossfeld liegen über die Herkunftsgruppen hinweg unabhängig vom Partnerschaftsstatus eng beisammen, mit Ausnahme der, westlichen' MigrantInnen aus NichtAnwerbestaaten und der Kinder von AussiedlerInnen (Unterschied 0,3 Punkte). 
Tabelle 28 - Sozio-ökonomische Unterschiede für MigrantInnen mit und ohne PartnerIn im Haushalt

\begin{tabular}{|c|c|c|c|c|c|c|c|c|c|c|}
\hline & & TÜRK & $\begin{array}{l}\text { ITAL, } \\
\text { GRIE, } \\
\text { SPAN \& } \\
\text { PORT }\end{array}$ & EX-JUG & AUSS & WEST & $\begin{array}{c}\text { ALLE } \\
\text { ZWEITE } \\
\text { GENERATION }\end{array}$ & $\begin{array}{c}\text { ALLE } \\
\text { ANDERTHALBTE } \\
\text { GENERATION }\end{array}$ & $\begin{array}{c}\text { ALLE } \\
\text { ERSTE } \\
\text { GENERATION }\end{array}$ & DTOM \\
\hline \multirow[t]{2}{*}{$\begin{array}{l}\text { MPS Mittel- } \\
\text { wert (Median) }\end{array}$} & $\begin{array}{l}\text { PartnerIn im } \\
\text { Haushalt }\end{array}$ & $\begin{array}{l}50,1^{* *} \\
(47,4)\end{array}$ & $\begin{array}{c}55,8 \text { n.s. } \\
(51,6)\end{array}$ & $\begin{array}{l}55,0^{*} \\
(51,4)\end{array}$ & $\begin{array}{c}63,0 * * \\
(60,4)\end{array}$ & $\begin{array}{c}68,4 * * \\
(61,2)\end{array}$ & $54,1 * *(51,5)$ & $51,0 * *(46,1)$ & $48,6 * *(41,1)$ & $\begin{array}{l}62,6^{* *} \\
(60,4)\end{array}$ \\
\hline & $\begin{array}{l}\text { Kein/e Part- } \\
\text { nerIn im } \\
\text { Haushalt }\end{array}$ & $\begin{array}{c}52,3 * * \\
(51,1)\end{array}$ & $\begin{array}{c}56,1 \text { n.s. } \\
(52,9)\end{array}$ & $\begin{array}{l}58,0^{*} \\
(53,2)\end{array}$ & $\begin{array}{l}57,2 * * \\
(53,2)\end{array}$ & $\begin{array}{l}57,2 * * \\
(53,9)\end{array}$ & $55,3 * *(51,1)$ & $53,0 * *(51,1)$ & $55,6 * *(47,4)$ & $\begin{array}{l}60,0 * * \\
(53,9)\end{array}$ \\
\hline \multirow{2}{*}{$\begin{array}{l}\text { Kat. Blossfeld } \\
\text { Mittelwert } \\
\text { (Median) }\end{array}$} & $\begin{array}{l}\text { PartnerIn im } \\
\text { Haushalt }\end{array}$ & $\begin{array}{c}2,7 \text { n.s. } \\
(3)\end{array}$ & $\begin{array}{c}3,1 \text { n.s. } \\
(3)\end{array}$ & $\begin{array}{c}3,0 \text { n.s. } \\
(3)\end{array}$ & $\begin{array}{c}3,5^{* *} \\
(4) \\
\end{array}$ & $\begin{array}{c}3,5^{* *} \\
(4) \\
\end{array}$ & 2,9 n.s. (3) & 2,9 n.s. (3) & $\begin{array}{c}2,6 * * \\
(2) \\
\end{array}$ & $\begin{array}{c}3,5^{* *} \\
(4) \\
\end{array}$ \\
\hline & $\begin{array}{l}\text { Kein/e Part- } \\
\text { nerIn im } \\
\text { Haushalt }\end{array}$ & $\begin{array}{c}2,6 \text { n.s. } \\
\text { (3) }\end{array}$ & $\begin{array}{l}3,0 \text { n.s. } \\
\text { (3) }\end{array}$ & $\begin{array}{l}3,0 \text { n.s. } \\
\text { (3) }\end{array}$ & $\begin{array}{c}3,2 * * \\
(3)\end{array}$ & $\begin{array}{c}3,2 * * \\
(3)\end{array}$ & 2,9 n.s. (3) & 2,9 n.s. (3) & $\begin{array}{c}2,8 * * \\
(3)\end{array}$ & $\begin{array}{c}3,2 * * \\
(4)\end{array}$ \\
\hline \multirow{2}{*}{$\begin{array}{l}\text { Kat. ISCED } \\
\text { Mittelwert } \\
\text { (Median) }\end{array}$} & $\begin{array}{l}\text { PartnerIn im } \\
\text { Haushalt }\end{array}$ & $2,8 * *(3)$ & $\begin{array}{c}3,1 \text { n.s. } \\
(3)\end{array}$ & $\begin{array}{c}3,2^{*} \\
(3)\end{array}$ & $\begin{array}{c}3,5^{*} \\
(4)\end{array}$ & $\begin{array}{c}3,8 \text { n.s. } \\
\text { (4) }\end{array}$ & $\begin{array}{c}3,0 * * \\
(3) \\
\end{array}$ & $\begin{array}{c}2,9 * * \\
(3) \\
\end{array}$ & $\begin{array}{c}2,8 * * \\
(3) \\
\end{array}$ & $\begin{array}{c}3,5^{* * *} \\
(3) \\
\end{array}$ \\
\hline & $\begin{array}{l}\text { Kein/e Part- } \\
\text { nerIn im } \\
\text { Haushalt }\end{array}$ & $3,0 * *(3)$ & $\begin{array}{l}3,1 \text { n.s. } \\
\text { (3) }\end{array}$ & $\begin{array}{l}3,4^{*} \\
(3)\end{array}$ & $\begin{array}{l}3,4^{*} \\
(3)\end{array}$ & $\begin{array}{c}3,8 \text { n.s. } \\
\text { (4) }\end{array}$ & $\begin{array}{c}3,2 * * \\
(3)\end{array}$ & $\begin{array}{c}3,0 * * \\
(3)\end{array}$ & $\begin{array}{c}3,2 * * \\
(3)\end{array}$ & $\begin{array}{c}3,5 * * \\
(3)\end{array}$ \\
\hline
\end{tabular}

T-Test Signifikanz $* * \mathrm{p}<0.01, * \mathrm{p}<0.05$, n.s. nicht signifikant

Mikrozensus 2005, FDZ der Statistischen Ämter des Bundes und der Länder, Eigene Berechnungen 
Für den nächsten Abschnitt, in dem die Integrationsmuster nur für MigrantInnen mit PartnerInnen im Haushalt untersucht werden, kann daher geschlussfolgert werden, dass, wenn durch die Beschränkung auf MigrantInnen mit PartnerInnen überhaupt ein Bias entsteht, dieser AussiedlerInnen und, westliche' MigrantInnen aus Nicht-Anwerbestaaten bevorzugt, alle anderen Herkunftsgruppen jedoch schlechter stellt. Daher kann eine systematische Verzerrung der Ergebnisse aufgrund der Einschränkung auf MigrantInnen mit PartnerInnen, wie sie bei Deutschen ohne Migrationshintergrund zu finden ist, ausgeschlossen werden.

Für Paare im DJI-Ausländersurvey stellt sich in Tabelle 29 die Situation im Ergebnis gleich dar. Bei der türkischen Herkunftsgruppe verfügen Personen, die in einer Partnerschaft leben, sowohl in der ersten/anderthalbten als auch in der zweiten Generation über ein niedrigeres Bildungsniveau als Personen ohne PartnerIn. Für die beiden anderen Herkunftsgruppen sind die Unterschiede jeweils nicht signifikant. Auch für das DJI-Datenset gibt es daher keine positive Verzerrung.

Tabelle 29 - Bildungsabschluss (ISCED): Partnerschaftsstatus

\begin{tabular}{|c|c|c|c|c|}
\hline & & TÜRK & ITAL & GRIE \\
\hline \multirow{2}{*}{ 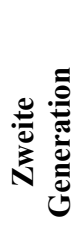 } & Ohne PartnerIn & $4,1 * *$ & 4,2 n.s. & 4,2 n.s. \\
\hline & Verpartnerte & $3,6^{* *}$ & 4,2 & 4,3 \\
\hline \multirow{2}{*}{ 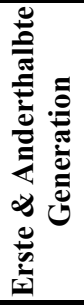 } & Ohne PartnerIn & $3,1^{*}$ & 3,5 n.s. & 3,5 n.s. \\
\hline & Verpartnerte & $2,6^{*}$ & 3,5 & 3,4 \\
\hline
\end{tabular}

T-Test Signifikanz $* * \mathrm{p}<0.01, * \mathrm{p}<0.05$, n.s. nicht signifikant Ausländersurvey 1997 des Deutschen Jugendinstituts, Eigene Berechnungen

Als erstes stellt sich die Frage, wie es in der zweiten Generation mit der Bereitschaft zu Partnerschaften mit Menschen anderer Herkunft aussieht. Allerdings wird für gewöhnlich nur spezifisch die Bereitschaft zur Ehe mit einer/m Deutschen abgefragt. Auch im DJIAusländersurvey liegen Zahlen nur zur Eheneigung vor. Tabelle 30 zeigt, dass diese Bereitschaft über die Generationen steigt: Während in der ersten Generation 36\% der MigrantInnen griechischer und türkischer Herkunft und 48\% derjenigen italienischer Herkunft auf jeden Fall oder möglicherweise zu einer Heirat mit einem/r deutschen PartnerIn bereit sind, steigt die 
Zahl in der zweiten Generation auf knapp 80\% für Personen italienischer, auf 72\% für Personen griechischer und auf gut 50\% für Personen türkischer Herkunft. Neben der insgesamt hohen Bereitschaft fällt der Anstieg von der ersten zur zweiten Generation für die türkische Herkunftsgruppe am geringsten aus. Knapp 5\% der MigrantInnen geben an, nicht heiraten zu wollen.

Tabelle 30 - Bereitschaft zur Ehe mit deutschem/r PartnerIn ohne Migrationshintergrund

\begin{tabular}{|c|c|c|c|c|}
\hline & & TÜRK & ITAL & GRIE \\
\hline \multirow{5}{*}{ U气 } & Auf jeden Fall & $12 \%$ & $27 \%$ & $22 \%$ \\
\hline & Möglicherweise & $41 \%$ & $52 \%$ & $50 \%$ \\
\hline & Wahrscheinlich nicht & $29 \%$ & $14 \%$ & $17 \%$ \\
\hline & Auf keinen Fall & $16 \%$ & $4 \%$ & $7 \%$ \\
\hline & Möchte nicht heiraten & $3 \%$ & $3 \%$ & $5 \%$ \\
\hline \multirow{5}{*}{ 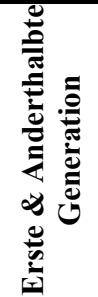 } & Auf jeden Fall & $3 \%$ & $6 \%$ & $8 \%$ \\
\hline & Möglicherweise & $33 \%$ & $42 \%$ & $38 \%$ \\
\hline & Wahrscheinlich nicht & $30 \%$ & $36 \%$ & $26 \%$ \\
\hline & Auf keinen Fall & $30 \%$ & $11 \%$ & $26 \%$ \\
\hline & Möchte nicht heiraten & $4 \%$ & $5 \%$ & $2 \%$ \\
\hline
\end{tabular}

Ausländersurvey 1997 des Deutschen Jugendinstituts, Eigene Berechnungen

Wie sieht es nun bei denjenigen aus, die tatsächlich in einer Partnerschaft leben? Die Zahlen aus dem Mikrozensus bieten eine einzigartige Gelegenheit, dieser Frage nachzugehen. Bisher konnte nämlich nur auf die offizielle Statstik der Eheschließungen zurückgegriffen werden. Da jedoch Herkunft immer weniger mit Staatsbürgerschaft korrespondiert, bergen diese Zahlen eine Reihe von Problemen: Heiratet z.B. ein Eingebürgerter eine Deutsche ohne Migrationshintergrund, dann ist dies keine binationale Ehe; heiratet er jedoch eine Türkin aus der Türkei, dann wird dies als gemischte Ehe gezählt (Sen, et al. 2001: 46). ${ }^{128}$ Tabelle 31 untersucht daher für die hier im Mikrozensus betrachteten MigrantInnen, welcher Generation ihre PartnerInnen angehören, unabhängig von deren Staatsbürgerschaft. Auf diese Zahl folgt der Prozentsatz der ,ko-ethnischen’ PartnerInnen unter den MigrantenpartnerInnen (also unter den PartnerInnen, die zur ersten, anderthalbten oder zweiten Generation gehören). Innerhalb der zweiten Generation haben MigrantInnen türkischer Herkunft am häufigsten PartnerInnen der ersten Generation, gefolgt von MigrantInnen aus Ex-Jugoslawien. Die zweite Generation türkischer Herkunft hat auch am seltensten PartnerInnen ohne Migrationshintergrund.

\footnotetext{
${ }^{128}$ Hinzu kommt, dass ein Großteil der MigrantInnen türkischer Herkunft in der Türkei heiratet und damit in der deutschen Statistik nicht auftaucht (Sen, et al. 2001: 46).
} 
Tabelle 31 - Herkunft und Generationsstatus der/s PartnerIn, MZ

\begin{tabular}{|c|c|c|c|c|c|c|c|c|c|c|c|}
\hline & & TÜRK & $\begin{array}{l}\text { \% GLEICHE } \\
\text { HERKUNFTS- } \\
\text { GRUPPE }\end{array}$ & $\begin{array}{l}\text { ITAL, } \\
\text { GRIE, } \\
\text { SPAN \& } \\
\text { PORT }\end{array}$ & $\begin{array}{l}\text { \% GLEICHE } \\
\text { HERKUNFTS- } \\
\text { GRUPPE }\end{array}$ & $\begin{array}{c}\text { EX- } \\
\text { JUG }\end{array}$ & $\begin{array}{l}\text { \% GLEICHE } \\
\text { HERKUNFTS- } \\
\text { GRUPPE }\end{array}$ & AUSS & $\begin{array}{l}\text { \% GLEICHE } \\
\text { HERKUNFTS- } \\
\text { GRUPPE }\end{array}$ & WEST & $\begin{array}{c}\text { \% GLEICHE } \\
\text { HERKUNFTS- } \\
\text { GRUPPE }\end{array}$ \\
\hline \multirow{4}{*}{ 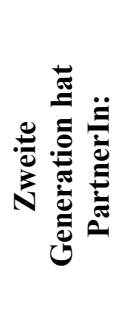 } & Zweite Generation & $34 \%$ & \multirow{3}{*}{$95 \%$} & $29 \%$ & \multirow{3}{*}{$74 \%$} & $20 \%$ & \multirow{3}{*}{$78 \%$} & $13 \%$ & \multirow{3}{*}{$70 \%$} & $9 \%$ & \multirow{3}{*}{$67 \%$} \\
\hline & $\begin{array}{l}\text { Anderthalbte Ge- } \\
\text { neration }\end{array}$ & $8 \%$ & & $7 \%$ & & $8 \%$ & & $10 \%$ & & 0 & \\
\hline & Erste Generation & $45 \%$ & & $20 \%$ & & $33 \%$ & & $13 \%$ & & $13 \%$ & \\
\hline & DTOM & $13 \%$ & & $44 \%$ & & $39 \%$ & & $64 \%$ & & $78 \%$ & \\
\hline \multirow{4}{*}{ 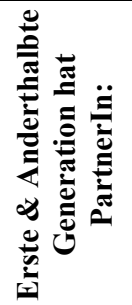 } & Zweite Generation & $19 \%$ & \multirow{3}{*}{$97 \%$} & $7 \%$ & \multirow{3}{*}{$85 \%$} & $8 \%$ & \multirow{3}{*}{$90 \%$} & $1 \%$ & \multirow{3}{*}{$78 \%$} & $2 \%$ & \multirow{3}{*}{$69 \%$} \\
\hline & $\begin{array}{l}\text { Anderthalbte Ge- } \\
\text { neration }\end{array}$ & $15 \%$ & & $4 \%$ & & $5 \%$ & & $9 \%$ & & $1 \%$ & \\
\hline & Erste Generation & $52 \%$ & & $60 \%$ & & $63 \%$ & & $79 \%$ & & $37 \%$ & \\
\hline & DTOM & $14 \%$ & & $29 \%$ & & $24 \%$ & & $11 \%$ & & $60 \%$ & \\
\hline
\end{tabular}

Mikrozensus 2005, FDZ der Statistischen Ämter des Bundes und der Länder, Eigene Berechnungen 
Im Vergleich der Herkunftsgruppen steigt nur bei MigrantInnen türkischer Herkunft der Anteil der Partnerschaften mit Deutschen ohne Migrationshintergrund von der ersten zur zweiten Generation nicht. Gleichzeitig haben MigrantInnen der ersten und anderthalbten Generation türkischer Herkunft häufiger als jede andere Gruppe PartnerInnen, die der zweiten Generation angehören. Dies verweist auf ein transnationales Partnerschaftsmuster, bei dem enge Kontakte zwischen dem Herkunftsland der Eltern und dem Land des Wohnsitzes der MigrantInnen bestehen. Darauf wird gleich bei den Daten des DJI noch näher einzugehen sein.

Der Anteil der ,ko-ethnischen' Beziehungen an den Partnerschaften mit MigrantInnen reduziert sich von der ersten und anderthalbten Generation zur zweiten Generation. Trotzdem bleibt er für die zweite Generation türkischer Herkunft auf einem sehr hohen Niveau: Bei 95\% der Partnerschaften gehört der/die PartnerIn der gleichen Herkunftsgruppe an. Am anderen Ende des Spektrums liegen die ,westlichen' MigrantInnen aus Nicht-Anwerbestaaten in der zweiten Generation, die eine/n PartnerIn mit Migrationshintergrund haben: Hier liegt der Anteil der ,ko-ethnischen' PartnerInnen bei nur 67\%. Zwischen einem Fünftel und einem Drittel der Ex-JugoslawInnen, ItalienerInnen, GriechInnen, SpanierInnen, PortugiesInnen und ,westlichen' MigrantInnen aus Nicht-Anwerbestaaten sowie der AussiedlerInnen der zweiten Generation gehen also ,ethnisch' gemischte Beziehungen ein, bei denen die beiden migrantischen PartnerInnen unterschiedlicher Herkunft sind. Dieses Phänomen der ,Regenbogen'Partnerschaften wurde bisher kaum diskutiert.

Transnationale Verbindungen und die Frage der Partnerschaften mit anderen MigrantInnen sollen nun anhand der Daten des DJI-Ausländersurveys noch genauer betrachtet werden. Zunächst zeigt Tabelle 32, dass der Prozentsatz von Partnerschaften mit MigrantInnen der ersten Generation aus dem Herkunftsland der Eltern in der zweiten Generation im Vergleich zur ersten und anderthalbten abnimmt, und zwar mit den bereits aus dem Mikrozensus bekannten Unterschieden zwischen den Herkunftsgruppen. Auch der Anteil ,ko-ethnischer' PartnerInnen aller Generationen liegt bei MigrantInnen der zweiten Generation 25 Prozentpunkte niedriger als in der ersten Generation. Bei MigrantInnen türkischer Herkunft verringert er sich jedoch nur um 12 Prozentpunkte und damit halb so stark wie bei den anderen Herkunftsgruppen. Für ,ko-ethnische' PartnerInnen aus dem Herkunftsland der Eltern erlaubt der DJIAusländersurvey darüber hinaus eine detailliertere Unterscheidung zwischen denjenigen, die zudem aus der gleichen Region wie die Eltern kommen, und denjenigen, die aus anderen Orten im Herkunftsland stammen. Erstere belegen besonders deutlich die transnationalen Kontakte der zweiten Generation. In der ersten und anderthalbten Generation stammen zwischen 
34\% und 45\% der PartnerInnen aus der gleichen italienischen, griechischen oder türkischen Region wie die Eltern der MigrantInnen. Für die zweite Generation ist ein starker Rückgang zu erwarten, da die Verbindungen zur Herkunftsregion der Eltern allein aufgrund der geographischen Distanz geringer ausfallen sollten. Für MigrantInnen der zweiten Generation griechischer und italienischer Herkunft entsprechen die Zahlen dieser Erwartung: Nur 6\% haben PartnerInnen, die aus der Herkunftsregion eines der Elternteile stammen. Bei der zweiten Generation türkischer Herkunft jedoch hat ein Viertel PartnerInnen aus der elterlichen Herkunftsregion. Dies verweist auf eine hohe Dichte der Interaktionen mit dem Herkunftsland und speziell mit der Herkunftsregion der Eltern noch in der zweiten Generation. ${ }^{129}$

Tabelle 32 - Herkunft und Generationsstatus der/s PartnerIn, DJI-Ausländersurvey

\begin{tabular}{|c|c|c|c|c|c|c|}
\hline & \multicolumn{3}{|c|}{$\begin{array}{c}\text { ZWEITE } \\
\text { GENERATION }\end{array}$} & \multicolumn{3}{|c|}{$\begin{array}{c}\text { ERSTE \& ANDERTHALBTE } \\
\text { GENERATION }\end{array}$} \\
\hline & TÜRK & ITAL & GRIE & TÜRK & ITAL & GRIE \\
\hline $\begin{array}{l}\text { PartnerIn gleiche Her- } \\
\text { kunftgruppe, im Herkunfts- } \\
\text { land geboren }\end{array}$ & $48 \%$ & $19 \%$ & $23 \%$ & $71 \%$ & $57 \%$ & $63 \%$ \\
\hline $\begin{array}{l}\text { PartnerIn gleiche Her- } \\
\text { kunftgruppe, in Deutschland } \\
\text { geboren }\end{array}$ & $27 \%$ & $21 \%$ & $28 \%$ & $16 \%$ & $8 \%$ & $13 \%$ \\
\hline $\begin{array}{l}\text { PartnerIn nicht gleiche Her- } \\
\text { kunftgruppe, in Deutschland } \\
\text { geboren }\end{array}$ & $23 \%$ & $54 \%$ & $47 \%$ & $11 \%$ & $33 \%$ & $23 \%$ \\
\hline $\begin{array}{l}\text { PartnerIn nicht gleiche Her- } \\
\text { kunftgruppe, im Ausland ge- } \\
\text { boren }\end{array}$ & $2 \%$ & $6 \%$ & $2 \%$ & $3 \%$ & $3 \%$ & $1 \%$ \\
\hline $\mathbf{N}$ & 220 & 219 & 189 & 121 & 107 & 105 \\
\hline $\begin{array}{l}\text { Aus gleicher Herkunftsregion } \\
\text { für alle Verpartnerten }\end{array}$ & $25 \%$ & $6 \%$ & $6 \%$ & $45 \%$ & $34 \%$ & $39 \%$ \\
\hline
\end{tabular}

Ausländersurvey 1997 des Deutschen Jugendinstituts, Eigene Berechnungen

Anfangs wurde erwähnt, wie weit verbreitet die Bevorzugung von PartnerInnen mit einem ähnlichen kulturellen Hintergrund ist. MigrantInnen der zweiten Generation, die in Deutschland als Paar leben, teilen den kulturellen Hintergrund des Aufwachsens und Schulbesuchs in Deutschland. Sie teilen auch die Erfahrung, in einer Familie mit migrierten Eltern aufgewachsen $\mathrm{zu}$ sein. Weniger eindeutig ist jedoch, ob sie auch die kulturellen Traditionen aus dem Heimatland der Eltern teilen. Für die Analyse der Muster wird der Begriff ,ko-ethnisch’ daher auf Beziehungen zwischen MigrantInnen im Aufnahmeland und ,ko-ethnischen’ PartnerInnen

\footnotetext{
${ }^{129}$ Aufgrund der Struktur der Datenerhebung im DJI-Ausländersurvey ist leider innerhalb der Gruppe der nicht ko-ethnischen PartnerInnen keine Unterscheidung in migrantische PartnerInnen (,Regenbogenpartnerschaften') und deutsche PartnerInnen ohne Migrationshintergrund möglich.
} 
der ersten Generation beschränkt, bei denen kulturelle Kenntnisse durch ihr Aufwachsen im Herkunftsland gesichert sind.

Gemäß Tabelle 33 erlangen die ,ko-ethnischen' PartnerInnen der ersten Generation von MigrantInnen aller Generationen geringere durchschnittliche Bildungs- und Ausbildungswerte als PartnerInnen, die nicht ,ko-ethnisch’ sind. ${ }^{130}$

Tabelle 33 - Bildungsabschluss (ISCED) PartnerIn gleiche Herkunftsgruppe

\begin{tabular}{|c|c|c|c|c|c|c|}
\hline & & TÜRK & $\begin{array}{l}\text { ITAL, GRIE, } \\
\text { SPAN \& PORT }\end{array}$ & EX-JUG & AUSS & WEST \\
\hline \multirow{2}{*}{ 泀 } & $\begin{array}{l}\text { PartnerIn nicht } \\
\text { gleiche Herkunfts- } \\
\text { gruppe }\end{array}$ & 4,7 & 5,3 & 5,6 & 5,9 & 6,3 \\
\hline & $\begin{array}{l}\text { PartnerIn gleiche } \\
\text { Herkunftsgruppe }\end{array}$ & 3,5 & 3,5 & 5,0 & 5,5 & 7,9 \\
\hline \multirow{2}{*}{ 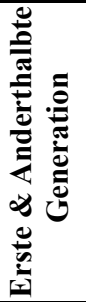 } & $\begin{array}{l}\text { PartnerIn nicht } \\
\text { gleiche Herkunfts- } \\
\text { gruppe }\end{array}$ & 3,9 & 5,3 & 5,0 & 5,4 & 6,8 \\
\hline & $\begin{array}{l}\text { PartnerIn gleiche } \\
\text { Herkunftsgruppe }\end{array}$ & 2,9 & 3,8 & 4,4 & 5,1 & 7,3 \\
\hline
\end{tabular}

Mikrozensus 2005, FDZ der Statistischen Ämter des Bundes und der Länder, Eigene Berechnungen

Dieses Ergebnis scheint die Annahme klassischer Integrationstheorien zu bestätigen, dass gemischte Partnerschaften mit Aufstiegsmobilität einhergehen. Unter dem Makrotrend können sich jedoch gegenteilige Effekte für eine signifikante Teilgruppe verbergen: So könnte es auch MigrantInnen geben, für die im Gegenteil ,ko-ethnische' Partnerschaften mit einer Statusverbesserung korrelieren. Bevor dieser Frage in Kapitel 4 durch die Analyse der Integrationsmuster auf der individuellen Ebene nachgegangen wird, werden Freundschaftsnetzwerke und ihre Wirkung betrachtet.

\subsubsection{Freundschaften}

Freundschaften sind neben Partnerschaften ein wichtiger Teil der sozialen Beziehungen. In ihnen werden Informationen ausgetauscht, Unterstützung, emotionale Nähe und Anerkennung sowie Hilfe bei praktischen Problemen gewährt. Freundschaften zwischen MigrantInnen und Deutschen ohne Migrationshintergrund sind damit zugleich ein Indikator für kulturelle Anpassung (und eine gewisse Offenheit auf Seiten der Nicht-MigrantInnen) und Förderer dersel-

\footnotetext{
${ }^{130}$ MigrantInnen aus, westlichen' Nicht-Anwerbestaaten folgen diesem Muster nicht.
} 
ben. Die Fehlerbalken in Abbildung 12 zeigen, wie sich der Anteil der ,ko-ethnischen' FreundInnen, also der FreundInnen gleicher Herkunft, unabhängig von der Staatsbürgerschaft über die Generationen entwickelt. Die Daten stammen aus dem DJI-Ausländersurvey; im Mikrozensus werden Freundschaften nicht erfragt. In allen drei Herkunftsgruppen sinkt der Anteil der ,ko-ethnischen' unter den bis zu benannten FreundInnen von der ersten und anderthalbten zur zweiten Generation signifikant. Allerdings gibt es große Unterschiede zwischen den Herkunftsgruppen: Personen türkischer Herkunft haben in der zweiten Generation durchschnittlich 60\% ,ko-ethnische' FreundInnen, was dem Prozentsatz bei MigrantInnen griechischer und italienischer Herkunft in der ersten und anderthalbten Generation entspricht. In der zweiten Generation haben Letztere nur noch gut 40\%, ko-ethnische' FreundInnen. Solche Unterschiede dürfen allerdings nicht ausschließlich voluntaristisch-individuell erklärt werden, wie es im Defizitansatz üblich ist („Personen türkischer Herkunft wünschen sich viele ,koethnische' FreundInnen“), sondern es müssen strukturelle Gegebenheiten berücksichtigt werden, z.B. eine begrenzte Bereitschaft von Deutschen ohne Migrationshintergrund, Freundschaften mit Personen türkischer Herkunft einzugehen oder aber beschränkte Möglichkeiten zur Freundschaft aufgrund nachbarschaftlicher und schulischer Segregation. Einige dieser Strukturaspekte werden in Kapitel 5 diskutiert.

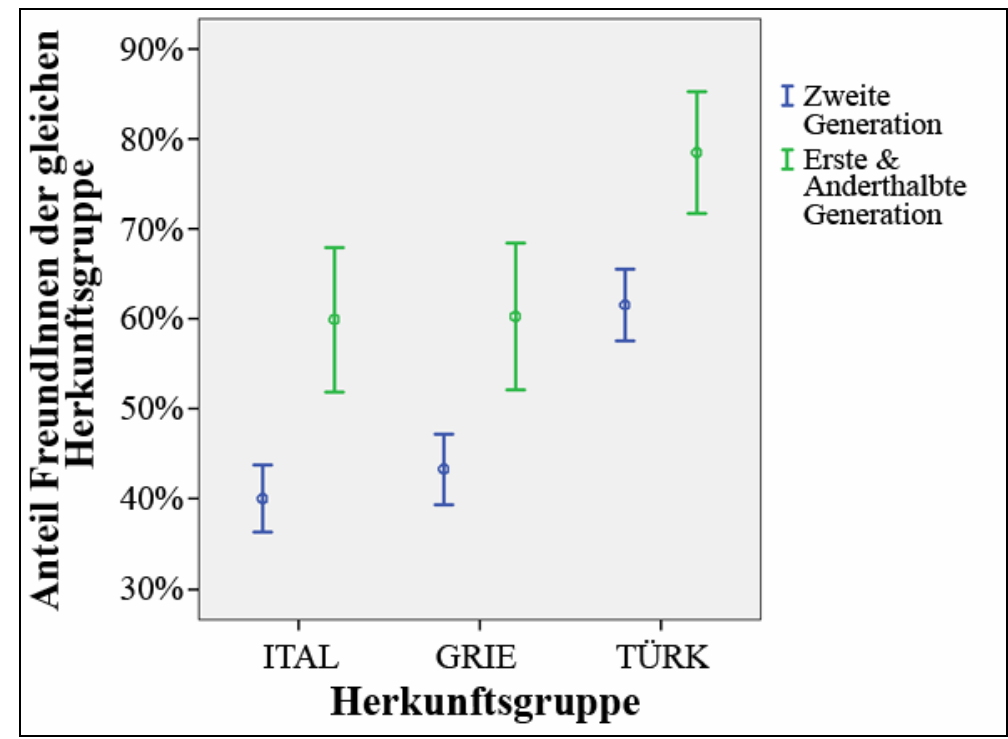

Ausländersurvey 1997 des Deutschen Jugendinstituts, Eigene Berechnungen

Abbildung 12 - FreundInnen der gleichen Herkunftsgruppe

Weiterhin gibt es einen sehr hohen Prozentsatz von MigrantInnen, die unter den 20 Personen, mit denen sie im Alltag zu tun haben, keine/n einzige/n FreundIn haben. Dies deutet auf eine ernstzunehmende Marginalisierung und/oder eine Beschränkung auf familiäre Kontakte hin. 
Der Anteil dieser im Alltag freundeslosen Personen beträgt in der ersten und anderthalbten Generation beinahe 50\%, in der zweiten Generation sinkt er auf 18\% bis 30\% (Abbildung 13).

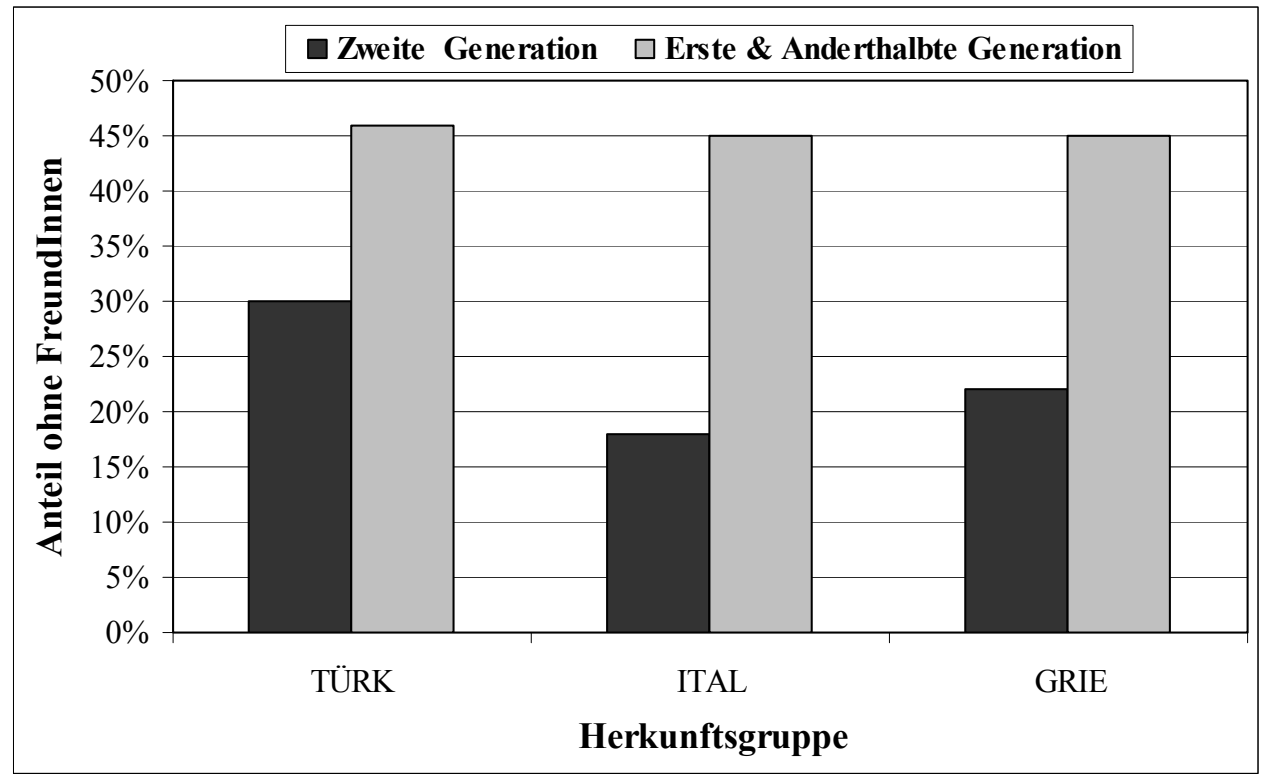

Ausländersurvey 1997 des Deutschen Jugendinstituts, Eigene Berechnungen

Abbildung 13 - Marginalisierung

Die Balkendiagramme der Abbildung 14 bringen die Zahl der sonst vernachlässigten sozialen Beziehungen zwischen MigrantInnen unterschiedlicher Herkunftsgruppen zu Tage. Deren Umfang ist, wie das Balkendiagramm zeigt, nicht unerheblich: Sowohl in der ersten und anderthalbten als auch in der zweiten Generation haben über 10\% der MigrantInnen mindestens eine/n migrantische/n FreundIn aus einer anderen Herkunftsgruppe.

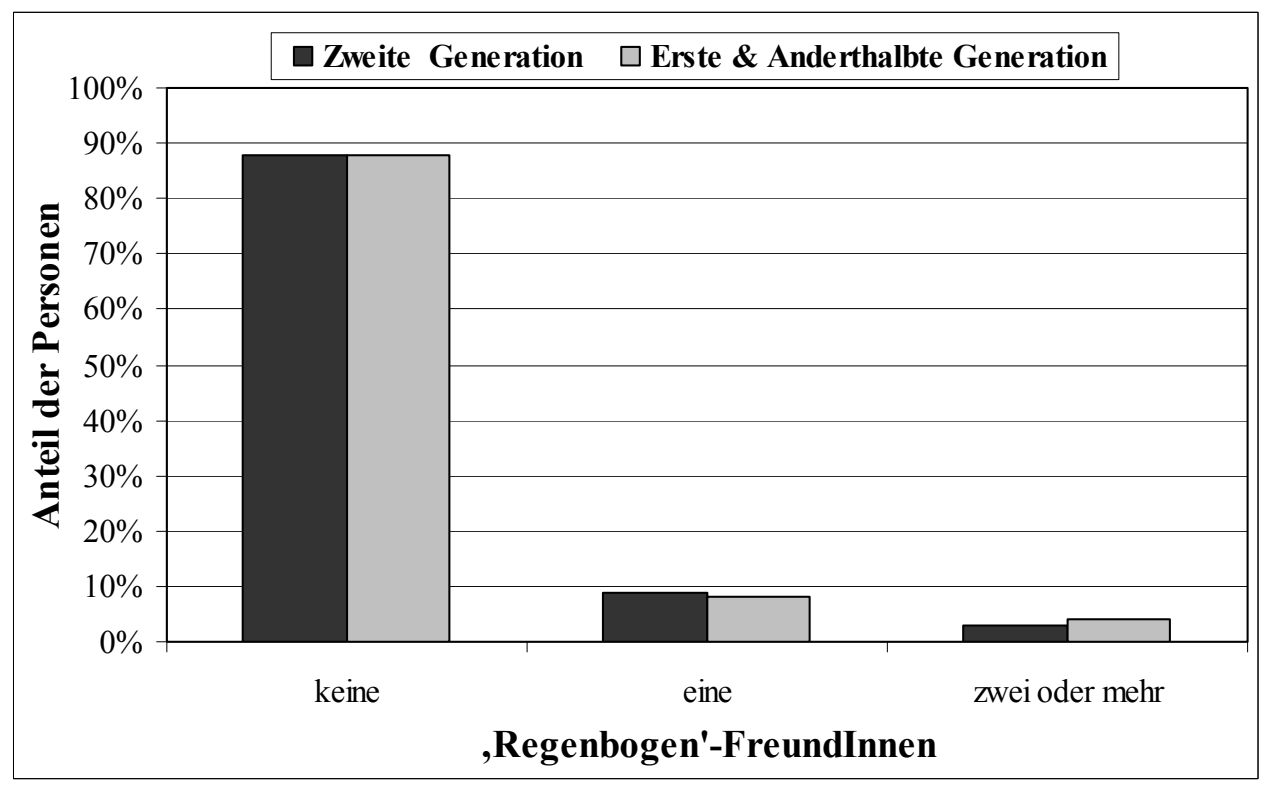

Ausländersurvey 1997 des Deutschen Jugendinstituts, Eigene Berechnungen

Abbildung 14 - ,Regenbogen'-FreundInnen 
Neben engen Freundschaften sagen vor allem Cliquen etwas über die sozialen Beziehungen der zweiten Generation aus. Aus qualitativen Studien ist die Existenz von subkulturellen Cliquen vor allem im Hiphop-Milieu, deren Mitglieder häufig verschiedenen Herkunftsgruppen angehören (sogenannte ,Regenbogen'-Gruppen) und die in ihren Einstellungen sowie ihrem Habitus den ,Mainstream' ablehnen, bekannt. Die Cliquenmitglieder entwickeln nicht zuletzt aus dem Gefühl der Diskriminierung heraus eine ,Oppositionskultur', die sich in einem Lebensstil bestehend aus Musik, Sport/Tanz und Graffiti sowie Mode äußert. (Nohl 2001, Lübcke 2007; Tietze 2006) ${ }^{131}$ Durch die Kommerzialisierung der Jugendkultur kann dieser Lebensstil vereinzelt zum Erwerb des Lebensunterhalts dienen. Die Cliquen agieren in einem Milieu, in dem Verbindungen in die Kriminalität bestehen. Die Mitglieder dieser hiphopnahen Gruppen sind von ihren Eltern unabhängig und unterliegen wenig sozialer Kontrolle durch Erwachsene.

Die ethnographischen Forschung zu Hiphop zeigt, dass habituelle Gemeinsamkeiten in diesen Gruppen wichtiger sind als ethnische Zugehörigkeiten (Nohl 2001: 193ff; Lübcke 2007), so dass die meisten Gruppen Mitglieder verschiedener Herkunftsgruppen, häufig auch der deutschen ohne Migrationshintergrund, enthalten. Der Habitus der Gruppenmitglieder umfasst weitläufige Bereiche des Lebens und stellt zeitliche, normative und materielle Anforderungen, die das Erfüllen der Forderungen des ,Mainstreams' unwahrscheinlich machen und somit z.B. langfristige Bildungsinvestitionen erschweren. Aus diesem Grund beschreibt Kraheck die Gruppen gleichzeitig als „soziale Stütze und Verführer“ (Kraheck 2004: 140). Die Cliquenmitglieder stehen zudem in einem Spannungsverhältnis zwischen oppositioneller Abgrenzung und Kommerzialisierung, so dass der von den Gruppen versprochene Halt mit einer Konsumnotwendigkeit zusammengehen kann (Tietze 2006: 157; Lübcke 2007: 302ff).

Interessant ist, dass diese Gruppen obwohl sie Teil einer globalen, in den urbanen Gebieten der USA entstandenen Kultur sind, auf einem stark lokalräumlichen Zugehörigkeitsgefühl basieren. Die räumliche Abgrenzung wird über einen bestimmten Slang verfestigt, gegenüber dem Leute aus wohlhabenderen Stadtvierteln z.B. ein ,sauberes“ Deutsch sprechen (Tietze 2006: 152). Das gemeinsame Kiezgefühl speist sich dabei auch aus erlebter Diskriminierung. So hat sich ein erheblicher Teil der Jugendlichen und jungen Erwachsenen bei der Arbeitsund Ausbildungsplatzsuche schon einmal aufgrund negativer Stereotype über ihren Stadtteil

\footnotetext{
${ }^{131}$ In der bisherigen Forschung sind diese multiethnischen Gruppen im Gegensatz zu Cliquen, die nur aus Mitgliedern einer Herkunftsgruppe bestehen, nur bei männlichen Jugendlichen untersucht worden. Inwieweit sie auch bei Mädchen und jungen Frauen existieren oder dort eher als Freundinnenkreis (vgl. den Dokumentarfilm Prinzessinnenbad (Blümner 2007)) auftreten, muss weiter untersucht werden.
} 
diskriminiert gefühlt (Tietze 2006: 153; Hampel 2006). Diese Diskriminierungserfahrung einigt die Jugendlichen mit Migrationshintergrund über die Grenzen der Herkunftsgruppen hinweg und schließt vereinzelt auch Deutsche ohne Migrationshintergrund ein. In den Gruppen werden teilweise negative Stereotype übernommen und positiv gewendet, wenn z.B. die Jugendlichen von sich als selbst als „Ghetto-Kids“ sprechen (Best 1999). In der empirischen Untersuchung dieser ,Oppositionskultur' zum ,Mainstream' grenzen sich die beschriebenen Gruppen stark nach unten ab und machen Front gegen „Penner, die Asozialen, die Junkies, den Abschaum, Leute aus dem Knast“ (Kraheck 2004: 153). Nur ein Teil der Gruppen der ,Oppositionskultur' ist zudem ,multiethnisch' aufgestellt, andere Gruppengrenzen verlaufen entlang der Herkunftsgruppen oder zwischen MigrantInnen und Nicht-MigrantInnen. Schließlich überführen Deutsche ohne Migrationshintergrund nicht selten den von ihnen erlebten sozial bedingten Ausschluss in ,ethnisch' diskriminierende Erklärungen wonach MigrantInnen deutschen Nicht-MigrantInnen aus sozial marginalisierten Schichten die Arbeitsplätze wegnehmen. Solche Erklärungen erschweren jedoch die Entstehung von ,Regenbogen'-Gruppen, die Nicht-MigrantInnen aus dem Viertel einschließen.

Bei diesen Untersuchungen zu den Gruppen der ,Oppositionskultur' stellt sich die Frage, inwieweit neben sozialen und herkunftsgruppenbezogenen Benachteiligungen negative Effekte auch direkt der räumlichen Konzentration zugeschrieben werden können. Die einzigen Studien, die eine eigenständige Wirkung von Konzentrationseffekten in sozial marginalisierten Stadtteilen für Deutschland umfassend untersucht haben, wurden von Dietrich Oberwittler ausgeführt (Oberwittler 2003; Oberwittler 2004; Oberwittler 2007). Oberwittler misst durch Daten des Statistischen Bundesamtes, der Polizei und durch flächendeckende Befragungen der Jugendlichen im Alter von 13 bis 16 Jahren in Köln und Freiburg den Einfluss der Konzentration von Armut auf delinquentes Verhalten von Jugendlichen. Er findet über die sich aus individuellen Faktoren ergebende Risikostruktur für Delinquenz (z.B. niedriger sozioökonomischer Status der Eltern, Migrationshintergrund) hinaus eine Erhöhung der Delinquenzwahrscheinlichkeit für Jugendliche, die ihren Alltag überwiegend in Stadtteilen mit Armutskonzentrationen verleben und deren FreundInnen aus diesem Stadtteil stammen. ${ }^{132}$ Dabei zeigt er auch, dass das Maß der sozialen Kontrolle diesen Prozess beeinflusst: Kennen die Eltern der Jugendlichen wiederum die Eltern der FreundInnen ihrer Kinder, sinkt bei Kontrolle einer Reihe sozioökonomischer und kultureller Variablen die Wahrscheinlichkeit, dass es von delinquenten Einstellungen zu delinquentem Verhalten kommt. Jugendliche, die nicht viel

\footnotetext{
${ }^{132}$ Oberwittler zeigt auch, dass diese sozialräumlichen Effekten der Wohngegend durch die Effekte der sozialen und ethnischen Zusammensetzung an den Schulen übertroffen werden. In Deutschland sind Schulen durchschnittlich stärker sozial und nach herkunftsgruppen segregiert als die Wohnviertel.
} 
Zeit in den betreffenden Stadttteilen verbringen, und deren FreundInnen in anderen Stadtteilen leben, weisen unter Kontrollbedingungen ebenfalls wesentlich weniger delinquentes Verhalten auf.

Dieser Abschnitt belegte den Rückgang von ,ko-ethnischen' Freundschaften von der ersten zur zweiten Generation, einen beachtlichen Prozentsatz an potenziell marginalisierten Personen sowie das Entstehen von ,Regenbogen'-Cliquen, deren Mitglieder in Stadtteilen mit einem erhöhten Anteil sozial marginalisierter Bevölkerung Elemente einer ,Oppositionskultur' aufweisen. Zudem wurden Belege für einen eigenständigen Effekt der Konzentration von Armut auf delinquentes Verhalten beschrieben. Können Freundschaften mit Mitgliedern der eigenen Herkunftsgruppe auch positive Effekte haben?

\subsubsection{Positive Konsequenzen ,ko-ethnischer' Freundschaften}

Wie die Auswirkung von Kenntnissen der Herkunftssprache oder einer Beschäftigung in der ,ethnischen Ökonomie' wird auch die Wirkung ,ko-ethnischer' Freundschaften kontrovers diskutiert. Verbessern sie das Familienklima und wirken sich positiv auf Selbstbild und Motivation der MigrantInnen der zweiten Generation aus oder behindern sie ein Vorankommen in Deutschland?

Hinsichtlich der Wirkung, ko-ethnischer' Freundschaften auf die Motivation zeigt sich in Tabelle 34 mit den Daten des DJI-Ausländersurveys, dass ein hoher Prozentsatz der FreundInnen der gleichen Herkunftsgruppe bei Kontrolle der sozioökonomischen Standardmerkmale positiv für die Selbstwirksamkeit ist. Selbstwirksamkeit wird über Zustimmung zu der Aussage „Erfolg hängt von der eigenen Leistung ab“ operationalisiert. MigrantInnen der zweiten Generation vertrauen ihrer eigenen Leistungsfähigkeit stärker, wenn sie einen höheren Anteil an FreundInnen der gleichen Herkunftsgruppe haben. Dies gilt unabhängig von der jeweiligen Gesamtzahl ihrer FreundInnen. Die Sprachkenntnisse sowohl im Deutschen als auch in der Herkunftssprache der Eltern sind in der Regression nicht signifikant. Der positive Effekt der ,ko-ethnischen' Freundschaften besteht besonders bei der türkischen Herkunftsgruppe.

In der nächsten Regression zeigt sich weiterhin, dass diese Form der Motivation sich positiv auf den Bildungs- und Berufsbildungsabschluss auswirkt, wenn die elterliche Bildung und die Zugehörigkeit zu Herkunftsgruppe und Geschlecht sowie die Sprachkenntnisse kontrolliert werden (Tabelle 35). Interaktionsvariablen mit den Herkunftsgruppen und dem Prozentsatz der ,ko-ethnischen' FreundInnen bzw. der Motivation waren in beiden Regressionen nicht signifikant. Bei einer direkten Regression des Prozentsatzes der ,ko-ethnischen’ FreundInnen 
auf die Bildungs- und Berufsergebnisse ist nun allerdings ein signifikanter negativer Effekt zu verzeichnen (Tabelle 36). Möglicherweise gibt es also eine dritte Variable, die alle drei Faktoren beeinflusst, etwa den sozioökonomischen Status im Freundeskreis.

Tabelle 34 - Auswirkungen Anteil FreundInnen der gleichen Herkunftsgruppe auf Selbstwirksamkeit

\begin{tabular}{|c|c|}
\hline & B (S.F.) \\
\hline ITAL- &,$- 19 * *(, 06)$ \\
\hline GRIE- &,$- 12 *(, 06)$ \\
\hline Beruf Eltern (in 6 Stufen) & ,00 n.s. $(, 02)$ \\
\hline Bildung Eltern (in 4 Stufen) &, $19 * *(, 04)$ \\
\hline Frauen $^{\circ}$ &,- 01 n.s. $(, 04)$ \\
\hline $\begin{array}{l}\text { Schriftliche Deutschkenntnisse } \\
\text { (in } 4 \text { Stufen) }\end{array}$ &, $16^{* *}(, 03)$ \\
\hline $\begin{array}{l}\text { Schriftliche Kenntnisse Her- } \\
\text { kunftssprache (in } 4 \text { Stufen) }\end{array}$ &,- 02 n.s. $(, 03)$ \\
\hline $\begin{array}{l}\text { Anteil FreundInnen gleiche Her- } \\
\text { kunftsgruppe }\end{array}$ &, $13 * *(, 05)$ \\
\hline Anzahl aller FreundInnen &,- 02 n.s. $(, 02)$ \\
\hline Konstante & 2,50 \\
\hline Korrigiertes $\mathbf{R}^{2}$ &, 05 \\
\hline
\end{tabular}

Koeffizienten (B) linearer Regression, Abhängige Variable Selbstwirksamkeit (in 4 Stufen)

$\left\ulcorner\right.$ Referenzkategorie: TÜRK; ${ }^{\circ}$ Referenzkategorie: Männer

** statistisch signifikant bei $\mathrm{p}<0.01,{ }^{*}$ statistisch signifikant bei $\mathrm{p}<0.05$, n.s. nicht signifikant

1997 des Deutschen Jugendinstituts, Eigene Berechnungen

Tabelle 35 - Auswirkungen Selbstwirksamkeit auf Bildungsabschluss (ISCED)

\begin{tabular}{|c|c|}
\hline & B (S.F.) \\
\hline ITAL- & -,10 n.s. $(, 09)$ \\
\hline GRIE- &,- 14 n.s. $(, 09)$ \\
\hline Beruf Eltern (in 6 Stufen) &, $07 * *(, 03)$ \\
\hline Bildung Eltern (in 4 Stufen) &, $78 * *(, 06)$ \\
\hline Frauen $^{\circ}$ &,- 10 n.s. $(, 07)$ \\
\hline $\begin{array}{l}\text { Schriftliche Deutschkenntnisse } \\
\text { (in } 4 \text { Stufen) }\end{array}$ &, $60 * *(, 06)$ \\
\hline $\begin{array}{l}\text { Schriftliche Kenntnisse Her- } \\
\text { kunftssprache (in } 4 \text { Stufen) }\end{array}$ &,- 06 n.s. $(, 05)$ \\
\hline Selbstwirksamkeit (in 4 Stufen) &, $16^{* *}(, 05)$ \\
\hline Konstante &, 05 \\
\hline Korrigiertes $\mathbf{R}^{2}$ &, 19 \\
\hline
\end{tabular}

Koeffizienten (B) linearer Regression, Abhängige Variable Bildungsabschluss (ISCED in 5 Stufen)

$\left\ulcorner\right.$ Referenzkategorie: TÜRK; ${ }^{\circ}$ Referenzkategorie: Männer

$* *$ statistisch signifikant bei $\mathrm{p}<0.01,{ }^{*}$ statistisch signifikant bei $\mathrm{p}<0.05$, n.s. nicht signifikant

1997 des Deutschen Jugendinstituts, Eigene Berechnungen 
Tabelle 36 - Auswirkungen Anteil FreundInnen der gleichen Herkunftsgruppe auf Bildungsabschluss (ISCED)

\begin{tabular}{|c|c|}
\hline & B (S.F.) \\
\hline ITAL- &,- 11 n.s. $(, 11)$ \\
\hline GRIE- &,- 05 n.s. $(, 11)$ \\
\hline Beruf Eltern (in 6 Stufen) & ,05 n.s. $(, 03)$ \\
\hline Bildung Eltern (in 4 Stufen) &, $81 * *(, 07)$ \\
\hline Frauen $^{\circ}$ &,- 05 n.s. $(, 09)$ \\
\hline $\begin{array}{l}\text { Schriftliche Deutschkenntnisse } \\
\text { (in } 4 \text { Stufen) }\end{array}$ &, $62 * *(, 07)$ \\
\hline $\begin{array}{l}\text { Schriftliche Kenntnisse Her- } \\
\text { kunftssprache (in } 4 \text { Stufen) }\end{array}$ &,- 08 n.s. $(, 06)$ \\
\hline $\begin{array}{l}\text { Anteil FreundInnen gleiche Her- } \\
\text { kunftsgruppe }\end{array}$ &,$- 33^{* *}(, 10)$ \\
\hline Anzahl aller FreundInnen &,- 02 n.s. $(, 02)$ \\
\hline Konstante &, 77 \\
\hline Korrigiertes $\mathbf{R}^{2}$ &, 17 \\
\hline
\end{tabular}

Koeffizienten (B) linearer Regression, Abhängige Variable Bildungsabschluss (ISCED in 5 Stufen)

- Referenzkategorie: TÜRK; ${ }^{\circ}$ Referenzkategorie: Männer

** statistisch signifikant bei $\mathrm{p}<0.01, *$ statistisch signifikant bei $\mathrm{p}<0.05$, n.s. nicht signifikant

1997 des Deutschen Jugendinstituts, Eigene Berechnungen

Frank Kalters Untersuchung hinsichtlich der Auswirkungen auf einen anderen sozioökonomischen Indikators, nämlich der Wahrscheinlichkeit, eine Angestellten- versus einer Arbeiterposition auf dem Arbeitsmarkt einzunehmen, zeigte keinen positiven Effekt für ein ,koethnisches' Freundesnetzwerk: Ein rein ,ko-ethnisches' Netzwerk hatte einen negativen Effekt auf die Wahrscheinlichkeit für eine Angestelltenposition; eine hohe Zahl von Deutschen ohne Migrationshintergrund unter den drei besten FreundInnen hingegen erhöhte signifikant die Chance auf eine Angestelltenposition und ließ die Benachteiligung der zweiten Generation türkischer Herkunft verschwinden. Auch ein gemischtes Freundesnetzwerk hatte im Vergleich zu einem nur aus Deutschen ohne Migrationshintergrund bestehenden Freundeskreis keinen positiven Effekt - allerdings auch keinen signifikant negativen. (Kalter 2004) Kalter geht jedoch nicht der Frage nach, ob Interaktionseffekte mit dem sozialen Status vorhanden sind: So könnte ein gemischtes oder ,ko-ethnisches' Netzwerk für niedrigqualifizierte MigrantInnen negativ, für höher qualifizierte aber positiv sein. Interessant ist in dieser Hinsicht, dass der Prozentsatz der MigrantInnen türkischer Herkunft, die keinen Kontakt zu deutschen MitschülerInnen ohne Migrationshintergrund haben, an Berliner Gymnasien und Hauptschulen gleichauf liegt, wobei dies einmal mit hohen und einmal mit niedrigen Bildungsleistungen zusammenfällt (Schneewind/Merkens 2001: 267). Eng damit verbunden ist die Frage der Ressourcen im Netzwerk. Denkbar wäre, dass sich hinter der ,ethnischen’ Beschreibung des 
Netzwerkes manchmal sozioökonomische Faktoren verbergen, so dass in ,ko-ethnischen' Netzwerken FreundInnen durchschnittlich aus einer niedrigeren sozialen Schicht kommen. Dies könnte über den Mangel an job-relevanten Informationen, Netzwerken und notwendigen Beziehungen negative Effekte dieser ,ko-ethnischen' Netzwerke erklären, ohne dass diese im engeren Sinne, ethnisch'-kulturell begründet wären. Um diese These zu belegen, müsste der sozioökonomische Status des Netzwerkes kontrolliert werden und der negative Effekt ,koethnischer' Netzwerke sich in dieser Kontrollbedingung verflüchtigen. Leider ist eine solche Überprüfung nicht möglich, da bisher in keiner Umfrage Bildungs- oder Berufsdaten zu den FreundInnen (oder deren Eltern) erhoben worden sind.

Dieser Abschnitt hat deutlich gemacht, dass der Anteil von PartnerInnen und FreundInnen der gleichen Herkunftsgruppe über die Generationen abnimmt. Dabei bestehen Unterschiede in den Herkunftsgruppen: Die türkische Herkunftsgruppe behält in der zweiten Generation die stärksten ,ko-ethnischen' Verbindungen. Zudem weist sie in der zweiten Generation den höchsten Anteil von PartnerInnen auf, die aus der gleichen Region im Herkunftsland stammen wie die Eltern der/s MigrantIn.

Nicht unerheblich ist bei allen MigrantInnengruppen der Anteil der Personen, die eine/n migrantische/n PartnerIn oder FreundIn anderer ,ethnischer' Herkunft haben, beispielsweise eine Migrantin türkischer Herkunft, die mit einer Migrantin jugoslawischer Herkunft befreundet ist. Diese Fälle wurden bisher noch nicht weiter untersucht. Auf der anderen Seite gibt es einen Prozentsatz an MigrantInnen, die unter den Personen, mit denen sie im Alltag zu tun haben, keine FreundInnen benennen können.

Schließlich scheint es in einigen urbanen Problemvierteln bei sozial deprivierten MigrantInnen die Tendenz zu geben, multiethnische Freundschaften mit anderen marginalisierten MigrantInnen (wie oben beschrieben) oder Deutschen ohne Migrationshintergrund zu unterhalten. Das Verbindende ist hier das Erleben von Chancenlosigkeit und das Gefühl, von den sozial Erfolgreicheren ausgeschlossen zu werden. Die bisher vorliegenden wissenschaftlichen Untersuchungen deuten dabei über individuelle Faktoren hinaus auf direkte negative Effekte der Konzentration von sozialer Benachteiligung hin.

,Ko-Ethnizität' fällt sowohl bei Partnerschaften als auch bei Freundschaften trotz einiger positiver Effekte (z.B. auf die Motivation) mit geringerem Status zusammen. Bei der Analyse der Integrationsmuster im nächsten Kapitel werde ich nun untersuchen, ob und inwieweit sich unter diesem durchschnittlich niedrigerem Wert unterschiedliche Wirkungen auf der Individualebene verbergen. 


\section{Empirie II: Individualebene der MigrantInnen - Integrations- muster}

In diesem Kapitel werden die aus den Theorien abgeleiteten Integrationsmuster für MigrantInnen der Altersgruppe von 20 bis 45 Jahren (Mikrozensus) und von 18 bis 25 Jahren (DJIAusländersurvey) untersucht. Aufgrund der Notwendigkeit, kulturelle Anpassung im Mikrozensus über die Partnerschaft zu operationalisieren, ist die Analyse auf MigrantInnen mit PartnerIn beschränkt. Wie ich im letzten Kapitel gezeigt habe, entstehen dadurch aber keine positiven Verzerrungen, da außer bei den ,westlichen' MigrantInnen aus NichtAnwerbestaaten und den Deutschen ohne Migrationshintergrund keine sozioökonomische Besserstellung der MigrantInnen mit PartnerIn vorliegt. Ein positiver Nebeneffekt ist, dass damit eine Gruppe untersucht wird, die in bisherigen Analysen wenig vorkam.

\subsection{Verteilung der Integrationsmuster}

\subsubsection{Operationalisierung}

Aus der aktuellen US-amerikanischen Theoriedebatte waren in Kapitel 2.4 drei Integrationsmuster herausgefiltert worden. Tabelle 1 beschrieb Indikatoren dieser Muster sowie eines Segregationsmusters für die vier Sachbereiche Sprache, Bildung, Beruf und Freund- bzw. Partnerschaften. Darauf aufbauend werden in diesem Abschnitt die vier Muster anhand der Variablen der beiden Datensets operationalisiert.

Wie in Abbildung 1 dargestellt, enthalten die Integrationsmuster zwei Achsen: eine der sozioökonomischen und eine der kulturellen Anpassung. Der sozioökonomische Status wird durch Bildung und Beruf der/s MigrantIn sowie durch das Bildungsniveau des/r PartnerIn erfasst. Der Einschluss der Bildungsabschlüsse der PartnerInnen ist sinnvoll, weil diese zu den familiären Ressourcen beitragen und dadurch den Lebensstil der untersuchten MigrantInnen beeinflussen. Da die im Ausländersurvey untersuchten Jugendlichen und jungen Erwachsenen sich zum großen Teil noch in der Ausbildung befinden, muss bei der Operationalisierung für den DJI-Ausländersurvey auf den Berufsindikator verzichtet werden. 
Kulturelle Anpassung auf der anderen Seite wird über die Sprachkenntnisse operationalisiert ${ }^{133}$ oder, wo dies nicht möglich ist, über den ,ko-ethnischen' Status der/s PartnerIn. Unter ,Ko-Ethnizität' wird dabei, wie im letzten Kapitel definiert, ein/e PartnerIn verstanden, der/die im Herkunftsland der Eltern geboren ist. Tabelle 37 verdeutlicht die Operationalisierung der Muster.

Tabelle 37 - Operationalisierung der Integrationsmuster

\begin{tabular}{|l|c|c|c|c|}
\hline & I & II & III & IV \\
\cline { 2 - 5 } & $\begin{array}{c}\text { KAT. ISCED } \\
\text { UND BLOSS- } \\
\text { FELD }\end{array}$ & STATUS & $\begin{array}{c}\text { PARTNERIN } \\
\text { GLEICHE HER- } \\
\text { KUNFTS-GRUPPE }\end{array}$ & $\begin{array}{c}\text { SCHRIFTLICHE } \\
\text { SPRACHKENNT- } \\
\text { NISSE }\end{array}$ \\
\hline Neoklassisch & $\geq 6$ & $\geq 8$ & $\begin{array}{c}\text { Nicht gleiche Her- } \\
\text { kunftsgruppe }\end{array}$ & Deutsch besser \\
\hline Pluralistisch & $\geq 6$ & $\geq 8$ & $\begin{array}{c}\text { Gleiche Herkunfts- } \\
\text { gruppe }\end{array}$ & $\begin{array}{c}\text { Herkunftssprache (der } \\
\text { Eltern) besser }\end{array}$ \\
\hline Abwärts & $\leq 5$ & $\leq 7$ & $\begin{array}{c}\text { Nicht gleiche Her- } \\
\text { kunftsgruppe }\end{array}$ & Deutsch besser \\
\hline Segregation & $\leq 5$ & $\leq 7$ & $\begin{array}{c}\text { Gleiche Herkunfts- } \\
\text { gruppe }\end{array}$ & $\begin{array}{c}\text { Herkunftssprache (der } \\
\text { Eltern) besser }\end{array}$ \\
\hline
\end{tabular}

Für die Analyse des Mikrozensus werden die Spalten I, II und III kombiniert ${ }^{134}$, im DJIAusländersurvey zum Vergleich die Spalten I und III sowie I und IV. Es wird also jedes Mal mindestens ein sozioökonomischer Indikator mit einer kulturellen Variable verbunden. Die Trenngröße (6 Punkte) von Spalte I folgt der aktuellen empirischen Ausbildungsforschung, welche gezeigt hat, dass ein abgeschlossener Hauptschulabschluss in Verbindung mit einer Lehre die Mindestanforderung für eine nicht-prekäre Partizipation auf dem Arbeitsmarkt darstellt (Solga 2005). Die Statusvariable in Spalte II umfasst den kategorisierten ISCED und den Blossfeld-Indikator der/s MigrantIn und den kategorisierten ISCED der/s PartnerIn. Diese Statusvariable erlaubt, die Bildung der/s PartnerIn einzurechnen, sie aber durch den Verbund mit den beiden anderen Variablen nicht genauso hoch $\mathrm{zu}$ bewerten wie die sozioökonomischen Merkmale der/s untersuchten MigrantIn selbst.

\footnotetext{
${ }^{133}$ Kulturelle Adaption liegt demnach vor, wenn die schriftlichen Deutschkenntnisse besser sind als die schriftlichen Kenntnisse der Sprache der Eltern. Sind die Kenntnisse in beiden Sprachen gleich gut, dann ist als Indikator der Sprachpraxis die Sprache ausschlaggebend, in der Unterhaltungssendungen meistens geschaut werden.

${ }^{134}$ In den wenigen Fälle, in denen anhand dieses Systems keine Zuordnung möglich ist, da Spalte I und II zu stark voneinander abweichen, kann ein fehlender Punkt beim Status (oder bei der ISCED/Blossfeld-Summe) durch eine erhöhte Punktzahl bei der anderen Variable ausgeglichen werden. Beträgt die Abweichung zwei Punkte oder mehr, wird der Fall der niedrigeren sozioökonomischen Gruppe und damit dem Abwärts- oder dem Segregationsmuster zugeschlagen.
} 


\subsubsection{Verteilung im Mikrozensus}

Wie sieht die Verteilung der Mustern im Mikrozensus aus? Tabelle 38 gibt diese ohne Trennung der Herkunftsgruppen wieder. Wie von der neoklassischen Integrationstheorie angenommen, ist das Muster des höheren sozioökonomischen Status und der kulturellen Adaption (= keine ,ko-ethnische' Beziehung) in der zweiten Generation stärker verbreitet als in der ersten und anderthalbten Generation. Der Umfang des umgekehrten Musters (Segregation) nimmt ab. Weiterhin existiert ein pluralistisches Muster, welches ,ko-ethnische' Partnerschaften mit gehobenem sozioökonomischem Status verbindet. Dieses ist zwar in der zweiten Generation weniger umfangreich als in der ersten, es findet sich aber dennoch bei $11 \%$ der MigrantInnen. Schließlich verbinden sich bei einem Viertel der MigrantInnen in der zweiten Generation ein niedriger sozioökonomischer Status mit einer bestimmten Form an kultureller Anpassung (Abwärts-Integration). Der Vergleich mit Deutschen ohne Migrationshintergrund zeigt, dass auch bei diesen ein Fünftel in diese sozioökonomisch problematische Gruppe fällt. $^{135}$

Tabelle 38 - Integrationsmuster: Generationen

\begin{tabular}{|l|c|c|c|c|}
\hline & $\begin{array}{c}\text { ZWEITE } \\
\text { GENERATION }\end{array}$ & $\begin{array}{c}\text { ANDERTHALBTE } \\
\text { GENERATION }\end{array}$ & $\begin{array}{c}\text { ERSTE } \\
\text { GENERATION }\end{array}$ & DTOM \\
\hline Neoklassisch & $46 \%$ & $41 \%$ & $27 \%$ & $80 \%$ \\
\hline Pluralistisch & $11 \%$ & $14 \%$ & $22 \%$ & - \\
\hline Abwärts & $27 \%$ & $25 \%$ & $26 \%$ & $20 \%$ \\
\hline Segregation & $16 \%$ & $20 \%$ & $25 \%$ & - \\
\hline
\end{tabular}

Mikrozensus 2005, FDZ der Statistischen Ämter des Bundes und der Länder, Eigene Berechnungen

Hinter diesem generationalen Muster können sich unterschiedliche Trends für die verschiedenen Herkunftsgruppen verbergen. Tabelle 39 schlüsselt daher die Verteilung der Integrationsmuster nach Herkunftsgruppen und Generationen auf. Die italienische, griechische und spanisch-portugiesische Herkunftsgruppe werden aufgrund der durch Berufs- und Partnerschaftsanforderung reduzierten Fallzahlen zusammen aufgeführt. Auf der rechten Seite der Tabelle sind die Ergebnisse für Männer und Frauen getrennt aufgeführt. Neoklassische Integration ist das vorherrschende Muster in der zweiten Generation: Es umfasst je nach Herkunftsgruppe zwischen einem Drittel und vier Fünftel der zweiten Generation.

\footnotetext{
135 Bei Personen ohne Migrationshintergrund wird automatisch von ausreichenden kulturellen Ressourcen ausgegangen. Dieses Pauschalurteil wird u.a. von neuen Bildungsstudien in Frage gestellt, die mangelnde Sprachkompetenzen im Deutschen auch bei Nicht-MigrantInnen aufzeigen (siehe Kapitel 3.3.1).
} 
Tabelle 39 - Integrationsmuster: Generationen, Herkunftsgruppen, Geschlechter

\begin{tabular}{|c|c|c|c|c|c|c|c|c|c|c|c|c|c|c|c|c|}
\hline & & \multicolumn{5}{|c|}{ MÄNNER UND FRAUEN } & \multicolumn{5}{|c|}{ FRAUEN } & \multicolumn{5}{|c|}{ MÄNNER } \\
\hline & & TÜRK & $\begin{array}{c}\text { ITAL, } \\
\text { GRIE, } \\
\text { SPAN } \\
\& \\
\text { PORT }\end{array}$ & $\begin{array}{c}\text { EX- } \\
\text { JUG }\end{array}$ & AUSS & WEST & TÜRK & $\begin{array}{l}\text { ITAL, } \\
\text { GRIE, } \\
\text { SPAN } \\
\& \\
\text { PORT }\end{array}$ & $\begin{array}{l}\text { EX- } \\
\text { JUG }\end{array}$ & AUSS & WEST & TÜRK & $\begin{array}{c}\text { ITAL, } \\
\text { GRIE, } \\
\text { SPAN } \\
\& \\
\text { PORT }\end{array}$ & $\begin{array}{c}\text { EX- } \\
\text { JUG }\end{array}$ & AUSS & WEST \\
\hline \multirow{4}{*}{ 窇 } & Neoklassisch & $32 \%$ & $56 \%$ & $48 \%$ & $73 \%$ & $77 \%$ & $32 \%$ & $54 \%$ & $49 \%$ & $66 \%$ & $67 \%$ & $31 \%$ & $59 \%$ & $47 \%$ & $79 \%$ & $87 \%$ \\
\hline & Pluralistisch & $14 \%$ & $5 \%$ & $18 \%$ & $2 \%$ & $7 \%$ & $13 \%$ & $5 \%$ & $16 \%$ & $2 \%$ & $11 \%$ & $16 \%$ & $5 \%$ & $20 \%$ & $2 \%$ & $3 \%$ \\
\hline & Abwärts & $27 \%$ & $33 \%$ & $23 \%$ & $23 \%$ & $16 \%$ & $32 \%$ & $38 \%$ & $24 \%$ & $30 \%$ & $22 \%$ & $23 \%$ & $28 \%$ & $23 \%$ & $17 \%$ & $10 \%$ \\
\hline & Segregation & $27 \%$ & $6 \%$ & $11 \%$ & $2 \%$ & - & $23 \%$ & $3 \%$ & $11 \%$ & $2 \%$ & - & $30 \%$ & $8 \%$ & $10 \%$ & $2 \%$ & - \\
\hline \multirow{4}{*}{ 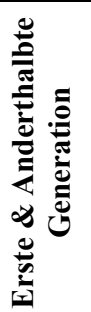 } & Neoklassisch & $14 \%$ & $24 \%$ & $23 \%$ & $26 \%$ & $60 \%$ & $16 \%$ & $24 \%$ & $21 \%$ & $26 \%$ & $58 \%$ & $12 \%$ & $25 \%$ & $25 \%$ & $26 \%$ & $64 \%$ \\
\hline & Pluralistisch & $8 \%$ & $12 \%$ & $19 \%$ & $29 \%$ & $23 \%$ & $7 \%$ & $10 \%$ & $16 \%$ & $25 \%$ & $22 \%$ & $9 \%$ & $15 \%$ & $24 \%$ & $34 \%$ & $25 \%$ \\
\hline & Abwärts & $44 \%$ & $31 \%$ & $28 \%$ & $17 \%$ & $13 \%$ & $45 \%$ & $39 \%$ & $34 \%$ & $21 \%$ & $16 \%$ & $40 \%$ & $18 \%$ & $20 \%$ & $12 \%$ & $8 \%$ \\
\hline & Segregation & $34 \%$ & $33 \%$ & $30 \%$ & $28 \%$ & $4 \%$ & $32 \%$ & $27 \%$ & $29 \%$ & $28 \%$ & $4 \%$ & $39 \%$ & $42 \%$ & $31 \%$ & $28 \%$ & $3 \%$ \\
\hline
\end{tabular}

Mikrozensus 2005, FDZ der Statistischen Ämter des Bundes und der Länder, Eigene Berechnungen 
Neben den MigrantInnen türkischer Herkunft (41\%) weisen die MigrantInnen, deren Eltern aus Ex-Jugoslawien stammen, den höchsten Prozentsatz ,ko-ethnischer' Partnerschaften auf (29\%). Allerdings gehen bei den MigrantInnen türkischer Herkunft in der zweiten Generation diese ,ko-ethnischen' Partnerschaften häufiger mit einem niedrigen sozioökonomischen Status einher als bei der ex-jugoslawischen Herkunftsgruppe (27\% statt 11\%). In der Herkunftsgruppe der AussiedlerInnen gibt es in der zweiten Generation die wenigsten ,ko-ethnischen' Partnerschaften. Dem Abwärts-Muster gehört ungefähr der gleiche Prozentsatz von Kindern von AussiedlerInnen, TürkInnen und Ex-JugoslawInnen an. Bei den MigrantInnen italienischer, griechischer, spanischer und portugiesischer Herkunft ist der Anteil der AbwärtsIntegration höher (33\%). Bezüglich der Geschlechterunterschiede ist festzuhalten, dass etwas mehr Frauen als Männer ein neoklassisches Muster zeigen (Ausnahme: ex-jugoslawische Herkunftsgruppe), annähernd gleiche Prozentsätze ein transnational-pluralistisches Muster und wesentlich weniger Frauen als Männer ein Muster der Abwärts-Integration. Auf der anderen Seite leben Frauen durchschnittlich häufiger segregiert als Männer, besonders Migrantinnen türkischer, italienischer, griechischer, spanischer und portugiesischer Herkunft.

In der Gegenüberstellung zur ersten und anderthalbten Generation fällt auf, dass in der zweiten Generation das neoklassische Muster wesentlich größer ausfällt, wohingegen das Muster der Segregation sich verringert. Hinsichtlich des pluralistischen Musters fällt der Vergleich gemischt aus: Während der Prozentsatz bei den Kindern der AussiedlerInnen, der ItalienerInnen, der GriechInnen, der SpanierInnen und PortugiesInnen und der , westlichen' MigrantInnen aus Nicht-Anwerbestaaten in der zweiten Generation sinkt, steigt er bei den MigrantInnen türkischer Herkunft und bleibt bei den Kindern der Ex-JugoslawInnen gleich. AbwärtsIntegration nimmt in der türkischen und ex-jugoslawischen Herkunftsgruppe in der zweiten Generation ab, steigt jedoch in der, westlichen' und der gemischten Herkunftsgruppe aus Südeuropa sowie bei den Kindern von AussiedlerInnen.

Während die Operationalisierung des sozioökonomischen Status für das in diesem Abschnitt beschriebene Integrationsmuster unproblematisch ist, da ISCED und Blossfeld weithin akzeptierte Standardindikatoren sind, bereitet die empirische Umsetzung des Konzepts kultureller Adaption mehr Schwierigkeiten. Sprachkenntnisse sind wegen ihrer generellen Veränderbarkeit ein guter Indikator für eine solche Anpassung. Leider werden sie im Mikrozensus nicht abgefragt. Da vor allem innerhalb von Familien kulturelle Traditionen gepflegt und kulturelle Ressourcen und Fähigkeiten erworben werden, wurden für die Analyse Beziehungsmuster als Proxy gewählt. Für MigrantInnen der ersten Generation, die schon im Herkunftsland in einer 
Partnerschaft lebten, führt diese Operationalisierung dazu, dass eine kulturelle Anpassung dann nur durch das Beenden der vorherigen Partnerschaft möglich wäre. Dieser Nachteil wird dadurch abgeschwächt, dass nur zwischen 6\% und 19\% der Migranten der verschiedenen Herkunftsgruppen und zwischen 23\% und 31\% der Migrantinnen zum Zeitpunkt der Zuwanderung nach Deutschland schon mit einer/m PartnerIn aus dem Herkunftsland verheiratet waren (Eigene Berechnungen, Scientific Use File Mikrozensus 2003).

\subsubsection{Verteilung im DJI-Ausländersurvey}

Um diesen kritischen Punkt genauer zu beleuchten, wurden im DJI-Ausländersurvey die Ergebnisse einmal mit einem sprachlichen und einmal mit einem partnerschaftlichen Kriterium gemessen. Dieser Vergleichs wird im Folgenden beschrieben, wobei sich zeigt, dass die Ergebnisse nahezu deckungsgleich sind (Tabelle 40).

Tabelle 40 - Integrationsmuster mit Kriterium Partnerschaft und Sprache

\begin{tabular}{|c|c|c|c|c|c|c|c|}
\hline & & \multicolumn{3}{|c|}{$\begin{array}{l}\text { KRITERIUM PART- } \\
\text { NERSCHAFT }\end{array}$} & \multicolumn{3}{|c|}{ KRITERIUM SPRACHE } \\
\hline & & TÜRK & ITAL & GRIE & TÜRK & ITAL & GRIE \\
\hline \multirow{4}{*}{ 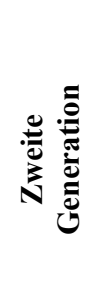 } & Neoklassisch & $23 \%$ & $49 \%$ & $47 \%$ & $26 \%$ & $48 \%$ & $46 \%$ \\
\hline & Pluralistisch & $13 \%$ & $5 \%$ & $7 \%$ & $10 \%$ & $5 \%$ & $8 \%$ \\
\hline & Abwärts & $30 \%$ & $33 \%$ & $32 \%$ & $31 \%$ & $36 \%$ & $34 \%$ \\
\hline & Segregation & $35 \%$ & $14 \%$ & $14 \%$ & $33 \%$ & $11 \%$ & $12 \%$ \\
\hline \multirow{4}{*}{ 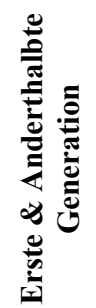 } & Neoklassisch & $9 \%$ & $25 \%$ & $16 \%$ & $6 \%$ & $19 \%$ & $8 \%$ \\
\hline & Pluralistisch & $11 \%$ & $17 \%$ & $15 \%$ & $14 \%$ & $22 \%$ & $24 \%$ \\
\hline & Abwärts & $23 \%$ & $19 \%$ & $21 \%$ & $14 \%$ & $13 \%$ & $14 \%$ \\
\hline & Segregation & $57 \%$ & $39 \%$ & $48 \%$ & $66 \%$ & $46 \%$ & $54 \%$ \\
\hline
\end{tabular}

Ausländersurvey 1997 des Deutschen Jugendinstituts, Eigene Berechnungen

In der zweiten Generation unterscheiden sich die Muster nur marginal, die größte Abweichung beträgt drei Prozentpunkte. In der ersten und anderthalbten Generation fallen die Unterschiede etwas größer aus. Hier sind die Muster des transnationalen Pluralismus und der Segregation im auf sprachlichen Kriterien basierenden Muster stärker vertreten als in dem, welches kulturelle Anpassung über die Herkunft der/s PartnerIn operationalisiert. Entsprechend fallen Abwärts-Integration und neoklassisches Muster geringer aus. Auf eine Besonderheit verweist das auf sprachlichen Kriterien basierende Muster: Der Anteil der pluralisti- 
schen Integration nimmt in allen Herkunftsgruppen über die Generationen ab. Nur bei der türkischen Herkunftsgruppe steigt er in dem anhand von Partnerschaft operationalisierten Mustern. In dem über Sprachkenntnisse konstruierten Muster stimmt der Trend im pluralistischen Bereich für die türkische Gruppe mit den anderen Herkunftsgruppen überein. Während also in den meisten Herkunftsgruppen die sprachliche Entwicklung mit der Partnerschaftswahl einhergeht, divergieren diese Indikatoren der kulturellen Anpassung bei der türkischen Herkunftsgruppe.

Darüber hinaus zeigen die Tabellen allgemeine Unterschiede zwischen der Verteilung der Muster mit den Daten des Mikrozensus und mit denen des DJI-Ausländersurveys. Diese Unterschiede sind vor allem der ungünstigeren sozioökonomischen Verteilung im DJIAusländersurvey geschuldet und haben nicht zuletzt mit dem niedrigen Alter der dort eingeschlossenen MigrantInnen zu tun. Schließlich fällt die kulturelle Anpassung im DJIAusländersurvey - unabhängig davon, ob sie über Partnerschaft oder über Sprachkenntnisse gemessen wird - geringer aus als im Mikrozensus. Insgesamt aber lassen sich in allen drei Operationalisierungen und bei beiden Datensets die gleichen Trends zwischen den Generationen und Herkunftsgruppen finden: Es gibt bei allen eine Entwicklung zu mehr neoklassischer Integration in der zweiten Generation und einen Rückgang der sozioökonomisch prekären Muster der Abwärts-Integration und der Segregation. Der Vergleich mit dem über die Sprachkenntnisse operationalisierten Muster sowie überhaupt mit den beiden Mustern im DJIAusländersurvey bestätigt also die Ergebnisse aus dem Mikrozensus und lässt die dort erzwungene Operationalisierung von kultureller Anpassung über die Herkunft der/s PartnerIn als tragfähige und robuste Lösung erscheinen.

Wie sieht es nun mit den von den Theorien gemachten Annahmen zu kausalen Zusammenhängen aus? Der nächste Abschnitt geht dieser Frage nach.

\subsection{Plausibilisierung der Muster}

Im Folgenden werden aus den in den Theorien postulierten Kausalmechanismen geeignete Variablen abgeleitet. Um die in den Theorien hergestellten Zusammenhänge zu plausibilisieren, werden in diesem Kapitel eine Reihe von bisher noch nicht untersuchten theorierelevanten Variablen betrachtet. Zunächst sollen jedoch die in den Theorien postulierten Kausalmechanismen wiederholt werden, um daraus geeignete Variablen abzuleiten.

Pluralistische Theorien gehen davon aus, dass sehr gute Kenntnisse in der Muttersprache der Eltern, ein großer Kreis von FreundInnen, die selbst oder deren Eltern aus dem gleichen Her- 
kunftsland stammen wie die eigenen Eltern, sowie enge Kontakte ins Herkunftsland der Eltern und/oder eine Erwerbstätigkeit in der, ethnischen Ökonomie' psychologische, soziale und finanzielle Ressourcen schaffen, die für eine Integration im Aufnahmeland positiv sind. Diese Ressourcen erlauben es, trotz kultureller Differenz einen nicht-prekären sozioökonomischen Status zu erlangen. Pluralistisch orientierten MigrantInnen ist ihre Familie wichtig, und die Beziehung zwischen Eltern und Kindern ist im Allgemeinen relativ funktional. Durch ein längeres Zusammenleben der Familienmitglieder, ist die Zahl der Angehörigen in pluralistischen Haushalten durchschnittlich höher. Die Teilnahme an ,ko-ethnischen' Vereinen ist hoch, und eine starke, zumeist multiple Identität bietet einen gewissen Schutz vor negativer Wahrnehmung von Diskriminierung.

Dagegen beschreibt die Abwärts-Integration Prozesse, in denen MigrantInnen der zweiten Generation, die aufgrund eines Rollentauschs in den isolierten Familien weder von ihren Eltern noch aus ,ko-ethnischen' Gruppen Unterstützung bekommen, sich stattdessen an anderen unterprivilegierten Jugendlichen in urbanen Problemkiezen orientieren. Dort entwickeln sie eine ,Oppositionskultur' gegen den ,Mainstream', die zum Gebrauch von Slang, geringer Leistungsmotivation und wenig Erfolg im institutionellen Bildungssystem führt. Sie werden Teil ,ethnisch' gemischter und sozial marginalisierter Unterschichten und von diesen als ähnlich wahrgenommen. Dieser Prozess betrifft Männer stärker als Frauen und Kinder von Alleinerziehenden stärker als MigrantInnen aus Familien mit zwei Elternteilen.

Gemäß der neoklassischen Integrationstheorie verbindet sich sozioökonomische Aufstiegsorientierung durchschnittlich mit einer Reihe von Anpassungsleistungen auf Seiten der MigrantInnen und einer veränderten Wahrnehmung von Differenzmarkern bei der Dominanzgesellschaft. Neoklassisch orientierte MigrantInnen sind motiviert und von ihrer Selbstwirksamkeit überzeugt, sie sprechen sehr gut Deutsch und ihr Freundeskreis besteht zu einem signifikanten Anteil aus Deutschen ohne Migrationshintergrund. Auch nehmen sie stärker an nichtmigrantischen als an ,ko-ethnischen' Vereinen teil. In ihren Augen überwiegen meritokratische Aspekte in der Gesellschaft, und sie nehmen relativ wenig Diskriminierung wahr. Im Bildungs- und Berufssystem erlangen sie nicht-prekären Status, was je nach Herkunft der Eltern häufig einen intergenerationalen Bildungsaufstieg impliziert.

Aufbauend auf diese kurze Zusammenfassung werden nun (folgende) theorierelevante Variablen analysiert: Neben Stadt-Land-Verteilung und Zahl der Haushaltsmitglieder interessieren das Bildungsverhältnis zwischen Kindern und Eltern sowie der Umfang der elterlichen Bildung. Im beruflichen Feld wird untersucht, ob sich hinsichtlich einer Beschäftigung im öffent- 
lichen Dienst und in der ,ethnischen' Ökonomie oder hinsichtlich einer Arbeitslosigkeit Unterschiede in den Durchschnittswerten für die Muster ergeben. Zusätzlich zur Sprachpraxis wird dann verglichen, ob in den Mustern Partnerschaften unterschiedlich stark transnational ausgerichtet sind und ob der Grad an Selbstwirksamkeit, Leistungsorientierung sowie die lokalen Identifizierungen voneinander abweichen. Dabei wird für die sprachlichen Variablen das auf Beziehungen basierende Muster verwendet, da sonst Tautologien entstünden. Auch eng auf die berufliche Position bezogene Variablen werden nur für das DJI Muster untersucht, bei dem keine Berufsvariablen eingeflossen sind.

Im Folgenden sollen diese Variablen, die in den von den Theorien beschriebenen Integrationsprozessen eine Rolle spielen, auf ihre Verteilung über die Muster untersucht werden. Es wird ausschließlich die zweite Generation in den beiden Altersgruppen (20 bis 45 Jahre im MZ, 18 bis 25 Jahre im DJI-Ausländersurvey) betrachtet, da diese im Zentrum des theoretischen Interesses steht. Während bisher die statische Verteilung der MigrantInnen auf die Muster betrachtet wurde, geht es also nun um die Plausibilisierung der theoretischen Annahmen über die Zusammenhänge im Muster. Bisher waren die Fragen: Wie verändert sich die Verteilung der Integrationsmuster über die Generationen? Bestehen in der Verteilung Unterschiede zwischen Männern und Frauen sowie zwischen den Herkunftsgruppen? Nun geht es darum, ob es in den erklärenden Variablen signifikante Abweichungen zwischen den vier Musterausprägungen gibt. Dies würde darauf hindeuten, dass die von den Theorien beschriebenen Prozessmechanismen tatsächlich wirken. Sicherheit in dieser Frage ließe sich nur durch Regressionen gewinnen, die für die drei Integrations- und das eine Segregationsmuster getrennt berechnet werden müssten, da die Muster nicht eindimensional in einer Hierarchie angeordnet werden können. Alternativ könnte eine multinominale logistische Regression durchgeführt werden (Andreß, et al. 1997). Gegen ein solches Vorgehen spricht jedoch, dass eine Reihe der wichtigen Variablen für die Regressionen gar nicht (z.B. sozioökonomischer Status des sozialen Netzwerkes, Familienbeziehungen) oder kaum (Indikatoren für ,ethnische Ökonomie', transnationale Verbindungen) in den Datensets vorhanden sind. Zudem müsste ein ernsthafter Theorietest wegen des Prozesscharakters der Theorien im Längsschnittverlauf stattfinden. Beides spricht für eine qualitative Analyse, um Daten in ausreichender Qualität und Tiefe zu gewinnen. Da eine solch umfangreiche empirische Untersuchung für diese Arbeit nicht möglich war, beschränkt sich das folgende Kapitel auf eine Plausibilisierung oder (vorläufige) Zurückweisung der Mechanismen der Muster. Hierfür sind Balkendiagramme für die nichtstetigen und Fehlerbalken für die stetigen Variablen gut geeignet, da sie signifikante Abweichungen auch grafisch aufbereiten. 
In den Fehlerbalken werden die Durchschnittswerte einer Variablen und das 95\% Konfidenzintervall um den Durchschnittswert als Balken in einer Grafik dargestellt. Der Balken umfasst somit den Wertebereich, in dem sich mit 95\% Wahrscheinlichkeit der wahre Durchschnittswert der Gesamtpopulation (nicht der Stichprobe) befindet. Die Länge des Fehlerbalkens hängt von der Varianz in der Stichprobe und dem Umfang der Stichprobe ab: Je geringer die Streuung ist und je mehr Fälle die Stichprobe enthält, umso kleiner ist das Konfidenzintervall. In diesem Fall kann der tatsächliche Durchschnittswert in der Gesamtpopulation also recht genau durch die Daten der Stichprobe bestimmt werden. Überschneiden sich die Fehlerbalken der verschiedenen Muster nicht, dann kann mit einer Fehlerwahrscheinlichkeit von 5\% $(\alpha=0,05)$ davon ausgegangen werden, dass die Durchschnittswerte in den Mustern bei der Gesamtpopulation signifikant voneinander abweichen. Überschneiden sie sich in Teilen, erhöht sich entsprechend die Wahrscheinlichkeit einer fehlerhaften Aussage über eine signifikante Abweichung.

Zunächst sollen sozialstrukturelle Standardvariablen wie Geschlecht, Alter und Stadt-LandVerteilung betrachtet werden. Danach folgen theorierelevante Variablen aus den Sachbereichen und abschließend Einstellungsmaße. Zuweilen führen die Daten des Mikrozensus und die des DJI-Ausländersurveys zu unterschiedlichen Ergebnissen. Es muss jedoch berücksichtigt werden, dass die Variablen im Mikrozensus und im DJI-Ausländersurvey zwar vergleichbar, aber nicht identisch sind. Die gestellten Fragen weichen z.T. in entscheidenden Wörtern voneinander ab. Zudem weisen die befragten Personen in den beiden Datensets signifikante Unterschiede auf: Die MigrantInnen der zweiten Generation im DJI-Ausländersurvey sind deutlich jünger. Hier fehlen die sozioökonomischen Spitzen nach unten und oben. Der Datensatz umfasst zudem nur Personen italienischer, griechischer und türkischer Herkunft, während im MZ weitere Herkunftsgruppen enthalten sind. Gehen die Trends in die gleiche Richtung, sind die Ergebnisse daher befriedigend, auch wenn es Unterschiede in den exakten Zahlen gibt. Entsprechen sich die Trends genau, wurde zugunsten der Übersichtlichkeit auf eine doppelte Darstellung verzichtet und es wurden die Ergebnisse nur eines Datensatzes dargestellt.

Hinsichtlich der Geschlechterverteilung ließen die Theorien mehr Männer als Frauen im Muster der Abwärts-Integration erwarten, und Abbildung 15 bestätigt dies. Frauen stellen entsprechend einen höheren Prozentsatz bei den anderen Mustern. 


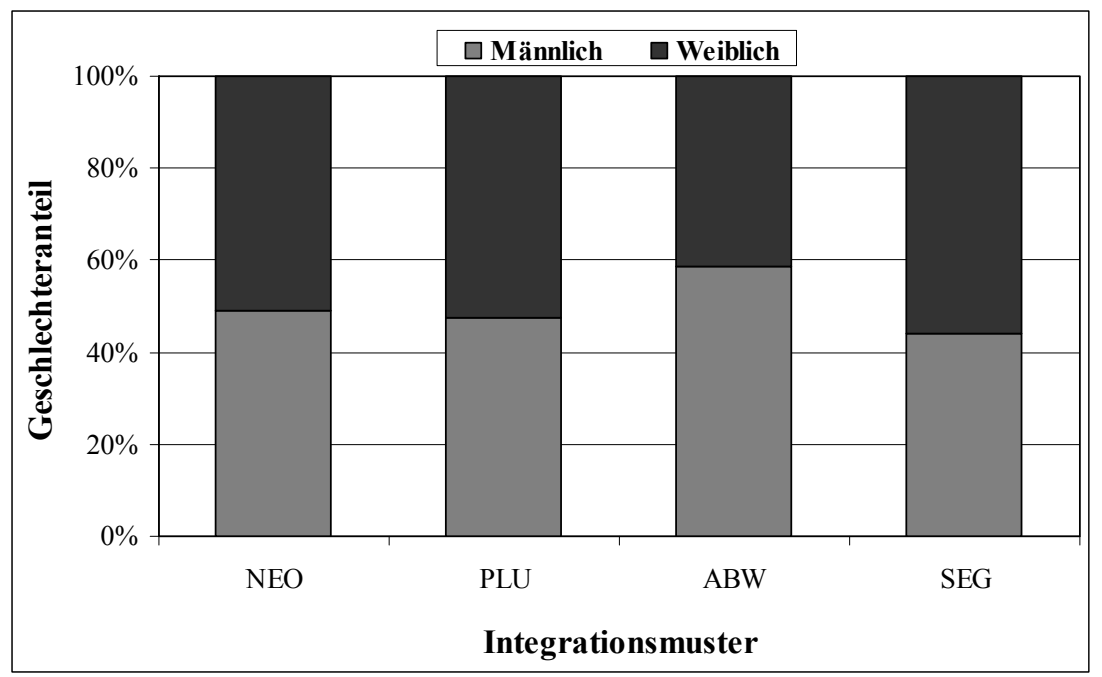

Mikrozensus 2005, FDZ der Statistischen Ämter des Bundes und der Länder, Eigene Berechnungen Abbildung 15 - Musteranalyse: Geschlechterverhältnis

Das Alter der MigrantInnen der zweiten Generation in den vier Mustern ist ungefähr gleich (Abbildung 16), der maximale Altersunterschied beträgt ein Jahr.

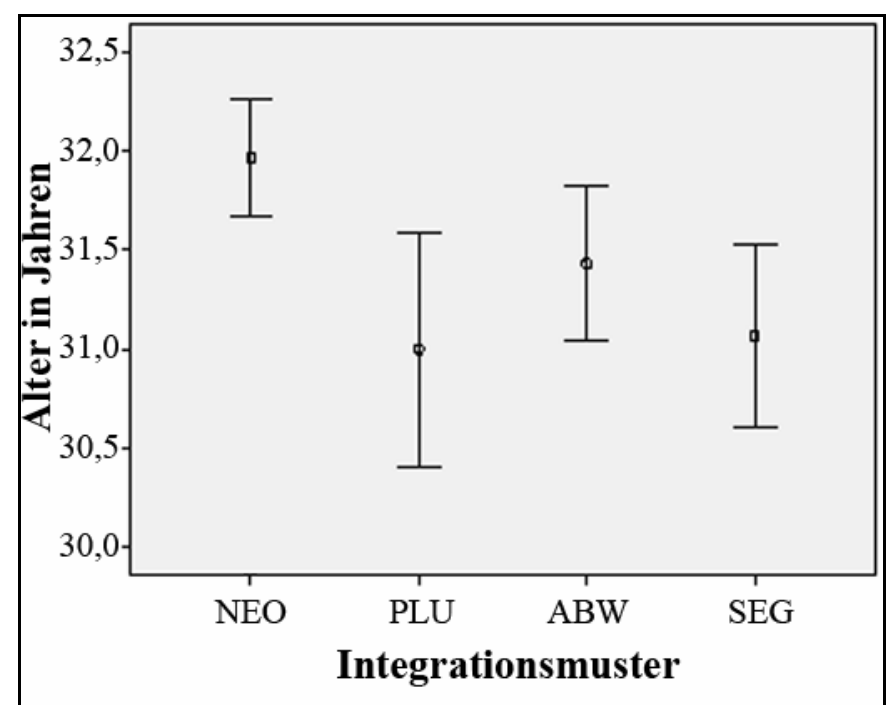

Mikrozensus 2005, FDZ der Statistischen Ämter des Bundes und der Länder, Eigene Berechnungen Abbildung 16 - Musteranalyse: Alter

70\% der MigrantInnen der zweiten Generation leben in großstädtischen, 30\% in ländlichen und kleinstädtischen Räumen. Nur das Muster der Abwärts-Integration weicht hiervon ab. Bei diesem leben mehr MigrantInnen in kleinstädtischer und ländlicher Umgebung. Dies widerspricht der Abwärts-Integrationstheorie, die vor allem MigrantInnen in urbanen Problemvierteln für gefährdet hält. (Abbildung 17) 


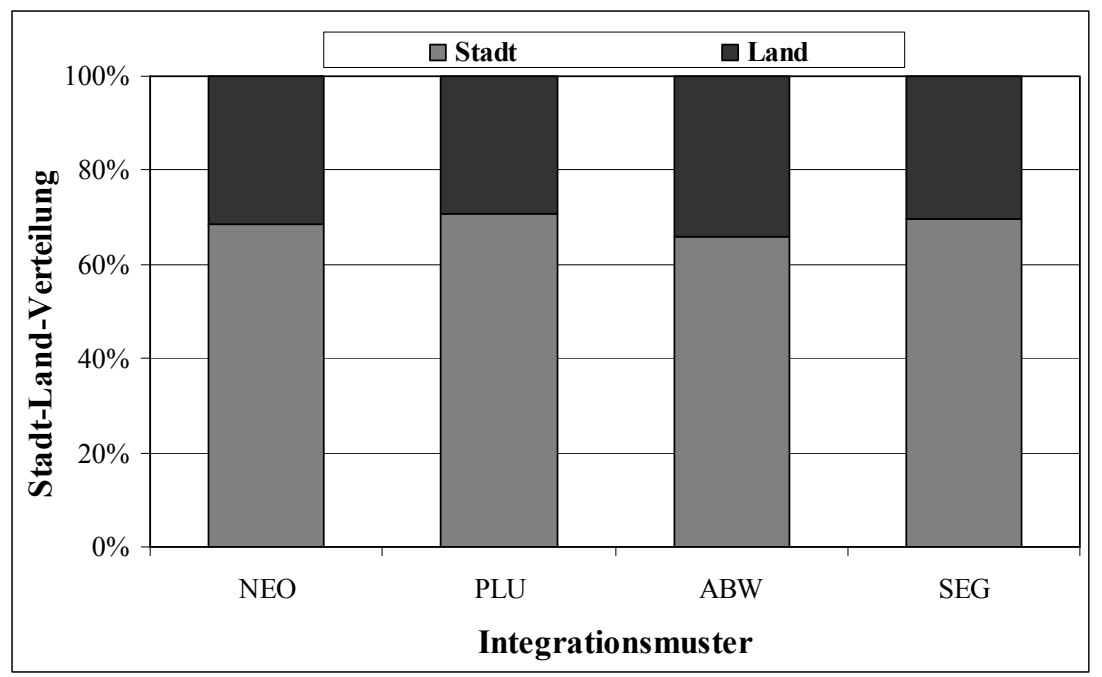

Mikrozensus 2005, FDZ der Statistischen Ämter des Bundes und der Länder, Eigene Berechnungen

Abbildung 17- Musteranalyse: Stadt-Land-Verteilung, MZ

Im DJI-Ausländersurvey fallen die Unterschiede zwischen den Mustern geringer aus als im Mikrozensus, nur Mitglieder des pluralistischen Musters leben durchschnittlich häufiger in städtischen Räumen. (Abbildung 18)

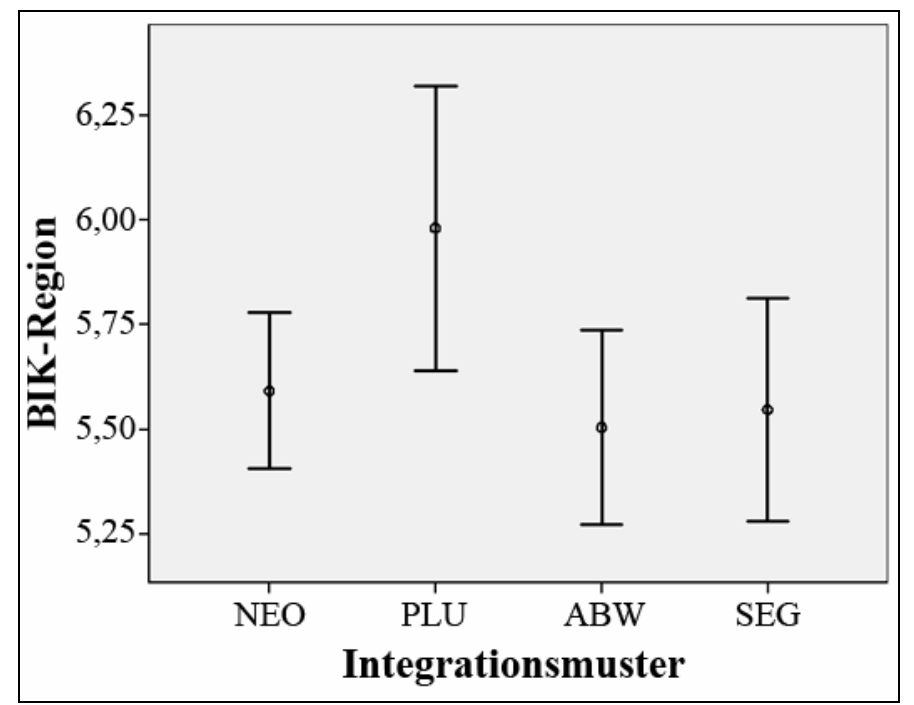

Ausländersurvey 1997 des Deutschen Jugendinstituts, Eigene Berechnungen

Abbildung 18 - Musteranalyse: Stadt-Land-Verteilung, DJI-Ausländersurvey

Die Frage der Staatsbürgerschaft wird in den U.S.-amerikanischen Integrationstheorien nicht explizit angesprochen. Dennoch mag ein Blick darauf aufgrund der jahrelang sehr hohen Hürden für den Erwerb der deutschen Staatsbürgerschaft interessant sein (Abbildung 19). Neben der Gruppe der AussiedlerInnen, die qua Definition deutsche StaatsbürgerInnen sind, weist die türkische Herkunftsgruppe mit Abstand den größten Anteil deutscher StaatsbürgerInnen auf. Wider Erwarten fällt der Anteil der deutschen StaatsbürgerInnen allerdings nicht für das Muster der neoklassischen Integration (46\%) besonders hoch aus, sondern für das plu- 
ralistische Muster (56\%). Die Gründe hierfür lassen sich selbstverständlich nicht aus der Grafik ablesen. Da türkischen Staatsangehörigen im Gegensatz zu EU-Bürgern eine Reihe von Möglichkeiten in Deutschland verschlossen sind, kann jedoch vermutet werden, dass ein Teil der Einbürgerungen mit dem pragmatischen Ergreifen von Chancen zu tun hat. Der Anteil der Personen mit doppelter Staatsbürgerschaft wird in der Grafik nicht extra ausgewiesen, da er in keinem Muster ein Prozent überschreitet.

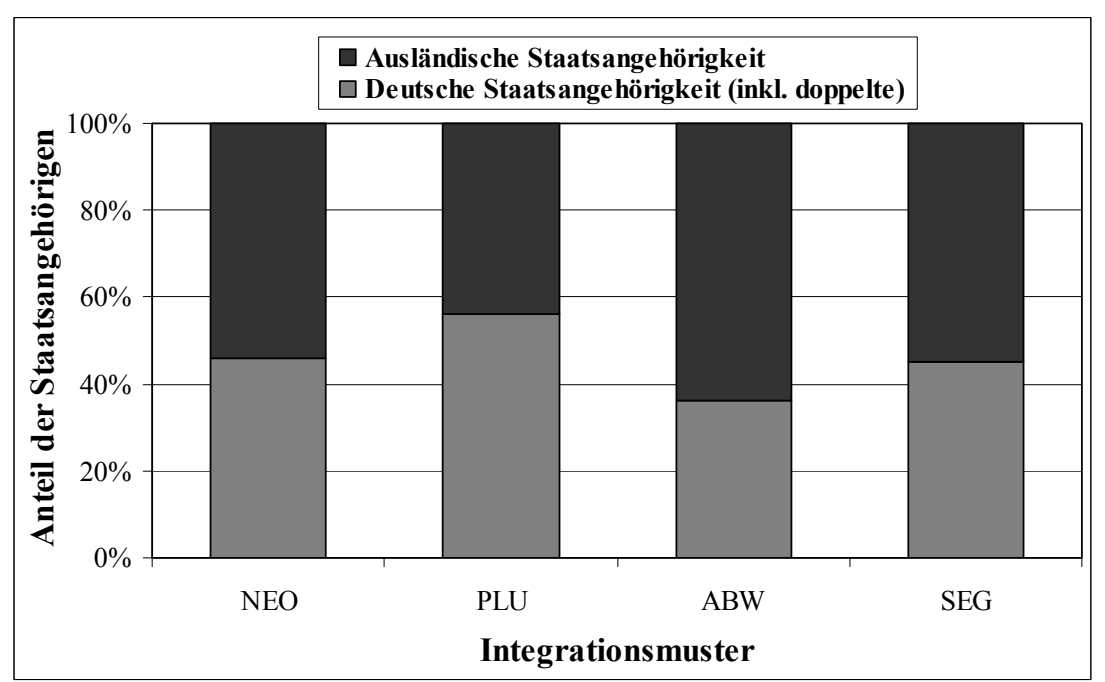

Mikrozensus 2005, FDZ der Statistischen Ämter des Bundes und der Länder, Eigene Berechnungen Abbildung 19 - Musteranalyse: Staatsbürgerschaften nur für türkische Herkunftsgruppe

Im Mikrozensus gibt es auch Angaben zur Anzahl der Haushaltsmitglieder (und damit in den meisten Fällen verbunden zur Zahl der Kinder). Abbildung 20 zeigt, dass die Zahlen über die Muster steigen: von durchschnittlich drei Haushaltsmitgliedern im neoklassischen Muster über 3,3 im pluralistischen auf 3,4 im Muster der Abwärts-Integration und 3,6 bei der Segregation. Dem entsprechen durchschnittliche Kinderzahlen von 0,9 pro Haushalt für das neoklassische Muster, 1,3 für das pluralistische Muster, 1,4 für die Abwärts-Integration und 1,5 für das segregierte Muster. 


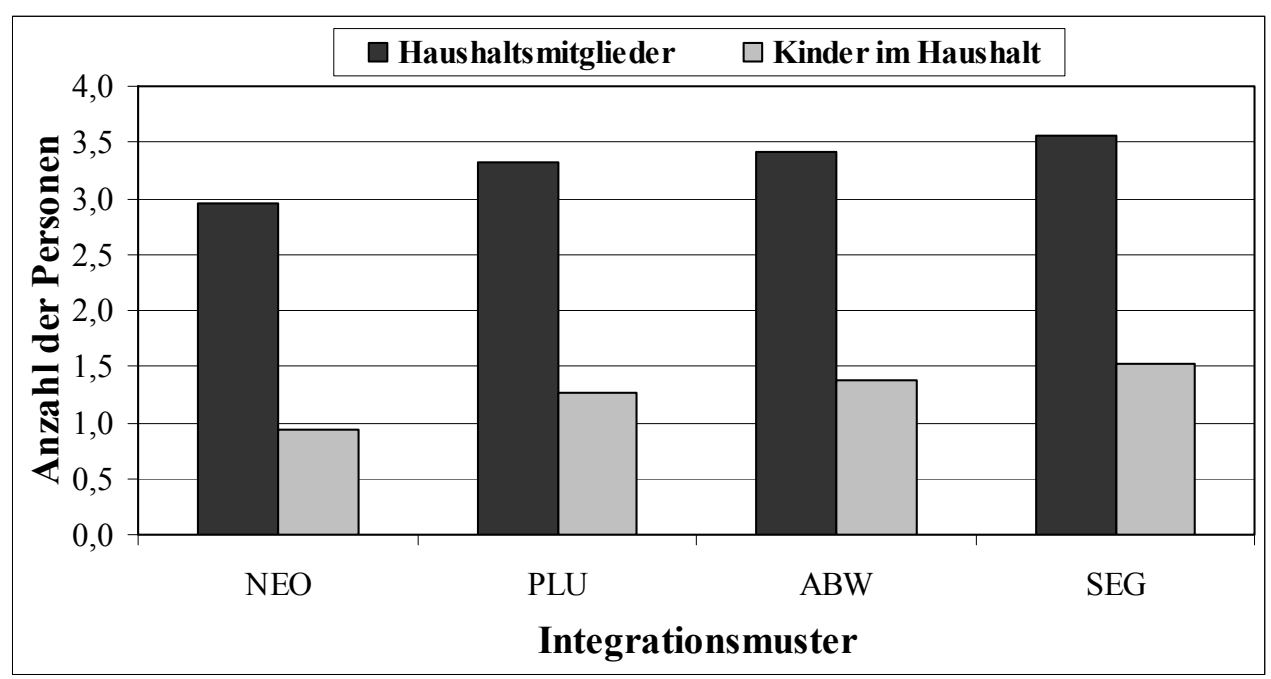

Mikrozensus 2005, FDZ der Statistischen Ämter des Bundes und der Länder, Eigene Berechnungen

Abbildung 20 - Musteranalyse: Haushaltsmitglieder und Kinder im Haushalt

Im Hinblick auf die Verteilung sprachlicher Variablen ${ }^{\mathbf{1 3 6}}$ verdeutlicht Abbildung 21 die Sprachpraxis am Beispiel von Unterhaltungssendungen. Die Durchschnittswerte bestätigen die Theorien: Bei Personen in Mustern, die keine kulturelle Anpassung enthalten, steigt der Anteil derjenigen, die die Sendungen häufiger in der Sprache der Eltern anschauen. Entsprechend werden Unterhaltungssendungen in Deutsch häufiger von MigrantInnen in der neoklassischen Integration und der Abwärts-Integration angesehen - den Mustern, die eine kulturelle Anpassung abbilden. Unabhängig von diesen Mustern werden in der zweiten Generation insgesamt Sendungen stärker auf Deutsch als in der Herkunftssprache angeschaut (Durchschnittswert größer 3).

Nun wäre es zu erwarten, dass die schriftlichen Kenntnisse im Deutschen dieser Sprachpraxis entsprechen. Wie in Abbildung $22 \mathrm{zu}$ sehen ist, schließen hier jedoch die MigrantInnen im Muster der pluralistischen Integration mit denen in den kulturell angepassten Mustern auf. Ihre schriftlichen Deutschkenntnisse entsprechen denjenigen der kulturell angepassten Abwärts-Integrierten ${ }^{137}$, hinter denen Segregierte klar zurückbleiben. Für die höher Qualifizierten bestimmt die Praxis also nicht die Kenntnisse. Bei den schriftlichen Kenntnissen in der Herkunftssprache fallen die Unterschiede geringer aus, und ein deutlicher Vorteil besteht theoriekonform - nur für die Gruppe der pluralistisch Integrierten. Auch hier bewegen sich die schriftlichen Kenntnisse auf einem hohen, nämlich durchschnittlich mindestens guten Niveau (Abbildung 23).

\footnotetext{
${ }^{136}$ Die Analyse wird mit dem Muster durchgeführt, das anhand der Partnerschaftsbeziehung konstruiert wurde. Mit dem auf den Sprachkenntnissen basierenden Muster wäre eine solche Analyse redundant.

${ }^{137}$ Hierbei handelt es sich ebenso wie bei dem Wort ,Segregierte' um sprachliche Verkürzungen zur Bezeichnung der Personen, die ein bestimmtes Integrationsmuster aufweisen. Sie dienen der Übersichtlichkeit und reklamieren keinesfalls einen umfassenden ontologischen Status.
} 


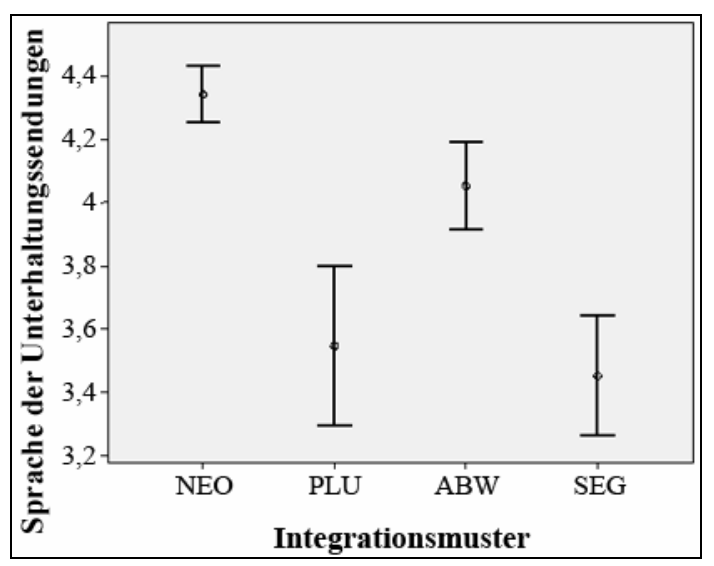

Abbildung 21 - Musteranalyse: Sprachpraxis durch Unterhaltungssendungen

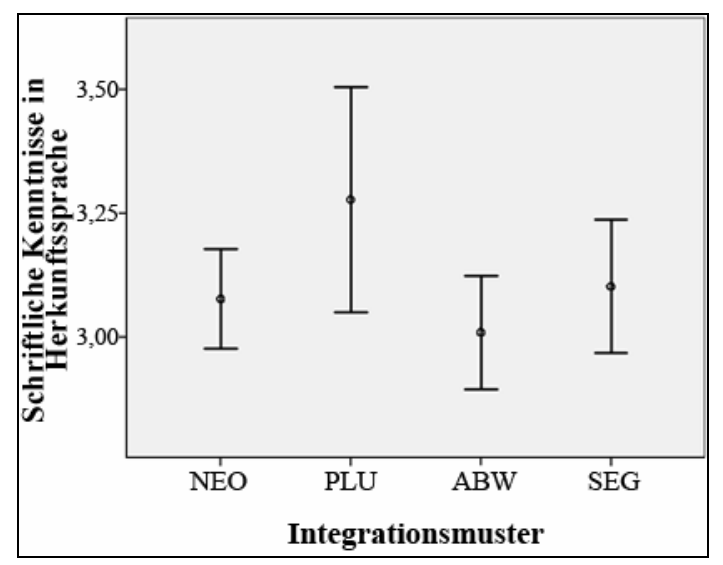

Abbildung 23 - Musteranalyse: Schriftliche Kenntnisse in Herkunftssprache

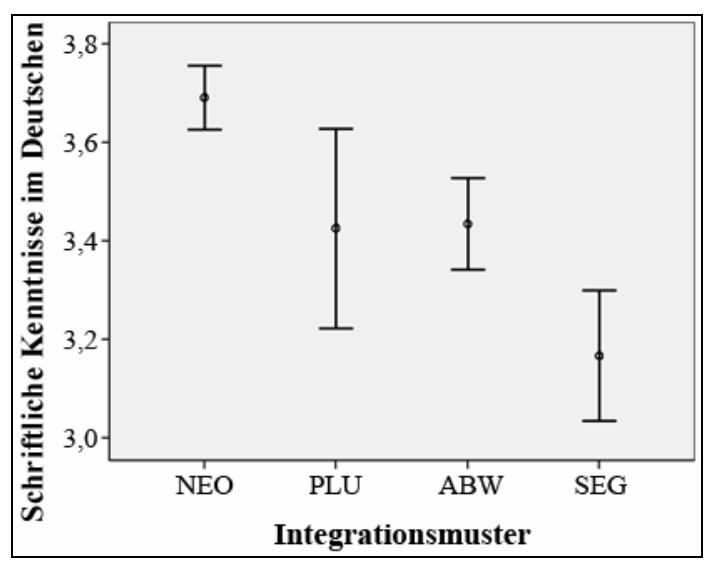

Abbildung 22 - Musteranalyse: Schriftliche Kenntnisse im Deutschen

Abbildung 21: Skala von 1 „Nur italienisch-, griechisch-, türkisch-sprachige Unterhaltungssendungen“ bis 5 „Nur deutschsprachige Unterhaltungssendungen"

Abbildung 22: Skala von 1 ,gar nicht“ bis 4 ,sehr gut"،

Abbildung 23: Skala von 1 ,gar nicht“ bis 4 ,sehr gut $^{6 *}$

Ausländersurvey 1997 des Deutschen Jugendinstituts, Eigene Berechnungen

Im Bereich der Bildung, stellt Abbildung 24 das Bildungsverhältnis vom Kind zum Vater dar. Das Verhältnis zur Mutter wurde außer Acht gelassen, da fast alle Kinder höher qualifiziert sind als ihre Mütter. Bildungsabstieg ist kaum zu finden, er bleibt in allen Mustern unter 4\%. Es gibt also den von der Abwärts-Integration befürchteten Bildungsabstieg nicht in signifikantem Maße. 


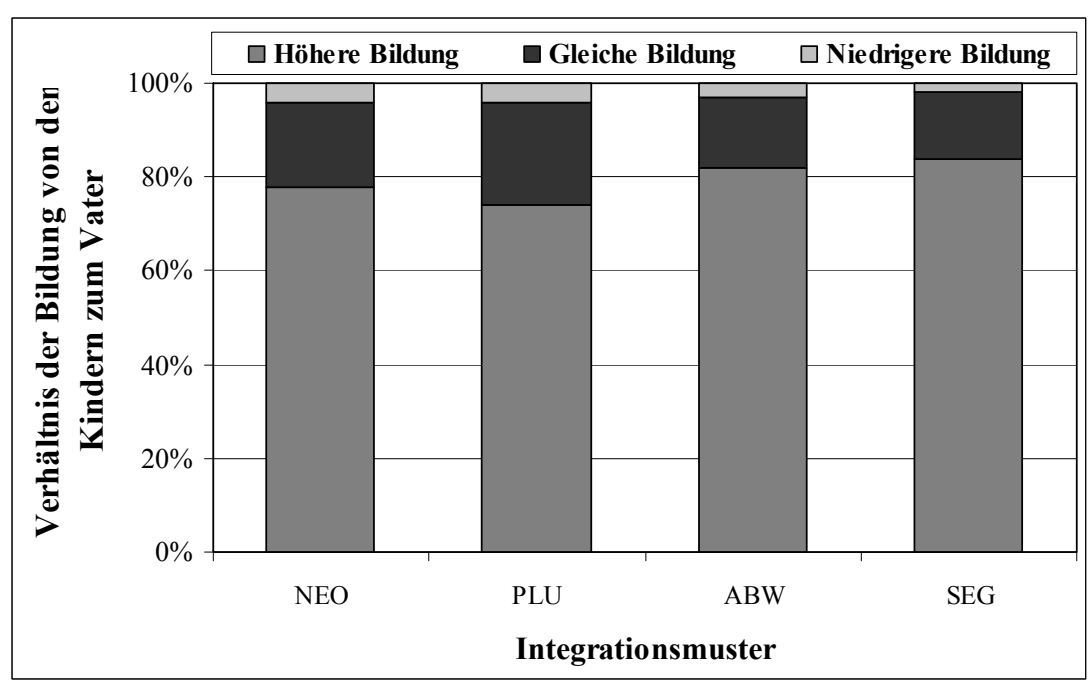

Ausländersurvey 1997 des Deutschen Jugendinstituts, Eigene Berechnungen

Abbildung 24 - Musteranalyse: Verhältnis der Bildung von den Kindern zum Vater

Die Fehlerbalken zu den Schuljahren des Vaters (Abbildung 25) zeigen neben zwei erwartbaren Ergebnissen eine Überraschung: Während Kinder im neoklassischen Muster Väter mit signifikant mehr Schuljahren haben und Kinder im segregierten Muster Väter mit signifikant weniger Schuljahren, liegen die Väter der Kinder sowohl im pluralistischen als auch im Abwärts-Muster hinsichtlich der Schuljahre gleichauf. Die Bildung der MigrantInnen der zweiten Generation ist in diesen beiden Gruppen jedoch sehr unterschiedlich. Hier müssen also, ganz theoriekonform, Faktoren gewirkt haben, die bei durchschnittlich gleichen Startbedingung in Bezug auf die väterliche Bildung zu divergierenden Ergebnissen für die Kinder geführt haben.

Für die berufliche Position wird in Abbildung 26 der aus den Theorien erwartbare Zusammenhang deutlich. Die DJI-Muster, in welche nur die Bildung der/s MigrantIn und der/s PartnerIn eingeflossen war, zeigen signifikant höhere Werte für den beruflichen Status im neoklassischen und im pluralistischen Muster und niedrigere für Abwärts-Integration und Segregation. 


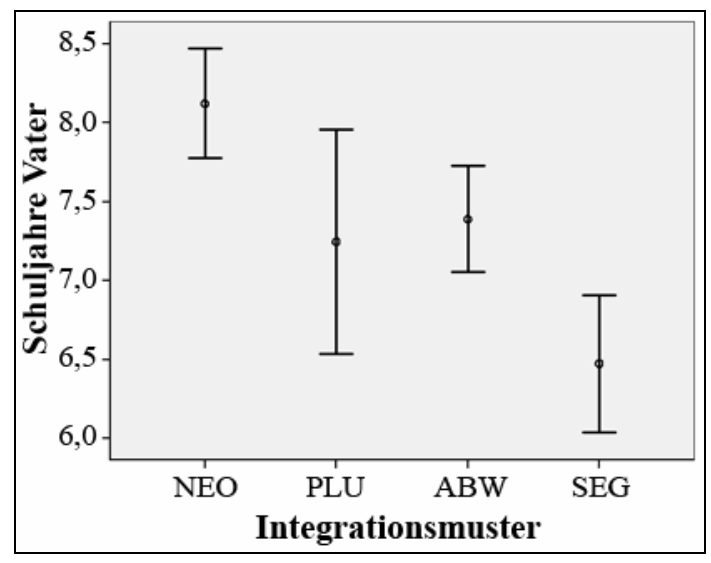

Ausländersurvey 1997 des Deutschen Jugendinstituts, Eigene Berechnungen

Abbildung 25 - Musteranalyse: Schuljahre des Vaters

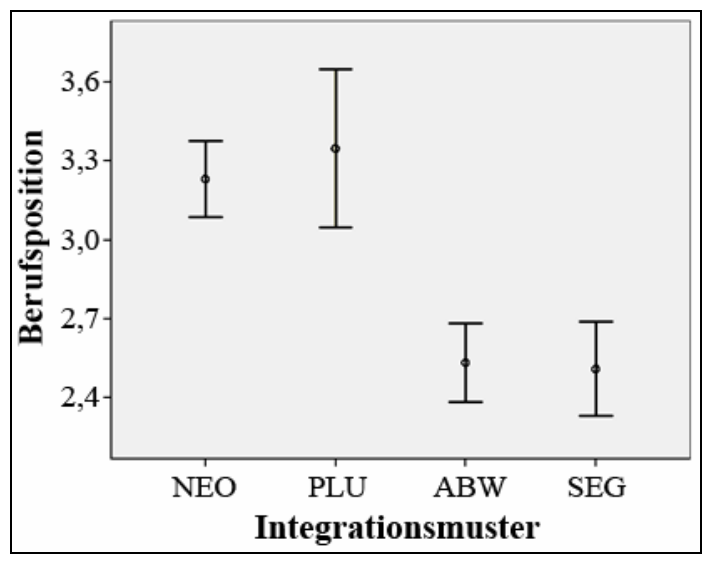

Ausländersurvey 1997 des Deutschen Jugendinstituts, Eigene Berechnungen

Abbildung 26 - Musteranalyse: Berufliche Position

Ähnlich sieht es bezüglich einer Beschäftigung im öffentlichen Dienst aus (Abbildung 27):

Auch hier zeigen sich höhere Prozentsätze (12\% und 13\%) für die ersten beiden Muster, niedrigere für das dritte und vierte Muster (5\% und 3\%).

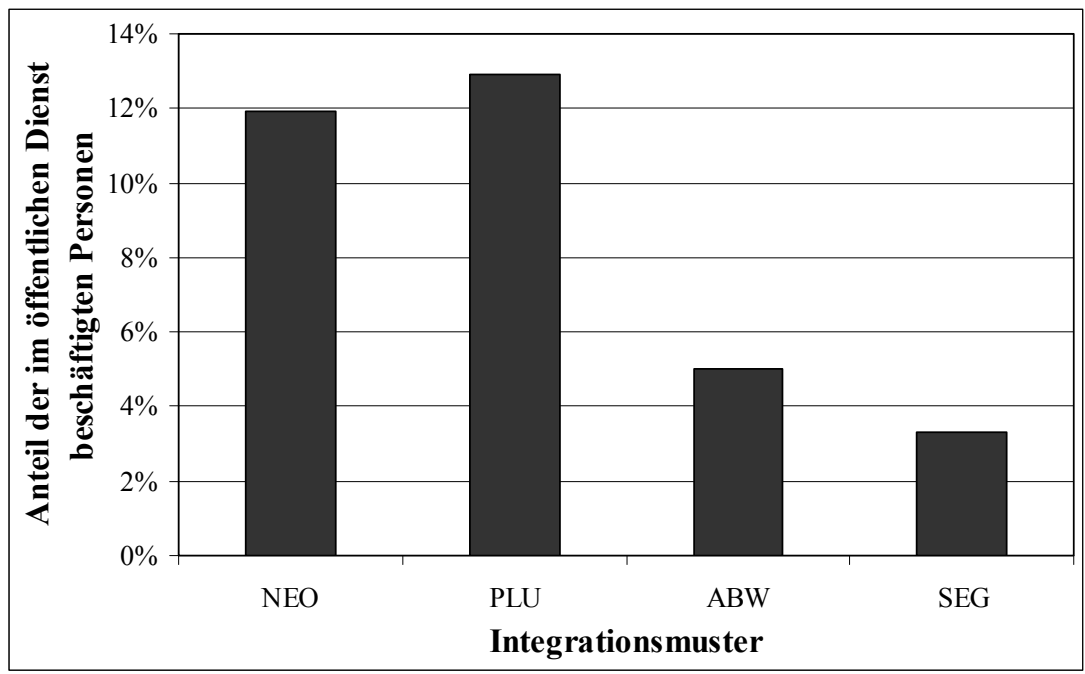

Mikrozensus 2005, FDZ der Statistischen Ämter des Bundes und der Länder, Eigene Berechnungen Abbildung 27 - Musteranalyse: Beschäftigung im öffentlichen Dienst

Arbeitslosengeld I oder II als Beispiel für staatliche Transferleistungen beziehen in den ersten beiden Mustern nur 3\% bzw. 4\%, jedoch 24\% der Abwärts-Integrierten und 26\% der Segregierten (Abbildung 28). 


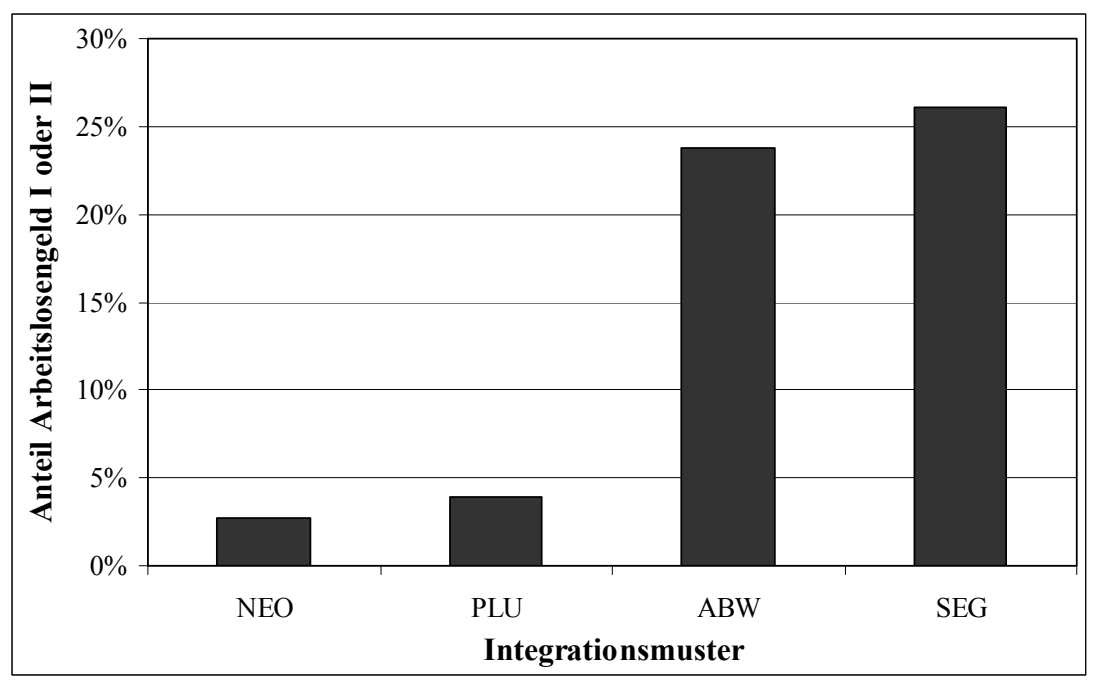

Mikrozensus 2005, FDZ der Statistischen Ämter des Bundes und der Länder, Eigene Berechnungen Abbildung 28 - Musteranalyse: Bezug von Arbeitslosengeld

Auch die Dauer der Arbeitslosigkeit bei Personen, die tatsächlich arbeitslos sind oder waren, unterscheidet sich signifikant: Während Abwärts-Integrierte und Segregierte durchschnittlich neun bis elf Monate arbeitslos waren, liegt die Dauer im neoklassischen und im pluralistischen Muster bei durchschnittlich fünf Monaten (Abbildung 29). Dies belegt nicht nur die erhöhte Wahrscheinlichkeit der beiden ersten Gruppen, arbeitslos zu werden, sondern auch die größere Schwierigkeit, wieder in Beschäftigung zurückzufinden.

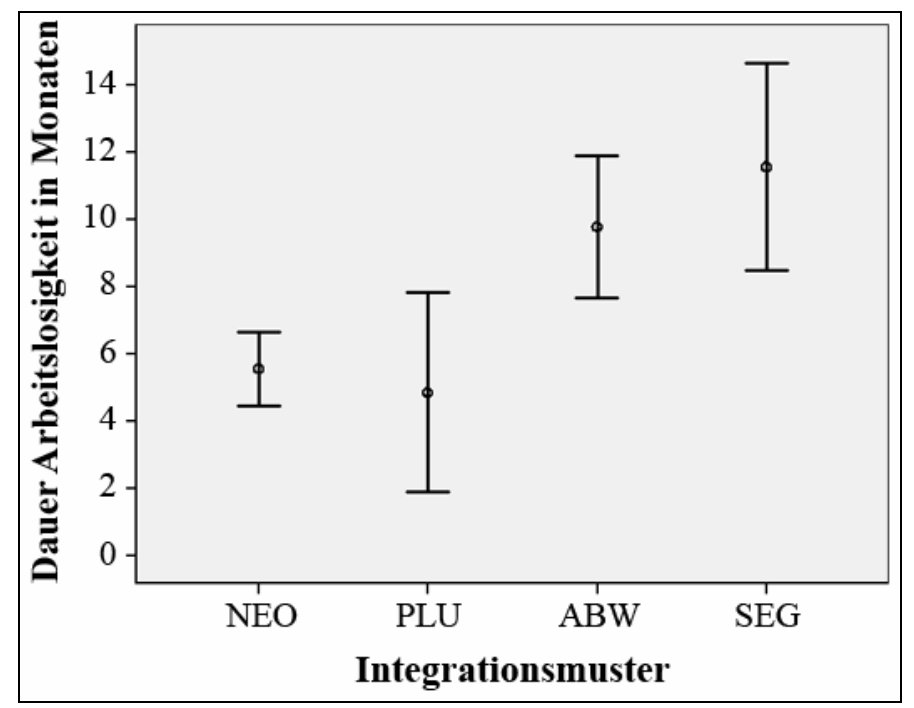

Ausländersurvey 1997 des Deutschen Jugendinstituts, Eigene Berechnungen Abbildung 29 - Musteranalyse: Länge der Arbeitslosigkeit

Eine Tätigkeit als Selbstständiger oder mithelfende/r Familienangehörige/r kommt einem Indikator für ,ethnische Ökonomie' am nächsten. Hier belegt Abbildung 30 einen erhöhten Prozentsatz im pluralistischen Muster (13\%) im Vergleich zu 8\% bei der Segregation und $11 \%$ bei der neoklassischen Integration. 


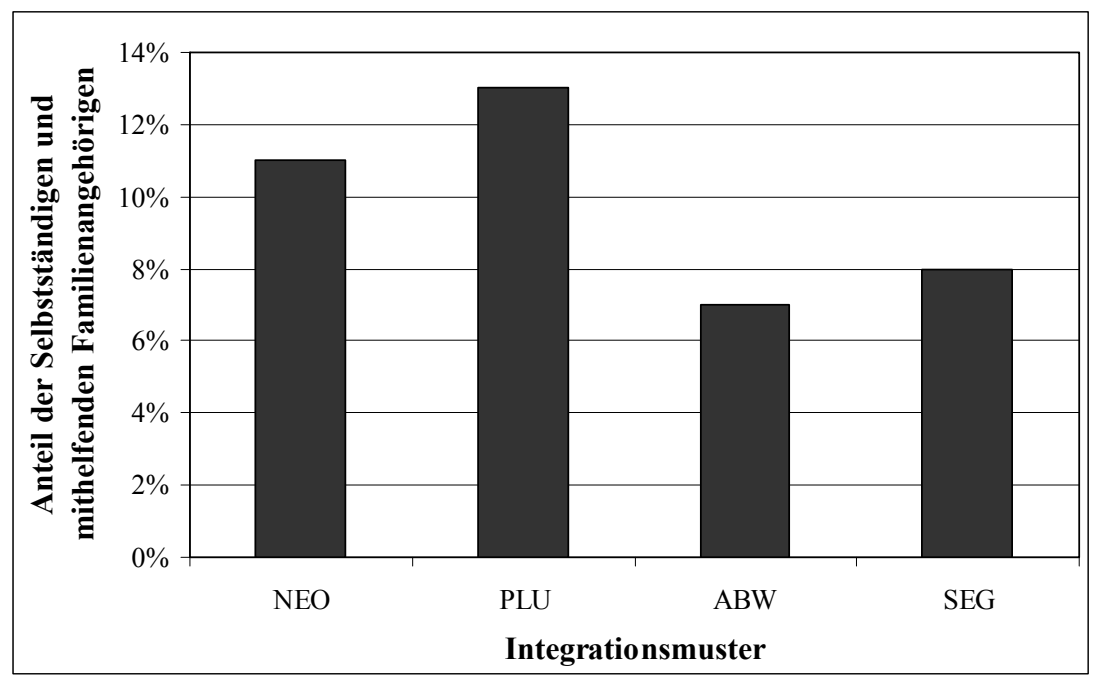

Mikrozensus 2005, FDZ der Statistischen Ämter des Bundes und der Länder, Eigene Berechnungen Abbildung 30 - Musteranalyse: Selbstständige und mithelfende Familienangehörige

Grundlegend anders stellt sich die Verteilung bezüglich der Arbeitssuche über persönliche Beziehungen dar. Diese erfolgt in den beiden Mustern niedriger Qualifikation wesentlich häufiger (12\% und 9\%) als in den Mustern höherer Qualifikation. So geben z.B. nur 4\% der MigrantInnen im pluralistischen Muster an, bei der Arbeitssuche auf persönliche Beziehungen zurückzugreifen. Die Suche über das eigene Netzwerk ist bei Personen mit geringerem sozioökonomischem Status nicht von Erfolg gekrönt und wird von den statushöheren MigrantInnen auffällig weniger durchgeführt (Abbildung 31).

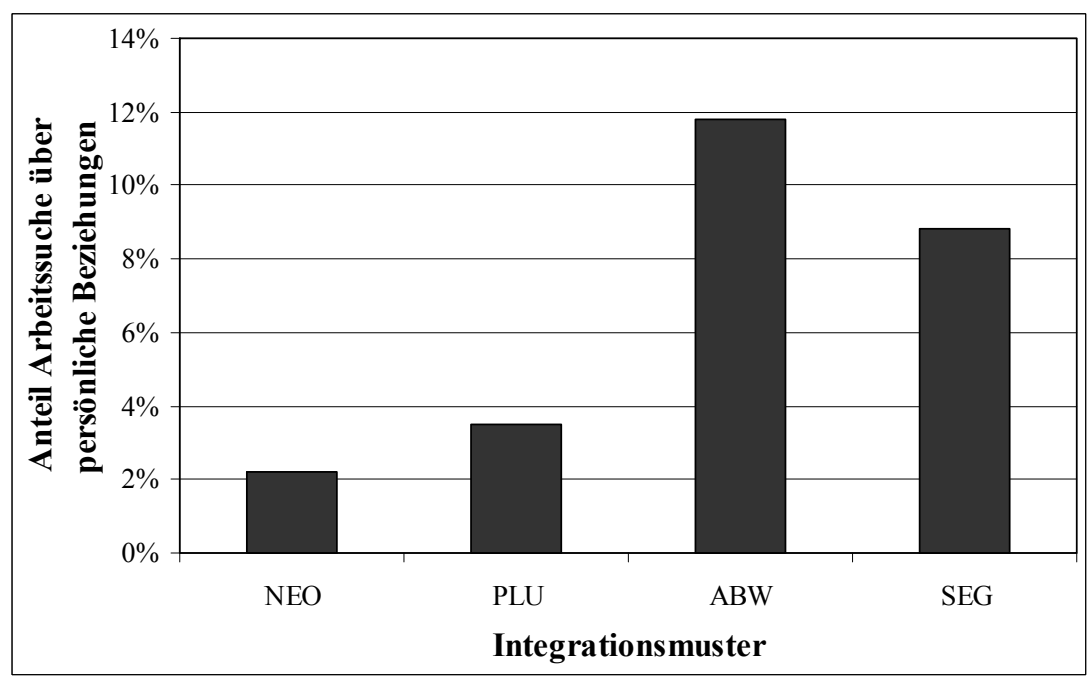

Mikrozensus 2005, FDZ der Statistischen Ämter des Bundes und der Länder, Eigene Berechnungen Abbildung 31 - Musteranalyse: Arbeitssuche über persönliche Beziehungen

Die meisten Variablen zur Partnerschaft sind schon in die Operationalisierung der Integrationsmuster eingeflossen. Zwei bisher noch nicht genutzte können jedoch als Indikatoren für pluralistische Partnerschaftsmuster gewertet werden und sollen daher hier untersucht werden. 
Es handelt sich um den Grad, in dem die eigene Partnerschaft von den Eltern bestimmt wird (Abbildung 32), und um die Frage, ob der/die PartnerIn aus der gleichen Herkunftsregion wie die Eltern kommt (Abbildung 37). Die Ergebnisse plausibilisieren die Theorien: Im pluralistischen und im segregierten Muster bestimmen die Eltern die Partnerschaften ihrer Kinder stärker als in den beiden anderen Mustern. ${ }^{138}$ Dem entsprechen hohe Prozentwerte für die Herkunft aus der gleichen Region. Da MigrantInnen im neoklassischen Muster und bei der Abwärts-Integration qua Definition nicht in einer Partnerschaft mit einer/m MigrantIn der ersten Generation leben, sind ihre Prozentsätze null. Für die beiden anderen Muster zeigt sich, dass etwa 40\% eine/n PartnerIn aus der Herkunftsregion der Eltern haben. Da die Werte für das pluralistische und das segregierte Muster mehr oder weniger gleichauf liegen, können die sozioökonomischen Unterschiede nicht durch diese Variablen der Verbundenheit zum Herkunftsland der Eltern erklärt werden.

In Bezug auf die Freundschaftsmuster gehen die pluralistische Theorie und das Muster der Segregation von einem höheren Anteil an FreundInnen der gleichen Herkunftsgruppe aus, das neoklassische Muster und die Abwärts-Integration vermuten einen geringeren Anteil und damit mehr deutsche FreundInnen ohne Migrationshintergrund. Diese theoretischen Vorhersagen werden in Abbildung 33 bestätigt; speziell zwischen neoklassischem Muster (30\% FreundInnen gleicher Herkunft) und segregiertem Muster (70\%) besteht ein großer Unterschied. In der Theorie der Abwärts-Integration wird zudem die Entstehung einer ,Regenbogen'-Peergruppe beschrieben, die Personen unterschiedlicher Herkunftsgruppen umfasst, die ihr geringer sozialer Status und eine Rebellion gegen den ,Mainstream' eint. Abbildung 34 weist theoriekonform einen signifikant höheren Anteil an Freundschaften unter Menschen mit Migrationshintergrund im Muster der Abwärts-Integration auf. Dies sind Freundschaften mit MigrantInnen der ersten, anderthalbten oder zweiten Generation, die jedoch einer anderen Herkunftsgruppen angehören, beispielsweise ist eine Migrantin türkischer Herkunft mit einem Migranten italienischer Herkunft befreundet. Auch Abbildung 35 zur Mitgliedschaft in deutschen Vereinen bringt zunächst theoriekonforme Ergebnisse: In den beiden Mustern, die kulturelle Anpassung verlangen, sind die MigrantInnen der zweiten Generation in mehr deutschen Vereinen Mitglied als die pluralistischen und segregierten MigrantInnen.

\footnotetext{
${ }^{138}$ Die Zahlen für das anhand der Sprachkenntnisse geschaffene Muster entsprechen diesen Werten. Sie werden daher nicht getrennt ausgewiesen.
} 


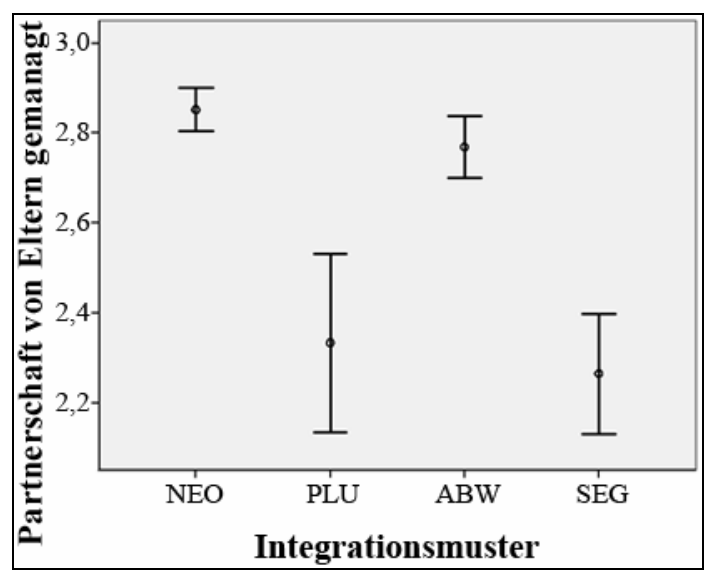

Abbildung 32 - Musteranalyse: Partnerschaft von Eltern gemanagt

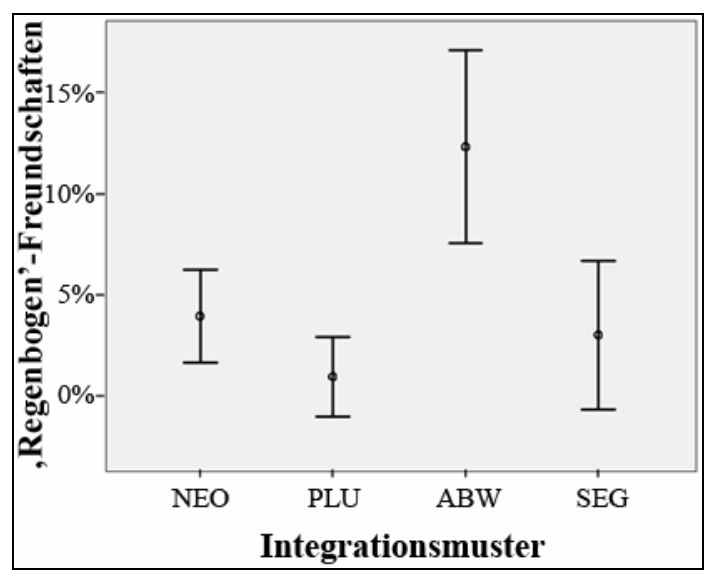

Abbildung 34 - Musteranalyse: ,Regenbogen'Freundschaften

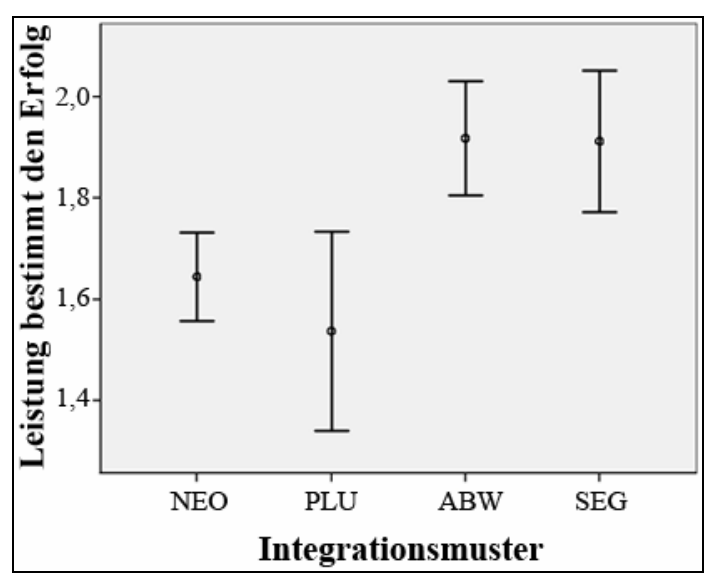

Abbildung 36 - Musteranalyse: Selbstwirksamkeit

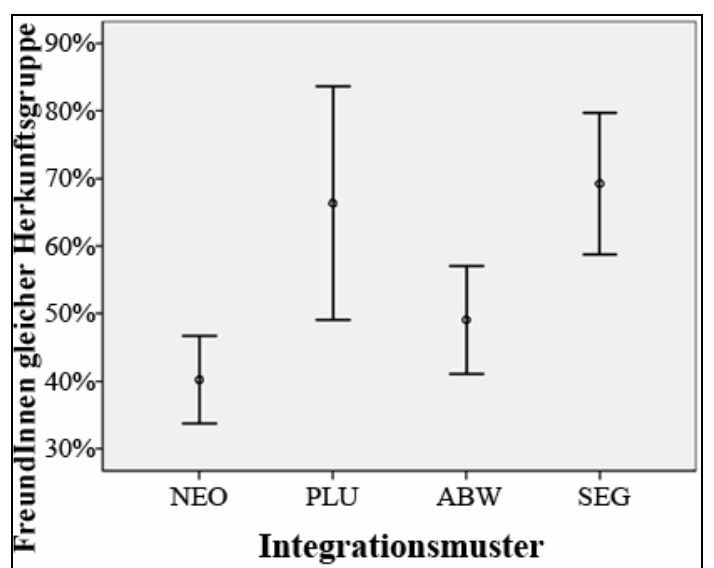

Abbildung 33 - Musteranalyse: FreundInnnen aus gleicher Herkunftsgruppe

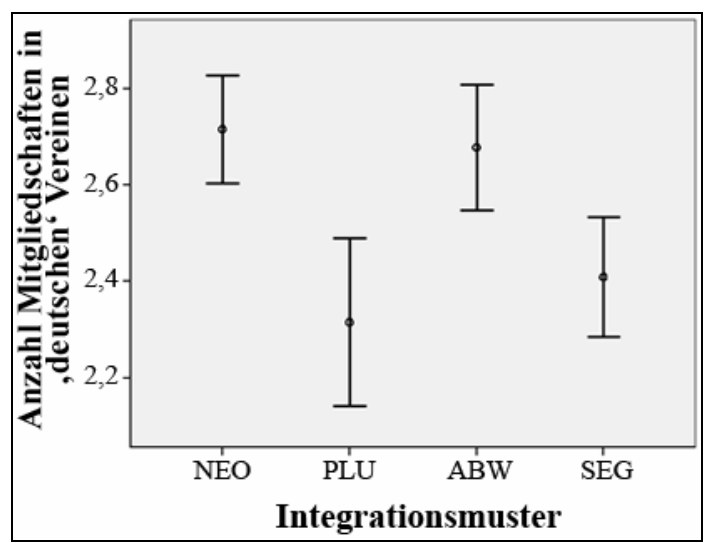

Abbildung 35 - Musteranalyse: Mitgliedschaften in ,deutschen' Vereinen

Abbildung 32: Skala von 1 „Eltern stark beteiligt“ bis 3 ,Eltern gar nicht beteiligt“

Abbildung 36: Skala von 1 ,stimme voll zu“ bis 4 „lehne ganz ab“"

Ausländersurvey 1997 des Deutschen Jugendinstituts, Eigene Berechnungen

Überraschend ist jedoch, dass die Zahl der Vereinsmitgliedschaften bei MigrantInnen der Abwärts-Integration beinahe genauso hoch ist wie bei MigrantInnen im neoklassischen Mus- 
ter. Hier hätte die ,Oppositionskultur’ eine größere Ferne zu Formen der organisierten Partizipation nahe gelegt. Allerdings schließen die Vereine beispielsweise auch Sportclubs ein und dürfen daher nicht als Indikator einer Zugehörigkeit zur Hochkultur missverstanden werden.

Der Bereich der Einstellungen wurde in der empirischen Analyse bisher nicht betrachtet. Nun soll dieser Bereich herangezogen werden, um die Muster zu plausibilisieren oder zu widerlegen. Dabei geht es um Lebensziele der MigrantInnen sowie um emotionale Beziehungen und die Wahrnehmung von Diskriminierung.

Abbildung 36 stellt die Überzeugung der Selbstwirksamkeit dar, gemessen am Grad der Zustimmung zu der Aussage „Leistung bestimmt den Erfolg“. Theoriekonform sind MigrantInnen im neoklassischen und pluralistischen Integrationsmuster stärker von ihrer Selbstwirksamkeit überzeugt und stimmen dieser Aussage mehr zu als MigrantInnen in den anderen beiden Mustern. Andere Indikatoren wie beispielsweise die Wahrnehmung von Autonomie entsprechen diesem Verhältnis und werden daher nicht extra ausgewiesen.

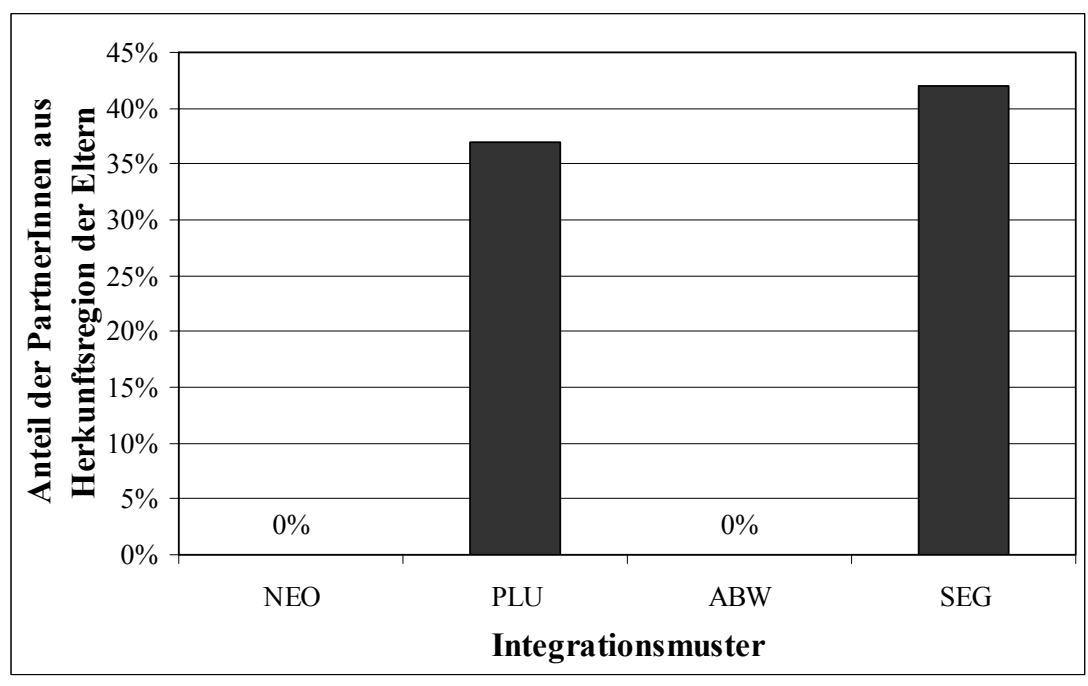

Ausländersurvey 1997 des Deutschen Jugendinstituts, Eigene Berechnungen

Abbildung 37 - Musteranalyse: PartnerIn aus Region der Eltern

Bei der Leistungsmotivation unterscheiden sich die Muster in interessanter Weise: Hier liegt das pluralistische Muster eindeutig vor allen anderen inklusive dem neoklassischen, obwohl dieses ihm in der sozioökonomischen Zusammensetzung gleicht (Abbildung 38). Pflichtbewusstheit als Lebensziel wird von pluralistischen MigrantInnen ebenfalls höher bewertet als von anderen, allerdings reichen segregierte MigrantInnen nahe an ihren Wert. Pluralistische und segregierte Theorien betonen ein Gefühl der Verpflichtung und Loyalität der MigrantInnen gegenüber ihren Herkunftsgruppen. Dieses scheint sich in den Fehlerbalken auszudrücken. (Abbildung 39) Im Hinblick auf das Lebensziel Hilfsbereitschaft (Abbildung 40) fällt 
auf, dass wiederum pluralistische und segregierte Muster höhere Durchschnitte aufweisen. Besonders sticht hervor, dass der Durchschnitt für die Abwärts-Integration tief liegt. Dies entspricht der Theorie, gemäß der sich Unterstützungsnormen für Abwärts-Integrierte auf die Clique beschränken und die ,ethnische Gemeinschaft' nicht einschließen.

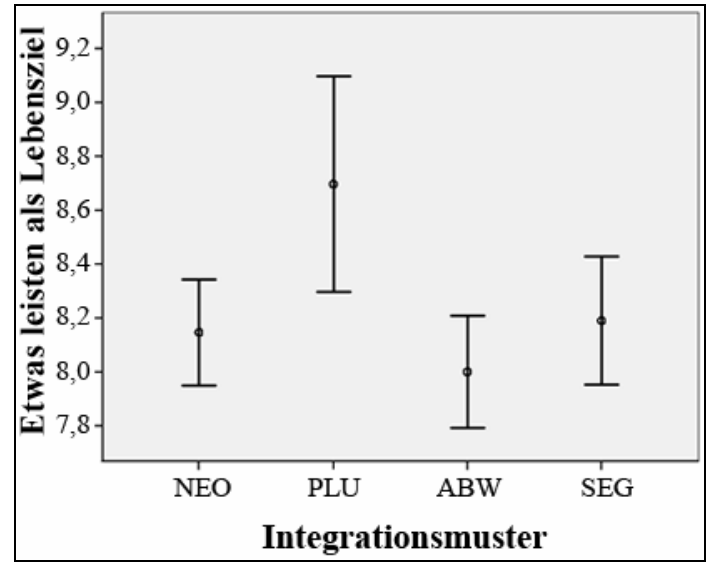

Abbildung 38 - Musteranalyse: Leistungsmotivation

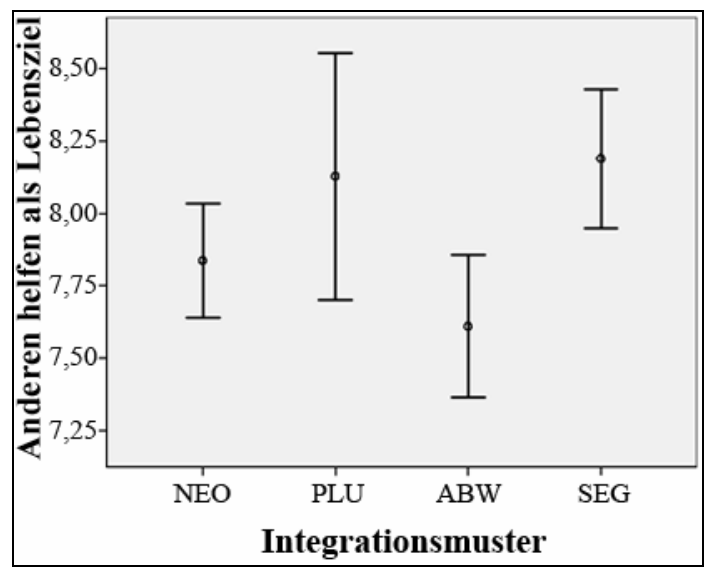

Abbildung 40 - Musteranalyse: Hilfsbereitschaft

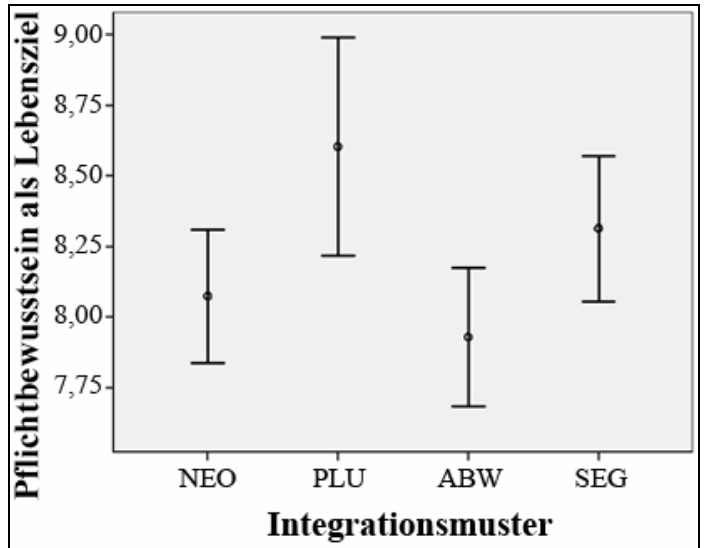

Abbildung 39 - Musteranalyse: Pflichtbewusstsein

Abbildung 38: Skala von 1 ,gar nicht wichtig“ bis 10 ,sehr wichtig“

Abbildung 39: Skala von 1 ,gar nicht wichtig“ bis 10 ,sehr wichtig“"

Abbildung 40: Skala von 1 ,gar nicht wichtig“ bis 10 ,sehr wichtig“"

Ausländersurvey 1997 des Deutschen Jugendinstituts, Eigene Berechnungen

Die Durchschnitte für die Wichtigkeit der Schul- und Berufsausbildung entsprechen ebenfalls den Theorien (Abbildung 41): MigrantInnen im neoklassischen und im pluralistischen Musters bewerten diesen Bereich als wichtiger. Der Bereich „Beruf und Arbeit“ wird analog bewertet und daher nicht extra ausgewiesen. Bezüglich des familiären Kontextes kehrt sich die Wichtigkeit theoriekonform um: Pluralistische und segregierte MigrantInnen halten Eltern und Geschwister für wichtigere Lebensbereiche als neoklassische und abwärtsintegrierte MigrantInnen (Abbildung 42). Es muss jedoch festgehalten werden, dass diese Unterschiede auf hohem Niveau liegen. Bei einem Maximalwert von sieben zeigen die Durchschnittswerte, dass Familie MigrantInnen in allen vier Mustern wichtig ist. 
Pluralistische Theorien gehen davon aus, dass es auch in der zweiten Generation Pendelmigration zwischen dem Herkunftsland der Eltern und dem Aufnahmeland gibt. Wie sieht dies für die im DJI-Ausländersurvey Interviewten aus? Durchschnittlich haben die Befragten knapp unter einem halben Jahr im Herkunftsland gelebt (Abbildung 43). ${ }^{139}$ Zwar liegen die Durchschnittswerte bei Pluralismus und Segregation etwas höher als bei den beiden anderen Mustern, aber diese Unterschiede sind nicht signifikant. Interessant ist nun, ob sich dennoch in einem subjektiven Heimatgefühl zum Herkunftsland der Eltern Unterschiede finden lassen. Solche Differenzen wären gemäß den Theorien durch die Unterschiede in der Sozialisation und im Zugehörigkeitsgefühl zur ,ethnischen Gemeinschaft' zu erwarten. Abbildung 44 zeigt, dass es keine signifikanten Unterschiede gibt, auch wenn Personen im pluralistischen Muster sich im Herkunftsland der Eltern ein wenig stärker zu Hause fühlen. Nota bene: Die Bezeichnung dieser Variablen lautet „Im Heimatland zu Hause fühlen“, das Wort ,Heimatland' war aber in der Frage nicht enthalten. Es ist interessant, wie hier normativ das Herkunftsland der Eltern als Heimatland gesetzt wird - und dies bei MigrantInnen, die zum großen Teil in Deutschland geboren sind.

Hinsichtlich der Diskriminierungswahrnehmung sollten gemäß den Theorien pluralistisch integrierte MigrantInnen stärker auf ,ethnische' Ressourcen zurückgreifen können und die Diskriminierung somit durchschnittlich besser verarbeiten können. Dies bedeutet natürlich nicht, dass sie weniger Diskriminierung erfahren; die Theorie sagt nur etwas zum Umgang mit Diskriminierung aus. Die Theorie der Abwärts-Integration auf der anderen Seite geht davon aus, dass die beschriebenen gemischten Peergruppen diskriminiert werden und Jugendliche und junge Erwachsene sich daher noch enger zusammenschließen. Abbildung 45 verdeutlicht, dass insgesamt relativ wenig Diskriminierung erlebt wird, der Durchschnitt liegt bei 4 Punkten, was einer Einschätzung von seltener Diskriminierung entspricht. Personen im pluralistischen und im segregierten Muster nehmen mehr Diskriminierung wahr, Personen der Abwärts-Integration jedoch nicht, was überraschend ist. Es könnte allerdings sein, dass Diskriminierung auf der Basis anderer Faktoren als der Nationalität wahrgenommen wird, z.B. über die Sozialschicht oder das Wohnviertel bei der Suche um einen Ausbildungsplatz, wo eine Adresse in einem Problemviertel als chancenmindernd erlebt werden könnte. Diese Formen der Diskriminierung wurden in den Datensets nicht gemessen.

\footnotetext{
${ }^{139}$ Bei der Beantwortung der Frage zählten nur ganze Lebensjahre, es war also nicht möglich, längere Urlaubszeiten aufzuaddieren.
} 


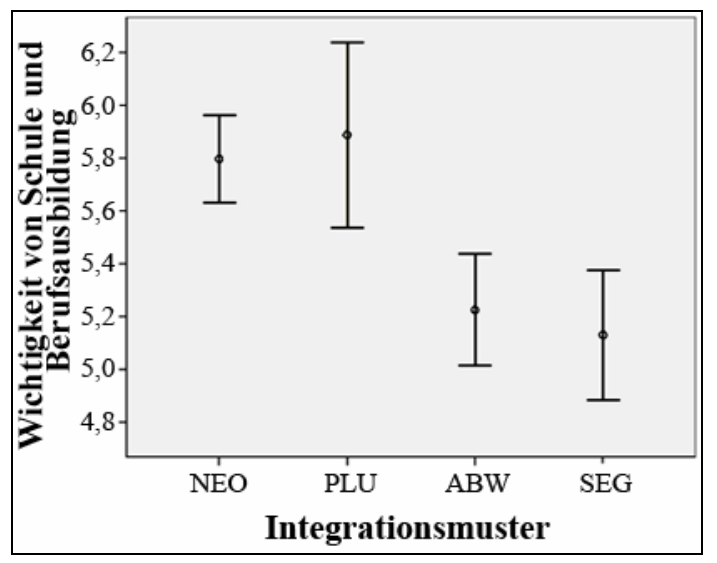

Abbildung 41 - Musteranalyse: Wichtigkeit von Schule und Berufsausbildung

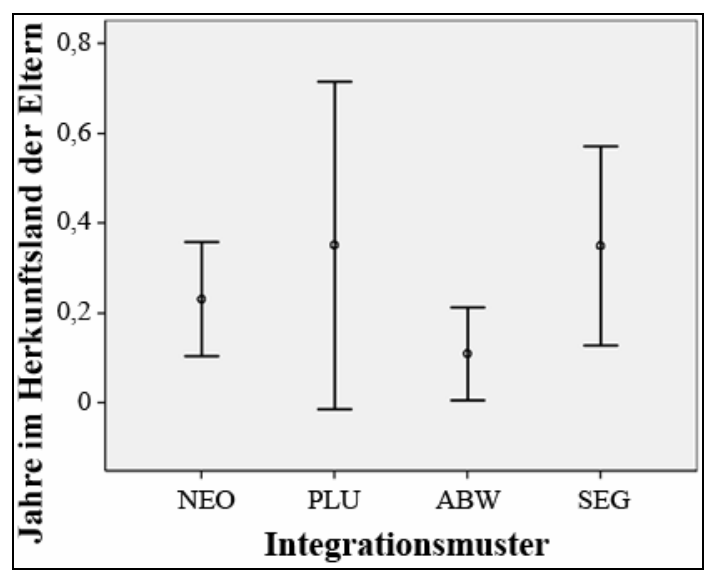

Abbildung 43 - Musteranalyse: Lebensjahre im Herkunftsland der Eltern

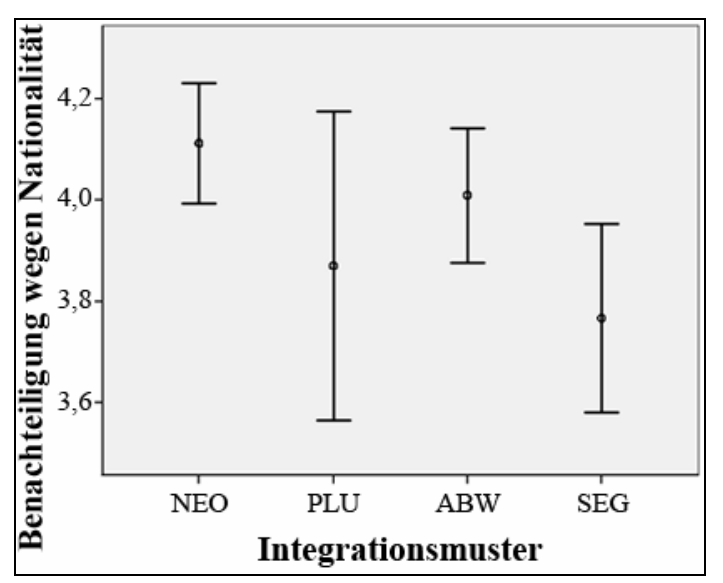

Abbildung 45 - Musteranalyse: Wahrnehmung von Diskriminierung

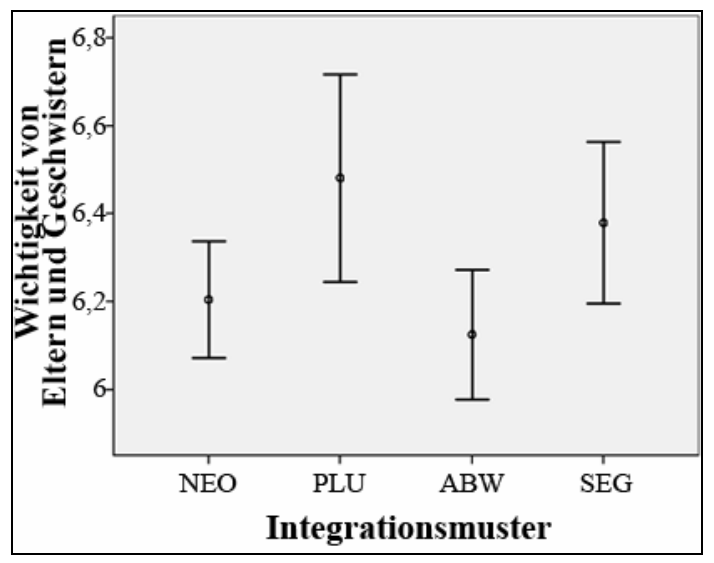

Abbildung 42 - Musteranalyse: Wichtigkeit von Eltern und Geschwistern

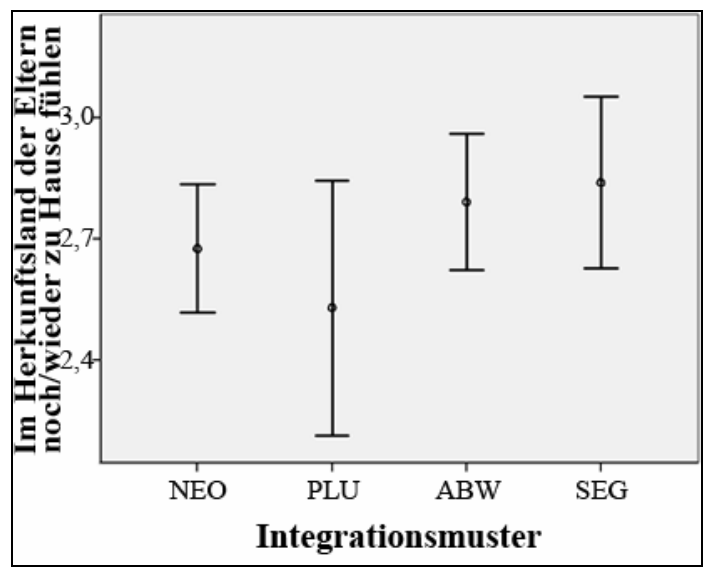

Abbildung 44 - Musteranalyse: Heimatgefühl im Herkunftsland der Eltern

Abbildung 41: Skala von 1 ,überhaupt nicht wichtig“ bis 7 ,sehr wichtig“"

Abbildung 42: Skala von 1 ,überhaupt nicht wichtig“ bis 7 ,sehr wichtig“"

Abbildung 43: Skala von 1 ,fühle mich sofort zu Hause“ bis 5 „fühle mich immer fremd“"

Abbildung 44: Skala von 1 ,sehr oft“ bis 5 ,nie“" Abbildung 45: Skala von 1 ,sehr oft“ bis 5 ,nie“"

Ausländersurvey 1997 des Deutschen Jugendinstituts, Eigene Berechnungen

Es bleibt also festzuhalten, dass ein Großteil der Fehlerbalken und Balkendiagramme bedeutsame Unterschiede zwischen den Mustern belegt haben, die den von den Theorien hypothetisierten Zusammenhängen entsprechen: Sprachpraxis und Sprachkenntnisse verlaufen wie er- 
wartet, ebenso die berufliche Position im DJI-Muster. ${ }^{140}$ Auch Transferleistungen und Arbeitslosigkeiten plausibilisieren die postulierten Zusammenhänge. Das Gleiche gilt für Freundschaften und Lebensziele sowie psychologische Dispositionen.

Über die verschiedenen Grafiken der Fehlerbalken wird deutlich, dass manchmal die statushohen Muster (Neoklassik und Pluralismus) zusammen ausschlagen und die statusniedrigen in die Gegenrichtung, in anderen Fällen aber die kulturell angepassten (neoklassische und Abwärts-Integration) synchron verlaufen. Dies belegt, dass die Status- und die Kultur-Achse keinesfalls zusammenfallen und eine Trennung, wie ich sie in dieser Arbeit vornehme, geboten ist.

Wie sieht es zusammenfassend mit Belegen für die einzelnen Integrationstheorien aus?

Zentral für die im Pluralismus beschriebenen Zusammenhänge sind Ressourcen, die aus der ,ethnischen Ökonomie’ erwachsen sollen. Als Indikator für eine solche ,ethnische’ Beschäftigung kommt in den Datensätzen der Prozentsatz der Selbstständigen in Frage. Diese sind erwartungsgemäß im transnational-pluralistischen Muster überrepräsentiert. Jedoch suchen pluralistisch integrierte MigrantInnen nicht wie vorhergesagt häufiger eine Arbeit über persönliche Beziehungen. Im Gegenteil, MigrantInnen der Abwärts-Integration und der Segregation tun dies stärker. Für die Bestimmung der eigenen Beziehung durch die Eltern und für PartnerInnen aus der Herkunftsregion der Eltern weist das pluralistische Muster einen höheren Prozentsatz auf als das neoklassische und die Abwärts-Integration. Allerdings besteht kein Unterschied zur Segregation, so dass diese Variablen nicht als Erklärung für eine Entwicklung der Muster (und des Statusunterschieds zwischen Pluralismus und Segregation) in Frage kommen. Bei den psychologischen Faktoren übersteigt die Leistungsmotivation der pluralistisch Integrierten die der MigrantInnen aller drei anderen Muster. Daneben geht Pluralismus von einer transnationalen Verbindung auch in der zweiten Generation aus. Die Analyse der Muster zeigte für den Pluralismus jedoch weder einen erhöhten Prozentsatz der Pendelmigration noch signifikant stärkere Heimatgefühle für das Herkunftsland der Eltern. Das vermutete transnationale Feld lässt sich bei den MigrantInnen in der zweiten Generation im DJI-Datensatz also nicht finden.

Im Muster der Abwärts-Integration findet sich wie erwartet ein erhöhter Prozentsatz an ,Regenbogen'-Freundschaften. Zudem fällt die Hilfsorientierung bei den MigrantInnen in diesem Muster geringer aus. Entgegen den theoretischen Annahmen fällt jedoch kein signifikanter

\footnotetext{
${ }^{140}$ Diese Basisdaten zum Beruf konnten nur für das DJI-Muster analysiert werden, da sie dort nicht in die Operationalisierung der Muster eingeflossen waren.
} 
Prozentsatz von Abwärts-Integrierten hinter das Bildungsniveau ihrer Eltern zurück. Interessant ist allerdings, dass die Väter der pluralistisch- und der Abwärts-integrierten MigrantInnen durchschnittlich die gleiche Anzahl an Schuljahren absolviert haben, diese gleiche Ausgangslage sich aber im Muster der Abwärts-Integration nicht in denselben Bildungs- und Berufserfolg übersetzt wie im pluralistischen. Schließlich war erwartbar, dass Abwärts-Integrierte sich stärker diskriminiert fühlen. Dies lässt sich anhand der Fehlerbalken zumindest für die Diskriminierung aufgrund der Nationalität nicht bestätigen.

Die von der neoklassischen Integrationstheorie erwartete sozioökonomische Besserstellung und kulturelle Anpassung über die Generationen lässt sich in den hier untersuchten Datensets belegen. Im Vergleich der vier Muster ist für neoklassische MigrantInnen Deutsch sowohl hinsichtlich der Sprachpraxis (gemessen an Unterhaltungssendungen) als auch der schriftlichen Kenntnisse dominant. Es werden häufiger als in den drei anderen Mustern Partner- und Freundschaften mit Deutschen ohne Migrationshintergrund geschlossen. Auch nehmen MigrantInnen in diesem Muster besonders rege an deutschen Vereinen teil. Es konnte gezeigt werden, dass in diesem Muster eine hohe Leistungsbereitschaft, vorausgesetzt, es findet nur wenig Diskriminierung statt, zum Ergreifen von Chancen und Statusaufstieg führt. Dieser Zusammenhang spiegelt sich in einem hohen $\mathrm{Ma}$ an Selbstwirksamkeit wider sowie in der Bedeutung, die MigrantInnen in diesem Muster dem Bildungs- und Berufsbereich zuschreiben.

Damit lässt sich eine grundsätzliche Plausibilisierung der Integrationsmuster festhalten (neoklassische Integration am meisten, pluralistische in weiten Teilen und Abwärts-Integration am wenigsten). In Kapitel 6.4 werden diese Muster mit den Ergebnissen zur Ähnlichkeitswahrnehmung und den Daten zu Rassismus auf Seiten der Dominanzbevölkerung zusammengeführt.

\subsection{Zwischenfazit zu den Integrationsmustern}

Die vorigen empirischen Abschnitte zu den Bereichen Sprache, Bildung, Arbeitsmarkt und soziale Beziehungen sowie die sich daraus ergebenden Integrationsmuster und die Analyse der Fehlerbalken haben Folgendes gezeigt:

1. MigrantInnen der zweiten Generation erlangen durchschnittlich einen höheren sozioökonomischen Status als MigrantInnen der ersten und anderthalbten Generation und gehen weniger ,ko-ethnische’ Partnerschaften ein als diese. Dieser Befund gilt für alle Herkunftsgruppen mit Ausnahme der ,westlichen' MigrantInnen aus NichtAnwerbestaaten. ,Westliche' MigrantInnen sind daher die einzige Gruppe, die von 
dem in den Medien und einigen wissenschaftlichen Berichten beschriebenen ,Niedergang' der zweiten Generation betroffen ist - allerdings auf hohem Niveau.141

2. Es gibt große Unterschiede zwischen den Herkunftsgruppen.142 Die türkische Herkunftsgruppe erzielt durchgängig den niedrigsten Status; jedoch sind die Kinder von Ex-JugoslawInnen und ItalienerInnen häufig nicht weit von diesen Ergebnissen entfernt. Zudem verringern sich die Unterschiede zwischen den Herkunftsgruppen in der zweiten Generation: Beispielsweise erreichen gut 30\% der türkischen MigrantInnen der ersten und anderthalbten Generation nur das niedrigste Bildungsniveau, während es bei den MigrantInnen italienischer Herkunft 10\% und bei denen griechischer Herkunft $13 \%$ sind. In der zweiten Generation jedoch schrumpft der Unterschied zwischen den MigrantInnen türkischer Herkunft und denen italienischer oder griechischer Herkunft auf einen einzigen Prozentpunkt (5\% gegenüber 4\%). (Tabelle 13)

3. Diese interethnische Diversität wird ergänzt durch eine bedeutsame intraethnische Varianz: Die Streuung der Mittelwerte innerhalb der Herkunftsgruppen fällt nicht wesentlich geringer als bei Nicht-MigrantInnen aus. Homogenisierende Aussagen z.B. über , die Türken' müssen daher mit Skepsis betrachtet werden.

4. MigrantInnen mit deutscher Staatsbürgerschaft sind hinsichtlich ihrer Bildungsabschlüsse und des Prestiges ihrer Berufe Deutschen ohne Migrationshintergrund ähnlicher als MigrantInnen mit ausländischer Staatsbürgerschaft. Die bis vor kurzem übliche Beschränkung auf ,AusländerInnen' in der deutschen Migrationsforschung verzerrt die empirischen Ergebnisse daher ins Negative. Dieser unvorteilhafte Bias wird durch den Ausschluss der Kinder von AussiedlerInnen in den empirischen Untersuchungen verstärkt, da AussiedlerInnen in der zweiten Generation durchschnittlich höhere sozialstrukturelle Positionen einnehmen als die meisten anderen Migrantengruppen. Eine Ausnahme zu diesem Befund der Besserstellung deutscher Staatsbürger bildet die Gruppe der, westlichen’ MigrantInnen aus Nicht-Anwerbestaaten. MigrantInnen mit hohem sozioökonomischem Status erwerben hier die deutsche Staatsbürgerschaft seltener, so dass die ausländischen StaatsbürgerInnen in dieser Herkunftsgruppe höhere ISCED-, Blossfeld- und MPS-Werte aufweisen als die Eingebürgerten.

\footnotetext{
${ }^{141}$ Da die empirische Analyse dieses Artikels sich auf die Alterskohorte der 1961-1985 Geborenen (bzw. die Teilgruppe 1971-1978 im DJI-Ausländersurvey) beschränkt, kann nicht ausgeschlossen werden, dass sich in einer jüngeren Altersgruppe in einem aktuellen Datensatz ein solcher, Niedergang' finden lässt.

${ }^{142}$ Meine empirische Untersuchung lässt daher vermuten, dass der angebliche ,Niedergang' der zweiten Generation, der sich beispielsweise in den bisherigen Auswertungen des national erweiterten Datensatzes von PISA 2003 (PISA 2003-E) abzeichnet, zum großen Teil durch Zusammensetzungseffekte erklärt werden kann (siehe hierzu die Diskussion in Kapitel 3.4.1). Eine Beantwortung dieser Frage wird jedoch erst nach einer Re-Analyse der bisher noch unter Verschluss gehaltenen PISA 2003-Daten möglich sein.
} 
5. Das weit verbreitete Stereotyp der schlecht gestellten Migrantinnen konnte für die zweite Generation nicht bestätigt werden. Im Bildungsbereich zum Beispiel blieben Migrantinnen der zweiten Generation aus der Türkei und Ex-Jugoslawien zwar ein wenig hinter den durchschnittlichen Ergebnissen der Migranten zurück, Frauen italienischer, griechischer, spanischer und portugiesischer Herkunft erzielten jedoch in etwa die gleichen Resultate wie die Männer.

6. Wenn Integrationsmuster auf der individuellen Ebene untersucht werden, zeigt sich, dass ein nicht-prekärer sozioökonomischer Status und kulturelle Anpassung nicht immer zusammenfallen. Zwischen 2 und 17\% aller MigrantInnen der zweiten Generation verbinden eine gesicherte sozialstrukturelle Positionierung mit fehlender kultureller Anpassung (erfasst durch eine Beziehung mit einem/r ,ko-ethnischen' PartnerIn der ersten Generation oder die Sprachkenntnisse). Gleichzeitig existiert eine Gruppe, bei der eine kulturelle Anpassung (ebenfalls über die Partnerschaft oder die Sprachkenntnisse erfasst) sich mit einem prekären sozioökonomischen Status verbindet.

7. Die quantitative Verteilung der Integrationsmuster gibt jedoch keine Auskunft über die Mechanismen, durch die sich eine pluralistische Orientierung mit einem gehobenen sozioökonomischen Status verbinden könnte bzw. kulturelle Anpassung mit einem prekären sozialstrukturellen Status. Die von den Theorien beschriebenen Mechanismen (z.B. Nutzung wirtschaftlicher, normativer und kognitiver Ressourcen eines transnationalen Raumes für den ersten Fall, dissonante Akkulturation und Oppositionsverhalten für den zweiten) konnten bei der Analyse theorierelevanter Variablen in den Fehlerbalken und Verteilungen teilweise plausibilisiert werden. Speziell trifft dies, wie oben gezeigt, auf psychologische Dispositionen und einen stärkeren Umgang mit MigrantInnen aus der gleichen Herkunftsgruppe im pluralistischen Muster und auf die Orientierung an der Peergruppe im Muster der Abwärts-Integration zu. Nicht bestätigt werden konnten die transnationalen Verbindungen in der zweiten Generation im Pluralismus sowie der Bildungsabstieg und das starke Diskriminierungsgefühl in der Abwärts-Integration. Die Existenz von gemischt ,ethnischen' Cliquen konnte in Ansätzen belegt werden. Das grundsätzliche Aufbrechen der sozioökonomischen und kulturellen Achse hat sich ebenfalls bewährt, die Variablen in den Fehlerbalken schlugen hinsichtlich der beiden Dimensionen unterschiedlich an.

Um diese Ergebnisse und die Plausibilisierung der Integrationsmuster in das in dieser Arbeit verwendete Integrationskonzept und die ihm zugrunde liegenden sozialkonstruktivistischen Prämissen einzubetten, wird im nächsten Kapitel der Blickwinkel geändert und die nicht- 
migrantische Dominanzbevölkerung untersucht. Während es also bisher in den Sachbereichen und bei den Integrationsmustern um Merkmale auf der Ebene der/s individuellen MigrantIn ging und Aggregationen von Merkmalen in bestimmten Herkunftsgruppen und Generationen der migrantischen Bevölkerung herausgearbeitet wurden, untersucht das nächste Kapitel Veränderungen über die Zeit in der Ähnlichkeitswahrnehmung von MigrantInnen durch die nichtmigrantische Dominanzbevölkerung sowie rassistische Einstellungen und Praktiken bei dieser Bevölkerungsgruppe. Gemäß meinen Prämissen hat Integration erst dann stattgefunden, wenn sich diese Ähnlichkeitswahrnehmung vergrößert hat. Daher ist es zwingend erforderlich, die Dominanzbevölkerung in den Blick zu nehmen und zu untersuchen, inwieweit sich ihre Wahrnehmungen und Fremdheitsmarkierungen verändert haben. Und schließlich findet der Integrationsprozess in einem Kontext statt, der unterschiedlich stark durch Rassismus geprägt ist. Darum soll Rassismus in seiner unterschiedlichen Bedeutsamkeit für verschiedene Gruppe analysiert und seine strukturelle Qualität erläutert werden. 


\section{Empirie III: Dominanzbevölkerung - Ähnlichkeitswahrneh- mung und Rassismus}

In den vorangegangenen Kapiteln wurden Sprachkenntnisse, Bildungs- und Berufsergebnisse sowie Freund- und Partnerschaftsmuster der zweiten Generation von MigrantInnen in Deutschland betrachtet. In einem zweiten Schritt ließen sich die einzelnen Fälle der Datensätze anhand von Sprach-, Partnerschafts-, Bildungs- und Berufskriterien den aus den Theorien abgeleiteten Integrationsmustern zuweisen. Laut dem sozialkonstruktivistischen Integrationsverständnis dieser Arbeit wird Integration jedoch als Vergrößerung der wahrgenommenen Ähnlichkeit konzeptionalisiert, die aus status- und/oder kulturgleich markierten Interaktionen erwächst (vgl. Kapitel 2.3). Bisher fehlt also ein Beleg dafür, dass eine Verbesserung in den Bildungsabschlüssen und auf dem Arbeitsmarkt sowie sehr gute Deutschkenntnisse und Partnerschaften mit Deutschen ohne Migrationshintergrund auch zu einem Anstieg der wahrgenommenen Ähnlichkeit führen. Dieser Nachweis soll in Kapitel 5.1 anhand einer Analyse von Umfragewerten der Dominanzbevölkerung zu ihrer Wahrnehmung von Ähnlichkeit mit verschiedenen MigrantInnengruppen aus der ALLBUS erbracht werden.

Der Integrationsprozess mit seinen Komponenten Status- und Spracherwerb, Aufbau sozialer Beziehungen (Freundschaften und Partnerschaften) und Wahrnehmung von Ähnlichkeit findet dabei nicht in einem Vakuum, sondern in einem vermachteten Raum statt, der in dieser Arbeit vor allem hinsichtlich der Diskriminierung aufgrund einer Markierung als MigrantIn und/oder ,fremd aussehender' Person untersucht wird. Hierfür wird der Begriff Rassismus verwendet, um zu verdeutlichen, dass es sich nicht allein um individuelle Einstellungen und Verhaltensweisen handelt; vielmehr sind diese Praktiken in gesellschaftliche Machtverhältnisse eingebettet, so dass Rassismus auch institutionelle und nicht-intentionale Formen der Diskriminierung einschließt. Dieser strukturelle Kontext wird in Kapitel 5.2 untersucht. Es zeigt sich, dass der Rassismus der Dominanzbevölkerung als Hintergrundvariable den Statuserwerb von MigrantInnen in allen Bereichen sowie die Markierung von Interaktionen als status- und kulturgleich beeinflusst, jedoch für verschiedene Gruppen in unterschiedlichem Maße.

In diesem Kapitel wechselt also die Perspektive: Während es zuvor um MigrantInnen der zweiten Generation sowohl auf einer individuellen als auch auf einer aggregierten Ebene ging, beschäftigt sich dieses Kapitel mit der nicht-migrantischen Dominanzbevölkerung: Wie hat sich deren Wahrnehmung von Ähnlichkeit gegenüber MigrantInnen verändert? Und inwieweit 
ist die Gesellschaft durchdrungen von Rassismus, so dass rassistische Diskriminierung zu einer Achse sozialer Ungleichheit wird?

\subsection{Wahrnehmung von Ähnlichkeit durch die Dominanzbevölke- rung}

In diesem Kapitel zeige ich, dass sich bei der nicht-migrantischen Dominanzbevölkerung die Wahrnehmung von Ähnlichkeit gegenüber verschiedenen MigrantInnengruppen in Deutschland über die Jahre verändert hat. Damit will ich belegen, dass Veränderungen in der Ähnlichkeitswahrnehmung grundsätzlich möglich sind und dass sie neben anderen Faktoren vom sozioökonomischen Status und den kulturellen Ressourcen von MigrantInnen abhängen, und zwar durch die Interaktionen, die dieser Status und diese Ressourcen ermöglichen. Ich erwarte also, dass sich durch den vergrößerten Anteil der zweiten Generation an allen MigrantInnen und ihren durchschnittlich höheren sozioökonomischen Status sowie ihre durchschnittlich stärkere kulturelle Anpassung an die nicht-migrantische Bevölkerung die Ähnlichkeitswahrnehmung bei der Dominanzbevölkerung gegenüber diesen MigrantInnengruppen über die Jahre vergrößert.

Diese vergrößerte Ähnlichkeitswahrnehmung wird sich gegenüber verschiedenen Herkunftsgruppen unterscheiden und von einer ganzen Reihe externer Faktoren bestimmt sein - als Beispiel sei nur die im Zuge der Attentate des 11. September 2001 gestiegene Islamophobie genannt. ${ }^{143}$ Sie variiert aber auch gegenüber verschiedenen Mitgliedern der gleichen Herkunftsgruppe (z.B. Männern und Frauen, Kopftuchträgerinnen und Frauen ohne Kopftuch). Und schließlich divergieren die Ähnlichkeitswahrnehmungen innerhalb der nicht-migrantischen Dominanzbevölkerung (z.B. zwischen Jungen und Alten, sich politisch eher links oder eher rechts Verortenden). Da eine solche Binnendifferenzierung in der empirischen Analyse aus Gründen der Komplexitätsreduktion nicht möglich ist, geht es im Folgenden um die durchschnittliche Wahrnehmung von Ähnlichkeit. Zudem erlauben die bestehenden Surveys nicht, die Wahrnehmung von Ähnlichkeit speziell gegenüber der zweiten Generation der jeweiligen Herkunftsgruppen abzufragen. ${ }^{144}$

\footnotetext{
${ }^{143}$ Weitere Beispiele sind die Diskussion um den EG-Beitritt der südeuropäischen ehemaligen Anwerbestaaten (Italien, Griechenland, Spanien und Portugal) und um die Verschärfung der Asylgesetzgebung Anfang der 1990er Jahre.

${ }^{144}$ Dabei ist zu beachten, dass solche Fragebögen, die zur Ähnlichkeitsmessung dienen, durch die Verwendung bestimmter Kategorien und Konzepte (z.B. „Asylbewerber“) selbst die Wahrnehmung von Ähnlichkeit beeinflussen. Sie sind also nicht nur passive Erhebungsinstrumente, sondern haben Anteil an den Prozessen der sozialen Konstruktion. Im Folgenden werden daher die Formulierungen aus den Fragebögen von mir auch im Text meiner Arbeit verwendet.
} 
Und schließlich betrachtete ich die Wahrnehmung von Ähnlichkeit auf Seiten der MigrantInnen nicht. Zwar halte ich MigrantInnen keinesfalls für passive Objekte, gehe aber davon aus, dass in einem vermachteten Raum die Wahrnehmung der mächtigeren Gruppe für Teilhabechancen entscheidender ist (extreme Beispiele hierfür sind ,Schwarze' im System der Apartheid, Juden und Jüdinnen im Nationalsozialismus). Allerdings kann in den meisten Fällen davon ausgegangen werden, dass eine vergrößerte Wahrnehmung von Ähnlichkeit auf Seiten der Dominanzbevölkerung mit einem entsprechend verstärkten Empfinden von Ähnlichkeit auf Seiten der MigrantInnenbevölkerung korrespondiert (Steinbach 2004: 180f). ${ }^{145}$

Wie also hat sich die Wahrnehmung von Ähnlichkeit bei der Dominanzbevölkerung gegenüber MigrantInnen über die Jahre verändert? Eine direkte Erhebung zu dieser Frage gab es nur in der Allgemeinen Bevölkerungsumfrage der Sozialwissenschaften (ALLBUS) in den Jahren 1996 und 2006. Darüber hinaus werden in der ALLBUS der Ähnlichkeit verwandte Aspekte seit 1980 erfasst, sodass sich mit diesem Datenset am ehesten eine Zeitreihe anfertigen lässt. Andere Längsschnittstudien, wie z.B. das Eurobarometer, verändern jedes Jahr die abgefragten Items, so dass die Kontinuität über die Zeit fehlt.

Als ein erster Indikator der Wahrnehmung von Ähnlichkeit oder Fremdheit sollen Heiratsnormen untersucht werden. Eine akzeptierende Haltung zur Einheirat eines Mitglieds einer anderen Gruppe in die eigene Familie wird dabei gemäß der Bogardus Social Distance Sca$l e^{146}$ als Ausdruck geringer Fremdheitswahrnehmung gewertet. Abbildung 46 zeigt einen deutlichen Rückgang in der Zustimmung zu der Aussage „Gastarbeiter [ab 1994: Ausländer] sollten sich ihre Ehepartner unter ihren eigenen Landsleuten auswählen“: ${ }^{147}$ Während 1980 noch beinahe die Hälfte der BundesbürgerInnen in Westdeutschland dieser Aussage zustimmten (Werte 5 bis 7), liegt die Zahl heute bei 14\%. In Ostdeutschland (ohne Berlin) befürwortet immer noch knapp ein Viertel der Bevölkerung das Endogamie-Gebot.

\footnotetext{
${ }^{145}$ Ein weiterer Grund für diese Aussparung liegt darin, dass bei der Analyse der Ähnlichkeitswahrnehmung auf Seiten der migrantischen Bevölkerung die Gefahr besteht, einen gesellschaftlichen Diskurs aufzugreifen, der den MigrantInnen die Verantwortung für die distanzierte Haltung der deutschen Nicht-Migrantenbevölkerung zuschiebt und diese als Integrationsunwilligkeit auf Seiten der MigrantInnen beschreibt.

${ }^{146}$ Die Bogardus Social Distance Scale besteht aus sieben Items, um die Bereitschaft von Menschen zu sozialen Kontakten mit Angehörigen einer anderen Gruppe zu messen. Sie reicht von Zustimmung zu der Aussage, dass Angehörige der anderen Gruppe aus dem eigenen Land ausgeschlossen werden sollten (= Indikator geringster Ähnlichkeitswahrnehmung), über Akzeptanz der/s Anderen als KollegIn im gleichen Beruf (mittlere wahrgenommene Ähnlichkeit) bis zur Akzeptanz von enger Freundschaft und schließlich Einheirat in die eigene Familie (=Indikator großer Ähnlichkeitswahrnehmung).

${ }^{147}$ Alle nachfolgenden Fragen werden in der ALLBUS nur deutschen StaatsbürgerInnen gestellt. Unter diesen befinden sich aber durch die Liberalisierung des Staatsangehörigkeitsrechts im Jahr 2000 auch zunehmend MigrantInnen der ersten und zweiten Generation. Deren Antworten auf diese Fragen gehen ebenso in die Daten ein. Die Veränderungen in der Ähnlichkeitswahrnehmung sind jedoch größer, als dass sie allein durch den gestiegenen Anteil der MigrantInnen an den deutschen StaatsbürgerInnen erklärt werden könnten.
} 
Wie hat sich neben dieser Bereitschaft nun der reale Kontakt zu anderen Herkunftsgruppen durch familiäre Beziehungen zwischen MigrantInnen und Nicht-MigrantInnen verändert? Abbildung 47 verdeutlicht, dass 1980 nur 5\% der BundesbürgerInnen ,persönlich Kontakt zu Gastarbeitern oder zu deren Familien [ab 1994: zu in Deutschland lebenden Ausländern] [...] in [i]hrer Familie oder anderen Verwandtschaft" hatten. Dieser Wert stieg über die Jahre kontinuierlich an, machte mit der Umstellung der Formulierung zu „AusländerInnen“ einen kleinen Sprung und hat sich in den letzten zwei Umfragen (2002 und 2006) in Westdeutschland (inklusive Berlin) bei 30\% eingependelt. In Ostdeutschland liegt der Satz mit 12\% niedriger. Dabei ist jedoch im Unterschied zu der vorherigen Frage, bei der es um Einstellungen ging, zu beachten, dass der Kontakt in Ostdeutschland allein schon wegen der geringeren Zahl der dort lebenden MigrantInnen geringer ausfallen muss als in Westdeutschland.

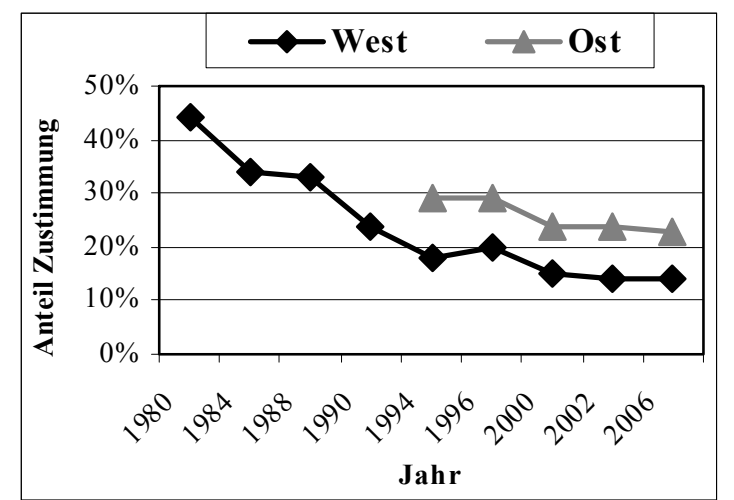

„Gastarbeiter [ab 1994 Ausländer] sollten sich ihre Ehepartner unter ihren eigenen Landsleuten auswählen." (Skala von 1 ,stimme überhaupt nicht $\mathrm{zu}^{\text {“ }}$ bis 7 ,stimme voll und ganz $\mathrm{zu}^{\text {“") }}$

ALLBUS 1980, 1984, 1988, 1990, 1994, 1996, 2000, 2002, 2006, Eigene Berechnungen

Abbildung 46 - Dominanzgesellschaft: Heiratsgebot

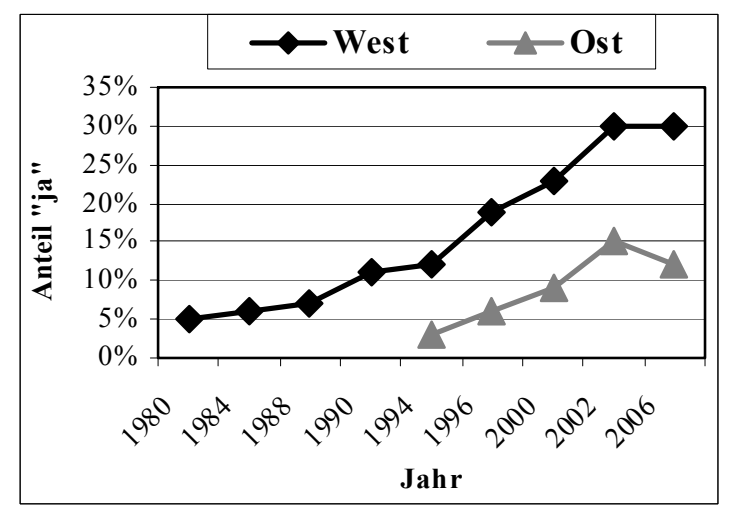

„Haben Sie persönlich Kontakte zu Gastarbeitern oder zu deren Familien [ab 1994 Ausländern] und zwar in Ihrer Familie oder näheren Verwandtschaft?"

ALLBUS 1980, 1984, 1988, 1990, 1994, 1996, 2000, 2002, 2006, Eigene Berechnungen

Abbildung 47 - Dominanzgesellschaft: Kontakt

Daten zu den direkten Indikatoren der Ähnlichkeitswahrnehmung liegen nur für zwei Zeitpunkte vor (Abbildung 48). 1996 und 2006 enthielt die ALLBUS einen umfassenden Fragenkatalog zu „Einstellungen gegenüber ethnischen Gruppen in Deutschland““. ${ }^{148}$ Auf die Frage, wie ähnlich oder unähnlich Angehörige verschiedener Gruppen ihnen seien, antworteten 1996 56\% der westdeutschen BundesbürgerInnen, zwischen ihnen und den in Deutschland leben-

\footnotetext{
${ }^{148}$ Dabei unterliegt der Fragebogen der häufigen Normalitätssetzung durch die dominante Gruppe, gemäß derer nur MigrantInnen einer ethnischen Gruppe angehören; die deutsche Herkunftsgruppe hingegen wird nicht als ethnische Gruppe konstituiert, sondern als quasi-natürliche Hintergrundsfolie, vor der sich die Anderen sichtbar abheben. Interessant ist auch, welche unterschiedlichen Gruppen dabei gebildet werden: Als Gruppenmerkmal dient auf der einen Seite Nationalität (ItalienerInnen), auf der anderen der Aufenthaltsstatus (AsylbewerberInnen) oder die Religionszugehörigkeit (Juden/Jüdinnen).
} 
den ItalienerInnen bestünden nur geringe Unterschiede; 22\% sahen mittlere, weitere 22\% starke Unterschiede. $\mathrm{Zu}$ den AussiedlerInnen in Deutschland sahen 34\% geringe, 24\% mittlere und $42 \%$ starke Unterschiede. Die Werte zu den in Deutschland lebenden TürkInnen deuten auf eine geringere Ähnlichkeitswahrnehmung hin: Hier sahen 15\% der westdeutschen BundesbürgerInnen geringe, 18\% mittlere und 67\% starke Unterschiede. Die geringste Ähnlichkeit wurde bei AsylbewerberInnen in Deutschland wahrgenommen: Hier gaben $8 \%$ geringe und 9\% mittlere Unterschiede an, $83 \%$ jedoch starke Unterschiede. Als letzte Gruppe wurde nach den Juden und Jüdinnen in Deutschland gefragt. Die wahrgenommene Ähnlichkeit fiel bei dieser Gruppe am größten aus. Zehn Jahre später hat sich die in diesen Zahlen zum Ausdruck gebrachte Fremdheitswahrnehmung gegenüber ItalienerInnen in Deutschland stark verringert. Die Zahlen bei AsylbewerberInnen und Juden/Jüdinnen in Deutschland sind leicht zurückgegangen, wohingegen die Wahrnehmung von Fremdheit gegenüber TürkInnen und gegenüber AussiedlerInnen geringfügig zugenommen hat. Die Entwicklung der Ähnlichkeitswahrnehmung von verschiedenen Herkunftsgruppen unterscheidet sich also signifikant.

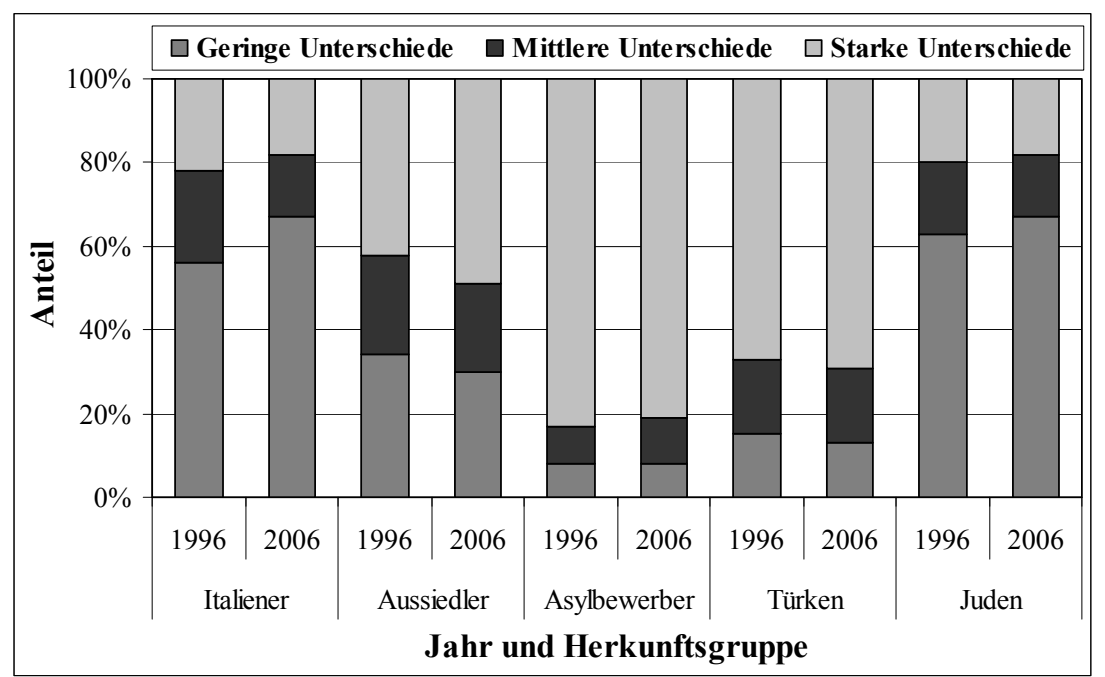

„Wie stark unterscheiden sich die in Deutschland lebenden [...] in ihrem Lebensstil von den Deutschen?“ (Skala von 1 ,überhaupt nicht“ bis 7 ,,sehr stark“)

ALLBUS 1996, 2006, Eigene Berechnung

Abbildung 48 - Dominanzgesellschaft: Lebensstilunterschiede

Die gleiche Tendenz ergibt sich bei der Frage, wie angenehm oder unangenehm die Einheirat von Angehörigen verschiedener Gruppen in die eigene Familie wäre (Abbildung 49). Während die Einheirat einer/s ItalienerIn auf einer 7-Punkte-Skala (mit $7=$ sehr angenehm) 1996 in Westdeutschland von deutschen StaatsbürgerInnen mit 4,4 bewertet wurde, stieg der Wert 2006 auf 4,8. In Ostdeutschland lauteten die entsprechenden Zahlen 3,8 und 4,3. Damit unterschieden sich die Zahlen im Jahr 2006 für ItalienerInnen nicht mehr von denen für Protestan- 
tInnen, bei denen eine Einheirat in die Familie 2002 ebenfalls mit einem durchschnittlichen Wert von 4,8 in Westdeutschland und 4,3 in Ostdeutschland gewünscht wurde (KatholikInnen: 4,9 und 4,2). Gegenüber AussiedlerInnen und TürkInnen lagen diese Werte der Einheirat niedriger. Das Schlusslicht bildete die durchschnittliche Haltung der Deutschen gegenüber ,AsylbewerberInnen'. Neben der signifikanten Zunahme einer positiven Bewertung einer Einheirat von ItalienerInnen weisen die Zahlen über die zwei Zeitpunkte eine relative Konstanz auf, mit leichten Zunahmen für TürkInnen und AsylbewerberInnen und einer leichten Abnahme für AussiedlerInnen. Damit bestätigen diese Zahlen die in Abbildung 48 abgebildete Entwicklung in der Ähnlichkeitswahrnehmung, wobei sie für die türkische Herkunftsgruppe jedoch eine etwas größere Ähnlichkeitswahrnehmung zeigen.

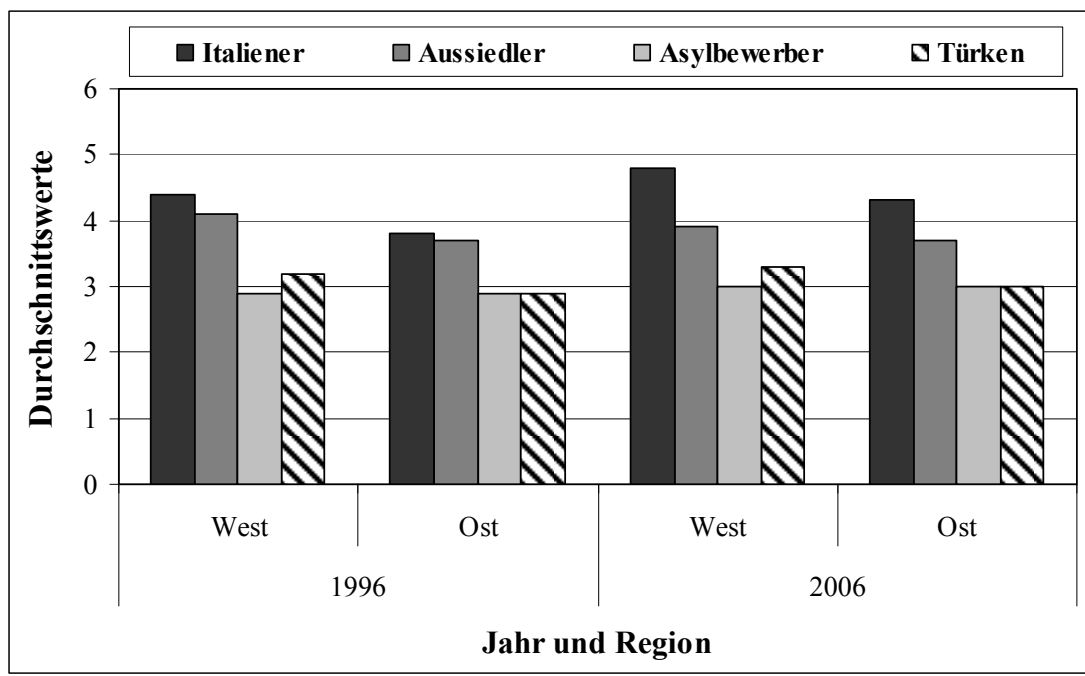

„Und wie wäre es, wenn ein Angehöriger einer dieser Gruppen in Ihre Familie einheiraten würde? Inwieweit wäre Ihnen das angenehm oder unangenehm?“ (Skala von 1 „wäre mir sehr unangenehm“ bis 7 ,wäre mir sehr angenehm")

ALLBUS 1996, 2006, Eigene Berechnung

\section{Abbildung 49 - Dominanzgesellschaft: Einstellung zu Einheirat}

Dass diese Fragen tatsächlich die Ähnlichkeitswahrnehmung erfassen können, bestätigt sich in den Antworten zu der Frage, ob Angehörige verschiedener Herkunftsgruppen die gleichen Rechte wie deutsche StaatsbürgerInnen bekommen sollten (Abbildung 50). Hier entspricht die Zustimmung (Werte 5 bis 7) zu gleichen Rechten für ItalienerInnen der für AussiedlerInnen. Die Fragen erfassen also tatsächlich die Ähnlichkeitswahrnehmung und nicht den durch Staatsangehörigkeit und Nationalität vorgegebenen rechtlichen Status, da dieser für AussiedlerInnen über ihre deutsche Staatsbürgerschaft de jure und de facto mit den deutschen StaatsbürgerInnen ohne Migrationshintergrund gleich auf liegen müsste, für ItalienerInnen als EUStaatsangehörige jedoch nicht. 


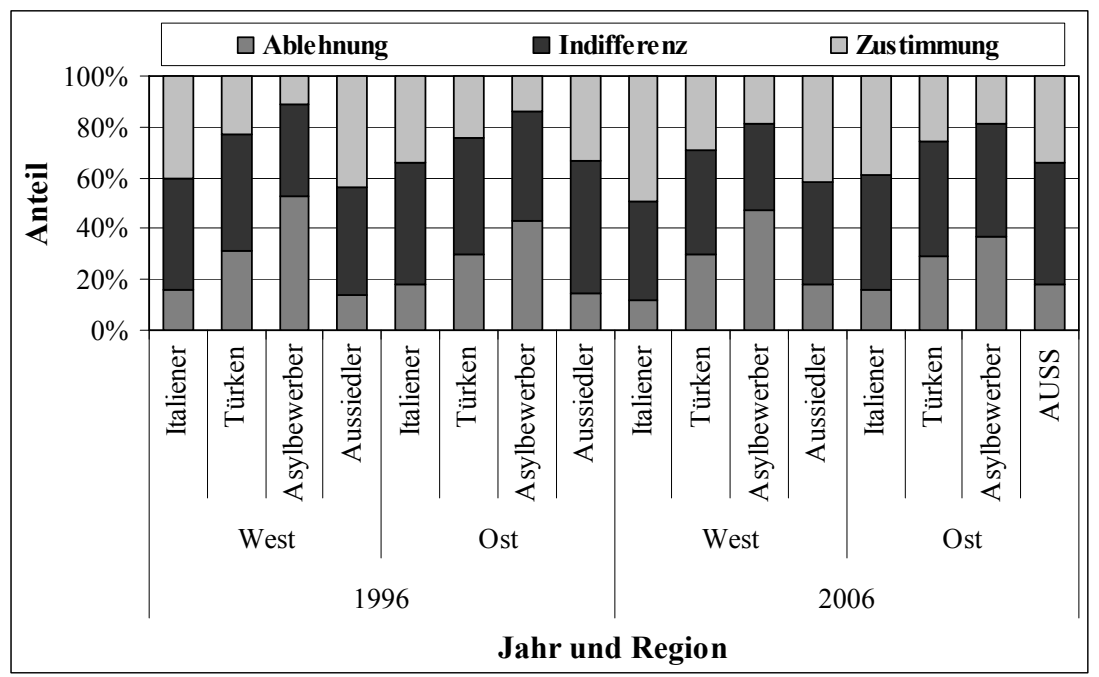

„Die in Deutschland lebenden [...] sollten in allen Bereichen die gleichen Rechte haben wie die Deutschen“ (Skala von 1 ,stimme überhaupt nicht zu“ bis 7 ,stimme voll und ganz $\mathrm{zu}^{\text {“) }}$

ALLBUS 1996, 2006, Eigene Berechnung

Abbildung 50 - Dominanzgesellschaft: Einstellung zur Gleichberechtigung

Wie sieht es mit Kriterien aus, die kulturelle Fremdheitszuschreibungen messen? Die Frage nach einer Bereicherung der Kultur in Deutschland durch die hier lebenden AusländerInnen trifft am ehesten die Idee eines Bedeutungsverlustes der zuvor vermeintlich festen kulturellen Grenzen. Bei dieser Frage erhöhte sich in Abbildung 51 der Anteil derjenigen, die die Kultur in Deutschland bereichert sahen, von 1996 zu 2006 und lag 2006 auf einer 7-Punkte-Skala bei 4,2 in Westdeutschland und 4,0 in Ostdeutschland.

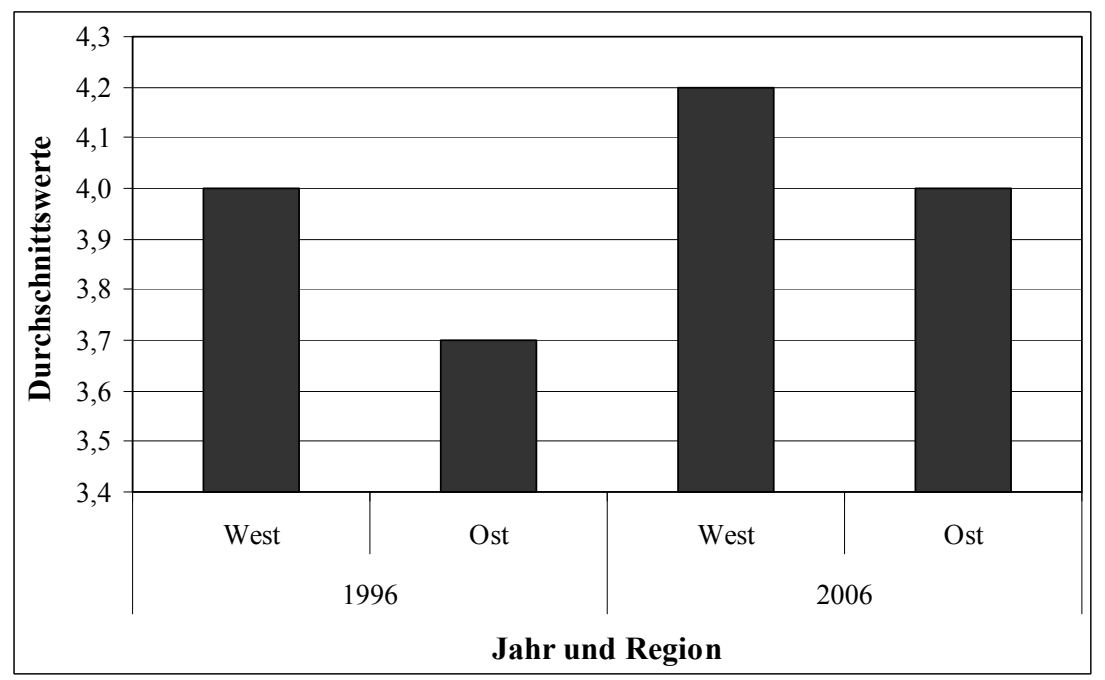

„Die in Deutschland lebenden Ausländer sind eine Bereicherung für die Kultur in Deutschland.“ (Skala von 1 ,stimme überhaupt nicht zu“ bis 7 ,stimme voll und ganz $\mathrm{zu}^{\circ}$ )

ALLBUS 1996, 2006, Eigene Berechnung

Abbildung 51 - Dominanzgesellschaft: Bereicherung der Kultur in Deutschland 
Interessant ist schließlich die Frage nach den Kriterien für eine Einbürgerung, da die Antworten auf die Bedingungen hindeuten, unter denen Nicht-MigrantInnen bereit sind, die ,Anderen' als Teil des ,Wir' zu akzeptieren. Dabei garantiert die deutsche Staatsbürgerschaft selbstverständlich noch keine volle Akzeptanz, wie die umfangreiche Diskriminierung von deutschen StaatsbürgerInnen mit Migrationshintergrund (und/oder ,dunkler Hautfarbe') zeigt. Es handelt sich also mathematisch gesprochen nicht um hinreichende Bedingungen für Akzeptanz, wohl aber um notwendige. Im Wandel dieser notwendigen Bedingungen zwischen 1996 und 2006 zeigt sich in Abbildung 52 eine Entwicklung, die die Wahrnehmung von Ähnlichkeit überhaupt erst ermöglicht: Von den sieben Kriterien haben drei in der öffentlichen Meinung für eine Einbürgerung an Bedeutung verloren: 2006 wird es für weniger wichtig gehalten, dass der/die MigrantIn in Deutschland geboren ist, dass er/sie deutscher Abstammung ist und dass er/sie lange in Deutschland gelebt hat. Alle drei sind Kriterien, die sich nicht nachträglich ändern lassen. Hingegen steigt das Gewicht der Kriterien, die grundsätzlich beeinflussbar sind, nämlich Deutsch zu sprechen, den Lebensstil anzupassen und für den eigenen Lebensunterhalt zu sorgen. Die Bedeutung angeborener (oder langfristig erworbener) Faktoren sinkt zugunsten von Kriterien, die von allen mehr oder weniger beeinflussbar sind. Die einzige Ausnahme zu diesem Trend ist das Kriterium der Mitgliedschaft in einer christlichen Kirche. Dessen Bedeutung steigt von 1996 auf 2006 leicht an. Hier schlägt sich möglicherweise der gesellschaftliche Diskurs zu den ,muslimischen Anderen' nach den Anschlägen des 11. September 2001 nieder.

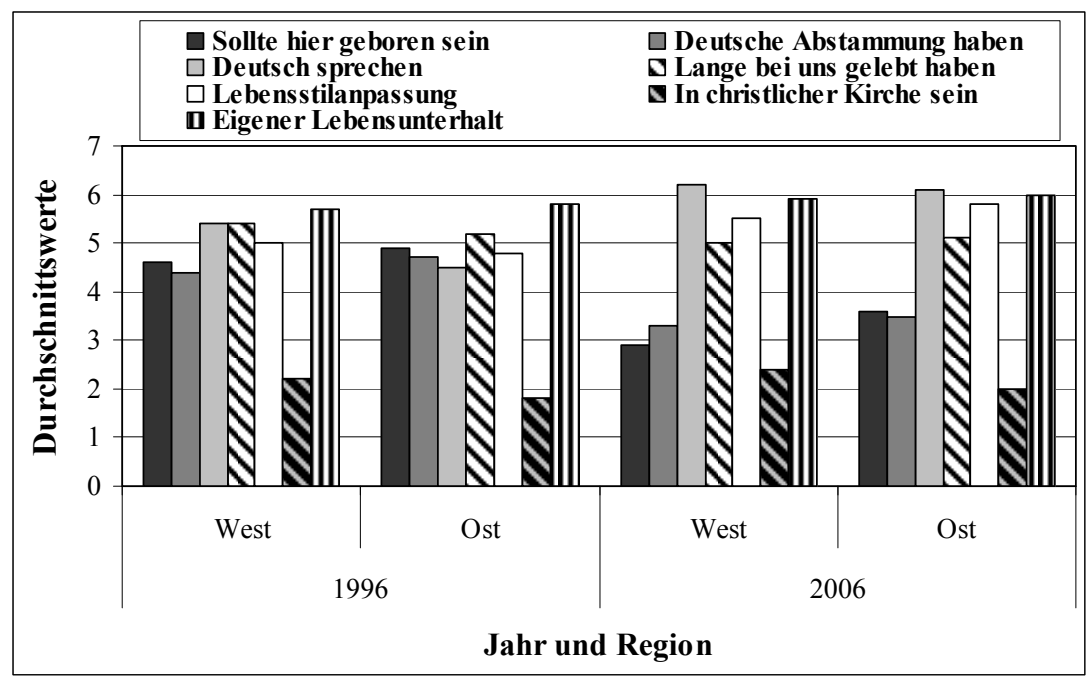

"Ich nenne Ihnen jetzt verschiedene Dinge, die bei der Entscheidung über die Vergabe der deutschen Staatsbürgerschaft eine Rolle spielen können. Bitte sagen Sie mir anhand der Skala, wie wichtig Ihrer Meinung nach diese Dinge jeweils sein sollten." (Skala von 1 "überhaupt nicht wichtig" bis zu 7 "sehr wichtig")

ALLBUS 1996, 2006, Eigene Berechnung

Abbildung 52 - Dominanzgesellschaft: Kriterien für Einbürgerung 
Schließlich möchte ich etwas detaillierter die Frage der Lebensstilanpassung betrachten, da diese in verschiedene Richtungen weist. Der Meinung, dass die in Deutschland lebenden AusländerInnen ,ihren Lebensstil ein bisschen besser an den der Deutschen anpassen“ sollten, waren 1980 66\% der Westdeutschen (Abbildung 53). 1994 sank dieser Wert bei den Westdeutschen auf unter 50\%; auch in Ostdeutschland lag er mit 54\% relativ niedrig. Danach steigt die Zustimmung zu dieser Aussage wieder: 2006 sprachen sich 79\% der Westdeutschen und 84\% der Ostdeutschen für ein bisschen mehr Anpassung aus. Diese Zustimmung wird von allen Bevölkerungsgruppen getragen: Während 1994 die Jüngeren, diejenigen mit höheren Bildungstiteln und diejenigen, die angaben, aus der Mittel- oder Oberschicht zu kommen, und/oder sich politisch eher links positionierten, eine Lebensstilanpassung für weniger nötig hielten, unterscheiden sich die verschiedenen Personengruppen heute in der Beantwortung dieser Frage kaum (Terwey/Scheuer 2007). Die Zunahme der Zustimmung zur Lebensstilanpassung ist damit vor allem Veränderungen bei diesen eher als tolerant geltenden Gruppen geschuldet. Die Forderung nach Anpassung ist in den letzten Jahren über alle Bevölkerungsgruppen hinweg Konsens geworden und entspricht dem dominanten politischen und medialen Diskurs.

Gleichzeitig steigt, wie oben gezeigt, die Bereitschaft, MigrantInnen bei Erfüllung bestimmter Bedingungen als ähnlich wahrzunehmen. Zudem betreffen diese Bedingungen zunehmend veränderbare Kriterien (z.B. Sprachkenntnisse). Keinen Rückschluss erlauben die Daten auf die Frage, ob phänotypische Differenzen (v.a. andere Hautfarbe) weiterhin als unverrückbare Markierungen von Andersartigkeit fungieren. Damit ist die zunehmende Anpassungsforderung eine zweischneidige Sache: Zum einen drückt sie eine generelle Bereitschaft aus, andere Menschen als Teil des, Wir' zu akzeptieren (zumindest ,Boundary Crossing') und zudem über die Kriterien, die ,Uns' ausmachen, zu diskutieren: Wie muss eine Deutsche oder ein Deutscher sein, welche verschiedenen Möglichkeiten gibt es? Dabei entstehen möglicherweise neue Identitätskonstellationen (,Boundary Shifting'). Auf der anderen Seite stellt sich jedoch die Frage, welche Kriterien im Sinne eines ,Boundary Blurrings' an Bedeutung verlieren und welche sich im Gegenzug als Markierungen von Fremdheit verfestigen. $\mathrm{Zu}$ dieser Frage gibt es keine empirischen Untersuchungen. Im ,Lifestyle'-Bereich hat es in den letzten Jahrzehnten sicherlich einige Verwischungen gegeben: kulinarisch, musikalisch, in der Mode (,Ethno-Look'). ${ }^{149}$ Allerdings scheint die Bereitschaft, die Bedeutung von Fremdheitsmarkierungen in größerem Umfang zu relativieren und Unterschiede zu einer persönlichen Präferenz, möglicherweise gar zu einer Privatangelegenheit zu erklären, insgesamt gering ausge-

\footnotetext{
${ }^{149}$ Für eine auch kritische Diskussion vergleiche Ha 2005.
} 
prägt. Hier besteht also die Gefahr, dass bestimmte Markierungen nicht an Bedeutung verlieren (d.h. ,Blurring' nicht stattfindet) und dadurch bestimmte Gruppen ausgeschlossen bleiben.

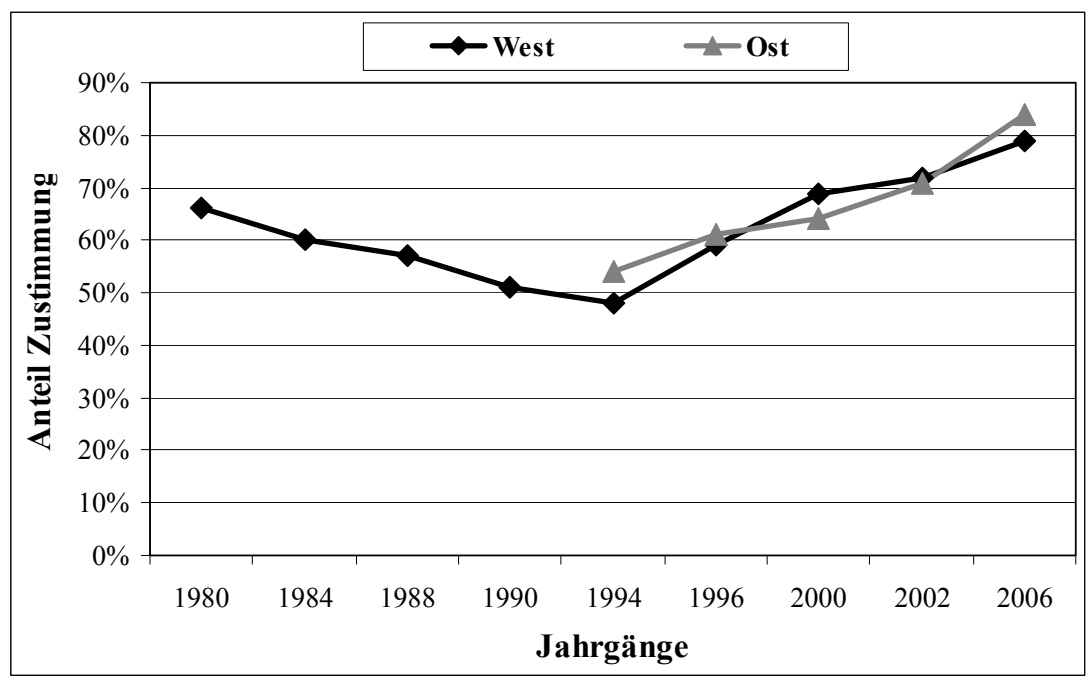

„Gastarbeiter sollten ihren Lebensstil ein bisschen besser an den der Deutschen anpassen.“ ALLBUS 1980, 1984, 1988, 1990, 1994, 1996, 2000, 2002, 2006, Eigene Berechnung

Abbildung 53 - Dominanzgesellschaft: Lebensstilanpassung

Die Frage nach der Lebensstilanpassung impliziert, dass die Deutschen einen Lebensstil hätten. Wie irrig diese Annahme ist, zeigt die Diskussion um eine deutsche Leitkultur (Merz 2000). In dieser Debatte konnten sich die BefürworterInnen einer Leitkultur nicht über Konkretisierungen des Lebensstils einigen, die über ein Bekenntnis zur demokratischfreiheitlichen Grundordnung, im Besonderen zum Grundgesetz, und gute Sprachkenntnisse im Deutschen hinausgehen. Eingeschlossen wurde darüber hinaus höchstens ein verantwortlicher Umgang mit der deutschen Geschichte, besonders dem Zweiten Weltkrieg und dem Holocaust. Solch minimale Übereinstimmung haben jedoch die wenigsten vor Augen, wenn sie die Frage mit dem Wunsch nach mehr Anpassung beantworten. Sie wünschen sich eine Anpassung an ihren Lebensstil und ignorieren dabei, dass sich dieser auch von dem anderer Deutscher ohne Migrationshintergrund unterscheidet. ${ }^{150}$

Schiffauer weist in seiner qualitativen Untersuchung auf diese Abgrenzungen zwischen verschiedenen Gruppen von Deutschen hin. In einer von ihm wiedergegebenen Sequenz aus einer Studie zu Schulkulturen grenzen sich linke, antifaschistische Gruppen von xenophoben Deutschen ab. Dadurch schaffen sie Identifikationsangebote und erleichtern MigrantInnen den $\mathrm{Zu}$ gang zu einer der nicht-migrantischen Gruppierungen in Deutschland - wenngleich einer rela-

\footnotetext{
${ }^{150}$ So denken FeministInnen bei der Frage der Anpassung des Lebensstils wahrscheinlich an andere Werte als Nationalkonservative.
} 
tiven Minderheit. (Schiffauer 2001: 244ff) Durch die Identifikation mit einer Gruppe, die sich explizit vom vermeintlichen ,Mainstream’ abgrenzt, entsteht die Möglichkeit, Teil der Mehrheitsgesellschaft zu werden.

Dass die Homogenität des ,Wir' brüchig ist, hatte, darauf sei hier nochmals hingewiesen, auch die Frage zu dem Angenehm/Unangenehm-Sein einer Einheirat von Angehörigen anderer Konfessionen gezeigt: Zwar wurde die Einheirat eines Muslims/einer Muslima in die eigene Familie als am wenigsten angenehm bewertet, aber auch ProtestantInnen und KatholikInnen stießen auf erhebliche Vorbehalte - ein Beispiel für die auch innerhalb der deutschen Bevölkerung ohne Migrationshintergrund bestehenden sozialen Distanzen, von den sozialen Distanzen zwischen Oberschicht und Prekarisierten sowie regionalen Abgrenzungen in Deutschland ganz zu schweigen.

Um nicht die Analyse unüberschaubar komplex werden zu lassen und die Qualität der vorhandenen Daten zu überfordern, ist auf sozioökonomische Binnendifferenzierungen im Vorhergehenden verzichtet worden. Am Ende dieses Kapitels soll jedoch anhand der SHELLJugendstudie noch auf das Alter als eine der wichtigen Variablen für unterschiedliche Haltungen eingegangen werden, da abweichende Einstellungen bei den Jüngeren möglicherweise einen Trend zum Ausdruck bringen.

Die 13. SHELL-Jugendstudie fand heraus, dass weit mehr als 50\% der Jugendlichen ohne Migrationshintergrund „Deutsche und Ausländer [als] eher ähnlich“ empfanden, und zwar im Bereich der Freizeit, der schulischen und beruflichen Qualifikation, in Fragen der eigenen Lebensführung und hinsichtlich Politik und Zukunftsgestaltung (Abbildung 54). Nur hinsichtlich eines Bereichs, zusammengesetzt aus Gewohnheiten hinsichtlich Ernährung, Kleidung, Familienleben und Religion, überwogen diejenigen, die mehr Unterschiede wahrnahmen, mit 60\%. (Münchmeier 2000: 248ff). Weiterhin dominierte bei den Jugendlichen die Einstellung, dass AusländerInnen und Deutsche ohne Migrationshintergrund voneinander lernen können: Dem stimmten knapp 70\% der Jungen und 77\% der Mädchen ohne Migrationshintergrund zu (Münchmeier 2000: 246). Zudem lehnten Jüngere auch in der ALLBUS eine Einheirat von Angehörigen verschiedener Zuwanderungsgruppen weniger ab (Koch/Wasmer 1997: 466). Diese Zahlen deuten auf einen zukünftigen gesellschaftlichen Wandel hin, auch wenn nicht ausgeschlossen ist, dass es sich neben einem Kohorteneffekt in Teilen um Alterseffekte handelt, die mit den Jahren nachlassen. 


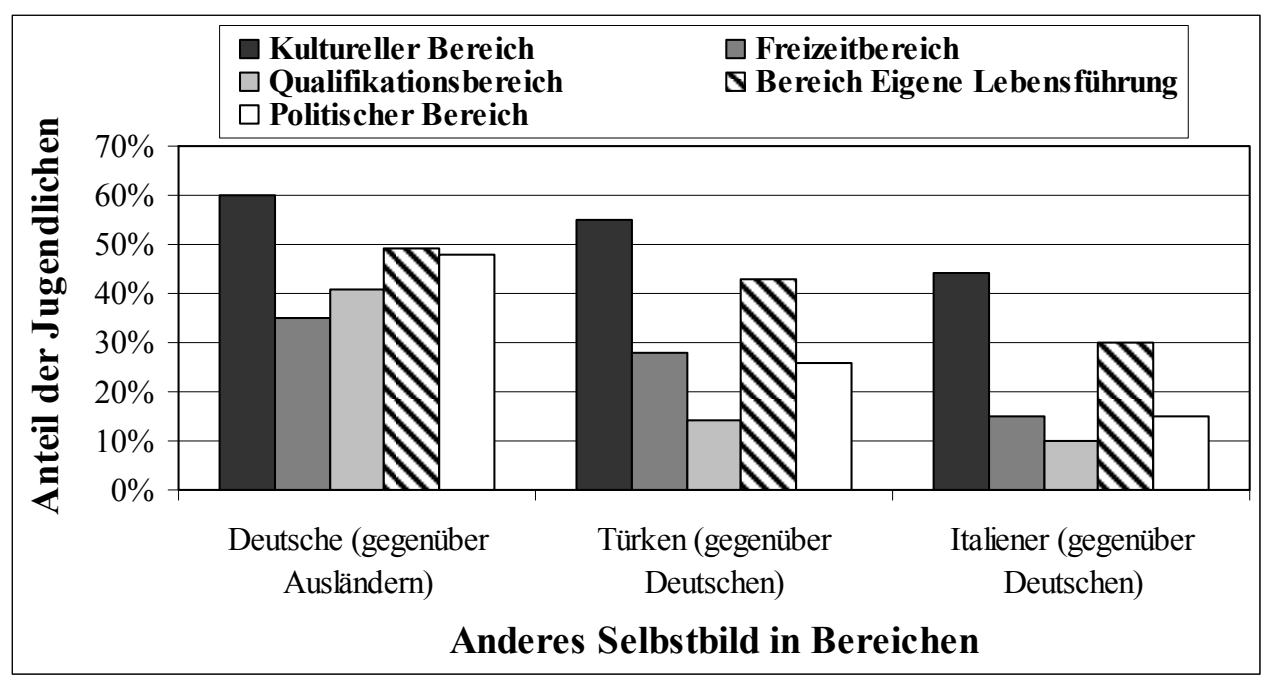

13. SHELL Jugendstudie (Münchmeier (2000): 248ff), Eigene Berechnungen

Abbildung 54 - Dominanzgesellschaft: Selbstbild Jugendliche

In diesem Abschnitt zeigte sich eine Zunahme der Ähnlichkeitswahrnehmung durch die nichtmigrantische Dominanzbevölkerung. Diese äußert sich u.a. in abnehmender Zustimmung zum Endogamie-Gebot, der verringerten Wahrnehmung von Unterschieden im Lebensstil und zunehmender Zubilligung von gleichen Rechten für einige Herkunftsgruppen. Bei den Jüngeren ist eine besonders große Ähnlichkeitswahrnehmung zu finden. Nach diesen Einstellungen und Wahrnehmungen geht es im nächsten Kapitel um Praktiken rassistischer Diskriminierung und um institutionellen Rassismus. Damit wird die strukturelle Ebene in den Blick genommen, die als Umgebung die Integrationsprozesse durchdringt.

\subsection{Rassismus als Struktur der Dominanzbevölkerung}

Rassismus als eine der strukturellen Variablen der Dominanzbevölkerung beeinflusst Integrationsprozesse mannigfaltig: den Erwerb von Ressourcen, die Überführung dieser Ressourcen in als status- und kulturgleich markierte Interaktionen und die Wahrnehmung der MigrantInnen als ähnlich durch die Dominanzbevölkerung. Dabei sind, wie in Kapitel 2.3 ausgeführt, Veränderungen in der Intensität und inhaltlichen Ausgestaltung des Rassismus schnell möglich, ausgelöst zum Beispiel durch weltpolitische Ereignisse und deren Interpretation oder durch Mobilisierungsstrategien von politischen Entrepreneuren. Solche kurzfristigen Schwankungen und ihre Auslöser sind jedoch nicht Gegenstand dieser Arbeit. In diesem Kapitel werden zunächst Veränderungen in den Selbstauskünften zu diskriminierenden Verhalten und Einstellungen beschrieben. Diese belegen zusammen mit der verstärkten Ähnlichkeitswahrnehmung eine Verringerung rassistischer Diskriminierung. In einem zweiten Schritt werden die Ergebnisse verschiedener Feldexperimente dargestellt. Diese bieten gegenüber den Selbst- 
auskünften zwei Vorteile: Zum einen kann eine Verzerrung der Daten durch soziale Erwünschtheit ausgeschlossen werden, da die einzelnen Mitglieder der Dominanzbevölkerung nicht im Vorhinein wissen, dass sie an einem Experiment teilnehmen. Zum anderen erlauben solche Experimente, die Reaktionen gegenüber Angehörigen verschieden markierter Gruppen zu differenzieren. Die in diesen Experimenten zum Ausdruck kommende rassistische Diskriminierung darf jedoch, wie gezeigt werden wird, nicht als individuell deviantes Verhalten interpretiert werden, sondern sie beinhaltet eine gewisse Rationalität, die sich durch die Struktur der sozialen Ungleichheit ergibt, von der Rassismus ein Teil ist. Schließlich wird in einem letzten Abschnitt die Absicherung und Reproduktion von Rassismus durch institutionelle und rechtliche Faktoren am Beispiel der Schule untersucht.

Der im vorigen Kapitel konstatierte Zunahme der wahrgenommenen Ähnlichkeit der migrantischen Bevölkerung auf Seiten der Dominanzbevölkerung entspricht eine verminderte Diskriminierung, die in den Daten der ALLBUS-Umfragen aus den Jahren 1996 und 2006 zum Ausdruck kommt. ${ }^{151}$ Um die soziale Erwünschtheit in den Antworten zu reduzieren, wurde zunächst untersucht, für wie wahrscheinlich ein Vorkommen von Diskriminierung gehalten wird (Tabelle 41).

Tabelle 41 - Dominanzgesellschaft: Einschätzung Wahrscheinlichkeit von Diskriminierung

\begin{tabular}{|c|c|c|c|c|c|}
\hline & & \multicolumn{2}{|c|}{1996} & \multicolumn{2}{|c|}{2006} \\
\hline & & WEST & OST & WEST & OST \\
\hline \multirow{3}{*}{$\begin{array}{l}\text { „Ein Gastwirt weigert sich, in } \\
\text { seinem Lokal Ausländer zu } \\
\text { bedienen“ }\end{array}$} & $\begin{array}{l}\text { Das kommt } \\
\text { nie/selten vor }\end{array}$ & $51 \%$ & $52 \%$ & $62 \%$ & $64 \%$ \\
\hline & $\begin{array}{l}\text { Das kommt hin } \\
\text { und wieder vor }\end{array}$ & $42 \%$ & $40 \%$ & $31 \%$ & $32 \%$ \\
\hline & $\begin{array}{l}\text { Das kommt (sehr) } \\
\text { oft vor }\end{array}$ & $7 \%$ & $8 \%$ & $7 \%$ & $4 \%$ \\
\hline \multirow{3}{*}{$\begin{array}{l}\text { „Eltern verbieten ihrer } \\
\text { 17jährigen Tochter die } \\
\text { Freundschaft mit einem tür- } \\
\text { kischen Jugendlichen“" }\end{array}$} & $\begin{array}{l}\text { Das kommt } \\
\text { nie/selten vor }\end{array}$ & $14 \%$ & $15 \%$ & $20 \%$ & $20 \%$ \\
\hline & $\begin{array}{l}\text { Das kommt hin } \\
\text { und wieder vor }\end{array}$ & $38 \%$ & $41 \%$ & $39 \%$ & $44 \%$ \\
\hline & $\begin{array}{l}\text { Das kommt (sehr) } \\
\text { oft vor }\end{array}$ & $48 \%$ & $44 \%$ & $41 \%$ & $36 \%$ \\
\hline \multirow{3}{*}{$\begin{array}{l}\text { „Ein Unternehmer, der Per- } \\
\text { sonal abbauen muss, entlässt } \\
\text { zuerst die ausländischen Ar- } \\
\text { beitnehmer“ }\end{array}$} & $\begin{array}{l}\text { Das kommt } \\
\text { nie/selten vor }\end{array}$ & $32 \%$ & $40 \%$ & $45 \%$ & $42 \%$ \\
\hline & $\begin{array}{l}\text { Das kommt hin } \\
\text { und wieder vor }\end{array}$ & $42 \%$ & $33 \%$ & $40 \%$ & $41 \%$ \\
\hline & $\begin{array}{l}\text { Das kommt (sehr) } \\
\text { oft vor }\end{array}$ & $26 \%$ & $27 \%$ & $15 \%$ & $17 \%$ \\
\hline
\end{tabular}

ALLBUS 1996 und 2006, Eigene Berechnungen

${ }^{151}$ Einschränkend muss festgehalten werden, dass in diesen Umfragen nur allgemein Diskriminierung gegenüber AusländerInnen erfasst und weder nach Herkunftsgruppe noch nach Generation unterschieden wird. 
Im Jahr 1996 dachte die Hälfte der Deutschen, dass ein Gastwirt sich hin oder wieder oder sogar (sehr) oft weigert, AusländerInnen in seinem Lokal zu bedienen. Zehn Jahre später waren dies reichlich zehn Prozentpunkte weniger. Auch dass Eltern ihrer 17-jährigen Tochter die Freundschaft mit einem türkischen Jugendlichen verbieten, wurde 2006 für weniger wahrscheinlich gehalten als 1996. Ebenso sah es mit der geschätzten Wahrscheinlichkeit dafür aus, dass ein Unternehmer, der Personal abbauen muss, zunächst die ausländischen Arbeitnehmer entlässt.

In einem zweiten Schritt wurde dann die Einstellung zu diesen diskriminierenden Aussagen abgefragt (Tabelle 42). Hier änderte sich allerdings in den zehn Jahren kaum etwas: Die Zustimmung zu Diskriminierung durch den Gastwirt sowie durch die Eltern blieb gleich. Nur die Diskriminierung durch den Unternehmer in Zeiten des Personalabbaus wurde 2006 stärker abgelehnt, und zwar um 5 Prozentpunkte in Ost- und 6 Prozentpunkte in Westdeutschland.

Tabelle 42 - Dominanzgesellschaft: Eigene Einstellung zu diskriminierenden Aussagen

\begin{tabular}{|c|c|c|c|c|c|}
\hline & & \multicolumn{2}{|c|}{1996} & \multicolumn{2}{|c|}{2006} \\
\hline & & WEST & OST & WEST & OST \\
\hline \multirow{3}{*}{$\begin{array}{l}\text { „Ein Gastwirt weigert } \\
\text { sich, in seinem Lokal } \\
\text { Ausländer zu bedie- } \\
\text { nen“6 }\end{array}$} & $\begin{array}{l}\text { Die meisten Deutschen finden das } \\
\text { überhaupt nicht in Ordnung }\end{array}$ & $62 \%$ & $52 \%$ & $63 \%$ & $59 \%$ \\
\hline & $\begin{array}{l}\text { Die meisten Deutschen finden das } \\
\text { eher nicht in Ordnung }\end{array}$ & $28 \%$ & $37 \%$ & $25 \%$ & $32 \%$ \\
\hline & $\begin{array}{l}\text { Die meisten Deutschen finden das } \\
\text { völlig/eher in Ordnung }\end{array}$ & $10 \%$ & $11 \%$ & $12 \%$ & $9 \%$ \\
\hline \multirow{3}{*}{$\begin{array}{l}\text { „Eltern verbieten ihrer } \\
\text { 17jährigen Tochter die } \\
\text { Freundschaft mit einem } \\
\text { türkischen Jugendli- } \\
\text { chen“" }\end{array}$} & $\begin{array}{l}\text { Die meisten Deutschen finden das } \\
\text { überhaupt nicht in Ordnung }\end{array}$ & $30 \%$ & $21 \%$ & $31 \%$ & $21 \%$ \\
\hline & $\begin{array}{l}\text { Die meisten Deutschen finden das } \\
\text { eher nicht in Ordnung }\end{array}$ & $40 \%$ & $41 \%$ & $37 \%$ & $42 \%$ \\
\hline & $\begin{array}{l}\text { Die meisten Deutschen finden das } \\
\text { völlig/eher in Ordnung }\end{array}$ & $30 \%$ & $38 \%$ & $32 \%$ & $37 \%$ \\
\hline \multirow{3}{*}{$\begin{array}{l}\text { „Ein Unternehmer, der } \\
\text { Personal abbauen } \\
\text { muss, entlässt zuerst } \\
\text { die ausländischen Ar- } \\
\text { beitnehmer“ }\end{array}$} & $\begin{array}{l}\text { Die meisten Deutschen finden das } \\
\text { überhaupt nicht in Ordnung }\end{array}$ & $39 \%$ & $23 \%$ & $45 \%$ & $28 \%$ \\
\hline & $\begin{array}{l}\text { Die meisten Deutschen finden das } \\
\text { eher nicht in Ordnung }\end{array}$ & $39 \%$ & $36 \%$ & $36 \%$ & $42 \%$ \\
\hline & $\begin{array}{l}\text { Die meisten Deutschen finden das } \\
\text { völlig/eher in Ordnung }\end{array}$ & $22 \%$ & $41 \%$ & $19 \%$ & $30 \%$ \\
\hline
\end{tabular}

ALLBUS 1996 und 2006, Eigene Berechnungen

In einem dritten Schritt wurde nach der Einschätzung der allgemeinen Meinung zu Diskriminierung in Deutschland gefragt (Tabelle 43). Die Befragten um ihre Einschätzung zur allgemeinen Meinung der Bevölkerung zu bitten, wird in sozialwissenschaftlichen Umfragen als geeignetes Instrument betrachtet, um die soziale Konditionierung von Antworten zu reduzie- 
ren: Während die Befragten die eigene diskriminierende Einstellung möglicherweise aufgrund eines empfundenen sozialen Drucks nicht preisgeben möchten, finden sie es meist unproblematisch, solche Einstellungen anderen zuzuschreiben. Grundsätzlich gilt, dass sich auch diese geschätzte diskriminierende Haltung der Allgemeinheit über die Jahre deutlich verringert hat. Darüber hinaus fällt jedoch auf, dass bei dieser Frage in der ALLBUS-Untersuchung durchschnittlich 20 Prozentpunkte mehr Personen angeben, dass die meisten Deutschen eine Diskriminierung im Gasthaus völlig oder eher in Ordnung fänden, als sie dies für sich selbst anführen. Bezüglich der elterlichen Diskriminierung einer Freundschaft der Tochter mit einem türkischen Jugendlichen beträgt dieser Unterschied zwischen 20 und 30 Prozentpunkten in Ost- und Westdeutschland, bezüglich des Personalabbaus durch den Unternehmer sogar 30-35 Prozentpunkte. Dennoch ergibt sich auch aus der Einschätzung der allgemeinen Meinung ein Rückgang der Diskriminierung zwischen 1996 und 2006, sowohl in Ost- als auch in Westdeutschland (mit Ausnahme des Gastwirt-Beispiels).

Tabelle 43 - Dominanzgesellschaft: Einschätzung allgemeine Meinung zu diskriminierenden Aussagen

\begin{tabular}{|c|c|c|c|c|c|}
\hline & & \multicolumn{2}{|c|}{1996} & \multicolumn{2}{|c|}{2006} \\
\hline & & WEST & OST & WEST & OST \\
\hline \multirow{3}{*}{$\begin{array}{l}\text { „Ein Gastwirt wei- } \\
\text { gert sich, in seinem } \\
\text { Lokal Ausländer zu } \\
\text { bedienen““ }\end{array}$} & $\begin{array}{l}\text { Die meisten Deutschen finden das } \\
\text { überhaupt nicht in Ordnung }\end{array}$ & $20 \%$ & $11 \%$ & $21 \%$ & $21 \%$ \\
\hline & $\begin{array}{l}\text { Die meisten Deutschen finden das } \\
\text { eher nicht in Ordnung }\end{array}$ & $51 \%$ & $52 \%$ & $48 \%$ & $53 \%$ \\
\hline & $\begin{array}{l}\text { Die meisten Deutschen finden das } \\
\text { völlig/eher in Ordnung }\end{array}$ & $29 \%$ & $37 \%$ & $31 \%$ & $26 \%$ \\
\hline \multirow{3}{*}{$\begin{array}{l}\text { „Eltern verbieten } \\
\text { ihrer 17jährigen } \\
\text { Tochter die Freund- } \\
\text { schaft mit einem } \\
\text { türkischen Jugend- } \\
\text { lichen“ }\end{array}$} & $\begin{array}{l}\text { Die meisten Deutschen finden das } \\
\text { überhaupt nicht in Ordnung }\end{array}$ & $6 \%$ & $4 \%$ & $11 \%$ & $9 \%$ \\
\hline & $\begin{array}{l}\text { Die meisten Deutschen finden das } \\
\text { eher nicht in Ordnung }\end{array}$ & $34 \%$ & $33 \%$ & $34 \%$ & $34 \%$ \\
\hline & $\begin{array}{l}\text { Die meisten Deutschen finden das } \\
\text { völlig/eher in Ordnung }\end{array}$ & $60 \%$ & $63 \%$ & $55 \%$ & $57 \%$ \\
\hline \multirow{3}{*}{$\begin{array}{l}\text { „Ein Unternehmer, } \\
\text { der Personal ab- } \\
\text { bauen muss, ent- } \\
\text { lässt zuerst die aus- } \\
\text { ländischen Arbeit- } \\
\text { nehmer“ }\end{array}$} & $\begin{array}{l}\text { Die meisten Deutschen finden das } \\
\text { überhaupt nicht in Ordnung }\end{array}$ & $9 \%$ & $7 \%$ & $13 \%$ & $11 \%$ \\
\hline & $\begin{array}{l}\text { Die meisten Deutschen finden das } \\
\text { eher nicht in Ordnung }\end{array}$ & $34 \%$ & $23 \%$ & $36 \%$ & $30 \%$ \\
\hline & $\begin{array}{l}\text { Die meisten Deutschen finden das } \\
\text { völlig/eher in Ordnung }\end{array}$ & $57 \%$ & $70 \%$ & $51 \%$ & $59 \%$ \\
\hline
\end{tabular}

ALLBUS 1996 und 2006, Eigene Berechnungen

Damit zeigt sich insgesamt eine Abnahme sowohl in der Häufigkeit, mit der diskriminierende Praktiken angenommen und Zustimmung zu diskriminierenden Aussagen geäußert wird, als auch in der Wahrscheinlichkeit, mit der diskriminierende Einstellungen bei anderen vermutet 
werden. Dieser Rückgang darf jedoch nicht über das weiterhin hohe Maß an Diskriminierung hinwegtäuschen.

Die Ergebnisse von Meinungsumfragen zu solch sensiblen Themen wie Diskriminierung sind aus sozialwissenschaftlicher Sicht mit einer gewissen Vorsicht zu betrachten, weil in den meisten ,westlichen' Gesellschaften heute ein Diskriminierungsverbot gilt, und diskriminierende Einstellungen von erheblichen Teilen der Bevölkerung als sozial unerwünscht wahrgenommen werden, womit die oben angesprochenen Diskrepanzen in den zustimmenden Werten erklärt werden können. Wie sieht es also mit dem beobachtbaren Verhalten aus? Bisher gibt es nur zwei Feldstudien, die quantitative Daten zu dieser Frage für Deutschland erhoben haben. Beide werden im Folgenden vorgestellt. Es handelt sich um die quasi-experimentellen Untersuchungen im Rahmen der Studie von Goldberg, Mourinho und Kulke für die International Labour Organisation (ILO) (Goldberg, et al. 1995) und um die Arbeit der beiden Sozialpsychologen Klink und Wagner (Klink/Wagner 1999). Beide Studien erlauben es, die Diskriminierung von Gruppen genauer zu untersuchen, die in unterschiedlicher Weise als fremd markiert werden: durch ,fremde Hautfarbe', Kleidung und Kopftuch, Akzent, migrantischklingende Nachnamen. Daraus können Unterschiede für die Generationen sowie Veränderungen in der Diskriminierung aufgrund von verschiedenen Verbindungen von Migrations- und sozialem Status abgeleitet werden.

Klink und Wagner (Klink/Wagner 1999) sammelten in 14 Feldstudien Daten über das Verhalten von Nicht-MigrantInnen zu den Bereichen Wohnungssuche, Hilfsbereitschaft, Service im Restaurant sowie informelles Marktverhalten in gestellten Situationen. Es konnten in 713 Fällen Beobachtungen gemacht werden. Insgesamt war in den Daten eine signifikante Diskriminierung der MigrantInnen zu beobachten. In einem Experiment riefen zum Beispiel jeweils eine Deutsche ohne Migrationshintergrund und eine durch Namen und Akzent als Angehörige der türkischen Minderheit markierte Frau in zufälliger Reihenfolge nacheinander bei den InserentInnen von Wohnungsgeboten an und erbaten einen Termin zur Besichtigung. Die biografischen Angaben der beiden Frauen sowie ihr Sprechtext für die Vorstellung entsprachen sich. Im Ergebnis bekamen die deutschen Frauen ohne Migrationshintergrund zu 81\% einen Termin, die als türkisch markierten Frauen nur ungefähr halb so häufig (44\%). Eine etwas geringere Differenz ergab sich bei demselben Experiment mit einem deutschen Mann ohne Migrationshintergrund und einem durch Namen und Akzent als Angehöriger der polnischen Minderheit markierten Mann: Die deutschen Männer ohne Migrationshintergrund bekamen zu $78 \%$ Besichtigungstermine, von den polnisch markierten Männern wurde nur gut die Hälfte zur Besichtigung eingeladen (55\%). Stellte sich der polnisch markierte Mann mit akademi- 
schem Titel („Dr. Kawowski“) vor, bekam er signifikant häufiger einen Termin. Darin zeigen sich graduelle Unterschiede der Diskriminierung, die zumeist nicht untersucht werden. Die Ergebnisse des Experiments spiegeln hingegen solche feinen Abstufungen der Diskriminierung aufgrund unterschiedlicher Ähnlichkeitswahrnehmung (hier: zwischen als türkisch bzw. polnisch Markierten, Statusniedrigeren und Statushöheren).

Für die vorliegende Arbeit zur zweiten Generation besonders relevant ist eine weitere Wohnungsstudie: Dabei erbaten Männer und Frauen, deren Namen eine türkische Herkunft nahelegte, akzentfrei einen Besichtigungstermin. Diese Kombination von akzentfreiem Deutsch und türkischem (oder anderem, ausländischem') Namen ist typisch für die zweite Generation. In der Studie von Klink und Wagner wurde dieser Gruppe im Unterschied zu den Türkinnen und den Polen mit Akzent genauso häufig ein Besichtigungstermin angeboten wie den Deutschen ohne Migrationshintergrund. Selbstverständlich bedeutet dies noch nicht, dass sie nach der Besichtigung auch in gleichem Maße den Zuschlag für die Wohnung bekommen hätten.

In einer zweiten Serie von Studien fragte eine Hilfesuchende nach dem Weg. Hier zeigte sich, dass Personen, die über Kleidung und Akzent als aus dem Nahen Osten kommend markiert wurden, signifikant weniger geholfen wurde als Deutschen ohne Migrationshintergrund. Besonders groß - nämlich 23 Prozentpunkte - war der Unterschied zwischen Frauen, die Kopftuch trugen und mit Akzent sprachen, und vorgeblich deutschen Frauen ohne Migrationshintergrund. Auch bei einer Studie mit vergessenen Briefen wurden Personen türkischer Herkunft stark diskriminiert: In einer Telefonzelle wurden zugeklebte und frankierte Briefe platziert. Diese waren in der Hälfte der Fälle an Personen mit deutsch klingenden Namen addressiert, in der anderen Hälfte an Personen mit türkisch klingenden Namen. Die Briefe enthielten keine Informationen zum Absender. Die Anzahl der Briefe, die in einen Briefkasten eingeworfen wurden und die AdressatInnen erreichten, unterschied sich signifikant: Bei den deutsch klingenden Namen erreichten 68\% den Adressaten, bei den türkisch klingenden nur 48\% - eine Differenz von 20 Prozentpunkten. Diese Diskriminierung von Personen türkischer Herkunft wurde auch in einem leicht modifizierten Feldexperiment deutlich: Die Briefe waren nun alle an „Dr. Stoecker, Anwalt“ adressiert, der Absender aber hatte einmal einen ,deutsch'-, einmal einen ,türkisch' klingenden Namen. Der Brief war frankiert, jedoch unverschlossen und enthielt eine Ermächtigung an Herrn Stoecker, den Briefeschreiber in einem Autounfall zu vertreten; weiterhin lag die Kopie eines Ausweises mit Foto bei. Die Zahl der NutzerInnen der Telefonzelle, die den Brief ignorierten, unterschied sich deutlich: Bei dem Absender mit ,deutschem' Namen passierten im Durchschnitt 3,8 Personen die Telefonzelle, ohne den Brief an sich zu nehmen, bei dem Absender mit ,türkisch klingendem' Namen (und 
entsprechendem Foto auf der Kopie des Ausweises) waren dies beinahe doppelt so viele (7,1 Personen).

Dass sich diese Diskriminierung keinesfalls auf MigrantInnen türkischer Herkunft beschränkt, belegt ein Experiment zur Hilfeleistung gegenüber einer Frau, die angab, ihre Brieftasche sei gestohlen worden. Bat in diesem Fall eine deutsche Frau ohne Migrationshintergrund um Kleingeld zum Telefonieren, wurde in der Hälfte aller Fälle nach Geld gesucht und/oder dieses ausgehändigt, bei einer als rumänisch markierten Frau nur in weniger als einem Viertel der Fälle.

Dagegen wurde in der oben beschriebenen Situation der Wegerfragung ein als Grieche markierter Mann im Durchschnitt nicht diskriminiert. Dies passt - bei aller Vorsicht hinsichtlich weitreichender Konklusionen - zu der theoretischen Annahme, dass rassistische Diskriminierung auf einem Hierarchieverhältnis aufbaut, wobei der EU-Bürger aus Griechenland höher bewertet wird als die Person aus dem ärmeren Rumänien, das zu jener Zeit kein EUMitgliedsland war.

Die Verbindung von Diskriminierung und sozialem Status wird in den folgenden zwei Experimenten deutlich: Bei verschiedenen gehobenen Restaurants ruft zunächst ein Chilene an und bittet um eine Tischreservierung, danach versucht ein Deutscher ohne Migrationshintergrund zu reservieren. Die Reservierungen des Letzteren wurden zu 100\% akzeptiert, die des Chilenen zu 92\%. Der Unterschied ist also geringer als in den anderen Diskriminierungsfällen. Noch deutlicher wird diese Verkettung mit dem sozioökonomischen Status bei der folgenden Studie: Hier versuchte eine deutsche Sekretärin, vor Ort für türkische oder deutsche Organisationen einen Tisch zu reservieren, in der Hälfte der Fälle für eine jeweilige Gewerkschaft, in der anderen für einen Anwaltsverein. Zwar zeigt sich auch hier das bekannte Muster, dass die Reservierungen für türkische Organisationen seltener angenommen wurden als die für deutsche Organisationen (80\% zu 100\%). Darüber hinaus unterscheidet sich die Wahrscheinlichkeit jedoch stark nach den beiden Organisationen: Die Reservierung für den türkischen Anwaltsbund wurde nur marginal weniger häufig angenommen als die für den deutschen, bei den Gewerkschaften war der Unterschied umso größer.

Schließlich wurden relativ persönliche Wahlentscheidungen auf einem informellen Markt abgefragt: Identische Mitfahrangebote von Deutschen ohne Migrationshintergrund oder von am Namen als Migranten türkischer Herkunft Erkennbaren wurden in dafür bereitstehenden Zettelkästen in einer deutschen Universität platziert. Bei Anruf wurde mitgeteilt, dass alle Plätze schon belegt seien und so die Möglichkeit gegeben, den jeweils anderen Anbieter anzurufen. 
57\% der Suchenden riefen ausschließlich den deutschen Fahrer ohne Migrationshintergrund an, 14\% nur den türkischen Anbieter; 29\% riefen beide an, von diesen kontaktierten jedoch $80 \%$ den deutschen Anbieter zuerst.

Die Ergebnisse dieser Feldexperimente zeigen also eine deutliche Diskriminierung. ${ }^{152} \mathrm{Ob}-$ wohl die Studie nicht speziell das Verhalten gegenüber MigrantInnen der zweiten Generation untersucht, lassen sich daraus doch Schlüsse zur Diskriminierung der zweiten Generation ziehen: Wohnungssuchende Personen mit ,türkisch' klingendem Nachnamen, die sich am Telefon akzentfrei in Deutsch verständigen konnten, erhielten genauso häufig eine Einladung zur Besichtigung wie Deutsche ohne Migrationshintergrund - ein deutlicher Unterschied zu denjenigen, die mit Akzent sprachen. Gleichzeitig zeigen die erhobenen Verhaltensdaten eine starke Diskriminierung von Frauen, die Kopftuch tragen - eine Diskriminierung, die am Telefon nicht zum Tragen kommen kann.

Ebenfalls belegt wurde in der Studie eine Minderung der Diskriminierung durch einen höheren sozioökonomischen Status. So wurden die Reservierungen für den türkischen Anwaltsverein häufiger akzeptiert als die für die türkische Gewerkschaft. Auch bei der Reservierung im Restaurant der gehobenen Preisklasse fand weniger Diskriminierung statt. Und schließlich half ein Doktortitel dem Migranten polnischer Herkunft bei der Wohnungssuche - er wurde signifikant häufiger zur Besichtigung der Wohnung eingeladen als ein migrantischer Bewerber polnischer Herkunft ohne Doktortitel.

Ausschließlich die zweite Generation betrachtete eine empirische Studie, die das Zentrum für Türkeistudien im Auftrag der International Labour Organization 1994 durchführte (Goldberg, et al. 1995). Durch Telefonanrufe und Initiativbewerbungen wurde die Ungleichbehandlungen von deutschen BewerberInnen und MigrantInnen der zweiten Generation türkischer Herkunft getestet.

In einer ersten Serie von Feldexperimenten wurde auf Stellenanzeigen für niedrigqualifizierte Jobs telefonisch geantwortet. Abwechselnd rief zuerst der deutsche Bewerber ohne Migrationshintergrund, dann der migrantische an und bat um einen Vorstellungstermin und umgekehrt. Die Migranten waren nur über ,typische türkische Namen' identifizierbar. Sie sprachen

\footnotetext{
${ }^{152}$ Eine Ausnahme hierzu war die Bestellung einer Cola in einer einfachen Kneipe durch einen Deutschen ohne Migrationshintergrund und einen als ,schwarz' markierten Menschen. Zwei Beobachter saßen in der Bar und stoppten die Zeit vom Eintritt der Person bis zu dem Zeitpunkt, zu dem der Kellner kam, um die Bestellung aufzunehmen. Weiterhin wurde die Zeit gemessen, bis die Rechnung gebracht wurde, nachdem diese angefragt worden war. Im Durchschnitt wurde hier die ,schwarz' markierte Person schneller bedient (62 Sek. vs. 71 Sek.). Schwierig zu interpretieren ist, ob schnelles versus langsames Bedienen tatsächlich auf fehlende Diskriminierung hinweist - oder z.B. auf den Wunsch, jemand als ,fremd' markierten schnell wieder loszuwerden.
} 
akzentfrei Deutsch und hatten ihre gesamte Bildung und Ausbildung in Deutschland erworben. Die Anrufer folgten identischen Sprechzetteln und wiesen Qualifikationen vor, die auf das Jobprofil maßgeschneidert waren. Die verschiedenen Antworten der ArbeitgeberInnen wurden auf einer Skala verortet; sie reichten von „kein Interesse“ über die Aufforderung, Unterlagen einzureichen, bis zu dem Angebot, sich später nochmals zu melden, einem konkreten Terminvorschlag oder sogar einem Jobangebot.

Bei den 175 gültigen gepaarten Anrufen (jeweils Deutscher ohne Migrationshintergrund und Migrant türkischer Herkunft) fand in 33 Fällen eine Diskriminierung zwischen deutschen und türkischen Bewerbern statt; beispielsweise wurde dem deutschen Bewerber ohne Migrationshintergrund ein Termin für ein Vorstellungsgespräch angeboten, der Bewerber türkischer Herkunft jedoch nur aufgefordert, seine Unterlagen einzureichen. Dabei ist zu beachten, dass die Einladung zum Interview nur ein erster Schritt zum Job ist. Im tatsächlichen Interview und in der Einstellungsentscheidung würde die Quote der Ungleichbehandlung möglicherweise noch höher ausfallen. Es zeigte sich jedoch schon in dieser ersten Stufe der telefonischen Terminvereinbarung eine statistisch signifikante Diskriminierung gegenüber den türkischen Bewerbern $(\alpha=0,05)$. Zwei Beispiele aus der Studie sollen dies veranschaulichen:

Beispiel 1: Ein Deutscher ohne Migrationshintergrund rief einen Arbeitgeber an, der Kräfte zur Auslieferung von Katalogen an Privathaushalte suchte. Er erhielt einen Termin für ein Vorstellungsgespräch. Als zehn Minuten später der Bewerber türkischer Herkunft anrief, wurde ihm gesagt, alle Stellen seien schon vergeben. Direkt im Anschluss rief nochmals der deutsche Bewerber ohne Migrationshintergrund unter einem anderen Namen an und erhielt abermals einen Vorstellungstermin.

Beispiel 2: In einer telefonischen Bewerbung für einen Job als Fotomodell rief zunächst der Migrant türkischer Herkunft an und gab auf die Frage nach seiner Größe 1,82 Meter an. Daraufhin sagte man ihm, dass die Mindestgröße 1,85 Meter sei. Im Anschluss rief der deutsche Bewerber an und erkundigte sich nach dem Job. Er gab an, 1,84 Meter groß zu sein, und bekam eine positive Antwort. Zur Kontrolle fragte er, ob 1,82 Meter auch noch in Ordnung seien, und dies wurde bejaht.

Das Untersuchungsdesign erlaubt somit, Diskriminierung zu erkennen, die - wie dies meist der Fall ist - nicht offen geschieht. Um Diskriminierung im Bereich der höher qualifizierten Jobs zu testen, wurde ein anderes Verfahren gewählt: Jeweils ein/e Deutsche/r und ein/e MigrantIn der zweiten Generation türkischer Herkunft schickten Initiativbewerbungen an insgesamt 2633 Firmen. Die Lebensläufe der beiden BewerberInnen waren identisch, es wurden 
nur die Namen der Schulen und Ausbildungsorte ausgetauscht. Gültig waren all jene Fälle, in denen beiden BewerberInnen geantwortet wurde (ca. 300). Bei diesen 300 ,gepaarten' Rückläufen war die Diskriminierungsrate gering, sie überschritt nicht das Signifikanzniveau $(\alpha=$ 0,05). Man kann jedoch im Unterschied zu den AutorInnen der Studie argumentieren, dass auch in den Fällen Diskriminierung vorlag, in denen überhaupt nur dem/der deutschen BewerberIn ohne Migrationshintergrund geantwortet wurde. Werden diese Fälle in die Berechnung eingeschlossen, steigt die Diskriminierungsrate auf ein signifikantes Niveau.

Die AutorInnen der Studie fanden zudem heraus, dass die Diskriminierung dort besonders ausgeprägt war, wo in den angestrebten Jobs direkter Kundenkontakt besteht. Möglicherweise wurden hier von den ArbeitgeberInnen im Interesse des Geschäfts auf vermeintlich rassistische Einstellungen der KundInnen Rücksicht genommen. Es würde sich dann um Beispiele ,vorauseilenden Gehorsams' handeln. Zudem war die Diskriminierung durch privatwirtschaftliche ArbeitgeberInnen höher als durch öffentliche.

Insgesamt zeigt sich in der Studie von Klink/Wagner und der von Goldberg et al., dass rassistische Diskriminierung existiert und sie gegenüber den verschieden markierten Gruppen unterschiedlich stark ausfällt. Besonders betroffen sind MigrantInnen türkischer Herkunft (speziell ,Kopftuchträgerinnen') sowie OsteuropäerInnen und MigrantInnen, die aus dem Nahen Osten kommen. Nicht betroffen sind hingegen EU-MigrantInnen (hier: griechischer Herkunft). Neben diesen Unterschieden bezüglich der Herkunftsgruppen treten in den beiden Studien Unterschiede im Hinblick auf die Generationen hervor: MigrantInnen der zweiten Generation, die akzentfrei Deutsch sprechen, wurden weniger, aber - so zeigt die ILO Studie - gleichwohl immer noch diskriminiert. Quer zu dieser Beobachtung liegt die besondere Diskriminierung von über ihr Kopftuch als fremd markierten Frauen, die unabhängig vom Generationenstatus stark ist. Schließlich mildert durchschnittlich ein höherer sozioökonomischer Status Diskriminierung, während ein niedriger sozialer Status sie verstärkt.

Die in der Studie von Klink/Wagner und der ILO-Studie erhobenen offensichtlichen Praktiken der Diskriminierung müssen als Teil des in Deutschland (und anderswo) herrschenden Rassismus verstanden werden. Erklärungen, die Diskriminierung als individuelles Fehlverhalten auffassen, greifen zu kurz. Eine solche Einschätzung ist aber in Deutschland weit verbreitet. Im Extremfall fasst sie Neonazis ausschließlich als individual-psychologisch gestörte Personen auf (Mecheril 2001) oder erklärt Rassismus zur zwangsläufigen Folge von sozioökonomischer Deprivation. Dazu stellt Anetta Kahane, Vorsitzende der Amadeu Antonio Stiftung zur Stärkung demokratischer Strukturen, ironisch fest: „Wenn in Großbritannien die Arbeitslosig- 
keit zunimmt, leben Lachse gefährlich, weil die arbeitslosen Briten dann vermehrt fischen gehen. [...] In Deutschland [jedoch] wird Rassismus als normale Reaktion auf soziale Verelendung gesehen“ (Kahane 2007: 34). Der hier verwendete Rassismus-Begriff erlaubt dagegen, über individuelles Diskriminieren hinaus Herrschaftsstrukturen und damit verknüpfte Ressourcen in Form von ökonomischem, sozialem, kulturellem und symbolischem Kapital zu analysieren.

Dieser Rassismus-Begriff orientiert sich an der oben vorgestellten Definition von Anja Weiß, die unter Rassismus Praktiken symbolischer Delegitimierung versteht, welche sich mit der Zeit zu negativem symbolischem Kapital verfestigt und damit zu einer Dimension von Ungleichheit wird (Weiß 2001: 351; s.o. Kapitel 2.3). Offensichtlich wird diese strukturelle Komponente von Rassismus auch bei der Diskriminierung innerhalb der eigenen Gruppe. So wollten in einer Untersuchung von 1.219 GrundschülerInnen vierter Klassen die deutschen SchülerInnen ohne Migrationshintergrund am liebsten neben anderen deutschen SchülerInnen ohne Migrationshintergrund sitzen - ganz gemäß der Bevorzugung der Ingroup in der Social Identity Theory der Sozialpsychologie (Tajfel 1982). Bei den Kindern türkischer Herkunft und den Kindern von AussiedlerInnen sah die Präferenz anders aus: Sie bevorzugten in diesem Alter keinesfalls Kinder der gleichen Herkunftsgruppe als SitznachbarInnen, sondern deutsche Kinder ohne Migrationshintergrund (Wagner, et al. 2001). Bei älteren Kindern der Jahrgangsstufen 9 und 10 fand sich eine solche Präferenz bei der Freundschaftswahl nicht mehr (Ramachers 1996). Möglicherweise äußern also jüngere Kinder das verinnerlichte negative symbolische Kapital direkter als ältere Kinder, denen dahinterliegende Ausschließungs- und Zuweisungslogiken bewusster sind.

Rassistische Diskriminierung ist als moralisch verwerflich kodiert. Auf der Ebene des Individuums lässt sie sich jedoch häufig rational, d.h. aus Interessen abgeleitet, begründen. Sie darf also nicht primär als Problem fehlender Aufklärung verstanden werden, sondern als Ausdruck struktureller Ungleichheit. In der Studie von Klink/Wagner zu den Mitfahrgelegenheiten beispielsweise handeln die Studierenden wahrscheinlich rational, wenn sie zunächst bei dem ,deutsch klingenden’ Anbieter anrufen: Bei längeren Mitfahrten werden Informationen ausgetauscht, es entstehen Freundschaften. In einer Gesellschaft aber, in der Ressourcen zwischen den Herkunftsgruppen ungleich verteilt sind, werden auf einem statistischen Durchschnitt basierende Annahmen über einzelne Gruppenmitglieder gemacht. Die Präferenz für eine Fahrt mit einem Unbekannten, der statistisch gesehen wahrscheinlich über mehr konvertible Ressourcen verfügt, ist somit je nach den weiteren individuellen Interessen rational. Benötigt je- 
mand zum Beispiel Hilfe bei den Türkisch-Hausaufgaben, müsste er/sie zuerst die Person mit dem ,türkisch klingenden' Namen anrufen.

In Deutschland ist dieses negative symbolische Kapital dabei so gelagert, dass es zu einer Gleichsetzung von Nationalität, Religion und ,Rasse'/Hautfarbe kommt. In der Eurobarometer-Umfrage des Jahres 2000 wurden die Interviewten gefragt, ob sie persönlich die Gegenwart von Menschen anderer Nationalität, ,Rasse' oder Religion störend finden. Der Prozentsatz der EinwohnerInnen Deutschlands, die die Anwesenheit der betreffenden Gruppe störend finden, ist für alle drei Kategorien gleich groß und beträgt 17 bzw. 18\% (Commission 2000: 88f). Darin unterscheidet sich Deutschland von anderen europäischen Ländern, in denen diese Kategorien der Markierung nicht in gleichem Maße gekoppelt sind. Möglicherweise deutet dies darauf hin, dass im deutschen Diskurs die Fremdheitsmarkierungen ,Ausländer', ,dunkelhäutig' und ,Muslim' gleichbedeutend verwendet und auch gleich bewertet werden. Dadurch würde ein ,Blurring' einer dieser Kategorien erschwert: Damit beispielsweise die Bedeutung von Religion als Fremdheitsmarkierung sinkt, müsste sie entweder von den beiden anderen Kategorien entkoppelt werden, oder aber die Bedeutung aller dreier müsste sich verringern.

Im Folgenden analysiere ich beispielhaft einige der institutionellen Faktoren im Feld der Bildung, die ungleiche Machtverhältnisse verfestigen. Dadurch soll exemplarisch gezeigt werden, dass institutionelle und rechtliche Faktoren zur Entstehung und Absicherung von Rassismus beitragen.

Das wichtigste Kennzeichen des deutschen Schulsystems ist seine Dreigliedrigkeit: Nach der vierten oder sechsten Klasse verteilen sich außer an Gesamtschulen die SchülerInnen auf Hauptschulen, Realschulen und Gymnasien. Die Bildungsforschung hat jedoch gezeigt, dass sich Unterschiede in den Kompetenzen durch das dreigliedrige System verstärken: So entwickelt sich beispielsweise bei Kontrolle der kognitiven Grundfähigkeiten statistisch betrachtet die Leseleistung auf der Hauptschule schlechter als auf der Realschule und auf dem Gymnasium nochmals besser als auf der Realschule (Schuemer, et al. 2004). Diese Verstärkung lässt sich auch aus der Gegenüberstellung der IGLU- (10-Jährige) und der PISA-Daten (15Jährige) ablesen: Bei den 10-Jährigen bewegen sich die Leistungsunterschiede im internationalen Vergleich im oberen Mittelfeld, bei den 15-Jährigen sind die Leistungsunterschiede in Deutschland größer als in jedem anderen Land (Schwippert, et al. 2003: 295f). SchülerInnen an Hauptschulen werden also im Vergleich zu SchülerInnen der beiden anderen Schultypen 
im Mittelwert weniger in ihrer Lernentwicklung unterstützt. An den Hauptschulen finden sich jedoch überproportional viele SchülerInnen mit Migrationshintergrund.

Durch die frühe Selektion des Schultyps kommt den Grundschulen eine bedeutsame Rolle zu. Inwieweit gelingt es, die Kinder bis zur vierten Klasse zu fördern, und wie werden Übergangsempfehlungen ausgestellt? In Studien zeigt sich, dass bei den Empfehlungen keinesfalls nur die schulischen Leistungen berücksichtigt werden. Soziale Herkunft ist bei Kontrolle der schulischen Leistungen ein signifikanter Prädiktor der Empfehlung (Kristen 2002; Bildungsberichterstattung 2006: 165 Gomolla/Radtke 2002: 89). Dies ist in Teilen sogar formal durch einen Entschluss der Kultusministerkonferenz aus den 1970er Jahren abgesichert, in dem diese empfahl, bei der Übergangsempfehlung neben den fachlichen Leistungen auch die für den Schulerfolg wichtigen allgemeinen Fähigkeiten zu berücksichtigen. So öffnete sie ein Hintertürchen, um den sozialen Status der Eltern einzubeziehen und eine darauf basierende Prognose über den zukünftigen Schulerfolg des Kindes abzugeben. (Schwippert, et al. 2004: 185) Dies wirkt sich für Kinder von MigrantInnen durch deren durchschnittlich niedrigere Ausstattung mit sozioökonomischen Ressourcen besonders negativ aus. Die Quoten des Übergangs auf die Hauptschule unterscheiden sich aber auch stark nach einzelnen Grundschulen: Während manche Elementarschulen beinahe komplette Jahrgänge für die Hauptschule empfehlen, ist dies in reicheren Stadtvierteln häufig exakt umgekehrt. Da MigrantInnen der zweiten Generation durch das historische Zuzugsmuster überdurchschnittlich häufig in ,Problemstadtvierteln' wohnen, trifft sie, ebenso wie die dort lebenden Kinder von ärmeren Deutschen ohne Migrationshintergrund, diese Diskriminierung häufiger.

Für Grundschulen bestehen zudem eigentlich verpflichtende Schuleinzugsbezirke, die zu einer sozialen Mischung im Sinne von Nachbarschaftsschulen führen sollen. Die Schuleinzugsbezirke sind indes häufig so vermessen, dass beispielsweise ein Komplex des sozialen Wohnungsbaus in einen Bezirk fällt, umliegende Reihenhäuser jedoch in einen anderen (Radtke 2004: 166). So werden trotz räumlicher Nähe aufgrund städtebaulicher Entwicklungen sozioökonomisch segregierte Schulen geschaffen. Hinzu kommt die räumliche Trennung von Bevölkerungsgruppen aufgrund von Pfadabhängigkeiten auf dem Wohnungsmarkt: Beispielsweise war zu den Hochzeiten der ,Gastarbeiter'-Anwerbung der Wohnraum in BerlinKreuzberg wegen der fehlenden Sanierung verglichen mit anderen Stadtvierteln billig. Durch Familiennachzug entstanden Konzentrationen (Kapphan 2001: 91f), die nun zu einer QuasiSegregation in den Schulen führen. Verstärkt wird diese Trennung an den Schulen durch Privatschulen (z.B. Waldorfschulen), für die die Bindung an die Schuleinzugsbezirke aufgehoben wird. Viele sozioökonomisch bessergestellte Eltern, die in ,gemischten' Stadtteilen leben 
und grundschulpflichtige Kinder haben, schicken diese daher auf Privatschulen. (Vermeintliche) Individuelle Nutzenmaximierung führt hier zu Segregation in den Schulen und damit kollektiv zu suboptimalen Ergebnissen. Der Trend zur Ausnahmeregelung von der Bezirksbindung verstärkt sich schließlich weiter durch die Spezialisierung von Schulen im Zuge des Wettbewerbs auch unter Schulen. Für Grundschulen, die ein besonderes Angebot vorweisen (z.B. Französischunterricht), kann nämlich ebenfalls eine Gestattung beantragt werden, die es erlaubt, die verpflichtenden Schuleinzugsbezirke zu umgehen. (Radtke 2004) Im Ergebnis führt dies dazu, dass schon an den Grundschulen und noch stärker an den weiterführenden Schulen SchülerInnen entlang des sozioökonomischen Status der Eltern und des Migrationshintergrunds segregiert werden. So stellt Stanat fest: „Festzuhalten ist aber, dass etwa ein Fünftel der Hauptschulen in Deutschland in problematischen Lernkontexten arbeitet, die durch einen sehr hohen Migrantenanteil in Verbindung mit niedrigem sozialen Status der Schüler, geringen kognitiven Grundfähigkeiten, häufigen Lernschwierigkeiten und Verhaltensproblemen gekennzeichnet sind. Über die ungünstigen individuellen Eingangsvoraussetzungen hinaus wirken sich solche Kontextbedingungen als zusätzliche Belastungen aus.“ (Stanat/Christensen 2006: 164)

Ein weiterer institutioneller Faktor, der diskriminierende Wirkung entfaltet, ist in den Halbtagsschulen zu sehen. Müssen erhebliche Lernschritte zu Hause bewältigt werden, vergrößert sich die Bedeutung der elterlichen Ressourcen für Hilfestellung, Beratung und Motivation. Dies verstärkt die Abhängigkeit schulischer Leistungen vom sozioökonomischen Status der Eltern: „Das andauernde Delegieren von Aufgaben in die schulfreie Zeit gewährt der privaten Sozialisation großen Spielraum, als Agent von In- und Exklusionen sowie effektiver Klassendistinktion wirken zu können.“ (Mannitz 2002a: 181f)

Die bisher vorgestellten institutionellen Mechanismen betreffen formal alle Kinder gleich, de facto betreffen die negativen Effekte jedoch Kinder aus unteren Schichten mehr als andere. Unter diesen Kindern sind MigrantInnen der zweiten Generation überproportional häufig zu finden. So entfalten vorgeblich neutrale Strukturen diskriminierende Wirkung und verfestigen die Strukturen sozialer Ungleichheit.

In einer vergleichenden Studie zeigten Schiffauer et al. schließlich, dass an deutschen Schulen eine Kultur wirkt, die sich grundlegend von der an britischen, französischen und niederländischen Schulen unterscheidet. Diese Kultur ist gekennzeichnet durch einen Mangel an explizi- 
ten Regeln. Die einzige Regel sei die Verpflichtung zur Verantwortung. ${ }^{153}$ Es herrschten Unklarheiten z.B. zum Gebrauch der Muttersprache, zum Tragen von Baseball-Kappen, zum Melden oder Einfach-drauf-los-Sprechen. Eindeutige Regeln, deren Einhaltung von allen eingefordert wird, fehlten. Diese Regellosigkeit „entspricht der politischen Kultur der Bundesrepublik, dem Einzelfall gerecht werden zu wollen und starren Regeln mit großer Skepsis zu begegnen“ (Sunier 2002b: 157). Im Alltag führt sie jedoch an den Schulen zu Unstimmigkeiten: Der eine Lehrer erlaubt die Kommunikation zwischen SchülerInnen türkischer Herkunft auf Türkisch, um beispielsweise Aufgaben zu erklären, die andere nicht. Beide LehrerInnen halten ihr Verhalten jedoch für selbstverständlich. In den unterschiedlichen Situationen sei, so beobachtet Schiffauer, das richtige Verhalten jenes, welches Verantwortung für die Situation übernimmt und zum geordneten Ablauf des Unterrichts beiträgt. Dies kann im einem Fall das Übersetzen der Aufgabe sein, im anderen Fall kann dieses Übersetzen störend und damit unverantwortlich sein. Die Lehrkraft entscheidet, in welche Kategorie das Verhalten fällt. (Schiffauer 2002: 7; Sunier 2002b: 157ff) Für Kinder aus Familien, in denen eine solche Verantwortungskultur nicht geübt wird und eher direktive Erziehungsstile herrschen, wie dies bei ärmeren Familien und Familien mit Migrationshintergrund durchschnittlich häufiger der Fall ist, ist eine solche Schulkultur schwer zu durchschauen und führt damit schnell zu Überforderung (Rolff 1997). Fehlende Kompetenz im Umgang mit dieser Regellosigkeit wird von den Lehrkräften jedoch zumeist dem Charakter und der Erziehung der SchülerInnen, nicht deren sozioökonomischem Status attribuiert: „Wenn SchülerInnen den an sie gerichteten Erwartungen des zivilen Umgangs nicht gerecht worden, galt dies also als Ausdruck einer defizitären Persönlichkeit: Da die Forderung nach situationsbezogener Angemessenheit des Verhaltens in der Berliner Schule nicht an konkreten Kriterien gemessen werden konnte, bestand der einzige Maßstab aus den persönlich verinnerlichten Werten, deren Vermittlung zunächst als Aufgabe der Eltern begriffen wurde.“ (Mannitz 2002b: 190) ${ }^{154}$

In den Schulen wird aber nicht nur die Fähigkeit der SchülerInnen mit Migrationshintergrund in Frage gestellt, sich jederzeit verantwortlich zu verhalten, auch ihre Erfahrungen und ihr

\footnotetext{
${ }^{153}$ Hierin sieht Schiffauer eine Übereinstimmung mit der politischen Kultur, die auch außerhalb der Schulen herrscht. Während Freiheit in Frankreich als Gleichheit, in Großbritannien als Unantastbarkeit, in den Niederlanden als Vernunft verstanden wird, assoziiert man sie in Deutschland mit Verantwortung. (Schiffauer 2001: 238)

${ }^{154}$ Dabei erstreckt sich dieses Misstrauen, dies sei am Rande festgehalten, über den schulischen Bereich hinaus: „Man traut den Immigranten nicht so richtig zu, sich im zivilgesellschaftlichen Austausch auf, angemessene' und ,verantwortliche' Weise einzubringen (und unterstellt Eigen- und Partikularinteressen, Unverantwortlichkeit usw.)." (Schiffauer 2001: 240) Deutlich äußert sich dies beispielsweise an dem zum Lackmustest erhobenen Kriterium der Gleichberechtigung zwischen den Geschlechtern. Hier wird MigrantInnen pauschal Rückständigkeit zugeschrieben und darüber ihre Eignung sich am politischen Geschehen überhaupt zu beteiligen, in Frage gestellt.
} 
biografischer Hintergrund werden abgewertet. Dies geschieht zum einen durch einen Unterrichtsstil, bei dem von vornherein feststeht, welches Ergebnis die SchülerInnen aus vorgeblich ergebnisoffenen Diskussionen mitnehmen sollen. Unerwartete Ideen und ein Hinterfragen der impliziten Annahmen werden in einem solchen pädagogischen System als Störungen wahrgenommen: „Der ausgeprägt normative Diskurs, der ein Korrelat zum Wahrheitsdiskurs zu sein scheint, macht es schwierig, aus Andersartigkeit positive Forderungen abzuleiten. Die kulturelle Differenz verdient allenfalls Verständnis, nicht aber gleiche Anerkennung.“ (Sunier 2002a: 250, vgl. auch Mannitz 2002c: 349) Zum anderen wertet auch der Lernstoff bestimmte Erfahrungen ab: „Die Aussparung der Migrationsgeschichte in den offiziellen Curricula und Schulbüchern produziert [...] eine Unterscheidung zwischen dem richtigen, dem harten Stoff der Deutschland ins Zentrum rückt - und dem Additiv, das irgendwie der Migrationssituation Rechnung trägt“" (Schiffauer 2001: 243). Aber diese geringe Wertschätzung betrifft nicht nur die historischen Erfahrungen. Sie lässt sich auch bei den Sprachkenntnissen finden: Beispielsweise gibt es die ,richtigen' Fremdsprachen Englisch, Französisch, Latein und die für schulische Zwecke zumeist , unnützen’ Sprachen wie Türkisch, Arabisch, Polnisch.

Hinzu kommt, dass manchmal selbst in bester Absicht Fremdheitszuschreibungen verfestigt werden. So beobachteten die FeldforscherInnen in dem Projekt von Schiffauer et al. eine Unterrichtsinteraktion, bei der die Lehrkraft die Situation der Juden und Jüdinnen in den 1930er Jahren in Deutschland mit der gegenwärtigen Situation der AusländerInnen in Deutschland gleichsetzte, um den Schulstoff zu aktualisieren und näher an die Lebenswirklichkeit der SchülerInnen heranzubringen. Dadurch wurde jedoch die Grenzziehung zwischen AusländerInnen und Deutschen verfestigt: Die AusländerInnen wurden auf ihren Status als ,Andere' verwiesen, die angeblich mehr Ähnlichkeit mit den verfolgten Juden und Jüdinnen im Dritten Reich hatten als mit den Deutschen ohne Migrationshintergrund zum Zeitpunkt der Jahrtausendwende. (Schiffauer 2001: 244ff) ${ }^{155}$

Dieser beispielhafte Einblick in die institutionellen und strukturellen Faktoren des Bildungssystems, die zu einer Abwertung der Fähigkeiten, Erfahrungen und (Wissens-)Hintergründe von SchülerInnen mit Migrationshintergrund führen, hat deutlich gemacht, dass Diskriminierung keinesfalls nur eine individuelle Verhaltensweise ist. Vielmehr ist sie eingebettet in Macht- und Herrschaftsverhältnisse. Verschiedene Gruppen sind gemäß ihrem Status unter-

\footnotetext{
${ }^{155}$ Selbst ein hoher sozialer Status schützt Individuen nicht vor einer solchen Fremdheitszuschreibung. Cem Özdemir beschreibt z.B., wie er bei einer Rückkehr nach Deutschland seinen Diplomatenpass zeigte, der ihn als Mitglied des deutschen Bundestages auswies, und daraufhin von dem Grenzschutzbeamten gefragt zu wurde, wie lange er denn bleiben wolle (Özdemir 1999: 12f).
} 
schiedlich davon betroffen. ${ }^{156}$ Damit geht Diskriminierung über individuelles deviantes Verhalten hinaus und wird als Rassismus zu einer Achse der sozialen Ungleichheit. Sie ist ein weitverbreitetes Phänomen, dem Angehörige dominanter Gruppen sich individuell nur schwerlich entziehen können. ${ }^{157}$ Mit den entsprechenden Fremdheitszuschreibungen werden MigrantInnen im alltäglichen Leben ständig konfrontiert. Diese strukturelle Sicht erlaubt es zudem, sozialen Status als weitere Achse in die Analyse einzubeziehen. So wurde gezeigt, dass ein höherer sozioökonomischer Status der/s MigrantIn in einigen Situationen die Diskriminierung abschwächen kann. ${ }^{158}$

Ausgehend von der Annahme, dass Integration kein einseitiger Prozess ist, bei dem MigrantInnen bestimmte Ressourcen erwerben und Anpassungen vornehmen, um dann integriert zu sein, sondern dass Integration vielmehr die Wahrnehmung von Ähnlichkeit auf Seiten der Dominanzbevölkerung gegenüber MigrantInnen bedeutet, wurde in diesem Kapitel der Blick auf die Dominanzbevölkerung gerichtet.

Es wurde gezeigt, dass sich parallel zur relativen Zunahme des Anteils der zweiten Generation an der Gruppe aller MigrantInnen und der sich daraus ergebenden durchschnittlich größeren Ressourcenausstattung der MigrantInnen die Wahrnehmung von Ähnlichkeit gegenüber MigrantInnen auf Seiten der Dominanzbevölkerung theoriekonform vergrößert hat. Allerdings unterschied sich diese Ähnlichkeitswahrnehmung hinsichtlich unterschiedlich (als ,TürkInnen' oder ,EU-BürgerInnen', als statusniedrig oder statushoch) markierter Gruppen deutlich.

Zeitgleich mit dieser verstärkten Ähnlichkeitswahrnehmung verringerte sich die in Umfragen angegebene Zustimmung zu rassistischer Diskriminierung. Um die Gültigkeit der zwei Integrationsmechanismen - als status- und/oder kulturgleich markierte Interaktion - zu überprüfen, wurden die Ergebnisse von Feldexperimenten beschrieben. Hier traten deutlicher als in den Zahlen zur Ähnlichkeitswahrnehmung Unterschiede für die als statusgleich bzw. statusungleich (respektive kulturgleich/kulturungleich) markierten Personen zu Tage, so dass die Integrationsmechanismen bestätigt werden konnten. So waren Frauen, die Kopftuch trugen, sowie Personen, die als der türkischen Herkunftsgruppe angehörig markiert waren, besonders von Diskriminierung betroffen. Aber auch OsteuropäerInnen und Personen aus dem Nahen

\footnotetext{
${ }^{156}$ Beispielsweise sind EngländerInnen trotz ihres Status als AusländerInnen in Deutschland selten von ,Ausländerfeindlichkeit' betroffen.

${ }^{157}$ Dieses Phänomen wird, in dem schon erwähnten Buch von Anja Weiß anschaulich belegt. Dort geht es um rassistisches Verhalten von MitgliederInnen anti-rassistischer Gruppen - deren „Rassismus wider Willen“ (so der Titel des Buchs; Weiß 2001).

${ }^{158}$ Auch Faktoren wie Geschlecht, Alter und physische Fitness spielen eine Rolle und modifizieren die Diskriminierung. Sie wurden hier jedoch aus Gründen der Komplexitätsreduktion nicht berücksichtigt.
} 
Osten erfuhren in den Feldexperimenten eine starke Diskriminierung. Für sozioökonomisch höhergestellte Personen war die Diskriminierung hingegen stark abgeschwächt.

Dies weist auf globale Hierarchieverhältnisse hin, aus denen Rassismus seine Virulenz bezieht. Gleichzeitig macht es deutlich, dass Rassismus strukturell als Hintergrundvariablen verstanden werden muss, die den gesamten Integrationsprozess beeinflusst. Statt einer individualisierenden und damit verharmlosenden Erklärung von Rassismus als deviantes Verhalten von Einzelpersonen öffnet ein solches strukturelles Verständnis von Rassismus den Blick auf (globale) Macht- und Herrschaftsverhältnisse. Dies erleichtert es, das Zusammenspiel von sozialem Status und Diskriminierung aufgrund von Fremdheitsmarkierungen durch den Migrationsstatus zu analysieren. So kann ein höherer sozialer Status eine Diskriminierung aufgrund des Migrationsstatus zum Teil verringern.

Im letzten Abschnitt wurde zudem am Feld der Schule beispielhaft gezeigt, wie durch institutionelle Mechanismen (Dreigliedrigkeit, Übergangsempfehlungen, Schuleinzugsbezirke, Halbtagsschulen, Schulkultur) Rassismus und soziale Ungleichheit geschaffen und verfestigt werden.

Im nächsten Kapitel werden die hier vorgestellten empirischen Befunde sowie die Ergebnisse der Analysen aus Kapitel 3 und 4 mit Blick auf die drei Integrationstheorien und die sie einenden Integrationsprämissen diskutiert. 


\section{Diskussion: Empirische Befunde und Plausibilisierung der Theorien}

In den vorangegangenen Kapiteln wurde die zweite Generation MigrantInnen im Alter von 20 bis 45 Jahren (im Mikrozensus) und von 18 bis 25 Jahren (im Ausländersurvey des Deutschen Jugendinstituts) hinsichtlich ihrer sozialstrukturellen und kulturellen Merkmale analysiert (Kapitel 3.3, 3.4, 3.5 und 3.6). Weiterhin wurde untersucht, ob und gegebenenfalls in welchem Umfang drei aus den theoretischen Debatten abgeleitete Integrations- und ein Segregationsmuster empirisch zu finden sind (Kapitel 4). Um die Validität des Konzepts von Integration als Zunahme der wahrgenommenen Ähnlichkeit zu plausibilisieren, wurden in Kapitel 5 Veränderungen in der Ähnlichkeitswahrnehmung über die Zeit sowie der strukturelle Kontext, innerhalb dessen Integrationsprozesse stattfinden, betrachtet.

In diesem Kapitel werden die Ergebnisse der vorliegenden Arbeit zusammengefasst und kritisch diskutiert. Konsequenzen daraus für den Integrationsdiskurs, die Migrationsforschung und die Integrationspolitik kommen im Schlusskapitel zur Sprache. Zunächst geht es um die wichtigsten Resultate aus den Sachbereichen (Sprache, Bildung, Beruf sowie Freund- und Partnerschaftsmuster): Welche Aussagen zur empirischen Situation der zweiten Generation lassen sich daraus treffen? Im Anschluss daran werden die aus den Theorien abgeleiteten Integrationsmuster und die ihnen zugrunde liegenden Annahmen über Integrationsmechanismen im Lichte der empirischen Ergebnisse diskutiert. Schließlich wird die Verbindung dieser sozioökonomischen und kulturellen Ergebnisse zur zweiten Generation mit Veränderungen bei der Dominanzbevölkerung beleuchtet und erörtert, welche Integrationstheorien sich für die Analyse der zweiten Generation von MigrantInnen in Deutschland eignen.

\subsection{Ergebnisse aus den Sachbereichen}

Die statistische Untersuchung erfolgte anhand der Daten des Mikrozensus 2005 und des Ausländersurveys des Deutschen Jugendinstituts. Der Mikrozensus 2005 erlaubt es erstmalig, MigrantInnen der zweiten Generation unabhängig von ihrer Staatsangehörigkeit zu identifizieren. Die Verwendung dieser beiden Datensätze zusammen kombiniert den Vorteil eines umfangreichen Samples für verschiedene Herkunftsgruppen bis in die Altersgruppe der 30bis 45-Jährigen (Mikrozensus) mit der Möglichkeit detaillierterer Analysen zu sozialen Beziehungen sowie zu Sprachkenntnissen und -praxis (Ausländersurvey). Zur Konstruktion der Integrationsmuster wurden individuelle sozioökonomische (Bildung und Beruf) und kulturelle 
Variablen (Sprachkenntnisse und Partnerwahl) für MigrantInnen türkischer, italienischer, griechischer, spanisch-portugiesischer und ex-jugoslawischer Herkunft sowie für Kinder von AussiedlerInnen aggregiert untersucht.

Es stellte sich heraus, dass MigrantInnen der zweiten Generation im Allgemeinen über gute bis sehr gute Kenntnisse der deutschen Sprache verfügen. Allerdings fallen die schriftlichen Kenntnisse durchschnittlich hinter die mündlichen Kenntnisse zurück (Tabelle 8). Anhand der Sprachstandserhebungen zur Einschulung lässt sich zeigen, dass auch bei 10\% der Kinder ohne Migrationshintergrund sprachliche Defizite im Deutschen bestehen. Da dieser Prozentsatz in armen Stadtvierteln höher ausfällt, ist eine Verbindung mit dem sozioökonomischen Status der Eltern wahrscheinlich. Die Kenntnisse in der Sprache der Eltern sind in der zweiten Generation gleichfalls durchschnittlich hoch, doch fällt die Differenz zwischen mündlichem und schriftlichem Können größer aus als im Deutschen. Auch sind im Allgemeinen die Deutschkenntnisse wesentlich besser als die Kenntnisse in der Muttersprache der Eltern: Der Anteil derjenigen, die in der Herkunftssprache der Eltern sehr gut schreiben können, liegt durchschnittlich 25 Prozentpunkte niedriger als der Anteil derjenigen, die im Deutschen sehr gut schreiben. Entgegen der These von der starken ,Heimatlandorientierung' von MigrantInnen türkischer Herkunft verfügt bei diesen im Vergleich mit MigrantInnen der zweiten Generation italienischer oder griechischer Herkunft der geringste Prozentsatz über sehr gute Kenntnisse in der Herkunftssprache der Eltern (Tabelle 10). Die MigrantInnen der zweiten Generation kommunizieren mit den Eltern hauptsächlich in deren Muttersprache, mit den Geschwistern spricht die Hälfte überwiegend Deutsch, die andere Hälfte die Sprache der Eltern (Tabelle 11).

Im Hinblick auf die Bildungsabschlüsse ist die zweite Generation wesentlich besser gestellt als die erste und die anderthalbte Generation, und zwar in allen Herkunftsgruppen außer den ,westlichen' MigrantInnen aus Nicht-Anwerbestaaten (britischer, französischer, schweizerischer Herkunft etc.) (Tabelle 13). Diese Verbesserung über die Generationen bestätigt sich in einer Regression, in die der Generationenstatus als Dummy-Variable eingeschlossen wurde (Tabelle 15).

Entgegen weit verbreiteten Annahmen ist die Bildungsaspiration in der zweiten Generation hoch. Gleichzeitig variiert die Motivation bei MigrantInnen der zweiten Generation stärker als bei Nicht-MigrantInnen, und es gibt eine Gruppe mit geringer (Berufsaus-)Bildung, die sich benachteiligt und aufgegeben fühlt. Die meisten migrantischen Eltern wünschen sich für ihre Kinder hohe Bildungsabschlüsse. Die tatsächlichen Unterstützungsleistungen der Eltern sind 
beschränkt, dies wird teilweise von Geschwistern ausgeglichen (s. Kapitel Motivation und Unterstützung).

Im beruflichen Bereich wurde zum einen die Erwerbsbeteiligung (erwerbstätig oder arbeitssuchend/-los) untersucht, zum anderen Berufsposition und Status. Die MigrantInnen der zweiten Generation spanischer/portugiesischer Herkunft weisen die höchste Rate an Erwerbsbeteiligung auf, gefolgt von denen griechischer und italienischer Herkunft. Die geringste Erwerbsbeteiligung zeigen die MigrantInnen der zweiten Generation türkischer Herkunft (Tabelle 18). Damit entspricht die Erwerbsbeteiligung der zweiten Generation - bis auf die türkische Herkunftsgruppe - der von Deutschen ohne Migrationshintergrund (oder übertrifft diese). Allerdings bestehen hier Geschlechterunterschiede: Während bei Frauen die Erwerbsbeteiligung in der zweiten Generation höher ist als in der ersten und der anderthalbten, sinkt sie bei Männern, am deutlichsten bei Migranten aus ,westlichen’ Herkunftsländern. Mit den Daten des Mikrozensus zeigt sich gemessen am Blossfeld-Berufsindikator sowie an der MagnitudePrestigeskala eine deutliche Besserstellung von der ersten und der anderthalbten zur zweiten Generation in allen Herkunftsgruppen bis auf die Gruppe der ,westlichen' MigrantInnen aus Nicht-Anwerbestaaten. Wie bei den Bildungsabschlüssen gibt es auch im beruflichen Sektor eine große Streuung: So ist zum Beispiel die Hälfte der grundsätzlich erwerbstätigen MigrantInnen türkischer Herkunft in der zweiten Generation entweder arbeitslos oder übt nur einfache Tätigkeiten aus, während ein Zehntel als (Semi-)Professionelle, ManagerInnen und IngenieurInnen tätig ist (Tabelle 19). Die Streuung über die Generationen nimmt im Allgemeinen zu und liegt in der zweiten Generation für die türkische und die ,westliche' Herkunftsgruppe am höchsten (Tabelle 24). Frauen sind in allen Herkunftsgruppen in den höheren Berufskategorien im Vergleich zu den Männern überrepräsentiert. Deutsche StaatsbürgerInnen mit Migrationshintergrund in der zweiten Generation sind auf dem Arbeitsmarkt erfolgreicher als ausländische StaatsbürgerInnen (Tabelle 21, Tabelle 22). MigrantInnen der zweiten Generation mit deutscher Staatsbürgerschaft erreichten mit Ausnahme der türkischen Herkunftsgruppe ähnliche Punktwerte auf der Magnitude-Prestigeskala wie Deutsche ohne Migrationshintergrund.

Von der ersten und anderthalbten Generation zur zweiten Generation steigt der Anteil der Partnerschaften mit Deutschen ohne Migrationshintergrund. Nur MigrantInnen türkischer Herkunft haben in der zweiten Generation nicht häufiger eine/n deutsche/n PartnerIn ohne Migrationshintergrund als in der ersten und der anderthalbten (Tabelle 31). Zudem stammen ihre PartnerInnen häufiger aus dem Herkunftsland und sogar der Herkunftsregion der Eltern: So hat ein Viertel der türkischen Herkunftsgruppe in der zweiten Generation PartnerInnen, die 
aus der gleichen Region in der Türkei kommen wie die Eltern der MigrantInnen. Bei der griechischen und der italienischen Herkunftsgruppe in der zweiten Generation sind dies weniger als $10 \%$.

Auch der Anteil der FreundInnen aus der gleichen Herkunftsgruppe sinkt über die Generationen. Gleichzeitig entsteht das Phänomen der ,Regenbogen'partner- und Freundschaften, d.h. der Freund- oder Partnerschaften mit MigrantInnen aus einer anderen Herkunftsgruppe. So haben über $10 \%$ der MigrantInnen der zweiten Generation mindestens eine/n migrantische/n FreundIn, der/die anderer Herkunft ist (Abbildung 13). Und von den , westlichen' MigrantInnen aus Nicht-Anwerbestaaten, die eine/n PartnerIn mit Migrationshintergrund haben, gehören über 30\% der PartnerInnen nicht derselben Herkunftsgruppe an. Auf der anderen Seite gab es aber auch einen nicht unerheblichen Anteil marginalisierter Personen, die über keine für sie signifikanten Freundschaften außerhalb der Familie verfügen (Abbildung 14). Und schließlich konnte aus bestehenden Studien gezeigt werden, dass lokale Konzentrationen von sozial marginalisierten Personen bei Kontrolle der individuellen Faktoren zu erhöhter Delinquenzwahrscheinlichkeit für die Jugendlichen dieser Stadtviertel führt (Kapitel 3.6.2).

Damit belegen die empirischen Ergebnisse der Sachbereiche die grundlegende Erwartung dieser Arbeit, dass die zweite Generation von MigrantInnen insgesamt positivere (und damit auch vielfältigere) Ergebnisse erzielt, als in vielen Untersuchungen zu AusländerInnen allgemein festgehalten wird. Vier Faktoren tragen hierzu bei: Verbesserungen zwischen den Generationen, systematische Unterschiede zwischen deutschen StaatsbürgerInnen und AusländerInnen, Differenzen zwischen den verschiedenen Herkunftsgruppen sowie große Streuungen innerhalb der Herkunftsgruppen. Im Folgenden werden diese vier Faktoren genauer dargestellt.

In allen durchgeführten Analysen nimmt die zweite Generation ${ }^{\mathbf{1 5 9}}$ in Bildung und Beruf statushöhere Positionen ein als die erste und die anderthalbte Generation (Tabelle 13, Tabelle 14, Tabelle 19, Tabelle 20). Meist gibt es einen kontinuierlichen Anstieg von der ersten zur anderthalbten und von dieser zur zweiten Generation (Tabelle 13, Tabelle 15, Tabelle 19, Tabelle 21, Tabelle 22). Dadurch verringert sich die Differenz zu Deutschen ohne Migrati-

\footnotetext{
${ }^{159}$ Wenn hier von Generationen gesprochen wird, dann handelt es sich - dies sei nochmals betont - nicht um Eltern-Kind-Generationen innerhalb einer Familie, sondern um Migrantengenerationen, d.h. um Personen der gleichen Jahrgänge, die entweder in Deutschland geboren bzw. bis zum Alter von sechs Jahren zugewandert sind (zweite Generation) oder zwischen dem 7. und dem 15. Lebensjahr (anderthalbte Generation) oder aber ab dem 16. Lebensjahr zugewandert sind (erste Generation). Wo ein direkter Vergleich der Eltern- und Kindergeneration möglich war, zeigt sich allerdings auch ein Aufstieg über diese Familiengenerationen. Dieser ist im Bildungsbereich in der türkischen Herkunftsgruppe am größten: Dort erlangen 72\% der MigrantInnen eine höhere Bildung als ihre Väter (Tabelle 17).
} 
onshintergrund bei der zweiten Generation signifikant. Nur bei der Gruppe der ,westlichen' MigrantInnen aus Nicht-Anwerbestaaten gibt es einen Statusrückgang über die Generationen. Damit ist sie die einzige der hier untersuchten Gruppen, auf die die seit der PISAUntersuchung kolportierte These des ,Niedergangs' der zweiten Generation zutrifft. Allerdings muss hier eher von einer Annäherung an die deutsche Vergleichsgruppe ohne Migrationshintergrund gesprochen werden, da diese MigrantInnen in der ersten Generation in fast allen Indikatoren die deutsche Vergleichsgruppe übertreffen.

Auch hinsichtlich kultureller Faktoren gibt es in der zweiten Generation durchschnittlich eine stärkere Anpassung: Die Sprachkenntnisse im Deutschen fallen höher aus, und es werden mehr Partnerschaften mit Deutschen ohne Migrationshintergrund eingegangen, auch erhöht sich der Anteil der FreundInnen ohne Migrationshintergrund. Nur bei den MigrantInnen türkischer Herkunft steigt der Anteil der Partnerschaften mit Deutschen ohne Migrationshintergrund in der zweiten Generation nicht über den in der ersten und anderthalbten Generation.

Die in Kapitel 2.5 hergeleitete Hypothese einer sozioökonomischen Verbesserung und stärkeren kulturellen Anpassung in der zweiten Generation kann folglich grundsätzlich bestätigt werden. Diese durchschnittliche Verbesserung spricht dafür, dass MigrantInnen in Deutschland Chancen erhalten und diese vielfach auch nutzen können.

Neben der intergenerationellen Verbesserung gibt es einen weiteren Grund, weshalb die hier untersuchte Gruppe der zweiten Generation einen höheren sozioökonomischen Status und eine stärkere kulturelle Anpassung aufweist als AusländerInnen in anderen Untersuchungen: In der vorliegenden Arbeit wurden MigrantInnen der zweiten Generation, die die deutsche Staatsbürgerschaft besitzen, eingeschlossen. ${ }^{160}$ Der Anteil der MigrantInnen mit deutscher Staatsbürgerschaft unterscheidet sich in den Herkunftsgruppen enorm: Bei den hier untersuchten 20- bis 45-Jährigen der zweiten Generation türkischer Herkunft haben über $40 \%$ die deutsche Staatsangehörigkeit, bei den EU-Staatsangehörigen aus ehemaligen Anwerbestaaten unter $10 \%$ und bei den Kindern von AussiedlerInnen qua Definition 100\% (Abbildung 4). ${ }^{161}$

MigrantInnen mit deutscher Staatsbürgerschaft erzielen über alle Herkunftsgruppen hinweg höhere durchschnittliche Bildungsniveaus (gemäß ISCED) und Berufspositionen (gemessen mit dem Blossfeld-Indikator und der MPS). Die MigrantInnen der zweiten Generation mit

\footnotetext{
${ }^{160}$ Es wurde davon ausgegangen, dass für deutsche StaatsbürgerInnen ihr Migrationshintergrund weiterhin relevant ist: So erleben auch sie Diskriminierung und wachsen in Familien auf, die eine Migrationserfahrung gemacht haben.

${ }^{161}$ Diese unterschiedlichen Quoten zwischen MigrantInnen türkischer Herkunft und beispielsweise denen italienischer Herkunft haben mit der Gewährung von Rechten auf der Basis der EU-Staatsbürgerschaft zu tun; diese macht es für ItalienerInnen weniger attraktiv, die deutsche Staatsbürgerschaft zu erwerben, da sie über eine Reihe der damit verbundenen Rechte und Vergünstigungen schon verfügen.
} 
deutscher Staatsbürgerschaft erreichen mit Ausnahme der türkischen Herkunftsgruppe ähnliche Punktewerte wie Deutsche ohne Migrationshintergrund (Tabelle 15, Tabelle 21, Tabelle 22). Eine Ausnahme bilden ,westliche' MigrantInnen aus Nicht-Anwerbestaaten, bei denen diejenigen, die weiterhin ausschließlich die ausländische Staatsbürgerschaft besitzen, im Durchschnitt einen höheren Bildungsstatus haben als die Eingebürgerten.

Zusätzlich zu diesen generellen Trends der Verbesserung in der zweiten Generation und des höheren sozioökonomischen Status für deutsche StaatsbürgerInnen unterscheiden sich die durchschnittlichen Ergebnisse für die Herkunftsgruppen und machen die zweite Generation vielfältig. Während üblicherweise nur eine, zwei oder maximal drei Herkunftsgruppen betrachtet werden, wurde in dieser Untersuchung auf eine repräsentative Bandbreite Wert gelegt, um einer Engführung z.B. auf die türkische Herkunftsgruppe entgegenzuwirken. In den Sachbereichen treten folgende Unterschiede zwischen den Herkunftsgruppen zu Tage:

Bei den Sprachkenntnissen ist der Anteil derjenigen, die sehr gut schreiben - sowohl im Deutschen wie in der Herkunftssprache - bei den MigrantInnen der zweiten Generation italienischer Herkunft am größten, gefolgt von denen griechischer Herkunft und schließlich denen türkischer Herkunft. Der hohe Anteil von MigrantInnen der zweiten Generation, die in beiden Sprachen sehr gut schreiben, verweist darauf, dass keine Verdrängung, sondern ein additiver Erwerb stattfindet (Tabelle 8, Abbildung 6, Tabelle 10).

Im Bildungsbereich erzielen, westliche' MigrantInnen aus Nicht-Anwerbestaaten die höchsten Abschlüsse. Es folgen die Kinder von AussiedlerInnen und dann die spanisch/portugiesische sowie die ex-jugoslawische Herkunftsgruppe. Etwas schlechter gestellt ist die griechische Herkunftsgruppe. Das Schlusslicht im Bildungsbereich bilden durchschnittlich die MigrantInnen türkischer und italienischer Herkunft (Abbildung 10).

Bei der Erwerbsbeteiligung ragen die MigrantInnen spanischer-portugiesischer Herkunft heraus. Deren Erwerbstätigenrate liegt deutlich über der der Nicht-MigrantInnen. Es folgt ein breites Mittelfeld; zehn Prozentpunkte niedriger liegt die türkische Herkunftsgruppe, welche in der zweiten Generation durchschnittlich die geringste Erwerbstätigkeit aufweist (Tabelle 18). Die berufliche Position nach dem Blossfeld-Indikator entspricht im Allgemeinen der Rangfolge der durchschnittlichen Bildungsabschlüsse. Die ,westlichen' MigrantInnen aus Nicht-Anwerbestaaten erzielen die positivste Verteilung, teilen sich diesen Platz allerdings mit den MigrantInnen spanisch-portugiesischer Herkunft, die bei den Bildungsabschlüssen weniger hohe Werte erzielen. An zweiter Stelle stehen die Kinder von AussiedlerInnen, gefolgt von den MigrantInnen ex-jugoslawischer, italienischer und griechischer Herkunft. Die 
MigrantInnen der zweiten Generation türkischer Herkunft erzielen die niedrigste BlossfeldVerteilung. Sie sind damit auch hier die Herkunftsgruppe mit dem geringsten Status. Während sie sich im Bildungsbereich diese Position noch mit MigrantInnen der zweiten Generation italienischer Herkunft teilen, können diese ebenso wie die spanisch-portugiesische Gruppe ihre schlechten bzw. mittleren Bildungsabschlüsse in relativ gute berufliche Positionen ummünzen, was der türkischen Herkunftsgruppe nicht gelingt. In Kapitel 3.5.3 wurde zudem gezeigt, dass die Rendite von Bildungsabschlüssen in der zweiten Generation höher ist als in der ersten. Allerdings sind die Einkommensverbesserungen durch höhere Bildungsabschlüsse für MigrantInnen auch in der zweiten Generation noch durchschnittlich geringer als für Deutsche ohne Migrationshintergrund. Hinsichtlich der Berufspositionen ergeben sich bei Kontrolle der Bildung (und des Geschlechts) nur für die Gruppe der türkischen StaatsbürgerInnen noch negative Effekte.

Angesichts dieser Unterschiede zwischen den Herkunftsgruppen darf aber nicht übersehen werden, dass die Vielfalt innerhalb der Herkunftsgruppen noch größer ist. Dies zeigt sich in der breiten Streuung der Ergebnisse der Sprachstandserhebung zur Einschulung, in der größeren Bandbreite der Ergebnisse von MigrantInnen gegenüber Nicht-MigrantInnen in der PISA-Untersuchung und schließlich in dem breiten Spektrum der Schulbildung von denjenigen, die nur über unzureichende Basisqualifikationen verfügen, auf der einen Seite und auf der anderen denjenigen mit Abitur, die eine höhere Studienneigung aufweisen als Deutsche ohne Migrationshintergrund mit Abitur (Abbildung 6, Tabelle 16, Kapitel 3.4.1). Am stärksten streuen die beruflichen Statuswerte für MigrantInnen der zweiten Generation türkischer oder ,westlicher' Herkunft (Tabelle 24). Stereotypisierende Zuschreibungen, gemäß denen für alle MigrantInnen türkischer Herkunft der Zugang zum Arbeitsmarkt prekär ist, lassen sich nicht bestätigen. Im Gegenteil, die Verteilung deckt die gesamte Bandbreite ab: ungefähr die Hälfte der grundsätzlich erwerbstätigen MigrantInnen türkischer Herkunft in der zweiten Generation ist entweder arbeitslos oder übt einfache Tätigkeiten aus, und 10\% liegen in den drei höchsten Blossfeld-Kategorien (z.B. ÄrztInnen, ArchitektInnen, SozialpädagogInnen) (Tabelle 19).

\subsection{Integrationsmuster}

Der theoretische Rahmen dieser Arbeit basierte auf der Annahme, dass assimilatorische Integrationsprozesse nicht der einzige Weg der Integration sind (und Segregation somit nicht die einzige Alternative), sondern es darüber hinaus für nicht vernachlässigbare Personengruppen Muster gibt, bei denen kulturelle Anpassung und sozioökonomischer Aufstieg nicht zusam- 
menfallen. So beschreibt die transnational-pluralistische Theorie Prozesse, bei denen ein nicht-prekärer sozioökonomischer Status mit kultureller Kompetenz in der Herkunftskultur der Eltern einhergeht, während im Muster der Abwärts-Integration trotz kultureller Anpassung (an eine sozial marginalisierte Schicht der Dominanzkultur) nur ein prekärer sozioökonomischer Status erlangt wird. Lässt sich diese Vielfalt der Integrationsmuster bestätigen? Zur Überprüfung dieser Frage bildete ich anhand von Kriterien aus den vier Bereichen Sprache, Bildung, Beruf und Partnerschaften Integrationsmuster, so dass jede/r einzelne MigrantIn auf der Basis ihrer/seiner Ausprägungen in diesen Bereichen einem der Muster zugewiesen werden konnte.

Wie von der neoklassischen Theorie angenommen, ist das Muster von höherem sozioökonomischem Status und kultureller Adaption in der zweiten Generation stärker verbreitet als in der ersten und der anderthalbten Generation; je nach Herkunftsgruppe trifft es auf 1/3 bis 4/5 der MigrantInnen der zweiten Generation zu. Der Umfang des umgekehrten, segregierten Musters verringert sich (Tabelle 38, Tabelle 39). Zudem findet sich ein pluralistisches Muster, welches ,ko-ethnische' Partnerschaften mit gehobenem sozioökonomischem Status verbindet. Dieses nimmt zwar ab, umfasst aber in der zweiten Generation noch $11 \%$ der MigrantInnen. MigrantInnen türkischer Herkunft weisen gemessen an Sprachkenntnissen oder Partnerschaften mit PartnerInnen der gleichen Herkunftsgruppe die geringste kulturelle Anpassung auf. Gleichzeitig fallen bei ihnen Partnerschaften mit PartnerInnen der gleichen Herkunftsgruppe seltener mit einem höheren sozioökonomischen Status zusammen als beispielsweise bei MigrantInnen ex-jugoslawischer Herkunft. ${ }^{162}$ Eine Beziehung mit einer/m PartnerIn aus dem Herkunftsland der Eltern bedeutet sozioökonomisch also nicht in allen Herkunftsgruppen dasselbe. Ein Viertel der MigrantInnen der zweiten Generation schließlich verbindet einen prekären sozioökonomischen Status mit kultureller Anpassung (Abwärts-Integration). Dem Abwärts-Muster gehört ungefähr der gleiche Prozentsatz an MigrantInnen der zweiten Generation türkischer, ex-jugoslawischer oder Aussiedler-Herkunft an. Etwas höher ist der Anteil der Abwärts-Integration bei der Gruppe der MigrantInnen italienischer, griechischer, spanischer und portugiesischer Herkunft. Während zusammengenommen bei MigrantInnen der zweiten Generation zwischen $16 \%$ und $54 \%$ in sozial prekären Verhältnissen leben, beträgt der entsprechende Anteil bei der Bevölkerung ohne Migrationshintergrund 20\%. Schließlich fallen theoriekonform mehr Männer als Frauen in das Muster der Abwärts-Integration. Frauen wei-

\footnotetext{
${ }^{162}$ Mit dem DJI-Ausländersurvey konnte kulturelle Anpassung auf zweierlei Weise operationalisiert werden: einmal über Sprachkenntnisse, ein anderes Mal über Partnerschaften (siehe Kapitel 4.1.1). Wenn im Folgenden sprachliche Variablen in den Mustern analysiert werden, dann basiert diese Untersuchung auf dem über Partnerschaften operationalisierten Muster und umgekehrt. Andernfalls entstünden Redundanzen, da die Kriterien der Operationalisierung das Ergebnis der Analyse vorwegnähmen.
} 
sen etwas häufiger als Männer ein neoklassisches Integrationsmuster auf, ungefähr gleich viele ein pluralistisches Muster. Umgekehrt leben - wie in der öffentlichen Debatte vermutet mehr Frauen als Männer segregiert. Dies trifft besonders auf Migrantinnen der zweiten Generation türkischer, italienischer, griechischer, spanischer und portugiesischer Herkunft zu.

Wie verändern sich die Anteile der Muster über die Generationen? Sowohl der Umfang von segregierten als auch der von pluralistischen Mustern nimmt im Allgemeinen in der zweiten Generation ab, wobei das pluralistische Muster auch in der zweiten Generation eine alternative Form der Statussicherung gegenüber assimilatorischen Wegen darstellt. Der Anteil des neoklassischen Musters steigt in allen Herkunftsgruppen von der ersten und anderthalbten zur zweiten Generation.

Die Analyse des DJI-Ausländersurveys bestätigt diese Ergebnisse und die Validität des Partnerschaftskriteriums zur Bildung der Muster, da die mit Partnerschafts- und die mit Sprachvariablen konstruierten Muster zu vergleichbaren Ergebnisse führen: Für die meisten Herkunftsgruppen geht sprachliche Anpassung mit der Wahl einer/s nicht-migrantischen PartnerIn einher. Nur bei der türkischen Herkunftsgruppe fällt die sprachliche Anpassung stärker aus als die Veränderung in der Partnerschaftswahl. Aus der Notwendigkeit, im Mikrozensus kulturelle Anpassung über den Partnerschaftsstatus zu operationalisieren, ergeben sich also keine systematischen Verzerrungen. Damit ist der Hintergrund der/s PartnerIn durchschnittlich ein adäquater Proxy für kulturelle Anpassung.

Diese Zusammenfassung zeigt, dass alle drei Integrationsmuster und das Segregationsmuster empirisch in der zweiten Generation vorkommen. Muster, bei denen kulturelle Anpassung nicht mit Statusaufstieg bzw. ausbleibende kulturelle Anpassung nicht mit einem prekären Status zusammenfällt, existieren in signifikantem Umfang. Dabei sah ich die Muster nicht als grundsätzlich konkurrierend an, sondern als jeweils kontext- und pfadabhängig für unterschiedliche Gruppen gültig. Entgegen verbreiteten Ansätzen gehe ich gerade nicht davon aus, dass ein Integrationsmuster für alle zutrifft, sondern dass sich die Vielfalt der zweiten Generation in unterschiedlichen Mustern widerspiegelt. Das empirische Vorliegen der theoretisch abgeleiteten Muster ist jedoch noch kein Beleg für die Gültigkeit der Theorien. Um diese zu plausibilisieren müssen die von den Theorien postulierten Mechanismen überprüft werden. Dabei geht es auch um die Frage, ob die Mechanismen wirklich bei denjenigen tragen, für die sie gelten sollen. Im nächsten Abschnitt wird daher diskutiert, inwieweit sich die den Integrationsmustern zugrunde liegenden kausalen Mechanismen (z.B. Vorteile für Bilinguale, oppositionelles Verhalten; s. Tabelle 1) bestätigen lassen. 


\subsection{Prüfung der Integrationsmuster}

In den Musteranalysen (Abbildung 21 bis Abbildung 45), die die Verteilung theorierelevanter Variablen zeigen, schlagen zum Teil die statushohen Muster (Neoklassik und Pluralismus) zusammen aus und die statusniedrigen in die Gegenrichtung, in anderen Fällen verlaufen aber die kulturell angepassten Muster (neoklassische und Abwärts-Integration) synchron. Dies bedeutet, dass die Status- und die Kultur-Achse keinesfalls zusammenfallen und die aus den Theorien abgeleitete Trennung der beiden Variablen empirisch belegt ist. Wie sieht es darüber hinaus mit den von den Theorien prognostizierten Prozessmechanismen aus? Diese werden im Folgenden für die drei Theorien einzeln diskutiert.

Pluralistische Theorien gehen davon aus, dass durch einen engen Zusammenhalt innerhalb herkunftsbezogener Gruppen und durch eine mögliche Entstehung transnationaler Räume zusätzliche Ressourcen für MigrantInnen generiert werden. Im Einzelnen schreiben sie Zweisprachigkeit, einer Beschäftigung in der ,ethnischen Ökonomie’ sowie engen innerfamiliären Beziehungen positive Effekte auf Bildung und Berufsposition und auf psychologische Dispositionen zu. Abbildung 22 und Abbildung 23 zeigen zusammengenommen, dass die Gruppe der Zweisprachigen im pluralistischen Muster am größten ist. Für sie konnten in dieser Untersuchung positive psychologische Effekte nachgewiesen werden: So fallen ihre Selbstwirksamkeit (d.h. ihre Überzeugung, gewünschte Ziele unter Einsatz von Ressourcen erreichen zu können) und die Selbsteinschätzung ihrer Fähigkeit, sich vor Pech zu schützen, höher aus und sie nehmen weniger rassistische Diskriminierung wahr als Deutsch- oder Herkunftssprachige (Abbildung 7, Abbildung 8, Abbildung 9). Eine Verbindung von Bilingualismus mit relativ konfliktfreien Familienbeziehungen lässt sich indes in dem Datenset kaum finden: Zwar geben Bilinguale für sich eine höhere Wichtigkeit der Familie an als Nicht-Bilinguale (Abbildung 10), Fragen direkt zum familiären Klima aber fehlen. Hinsichtlich des sozioökonomischen Status ergibt sich für die Bilingualen eine leichte, aber nicht signifikante Besserstellung (Abbildung 11, Tabelle 12). In der bisher größten deutschen Untersuchung zu dieser Frage (DESI) wurde zwar für die Schulleistungen im Deutschen keine Besserstellung der Bilingualen gefunden, im Fach Englisch aber lagen die Leistungen der Bilingualen vor denen der rein Deutschsprachigen und derer, die zu Hause nur in einer anderen Fremdsprache kommunizierten. Bei Kontrolle des sozioökonomischen Status vergrößerte sich dieser positive Effekt weiter. Während die vermuteten positiven psychologischen Effekte für Zweisprachige sich also finden lassen, sind ihre schulischen Leistungen nur in Teilen besser. 
Im Vergleich der vier Muster fallen im pluralistischen Muster die Werte zum Bezug von Arbeitslosengeld (Abbildung 28) und zur Länge der Arbeitslosigkeit (Abbildung 29) theoriekonform niedrig und der Prozentsatz der Selbstständigen wie erwartet hoch aus (Abbildung 30). Unvermutet viele pluralistisch Integrierte sind im öffentlichen Dienst beschäftigt (Abbildung 27), und dies, obwohl für eine solche Beschäftigung von der Notwendigkeit einer kulturellen Anpassung ausgegangen werden kann. Bei den Selbstständigen zeigte sich in Kapitel 3.5 eine große Diversität, die von Ein-Mann/Frau-Betrieben bis zu kapitalintensiven Unternehmen mit mehreren Hundert Beschäftigten reicht. Eine Beschäftigung in der ,ethnischen Ökonomie' stärkt zudem den familiären Zusammenhalt. In meinen Daten lässt sich bei Kontrolle der Ergebnisse anhand der Variablen Bildung, Geburtskohorte und Herkunftsgruppe ein positiver Einkommenseffekt für eine selbstständige Beschäftigung in der ,ethnischen Ökonomie' finden (Tabelle 25, Tabelle 26). Essers These der, ethnischen Ökonomie als Mobilitätsfalle' lässt sich nicht halten.

Pluralistisch Integrierte ${ }^{163}$ haben mehr FreundInnen, die der gleichen Herkunftsgruppe angehören, und ihre partnerschaftlichen Beziehungen werden stärker durch die Eltern gemanagt als in den anderen Mustern (Abbildung 32, Abbildung 33). Zudem stammt ein höherer Prozentsatz der PartnerInnen aus der gleichen Herkunftsregion wie die Eltern (Abbildung 37). Die entsprechende Zahl für die segregierten MigrantInnen ist gleich hoch. Es stellt sich somit die Frage, welche Faktoren in dem einen Fall zu einem gefestigten sozioökonomischen Status führen, im anderen jedoch zu einem prekären Status. Ein erklärender Faktor könnte der soziale Status der FreundInnen sein, wobei sozial höhergestellte FreundInnen derselben Herkunftsgruppe positive Effekte auf die Berufsposition hätten und sozial prekär gestellte FreundInnen negative Auswirkungen. Dieser hypothetisierte Zusammenhang kann jedoch nicht überprüft werden, da in den Datensets keine Informationen zum sozialen Status der Freundschaftsnetzwerke vorliegen. Das genaue Zusammenspiel der Faktoren, das eine kausale Erklärung für den teilweisen pluralistischen Erfolg liefern könnte, bleibt also offen. Psychologische Aspekte tragen indes zur Erklärung bei: So ist die Leistungsmotivation sowie die Selbstwirksamkeit und die Zufriedenheit mit dem eigenen Leben bei pluralistisch Integrierten höher als in den anderen Mustern (Abbildung 36, Abbildung 38), aber auch Pflichtbewusstsein und Hilfsorientierung fallen hoch aus (Abbildung 39, Abbildung 40). Diese Gruppe geht folglich durchschnittlich positiver gestimmt an Herausforderungen im schulischen und beruflichen Bereich heran.

${ }^{163}$ Hierbei handelt es sich - wie auch bei den folgenden Mustern - um eine verkürzte Formulierung zur Bezeichnung von MigrantInnen der zweiten Generation, die sich dem Muster der pluralistischen Integration zuweisen lassen. Diese Verkürzung soll aber nicht zu einer neuen Fremdheitsmarkierung führen. 
Belege für die Existenz transnationaler Räume konnten durch die quantitative Analyse kaum gewonnen werden. Zwar haben die pluralistisch Integrierten im Durchschnitt etwas mehr Zeit im Heimatland der Eltern verbracht, Pendelmigration oder lange Auslandsaufenthalte mit Schulbesuch lassen sich aber kaum finden (Abbildung 43). Eine Untersuchung transnationaler Räume ist auch wegen der Struktur der quantitativen Datensets kaum möglich. In den Fragebögen wird von binären Entscheidungen ausgegangen, bei denen Migration einmalig von einem Land in ein anderes stattfindet. Ein Leben in nationalstaatlich nicht eindeutig verortbaren Räumen wird durch die Art der Fragen von vornherein ausgeschlossen. Für eine genauere Analyse der kausalen Mechanismen wären also qualitative Daten zu den Ressourcen der FreundInnen, dem Familienklima und der Verortung in einem transnationalen Feld nötig sowie Längsschnittstudien, bei denen MigrantInnen über Jahre hinweg begleitet werden, so dass die Wirkungsrichtung der Variablen in den Paneldaten analysiert werden kann.

Die Kritik, dass pluralistische Muster nur für Angehörige der Oberschicht realisierbar seien (z.B. für Diplomatenkinder), konnte entkräftet werden: Die sozioökonomische Bandbreite der im pluralistischen Muster vertretenen MigrantInnen ist zu groß, als dass diese Kritik zutreffen könnte. Allerdings ist eine insgesamt größere Ressourcenausstattung, insbesondere sehr gute Deutschkenntnisse zusätzlich zu sehr guten Kenntnissen in der Herkunftssprache, für Integration nach dem pluralistischen Muster förderlich, da sie den pluralistisch Integrierten erlaubt, sich ihnen bietende Chancen zu ergreifen.

Auch im Muster der Abwärts-Integration belegen die Durchschnittswerte verschiedener Variablen die theoretisch postulierten Zusammenhänge. So haben in dem auf Partnerschaft basierenden Muster Abwärts-Integrierte nur schlechte Kenntnisse in der Herkunftssprache der Eltern, und ihre schriftlichen Kenntnisse im Deutschen sind mittelmäßig (Abbildung 22, Abbildung 23). Wie in der Theorie vermutet, existiert in der deutschen Bevölkerung ohne Migrationshintergrund ein Segment, das nur über mangelhafte Kenntnisse im Deutschen verfügt (Kapitel 3.3). Nicht untersucht wurde das Vorhandensein eines Slangs bei den Jugendlichen und Erwachsenen, da die Datensets entsprechende Items nicht einschließen.

Bildung und Beruf werden von den Abwärts-Integrierten im Vergleich zu den anderen beiden Integrationsmustern theoriekonform als nicht so wichtig angesehen (Abbildung 41). Ihre Lage auf dem Arbeitsmarkt ist vergleichsweise prekär (Abbildung 28, Abbildung 29). Auch glauben sie weniger daran, dass Leistung den Erfolg bestimmt (Abbildung 36). Zusammengenommen deutet dies auf das Vorliegen einer ,Oppositionskultur' hin. Allerdings gibt es entgegen den theoretischen Annahmen in diesem Muster keinen signifikanten Anteil von 
MigrantInnen, der hinter das Bildungsniveau des Vaters ${ }^{164}$ zurückfällt (Abbildung 24). Dies widerspricht einem vom Muster hypothetisierten Zusammenhang, hängt aber mit dem vorherrschend niedrigen Bildungsniveau der Väter zusammen. Auffallend ist, dass die Väter der pluralistisch Integrierten und die der Abwärts-Integrierten ungefähr die gleiche Anzahl an Schuljahren absolviert haben, die Bildungsergebnisse der Kinder jedoch sehr unterschiedlich ausfallen. Auch sind nicht wie erwartet die Großstädte Zentren der Abwärts-Integration, sondern es leben fünf Prozentpunkte mehr Abwärts-Integrierte auf dem Land und in Kleinstädten als in den anderen drei Mustern (Abbildung 17). Kinder von Alleinerziehenden tun sich, wie im Bildungskapitel besprochen, nach Kontrolle des sozioökonomischen Status in der Schule nicht schwerer (PISA-Konsortium 2004: 228).

Theoriekonform ist der Anteil der Männer in der Gruppe der Abwärts-Integration besonders hoch. Zudem unterhalten die MigrantInnen der zweiten Generation in diesem Muster in hohem Maße Freundschaften mit MigrantInnen, die einer anderen Herkunftsgruppe angehörten (,Regenbogen'-Freundschaften) (Abbildung 34), der Anteil der Freundschaften mit MigrantInnen aus der gleichen Herkunftsgruppe ist niedrig (Abbildung 33). Allerdings sind MigrantInnen der zweiten Generation im Muster der Abwärts-Integration stärker als von der Theorie erwartet in deutschen Vereinen aktiv (Abbildung 35). Insgesamt ist ihr Lebensumfeld von der restlichen Gesellschaft nicht in dem Maße getrennt, wie dies die im US-amerikanischen Kontext entstandenen Theorien über die ,inner-city ghettos' behaupten.

Hinsichtlich psychologischer Faktoren geben MigrantInnen in dieser Gruppe dem Lebensziel „Anderen helfen“ vergleichsweise wenig Bedeutung, und die Einschätzung ihrer Selbstwirksamkeit und die Zufriedenheit mit dem eigenen Leben fällt geringer aus als in den anderen Mustern (Abbildung 40, Abbildung 36). Das Maß der Diskriminierung aufgrund ihrer Nationalität halten sie durchschnittlich für gering (Abbildung 45). Eine Frage zu Diskriminierung aufgrund sozialer Herkunft wurde leider nicht gestellt, so dass ein Vergleich nicht möglich ist. Auch zur Frage nach der Verbrüderung/Verschwesterung mit Deutschen ohne Migrationshintergrund aus einer ähnlichen sozialen Lage liegen zu wenig Informationen vor: Demnach scheint es im Hiphop-Milieu gemeinsame Cliquenbildung zwischen verschiedenen Herkunftsgruppen und teilweise auch zwischen MigrantInnen und Nicht-MigrantInnen zu geben. Zudem konnten Konzentrationseffekte von sozial marginalisierten Personen auf delinquentes Verhalten belegt werden.

\footnotetext{
${ }^{164} \mathrm{Da}$ die durchschnittlichen Bildungsabschlüsse der Mütter geringer ausfielen als die der Väter, wurden in dieser Arbeit die väterlichen Bildungsabschlüsse als anspruchsvollere Kriterien für eine Verschlechterung in den Bildungsergebnissen von der Eltern- zur Kindergeneration gesetzt.
} 
Wie sieht es schließlich mit dem Muster der neoklassischen Integration aus? In diesem wird den individuellen rationalen Wahlentscheidungen großes Gewicht gegeben; sie sollen in einem relativ diskriminierungsfreien Raum zu kultureller Anpassung und sozioökonomischem Aufstieg führen. Im Vergleich mit den anderen Mustern zeigt sich bei der neoklassischen Integration eine deutlichere kulturelle Anpassung an Deutsche ohne Migrationshintergrund hinsichtlich der Kinderzahl (Abbildung 1) sowie des Spracherwerbs und -gebrauchs (Abbildung 21, Abbildung 22). Die Kenntnisse in der Herkunftssprache der Eltern fallen durchschnittlich geringer aus als bei pluralistisch integrierten und segregierten MigrantInnen (Abbildung 23). Gleichzeitig werden Freundschaften vergleichsweise häufig mit Deutschen ohne Migrationshintergrund eingegangen und Mitgliedschaften in deutschen Vereinen gepflegt (Abbildung 33, Abbildung 35). MigrantInnen im neoklassischen Muster sind leistungsbereit und geben eine starke Selbstwirksamkeit an, allerdings nicht im selben Maße wie pluralistisch Integrierte (Abbildung 36, Abbildung 38). Die Bildungsabschlüsse verbessern sich innerhalb der Familien häufig von Vätern zu Kindern, und neoklassisch Integrierte äußern eine hohe Bildungsund Berufsaspiration (Abbildung 24, Abbildung 41). Sie sind relativ häufig im öffentlichen Dienst beschäftigt (Abbildung 27). Bei der Untersuchung zum Übergang auf eine weiterführende Schule sowie zu einem Ausbildungsplatz zeigte sich zudem, dass in einem höheren schulischen Leistungssegment Entscheidungen meritokratischer erfolgen als in einem weniger qualifizierten Segment, in dem Zuschreibungen aufgrund des sozialen Status und der kulturellen Herkunft Entscheidungen über die Übergangsempfehlung und den Erhalt eines Ausbildungsplatzes stärker verzerren (Kapitel 3.4). Diese Tendenz erspart möglicherweise den durchschnittlich sozial höher gestellten neoklassisch Integrierten einige Diskriminierungserfahrungen.

Damit sind die kausalen Mechanismen in Bezug auf die neoklassische Integration bestätigt worden; in Bezug auf den Pluralismus treffen sie in großen Teilen zu, auch wenn Details des kausalen Zusammenspiels offenbleiben. Bei der Abwärts-Integration wurden die Mechanismen für die MigrantInnen bestätigt, das Aufgehen in einer, Oppositionskultur' zusammen mit deutschen Nicht-MigrantInnen allerdings bleibt unsicher, so dass das Funktionieren dieses Musters am wenigsten belegt wurde. Da das Muster der neoklassischen Integration in allen Herkunftsgruppen das bedeutsamste war, ist erstaunlich, wie wenig prominent es in der öffentlichen Wahrnehmung ist. Dies erklärt sich zum Teil durch die Beschränkung der Diskussion auf aus dem ländlichen Raum zugewanderte TürkInnen, während diese Arbeit auf Repräsentativität der Ergebnisse abzielte. Für das transnational-pluralistische Muster verweist der beschränkte Umfang erhältlicher Daten auf ein geringes öffentliches Interesse an den positi- 
ven Effekten solcher Lebensweisen sowie eine fehlende Förderung dieser Ressourcen. Somit kann für Deutschland von ungenutzten transnational-pluralistischen Potenzialen bei der Integration von MigrantInnen der zweiten Generation ausgegangen werden.

\subsection{Integration und Dominanzbevölkerung}

Die drei Integrationstheorien eint, wie herausgearbeitet wurde, ein Verständnis, gemäß dem Integration nicht den Erwerb bestimmter Ressourcen und bestimmte Anpassungsleistungen auf Seiten der MigrantInnen bedeutet, sondern sich in einer Zunahme der wahrgenommenen Ähnlichkeit der MigrantInnen durch die Dominanzbevölkerung misst. ${ }^{165}$

Kapitel 5 ging diesen Veränderungen der Wahrnehmung nach. Es zeigte sich, dass Hand in Hand mit der gestiegenen sozioökonomischen und kulturellen Ressourcenausstattung durch die prozentuale Zunahme der zweiten Generation an der Gruppe der MigrantInnen insgesamt auch die Dominanzbevölkerung durchschnittlich eine größere Ähnlichkeit mit MigrantInnen wahrnimmt. Dies drückt sich in den erhöhten Prozentsätzen derjenigen aus, die zwischen Deutschen ohne Migrationshintergrund und den in Deutschland lebenden ItalienerInnen, TürkInnen und SpätaussiedlerInnen nur geringe Unterschiede sehen - Prozentsätze, die besonders für ItalienerInnen von 1996 zu 2006 deutlich gestiegen sind (Abbildung 48). Anhand dieser Daten lässt sich festhalten, dass Integration - gemessen an der wahrgenommenen Ähnlichkeit - sich über die Jahre insgesamt vergrößert hat, ${ }^{166}$ dass sie jedoch, wie dies auch schon in den empirischen Kapiteln zur Ressourcenausstattung der MigrantInnen zu sehen war, für verschieden markierte Gruppen in unterschiedlichem Maße stattfindet: für Kinder von ,GastarbeiterInnen' aus Ländern, die mittlerweile zur EU gehören, durchschnittlich wesentlich stärker als für Kinder der türkischen Herkunftsgruppe. Der gleiche Trend zunehmender Ähnlichkeitswahrnehmung äußert sich in der nachlassenden Zustimmung zum Endogamie-Gebot und dem verstärkten Kontakt mit MigrantInnen in der eigenen Familie (Abbildung 46). Auch die Zahlen zur Erwünschtheit von Einheiraten in die eigene Familie spiegeln diese Tendenz: Der Prozentsatz der Dominanzbevölkerung, der eine solche Einheirat angenehm fände, nimmt über die Jahre zu. Am stärksten gilt dies für eine Einheirat einer/s ItalienerIn; für diese Gruppe können die Zustimmungswerte nicht mehr von denen für Nicht-MigrantInnen unterschieden

\footnotetext{
${ }^{165}$ Dabei wird angenommen, dass dieser vergrößerten Ähnlichkeitswahrnehmung auf Seiten der Dominanzbevölkerung auch eine vergrößerte Ähnlichkeitswahrnehmung bei den MigrantInnen entspricht. Diese wurde jedoch aus den in Kapitel 5.1 erläuterten Gründen nicht untersucht.

${ }^{166}$ Zudem weisen jüngere Personen der Dominanzbevölkerung durchschnittlich eine stärkere Ähnlichkeitswahrnehmung auf (Abbildung 54).
} 
werden, die in der Umfrage anhand von Religion (ProtestantInnen, KatholikInnen) markiert wurden. Für die Gruppe türkischer Herkunft nehmen die Zahlen nur leicht zu, für die der SpätaussiedlerInnen sogar leicht ab (Abbildung 49).

Diese Daten sprechen dafür, dass es für bestimmte Gruppen (z.B. ItalienerInnen, aber auch SpanierInnen und GriechInnen) zu einem ,Boundary Shifting' gekommen ist, gemäß dem diese Gruppen nun als so ähnlich wahrgenommen werden, dass sie geschlossen zur Dominanzbevölkerung gezählt werden. Weiterhin wahrgenommene Unterschiede im Verhältnis zu diesen Gruppen werden in den Raum des Privaten und dort bestehender Präferenzen und Lebensstilvorlieben verwiesen. ,Westliche' MigrantInnen aus Nicht-Anwerbestaaten wie den USA und Frankreich fallen ebenfalls in diese Gruppe. In Umfragen lassen sich keine Items speziell zu dieser Gruppe finden, was darauf hindeutet, dass die Ähnlichkeit dieser Gruppe als Selbstverständlichkeit gewertet wird. Die Kategoriensetzung in den Fragebögen verstärkt diese Selbstverständlichkeit. Für als ,Schwarze' markierte AmerikanerInnen oder als ,AsiatInnen' markierte JapanerInnen stellt sich die Situation nochmals anders dar, wie die Diskussion zu Rassismus weiter unten zeigt.

Wie sieht es mit dem ,Blurring' aus, dem Bedeutungsverlust bestimmter Kriterien, die als Fremdheitsmarkierung dienen? ${ }^{167}$ Hier zeigt sich bei der Frage nach den für den Erwerb der deutschen Staatsbürgerschaft zu erfüllenden Bedingungen, dass die Bedeutung der angeborenen oder unveränderlichen Kriterien (z.B. Geburt in Deutschland, deutsche Eltern und Großeltern) sinkt, die der erwerbbaren (z.B. Sprachkenntnisse, Lebensstilanpassung, Sicherung des eigenen Lebensunterhalts) hingegen steigt (Abbildung 52). Da davon ausgegangen werden kann, dass Befragte anderen Personen die Staatsbürgerschaft am ehesten dann zuerkennen, wenn sie diese als ähnlich wahrnehmen, können die hier genannten Kriterien auch als Ähnlichkeitskriterien gewertet werden. Damit zeichnet sich ein ,Blurring' zumindest des Kriteriums des In-Deutschland-aufgewachsen-Seins ab. Wie es sich mit anderen Kriterien (z.B. dem Aussehen/der ,Rasse') verhält, wurde leider nicht erhoben. Kulturellen (Sprachkenntnisse, Lebensstilanpassung) und sozioökonomischen Kriterien (eigener Lebensunterhalt) wird allerdings im Jahr 2006 im Vergleich zu 1996 ein größeres Gewicht zugesprochen. Sie werden also nicht verwischt, sondern im Gegenteil verfestigt. Auch dem Kriterium der Mitgliedschaft in einer christlichen Kirche wird etwas mehr Bedeutung zugeschrieben. Hier scheint sich eine Markierung als ChristIn/Nicht-ChristIn (bzw. wahrscheinlich eher Nicht-MuslimIn/MuslimIn)

\footnotetext{
${ }^{167}$ Die dritte Möglichkeit des ,Boundary Shiftings' ist ein individueller Prozess, der daher hier nicht weiter untersucht wurde. Er hängt von der jeweiligen Ressourcenausstattung der/s MigrantIn ab. Im deutschen Fall werden diese individuellen Bemühungen zudem durch auf phänotypischen Kriterien basierenden Fremdheitsmarkierungen eingeschränkt.
} 
durchzusetzen, die zunehmend als Fremdheitszuschreibung dient und eine Trennlinie schafft, die an Bedeutung gewinnt.

Insgesamt profitiert die zweite Generation von diesen Entwicklungen: Während Kriterien, die sie nicht erfüllen kann, für die Ähnlichkeitswahrnehmung an Bedeutung verlieren (,Blurring'), steigt das Gewicht derjenigen Kriterien, die die zweite Generation im Mittelwert in höherem Maße als die erste Generation erfüllt (bessere Deutschkenntnisse und höherer sozioökonomischer Status). Eine bedeutsame Abweichung von dieser allgemeinen Tendenz besteht jedoch für Personen, die als MuslimInnen markiert werden. Gegenüber diesen Personen hat sich die Wahrnehmung von Ähnlichkeit bei der Dominanzbevölkerung nicht vergrößert.

Wie lassen sich nun diese allgemeinen Wahrnehmungen von MigrantInnen auf spezifische Wahrnehmungen von MigrantInnen in einem der vier Integrationsmuster herunterbrechen? $\mathrm{Zu}$ dieser Frage liegen keine hinreichend aufgeschlüsselten Umfragewerte vor. Daher können Schlussfolgerungen nur aus den Daten insgesamt gezogen werden. Für das Muster der neoklassischen Integration ist die Frage einfach zu beantworten: Dieses Muster basiert auf einer Verbesserung im sozioökonomischen Status und einer kulturellen Anpassung. Beiden Faktoren kommt bei der Ähnlichkeitswahrnehmung große Bedeutung zu, daher kommt es für dieses Muster durchschnittlich zu einer vergrößerten Ähnlichkeitswahrnehmung. Das Muster der fehlenden Integration - der Segregation - baut auf den gleichen Faktoren mit negativen Vorzeichen auf und führt daher zu einer reduzierten Ähnlichkeitswahrnehmung.

Für das pluralistische Muster, das sozioökonomischen Aufstieg mit kultureller Differenz verbindet, ist ein ,Blurring' der kulturellen Kriterien wichtig. Dieses findet sich in den Umfragen hinsichtlich Sprachkenntnissen und Lebensstilanpassung nicht. Allerdings zeigt die empirische Analyse auch, dass pluralistisch Integrierte im Durchschnitt neben sehr guten Kenntnissen in der Herkunftssprache der Eltern auch über (sehr) gute Deutschkenntnisse verfügten. Das Sprachkriterium wird daher für die meisten pluralistisch Integrierten nicht zum Problem. Anders sieht es mit der Lebensstilanpassung aus. Was aber genau bedeutet Lebensstilanpassung? Wie gezeigt wurde, sind einzelne Aspekte davon unerheblich geworden: Essgewohnheiten, Musik und Umgangsformen haben sich internationalisiert und weisen daher eine gröBere Bandbreite auf. Andere Bereiche haben sich in ihrer Funktion als Fremdheitsmarkierung verhärtet (z.B. religiöse Praktiken). Zudem bestehen in dieser Frage große Unterschiede zwischen der Dominanzbevölkerung in ländlichen Räumen und in Großstädten. GroßstädterInnen sehen wegen der größeren Diversität der Lebensstile innerhalb der Dominanzbevölkerung auch ein größeres Spektrum von Lebensstilen bei den MigrantInnen als ,kulturgleich', wäh- 
rend auf dem Land möglicherweise schon ein Mitglied der Dominanzbevölkerung aus einem anderen Bundesland als ,kulturungleich' wahrgenommen wird. Für das pluralistische Integrationsmuster wären also genauere Untersuchungen darüber nötig, wie Status und Kultur bei der Ähnlichkeitswahrnehmung zusammenspielen und wann welche Aspekte der beiden Faktoren salient werden.

Das Muster der Abwärts-Integration schließlich verbindet einen niedrigen sozioökonomischen Status mit kultureller Anpassung an eine marginalisierte Gruppe der Dominanzbevölkerung. In dieser Arbeit konnte zwar die grundsätzliche Existenz solcher Lebensweisen und darauf basierender Cliquen, Peergruppen und Banden belegt werden, es lagen jedoch keine Umfragedaten zu Wahrnehmungen bei diesem Segment der Dominanzbevölkerung vor. Daher kann auf der Basis der hier vorgestellten Empirie nur ansatzweise plausibilisiert werden, dass sich innerhalb dieser Gruppen die Wahrnehmung von Ähnlichkeit verstärkt. Eine solche Ähnlichkeitswahrnehmung basiert, wie gezeigt wurde, auf der Abgrenzung gegenüber anderen Gruppen (z.B. den verwöhnten ,Muttersöhnchen') und einer starken lokalen Identität (,Wir Wilhelmsburger' gegen die anderen Hamburger). Selbstverständlich ist damit nur die Integration in eine Subgruppe der Gesellschaft gelungen, die zudem für ihre Mitglieder (egal ob migrantisch oder aus der Dominanzbevölkerung) das Stigma der Minderwertigkeit trägt.

Ähnlichkeitswahrnehmung entsteht gemäß der Theorie von Sherif und Tajfel auf Interaktionen, die auf der Basis eines als ähnlich markierten Status oder einer als ähnlich markierten Kultur stattfinden. Beim (realen oder imaginierten) Zusammentreffen von MigrantInnen und Dominanzbevölkerung können jedoch die migrantischen Ressourcen nicht unmittelbar in status- oder kulturgleich markierte Interaktionen überführt werden. Vielmehr wirkt struktureller Rassismus auf diese Interaktionen und beeinflusst die Übersetzung der Ressourcen. Daher werden im letzten Abschnitt dieses Kapitels die Ergebnisse zum Zusammenhang von Integration und Rassismus diskutiert.

Durchschnittlich geht die vergrößerte Ähnlichkeitswahrnehmung mit einer verringerten Zustimmung zu rassistischer Praktiken einher (Tabelle 42). Durch die Auswertung der Feldexperimente von Klink/Wagner (1999) und Goldberg et al. (1995) in Kapitel 5.2 habe ich versucht, die Integrationsmechanismen der kultur- und/oder statusgleich markierten Interaktionen zu beleuchten. Die Feldexperimente erlauben den direkten Vergleich zwischen kultur- und/oder statusungleich markierten und kultur- und/oder statusgleich markierten Interaktionen. Es zeigt sich, dass in Kommunikationen per Telefon akzentfreies Deutsch zu kulturgleich markierten Interaktionen führt. Die ursprüngliche ,Grundierung' des Gesprächs durch die Vorstellung mit 
einem ausländisch klingenden Namen entfaltet keine diskriminierende Wirkung, und rassistische Diskriminierung findet nicht in einem statistisch signifikanten Maß statt. ${ }^{168}$ Auch zeigen die drei Feldexperimente zum sozialen Status (Termin zur Wohnungsbesichtigung einmal von Herrn Kawowski, einmal von Dr. Kawowski angefragt, Tischreservierung in einem teuren und in einem gewöhnlichen Restaurant sowie Tischreservierung für eine deutsche bzw. eine türkische Gewerkschaft versus einen deutschen bzw. einen türkischen Anwaltsbund) eine deutlich geringere Diskriminierung für die statushöhere Gruppe und den statushöheren Ort. Während diese Experimente ebenso wie der Trend der verringerten Zustimmung zu rassistischen Aussagen für die Gültigkeit der in dieser Arbeit vorgestellten Integrationsmechanismen der kultur- und statusgleich markierten Interaktionen sprechen, verweisen die Feldexperimente in den als ,fremd' markierten Interaktionen auf ein hohes Maß rassistischer Diskriminierung. Dass es sich dabei nicht (nur) um individuelles Fehlverhalten handelt, wurde in der Untersuchung des Schulbereichs im Hinblick auf institutionelle Mechanismen von Rassismus deutlich: An Schulen und im schulischen Umfeld führen eine Reihe von institutionellen, rechtlichen und kulturellen Strukturen zu einer Auf- bzw. Abwertung bestimmter Ressourcen. Darüber werden Über- und Unterordnungen zugewiesen und verfestigt. Die Strukturen erlauben also nicht allen Kindern in gleichem Maße, ihre Ressourcen zu nutzen. Beispielsweise verhindern nicht ausgesprochene und zudem in sich unstimmige Regeln über das, was ein/e gute/r SchülerIn tut oder nicht tut, faire Wettbewerbsbedingungen für alle. Auch treffen institutionelle Mechanismen wie Halbtagsschulen, die Aufhebung von Schulbezirksbindungen oder ,prognostizierende' Übergangsempfehlungen MigrantInnen vermittelt über ihren durchschnittlich niedrigeren sozioökonomischen Status stärker als die Dominanzbevölkerung, obwohl formal eine Gleichbehandlung von MigrantInnen und Dominanzbevölkerung herrscht. Kapitel 5.2 hat somit herausgestellt, dass Rassismus bestimmten Personen negative Kapitalien zuweist, die akkumuliert werden und sich aggregiert zu einer Struktur sozialer Ungleichheit verfestigen. Neben der individuellen Ebene rassistischer Praktiken besteht Rassismus auch aus einer strukturellen Ebene sozialer Ungleichheit, welche den Erwerb und Einsatz von Ressourcen der MigrantInnen beeinflusst.

Das Kapitel bestätigte somit das in dieser Arbeit zugrunde gelegte Integrationsverständnis, gemäß dem Integration nicht einseitig von den MigrantInnen abhängt. Vielmehr erwerben MigrantInnen in einem rassistisch durchdrungenen Umfeld Ressourcen und überführen diese in status- und kulturgleich markierte Interaktionen. Solche Interaktionen vergrößern die wahr-

\footnotetext{
${ }^{168}$ Diese fehlende statistische Signifikanz sollte aber nicht vergessen lassen, dass jeder einzelne Fall, in dem Diskriminierung erfolgt, relevant ist und verhindert werden muss.
} 
genommene Ähnlichkeit auf Seiten der nicht-migrantischen Dominanzbevölkerung, wobei die Interaktionen von Rassismus beeinflusst sind. Und schließlich wirken sowohl die Ressourcenverteilung selbst als auch die Ähnlichkeitswahrnehmungen auf den Rassismus in einer Gesellschaft zurück. Allerdings entwickelt sich Rassismus in Gesellschaften keinesfalls stetig, sondern kann von politischen EntrepreneurInnen durch eine bestimmte Interpretation externer Ereignisse kurzfristig verstärkt werden. ${ }^{169}$ Auf den Prozess der Integration wirkt also eine Reihe wenig kalkulierbarer Faktoren ein. Der üblicherweise herausgegriffene Ressourcenerwerb auf Seiten der MigrantInnen (der zumeist als defizitär beschrieben wird) ist nur ein Faktor unter mehreren. Gleichzeitig determiniert die rassistische Struktur weder den Ressourcenerwerb der MigrantInnen noch die Interaktionen und die Ähnlichkeitswahrnehmung vollständig. Sie stellt vielmehr das Umfeld dar, in dem AkteurInnen ihre Identitäten, Interessen und Präferenzen entwickeln. Dem willentlichen Handeln der AkteurInnen fällt in der Empirie dieser Arbeit jedoch weniger Bedeutung zu, als ihm gemäß dem theoretischen Konzept gebührte, da das quantitative Design der Arbeit größtenteils auf aggregierten oder sogar strukturellen Daten basiert.

Zusammengenommen wurde somit, auch mithilfe des Einschlusses deutscher StaatsbürgerInnen mit Migrationshintergrund, die Besserstellung der zweiten Generation im Vergleich zur ersten und anderthalbten Generation belegt. Eine große Vielfalt der Ergebnisse zwischen, vor allem aber innerhalb der Herkunftsgruppen wurde nachgewiesen. Auch Integrationsmuster, bei denen Kultur- und Statusvariablen nicht Hand in Hand gehen, existieren. Im Hinblick auf die Mechanismen hinter diesen Integrationsmustern muss jedoch eine gemischte Bilanz gezogen werden: Für das neoklassische Muster können diese problemlos belegt werden. Beim pluralistischen Muster lassen sich einige untermauern (z.B. positive Effekte von Zweisprachigkeit), für andere fehlen jedoch Daten, um sie zu untersuchen (z.B. zu Ressourcen der ,koethnischen' FreundInnen oder Familienklima sowie Längsschnittdaten allgemein). Bei der Abwärts-Integration konnten nur wenig Belege für eine gemeinsame ,Oppositionskultur' von MigrantInnen und Nicht-MigrantInnen und eine Ähnlichkeitswahrnehmung innerhalb dieser Gruppe gefunden werden.

Schließlich wurde das hier zugrunde gelegte Integrationsverständnis untermauert: Der Zunahme und Besserstellung der zweiten Generation entspricht ein Trend der vergrößerten Ähnlichkeitswahrnehmung bei der Dominanzbevölkerung. Für die einzelnen Muster konnte der Integrationsprozess jedoch unterschiedlich gut nachgewiesen werden: Die integrative Wir-

\footnotetext{
${ }^{169}$ Die Wirkung der kollektiven Interpretation von Ereignissen wie den Attentaten des 11. September 2001 auf Rassismus zu untersuchen, war jedoch nicht das Anliegen dieser Arbeit.
} 
kung von kultur- und statusgleich markierten Interaktionen für das neoklassische Muster war durch die Feldexperimente schlüssig zu belegen. Beim pluralistischen Muster blieb unklar, welche kulturellen Faktoren dafür zwingend erforderlich sind (d.h. bei welchen entschieden kein ,Blurring' stattfindet) und wann entweder die Status- oder die Kulturkriterien salienter werden. Am schlechtesten konnten die Integrationsmechanismen für das Muster der AbwärtsIntegration belegt werden, da Wahrnehmungsdaten für die betreffende Subkultur der Dominanzbevölkerung nicht vorliegen.

Im Folgenden soll nun erörtert werden, was diese Ergebnisse für den Integrationsdiskurs, die Migrationswissenschaften und die Politik bedeuten. 


\section{Fazit: MigrantInnen der zweiten Generation - Abgehängt, chancenlos, unwillig?}

Von MigrantInnen der zweiten Generation wird kolportiert, dass sie geringere schulische Leistungen als die erste Migrantengeneration erbrächten und daher schlechte Chancen auf dem Arbeitsmarkt hätten; sie sprächen nur mangelhaft Deutsch und verweigerten sich den Aufnahmebemühungen der Mehrheitsgesellschaft, stattdessen zögen sie ein Leben in parallelen Subkulturen vor (PISA-Konsortium 2004; Kelek 2005). Dabei handelt es sich mit ca. 3,3 Millionen Personen um eine signifikante Gruppe in Deutschland, deren relativer Anteil an allen MigrantInnen aufgrund der restriktiven deutschen Zuwanderungspolitik und damit einhergehenden geringen Zuzugszahlen weiter steigt (vgl. Kapitel 3.2).

Mit dieser zweiten Generation setzte sich dieses Buch auseinander und untersuchte die Stichhaltigkeit obiger negativer Berichterstattung. Während bisherige Untersuchungen auch aufgrund einer selektiver Datenlage weitestgehend auf junge MigrantInnen mit ausländischer Staatsangehörigkeit aus wenigen Herkunftsgruppen (zumeist Türkei und Italien) beschränkt waren, betrachtete diese Studie mit den 20- bis 45-Jährigen (DJI-Survey: 18 bis 25 Jahre) ein höheres Alterssegment aus verschiedenen Herkunftsgruppen: Neben den ehemaligen ,Anwerbestaaten' (Türkei, Italien, Griechenland, Spanien, Portugal, Ex-Jugoslawien) wurden auch die Kinder von AussiedlerInnen untersucht sowie MigrantInnen aus, westlichen' Staaten wie Österreich, Frankreich, den Niederlanden und den USA, mit denen keine Anwerbeabkommen bestanden. Dabei wurden in allen Herkunftsgruppen auch deutsche Staatsangehörige betrachtet. Die Variable Staatsangehörigkeit ist bedeutsam, da MigrantInnen mit deutscher Staatsbürgerschaft durchschnittlich einen höheren sozioökonomischen Status aufweisen als ausländische StaatsbürgerInnen. Der Anteil der deutschen Staatsangehörigen variiert indes stark zwischen den Herkunftsgruppen: Während bei den 20- bis 45-Jährigen MigrantInnen der zweiten Generation türkischer Herkunft $40 \%$ die deutsche Staatsbürgerschaft besitzen, sind dies bei denen mit Eltern aus Anwerbestaaten, die heute zur EU gehören, nur 10\%. Bei der türkischen Herkunftsgruppe entfielen also in den bisherigen Untersuchungen, die sich auf ausländische Staatsangehörige beschränkten, besonders viele sozioökonomisch erfolgreiche MigrantInnen.

\subsection{Forschungsinteresse der Arbeit}

Im Zentrum der Untersuchung standen wie in Kapitel 1.1 erläutert zwei Forschungsfragen: 
- Inwiefern sind MigrantInnen der zweiten Generation in Deutschland integriert?

- Was sind Schwachstellen bisheriger Integrationstheorien (und wie können sie überwunden werden)? Welche Integrationstheorien eignen sich für die Analyse der zweiten Generation in Deutschland?

Hinsichtlich der ersten Frage sollte untersucht werden, ob die v.a. seit den PISAUntersuchungen in den Raum gestellte These eines ,Niedergangs' der zweiten Generation zutrifft. Zudem interessierte, ob die veränderte Erhebung im größten deutschen Datenset, dem Mikrozensus, mit der neu geschaffenen Möglichkeit, MigrantInnen unabhängig von Staatsbürgerschaftsstatus und dem Zusammenleben mit den Eltern zu identifizieren, andere empirische Ergebnisse zu Tage bringt als bisherige ,Ausländerstudien'. Meine Vermutung war, dass entgegen den Ergebnissen, zu denen die mediale Fokussierung auf MigrantInnen türkischer Herkunft aus marginalisierten sozialen Schichten führt, diese Untersuchung eine große Bandbreite der Ressourcenausstattung für die zweite Generation zeigen würde.

Für den zweiten Fragekomplex wurden aufbauend auf der Diskussion der problematischen Aspekte bestehender Theorien (Tendenz zur Reifizierung, Homogenisierung und Teleologie) in Kapitel 2.3 folgende Integrationsprämissen entwickelt: Veränderungen finden nicht einseitig bei MigrantInnen statt, sondern zweiseitig auch auf Seiten der Bevölkerung ohne Migrationshintergrund. Integration konzeptionalisiere ich sozialkonstruktivistisch als Zunahme der Ähnlichkeitswahrnehmung der migrantischen Bevölkerung durch die Dominanzbevölkerung. ${ }^{170}$ Dafür müssen bestimmte Kriterien der Grenzziehung entweder an Bedeutung verlieren (,Boundary Blurring'), als pauschal für ganze Gruppen erfüllt angesehen werden (,Boundary Shifting') oder von Einzelnen erfüllbar sein (,Boundary Crossing') (Bauböck 1995). Sozioökonomischer Statuserwerb muss gemäß meiner Prämissen nicht mit kultureller Anpassung einhergehen, sondern kann auch ohne diese stattfinden (und umgekehrt). Dabei sind sowohl die Erwerbung von Ressourcen als auch die Ähnlichkeitswahrnehmung von existierenden rassistischen Strukturen und Praktiken in der deutschen Gesellschaft beeinflusst. Aus der gegenwärtig in den USA geführten Debatte arbeitete ich drei Theoriestränge heraus (neoklassische Integration, Abwärts-Integration, transnational-pluralistische Integration), die mit den

\footnotetext{
${ }^{170}$ Selbstverständlich muss diese Ähnlichkeitswahrnehmung auf Seiten der Dominanzbevölkerung mit einer eben solchen vergrößerten Wahrnehmung von Ähnlichkeit auch bei den MigrantInnen gegenüber der nichtmigrantischen Bevölkerung einhergehen. In einem hierarchischen Gesellschaftssystem ist jedoch die Wahrnehmung der dominanten Gruppe die machtvollere. Daher wurde in dieser Studie ausschließlich die Wahrnehmung bei der Bevölkerung ohne Migrationshintergrund untersucht. Der Begriff der Dominanzbevölkerung soll dabei dieses in vielen Aspekten zwischen MigrantInnen und Deutschen ohne Migrationshintergrund bestehende Hierarchieverhältnis deutlich zum Ausdruck bringen.
} 
von mir entwickelten Integrationsprämissen kompatibel sind und ging davon aus, dass sich diese Theorien auf Deutschland übertragen lassen. (vgl. Kapitel 2.6)

Im Folgenden wird erörtert, welche dieser Annahmen in der Arbeit bestätigt werden konnten, welche widerlegt wurden und wo Einschränkungen gemacht werden müssen. Zunächst werden die Ergebnisse der empirischen Untersuchung, die im vorherigen Kapitel ausführlich diskutiert worden sind, pointiert zusammengefasst, um darauf aufbauend die Gültigkeit der in dieser Arbeit verwendeten Integrationstheorien zu diskutieren und das hier entwickelte Integrationsverständnis zu stärken. Im Anschluss wird dargestellt, über welche Gruppen und Bereiche dieses Buch Aussagen zulässt und welche Beschränkungen sie hat. Damit soll zum einen die Reichweite der Ergebnisse klarer umrissen und zum anderen die Frage aufgeworfen werden, welche Probleme bei der Übertragung US-amerikanischer Theorien auf Deutschland entstehen. Schließlich werden sich aus dieser Arbeit ergebende Konsequenzen für den Integrationsdiskurs aufgezeigt und drei politische Herausforderungen abgeleitet.

\subsection{Zusammenfassung empirischer Ergebnisse}

Die Untersuchung zeigte, dass entgegen geläufiger Darstellungen MigrantInnen der zweiten Generation eine heterogene Gruppe sind, die sowohl zwischen als auch innerhalb der Herkunftsgruppen eine große Vielfalt aufweist. Zwar ist der Abstand zwischen den Durchschnittswerten für die türkische Herkunftsgruppe und der jeweils am besten abschneidenden Herkunftsgruppe (zumeist die ,westliche', aber auch die Kinder von AussiedlerInnen oder die spanisch/portugiesische Herkunftsgruppe) groß, noch bedeutsamer jedoch ist die Bandbreite der Ergebnisse innerhalb der verschiedenen Herkunftsgruppen. Diese Streuung innerhalb der Gruppen fällt nur unwesentlich geringer aus als bei Deutschen ohne Migrationshintergrund. Wo es also vor zehn Jahren in der Integrationsforschung um die Anerkennung der Differenz ging (,MigrantInnen verfügen aufgrund der Migrationserfahrung über spezifische, ihnen eigene Probleme und Ressourcen“), betont diese Arbeit die Vervielfältigung der Differenzen innerhalb der zweiten Migrantengeneration (,Die Probleme und Chancen für diese Gruppe sind unterschiedlich und entlang nicht-migrationsspezifischer Faktoren gebrochen"). Eine Gleichsetzung von MigrantInnen mit MuslimInnen oder speziell mit MigrantInnen türkischer Herkunft, die marginalisierten sozialen Schichten angehören, wirkt in dieser Verkürzung verzerrend. Auch die These des ,Niedergangs' der zweiten Generation lässt sich nicht halten: Durchschnittlich erreicht die zweite Generation der Jahrgänge 1961-1985 einen höheren sozioökonomischen Status als die erste und die anderthalbte Generation derselben Jahrgänge. 
Nur bei den MigrantInnen aus ,westlichen' Herkunftsländern, mit denen keine Anwerbeabkommen bestanden (z.B Niederlande, USA, Großbritannien) ist der sozioökonomische Status in der zweiten Generation niedriger als in der ersten und anderthalbten. Allerdings findet dieser Rückgang auf hohem Niveau statt und eignet sich nicht als Beleg für Segregationstendenzen. Diese Befunde dürfen dennoch nicht über die reale Existenz einer Gruppe hinwegtäuschen, die keine oder sehr schlechte Schulabschlüsse erwirbt, unfreiwillig prekär oder gar nicht am Arbeitsmarkt teilnimmt und denen die für gesellschaftliche Partizipation erforderlichen schriftlichen Deutschkenntnisse fehlen. Angehörige dieser Gruppe werden zudem häufig rassistisch als fremd markiert werden und leben in benachteiligten Stadtteilen, so dass zur individuellen Benachteiligung eine sozialräumliche hinzukommt. Als Prototyp dieser Gruppe muss, wenn auch keinesfalls ausschließlich, ein Teil der türkischen Herkunftsgruppe angesehen werden. Gleichwohl gilt: MigrantInnen dieser Gruppe sind für die zweite Generation insgesamt nicht repräsentativ.

Dieses Ergebnis, das insgesamt positiver und unspektakulärer ausfällt als aufgrund der Medienberichterstattung und bisheriger Studien zu AusländerInnen erwartbar, ist, wie gezeigt wurde, neben der Differenzierung nach Generationen auf den Einschluss deutscher StaatsbürgerInnen in das Sample zurückzuführen, da diese durchschnittlich einen höheren sozioökonomischen Status (gemessen durch Bildungs- und Berufsindikatoren) und mehr kulturelle Anpassung (gemessen durch Sprachkenntnisse und Partnerschaftswahl) aufweisen. ${ }^{171}$ Das sozioökonomische Niveau gehoben hat auch der Einschluss älterer MigrantInnen der zweiten Generation und überhaupt die Untersuchung der Positionierung am Arbeitsmarkt. Eine solche Analyse wurde durch die Veränderungen in der amtlichen Statistik ermöglicht, durch die seit 2005 im Mikrozensus MigrantInnen mit deutscher Staatsbürgerschaft sowie die zweite Generation, die nicht mehr bei ihren Eltern lebt, identifiziert werden können.

Weiterhin konnte anhand der Daten des Mikrozensus und des DJI-Ausländersurveys die Existenz der aus den US-amerikanischen Theoriedebatten abgeleiteten Integrationsmuster (neoklassische und transnational-pluralistische Integration sowie Abwärts-Integration) (vgl. Kapitel 4) belegt werden: Ungefähr die Hälfte der MigrantInnen der zweiten Generation folgt einem neoklassischen Integrationsmuster, bei dem eine erfolgreiche Teilnahme im Bildungssystem und auf dem Arbeitsmarkt zusammenfällt mit sehr guten schriftlichen Deutschkenntnissen oder Beziehungen zu LebenspartnerInnen, die selbst nicht aus dem Herkunftsland der Eltern migriert sind. Am anderen Ende des Spektrums befinden sich segregierte MigrantInnen

\footnotetext{
${ }^{171}$ Bei MigrantInnen aus ,westlichen' Nicht-Anwerbestaaten haben diejenigen mit deutscher Staatsbürgerschaft allerdings einen niedrigeren sozioökonomischen Status als die ausländischen Staatsangehörigen.
} 
der zweiten Generation mit sehr guten Sprachkenntnissen in der Muttersprache der Eltern und geringen Deutschkenntnissen oder selbst zugewanderten PartnerInnen der gleichen Herkunftsgruppe, welche nicht die notwendige Bildung erworben haben, um gesichert am Arbeitsmarkt teilzunehmen, und in Folge arbeitslos sind oder als ungelernte ArbeiterInnen oder in einfachen Dienstleistungsjobs beschäftigt sind. Während der Anteil der neoklassischen Integration von der ersten und anderthalbten Generation zur zweiten Generation stark zunimmt, verringert sich der Prozentsatz der Segregation.

Darüber hinaus lassen sich zwei Integrationsmuster finden, welche die gewöhnlich angenommene Verschränkung von sozioökonomischem Aufstieg und kultureller Anpassung aufbrechen: Auf der einen Seite gibt es eine Gruppe pluralistischer MigrantInnen, die mit selbst zugewanderten PartnerInnen der gleichen Herkunftsgruppe zusammenleben (oder sehr gute Kenntnisse in der Elternsprache haben) und hinreichend Bildung für eine nachhaltige Beteiligung auf dem Arbeitsmarkt erlangt haben. Auf der anderen Seite beendet ein Viertel der MigrantInnen der zweiten Generation die Bildungskarriere so frühzeitig, dass sie nur unsichere Positionen auf dem Arbeitsmarkt einnehmen kann. Diese Gruppe lebt jedoch mit PartnerInnen zusammen, die nicht MigrantInnen der ersten Generation sind, oder hat im Deutschen bessere Sprachkenntnisse als in der Herkunftssprache. Durch die empirische Existenz dieser Muster konnte belegt werden, dass sozioökonomischer Status und kulturelle Anpassung im Integrationsprozess zwei eigenständige Achsen sind, die häufig, aber nicht zwangsläufig zusammengehen und für einen signifikanten Anteil auch voneinander getrennt sind. Durch die Pluralisierung der Integrationstheorien eröffnet sich die Chance, statt von einer einheitlichen Migrantengruppe von einzelnen MigrantInnen mit gesellschaftlich unterschiedlich bewerteten Ressourcen auszugehen und das Zusammenspiel von Migrationsstatus, sozialer Schicht, Zuschreibungen an Hautfarbe/Religionszugehörigkeit zu analysieren. Die Erweiterung der Integrationstheorien erlaubt es also, eine verbreitete kulturalistische Engführung aufzubrechen.

Neben der empirischen Existenz dieser Integrationsmuster in Deutschland und der Verteilung der zweiten Generation auf die Muster wurde in diesem Buch versucht, die Mechanismen ihrer Entstehung zu plausibilisieren - mit unterschiedlichem Erfolg. Für das neoklassische Muster konnte gezeigt werden, wie MigrantInnen bei Wahrnehmung relativer Diskriminierungsfreiheit Möglichkeiten ergreifen und hohe Aspirationen in bessere Bildungsabschlüsse und Jobs übersetzen können. Dieser Prozess wird unterstützt durch die relative Diskriminierungsfreiheit und größere Meritokratie im höheren schulischen Leistungsspektrum. Die im pluralistischen Muster angenommene Rendite von Zweisprachigkeit und einer Beschäftigung in der ,ethnischen Ökonomie' für den Status und das Einkommen wurde direkt oder vermittelt 
über psychologische Effekte nachgewiesen. Die Existenz transnationaler Räume konnte jedoch - nicht zuletzt aufgrund der Struktur der quantitativen Daten, die ein Entweder-oder erzwingen - nicht belegt werden. Offen blieben auch die kausale Richtung der Effekte (ist es beispielsweise die Zweisprachigkeit, die ein positives Familienklima bewirkt oder führt der Familienzusammenhalt zur Zweisprachigkeit?) und die Frage, ob ein bestimmter sozioökonomischer Mindeststandard nötig ist, damit positive Effekte einer solchen bikulturellen Orientierung entstehen können. Die Mechanismen der Abwärts-Integration schließlich konnten in Teilen belegt werden. MigrantInnen in diesem Muster weisen oppositionelles Verhalten gegenüber Institutionen und Personen des ,Establishments' auf, welches sich in geringen Bildungsaspirationen und niedriger Selbstwirksamkeit sowie der Wahrnehmung eines hohen Maßes an Diskriminierung ausdrückt, und es konnten musterkonform Belege für diese Form der Integration in multiethnischen Peergruppen (,Regenbogen'-Gruppen) gefunden werden. Die genauen Prozesse der Ähnlichkeitswahrnehmung in diesen Gruppen ebenso wie der Mechanismus der dissonanten Akkulturation in den Familien bleiben aber offen.

In Bezug auf das hier verwendete sozialkonstruktivistische Integrationskonzept konnte gezeigt werden, dass in dem Maße, in dem sich der Anteil der zweiten Generation an der migrantischen Bevölkerung erhöht und sich damit eine durchschnittliche sozioökonomische Besserstellung und größere kulturelle Anpassung ergibt, auch die Ähnlichkeitswahrnehmung auf Seiten der Dominanzbevölkerung zunimmt. Darüber hinaus muss differenziert werden: Die beschriebenen experimentellen Untersuchungen zeigten eine starke Reduktion von Diskriminierung in den als status- und/oder kulturgleich markierten Interaktionen, ${ }^{172}$ wohingegen als ungleich markierte Interaktionen weiterhin ein hohes Maß rassistischer Diskriminierung aufwiesen. Auch die Verfestigung bestimmter Fremdheitsmarkierungen (z.B. Kopftücher) wurde belegt. Es wurde argumentiert, dass Rassismus eine Struktur sozialer Ungleich darstellt, die über individuelles rassistisches Verhalten (und dessen Aggregation) hinausgeht. So konnte speziell für das Bildungssystem gezeigt werden, wie aus institutionellen, rechtlichen und kulturellen Gründen bestimmte Ressourcen systematisch auf- und andere systematisch abgewertet werden.

Hinsichtlich der Kriterien von Ähnlichkeitszuschreibungen belegte die empirische Untersuchung eine hohe Bedeutung sozioökonomischer wie sprachlicher Normen (z.B. nichtprekärer sozioökonomischer Status, sehr gute Deutschkenntnisse). Die Wirkung anderer Markierungen wie Religion, Kleidung, Hautfarbe ist weniger klar. Meine Untersuchung deutet darauf hin, dass für Personen, die nicht über das Aussehen als fremd markiert sind (z.B. Po-

\footnotetext{
${ }^{172}$ Für die Einführung in dieses Konzept vergleiche Kapitel 2.3.4.
} 
IInnen), ein Überwinden der Grenze zwischen Fremdem und Eigenem grundsätzlich möglich ist (,Boundary Crossing'). Die Gruppe der MigrantInnen der zweiten Generation italienischer (aber auch spanischer, portugiesischer) Herkunft befindet sich auf dem Weg eines ,Boundary Shifting', d.h. die Grenze der Fremdheitsmarkierung verändert sich so, dass die gesamte Gruppe durchschnittlich als Teil des Nicht-Fremden betrachtet wird. Hinsichtlich des ,Boundary Blurring' muss festgehalten werden, dass ein solches zur Zeit in Bezug auf Markierungen von angenommener islamischer Religion durch die Mehrheitsbevölkerung nicht stattfindet. Die Möglichkeiten eines Bedeutungsverlustes von Fremdheitsmarkierungen (,Blurring') unterliegen jedoch dem gesellschaftlichen Wandel, aus der aktuellen Situation darf daher kein quasi-natürlicher Status der Kriterien abgeleitet werden. Diese haben und werden sich weiter wandeln und sind somit Ansatzpunkt für politisches Handeln. Sie verweisen indes auf gegenwärtige (globale) Hierarchieverhältnisse und beeinflussen Integrationsprozesse.

Grundsätzlich gelang es, für den amerikanischen Kontext entwickelte Integrationstheorien auf Deutschland zu übertragen und diese helfen auch den Blick für eine vielfältige Realität zu öffnen. Es ließ sich darüber zudem eine ähnliche Funktion von Sprache und Religion in den USA und in Deutschland hinsichtlich von Integration zeigen. Zolberg und Woon hatten in dem Artikel Why Islam is Like Spanish argumentiert, dass die Debatte um die spanischsprachigen MigrantInnen in den USA der um die muslimischen MigrantInnen in Europa ähnele (Zolberg/Woon 1999). In dem empirischen Teil zur Wahrnehmung in diesem Buch wurde gezeigt, wie Markierungen als gläubige/r MuslimIn (z.B. Markierung als ,Kopftuchträgerin') zu einer ähnlich großen Fremdheitszuschreibung führen, wie dies in den USA für Markierungen als Spanischsprachige/r der Fall ist. Es gleichen sich also die Mechanismen, auch wenn die Fremdheitskonstruktionen selbst unterschiedlich sind. In beiden Fällen steht hinter diesen kulturalistischen Fremdheitsmarkierungen auch Rassismus. Dabei scheinen in Deutschland kulturelle Erklärungen der Fremdheit, die immer auch die Zuschreibung einer ,Mitschuld' der/s MigrantIn an seiner/ihrer Fremdheit implizieren, besonders weit verbreitet zu sein. Ein klarerer Blick auf den Rassismus in Deutschland würde es hingegen erzwingen, Machtverhältnisse in den Fokus der Analyse zu stellen und über ihre politische Gestaltbarkeit sowie die Frage ihrer Erwünschtheit zu diskutieren.

Als Grenze der Übertragbarkeit bleibt jedoch festzuhalten, dass es keine deutsche Bevölkerungsgruppe ohne Migrationshintergrund von signifikanter Größe gibt, die dauerhaft als fremd markiert würde, wie dies für ,Schwarze' in den USA der Fall ist. Hinzu kommt, dass auch die Wohnsituationen in deutschen Großstadtvierteln aufgrund der bisher größeren 
Durchmischung (noch) nicht der amerikanischen Situation entspricht. ${ }^{173}$ In dem empirischen Teil dieser Arbeit wurden ,Regenbogen'-Gruppen analysiert, in denen es zu einer Solidarisierung zwischen Jugendlichen mit und ohne Migrationshintergrund aufgrund fehlender Perspektiven kommt. Zudem wurden negative Effekte aufgrund der Konzentration von Benachteiligung bestätigt. Obwohl damit die grundsätzliche Existenz des Musters der AbwärtsIntegration empirisch belegt ist, müssen die Mechanismen der Abgrenzung und des Einschlusses unter diesen anderen Bedingungen in Deutschland für die Theorie der AbwärtsIntegration noch näher untersucht werden. In einer medial globalisierten Welt kann allerdings eine ,schwarze Oppositionskultur' auch ohne reale Existenz in Deutschland als Referenzpunkt für MigrantInnen im Muster der Abwärts-Integration dienen.

Als weiterer Unterschied wurden in Deutschland aus historischen Gründen keine ,Affirmative Action'-Programme entwickelt. Dadurch fehlen für MigrantInnen geförderte Mechanismen des Aufstiegs (,Empowerment'), wobei sich zur Zeit durch Selbstverpflichtungen von Unternehmen und Verwaltungen die beruflichen Chancen für qualifizierte MigrantInnen vergrößern. ${ }^{174}$ Die Mobilitätsförderung durch die EU erleichtert zudem innerhalb der Union das Entstehen transnationaler Räume und wertet bikulturelle Kompetenzen für den Kreis der UnionsbürgerInnen auf. Gegenüber Angehörigen aus Drittstaaten verfestigt sich jedoch die Abgrenzung und führt auch zu einer Trennung im Inneren, wie z.B. in der Diskussion um den Verweis auf das „,christliche Erbe Europas“ im Entwurf der EU Verfassung geschehen.

Die empirische Untersuchung in diesem Buch

- widerspricht damit der These eines allgemeinen ,Niedergangs' der zweiten Generation, vielmehr

- zeigt sie, dass dieser Niedergang auf Zusammensetzungseffekten beruht und belegt bei Einschluss von deutschen StaatsbürgerInnen mit Migrationshintergrund und einem älteren Segment in die Daten eine durchschnittliche Verbesserung für die zweite Generation, v.a. aber eine große Vielfalt innerhalb dieser Gruppe; ${ }^{175}$

\footnotetext{
${ }^{173}$ So liegt beispielsweise in den Berliner Stadtvierteln Kreuzberg, Neukölln, Wedding der Anteil der türkischen StaatsbürgerInnen bei $16 \%$ der Bevölkerung, die Anteile der ausländischen Bevölkerung insgesamt bei über 30\% (Kapphan 2001: 103). Bei Einschluss der deutschen StaatsbürgerInnen mit Migrationshintergrund und der Berücksichtigung von Konzentrationen innerhalb der Stadtviertel, z.B. in Neukölln das Rollberge Viertel, kommt der Prozentsatz den Zahlen für US-amerikanische, innerstädtische , Ghettos' näher.

${ }^{174}$ So haben sich eine Reihe von lokalen Verwaltungen das Ziel gesetzt, mehr MigrantInnen zu rekrutieren, und die Begabtenförderungswerke versuchen aktiv, den Prozentsatz von StipendiatInnen mit Migrationshintergrund zu erhöhen (Buntrock 2006; Niedersachsen 2008).

${ }^{175}$ Die Vielfalt bedeutet jedoch auch, dass es die medial als ,problematisch' dargestellten Gruppen real gibt, nur können sie weder als Durchschnitt noch als typische VertreterInnen der zweiten Generation gelten.
} 
- verdeutlicht mit Hilfe eines sozialkonstruktivistischen Integrationsbegriffs die Mehrseitigkeit und strukturelle Bedingtheit von Integration sowie den Wandel im Integrationsverständnis;

- dokumentiert empirisch die Pertinenz der aus den US-amerikanischen Theorien entwickelten Integrationsmuster für den deutschen Kontext trotz oben beschriebener Probleme der Übertragbarkeit und

- eröffnet durch diese Erweiterung der Integrationstheorien die Möglichkeit, die paradigmatische Verschränkung von Status und Kultur, wie sie in assimilatorischen Theorien vorgenommen wird, aufzulösen und stattdessen das vielfältige Zusammenspiel dieser und weiterer Faktoren zu betrachten.

Was bedeuten diese Ergebnisse für die im Entstehen begriffenen Migrationswissenschaften, die den gesellschaftlichen Diskurs beeinflussen und von ihm beeinflusst sind?

\subsection{Theoretische Verortung in den Migrationswissenschaften}

Das in dieser Arbeit entwickelte Integrationskonzept stellt drei in den Migrationswissenschaften weit verbreitete Annahmen in Frage:

Erstens stellt es der Einseitigkeit von Integrationsprozessen (wie sie z.B. Esser beschreibt) ein Konzept entgegen, das Integration über vergrößerte Ähnlichkeitswahrnehmung definiert. Damit können die Debatte über die Bringschuld der MigrantInnen und der Fokus auf ihre Defizite überwunden werden. Vielmehr betont das Konzept die wechselseitige Konstruktion und Verfestigung von Fremdheit in einem System globaler Machtverhältnisse. ${ }^{176}$

Zweitens wird zumeist von einer Dichotomie von Integration und Desintegration ausgegangen. Ich habe diese binäre Sichtweise in der vorliegenden Arbeit durch das Aufspannen einer Vier-Felder-Matrix mit zwei getrennten Achsen zurückgewiesen. Damit stellt sich jedoch auch die normative Frage, ob die Forderung nach Integration immer positiv zu bewerten ist, besonders wenn kulturelle Anpassung nicht mit einer sozioökonomischen Verbesserung zusammengeht. Diese Dichotomie ist zudem eingeschrieben in die Erhebung der empirischen Daten selbst, die häufig nur ein Entweder-oder zulassen: So mussten sich in Heitmeyers Studie die Jugendlichen türkischer Herkunft entscheiden, ob sie sich ,unter Deutschen wohler als unter Türken“ oder „unter Türken wohler als unter Deutschen“ fühlten (Heitmeyer, et al.

\footnotetext{
${ }^{176}$ In der empirischen Untersuchung wurde jedoch nur eine Seite dieses gegenseitigen Prozesses betrachtet, nämlich die Ähnlichkeitswahrnehmung bei der Dominanzbevölkerung, weil diese in einem hierarchisch strukturierten Gesellschaftssystem durch ihre Konsequenzen die Bedeutsamere ist.
} 
1997: 248). Diese Binarität greift zu kurz, weil Menschen zu multiplen und komplexen Identifikationen und Lebensformen fähig sind und diese alltäglich leben.

Sie greift aber auch deswegen zu kurz, weil drittens die dahinter stehende Homogenitätsannahme abgelehnt werden muss. Für die MigrantInnen wurde in dieser Arbeit die große Vielfalt allein innerhalb der zweiten Generation deutlich. Allerdings besteht diese Vielfalt auch mit Blick auf die Dominanzbevölkerung. Dies zeigt sich sozioökonomisch u.a. an zunehmender Einkommensungleichheit und steigender Armut (besonders Kinderarmut), ${ }^{177}$ kulturell z.B. an der Varianz der Deutschkenntnisse: Wie im Kapitel zu Sprachkenntnissen dargelegt, verfügt ein signifikanter Teil der deutschen Kinder ohne Migrationshintergrund bei der Einschulung nicht über ausreichende Deutschkenntnisse. Diese ungleiche Ressourcenverteilung schlägt sich in unterschiedlichen Fähigkeiten nieder, mit den Herausforderungen von gesellschaftlichem, ökonomischem und sozialem Wandel, von Flexibilität und individueller (Vor-) Sorge umzugehen.

Aus der Heterogenität auf Seiten der Dominanzbevölkerung ergibt sich die Frage nach dem ,Worein': In welchen Teil der Gesellschaft sollen sich MigrantInnen integrieren oder integriert werden? Die drei in dieser Arbeit zugrunde gelegten Integrationsmuster geben unterschiedliche Antworten: in die gesamte Bandbreite der Dominanzgesellschaft (Neoklassische Integration), in marginalisierte soziale Schichten mit wenig Möglichkeiten für Aufstieg und für nachhaltige, nicht-prekäre Lebensweise (Abwärts-Integration) oder in herkunftsgruppenbezogene Räume (Pluralismus). Diese drei Möglichkeiten sind unter normativen Gesichtspunkten verschieden zu bewerten: Während die neoklassische Integration aufgrund des breiten Spektrums der Ergebnisse, die der nicht-migrantischen Bevölkerung entsprechen, genauso wie diese zu bewerten ist und deswegen hier nicht weiter behandelt wird, hängt die Einschätzung für die pluralistische Theorie von der Freiwilligkeit eines Lebens in herkunftsbezogenen Strukturen ab. Pluralistische Lebensweisen sollten nicht romantisiert werden. Soziale Kontrolle und enge Familienbeziehungen haben ihre Negativa, manche Aspekte dieser pluralistischen Kulturen schränken einige ihrer Mitglieder in ihren Entfaltungsmöglichkeiten ein. Zudem dürfen pluralistische Integrationsoptionen nicht als Ersatz für redistributive Maßnahmen des Staates missverstanden werden. Für die Abwärts-Integration schließlich fällt die Bewertung negativ aus: Die Chancen auf Teilhabe und Entwicklung sind gering. Auf jeden

\footnotetext{
${ }^{177}$ Der GINI Koeffizient misst die Ungleichheit in der Verteilung der Markteinkommen und Renten. Er stieg in Westdeutschland von 1991 bis 2002 von 0,319 auf 0,368. Noch stärker fällt die Ungleichheit bei den Vermögen aus (bpb 2004: 626). Die Zahl der Kinder, die in armen Haushalten aufwachsen, hat sich laut Bericht des Deutschen Kinderhilfswerkes in den letzten zehn Jahren verdoppelt. Im Jahr 2007 wuchsen demnach 14\% der Kinder in Deutschland in Armut auf. (Kinderhilfswerk 2007)
} 
Fall muss damit insgesamt festgehalten werden, dass einseitige, auf kulturelle Faktoren verengte Integration sozioökonomisch betrachtet nicht immer wünschenswert ist.

Wie verortet sich der in dieser Arbeit entwickelte Integrationsansatz in den Migrationswissenschaften?

Schlechtere sozioökonomische Ergebnisse für MigrantInnen im Vergleich zur nichtmigrantischen Bevölkerung werden in der Migrationsforschung über vier verschiedene Faktoren allein oder in Kombination erklärt: (1) durch Probleme von durchschnittlich statusniedrigen Personen im Aufnahmeland, also durch die soziale Lage, (z.B. Kalter, et al. 2007), (2) durch kulturelle Differenzen (z.B. Heitmeyer, et al. 1997; Kelek 2002), (3) durch eine Entwertung von Ressourcen (Sozialkapital, Sprache) durch die Migration (z.B. Kalter, et al. 2007, Granovetter 1973) oder (4) durch rassistische Diskriminierung (Wacquant 2006; Terkessidis 2004). Die in der vorliegenden Arbeit entwickelten Integrationsmuster betonen die Bedeutung sozioökonomischer Faktoren zusammen mit der Strukturvariable Rassismus über kulturbasierten Erklärungen. Wo kulturelle Faktoren eine Rolle spielen (,Oppositionskultur') wird nicht auf ,ethnische Kulturen' zurückgegriffen, sondern auf schichtspezifische. Die Migrationserfahrung wird im Zusammenspiel der Faktoren zwar in meinen Mustern weiterhin als wichtig angesehen, ihr kommt in der zweiten Generation indes keine entscheidende Bedeutung mehr zu. Die hier entwickelten Integrationsmuster wenden sich damit auch gegen einen Integrationsdiskurs, der vermeintliche kulturelle Differenzen reifiziert und daraus auf individuelle Rückständigkeit und Unwilligkeit schließt. Die theoretische Ausrichtung beschränkt dabei die Faktoren, die überhaupt in den Blick genommen werden. Die stärkere Betonung sozioökonomischer Faktoren leistet einen Ausgleich zu üblichen kulturalistischen Erklärungsversuchen. Jede Erklärung dieser komplexen Phänomene muss jedoch multikausal angelegt sein.

Im folgenden Abschnitt werden deshalb die Limitierungen der vorliegenden Arbeit diskutiert, um den kritischen Blick für das hier verwendete Forschungsdesign zu schärfen.

\subsection{Begrenzungen der Untersuchung}

In dieser Arbeit wurden aus den US-amerikanischen Integrationsdebatten drei Integrationsmuster entwickelt und auf Deutschland übertragen, um in der deutschen Diskussion blinde Flecken erkennbar zu machen und der ideologischen Verkürzung auf eine Problemgruppe entgegenzutreten. In der Debatte in Deutschland wird nämlich der Prototyp der/s muslimischtürkischen MigrantIn aus sozial marginalisierten Schichten gesetzt, der zwar real existiert, a- 
ber in keinem realistischen Verhältnis zur Empirie steht und daher in seiner ideologischen Dimension verstanden werden muss. Die Übertragung auf den deutschen Kontext gelang in Teilen, es gerieten dadurch aber auch Spezifika des deutschen Kontextes aus dem Blick. So wäre es für eine Fortentwicklung der Theorien nötig, diese stärker an die Historie Deutschlands, an die Einwanderungsentwicklung und an damit zusammenhängende rechtlichen Faktoren anzupassen. Einer der wichtigsten Unterschiede zu den USA ist die spezifische deutsche nationale Identität. Das ist zum einen eine abwehrend ethno-national konnotierte Identität, die auf der mythischen Idee einer völkischen Nation basiert und ,Fremdes' als Bedrohung versteht. Wo Identität jedoch nicht als erarbeitete Leistung verstanden wird, sondern als Ergebnis von Ursprungsbindungen, fühlt sie sich durch Zuwanderung besonders bedroht (Fijalkowski 2001: 163).

Darüber hinaus ist die deutsche Identität aber auch eine mehrfach gebrochene und unsichere Identität. Viele Anknüpfungspunkte scheinen durch den Zweiten Weltkrieg und Holocaust sowie die ehemalige Teilung in Ost- und Westdeutschland zerstört, was zu Distanzierungen bis hin von der eigenen Sprache führt. ${ }^{178}$ Wenn also MigrantInnen der zweiten Generation bei der Fußballweltmeisterschaft für die jeweiligen Gegner der deutschen Nationalmannschaft Partei ergreifen (im Gegensatz zu ihren Eltern, die im Allgemeinen Fans der deutschen Nationalmannschaft sind) (Öner/Laschet 2007) dann hat dies nicht zuletzt mit dem Kampf um eine deutsche Identität zu tun. Dieser findet im Feld einer Ablehnung des Deutschseins auch bei vielen Deutschen ohne Migrationshintergrund statt, von denen ein nicht unerheblicher Teil ebenfalls die jeweils gegnerische Mannschaft unterstützt. Typisch deutsch zu sein, bedeutet aus dieser Sicht, nicht deutsch sein zu wollen.

MigrantInnen werden also sowohl mit diesem ambivalenten Selbstverhältnis der Deutschen als mit dem exkludierenden ethno-nationalen Verständnis konfrontiert. Es überrascht kaum, wenn manche diesen Ballast zu umgehen suchen. So stellte eine junge Migrantin der zweiten Generation im Gruppeninterview fest: „Ich möchte mich aber auch irgendwie nicht als Deutsche fühlen, weil ,typisch deutsch’ schreckt irgendwie ab“ (Schiffauer 2001: 244).

Für eine Adaption der drei amerikanischen Integrationstheorien auf die Verhältnisse in Deutschland ist es also wichtig, die (fehlende) Attraktion dieser deutschen Identität und die ihr immer noch innewohnenden primordialen Ausschließungen besser zu verstehen und zu untersuchen, welche inkludierenden oder exkludierenden Wirkungen Ersatzidentifikationen

\footnotetext{
${ }^{178}$ Jürgen Trabant spricht in einem Artikel in der Frankfurter Allgemeinen Zeitung vom 28.09.07 von einer „Sprachscham“ der Deutschen.
} 
über Wirtschaftswachstum und materiellen Wohlstand, regionale und lokale Identitäten (,Wir Bayern“, „Wir Berliner“, „Wir Neuköllner“) für MigrantInnen der zweiten Generation haben.

Gerade mit Blick auf Identitäten muss als Einschränkung einer quantitativen Untersuchung festgehalten werden, dass die Vorgabe von Kategorien bestimmte Realitäten schafft. Einige theorierelevante Variablen fehlen in den Datensätzen gänzlich (z.B. Angaben zum sozioökonomischen Status von FreundInnen, Familienbeziehungen, Wahrnehmung innerhalb der ,Regenbogen'-Gruppen), so dass hinsichtlich dieser Aspekte keine Analyse möglich ist. Darüber hinaus mangelt es an Daten für Mehrebenenanalysen, beispielsweise kombinierten Informationen zu individuellen schulischen Leistungen, Hintergrund der Eltern und schulischer Diskriminierung. Solche Mehrebenenanalysen verbinden Akteurshandeln mit Strukturen und erleichtern damit eine sozialkonstruktivistische Analyse. Besonders bedeutsam in einer quantitativen Untersuchung ist aber die Wirkungsmächtigkeit der Kategorien selbst in der Schaffung sozialer Realitäten. Statistik dient der „Bio-Macht“, die Subjekte an einer Norm misst und ausrichtet (Foucault 1977). Welche Forschung wird in der „Normalisierungsgesellschaft“ (Foucault 1977: 172) von den statistischen Kategorien ermöglicht, welche lässt sich nicht verfolgen? ${ }^{179}$ In der Arbeit habe ich an verschiedenen Stellen darauf aufmerksam gemacht, dass soziale Realität durch die statistischen Kategorien nur in bestimmten Weisen wahrgenommen werden konnte und dadurch ein Wahrnehmen und Denken innerhalb bestimmter Bahnen konstruiert wurde. Durch diese kritische Reflexion soll das Entstehen falscher neuer Eindeutigkeiten vermieden werden.

Abschließend möchte ich auf zwei spezifische Limitierungen dieser Arbeit hinweisen:

Zum einen habe ich die Frage einer islamistischen Extremisierung der zweiten Generation außen vor gelassen. Die Bearbeitung dieser Frage hätte die Untersuchung automatisch auf MigrantInnen muslimischer Herkunft beschränkt und damit die Tendenz, MigrantInnen türkischer Herkunft als Pars pro Toto für alle ZuwanderInnen in Deutschland heranzuziehen, weiter Vorschub geleistet. Auch wäre ich damit dem in meinen Augen unerfreulichen Trend gefolgt, Integrationsfragen vom Sicherheitsdiskurs überlagern zu lassen. Islamismus sollte nicht als Integrationsproblem diskutiert werden, sondern als globale politische Ideologie ernst genommen werden.

Zum anderen lassen sich zwar die vermeintlichen Verschlechterungen von erster und anderthalbter Generation zur zweiten Generation in den Analysen der PISA Daten größtenteils

\footnotetext{
${ }^{179}$ Daran schließen sich auch gesellschaftspolitische Fragen an: Wieso nimmt z.B. der Mikrozensus gerade Anfang der 2000er Jahre den Migrationshintergrund in den Blick? Welche politischen Ziele werden damit verfolgt?
} 
auf Zusammensetzungseffekte zurückführen und die Annahme des ,Niedergangs' damit zurückweisen (vgl. Kapitel 3.4.1), doch kann ich nicht ausschließen, dass die sozioökonomische Situation der jüngeren Geburtsjahrgänge schlechter ist als die in meiner relativ alten Altersgruppe (20 bis 45 Jahre im Mikrozensus und 18 bis 25 im DJI-Survey, letzterer zudem schon Mitte der 1990er Jahr erhoben). Diese jüngeren Geburtsjahrgänge sind in den 1990er Jahren nach der Wende aufgewachsen, wo sich die Situation auf dem Arbeitsmarkt speziell für niedrigqualifizierte MigrantInnen dramatisch verschlechtert hat und einige soziale Leistungen verringert worden sind (z.B. Dauer des Sprachförderunterricht), obwohl sich seit 1998 der politische Integrationsdiskurs durch die Reform des Staatsangehörigkeitsrechts und das neue Zuwanderungsgesetz geöffnet hat. Hinzu kommt die durch die Wiedervereinigung entstandene Welle deutschen Nationalismus, der in Zusammenhang mit dem politischen Diskurs zur Novellierung der Asylgesetzgebung und rassistischen Gewalttaten (z.B. Mölln, Solingen $^{180}$ ) v.a. MigrantInnen türkischer Herkunft tief verunsichert und ein Gefühl der Exklusion hervorgerufen hat (Çil 2007). ${ }^{181}$ Auch Kinder der SpätaussiedlerInnen aus Kasachstan und Sibirien (Wellen ab 1992), also die zweite Generation dieser Herkunftsgruppe, sind in meiner empirischen Untersuchung aufgrund der Altersbeschränkung noch nicht enthalten. Bisherige Studien zeigen für sie jedoch eine schwierige sozioökonomische Eingliederung mit geringen Bildungsabschlüssen (Oswald 2006: 213). Auch aufgrund der sich verstärkenden sozioökonomischen Disparitäten innerhalb der Dominanzbevölkerung hinsichtlich (Berufs-)Bildung, Einkommen und Vermögen müsste die Wirkung all dieser neueren Entwicklungen auf die jüngere Altersgruppe noch untersucht werden. Allerdings empfiehlt es sich, abzuwarten, bis diese auf dem Arbeitsmarkt angekommen sind, da ein Nachholen der Bildungsabschlüsse bei MigrantInnen stärker als in der Dominanzbevölkerung verbreitet ist (Baden-Württemberg 2008; Berlin 2003). Eine solche Untersuchung sollte zudem nicht nur ausländische Staatsangehörige betrachten, da schon in meiner Untersuchung beachtliche Verbesserungen durch den Einschluss deutscher StaatsbürgerInnen gezeigt wurden und diese für die jüngeren Altersgruppen, in denen der Anteil der deutschen StaatsbürgerInnen (z.T. ab Geburt) weiter steigt, noch stärker ausfallen werden.

\footnotetext{
${ }^{180}$ In schleswig-holsteinischen Stadt Mölln wurden am 23. November 1992 drei Migrantinnen türkischer Herkunft bei einem rassistisch motivierten Brandanschlag getötet. Ebenfalls durch einen Brandanschlag wurden in Solingen am 29. Mai 1993 fünf Migrantinnen türkischer Herkunft ermordet.

${ }^{181} \mathrm{Zu}$ den Ausmaßen und Ursachen des widererstarkenden völkischen Nationalismus vergleiche Funke 2002 und zur vergrößerten politischen Opportunität antisemitischer Vorurteile Rensmann 2004.
} 


\subsection{Trends im deutschen Integrationsdiskurs}

Welche Veränderungen im Integrationsdiskurs legen die beschriebenen Ergebnisse für die zweite Generation, v.a. die Besserstellung im Vergleich zu anderen Generationen und die große Vielfalt innerhalb der zweiten Generation, nahe?

Zum einen wird sich der Diskurs mittelfristig von Ausländer- oder Migrantenfragen zu einer Diskussion über Minderheiten wenden. Dazu trägt auch bei, dass mittlerweile mehr deutsche StaatsbürgerInnen mit Migrationshintergrund (8 Millionen) in Deutschland leben als ausländische StaatsbürgerInnen (7,3 Millionen) (Bildungsberichterstattung 2006: 140). Zudem werden sich neue Koalitionen bilden: Schon jetzt versucht beispielsweise der Zentralrat der Juden in Deutschland, mit der Türkischen Gemeinde in Deutschland in einzelnen Sachfragen eine gemeinsame Interessengruppe zu bilden. ${ }^{182}$ Mit der zweiten Generation und der Thematisierung von Minderheiten wird aber auch die Frage von Anerkennung an Bedeutung gewinnen. Eine einfache Akzeptanz von Minderheiten im Sinne von Toleranz wird für ein gleichberechtigtes Miteinander nicht ausreichen, vielmehr muss es um Respekt und Wertschätzung (auch für die Differenz) sowie um Teilhabegerechtigkeit gehen. ${ }^{183}$

Damit diese gesellschaftliche Anerkennung gegeben werden kann, muss der Integrationsdiskurs sich jedoch wegbewegen von der Funktion der Selbstvergewisserung der Dominanzgesellschaft über das Fremde und damit das Eigene hin zu einer Auseinandersetzung über gesellschaftlich erwünschte Ziele der Ressourcen- und Chancenverteilung. Noch hat der Integrationsdiskurs weniger mit MigrantInnen zu tun als mit deutscher Befindlichkeit, bei der die Dominanzbevölkerung erst über das als fremd Markierte das Eigene erkennt (Claessens 1991). Die Identitätsnöte der deutschen Dominanzbevölkerung in der Nach-Auschwitz-Zeit wurden oben schon thematisiert. In einer solchen Situation der Unsicherheit wird Anderssein schnell als Bedrohung aufgefasst, da es nicht auf einen gefestigten Boden trifft. ${ }^{184}$ Die Leitkulturdebatte hat dabei gezeigt, wie dünn der Konsens über die deutsche Identität ist: Über deutsche Sprachkenntnisse und ein Bekenntnis zum Grundgesetz - also die Habermas'schen Verfahrenswerte - hinaus konnte man sich in der Debatte auf keine weiteren Werte einigen. Dennoch wird von konservativer Seite immer noch gefordert, christliche Werte als Teil der

\footnotetext{
${ }^{182}$ Laut persönlichen Mitteilungen von Stephan Kramer, Generalsekretär des Zentralrats der Juden in Deutschland, am 10. März 2005 in Cambridge (USA), sowie von Kenan Kolat, Bundesvorsitzender der Türkischen Gemeinde in Deutschland, am 28. November 2007 in Berlin.

${ }^{183}$ In dieser Hinsicht sollten auch Islamisierungstendenzen als Konflikt um (globale) Anerkennung verstanden werden (Tietze 2006: 173)).

${ }^{184}$ Wobei bei dieser Funktion von Fremdheit offen bleibt, ob dies über ein generalisiertes Fremdes zu geschehen hat oder über multiple Formen verschiedener Fremdheiten, die aber auch teilweise mit Vertrautheiten zusammengehen. Oben beschriebenes ,Blurring', also der Bedeutungsverlust bestimmter Fremdheitsmarkierungen, wäre wahrscheinlicher, wenn es jeweils um einzelne Aspekte der Fremdheit ginge.
} 
Leitkultur zu verstehen (Lammert 2005). Die daraus folgende Exklusion betrifft vor allem jene MigrantInnen, die über ihre vermeintliche muslimische Glaubenszugehörigkeit als fremd markiert werden.

Gemäß meiner Prognose wird der Integrationsdiskurs also gleichberechtigter und weniger paternalistisch, dafür aber auch konfliktiver werden. Statt eines Sprechens über Minderheiten sollte mit ihnen gesprochen werden und dies nicht innerhalb einer Nische sondern im Raum der normalen politischen Auseinandersetzung.

\subsection{Politische Herausforderungen}

Aus meiner Arbeit und den prognostizierten Änderungen des Integrationsdiskurses ergeben sich drei Forderungen an die Politik:

Zum einen müssen politische Diskurse, die Integration als kulturelles Problem bestimmter Herkunftsgruppen erklären, zurückgedrängt werden. Eine solche Abkehr von kulturalistischen Erklärungen würde es erlauben, die Probleme der Abwärts-Integration - bei diesem Muster führt kulturelle Anpassung nicht zum Erwerb nachhaltiger sozioökonomischer Positionen - stärker im öffentlichen Bewusstsein zu verankern und den Blick auf allgemeine Fragen der Integration in sich diversifizierenden Gesellschaften mit zunehmender sozialer Ungleichheit zu richten. Bisher gängige kulturalistische Erklärungen erschweren es zudem, Integration als wechselseitigen Prozess wahrzunehmen, da Probleme einseitig auf vermeintliche kulturelle Defizite von MigrantInnen zurückgeführt werden. Eine größere Vorsicht mit solchen Erklärungen würde schließlich doppelte Maßstäbe vermeiden, bei denen dieselben Aktivitäten, wenn sie von Angehörigen der Dominanzbevölkerung ausgeführt werden als individualpsychologisches oder als Schichtenproblem aufgefasst werden, bei MigrantInnen jedoch als kulturelles. So stellt der nordrhein-westfälische Minister für Generationen, Familie, Frauen und Integration Laschet kritisch fest: „Wenn ein deutscher Mann seine Frau aus Eifersucht tötet, dann ist dies eine „Familientragödie“. Wenn das ein türkischer Mann macht, ist es ein Ehrenmord.“(Öner/Laschet 2007)

Aufbauend auf diese politische Zurückweisung kulturalistischer Erklärungen, sollte die Diskussion über Integration in generalisierte Kategorien gesellschaftlicher Selbstbeobachtung z.B. zu sozialer Ungleichheit und Chancengerechtigkeit eingebaut werden. Statt eine spezielle Integrationspolitik zu betreiben, muss diese Teil der Bildungs-, Sozial- und Wohnungspolitik werden, was auch ihre Akzeptanz bei der Dominanzbevölkerung erhöhen würde. Eine Politik für , unsere ausländischen Mitbürger und Mitbürgerinnen' wie sie noch zu Beginn der 1990er 
Jahre betrieben wurde (Brubaker 2003: 46), ist mittlerweile diskreditiert, aber auch eine separate Politik für ,Zuwanderer und Zuwanderinnen' birgt wie alle ,getrennt-aber-gleich' Politiken (,separate but equal') die Gefahr der ungleichen Chancenverteilung. Und selbst ein System der formalen Gleichheit kann Ungleichheit produzieren, wenn sich nämlich die Ausgangsbedingungen systematisch unterscheiden. Daher wird ein Mainstreaming von Integrationspolitik wichtig. Mainstreaming bedeutet, Entscheidungen auf ihre jeweiligen Wirkungen für unterschiedliche Gruppen zu untersuchen. Sind MigrantInnen und Nicht-MigrantInnen beispielsweise von der erhöhten Konkurrenz zwischen Schulen, die durch die Einführung standardisierter Tests entstanden ist, gleich betroffen oder benachteiligt das seitdem zu beobachtende ,Rosinenpicken' in Bezug auf SchülerInnen bestimmte Gruppen? Durch Mainstreaming werden auch indirekte Diskriminierungen offen gelegt, die einem meritokratischen System im Wege stehen. In einer globalisierten Welt mit ihren komplexen Anforderungen, müssen unterschiedliche Kompetenzen, z.B. pluralistisch integrierter MigrantInnen, in gleichem Maße wahrgenommen und gefördert werden, so dass alle Gruppen ihre Fähigkeiten in die Gesellschaft einbringen können.

Die Auswirkungen solcher und anderer Verwaltungsentscheidungen können allerdings nur überprüft werden, wenn entsprechende Daten vorliegen. Um diese zu erhalten, muss in Kernbereichen wie der Bildung, des Gesundheitssystems das Instrument des Ethnic Monitoring ${ }^{185}$ eingeführt werden. Beim Ethnic Monitoring werden Gruppenzugehörigkeiten in statistischen Erhebungen erfasst und als ein Kriterium analysiert, so dass eine eventuelle ungleiche Wirkung administrativer Maßnahmen für verschiedene Gruppen überprüft und ihr gegebenenfalls entgegen gesteuert werden kann. Hinter jedem Mainstreaming müssen jedoch bestimmte Werte als Zielvorgabe stehen. Daher wird eine politische Verständigung über gesellschaftlich angestrebte Entwicklungen nötig: Wie meritokratisch und ,color blind' soll die Gesellschaft sein? Wie viele reale Chancen soll sie benachteiligten Individuen, z.B. über , affirmative action', bieten? Und was soll sie über die Anerkennung von Differenz und Komplexitätstoleranz hinaus zusammenhalten?

Schließlich erfolgt die Diversifizierung von Gesellschaften (u.a. durch Migration) nicht ohne Kosten, z.B. einer Reduktion des interpersonalen Vertrauens, für die ein Ausgleich geschaffen werden muss. So hat die Forschung von Putnam in ,ethnisch ${ }^{186}$ diversen Orten in den USA gezeigt, dass bei Kontrolle des durchschnittlichen sozioökonomischen Status, der Größe der

${ }^{185}$ Für eine Darstellung des Instruments Ethnic Monitoring vergleiche FitzGerald/Sibbitt 1997 und Krizsan 2001.

${ }^{186}$ Putnam verwendet die Begriffe ,Ethnizität' und ,Rasse', wie in US-amerikanischer Forschung häufig der Fall, synonym. 
Orte etc., Menschen anderen Menschen in ,ethnisch' diversen Orten weniger vertrauen als an ,ethnisch' homogeneren Orten. Und zwar vertrauen sie nicht nur Angehörigen einer anderen ,Rasse' weniger, sie vertrauen auch Personen ihrer eigenen ,Rasse' weniger. (Putnam 2007) Ein solcher Vertrauensverlust hat Auswirkungen auf das Sozialkapital vor Ort, erhöht die Transaktionskosten und macht staatliches Eingreifen zur Lösung von Konflikten und zur Bereitstellung von Gütern nötiger. ${ }^{187}$ Fügt man zu diesen Ergebnissen den generellen Trend zur Reduktion von Sozialkapital in Gesellschaften mit größerer sozioökonomischer Disparität hinzu - und die deutsche Gesellschaft macht Schritte in diese Richtung - dann wird es zentral,

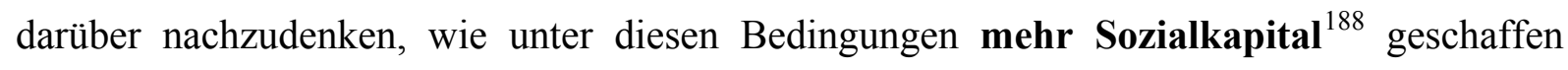
werden kann. Der Erhalt und die Stärkung des Sozialkapitals ist auch deswegen zentral, weil es dieses Zusammengehörigkeitsgefühls bedarf, um nachhaltig die Zustimmung zu Umverteilungsmaßnahmen zu sichern (Luttmer 2001; Costa/Kahn 2003). ${ }^{189}$ Umverteilung wird jedoch weiterhin, wenn nicht zunehmend nötig sein, um sozial Benachteiligte über, Empowerment' wirklich gleichberechtigt am gesellschaftlichen Leben teilhaben zu lassen.

Um dieser Verringerung von interpersonalem und institutionellem Vertrauen entgegen zu wirken, sollte Partizipation in allen ihren Facetten gestärkt werden. Vier Bereichen kommt dabei zentrale Bedeutung zu. Erstens ist dies der politische Bereich. Gesine Schwan hat in ihrem Buch Politik und Schuld untersucht, wie demokratische politische Identitäten entstehen und festgestellt, dass der politischen Teilhabe in pluralistischen Institutionen eine zentrale Rolle zukommt, um Vertrauen zu stärken (Schwan 1997). Politische Partizipation erfordert zumeist den Besitz der deutschen Staatsbürgerschaft. Eine größere Verbreitung der deutschen Staatsangehörigkeit unter MigrantInnen wird durch das Verbot einer doppelten Staatsangehörigkeit erschwert. Soll politische Partizipation also gestärkt werden, muss der Erwerb der doppelten Staatsbürgerschaft erleichtert werden. ${ }^{190}$

Zweitens ist der Bildungsbereich mit den öffentlichen Schulen, in denen Kinder verschiedener sozialer Schichten sich treffen und gemeinsam lernen, wichtig. Die Auseinandersetzung mit

\footnotetext{
${ }^{187}$ Kenneth Arrow hat aus diesem Grund Vertrauen als wichtiges Schmiermittel eines sozialen Systems bezeichnet (Arrow 1974: 23).

${ }^{188}$ Wichtig ist, dabei eine ausgewogene Mischung zwischen „Bonding“ Sozialkapital, d.h. Verbindungen zwischen sich hinsichtlich sozioökonomischen Status, ,ethnischer’ Herkunft, Religion und anderen Faktoren ähnlichen Menschen und „Bridging“ Sozialkapital, welches Verbindungen zwischen diesen Gruppen meint, zu erzielen (Warren, et al. 2002).

${ }^{189}$ Für eine Diskussion der institutionellen Faktoren, die die Wirkung von ethnischer Vielfalt auf den Wohlfahrtsstaat beeinflussen siehe (Seekings 2004 und Banting/Kymlicka 2004).

${ }^{190}$ Alternativ könnten auch Partizipationsrechte von der Staatsbürgerschaft entkoppelt und wohnortsabhängig gemacht werden. Die politische Zustimmung zu einer solchen Lösung fällt jedoch im Vergleich zur doppelten Staatsangehörigkeit deutlich geringer aus. Daher wird diese Option hier nicht weiter ausgeführt.
} 
bisher unbekannten Lebensweisen in einer Institution, die auf die Durchsetzung von Gleichberechtigung achtet, stärkt das interpersonale Vertrauen und erschwert es, bestimmte Gruppen als grundsätzliche Fremde zu konstruieren. Dies setzt jedoch voraus, dass Schulen interkulturelles Lernen fördern und ihre SchülerInnen zur kritischen Reflexion von gesellschaftlichen Machtverhältnissen anleiten.

Drittens ist ein attraktiver, für alle zugänglicher öffentlicher Raum bedeutsam. In ihm kommen auch dann verschiedene Gruppen zusammen, wenn diese in privaten und beruflichen $\mathrm{Zu}$ sammenhängen voneinander getrennt sind. Durch diesen Raum wird überhaupt erst die Möglichkeit vertrauensschaffender Interaktionen gelegt. Die zunehmende Kommodifizierung des öffentlichen Raumes mit einhergehender Reglementierung des Zugangs (z.B. Malls) muss also aufgehalten werden.

Und schließlich ist Teilhabe an einem relativ diskriminierungsfreien Arbeitsmarkt, auf dem menschenwürdige Bedingungen herrschen, bedeutsam. Als Konsequenz für die Arbeitsmarktpolitik bedeutet dies zum Beispiel, einen umfangreichen staatlichen Arbeitsmarkt mit Vollzeitbeschäftigung und existenzsichernder Entlohnung (also nicht 1 Euro Jobs) als dauerhafte Alternative zu privaten Arbeitsverhältnissen speziell für Niedrigqualifizierte zu etablieren.

Alle vier Bereiche sind zentral, um durch ein tätiges Erleben der eigenen Kompetenz und der Gestaltbarkeit sozialer und physischer Lebenszusammenhänge Vertrauen aufzubauen. Institutionen müssen dabei ein Umfeld befördern, in dem sich ein „Kernbestand von ethischen Motivationen und psychischen Dispositionen“ bei den BürgerInnen herausbildet, welches die „gegenseitige Anerkennung und Sicherung der gleichen Freiheit und Würde aller Menschen“ zum Ziel hat (Schwan/Schwelling 2006: 16).

Viele dieser Aspekte betreffen aber nicht ausschließlich MigrantInnen - und diese zudem nicht alle gleichermaßen wie ich in dieser Arbeit gezeigt habe - sondern auch Angehörige sozial marginalisierter Schichten. So sollte z.B. die Vorherrschaft kulturalistischer Erklärungen nicht nur hinsichtlich sozioökonomischer Benachteiligungen vieler MigrantInnen zurückgewiesen werden, sondern auch in der sich aktuell verbreitenden Variante der Unterschichtendebatte: Diese erklärt die realen Probleme von marginalisierten Bevölkerungsgruppen durch eine fehlende Sozialisation in basalen Kulturwerten und bewertet die Gruppen daher moralisch. Eine solche Strategie dient der Distinktion zwischen Privilegierten und Benachteiligten und etabliert eine vermeintlich grundsätzliche Andersartigkeit zwischen ihnen. In den USA hat sich die kulturelle und moralische Verbrämung von Armut seit der Regierungszeit von Präsident Reagan verfestigt und in weiten Teilen zu einer veränderten Wahrnehmung von 
Armut als Unwürdigkeit geführt (Piven/Cloward 1993; Piven/Cloward 1985). Der Diskurs in Deutschland unterscheidet sich aufgrund der historischen Unterstützung für die staatlichen Wohlfahrtssysteme noch immer von dem US-amerikanischen, tendiert aber, z.B. in der Diskussion um Leistungseliten und Unterschichtenfernsehen, in ähnliche Richtungen. Dabei verdeckt die ideologische Absicherung eine auch in Deutschland zunehmend reale Schließung zwischen sozialen Schichten (Hartmann 2002; Hartmann/Kopp 2001). Wo jedoch keine grundsätzliche Offenheit besteht und sich sowohl Privilegierung als auch Unterprivilegierung vererben, schwindet die Solidarität. Die in diesem Abschnitt erhobenen politischen Herausforderungen gehen also weit über das Verhältnis von MigrantInnen und Nicht-MigrantInnen hinaus.

\subsection{Zusammenfassung}

Die Stärke der vorliegenden Arbeit liegt darin, mit der statistisch nachweisbaren Besserstellung sowie dem Herausarbeiten der großen Vielfalt innerhalb und zwischen den Herkunftsgruppen und den theoretischen Integrationsmustern (neoklassische und pluralistische Integration, Abwärts-Integration) neue empirische Erkenntnisse zur zweiten Generation vorzulegen. Darüber hinaus wurden durch die Plausibilisierung der Integrationsmuster auch solche Wege von Integration aufgezeigt, bei denen kulturelle und sozioökonomische Faktoren nicht Hand in Hand gehen. Dadurch wird eine Abkehr von kulturalistischen und auf Homogenitätsannahmen basierenden Ansätzen sowie von Defizitansätzen nahe gelegt. Diese Ergebnisse konnten durch die quantitative Analyse größerer Datensets erlangt werden und daher war dieser quantitative Ansatz für den Zweck der Arbeit zielführend. Allerdings wurden in dieser Arbeit auch Fragen berührt, die nur qualitativ und mit Paneldaten angemessen zu untersuchen wären: Dies betrifft einige Mechanismen der Muster (Familienklima, transnationale Räume, Wahrnehmung in ,Rainbowgruppen') und die Frage nach der Entwicklung von Markierungen (dem ,Blurring' oder Ausbleiben desselben). Eine weitere interessante Frage für zukünftige Forschung betrifft lokale Auswirkungen von sich rasch entwickelnder Diversität mit einer Vielzahl von verschiedenen Gruppen (,Superdiversity’, vgl. Vertovec 2007).

Abgehängt, chancenlos, unwillig - so die Frage im Titel dieser Arbeit - ist die zweite Generation sicherlich nicht: Sie ist Teil der durchschnittlichen Gesellschaft, auch wenn es Gruppen gibt, die den Anschluss an gesellschaftliche Entwicklungen und die Möglichkeit, an diesen zu partizipieren, verlieren. Allerdings finden sich solche Gruppen auch bei der NichtMigrantenbevölkerung. Die Chancen auf Teilhabe fallen je nach Ressourcen der MigrantIn- 
nen unterschiedlich aus. Doch existieren Entwicklungspotenziale, die nicht zuletzt aufgrund des Rassismus der Dominanzbevölkerung ungenutzt bleiben. Auch hinsichtlich ihrer Bildungsmotivation unterscheiden sich MigrantInnen der zweiten Generation. Durchschnittlich ist diese hoch und der Wunsch nach gesellschaftlicher Anerkennung weit verbreitet. Eine ernsthafte Anerkennung aber wird nicht ohne den Abbau von Privilegien auf Seiten der Dominanzbevölkerung zu erzielen sein. Daraus rührt auch ein Teil der Unwilligkeit der Dominanzbevölkerung, in dieser neuen politischen Situation anzukommen und Ressourcen gerechter zu verteilen.

Indes ist ein einfaches Anpassen der MigrantInnen an die Dominanzbevölkerung, wie in dem Cartoon eingangs gefordert, nicht nur mit Hinblick auf die sich ausdifferenzierende Dominanzbevölkerung schwerlich möglich, sondern führt für bestimmte Gruppen zudem zu reduzierten Chancen. Und so wird es zentral zu überlegen, wohin die gesellschaftliche Entwicklung gehen soll. Wie es schon die Katze in Alice im Wunderland auf den Punkt bringt:

"Would you tell me, please, which way I ought to go from here?" [asked Alice] "That depends a good deal on where you want to get to," said the Cat. Alice in Wonderland, Kapitel VI 


\section{Literaturverzeichnis}

Abramson, Harold J. 1980: Assimilation and Pluralism, in: Thernstrom, Stephen (Hrsg.): Harvard Encyclopedia of American Ethnic Groups, Cambridge, 150-160.

Acs, Gregory/Philipps, Katherin Ross/McKenzie, Daniel 2000: Playing by the Rules, but Losing the Game. Americans in Low-Income Working Families, Washington D.C.

Adler, Emanuel 2001: Constructivism an International Relations, in: Carlsnaes, Walter/Risse, Thomas/Simmons, Beth A (Hrsg.): Handbook of International Relations, London, Thousand Oaks, New Delhi, 95-118.

Akbulut, Nazire 1993: Zur aktuellen Lage türkischer Studentinnen an der Freien Universität Berlin. Lebensbedingungen, Eigenbild, Vorurteile. Eine empirische Untersuchung im Auftrag der Kommission der Nachwuchswissenschaftlerinnen der FU Berlin, in: Interkulturell, Kulturen in Europa 1/2/3: 235-255.

Alba, Richard/Handl, Johann/Mueller, Walter 1994: Ethnische Ungleichheit im deutschen Bildungssystem, in: Koelner Zeitschrift fuer Soziologie und Sozialpsychologie 46: 2, 209-237.

Alba, Richard/Nee, Victor 1997: Rethinking Assimilation Theory for a New Era of Immigration, in: International Migration Review 31: 4, 826-874.

Alba, Richard/Nee, Victor 2003: Remaking the American Mainstream. Assimilation and Contemporary Immigration, Cambridge and London.

Albert, Mathias/Gensicke, Thomas/Hurrelmann, Klaus/Leven, Ingo/Linssen, Ruth/Picot, Sibylle/Quellenberg, Holger 2004: Jugend 2004, 14. Shell Studie. Zusammenfassung und Hauptergebnisse (http://www.shelljugendstudie.de/download/hauptergebnisse 2002.pdf), 21.08.05.

Alisch, Monika/Dangschat, Jens S. 1998: Armut und soziale Integration. Strategien sozialer Stadtentwicklung und lokaler Nachhaltigkeit, Opladen.

Allmendinger, Jutta/Aisenbrey, Silke 2002: Soziologische Bildungsforschung, in: Tippelt, Rudolf (Hrsg.): Handbuch für Bildungsforschung, Opladen, 41-60.

Altvater, Elmar/Mahnkopf, Birgit 2002: Grenzen der Globalisierung - Ökonomie, Ökologie und Politik in der Weltgesellschaft, Münster.

Andreß, Hans-Jürgen 1997: Armut in Deutschland: Prozesse sozialer Ausgrenzung und die Entstehung einer neuen "Underclass"? Vorläufige Antworten auf der Basis von Umfragedaten, in: Soziale Probleme 8: 1, 3-39.

Andreß, Hans-Jürgen/Hagenaargs, Jacques/Kühnel, Steffen 1997: Analyse von Tabellen und kategorialen Daten. Log-lineare Modelle, latente Klassenanalyse, logistische Regressionen und GSK-Ansatz, Berlin. 
Appadurai, Arjun 1996: Modernity at Large: Cultural Dimensions of Globalization, Minneapolis.

Archdeacon, Thomas J. 1992: Reflections on Immigration to Europe in Light of U.S. Immigration History, in: International Migration Review 26: 2, 525-548.

Arrow, Kenneth 1974: The Limits of Organization, New York.

Bade, Klaus J. 1983: Vom Auswanderungsland zum Einwanderungsland? Deutschland 1880 bis 1980, Berlin.

Bade, Klaus J. 2007: Leviten lesen: Migration und Integration in Deutschland, in: IMIS (Hrsg.): Klaus J. Bade, Leviten lesen: Migration und Integration in Deutschland. Abschiedsvorlesung 27.6.2007 mit Grußworten und ausgewähltem Schriftenverzeichnis (=IMIS Beiträge Heft 31), Osnabrück, 43-64.

Bade, Klaus J./Oltmer, Jochen (Hrsg.) 1999: Aussiedler: deutsche Einwanderer aus Osteuropa (IMIS/Schriften, Bd.8), 1999, Osnabrück.

Baden-Württemberg, Statistisches Landesamt 2008: Zweiter Bildungsweg: Gut 6200 Erwachsene besuchen derzeit eine Abendschule oder ein Kolleg (Pressemitteilung 117/2008, 11. April 2008, http://www.statistik.baden-

wuerttemberg.de/Pressemitt/2008117.asp, 22.04.08), Stuttgart.

Baltzell, Edward Digby 1964: The Protestant Establishment: Aristocracy \& Caste in America, New York.

Bandura, Albert 1986: Social Foundations of Thought and Action. A Cognitive Social Theory, Englewood Cliffs.

Banting, Keith/Kymlicka, Will 2004: Do Multiculturalist Policies Erode the Welfare State? (Paper for the Conference "Does Cultural Diversity Undermine Economic Solidarity", Harvard University, 2 May 2005), Cambridge.

Barry, Brian 2001: Culture and Equality. An Egalitarian Critique of Multiculturalism, Cambridge.

Basch, Linda/Glick Schiller, Nina/Blanc-Szanton, Cristina 1994: Nations Unbound: Transnational Projects, Postcolonial Predicaments, and Deterritorialized Nation States, Langhorne, PA.

Bauböck, Rainer 1995: The integration of immigrants (=Reprint of Council of Europe CDMG (94) 25 E), Wien.

Beauftragte der Bundesregierung für Migration, Flüchtlinge und Integration 2007: Bericht der Beauftragten der Bundesregierung für Migration, Flüchtlinge und Integration über die Lage der Ausländerinnen und Ausländer in Deutschland, Berlin.

Beck, Ulrich 1999: Die Zukunft der Arbeit oder die Politische Ökonomie der Unsicherheit, in: Berliner Journal für Soziologie 9: 4, 467-478. 
Beck, Ulrich/Beck-Gernsheim, Elisabeth 1994: Riskante Freiheiten. Gesellschaftliche Individualisierungsprozesse in der Moderne, Frankfurt.

Bell, Daniel 1976: The Coming of Post-Industrial Society. A Venture in Social Forecasting, New York.

Bell, Daniel 1994: The coming of post-industrial society, in: Grusky, David B.

(Hrsg.): Social stratification: Class, race, and gender in sociological perspective, Boulder, 686-697.

Benton, Gregor 2003: Chinese Transnationalism in Britain. A Longer History, in: Identities. Global Studies in Culture and Power 10: 3, 347-375.

Berger, Peter L./Luckmann, Thomas 1977: Die gesellschaftliche Konstruktion der Wirklichkeit, Frankfurt am Main.

Berlin, Statistisches Landesamt 2003: Zweiter Bildungsweg 2002: Mehr Teilnehmer in den Kursen des Zweiten Bildungsweges (http://www.statistikberlin.de/pms2000/sg08/2003/03-07-10a.html, 22.04.08), Berlin.

Best, Ulrich 1999: Kreuzberg und Kreuzberger. Identität und Lebenswelt unter dem Ghetto-Stigma. Eine diskursanalytische Interpretation (Diplomarbeit am Geographischen Institut der Freien Universität Berlin), Berlin.

Bialystok, Ellen 1994: In other words: the science and psychology of second-language acquisition, New York.

Bildungsberichterstattung, Autorengruppe 2008: Bildung in Deutschland 2008. Ein indikatorengestützter Bericht mit einer Analyse zu Übergängen im Anschluss an SekundarbereichI, Bielefeld.

Bildungsberichterstattung, Konsortium 2006: Bildung in Deutschland. Ein indikatorgestützter Bericht mit einer Analyse zu Bildung und Migration, Gütersloh.

Blossfeld, Hans Peter 1985: Bildungsexpansion und Berufschancen, Frankfurt.

Blümner, Bettina 2007: Prinzessinnenbad, Berlin.

$B M A S$ (Hrsg.) 1996: Situation der ausländischen Arbeitnehmer und ihrer Familienangehörigen in der Bundesrepublik Deutschland, 1996, Bonn.

$B M B F$ 2006: Berufsbildungsbericht 2006, Berlin.

Bonacich, Edna 1972: A Theory of Ethnic Antagonism. The Split Labor Market, in: American Sociological Review 37: 5, 547-559.

Bonacich, Edna 1987: 'Making it' in America. A Social Evaluation of the Ethnics of Immigrant Entrepreneurship, in: Sociological Perspectives 30: 446-466.

Bonacich, Edna/Modell, J 1980: The Economic Basis of Ethnic Solidarity: Small Business in the Japanese-American Community, Berkeley. 
Bös, Mathias 2005: Rasse und Ethnizität. Zur Problemgeschichte zweier Begriffe in der amerikanischen Soziologie, Wiesbaden.

Bourdieu, Pierre 1987: Die feinen Unterschiede. Kritik der gesellschaftlichen Urteilskraft, Frankfurt am Main.

bpb 2004: Datenreport 2004, Bonn.

Breton, Raymond 1964: Institutional Completeness of Ethnic Communities and the Personal Relations of Immigrants, in: The American Journal of Sociology 70: 2, 193 205.

Brubaker, Rogers 2003: The Return of Assimilation? Changing Perspectives on Immigration and its Sequels in France, Germany, and the United States, in: Joppke, Christian/Morawska, Ewa (Hrsg.): Toward Assimilation and Citizenship: Immigrants in Liberal Nation-States, Houndmills, Basingstoke, 39-58.

Bundesagentur 2005: Strukturmerkmale der arbeitslosen Ausländer/Aussiedler/Asylbewerber in der Region Berlin-Brandenburg. Ergebnisse der Bestände an Arbeitslosen (Bundesagentur für Arbeit), Berlin.

Bundesamt, Statistisches 2000: Datenreport 1999. Zahlen und Fakten über die Bundesrepublik Deutschland, Wiesbaden.

Bundesamt, Statistisches 2001: Bildung und Kultur (=Fachserie 11, Reihe 1, Allgemeinbildende Schulen), Wiesbaden.

Bundesamt, Statistisches 2008: Statistisches Jahrbuch 2008, Wiesbaden.

Bundesregierung 2004a: Gesetz zur Steuerung und Begrenzung der Zuwanderung und zur Regelung des Aufenthalts und der Integration von Unionsbürgern und Ausländern (Zuwanderungsgesetz), in: Bundesgesetzblatt 2004/I 41, 5. August 2004 1950-2011.

Bundesregierung 2004b: Migrationsreport 2004. Bericht des Sachverständigenrates für Zuwanderung und Integration im Auftrag der Bundesregierung in Zusammenarbeit mit dem europäischen forum für migrationsstudien (efms) an der Universität Bamberg (http://www.bmi.bund.de/cln_006/nn_122688/Internet/Content/Common/Anlagen/Th emen/Auslaender Fluechtlinge Asyl/DatenundFakten/Migrationsbericht 2004,tem plateId=raw,property=publicationFile.pdf/Migrationsbericht 2004, 18. 12. 2004).

Buntrock, Tanja 2006: Berliner Polizei soll internationaler werden (18.11.06), Berlin.

Castel, Robert 2000: Die Metamorphosen der sozialen Frage. Eine Chronik der Lohnarbeit, Konstanz.

Castro Varela, María do Mar 2007: Unzeitgemäße Utopien. Migrantinnen zwischen Selbsterfindung und gelehrter Hoffnung, Bielefeld. 
Cavalli-Wordel, Alessandra 1989: Schicksale italienischer Migrantenkinder. Eine Fallstudie zur Schul- und Familiensituation, Weinheim.

CBS 2006: CBS News Poll

(http://www.cbsnews.com/stories/2006/04/12/national/main1494697.shtml), 20.

05.07 .

Chiswick, Barry R. 1978: The Effect of Americanization on the Earnings of ForeignBorn Men, in: Journal of Political Economy 86: 897-921.

Chlosta, Christoph/Ostermann, Torsten 2005: Warum fragt man nach der Herkunft, wenn man die Sprache meint? Ein Plädoyer für eine Aufnahme sprachbezogener Fragen in demographische Untersuchungen, in: BMBF (Hrsg.): Migrationshintergrund von Kindern und Jugendlichen. Wege zur Weiterentwicklung der amtlichen Statistik (=Bildungsreform Band 14), Bonn, Berlin, 55-65.

Çil, Nevim 2007: Topographie des Außenseiters. Türkische Generationen und der deutsch-deutsche Wiedervereinigungsprozess, Berlin.

Claessens, Dieter 1991: Das Fremde, Fremdheit und Identität, in: Schäffter, Ortfried (Hrsg.): Das Fremde. Erfahrungsmöglichkeiten zwischen Faszination und Bedrohung, Opladen, 45-55.

Collins, Patricia Hill 1990: Black Feminist Thought: Knowledge, Consciousness, and Empowerment, Boston.

Commission, European 2000: Eurobarometer. Public opinion in the European Union. Report Number 53, Brussels.

Conzen, Kathleen Neils 1991: Mainstreams and Side Channels. The Localization of Immigrant Cultures, in: Journal of American Ethnic History 11: Fall, 5-20.

Coser, Lewis 1977: Masters of Sociological Thought. Ideas in Historical and Social Context, New York.

Costa, Dora L./Kahn, Matthew E. 2003: Civic Engagement and Community Heterogeneity: An Economist's Perspective, in: Perspectives on Politics 103-111.

Crul, Maurice/Vermeulen, Hans 2003: The Second Generation in Europe, in: International Migration Review 37: 4, 965-986.

Cummins, J. 1979a: Cognitive/academic language proficiency, linguistic interdependence, the optimum age question, and some other matters, in: Working Papers on Bilingualism 19: 121-129.

Cummins, J. 1979b: Linguistic interdependence and the educational development of bilingual children, in: Review of Educational Research 49: 222-251.

Deane, Claudia/Fears, Darryl 2006: Views of Islam (=Washington Post - ABC News Poll), in: Washington Post 9 März, A01. 
Denzin, Norman 1970: The Research Act. A Theoretical Introduction to Sociological Methods, Chicago.

DeSipio, Louis/de la Garza, Rodolfo O. 1998: Making Americans, Remaking Amercia. Immigration and Immigrant Policy, Boulder.

Deutsch, Karl W. 1957: Political Community and the North Atlantic Area. International Organization in the Light of Historical Experience, Princeton.

Dicke, Hugo 2004: Der Europäische Binnenmarkt, in: Weidenfeld, Werner (Hrsg.): Die Europäische Union. Politisches System und Politikbereiche, Gütersloh, 223-241.

Diefenbach, Heike 2005: Schulerfolg von ausländischen Kindern und Kindern mit Migrationshintergrund als Ergebnis individueller und institutioneller Faktoren, in: BMBF (Hrsg.): Migrationshintergrund von Kindern und Jugendlichen. Wege zur Weiterentwicklung der amtlichen Statistik (=Bildungsreform Band 14), Bonn, Berlin, 43-54.

Diehl, Claudia 1998: Die soziale und politische Partizipation von Zuwanderen in der Bundesrepublik Deutschland, Bonn.

Diehl, Claudia 2002: Die Partizipation von Migranten in Deutschland. Rückzug oder Mobilisierung?, Opladen.

Döbert, Marion/Hubertus, Peter 2000: Ihr Kreuz ist die Schrift. Analphabetismus und Alphabetisierung in Deutschland, Münster.

Drake, St. Clair/Cayton, Horace R. 1945: Black metropolis; a study of Negro life in a northern city, New York, Harcourt.

Eberhard, Verena/Krewerth, Andreas/Ulrich, Joachim Gerd 2005: "Man muss geradezu perfekt sein, um eine Ausbildungsstelle zu bekommen". Die Situation auf dem Lehrstellenmarkt aus Sicht der Lehrstellenbewerber, in: Berufsbildung in Wissenschaft und Praxis 3, 10-13.

Eberhard, Verena/Krewerth, Andreas/Ulrich, Joachim Gerd 2006: Mangelware Lehrstelle. Zur aktuellen Lage der Ausbildungsplatzbewerber in Deutschland, Bielefeld.

efms 2001: Final Report. Effectiveness of National Integration Strategies Towards Second Generation Migrant Youth in a Comparative European Perspective - EFFNATIS, Bamberg.

Ehmann, Christoph 2001: Bildungsfinanzierung und soziale Gerechtigkeit. Vom Kindergarten bis zur Weiterbildung, Bielefeld.

Elias, Norbert/Scotson, John L. 1993: Etablierte und Außenseiter, Frankfurt.

Engelmann, Bernt 2001: Du Deutsch? Geschichte der Auslaender in Deutschland, Goettingen. 
Ermish, John/Francesconi, Marco/Siedler, Thomas 2006: Intergenerational Mobility and Martial Sorting, in: The Economic Journal 116: 659-679.

Eryilmaz, Aytac/Jamin, Mathilde (Hrsg.) 1998: Fremde Heimat. Eine Geschichte der Einwanderung. Ruhrlandmuseum Essen, 1998, Essen.

Espiritu, Yen Le 2001: "We Don't Sleep around like White Girls Do": Family, Culture, and Gender in Filipina American Lives, in: Signs 26: 2, 415-440.

Esser, Hartmut 1979: Ansätze zur Erklärung der Integration von Migranten - Theoretische Grundlagen zur Erforschung des Verhaltens ausländischer Arbeitnehmer der Bundesrepublik Deutschland, in: Esser, Hartmut/Gaugler, Eduard/Neumann, KarlHeinz (Hrsg.): Arbeitsmigration und Integration. Sozialwissenschaftliche Grundlagen, Königstein, 1-166.

Esser, Hartmut 1980: Aspekte der Wanderungssoziologie. Assimilation und Integration von Wanderern, ethnischen Gruppen und Minderheiten., Darmstadt, Neuwied.

Esser, Hartmut 2001: Integration und ethnische Schichtung (Arbeitspapier - Mannheimer Zentrum für Europäische Sozialforschung, Nr. 40) (www.mzes.unimannheim.de/publications/wp/up-40.pdf, 05.08.2005), Mannheim.

Esser, Hartmut 2003: Does the New Immigration Require a New Theory of Intergenerational Integration (Arbeitspapiere - Mannheimer Zentrum für Europäische Sozialforschung 71), Mannheim.

Esser, Hartmut 2006: Migration, Sprache und Integration (=AKI Forschungsbilanz 4), Berlin.

EU 2004: Gleichstellung sowie Bekämpfung von Diskriminierungen in einer erweiterten Europäischen Union (Grünbuch http://europa.eu.int/comm/employment_social/fundamental_rights/pdf/pubst/grpap04 de.pdf), Brüssel.

Faist, Thomas 2000a: Grenzen überschreiten. Das Konzept Transstaatliche Räume und seine Anwendungen, in: Faist, Thomas (Hrsg.): Transstaatliche Räume: Politik, Wirtschaft und Kultur in und zwischen Deutschland und der Türkei, Bielefeld, 9-56.

Faist, Thomas 2000b: Jenseits von Nation und Postnation. Eine neue Perspektive für die Integrationsforschung, in: Faist, Thomas (Hrsg.): Transstaatliche Räume. Politik, Wirtschaft und Kultur in und zwischen Deutschland und der Türkei, Bielefeld, 339392.

Faist, Thomas 2000c: The volume and dynamics of international migration and transnational social spaces, Oxford.

Faist, Thomas 2004a: The Border-Crossing Expansion of Social Spaces: Concepts, Questions and Topics, in: Faist, Thomas/Oezveren, Eyuep (Hrsg.): Transnational Social Spaces. Agents, Networks and Institutions, Aldershot, 1-34. 
Faist, Thomas 2004b: Towards a Political Sociology of Transnationalization. The State of the Art in Migration Research, in: Archives Europeennes de Sociologie 45: 3, 331-366.

Falk, Svenja 1998: Dimensionen kurdischer Ethnizität und Politisierung - Eine Fallstudie ethnischer Gruppenbildung in der Bundesrepublik Deutschland, Baden-Baden.

Fanon, Frantz 1969: Peau noire, masques blancs, Paris.

Farley, Reynolds/Alba, Richard 2002: The New Second Generation in the United States, in: International Migration Review 36: 3, 669-701.

Fijalkowski, Jürgen 1997: Integrationspolitik im europäischen Vergleich, in: Angenendt, Steffen (Hrsg.): Migration und Flucht. Aufgaben und Strategien für Deutschland, Europa und die internationale Gemeinschaft, Bonn, 154-170.

Fijalkowski, Jürgen 2001: Die ambivalente Funktion der Selbstorganisation ethnischer Minderheiten. Das Beispiel Berlin, in: Gesemann, Frank (Hrsg.): Migration und Integration in Berlin. Wissenschafliche Analysen und politische Perspektiven, Opladen, 163-182.

FitzGerald, Marian/Sibbitt, Rae 1997: Ethnic Monitoring in Police Forces. A Beginning (=Home Office Research Study 173), London.

Foner, Nancy 1997: What's New about Transnationalism? New York's Immigrants Today and at the Turn of the Century, in: Diaspora 6: 3, 355-375.

Fong, Eric/Ooka, Emi 2002: The Social Consequences of Participating in the Ethnic Economy, in: International Migration Review 36: 1, 125-146.

Fordham, Signithia/Ogbu, John 1986: Black students' school success. Coping with the "burden of acting White", in: Urban Review 18: 176-206.

Foucault, Michel 1977: Sexualität und Wahrheit Bd. 1, Frankfurt am Main.

Friedberg, Rachel M. 2000: You Can't Take It with You? Immigration Assimilation and the Portability of Human Capital, in: Journal of Labor Economics 18: 221-251.

Friedmann, Milton 1962: Capitalism and Freedom, Chicago.

Friese, $P$ 1998: Besonderheiten in den Familienstrukturen ausländischer Migrantenfamilien, in: Main, Kommunale Ausländerinnen und Ausländervertretung (KAV) der Stadt Frankfurt am (Hrsg.): Sonderschulen - Schulen für Migrantenkinder?, Mönchengladbach, 183-197.

Frietsch, Rainer/Wirth, Heike 2001: Die Übertragung der Magnitude-Prestigeskala von Wegener auf die Klassifizierung der Berufe, in: ZUMA-Nachrichten 48: 139-163.

Funke, Hajo 2002: Paranoia und Politik. Rechtsextremismus in der Berliner Republik, Berlin. 
Fürstenau, Sara 2004: Mehrsprachigkeit als Kapital im transnationalen Raum, Münster, New York, München, Berlin.

Gans, Herbert 1992: Second Generation Decline. Scenarios for the Economic and Ethnic Futures of Post-1965 American Immigrants, in: Ethnic and Racial Studies 15: April, 173-192.

Gans, Herbert 1999: Toward a Reconciliation of "Assimilation" and "Pluralism". The Interplay of Acculturation and Ethnic Retention, in: Hirschman, Charles/Kasinitz, Philip/DeWind, Josh (Hrsg.): The Handbook of International Migration: The American Experience, New York, 161-171.

Ganzeboom, Harry H.G./De Graaf, Paul/Treiman, Donald J. 1992: A Standard International Socio-Economic Index of Occupational Status, in: Social Science Research 21: $1-56$.

Gerstle, Gary/Mollenkopf, John (Hrsg.) 2001: E Pluribus Unum? Contemporary and Historical Perspectives on Immigrant Political Incorporation, 2001, New York.

Gestring, Norbert/Janßen, Andrea/Polat, Ayca/Siebel, Walter 2004: Die zweite Generation türkischer Migranten, in: Einblicke 40: 8-11.

Glatzer, Wolfgang 2004: Integration und Partizipation junger Ausländer vor dem Hintergrund ethnischer und kultureller Identifikation. Ergebnisse des Integrationssurveys des BiB, Wiesbaden.

Glazer, Nathan/Moynihan, Daniel Patrick 1963: Beyond the Melting Pot. The Negroes, Puerto Ricans, Jews, Italians, and Irish of New York City, Cambridge.

Glick Schiller, Nina/Basch, Linda/Blanc-Szanton, Cristina (Hrsg.) 1992a: Towards a Transnational Perspective on Migration. Race, Class, Ethnicity, and Nationalism Reconsidered (=Annals of the New York Academy of Sciences, vol. 645), 1992a, New York.

Glick Schiller, Nina/Basch, Linda/Blanc-Szanton, Cristina 1992b: Transnationalism: A New Analytic Framework for Understanding Migration, in: Glick Schiller, Nina/Basch, Linda/Blanc-Szanton, Cristina (Hrsg.): Towards a transnational perspective on migration. Race, Class, Ethnicity, and Nationalism Reconsidered (=Annals of the New York Academy of Science, vol. 645), New York, 1-24.

Glick Schiller, Nina/Basch, Linda/Blanc-Szanton, Cristina 1997: From Immigrant to Transmigrant. Theorizing Transnational Migration, in: Pries, Ludger (Hrsg.): Transnationale Migration (Sonderband 12, Soziale Welt), Baden-Baden, 121-140.

Goebel, Jan/Habich, Roland/Krause, Peter 2004: Einkommen - Verteilung, Armut und Dynamik, in: Bundesamt, Statistisches (Hrsg.): Datenreport 2004, Bonn, 623638.

Gogolin, Ingrid 1994: Der monolinguale Habitus der multilingualen Schule, Münster, New York. 
Goldberg, Andreas/Mourinho, Dora/Kulke, Ursula 1995: Arbeitsmarkt Diskriminierung gegenüber ausländischen Arbeitnehmern, Geneva.

Goldring, Luin 1997: Power and Status in Transnational Social Spaces, in: Pries, Ludger (Hrsg.): Transnationale Migration (=Sonderband 12, Soziale Welt), BadenBaden, 179-195.

Gomolla, Mechthild/Radtke, Frank-Olaf 2002: Institutionelle Diskriminierung. Die Herstellung ethnischer Differenz in der Schule, Opladen.

Gordon, Milton 1964: Assimilation in American Life: The Role of Race, Religion, and National Origins, New York.

Gould, M. 1999: Race and theory: Culture, poverty, and adaptation to discrimination in Wilson and Ogbu, in: Sociological Theory 17: 171-200.

Granato, Nadia 2003: Ethnische Ungleichheit auf dem deutschen Arbeitsmarkt, Opladen.

Granato, Nadia/Kalter, Frank 2001: Die Persistenz ethnischer Ungleichheit auf dem deutschen Arbeitsmarkt: Diskriminierung oder Unterinvestition in Humankapital?, in: Kölner Zeitschrift für Soziologie und Sozialpsychologie 53: 497-520.

Granovetter, Mark S. 1973: The Strength of Weak Ties, in: American Journal of Sociology 78: 6, 1360-1380.

Guarnizo, Luis Eduardo 2001: On the Political Participation of Transnational Migrants: Old Practices and New Trends, in: Gerstle, Gary/Mollenkopf, John (Hrsg.): E Pluribus Unum? Contemporary and Historical Perspectives on Immigrant Political Incorporation, New York, 213-263.

Ha, Kien Nghi 1999: Ethnizität und Migration, Münster.

Ha, Kien Nghi 2005: Hype um Hybridität. Kultureller Differenzkonsum und postmoderne Verwertungstechniken im Spätkapitalismus, Bielefeld.

Haas, Peter 1992: Introduction: Epistemic Communities and International Policy Coordination, in: International Organization 46: 1, 1-35.

Hailbronner, Kay/Renner, Günther 2005: Staatsangehörigkeitsrecht, Kommentar, München.

Hammar, Tomas 1989: Comparing European and North American International Migration, in: International Migration Review 23: 3, 631-637.

Hampel, Martin 2006: Vage Aussichten auf einen Job. Das Berufsvorbereitungsjahr verstehen viele Jugendlichen nicht als Chance, sondern allein als verlorene Zeit, in: Frankfurter Rundschau 78: 1. April, 3.

Hartmann, Michael 2002: Der Mythos von den Leistungseliten. Spitzenkarrieren und soziale Herkunft in Wirtschaft, Politik, Justiz und Wissenschaft, Frankfurt. 
Hartmann, Michael/Kopp, Johannes 2001: Elitenselektion durch Bildung oder durch Herkunft? Promotion, soziale Herkunft und der Zugang zu Führungspositionen in der deutschen Wirtschaft, in: Kölner Zeitschrift für Soziologie und Sozialpsychologie 3, 436-466.

Haug, Sonja 2004: Soziale Integration durch soziale Einbettung in Familie, Verwandtschafts- und Freundesnetzwerke, in: Zeitschrift für Bevölkerungswissenschaft 29: 2, 163-192.

Häußermann, Hartmut 1998: Zuwanderung und die Zukunft der Stadt. Neue ethnischkulturelle Konflikte durch die Entstehung einer neuen sozialen "underclass"?, in: Heitmeyer, Wilhelm/Dollase, Rainer/Backes, Otto (Hrsg.): Die Krise der Städte. Analysen zu den Folgen desintegrativer Stadtentwicklung für das ethnisch-kulturelle Zusammenleben, Frankfurt am Main, 145-175.

Häußermann, Hartmut/Kapphan, Andreas 2002: Berlin: Von der geteilten zur gespaltenen Stadt? Sozialräumlicher Wandel seit 1990, Opladen.

Heine, Christopher/Spangenberg, Heike/Sommer, D. 2006: Studienberechtigte 2004 (=Unveröffentlichter HIS Projektbericht), Hannover.

Heitmeyer, Wilhelm 1998: Versagt die "Integrationsmaschine" Stadt? Zum Problem der ethnisch-kulturellen Segregation und ihrer Konfliktfolgen, in: Heitmeyer, Wilhelm/Dollase, Rainer/Backes, Otto (Hrsg.): Die Krise der Städte. Analysen zu den Folgen desintegrativer Stadtentwicklungen für das ethnisch-kulturelle Zusammenleben, Frankfurt, 443-467.

Heitmeyer, Wilhelm (Hrsg.) 2004: Deutsche Zustaende. Folge 3, 2004, Frankfurt am Main.

Heitmeyer, Wilhelm/Anhut, Reimund (Hrsg.) 2000: Bedrohte Stadtgesellschaften. Soziale Desintegrationsprozesse und ethnisch-kulturelle Konfliktkonstellationen, 2000, Weinheim, München.

Heitmeyer, Wilhelm/Collmann, Birgit/Conrads, Jutta/Matuschek, Ingo/Kraul, Dietmar/Kühnel, Wolfgang/Möller, Renate/Ulbrich-Hermann, Matthias 1995: Gewalt. Schattenseiten der Individualisierung bei Jugendlichen aus unterschiedlichen Milieus, Weinheim und München.

Heitmeyer, Wilhelm/Mueller, Joachim/Schröder, Helmut 1997: Verlockender Fundamentalismus. Tuerkische Jugendliche in Deutschland, Frankfurt.

Herrmann, Dietrich 1996: Be an American! Amerikanisierungsbewegung und Theorien zur Einwandererintegration, Frankfurt, New York.

Herwartz-Emden, Leonie/Westphal, Manuela 2002: Integration junger Aussiedler: Entwicklungsbedingungen und Akkulturationsprozesse, in: Oltmer, Jochen (Hrsg.): Migrationsforschung und Interkulturelle Studien (IMIS Schriften II), Osnabrück, 229259. 
Hillmann, Felicitas 2001: Struktur und Dynamik der Arbeitsmarktintegration der ausländischen Bevölkerung in Berlin, in: Gesemann, Frank (Hrsg.): Migration und Integration in Berlin. Wissenschaftliche Analysen und politische Perspektiven, Opladen, 185-208.

Hillmann, Felicitas 2006: Ethnische Ökonomien - Kontext, Ausprägungen und Potenziale, Wirtschaft in Neukölln: Auf dem Weg in die Vielfalt, Berlin, 11-19.

Hoffmann-Nowotny, Hans-Joachim 1973: Soziologie des Fremdarbeiterproblems. Eine theoretische und empirische Analyse am Beispiel der Schweiz, Stuttgart.

Holtappels, Heinz Günter/Klieme, Eckhard/Rauschenbach, Thomas/Stecher, Ludwig (Hrsg.) 2007: Ganztagsschule in Deutschland. Ergebnisse der Ausgangserhebung der "Studie zur Entwicklung von Ganztagsschulen" (StEG), 2007, Weinheim.

Holtbrügge, H 1975: Türkische Familien in der Bundesrepublik, Duisburg.

hooks, bell 1989: Talking Back: Thinking Feminist, Thinking Black, Boston.

Huntington, Samuel 2004: The Hispanic Challenge, in: Foreign Policy 141:

March/April, 30-46.

Ignatiev, Noel 1995: How the Irish Became White, New York.

Itzigsohn, Jose/Dore Cabral, Carlos/Hernandez Medina, Esther/Vazquez, Obed 1999: Mapping Dominican transnationalsm: narrow and broad transnational practices, in: Ethnic and Racial Studies 22: 316-339.

Joppke, Christian 1996: Multiculturalism and Immigration: A Comparison of the United States, Germany and Great Britain, in: Theory and Society 25: 4, 449-500.

Kahane, Anetta 2007: Anetta Kahane - Niklas Frank: Zivilcourage, in: chrismon 11, 31-36.

Kalter, Frank 2004: The Second Generation in the German Labor Market. What Is Wrong with Turks? (=Paper presented at "The Next Generation: Immigrant Youth and Families in Comparative Perspective, Harvard University, October 29-30, 2004), Cambridge.

Kalter, Frank/Granato, Nadia 2002: Demographic Change, Educational Expansion, and Structural Assimilation of Immigrants. The Case of Germany, in: European Sociological Review 18: 2, 199-216.

Kalter, Frank/Granato, Nadia/Kristen, Cornelia 2007: Disentangling recent trends of the second generation's structural assimilation in Germany, in: Scherer, Stefani/Pollak, Reinhard/Otte, Gunnar/Gangl, M. (Hrsg.): From origin to destination. Trends and mechanisms in social stratification research, Frankfurt, 214-245.

Kapphan, Andreas 2001: Migration und Stadtentwicklung. Die Entstehung ethnischer Konzentrationen und ihre Auswirkungen, in: Gesemann, Frank (Hrsg.): Migration und 
Integration in Berlin. Wissenschaftliche Analysen und politische Perspektiven, Opladen, 89-108.

Karakasoglu-Aydin, Yasemin 2000: Studentinnen türkischer Herkunft an deutschen Universitäten unter besonderer Berücksichtigung der Studierenden pädagogischer Fächer, in: Attia, Iman/Marburger, Helga (Hrsg.): Alltag und Lebenswelten von Migrantenjugendlichen, Frankfurt, 101-126.

Kasinitz, Philip/Mollenkopf, John/Waters, Mary C. (Hrsg.) 2004: Becoming New Yorkers. Ethnographies of the New Second Generation, 2004, New York.

Kazis, Richard/Miller, Marc S. (Hrsg.) 2001: Low-Wage Workers in the New Economy, 2001, Washington D.C.

Kelek, Necla 2002: Islam im Alltag. Islamische Religiösität und ihre Bedeutung in der Lebenswelt von Schülerinnen und Schülern türkischer Herkunft, Münster, New York.

Kelek, Necla 2005: Die fremde Braut. Ein Bericht aus dem Inneren des türkischen Lebens in Deutschland, Köln.

Keller, Carsten 1999: Armut in der Stadt. Zur Segregation benachteiligter Gruppen in Deutschland, Opladen, Wiesbaden.

Keohane, Robert/Nye, Joseph (Hrsg.) 1972: Transnational Relations and World Politics, 1972, Cambridge.

Kinderhilfswerk, Deutsches 2007: Kinderreport Deutschland. Daten, Fakten, Hintergründe, Seelze.

Klieme, Eckhard/Eichler, Wolfgang/Helmke, Andreas/Lehmann, Rainer H. 2006: Unterricht und Kompetenzerwerb in Deutsch und Englisch. Zentrale Befunde der Studie Deutsch Englisch Schülerleistung International (DESI), Frankfurt am Main.

Klinger 2003: Ungleichheit in den Verhältnissen von Klasse, Rasse und Geschlecht, in: Knapp, Gudrun-Axeli/Wetterer, Angelika (Hrsg.): Achsen der Differenz, Münster, 14-48.

Klink, Andreas/Wagner, Ulrich 1999: Discrimination Against Ethnic Minorities in Germany: Going Back to the Field, in: Journal of Applied Social Psychology 29: 2, 402-423.

Klinkhammer, Gritt 2000: Moderne Formen islamischer Lebensführung. Eine qualitative Untersuchung zur Religiosität sunnitisch geprägter Türkinnen der zweiten Generation in Deutschland, Marburg.

KMK 1996: Empfehlung "Interkulturelle Bildung und Erziehung" der KMK vom 25.10.1996 (http://www.kmk.org/doc/beschl/671-1_Interkulturelle\%20Bildung.pdf), 14.01.08.

KMK 2005: Sonderpädagogische Förderung in Schulen 1994 bis 2003, Bonn. 
Knapp, Gudrun-Axeli/Wetterer, Angelika (Hrsg.) 2003: Achsen der Differenz. Gesellschaftstheorie und feministische Kritik II, 2003, Münster.

Koch, A./Wasmer, M. 1997: Einstellungen der Deutschen gegenüber verschiedenen Zuwanderergruppen, in: Bundesamt, Statistisches (Hrsg.): Datenreport, Wiesbaden, 457-467.

Kontos, Maria 2005: Migrantenökonomie - Ausweg aus der Armut, in: Migration und Soziale Arbeit 27: 3/4, 211-218.

Koopmans, R./Statham, P. 1999: Challenging the Liberal Nation-State? Postnationalism, Multiculturalism, and the Collective Claims Making of Migrants and Ethnic Minorities in Britain and Germany, in: American Journal of Sociology 105: 3, 652-696.

Kraheck, Nicole 2004: Karrieren jenseits normaler Erwerbsarbeit. Lebenslagen, Lebensentwürfe und Bewältigungsstrategien von Jugendlichen und jungen Erwachsenen in Stadtteilen mit besonderem Erneuerungsbedarf, München.

Kristen, Cornelia 1999: Bildungsentscheidungen und Bildungsungleichheit - ein Ueberblick ueber den Forschungsstand (=Arbeitspapiere - Mannheimer Zentrum fuer Europaeische Sozialforschung, Nr. 5, 1999), Mannheim.

Kristen, Cornelia 2002: Hauptschule, Realschule oder Gymnasium?, in: Kölner Zeitschrift für Soziologie und Sozialpsychologie 54: 3, 534-552.

Kristen, Cornelia 2004: Ethnic school choice and school segregation (Ph.D. thesis, Mannheim), Mannheim.

Kristen, Cornelia/Granato, Nadia 2005: Bildungsinvestitionen in Migrantenfamilien, in: BMBF (Hrsg.): Migrationshintergrund von Kindern und Jugendlichen. Wege zur Weiterentwicklung der amtlichen Statistik, Bonn, Berlin, 25-42.

Krizsan, Andrea (Hrsg.) 2001: Ethnic Monitoring and Data Protection. The European Context, 2001, Budapest.

Krohne, Julia/Meier, Ulrich/Tillmann, Klaus-Jürgen 2004: Sitzenbleiben, Geschlecht und Migration - Klassenwiederholungen im Spiegel der PISA-Daten, in: Zeitschrift für Pädagogik 50: 3, 373-390.

Kronauer, Martin 2002: Exklusion. Die Gefährdung des Sozialen im hoch entwickelten Kapitalismus, Frankfurt am Main, New York.

Lajios, Konstantin (Hrsg.) 1991: Die zweite und dritte Ausländergeneration. Ihre Situation und Zukunft in der Bundesrepublik Deutschland., 1991, Opladen.

Lammert, Norbert 2005: Auch die EU braucht ein ideelles Fundament. Gastkommentar: Leitkultur, in: Die Welt 13.12.2005: Berlin.

Lamont, Michèle/Molnar, Virág 2002: The Study of Boundaries in the Social Sciences, in: Annual Review Sociology 28: 167-195. 
Lau, Jörg 2008: Wir wollen hier rein! Die Deutschtürken fühlen sich unerwünscht. Sie möchten dazugehören und fürchten doch den Identitätsverlust, in: DIE ZEIT 12: 13. März 2008, 6-8.

Lauria-Perricelli, Antonio 1992: Towards a Transnational Perspective on Migration: Closing Remarks, in: Glick Schiller, Nina/Basch, Linda/Blanc-Szanton, Cristina (Hrsg.): Towards a Transnational Perspective on Migration. Race, Class, Ethnicity, and Nationalism Reconsidered (=Annals of the New York Academy of Sciences Vol. 645), New York, 251-258.

Lehmann, R.H./Ivanov, S./Hunger, S./Gänsfuß, R. 2004: Untersuchung der Leistungen, Motivation und Einstellungen zu Beginn der beruflichen Ausbildung (ULME I), Hamburg.

Lenneberg, Eric H. 1967: Biological foundations of language, New York.

Levitt, Peggy 2001: The Transnational Villagers, Berkeley.

Levitt, Peggy/Waters, Mary C. (Hrsg.) 2002a: The Changing Face of Home. The Transnational Lives of the Second Generation, 2002a, New York.

Levitt, Peggy/Waters, Mary C. 2002b: Introduction, in: Levitt, Peggy/Waters, Mary C. (Hrsg.): The Changing Face of Home. The Transnational Lives of the Second Generation, New York, 1-30.

Lewis, Oscar 1965: La Vida: A Puerto Rican Family in the Culture of Poverty, New York.

Lewis, Oscar 1966: The Culture of Poverty, in: Scientific American 215: 19-24.

Lewis, Oscar 1998: The Culture of Poverty, in: Moynihan, Daniel Patrick (Hrsg.): On Understanding Poverty: Perspectives from the Social Sciences, New York, 178-200.

Light, Ivan 1972: Ethnic Enterprise in America. Business and Welfare among Chinese, Japanese, and Blacks, Berkeley.

Light, Ivan/Sabagh, G./Bozorgnehr, M./Der-Martirosian, C. 1994: Beyond the Ethnic Enclave Economy, in: Social Problems 41: 65-80.

Linsenmann, Ingo 2004: Die Bildungspolitik der Europäischen Union, in: Weidenfeld, Werner (Hrsg.): Die Europäische Union. Politisches System und Politikbereiche, Gütersloh, 332-341.

Lübcke, Claudia 2007: Jugendkulturen junger Muslime in Deutschland, in: von Wensierski, Hans-Jürgen/Lübcke, Claudia (Hrsg.): Junge Muslime in Deutschland. Lebenslagen, Aufwachsprozesse und Jugendkulturen, Opladen, 285-318.

Lundy, Garvey 2003: The Myths of Oppositional Culture, in: Journal of Black Studies 33: $4,450-467$. 
Lüttinger, Paul 1989: Integration der Vertriebenen. Eine empirische Analyse, Frankfurt, New York.

Luttmer, Erzo 2001: Group Loyalty and the Taste for Redistribution, in: Journal of Political Economy 109: 3, 500-528.

Mahnig, Hans 2001: "Ist Deutschland wirklich anders?" Die deutsche Integrationspolitik im europäischen Vergleich, in: Currle, Edda/Wunderlich, Tanja (Hrsg.): Deutschland - ein Einwanderungsland? Rückblick, Bilanz und neue Fragen, Stuttgart, 159187.

Mammey, Ulrich/Sattig, Jörg 2002: Zur Integration türkischer und italienischer junger Erwachsener in die Gesellschaft der Bundesrepublik Deutschland. Der Integrationssurvey des BiB. Projekt- und Materialdokumentation (=Materialien zur Bevölkerungswissenschaft, Heft 105a), Wiesbaden.

Mannheim, Karl 1964: Das Problem der Generation, in: Wolff, Kurt H. (Hrsg.): Wissenssoziologie. Auswahl aus dem Werk, Neuwied, Berlin, 509-565.

Mannitz, Sabine 2002a: Auffassungen von kultureller Differenz: Identitätsmanagement und diskursive Assimilation, in: Schiffauer, Werner (Hrsg.): Staat - Schule Ethnizität. Politische Sozialisation von Immigrantenkindern in vier europäischen Ländern, Münster, New York, 255-320.

Mannitz, Sabine 2002b: Disziplinarische Ordnungskonzepte und zivile Umgangsformen in Berlin und Paris, in: Schiffauer, Werner/Baumann, Gerd/Kastoryano, Riva/Vertovec, Steven (Hrsg.): Staat-Schule-Ethnizität. Politische Sozialisation von Immigrantenkindern in vier europäischen Ländern., Münster, New York, München, Berlin, 161-219.

Mannitz, Sabine 2002c: Einschränkungen, Konvergenz und Cross-Over, in: Schiffauer, Werner/Baumann, Gerd/Kastoryano, Riva/Vertovec, Steven (Hrsg.): Staat-SchuleEthnizität. Politische Sozialisation von Immigrantenkindern in vier europäischen Ländern., Münster, New York, München, Berlin, 323-357.

Martiniello, Marco 2005: Political Participation, Mobilization and Representation of Immigrants and Their Offspring in Europe (= Konferenzbeitrag für "Immigrant Political Incorporation", 22-23 April 2005, Harvard University), Cambridge.

Mattes, Monika 2005: "Gastarbeiterinnen" in der Bundesrepublik. Anwerbepolitik, Migration und Geschlecht in den 50er bis 70er Jahren, Frankfurt am Main, New York.

McGuire, Therese/Papke, Leslie E. 2008: Local Funding of Schools. The property tax and its alternatives, in: Ladd, Helen F./Fiske, Edward B. (Hrsg.): Handbook of Research in Education Finance and Policy, New York, 357-372.

McPherson, Miller/Smith-Lovin, Lynn/Cook, James M. 2001: Birds of a Feather: Homophily in Social Networks, in: Annual Review Sociology 27: 415-444.

Mecheril, Paul 2001: Psychologische Erklärungsansätze zum Rassismus. Einige Problematisierungen, in: IDA-NRW (Hrsg.): Überblick 4/2001, NRW, 6-9. 
Mengering, Fred 2005: Bärenstark - Empirische Ergebnisse der Berliner Sprachstandserhebung an Kindern im Vorschulalter, in: Zeitschrift für Erziehungswissenschaft 8: 2, 241-262.

Menschenrechtskommission, UN 1996: Bericht des UN Sonderberichtserstatters ueber gegenwaertige Formen des Rassismus, der Rassendiskriminierung, Fremdenfeindlichkeit und verwandter Intoleranz, Maurice Glele-Ahanhanzo, ueber seine Reise vom 18.-27.09.1995 nach Deutschland (E/CN.4/1996/72/Add2) (http://www.unimarburg.de/dir/MATERIAL/DOKU/international/ahanhanzo.html), 01.05.05.

Merz, Friedrich 2000: Die Entstehung von Parallelgesellschaften ist nicht zu dulden (Die Welt 25.10.2005), Berlin.

Mittag, Hartmut/Weidacher, Alois 2000: Methodische Aspekte der Untersuchung: Stichproben, Erhebungsinstrumente, Analyseverfahren, in: Weidacher, Alois (Hrsg.): In Deutschland zu Hause. Politische Orientierungen griechischer, italienischer, türkischer und deutscher junger Erwachsener im Vergleich. DJI-Ausländersurvey, Opladen, 273-281.

Morawska, Ewa 2003: Immigrant Transnationalism and Assimilation: A Variety of Combinations and the Analytic Strategy it Suggests, in: Joppke, Christian/Morawska, Ewa (Hrsg.): Toward Assimilation and Citizenship: Immigrants in Liberal NationStates, Houndmills, Basingstoke, 133-176.

Münchmeier, Richard 2000: Miteinander - Nebeneinander - Gegeneinander? Zum Verhältnis zwischen deutschen und ausländischen Jugendlichen, in: Shell, Deutsche (Hrsg.): Jugend 2000, Opladen, 221-260.

Nauck, B. 2001: Social Capital, Intergenerational Transmission and Intercultural Contact in Immigrant Families, in: Journal of Comparative Family Studies 32: 4, 465-488.

Nauck, Bernhard/Steinbach, Anja 2001: Intergeneratives Verhalten und Selbstethnisierung von Zuwanderern. Expertise für die Unabhängige Kommission "Zuwanderung", Chemnitz.

Neumann, U. 1980: Erziehung ausländischer Kinder, Düsseldorf.

Newman, Katherine 1999: No Shame in My Game: The Working Poor in the Inner City, New York.

Niedersachsen, Polizei 2008: Sie interessieren sich für den Polizeiberuf und haben einen Migrationshintergrund? (http://www.polizei-

$\underline{\text { studi- }}$

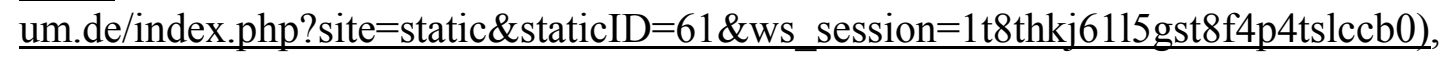
Hannover.

Nohl, Arnd-Michael 2001: Migration und Differenzerfahrung. Junge Einheimische und Migranten im rekursiven Milieuvergleich, Opladen. 
Norusis, Marija J. 2003: SPSS 12.0 Statistical Procedures Companion, Upper Saddle River.

Oberwittler, Dietrich 2003: Stadtstruktur, Freundeskreis und Delinquenz: Eine Mehrebenenanalyse zu sozialökologischen Kontexteffekten auf schwere Jugenddelinquenz (= Sonderheft), in: Kölner Zeitschrift für Soziologie und Sozialpsychologie 43: 135170 .

Oberwittler, Dietrich 2004: A Multilevel Analysis of Neighbourhood Contextual Effects on Serious Juvenile Offending. The Role of Subcultural Values and Social Disorganization, in: European Journal of Criminology 1: 2, 201-235.

Oberwittler, Dietrich 2007: The Effects of Ethnic and Social Segregation on Children and Adolescents: Recent Research and Results from a German Multilevel Study, Berlin.

OECD 1999: Classifying Educational Programmes. Manual for ISCED-97 Implementation in OECD Countries, Paris.

OECD 2003: Urban Renaissance Studien: Berlin. Wege zu einer integrativen Strategie für sozialen Zusammenhalt und wirtschaftliche Entwicklung, Paris.

Oenning, Ralf-Karl 1990: "Du da mitti polnischen Farben". Sozialisationserfahrungen von Polen im Ruhrgebiet 1918-1939, Muenster, New York.

Ofner, Ulrike Selma 2003: Akademikerinnen türkischer Herkunft. Narrative Interviews mit Töchtern aus zugewanderten Familien, Berlin.

Ogbu, John 1991: Low performance as adaptation: The case of Blacks in Stockton, California, in: Gibson, M.A. /Ogbu, John (Hrsg.): Minority status and schooling, New York, 249-285.

Ohliger, Rainer/Motte, Jan/von Oswald, Anne 1999: 50 Jahre Bundesrepublik - 50 Jahre Einwanderung, in: Ohliger, Rainer/Motte, Jan/von Oswald, Anne (Hrsg.): 50 Jahre Bundesrepublik - 50 Jahre Einwanderung: Nachkriegsgeschichte als Migrationsgeschichte, Frankfurt, 15-30.

Öner, Ihsan/Laschet, Armin 2007: Das Megathema des nächsten Jahrzehnts, in: Süddeutsche Zeitung 12.06.07, 6-7.

Osterman, Paul 1980: Getting Started: The Youth Labor Market, Cambridge.

Oswald, Ingrid 2006: Neue Migrationsmuster. Flucht aus oder in "Überflüssigkeit"?, in: Bude, Heinz/Willisch, Andreas (Hrsg.): Das Problem der Exklusion. Ausgegrenzte, Entbehrliche, Überflüssige, Hamburg, 200-224.

Özcan, Veysel/Seifert, Wolfgang o.J.: Selbständigkeit von Ausländern in Deutschland - Zeichen der Integration oder Marginalisierung (www.networkmigration.org/miginfo/dokumente und materialien/deutschland/docs/oezcan_selbstaendigke it.pdf), Berlin. 
Özdemir, Cem 1999: Currywurst und Döner. Integration in Deutschland, Bergisch Gladbach.

Park, Robert Ezra 1930: Assimilation, Social, in: Seligman, Edwin/Johnson, Alvin (Hrsg.): Encyclopedia of the Social Sciences, New York, 281-283.

Park, Robert Ezra 1937: Introduction, in: Adams, R.C. (Hrsg.): Interracial Marriage in Hawaii. A Study of the Mutually Conditioned Processes of Acculturation and Amalgamation, New York, vii-xiv.

Park, Robert Ezra 1950: Race and Culture, Glencoe, Ill.

Park, Robert Ezra/Burgess, Ernest W. 1921 (2nd ed. 1924): Introduction to the Science of Sociology, Chicago.

Pecoud, Antoine 2004: Entrepreneurship and Identity: Cosmopolitanism and Cultural Competencies Among German-Turkish Businesspeople in Berlin, in: Journal of Ethnic and Migration Studies 30: 1, 3-20.

Pilz, Frank 2004: Der Sozialstaat. Ausbau - Kontroversen - Umbau, Bonn.

PISA-Konsortium 2004: PISA 2003. Der Bildungsstand der Jugendlichen in Deutschland - Ergebnisse des zweiten internationalen Vergleichs, Münster, New York, München, Berlin.

Piven, Frances Fox/Cloward, Richard A. 1985: New Class War. Reagan's attack on the welfare state and its consequences, New York.

Piven, Frances Fox/Cloward, Richard A. 1993: Regulating the Poor. The Functions of Public Welfare, New York.

Plessner, Helmuth 1959: Die verspätete Nation. Über die politische Verführbarkeit bürgerlichen Geistes, Frankfurt.

Portes, Alejandro (Hrsg.) 1996: The new second generation, 1996, New York.

Portes, Alejandro 1999: Conclusion: Towards a new world- the origins and effects of transnational activities, in: Ethnic and Racial Studies 22: 2, 463-477.

Portes, Alejandro/Bach, $R$ L 1985: The Latin journey: Cuban and Mexican Immigrants in the United States, Berkeley.

Portes, Alejandro/Guarnizo, Luis Eduardo/Landolt, Patricia 1999: The study of transnationalism: pitfalls and promise of an emergent research field, in: Ethnic and Racial Studies 22: 2, 217-237.

Portes, Alejandro/Rumbaut, Ruben G. 1996: Immigrant America. A Portrait, Berkeley, Los Angeles, London. 
Portes, Alejandro/Rumbaut, Ruben G. 2001: Legacies: The story of the immigrant second generation, Berkeley.

Portes, Alejandro/Zhou, M. 1993: The New Second Generation: Segmented Assimilation and Its Variants, in: Annals of the American Academy of Political and Social Scienes 530 (November): 74-96.

Pott, Andreas 2002: Räumliche Differenzierung und der Bildungsaufstieg in der zweiten Migrantengeneration, in: Löw, Martina (Hrsg.): Differenzierungen des Städtischen, Opladen, 87-105.

Pries, Ludger 1997: Neue Migration im transnationalen Raum, in: Pries, Ludger (Hrsg.): Transnationale Migration (Sonderband 12, Soziale Welt), Baden-Baden,

Putnam, Robert B. 2007: E Pluribus Unum: Diversity and Community in the Twentyfirst Century. The 2006 Johan Skytte Prize Lecture, in: Scandinavian Political Studies 30: 2, 137-174.

Pütz, Robert 2005: Marginalisierte Unternehmer: Armut als Bestandteil der Migrantenökonomie, in: Migration und Soziale Arbeit 27: 3/4, 202-210.

Radtke, Frank-Olaf 2004: Die Illusion der meritokratischen Schule. Lokale Konstellationen der Produktion von Ungleichheit im Erziehungssystem, in: Bade, Klaus J./Bommes, Michael (Hrsg.): Migration - Integration - Bildung. Grundfragen und Problembereiche (=IMIS-Beiträge 23), Osnabrück, 143-178.

Raiser, Ulrich 2007: Erfolgreiche Migranten im deutschen Bildungssystem - es gibt sie doch Lebensläufe von Bildungsaufsteigern türkischer und griechischer Herkunft, Münster.

Ramachers, G. 1996: Konflikte und Konfliktbewältigung in intra- und interkulturellen Freundschaften, Frankfurt.

Reich, Hans H./Roth, Hans-Joachim 2002: Spracherwerb zweisprachig aufwachsender Kinder und Jugendlicher. Ein Überblick über den Stand der nationalen und internationalen Forschung, Hamburg.

Rensmann, Lars 2004: Demokratie und Judenbild. Antisemitismus in der politischen Kultur der Bundesrepublik Deutschland, Wiesbaden.

Risse, Thomas 2002: Transnational Actors and World Politics, in: Carlsnaes, Walter/Risse, Thomas/Simmons, Beth A. (Hrsg.): Handbook of International Relations, London, Thousand Oaks, New Delhi, 255-274.

Rolff, Hans-Günter 1997: Sozialisation und Auslese durch die Schule (= überarbeitete Neuausgabe), Weinheim, München.

Rommelspacher, Birgit (Hrsg.) 1995: Dominanzkultur. Texte zu Fremdheit und Macht, 1995, Berlin. 
Rosen, $B C$ 1959: Race, ethnicity, and the achievement syndrome, in: American Sociological Review 24: 47-60.

Rumbaut, Ruben G. 2001: Assimilation of Immigrants, in: Smelser, Neil J./Baltes, Paul B. (Hrsg.): International Encyclopedia of the Social and Behavioral Sciences, 2, Amsterdam, Paris, New York, Oxford, Shannon, Singapore, Tokyo, 845-849.

Salentin, Kurt/Wilkening, Frank 2003: Ausländer, Eingebürgerte und das Problem einer realistischen Zuwanderer-Integrationsbilanz, in: Kölner Zeitschrift für Soziologie und Sozialpsychologie 55: 2, 278-298.

Sanders, Jimy M. 2002: Ethnic Boundaries and Identity in Plural Societies, in: Annual Review Sociology 28: 327-357.

Schäfer, Claus 2003: Effektiv gezahlte Niedriglöhne in Deutschland, in: WSI Mitteilungen 7: 420-428.

Schaich, Eberhard 1990: Schätz- und Testmethoden für Sozialwissenschaftler, München.

Schiffauer, Werner 2001: Staat - Schule - Ethnizität, in: Gesemann, Frank (Hrsg.): Migration und Integration in Berlin. Wissenschaftliche Analysen und politische Perspektiven, Opladen, 233-250.

Schiffauer, Werner 2002: Einleitung: Nationalstaat, Schule und politische Sozialisation, in: Schiffauer, Werner/Baumann, Gerd/Kastoryano, Riva/Vertovec, Steven (Hrsg.): Staat-Schule-Ethnizität, Münster, New York, München, Berlin, 1-19.

Schimpl-Neimanns, Bernd 2003: Mikrodaten-Tools: Umsetzung der Berufsklassifikation von Blossfeld auf die Mikrozensen von 1973-1998 (ZUMA-Methodenbericht 2003/10), Mannheim.

Schmitter Heisler, Barbara 1992: The Future of Immigrant Incorporation: Which Models? Which Concepts?, in: International Migration Review 26: 2, 623-645.

Schneewind, Julia/Merkens, Hans 2001: Inklusion und Exklusion von Mitgliedern der türkischen Minorität in Schulklassen, Opladen, 251-270.

Schneider, Eric C. 1999: Vampires, Dragons, and Egyptian Kings. Youth Gangs in Postwar New York, Princeton.

Schroedter, Julia H./Lechert, Yvonne/Lüttinger, Paul 2006: Die Umsetzung der Bildungsskala ISCED-1997 für die Volkszählung 1970, die MikrozensusZusatzerhebung 1971 und die Mikrozensen 1976-2004 (=ZUMA Methodenbericht 2006/08), Mannheim.

Schuemer, G./Tillmann, K.-J./Weiss, M. (Hrsg.) 2004: Die Institution Schule und die Lebenswelt der Schueler - vertiefende Analysen der PISA 2000 Daten zum Kontext von Schuelerleistungen, 2004, Wiesbaden.

Schütz, Alfred 1932: Der sinnhafte Aufbau der sozialen Welt, Wien. 
Schütz, Alfred 1984: Strukturen der Lebenswelt, Frankfurt.

Schwan, Gesine 1997: Politik und Schuld, Frankfurt.

Schwan, Gesine/Schwelling, Birgit 2006: Einleitung, in: Schwan, Gesine/Holzer, Gerzy/Lavabre, Marie-Claire/Schwelling, Birgit (Hrsg.): Demokratische politische Identität: Deutschland, Polen und Frankreich, Wiesbaden, 9-34.

Schwippert, Knut/Bos, Wilfried/Lankes, Eva-Maria 2003: Heterogenität und Chancengleichheit am Ende der vierten Jahrgangsstufe im internationalen Vergleich, in: Bos, Wilfried/Lankes, Eva-Maria/Prenzel, Manfred/Schwippert, Knut/Walther, Gerd/Valtin, Renate (Hrsg.): Erste Ergebnisse aus IGLU. Schülerleistungen am Ende der vierten Jahrgangsstufe im internationalen Vergleich, Münster, 265-302.

Schwippert, Knut/Bos, Wilfried/Lankes, Eva-Maria 2004: Heterogenität und Chancengleichheit am Ende der vierten Jahrgangsstufe in den Ländern der Bundesrepublik Deutschland und im internationalen Vergleich, in: Bos, Wilfried/Lankes, EvaMaria/Prenzel, Manfred/Schwippert, Knut/Valtin, Renate/Walther, Gerd (Hrsg.): IGLU. Einige Länder Bundesrepublik Deutschland im nationalen und internationalen Vergleich, Münster, 165-190.

Seekings, Jeremy 2004: Institutional Design, Cultural Diversity and Economic Solidarity: A Comparison of South Africa, Brazil and Nigeria, in: van Parijs, Philippe (Hrsg.): Cultural Diversity versus Economic Solidarity, Brussels, 105-142.

Sen, Amartya 2000: Development as Freedom, New York.

Sen, Faruk/Sauer, Martina 2005: Türkische Unternehmer in Berlin. Struktur - Wirtschaftskraft - Problemlagen (=Berliner Beiträge zur Integration und Migration), Berlin.

Sen, Faruk/Sauer, Martina/Halm, Dirk 2001: Intergeneratives Verhalten und (Selbst)Ethnisierung von türkischen Zuwanderern. Gutachten des ZfT für die Unabhängige Kommission "Zuwanderung"

(http://www.bmi.bund.de/Internet/Content/Common/Anlagen/Themen/Zuwanderung/ DatenundFak-

ten/ZfT_pdf,templateId=raw,property=publicationFile.pdf/ZfT_pdf.pdf, 28.11.04), Berlin.

Senatsverwaltung für Bildung, Jugend und Sport Berlin 2003: Bärenstark. Berliner Sprachstandserhebung für künftige Erstklässler. Pressekonferenz 16. Mai 2003 (http://www.efb-berlin.de/download/baerenstark2003 web.pdf), Berlin.

Senatsverwaltung für Bildung, Jugend und Sport Berlin 2006a: Detailergebnisse Deutsch Plus (http://www.berlin.de/sen/bildung/schulqualitaet/lernausgangsuntersuchungen/, 16.01.08), Berlin.

Senatsverwaltung für Bildung, Jugend und Sport Berlin 2006b: Ergebnisse Deutsch Plus 2004-2006 
(http://www.berlin.de/sen/bildung/schulqualitaet/lernausgangsuntersuchungen/) (19.06.07), Berlin.

Senol, Sengül 1992: Kurden in Deutschland. Fremde unter Fremden, Frankfurt am Main.

Shibutani, Tomatsu/Kwan, Kian 1965: Ethnic stratification, New York.

Simon, Patrick 2003: France and the Unknown Second Generation: Preliminary Results on Social Mobility, in: International Migration Review 37: 4, 1091-1119.

Skutnabb-Kangas, Tove/Toukomaa, Pertti 1976: Teaching migrant children's mother tongue and learning the language of the host country in the context of the sociocultural situation of the migrant family (Report written for Unesco, University of Tampere, Department of Sociology and Social Psychology, Research Reports 15), Tampere.

Smith, Robert 1998: Social Location, Generation and Life Course as Social Processes Shaping Second Generation Transnational Life (=Paper presented at Transnationalism and the Second Generation Conference, Harvard University), Harvard University, Cambridge.

Smith, Robert 2003: Diasporic Memberships in Historical Perspective: Comparative Insights from the Mexican, Italian and Polish Cases, in: International Migration Review 37: 3, 724-759.

Solga, Heike 2005: Ohne Abschluss in die Bildungsgesellschaft. Die Erwerbschancen gering qualifizierter Personen aus soziologischer und ökonomischer Perspektive, Opladen.

Stanat, Petra/Christensen, Gayle 2006: Where immigrant students succeed - A comparative review of performance and engagement in PISA 2003, Paris.

Steinbach, Anja 2004: Soziale Distanz. Ethnische Grenzziehung und die Eingliederung von Zuwanderern in Deutschland, Opladen.

Straßburger 2004: Transnational Ties of the Second Generation: Marriages of Turks in Germany, in: Faist, Thomas/Özveren, Eyüp (Hrsg.): Transnational social spaces: agents, networks and institutions, Aldershot, 211-231.

Suarez-Orozco, Marcelo 1987: Towards a psychosocial understanding of Hispanic adaptation to American schooling, in: Trueba, Henry T. (Hrsg.): Success or failure? Learning the languages of minority students, New York, 156-168.

Sullivan, Mercer 1989: 'Getting Paid': Youth Crime and Work in the Inner City, Ithaca.

Sunier, Thijl 2002a: Argumentationsstrategien, in: Schiffauer, Werner/Baumann, Gerd/Kastoryano, Riva/Vertovec, Steven (Hrsg.): Staat-Schule-Ethnizität. Politische Sozialisation von Immigrantenkindern in vier europäischen Ländern., Münster, New York, München, Berlin, 221-253. 
Sunier, Thijl 2002b: Landessprache und Muttersprache, in: Schiffauer, Werner/Baumann, Gerd/Kastoryano, Riva/Vertovec, Steven (Hrsg.): Staat-SchuleEthnizität. Politische Sozialisation von Immigrantenkindern in vier europäischen Ländern., Münster, New York, München, Berlin, 141-160.

Swidler, Anne 1986: Culture in Action: Symbols in Strategies, in: American Sociological Review 51: 2, 273-286.

Szydlik, Marc 1996: Ethnische Ungleichheit auf dem deutschen Arbeitsmarkt, in: Kölner Zeitschrift für Soziologie und Sozialpsychologie 48: 4, 658-676.

Tajfel, Henri (Hrsg.) 1982: Social identity and intergroup relations, 1982, Cambridge, New York.

Teckenberg, Wolfgang 1999: Sozialstruktur und Partnerwahl, Opladen.

Terkessidis, Mark 2004: Die Banalität des Rassismus. Migranten der zweiten Generation entwickeln eine neue Perspektive, Bielefeld.

Terwey, Michael/Scheuer, Angelika 2007: Etwas mehr Anpassung gewünscht, in: Informationsdienst Soziale Indikatoren 38: 12-14.

Thränhardt, Dietrich/Böcker, Anita 2003: Einbürgerung und Mehrstaatigkeit in Deutschland und den Niederlanden, in: Thränhardt, Dietrich/Hunger, Uwe (Hrsg.): Migration im Spannungsfeld von Globalisierung und Nationalstaat (=Leviathan Sonderheft 22/2003), Wiesbaden, 117-134.

Tietze, Nikola 2006: Ausgrenzung als Erfahrung. Islamisierung des Selbst als Sinnkonstruktion in der Prekarität, in: Bude, Heinz/Willisch, Andreas (Hrsg.): Das Problem der Exklusion. Ausgegrenzte, Entbehrliche, Überflüssige, Hamburg, 147-173.

Tucci, Ingrid 2005: Armutsentwicklung und Arbeitsmarktlage von Migranten und Migrantinnen, in: Migration und Soziale Arbeit 27: 3/4, 184-193.

Ulrich, Joachim Gerd 2006: Aktuelle Ausbildungschancen von Jugendlichen mit Migrationshintergrund (6. Fachtagung Berufsorientierung - Berufsvorbereitung - Berufsausbildung, http://www.swaprogramm.de/tagungen/hamburg/folien ulrich ag2.pdf, 31.07.06), Wiesbaden.

Ulrich, Joachim Gerd/Granato, Mona 2006: "Also, was soll ich noch machen, damit die mich nehmen?". Jugendliche mit Migrationshintergrund und ihre Ausbildungschancen, in: FES (Hrsg.): Kompetenzen stärken, Qualifikationen verbessern, Potenziale nutzen. Berufliche Bildung von Jugendlichen und Erwachsenen mit Migrationshintergrund (=Dokumentation einer Fachkonferenz der Friedrich-Ebert-Stiftung und des Bundesinstituts für Berufsbildung), Bonn, 30-50.

Unabhängige Kommission, "Zuwanderung" 2001: Zuwanderung gestalten, Integration fördern. Bericht der unabhängigen Kommission "Zuwanderung", Berlin. 
UNESCO 2000: International Standard Classification of Education. ISCED 1997, Paris.

Vasquez, Manuel/Gomez, Ileana 2001: Saving Souls Transnationally: Pentecostalism and Gangs in El Salvador and the United States, in: Vasquez, Manuel/Marquardt, Marie Friedmann (Hrsg.): Globalizing the Sacred. Religion across the Americas, New Brundswick, New Jersey, London, 119-144.

Vertovec, Steven 1999: Conceiving and researching transnationalism, in: Ethnic and Racial Studies 22: 2, 447-462.

Vertovec, Steven 2007: Super-diversity and its implications, in: Ethnic and Racial Studies 29: 6, 1024-54.

von Below, Susanne 2003: Schulische Bildung, berufliche Ausbildung und Erwerbstätigkeit junger Migranten. Ergebnisse des Integrationssurveys des BiB, Wiesbaden.

von Loeffelholz, Hans Dietrich/Hernold, Peter 2001: Berufliche Integration von Zuwanderern (=Gutachten im Auftrag der Unabhängigen Kommission "Zuwanderung" beim Bundesminister des Innern; http://www.bmi.bund.de/cln_007/nn_165090/Internet/Content/Common/Anlagen/Def ault/RWI_pdf,templateId=raw,property=publicationFile.pdf/RWI_pdf, 28.11.2004), Essen.

Wachendorfer, Ursula 2001: Weiß-Sein in Deutschland. Zur Unsichtbarkeit einer herrschenden Normalität, in: Arndt, Susan (Hrsg.): AfrikaBilder. Studien zu Rassismus in Deutschland, Münster, 87-101.

Wacquant, Loic J.D. 2006: Parias Urbains. Ghetto, Banlieue, Etat, Paris.

Wacquant, Loic J.D./Wilson, William Julius 1989: The Cost of Racial and Class Exclusion in the Inner City, in: Annals of the American Academy of Political and Social Scienes 501: January, 8-25.

Wagner, Ulrich/Van Dick, R./Petzel, R./Auernheimer, G. 2001: Der Umgang von Lehrerinnen und Lehrern mit interkulturellen Konflikten, in: Auernheimer, G./Van Dick, R./Wagner, U./Petzel, R. (Hrsg.): Interkulturalität im Arbeitsfeld Schule, Opladen, 17-40.

Wakeman, Frederic 1988: Transnational and comparative research, in: Items 42: 4, 85-88.

Waldinger, Roger/Feliciano, Cynthia 2004: Will the new second generation experience 'downward assimilation'? Segmented assimilation re-assessed, in: Ethnic and Racial Studies 27: 3, 376-402.

Waldinger, Roger/Fitzgerald, David 2004: Transnationalism in Question, in: American Journal of Sociology 109: 5, 1177-1195.

Warner, W. Lloyd/Srole, Leo 1945: Social Systems of American Ethnic Groups, New Haven. 
Warren, Mark R./Thompson, J. Phillip/Saegert, Susan 2002: The Role of Social Capital in Combating Poverty, in: Saegert, Susan/Thompson, J. Phillip/Warren, Mark R. (Hrsg.): Social Capital and Poor Communities, New York, 1-29.

Waters, Mary C. 1996: Ethnic and Racial Identities of Second-Generation Black Immigrants in New York City, in: Portes, Alejandro (Hrsg.): The New Second Generation, New York, 171-196.

Waters, Mary C. 1999: Black Identities. West Indian Immigrant Dreams and American Realities, New York.

Weber, Max 1985: Wirtschaft und Gesellschaft. Grundriß der verstehenden Soziologie (=5. revidierte Auflage), Tübingen.

Wegener, Bernd 1988: Kritik des Prestiges, Opladen.

Weidacher, Alois (Hrsg.) 2000: In Deutschland zu Hause. Politische Orientierungen griechischer, italienischer, türkischer und deutscher junger Erwachsener im Vergleich. DJI-Ausländersurvey, 2000, Opladen.

Weiß, Anja 2001: Rassismus wider Willen. Ein anderer Blick auf eine Struktur sozialer Ungleichheit, Opladen.

Weißhaupt, Horst 1996: Innerstädtische Disparitäten des Schulbesuchs. Ein Forschungsüberblick, in: Die Deutsche Schule 88: 1, 56-65.

Wendt, Alexander 1995: Constructing International Politics, in: International Security 20: $1,71-81$.

Wiley, Norbert F. 1967: Ethnic Mobility Trap, in: Social Problems 605: 2, 147-159.

Wilpert, Czarina 1980: Die Zukunft der Zweiten Generation. Erwartungen und Verhaltensmöglichkeiten ausländischer Kinder, Königstein.

Wilson, William Julius 1980: The declining significance of race. Blacks and Changing American Institutions, Chicago and London.

Wilson, William Julius 1987: The Truly Disadvantaged. The Inner City, the Underclass, and Public Policy, Chicago and London.

Wilson, William Julius 1996: When Work Disappears: The World of the New Urban Poor, New York.

Worbs, Susanne 2003: The Second Generation in Germany: Between School and Labor Market, in: International Migration Review 37: 4, 1011-1038.

Worbs, Susanne/Heckmann, Friedrich 2003: Islam in Deutschland: Aufarbeitung des gegenwärtigen Forschungsstandes und Auswertung eines Datensatzes zur zweiten Migrantengeneration, in: BMI (Hrsg.): Islamismus - Texte zur Inneren Sicherheit, Berlin, 133-220. 
ZfT 2003: Die Wirtschaftskraft der türkischen Selbständigen in Deutschland, NRW und der Europäischen Union, Essen.

Zhou, Min 2004: Revisiting Ethnic Entrepreneurship: Convergencies, Controversies, and Conceptual Advancements, in: International Migration Review 38: 3, 1040-1075.

Zhou, Min/Logan, John R. 1989: Returns on Human Capital in Ethnic Enclaves, in: American Sociological Review 54: 809-820.

Zimmermann, Klaus F./Hinte, Holger 2004: Zuwanderung und Arbeitsmarkt. Deutschland und Dänemark im Vergleich, Berlin.

Zolberg, Aristide R. 1989: The Next Waves: Immigration Theory for a Changing World, in: International Migration Review 20: 151-169, 151-169.

Zolberg, Aristide R./Woon, Long Litt 1999: Why Islam Is Like Spanish: Cultural Incorporation in Europe and the United States, in: Politics and Society 27: March, 5-38. 


\section{Weitere Verzeichnisse}

\section{Abkürzungsverzeichnis}

$\begin{array}{ll}\text { ABW } & \text { Abwärts-Integration } \\ \text { AFRI } & \text { Afrikanische Herkunftsgruppe } \\ \text { ALLBUS } & \text { Allgemeine Bevölkerungsumfrage } \\ \text { ASIA } & \text { Asiatische Herkunftsgruppe } \\ \text { AUSL } & \text { Ausländische Staatsangehörige } \\ \text { AUSS } & \text { Herkunftsgruppe der AussiedlerInnen } \\ \text { BMBF } & \text { Bundesministerium für Bildung und Forschung } \\ \text { BMAS } & \text { Bundesministerium für Arbeit und Soziales } \\ \text { DESI } & \text { Deutsch Englisch Schülerleistung International } \\ \text { DEUT } & \text { Deutsche Staatsangehörige } \\ \text { DJI } & \text { Deutsches Jugendinstitut, Ausländersurvey } \\ \text { DTOM } & \text { Deutsche ohne Migrationshintergrund } \\ \text { EFFNATIS } & \text { Effectiveness of National Integration Strategies Towards Sec- } \\ & \text { ond Generation Migrant Youth in a Comparative European Per- } \\ \text { EU } & \text { spective } \\ \text { EX-JUG } & \text { Europäische Kommission } \\ \text { GRIE } & \text { Ex-Jugoslawische Herkunftsgruppe } \\ \text { ILO } & \text { Griechische Herkunftsgruppe } \\ \text { ISCED } & \text { International Labour Organisation } \\ \text { ISEI } & \text { International Standard Classification of Education } \\ \text { ITAL } & \text { International Socio-Economic Index of Occupational Status } \\ \text { MPS } & \text { Italienische Herkunftsgruppe } \\ \text { MZ } & \text { Magnitude-Prestigeskala } \\ \text { NEO } & \text { Mikrozensus } \\ \text { O-EUR } & \text { Neoklassisches Muster } \\ \text { PISA } & \text { Osteuropäische Herkunftsgruppe } \\ \text { PLU } & \text { Programme for International Student Assessment } \\ \text { PORT } & \text { Pluralistisches Muster } \\ \text { SEG } & \text { Portugiesische Herkunftsgruppe } \\ \text { SOEP } & \text { Muster der Segregation } \\ \text { SPAN } & \text { Sozio-Oekonomische Panel } \\ \text { TIES } & \text { Spanische Herkunftsgruppe } \\ \text { TÜRK } & \text { The Integration of the European Second Generation } \\ \text { ZUMA } & \text { Zürische Herkunftsgruppe } \\ & \end{array}$




\section{Abbildungsverzeichnis}

ABBILDUNG 1 - VIER-FELDER-MATRIX …................................................................................ 58

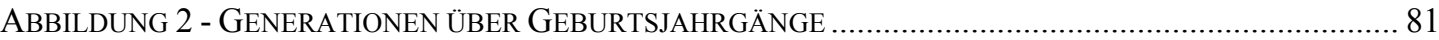

ABBILDUNG 3 - ZWEITE GENERATION NACH HERKUNFTSGRUPPEN ...................................................... 82

ABBILDUNG 4 - STAATSBÜRGERSCHAFT: GENERATIONEN UND HERKUNFTSGRUPPEN............................ 83

ABBILDUNG 5 - FÖRDERBEDARF IM DEUTSCHEN: PUNKTWERTE DER KINDER DEUTSCHER

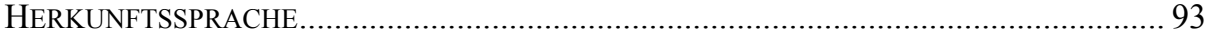

ABBILDUNG 6 - FÖRDERBEDARF IM DEUTSCHEN: PUNKTWERTE DER KINDER NICHT-DEUTSCHER

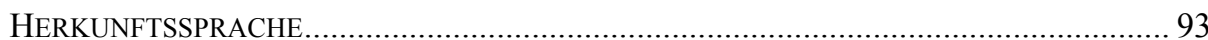

ABBILDUNG 7 - ZWEISPRACHIGKEIT: KONTROLLÜBERZEUGUNG ...................................................... 99

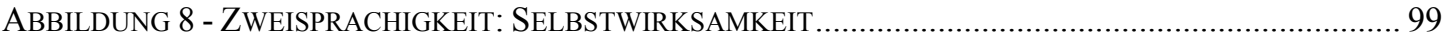

ABBILDUNG 9 - ZWEISPRACHIGKEIT: BENACHTEILIGUNG AUFGRUND NATIONALITÄT .......................... 99

ABBILDUNG 10 - ZWEISPRACHIGKEIT: WICHTIGKEIT VON ELTERN UND GESCHWISTERN.......................99

ABBILDUNG 11 - ZWEISPRACHIGKEIT: BILDUNGSABSCHLÜSSE ...................................................... 99

ABBILDUNG 12 - FREUNDINNEN DER GLEICHEN HERKUNFTSGRUPPE .......................................... 152

ABBILDUNG 13 - MARGINALISIERUNG ................................................................................. 153

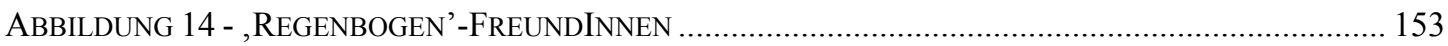

ABBILDUNG 15 - MUSTERANALYSE: GESCHLECHTERVERHÄLTNIS ............................................. 170

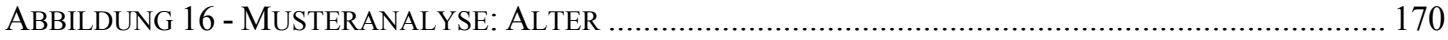

ABBILDUNG 17- MuSTERANALYSE: STADT-LAND-VERTEILUNG, MZ .............................................. 171

ABBILDUNG 18 - MUSTERANALYSE: STADT-LAND-VERTEILUNG, DJI-AUSLÄNDERSURVEY................. 171

ABBILDUNG 19 - MUSTERANALYSE: STAATSBÜRGERSCHAFTEN NUR FÜR TÜRKISCHE HERKUNFTSGRUPPE

ABBILDUNG 20 - MUSTERANALYSE: HAUSHALTSMITGLIEDER UND KINDER IM HAUSHALT ................... 173

ABBILDUNG 21 - MUSTERANALYSE: SPRACHPRAXIS DURCH UNTERHALTUNGSSENDUNGEN................. 174

ABBILDUNG 22 - MUSTERANALYSE: SCHRIFTLICHE KENNTNISSE IM DEUTSCHEN ............................ 174

ABBILDUNG 23 - MUSTERANALYSE: SCHRIFTLICHE KENNTNISSE IN HERKUNFTSSPRACHE .................. 174

ABBILDUNG 24 - MUSTERANALYSE: VerHÄLtNis DER BILDUNG VON DEN KINDERN ZUM VATER ....... 175

ABBILDUNG 25 - MUSTERANALYSE: SCHULJAHRE DES VATERS ........................................................ 176

ABBILDUNG 26 - MUSTERANALYSE: BERUfLICHE PoSITION .......................................................... 176

ABBILDUNG 27 - MUSTERANALYSE: BESCHÄFTIGUNG IM ÖFFENTLICHEN DiENST ............................. 176

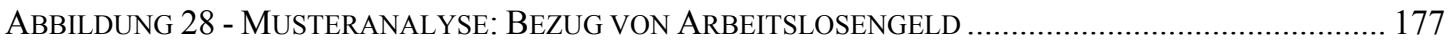

ABBILDUNG 29 - MUSTERANALYSE: LÄNGE DER ARBEITSLOSIGKEIT ............................................... 177

ABBILDUNG 30 - MuSTERANALYSE: SELBSTSTÄNDIGE UND MitHELFENDE FAMILIENANGEHÖRIGE..... 178

ABBILDUNG 31 - MuSTERANALYSE: ARBEITSSUCHE ÜBER PERSÖNLICHE BEZIEHUNGEN..................... 178

ABBILDUNG 32 - MUSTERANALYSE: PARTNERSCHAFT VON ELTERN GEMANAGT ................................ 180

ABBILDUNG 33 - MUSTERANALYSE: FREUNDINNNEN AUS GLEICHER HERKUNFTSGRUPPE................... 180

ABBILDUNG 34 - MUSTERANALYSE: ,REGENBOGEN'-FREUNDSCHAFTEN.......................................... 180

ABBILDUNG 35 - MUSTERANALYSE: MITGLIEDSCHAFTEN IN ,DEUTSCHEN’ VEREINEN ....................... 180

ABBILDUNG 36 - MUSTERANALYSE: SELBSTWIRKSAMKEIT ............................................................ 180

ABBILDUNG 37 - MuSTERANALYSE: PARTNERIN AUS REgION DER ELTERN....................................... 181

ABBILDUNG 38 - MUSTERANALYSE: LEISTUNGSMOTIVATION ....................................................... 182

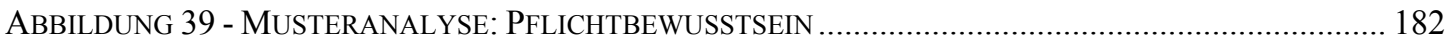

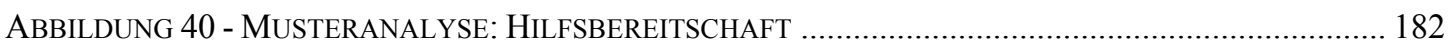

ABBILDUNG 41 - MuSTERANALYSE: WiChTIGKEIT VON SCHULE UND BERUFSAUSBILDUNG................. 184

ABBILDUNG 42 - MUSTERANALYSE: WiCHTIGKEIT VON ELTERN UND GESCHWISTERN........................ 184

ABBILDUNG 43 - MUSTERANALYSE: LEBENSJAHRE IM HERKUNFTSLAND DER ELTERN........................ 184

ABBILDUNG 44 - MUSTERANALYSE: HEIMATGEFÜHL IM HERKUNFTSLAND DER ELTERN...................... 184

ABBILDUNG 45 - MUSTERANALYSE: WAHRNEHMUNG VON DISKRIMINIERUNG................................. 184

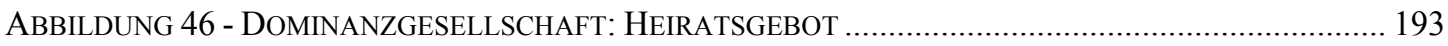

ABBILDUNG 47 - DOMINANZGESELLSCHAFT: KONTAKT .................................................................. 193

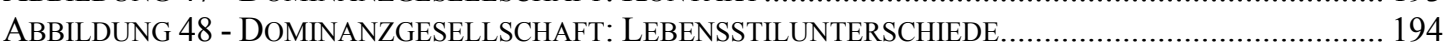

ABBILDUNG 49 - DOMINANZGESELLSCHAFT: EINSTELLUNG ZU EINHEIRAT ...................................... 195

ABBILDUNG 50 - DOMINANZGESELLSCHAFT: EINSTELLUNG ZUR GLEICHBERECHTIGUNG.................... 196

ABBILDUNG 51 - DOMINANZGESELLSCHAFT: BEREICHERUNG DER KULTUR IN DEUTSCHLAND ............ 196

ABBILDUNG 52 - DOMINANZGESELLSCHAFT: KRITERIEN FÜR EINBÜRGERUNG ................................... 197

ABBILDUNG 53 - DOMINANZGESELLSCHAFT: LEBENSSTILANPASSUNG …......................................... 199

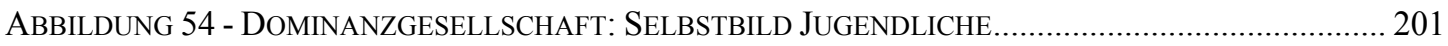




\section{Tabellenverzeichnis}

TABELLE 1 - PROGNOSTIZIERTE INTEGRATIONSMUSTER ......................................................................62

TABELLE 2 - MigRANTINNEN: GENERATIONEN UND HERKUNFTSGRUPPEN ................................................76

TABELLE 3 - FALLZAHLEN IM DJI-AUSLÄNDERSURVEY NACH GENERATIONEN UND

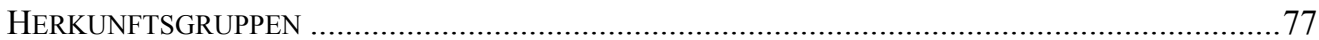

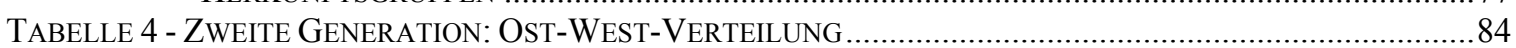

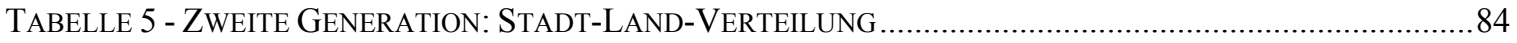

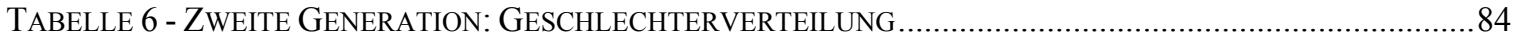

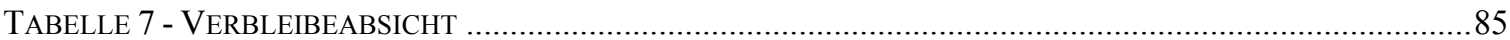

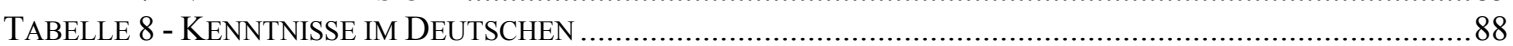

TABELLE 9 - FÖRDERBEDARF IM DEUTSCHEN: STADTVIERTEL UND KITABESUCH …...............................91

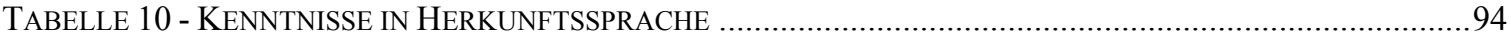

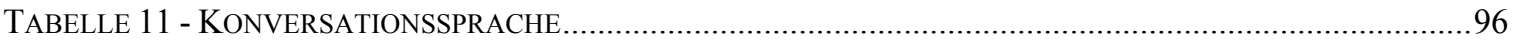

TABELLE 12 - AUSWIRKUNGEN ZWEISPRACHIGKEIT AUF BILDUNGSABSCHLUSS (ISCED) ............................100

TABELLE 13 - BILDUNGSABSCHLUSS (ISCED) : GENERATIONEN, HERKUNFTSGRUPPEN, GESCHLECHTER ... 105

TABELLE 14 - VERGLEICH BILDUNGSABSCHLUSS (ISCED) IM MZ UND DJI-AUSLÄNDERSURVEY...............106

TABELlE 15 - BILDUNGSABSCHLUSS (ISCED): GENERATIONEN, HERKUNFTSGRUPPEN,

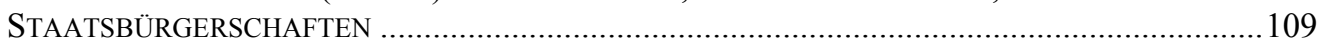

TABELLE 16 - GENERATIONEN AUF PISA MATHEMATIK- UND LESESKALA ..............................................111

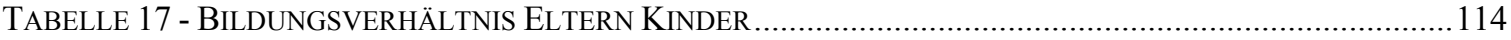

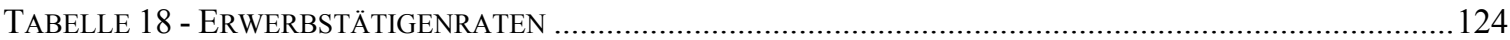

TABELlE 19 - BERUfSPOSITION (BLOSSFELD): GENERATIONEN, HERKUNFTSGRUPPEN, GESCHLECHTER......128

TABelle 20 - Berufsposition (Angestelltenstatus): Generationen, Herkunftsgruppen, GESCHLECHTER

TABelle 21 - Berufsposition (Blossfeld): Generationen, HerkunftSGRUPPEN, STAATSBÜRGERSCHAFTEN ....

TABElle 22 - BerufsStATUS (MPS): GENERATIONEN, HERKUNFTSGRUPPEN,

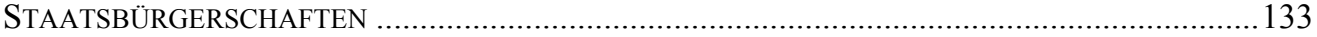

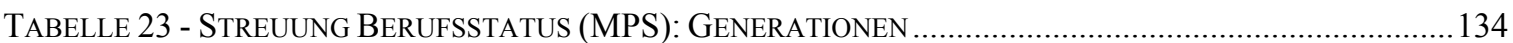

TABELLE 24 - STREUUNG BERUFSSTATUS (MPS): HERKUNFTSGRUPPEN UND GENERATIONEN.....................135

TABELLE 25 - AUSWIRKUNG ,ETHNISCHE ÖKONOMIE’ AUF EINKOMMEN, MZ ........................................140

TABELLE 26 - AUSWIRKUNG ,ETHNISCHE ÖKONOMIE’ AUF EINKOMMEN, DJI-AUSLÄNDERSURVEY .............141

TABELLE 27 - FALLZAHLEN PARTNERIN IM HAUSHALT ....................................................................... 144

TABELLE 28 - SOZIO-ÖKONOMISCHE UNTERSCHIEDE FÜR MIGRANTINNEN MIT UND OHNE PARTNERIN IM HAUSHALT

TABELLE 29 - BILDUNGSABSCHLUSS (ISCED): PARTNERSCHAFTSSTATUS.............................................. 146

TABELLE 30 - BEREITSCHAFT ZUR EHE MIT DEUTSCHEM/R PARTNERIN OHNE

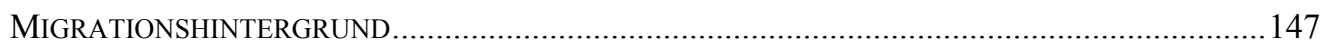

TABELLE 31 - HERKUNFT UND GENERATIONSSTATUS DER/S PARTNERIN, MZ .......................................... 148

TABELlE 32 - HERKUNFT UND GENERATIONSSTATUS DER/S PARTNERIN, DJI-AUSLÄNDERSURVEY .............150

TABELLE 33 - BILDUNGSABSCHLUSS (ISCED) PARTNERIN GLEICHE HERKUNFTSGRUPPE ..........................151

TABELLE 34 - AUSWIRKUNGEN ANTEIL FREUNDINNEN DER GLEICHEN HERKUNFTSGRUPPE AUF

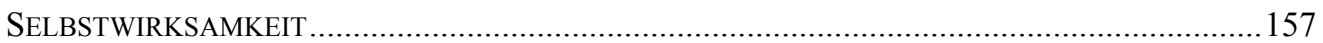

TABELlE 35 - AuSWIRKUNGEN SELBSTWIRKSAMKEIT AUF BILDUNGSABSCHLUSS (ISCED)......................157

TABELLE 36 - AUSWIRKUNGEN ANTEIL FREUNDINNEN DER GLEICHEN HERKUNFTSGRUPPE AUF

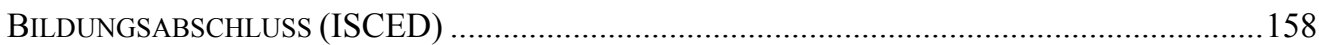

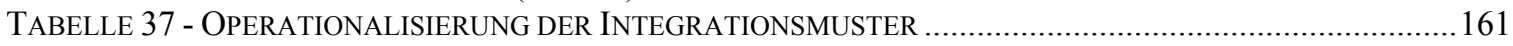

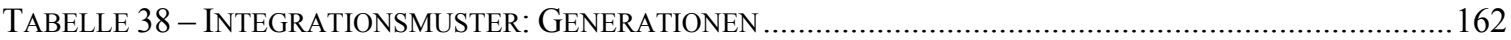

TABELLE 39 - INTEGRATIONSMUSTER: GENERATIONEN, HERKUNFTSGRUPPEN, GESCHLECHTER .................163

TABELLE 40 - INTEGRATIONSMUSTER MIT KRITERIUM PARTNERSCHAFT UND SPRACHE ..............................165

TABELLE 41 - DOMINANZGESELLSCHAFT: EINSCHÄTZUNG WAHRSCHEINLICHKEIT VON

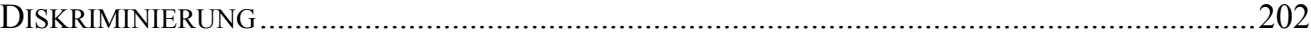

TABELLE 42 - DOMINANZGESELLSCHAFT: EIGENE EINSTELLUNG ZU DISKRIMINIERENDEN AUSSAGEN........203

TABELlE 43 - DOMINANZGESELLSCHAFT: EINSCHÄTZUNG ALLGEMEINE MEINUNG ZU DISKRIMINIERENDEN AUSSAGEN 